
1C. Cox Charleston, levva.

1943

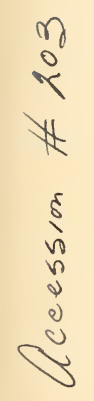





\title{
A Symposium on HUMAN MALARIA \\ With Special Reference to North America and the Caribbean Region
}

Publication of the American Association for the Advancement of Science

No. 15

\author{
Publication Committee \\ MARK F. BOYD, Chairman \\ MALCOLM H. SOULE, Secretary \\ L. T. COGGESHALL \\ CHARLES F. CRAIG \\ W. H. TALIAFERRO \\ L. L. WILLIAMS, JR.
}

Edited by

FOREST RAY MOULTON

American Association for the Advancement of Science,

Smithsonian Institution Building, Washington, D. C. 
Copyright, 1941, by THE AMERICAN ASSOCIATION FOR THE ADVANCEMENT OB SCIRNCE Reprinted June, 1942 


\section{FOREWORD}

This volume on Human Malaria is the eighth symposium in the field of public health published by the American Association for the Advancement of Science. The program for this symposium was organized by a joint committee from the Section on Medical Sciences of the Association, the American Society of Parasitologists, the American Society of Tropical Medicine and the National Malaria Committee. The organizing committee invited the contributions and made editorial revisions of the papers after they were presented at the Philadelphia meeting of the Association Dec. 30, 1940-Jan. 1, 1941 . As the volume now appears, it presents a systematic, comprehensive, authoritative and thoroughly documented discussion of the problems of human malaria in North America and the Caribbean region.

Although malaria has been known from antiquity and is believed to have had important effects on history, its cause and the mode of its transmission remained quite unknown until about fifty years ago. The discovery of the causative parasites in the blood of persons suffering from the disease was followed in a few years by proofs that the organisms live a part of their life cycle in Anopheles mosquitoes which transmit them to human beings. Immediately after these discoveries several great fields were opened for investigation-that of the distribution, ecology and habits of many species and subspecies of anopheline mosquitoes; that of the morphology, life cycle and physiology of several species of plasmodia; that of the nature and effects of natural and synthetic drugs in curing or preventing the disease; that of determining the seriousness of malaria as a public health, social and economic problem; and that of devising scientific locally coopera- tive, governmental, eqonomically feasible and otherwise workable methods of controlling the disease. These and related subjects are treated in this volume.

It should not be assumed that the answers to all the questions considered in this book are known. If they were it might be less valuable than it is. It comes after an enormous amount of work on malaria has been done and when the avenues to future progress in its study appear to be pretty well understood. One of its purposes is to point out these promising avenues, as well as to present the conclusions that are well established. It points out, too, many almost unexplored fields, such as the production of culture media in which to grow and study the parasites, the development of vaccines and the synthesis of prophylactic drugs.

This volume is especially timely because of the new naval bases being constructed in lower latitudes by the United States Government, and of the numerous military and aviation training camps being established in the southern states where malaria is prevalent. The Association and the cooperating societies sincerely thank the participants in this symposium for their contribution to it and to national defense. The phrase "national defense" in this statement does not apply only to present international problems, but to the continuing problem of defense against one of the most serious of the diseases that afflict mankind. The Association is especially grateful to the organizing committee (The Publication Committee) whose names appear on the title page, for without their guidance and individual papers it could not have made available this contribution to the advancement of science.

\section{F. R. Moulton}





\section{LIST OF CONTRIBUTORS}

Justin Meredith ANDREws, Sc.D.

Director, Division of Malaria and Hookworm Service, Georgia Department of Public Health, Atlanta, Ga.

Marshatl A. Barber, A.M., Ph.D.

U. S. Public Health Service, Memphis, Tenn.

MARK F. BOYD, M.D., M.S., C.P.H.

Staff Member, International Health Division, The Rockefeller Foundation, Director, Station for Malaria Research, Tallahassee, Fla.

George Hirst Bradley, B.S.

Entomologist, U. S. Department of Agrieulture, Bureau of Entomology and Plant Quarantine, Orlando, Fla.

Paul Roberts Cannon, Ph.D., M.D.

Professor of Pathology, The University of Chicago, Chicago, Ill.

Herbert Charles Clark, M.D.

Director, Gorgas Memorial Laboratory, Apartado 1252, Panama, R. P.

G. Robert Coatnex, M.A., Ph.D.

Associate Protozoologist, U. S. Public Health Service, Columbia, S. Car.

Lowelu T. Coggeshall, A.M., M.D.

Staff Member, International Health Division, The Rockefeller Foundation, New York, N. Y.

Charles Franklin Craig, M.D., M.A., D.S.M.

Colonel, U. S. Army, Retired; Emeritus Professor of Tropical Medicine, Medical School, The Tulane University of Louisiana; San Antonio, Texas

John Edward Elmendorf, JR., M.D.

Staff Member, International Health Division, The Rockefeller Foundation, Escambia County Health Department, Pensacola, Fla.

Ernest Carroll Faust, M.A., Ph.D.

Professor of Parasitology, Department of Tropical Medicine, The Tulane University of Louisiana, New Orleans, La.

Joseph M. Ginsburg, M.S., Ph.D.

Biochemist and Associate Professor of Entomology, Rutgers University, New Jersey Agricultural

Experiment Station, New Brunswick, N. J.
Lewis Wendell Hackett, M.D., Dr.P.H. Assistant Director, International Health Division, The Rockefeller Foundation, New York, N. Y. (Buenos Aires, Argentina)

Redginat Hewitt, M.S., D.Sc.

Junior Malariologist, Health and Safety Department, Tennessee Valley Authority, Wilson Dam, Ala.

E. Harold Hinman, M.S., Ph.D., M.D.

Senior Biologist, Health and Safety Department, Tennessee Valley Authority, Wilson Dam, Ala.

Clay G. Huff, Sc.D.

Professor of Parasitology and Secretary of the Department of Bacteriology and Parasitology, The University of Chicago, Chicago, Ill.

Danter M. Jobbins, M.Sc.

Senior Medical Entomologist, Gorgas Memorial Laboratory, Apartado 1252, Panama, R. P.

H. A. Johnson, B.S.

Sanitary Engineer and Executive Officer, Office of Malaria Investigations, U. S. Public Health Service, Washington, D. C.

\section{Calvin C. KiKer}

Sanitary Engineer, Health and Safety Department, Tennessee Valley Authority, Wilson Dam, Ala.

Whllard V. King, Ph.D.

Senior Entomologist, U. S. Department of Agriculture, Bureau of Entomology and Plant Quarantine, Orlando, Fla.

Stuart Fordyce Kitchen, M.D.

Staff Member, International Health Division, The Rockefeller Foundation, Tallahassee, Fla.

WILLIAM H. W. Komp, M.S.

Senior Medical Entomologist, U. S. Public Health Service, Gorgas Memorial Laboratory, Panama, R. P.

H. W. Kumm, M.D.

Staff Member, International Health Division, The Rockefeller Foundation, New York, N. Y. (San Salvador, El Salvador, C. A.)

LucILE LOGaN

Microscopist, Station for Malaria Research, Tallahassee, Fla. 
John MaIER, M.D.

Staff Member, International Health Division, The Rockefeller Foundation, New York, N. Y.

Reginald D. Manweil, A.M., Sc.D.

Professor of Zoology, Syracuse University, Syracuse, N. Y.

Robert Matheson, M.S., Ph.D.

Professor of Entomology, Cornell University, Ithaca, N. Y.

Henry Edmund Meleney, M.D.

Associate Professor of Preventive Medicine and Public Health, Vanderbilt University School of Medicine, Nashville, Tenn.

Hans Molitor, M.D.

Director, Merck Institute for Therapeutic Research, Rahway, N. J.

Erwin Eluis Nerson, Ph.D., M.D.

Professor of Pharmacology, The Tulane University of Louisiana School of Medicine, New Orleans, La.

NeLson H. Rector, B.E.

Assistant State Director of Malaria Control, Mississippi State Board of Health, Jackson, Miss.

Wriltam Brinson Redmond, M.S., Ph.D.

Assistant Professor of Biology, Emory University, Ga.

Lloyd E. Rozeboom, Sc.D.

Associate in Medical Entomology, The Johns Hopkins University School of Hygiene and Public Health, Baltimore, Md.

\section{Whlem Rudolfs, Ph.D.}

Department of Water Supply and Sewage Disposal, Rutgers University, New Jersey Agricultural Experiment Station, New Brunswick, N. J.
PAUl FarR Russerl, M.D., M.P.H.

Staff Member, International Health Division, The

Rockefeller Foundation; Officer-in-Charge, $\mathrm{Ma}$ laria Investigations, Pasteur Institute, Coonoor, Madras, India.

James Stevens Simmons, M.D., Ph.D., Sc.D., D.P.H.

Lieutenant Colonel, U. S. Army Medical Corps; Chief Division of Preventive Medicine, Office of The Surgeon General U. S. Army, Washington, D. C.

Warren Kmwell Stratman-Thomas A.M., Рн.D., M.D., D.T.M. and H.

Malariologist, State Department of Public Health, Nashville, Tenn.

William Hay Taliaferro, Ph.D.

Professor of Parasitology, Chairman Department of Bacteriology and Parasitology, and Dean Division of Biological Sciences, The University of Chicago, Chicago, Ill.

Robert Briggs Watson, M.D., M.P.H.

Chief, Laboratory Services Staff and Senior Malariologist, Health and Safety Department,

Tennessee Valley Authority, Wilson Dam, Ala.

\section{AIMEe Wricox}

Assistant Technologist, Malaria Investigations, U. S. Public Health Service, Memphis, Tenn.

Louis L. Whliams, JR., M.D.

Senior Surgeon, U. S. Public Health Service, National Institute of Health, Bethesda, Md.

Martin D. Young, M.S., Sc.D.

Associate Zoologist, Malaria Research Laboratory, Malaria Investigations, U. S. Public Health Service, Columbia, s. Car. 


\section{TABLE OF CONTENTS}

\section{INTRODUCTION}

Historical Introduction to the Symposium on Malaria. Mark F. Boyd

\section{PARASITOLOGY}

The Distribution of Malaria in North America, Mexico, Central America and the West Indies. ERnest CarROLL FAUST

The Taxonomy of the Human Malaria Parasites with Notes on the Principal American Strains. G. ROBERT Coatney and Martin D. Young ...

The Morphology, Life Cycle and Physiology of Plasmodium malariae (Grassi and Feletti, 1890). MarTin D. Young and G. Robert Coatney......
The Morphology, Life Cycle and Physiology of Plasmodium vivax. Reginald D. Manwell

8 The Morphology, Life Cycle and Physiology of Plasmodium falciparum. S. F. KITCHEN

19 The Detection and Differential Diagnosis of Malarial Parasites in the Schizogonous and Sporogonous Cycles. AImeE Wricox and Lucme LOGAN

\section{ANOPHELINE VECTORS}

General Morphology of Anopheles and Classification of the Nearetic Species. W. V. KING and G. H. BRADLEY

Distribution of the Nearctic Species of Anopheles. W. V. KING and G. H. BRADLEY

Bionomics and Ecology of Nearctic Anopheles. G. H. Bradley and W. V. KING

The Classification and Identification of the Anopheles Mosquitoes of Mexico,
Central America, and the West Indies. W. H. W. KoMP .

63 Distribution and Ecology of the Anopheles Mosquitoes of the Caribbean Region. L. E. RоZевоом

71 Factors Influencing Infection of Anopheles with Malarial Parasites. Clay G. Huff

79 The Transmission of Malaria by the Anopheles Mosquitoes of North America. James Stevens Simmons 113

\section{EPIDEMIOLOGY}

Cyclical Variation in the Incidence of Malaria. Charles F. Craig 131

Topographical and Related Factors in the Epidemiology of Malaria in North America, Central America, and the West Indies. RoBert
Briggs Watson and Redginal HEwITT …......................................................... 135

Malaria and the Community. L. W. HACKETT

The Role of Anophelines in the Epidemiology of Malaria. ROBERT MATHESON

\section{SYMPTOMATOLOGY}

The Infection in the Intermediate Host: Symptomatology, General Considerations. Mark F. BoYd ........ 163

The Infection in the Intermediate Host: Symptomatology, Vivaz Malaria. WARREN K. StratmanTHOMAS 183

The Infection in the Intermediate

Host: Symptomatology, Quartan Malaria. S. F. KITCHEN ........................ 190 Infection in the Intermediate Host: Symptomatology, Falciparum Malaria. S. F. KITCHEN .............................. 196 The Infection in the Intermediate Host: Blackwater Fever. S. F. KITCHeN 208 


\section{PATHOLOGY AND IMMUNITY}

Some Pathologic Aspects of Human Malaria. PAUL R. CANNON

The Physiological Pathology of Malaria. Henry E. MELENEY

Immunity to Human Malaria: Char- acteristies of Immunity. W. B. REDMOND .......................................................... 231

The Cellular Basis for Immunity in Malaria. WILLIam H. TAliaferro..... 239 Humoral Immunity in Malaria. L. T. CoggeshaLL

\section{TREATMENT}

Cinchona and Its Alkaloids in the Treatment of Malaria. ERwIN E. NeLSON

Antimalarials Other Than Quinine. HANS MoLITOR

Experimental Chemotherapy in Malaria. JoHN MAIER
A Summary of Ten Years of Observations on Malaria in Panama with 255 Reference to Control with Quinine, Atabrine, and Plasmochin, Without Anti-Mosquito Measures. HERBERT C. Clark and Whliam H. W. KомP

\section{CONTROL AND ERADICATION}

General Considerations in Planning Malaria Control. Justrin ANDREwS... 285

Malaria Survey-Methods and Procedures. JoHN E. ElmendoRF, JR. 295

Methods Directed Against Adult Mosquitoes in the Control and Eradication of Malaria. DANIEL M. JobBINS 302

Housing with Special Reference to Mosquito-Proofing for Malaria Control. Calvin C. Kiker 308

Drainage and Filling Methods for Mosquito and Malaria Control. Neuson H. RECTOR 315

The Management of Water for Malaria Control. E. Harold Hinman 324

Petroleum Products for Mosquito Con- trol. Josepr M. Ginsburg and WILLEM RUDOLFS 333

Paris Green (Aceto Arsenite of Copper) and Other Stomach Poisons as Larvicides Against Mosquito Larvae. M. A. BARBER

Naturalistic Methods of Malaria Control. Paul F. Russell 347

Adaptability of Control Measures to the Nearctic Fauna of Anopheles Mosquitoes. Henry A. JoHnson ...... 353

The Adaptability of Control Measures to the Malaria Vectors of the Caribbean Region. HeNry W. KumM ..... 359 The Anti-Malaria Program in North America. Louis L. Williams, JR. ...... 365

\section{BIBLIOGRAPHY}




\title{
HISTORICAL INTRODUCTION TO THE SYMPOSIUM ON MALARIA
}

\author{
By MARK F. BOYD \\ INTERNATIONAL HEALTH DIVISION, ROCKEFELLER FOUNDATTON, TALLAHASSEE, FLORIDA
}

\section{Definition}

$I_{N}$ current usage of the past century the word malaria has come to designate any member of a group of chronic infections of vertebrates produced by several species of protozoan parasites belonging to the family Plasmodidae. Parasites of this family are known from a wide variety of hosts, including various species of lizards, birds and mammals. Not to any species included in the last group of hosts are these infections more important than to the human, in which they are the cause of widespread morbidity and mortality. The human infections are attributable to three or more species of these parasites, which for precision should be distinguished by the name of the causative parasite as falciparum malaria, vivax malaria and malariae malaria, although for euphony it is desirable to substitute quartan malaria for the last term.

\section{Derivation}

During most of the past century these diseases were generically known as malarial fevers, the term malaria (Italian, mal aria $=$ bad air), as well as miasma, being used to designate the supposed exciting agent of these as well as other diseases.

In this sense the former word was introduced into English medical literature by Macculloch (1829) who states : "It has long been familiar to physicians that there was produced by wet lands, or by marshes and swamps, a poisonous and acriform substance, the cause, not only of ordinary fevers, but of intermittent; and to this unknown agent of disease the term marsh miasma has been applied. . . . This is the unseen, and still unknown, poison to which Italy applies the term that I have borrowed, Malaria." Perhaps the best, as well as one of the last, expressions of the old viewpoint is afforded by the following quotation from Sternberg (1884): "The various types of intermittent and remittent fever which are cured by quinine are by common consent recognized as due to malarial poisoning, and ... we must insist that the prevalence of periodic fever be taken as the test of the presence of malaria."

\section{SYNONYMY}

The antiquity of the association of the human race with malaria has brought this disease under the observation of countless generations of physicians, many of whom left a record of their astute observations in a rich literature. Unfortunately students of modern medicine are but slightly if at all conversant with the older writers, particularly those preceding Laveran, and thus fail to appreciate the substantial character of the contributions which have come down to us from the past. In extenuation it must be admitted that with the introduction of modern scientific methods to the study of biology, the theory of medicine became revolutionized. These changes find their expression in medical terminology, so that it is often difficult for the physician with modern training to grasp fully the significance of the older writings. A brief consideration of nosological synonymy may therefore be useful.

The different kinds of malarial infection, designated according to the modern etiological classification previously given are more or less closely represented by the following equivalents in the older literature:

Vivax malaria: Tertian fever, benign tertian fever, simple intermittent fever, paroxysmal fever, tertian ague, chills and fever, fever and ague. 
Quartan malaria: Quartan fever, quartan ague, simple intermittent fever, paroxysmal fever, chills and fever.

Falciparum malaria: Tertian fever, estivo-autumnal malaria, malignant tertian fever, remittent fever (including bilious, congestive and malignant types), continued malarial fever, pernicious fever, congestive intermittent fever, pernicious intermittent fever, congestive fever, congestive chills.

The diagnosis of remittent fever has been applied to many a case of typhoid, while yellow fever has masqueraded as bilious remittent fever.

The regularity of the recurring quartan, tertian and quotidian paroxysms described in the Hippocratic writings (Jones 1923) are sufficiently pathognomonic to warrant the identification of quartan malaria, but less clearly distinguish vivax from falciparum malaria. The identity of falciparum malaria did not clearly emerge from the background of other continued fevers until the use of cinchona became general. The tertian similarity of the paroxysms of the last two diseases makes their distinction on clinical grounds alone difficult, as is illustrated by the following fragmentary descriptive quotations from Jackson (1791): "The type was frequently double tertian, or quotidian; the remissions were indistinct; the bilious vomitings and purgings were often excessive, and marks of malignancy appeared in several instances. ... the remissions were generally obscure, but ... the type changed frequently from double to single tertian; at the same time the intermissions became clear and distinct." These words are evidently descriptive of falciparum malaria.

James (1929) has pointed out that the word ague, now a widespread word for malaria particularly among the laity, was not always used in such a limited sense. Derived from febris acuta, it was originally applied to any acute, and most commonly to a continued, fever. Its signifcance must therefore be interpreted with caution:

In addition to the foregoing, the follow- ing broader terms, viz., marsh fever, swamp fever, paludal fever and paludism, may be taken as generic references to malaria. Hemorrhagic malarial fever, bilious fever, melanuric malarial fever, or blackwater fever, is a condition of obscure origin, although generally regarded as a manifestation of malaria infection. According to Stephens (1937), the relative frequency with which it is associated with infections produced by different species of parasites is proportional to the general local distribution of these species.

\section{INFERENTIAL REFERENCES}

In addition to the foregoing terms there are many allusions in the literature of significance in connection with our subject. Principal among these is the stigma or repute of long-continued insalubrity attributed to many places. Although it is probable that in rural regions the presence of malaria alone may be safely inferred from such references, it is likely that in many urban centers the blame was also shared with yellow fever. Similarly the presence of malaria and yellow fever is also inferable from references to acelimation fever, "acclimatization" among, and the "seasoning" of, immigrants. The significance of those expressions is well brought out by the following quotation from Kalm (1770) speaking of "fever and ague" in the southern part of New Jersey : "Strangers who arrive here are commonly attacked by this sickness the first or second year after their arrival, and it acts more violently upon them than upon the natives, so that they sometimes die of it. But if they escape the first time, they have the advantage of not being visited again the next year, or perhaps ever. It is commonly said here that strangers get the fever to accustom them to the climate." Or by a statement from Cumming (1810) : "All newcomers are subject to what is called a seasoning, after which, though they may be annually attacked by this scourge of the climate, it rarely confines them longer than a few days." 


\section{Antiquity of Malaria Infections}

Those who desire to pursue this fascinating subject may find an introduction in Hirsch (1883), in the opening chapter of Ross (1910), in Jones (1909), in Dock (1931) and in Hoops (1934).

\section{Progress in Basic Scientific Knowledee}

Perhaps the earliest verification in the United States of Laveran's discovery of the malarial parasite was effected by Sternberg (1886), who, recently returned from studies under Marchiafava and Celli, demonstrated the parasites to $\mathrm{Dr}$. Wm. H. Welch in the latter's laboratory, in the blood of an active clinical case. It was several years before effective staining techniques were introduced, and in those early years, on the infrequent occasions when microscopical diagnosis was practiced, it was commonly done with fresh blood. Attention was naturally more directed to the large parasites, and the type of preparation employed facilitated exflagellation of the microgametocytes. The nature of the process was not understood until MacCallum (1897) saw a free flagellum enter a quiet spherical form, and recognized the signifcance of the act. Among other early observers of the parasites, Opie, Dock and Thayer should be mentioned.

Long before it became possible to differentiate surely between yellow fever and falciparum malaria (bilious remittent fever) a number of physicians in the Americas had come to suspect that mosquitoes were involved in the transmission of both diseases. Perhaps the first to advance such an idea was Nott (1848), who was followed by Beauperthay (1854). The most practical development of this idea was effected by King (1883), who advanced the following propositions :

(1) The malaria season corresponds to the season of mosquito abundance; (2) malarial country is suitable for mosquito breeding; (3) similar conditions afford protection against malaria and against mosquitoes; (4) exposure to night air means exposure to mosquitoes; (5) soldiers, tramps and fishermen are particularly susceptible to malaria and are especially exposed to mosquitoes at night; (6) turning up the soil or making excavations in previously healthy districts is often followed by malaria; (7) coincidence of malaria and mosquitoes, increase of both in late summer and autumn.

It is likely that the demonstration of the tick transmission of Texas fever of cattle by Smith and Kilborne, in 1893, may have influenced Manson in his inference that malarial gametocytes achieve their destiny in mosquitoes. The first attempt to verify the work of Ross and of Grassi with American anophelines was reported by Thayer (1900), who infected A. quadrimaculatus with $P$. vivax and $P$. falciparum, and in the following year Waldert (1901) infected this species with the latter parasite. However, these American students discontinued their observations as soon as eysts were detected on the stomach walls. The results reported by some subsequent observers were erroneous or inconclusive (Beyer et al., 1902a), and not until the work of King (1916b) was the susceptibility of the three commonest North American anophelines adequately studied.

Probably the earliest work incriminating any Neotropical species was carried out by Darling (1910), as a result of which the importance of $A$. albimanus in malaria transmission in the Caribbean region was ascertained. These studies led to the development of a program in the Canal Zone which concentrated the attack on malaria to this species of anopheline.

Prior to 1900 the anophelines, as well as mosquitoes in general, had received only cursory attention from American entomologists, and it is noteworthy that of the 5 species described from North America and the Caribbean up to that year only two had been described by an American student. Those known at that time were :

$\begin{array}{lll}\text { albimanus } & \text { Wiedeman, } & 1821 \\ \text { punctipennis } & \text { Say, } & 1823 \text { (1819) } \\ \text { quadrimaculatus } & \text { Say, } & 1824 \\ \text { argyritarsis } & \text { Robineau-Desvoidy, } & 1827 \\ \text { crucians } & \text { Wiedeman, } & 1828\end{array}$


Following the incrimination of mosquitoes in the transmission of yellow fever and malaria, a keen demand arose for information relating to the life history of mosquitoes and of measures for their control. In part this was supplied by L. O. Howard (1900) through the publication of Bull. 25, N.S., of the Division of Entomology, which marked the beginning of an extensive literature reporting the results of widespread research.

From these studies it has been found that the various anopheline species have a very diverse ecology, especially exhibiting striking differences in the water collections favorable for breeding, and as a consequence many of the paradoxical differences in the epidemiological picture of malarial incidence in different parts of the world have been explained. These studies emphasize the fact that effective antianopheline work requires an extensive knowledge of the bionomics of local vectors, and that control measures must be adapted to the character of the local problem arising from these peculiarities.

\section{Progress in Treatment and Prevention}

The tremendous importance of the disclosure to European medicine that the bark of a Peruvian tree could cure intermittent fevers should be stressed (Dock 1931). Attention may be called to the study by Paz Soldán (1938), which indicates that the account of this event by Markham (1880) is historically inaccurate. Paz Soldán presents a doçument which was the official day-by-day account of the administration of Don Fernando de Cabrera y Bovadilla, the Count of Chinchón, as viceroy from 1629 to 1639 , during the greater portion of which period he suffered from repeated attacks of recurring fevers. Nothing in the chronicle indicates that his wife, the countess Doña Francisca de Rivera, ever suffered from malaria, although it appears that by the year 1639, the viceroy had regained good health. Tantalizingly, the narrative does not disclose whether he had been given the powders of Loxa bark, which according to the tradition had been sent in 1638 to Don Juan de Vega, his physician in Lima, by Don Juan Lopez de Cannizares. It is alleged that previously, as early as 1630 , Don Juan Lopez himself had been cured of an intermittent fever by the use of the bark.

The extent to which the bark came into general use as a febrifuge in the Americas during the next century and a half is obscure. The buccaneer surgeon, Lionel Wafer (1699), appears to have become familiar with the Peruvian or Jesuits' bark in his cruise down the west coast of South America in 1680-81, as he speaks of observing it brought into Arica, Peru, on mule back for export. He states in the account of his adventures that, "We brought away with us several bundles of this bark, and I found it to be the right sort, by the frequent use I made of it in Virginia and elsewhere." He was in Virginia in 1682-83 and again in 1688-90. Peter Kalm (1770), the celebrated Swedish botanist, traveled extensively in the middle Atlantic colonies in 1748-50 and mentions the use of Jesuits' bark in the treatment of ague. He states that this was formerly a certain remedy, but that at the time of his visit it was not always effective, though genuine and selected. Further on he says that the bark can seldom be secured unadulterated, and describes the manner in which it was effectively used. According to Humboldt (1852), at the end of the 18th century the people of America (South) had a most inveterate prejudice against the employment of the different kinds of cinchona. According to Juan and Ullua (1806), the aversion to the use of the bark in Guayaquil was due to the notion that on account of its hot quality it could have no good effect in that climate. As early as $\mathbf{1 7 7 6}$ the Continental Congress ordered the medical committee to forward 300 pounds of Peruvian bark to the southern department for the use of the troops (Blanton 1931). Jackson (1791), a British army surgeon attached to one of the regiments of Lord Cornwallis' army, made extensive use of Peruvian bark in 
treating the abundant intermittent fevers from which the British army suffered in its southern campaign. At any rate, its use in the United States appears to have become widespread subsequent to the revolution.

Quinine was prepared commercially in Philadelphia as early as 1823, three years after its isolation (Hogstad 1931). The earliest report of its employment in the United States that has come to our attention is by Henry Perrine (1826), a physician of Natchez, Miss. He employed from 6 to 12 grains every 2 to 3 hours at any period of the fever, continued until its symptoms in pulse and skin were subdued, repeating if the return of the fever was suggested. Editorial comment in 1850 (Fenner 1850), eredits Perrine and other Natchez physicians with the use of quinine as early as 1823. MeGown (1849) states that Dr. Thomas Fearn of Huntsville, Ala., employed quinine during the epidemic of fatal fevers which prevailed at that place during 1824 and 1825, and gives him the credit as the first to use large doses of this alkaloid. Although the use of the alkaloid for the treatment of intermittents appears to have become quite general during the next two decades, some physicians were reluctant to employ it in remittent fevers. However, by this time daily doses of from 15 to 30 grains, given during the remission in the former, were regarded as a desirable practice.

Quinine soon became an ingredient of proprietary fever remedies, one of the earliest of which, Dr. Sappington's "AntiFever Pills," was extensively sold throughout the Mississippi Valley and the Southwest during the 30 's and 40 's (Terry 1931).

According to Terry, Dr. Sappington, as early as 1844, advised the prophylactic use of quinine. This possibility, however, did not appear to attract much attention until the decade immediately before the Civil War. Merritt (1861) claims that while surgeon to a mining company in Panama in 1850 he obliged all employees to take a daily dose of 5 grains before breakfast. The crews of the vessels operated by the Panama Railway Company were required in $\mathbf{1 8 5 3}$ to take quinine while in the port of Aspinwall (Anon. 1861). De Saussure (1860) reported its successful employment as a prophylaxis among the negro slaves engaged in the construction of the Charleston and Savannah Railway. It was extensively, although not systematically, employed for this purpose during the Civil War.

Mass treatment of infected persons as a public health measure was attempted in a large area in the Mississippi delta by Bass from 1916 to 1918 . From the experience gained in 1916-17, a regimen of quinine administration was developed which became widely known as the "Standard Treatment." This was gratuitously distributed to infected persons in the study area in 1918 when 40 per cent of the population experienced malarial attacks. In the following year only 4 per cent of the population had attacks. However, when the people were subsequently urged to purchase the treatment, the consumption of quinine was disappointing, which probably explains why the program never gained ground (Bass 1920).

The XIXth century witnessed many examples of the deliberate application of drainage for the prevention of intermittent and remittent fevers. We have no means of appraising the volume of such work, but sufficient accounts are preserved in the literature to indicate that it was considerable. One of the earliest significant instances is afforded by the action of the city of Savannah, Ga., with relation to rice culture. In 1817 , at a town meeting, the city appropriated $\$ 70,000$ to purchase the right of cultivation of rice, in wet culture of such of the tidal swamps as were adjacent to the city. The policy was sustained at a referendum in 1821. A marked reduction in deaths from "autumnal diseases" immediately resulted (Daniell 1826). The interest in the subject of defective drainage as a cause of excessive mortality is shown by the systematic medical survey of the state of New York reported in 1832 (Anon. 
1832), as well as a second survey of the same state later conducted by J. M. Smith (1860). Dr. Garrish (1879) relates that a system of drainage was installed to improve the healthfulness of the unnamed town of his residence, on one of the tributaries of the White River in Indiana, which resulted in the diminution of ague to insignificant incidence. Continued interest in the subject is shown by a symposium on drainage and public health that was held at one of the meetings of the American Medical Association, which largely fills the volume of transactions for 1874. To this Kedzie (1874) contributes a statement that in 1857 the State of Michigan adopted legislation to encourage the drainage of swamps, marshes and lowlands, and estimated that in the 20 years preceding his report, no less than 20,000 miles of ditches had been dug. The motive, of course, was agricultural reclamation, yet a profound effect on health was noted. He stated that as drainage became more perfect, a diminution of malarial diseases was observed, and that the fevers did not yield as readily to antipaludic remedies, there being observed an increase of the continued fevers and a diminution of the intermittents.

With the discovery of the transmission of the parasite by anopheline mosquitoes, drainage as a means of preventing malaria was removed from the field of empirical sanitary procedures, and developed a significance of its own, i.e., a means for the elimination of anopheline breeding places. In conjunction there also developed, as a natural corollary, practices to prevent anopheline breeding. The earliest application of drainage for malarial control with this orientation was effected during the American occupation of Cuba subsequent to 1900 . This early demonstration of the practical value of the discovery of Ross was directed by Col. W. C. Gorgas, assisted by J. A. LePrince (LePrince and Orenstein 1916), providing an apprenticeship for their later effective control of yellow fever and malaria in the Canal Zone (Gorgas, $1910 ; 1915)$.

The immediate effect of this discovery and of the later demonstrations in Havana and Panama was practically nil on the continental United States. Perhaps the most distinctive project of an antimalarial character was the program executed on Staten Island between 1901 and 1910 by A. H. Doty (Howard et al. 1912b), health officer of the port of New York. In addition a small amount of isolated work was executed in widely scattered localities by local initiative, which was doubtless productive of much good; but even at this early date these steps tended to exhibit the characteristic of a general attack on all mosquitoes, a trend which continued until comparatively recent years and has undoubtedly retarded the control of malaria.

Finally, however, the Panama demonstrations commenced to bear fruit in the United States, and R. H. von Ezdorf and H. R. Carter of the Public Health Service, the latter a close associate of Gorgas, initiated field studies of malaria in the south in 1912 and 1913. These early studies resulted in practical demonstrations of antimalarial measures at Roanoke Rapids, N. C., and Electric Mills, Miss. (von Ezdorf 1916), which really initiated serious malarial control work in the South.

A decided impetus was given to interest in malarial control by the program executed in extra cantonment zones during 1917 and 1918 while the United States was engaged in the first World War. This program, executed by the Public Health Service, extended to 43 areas in 15 states, over a total area of about 1,200 square miles. Apart from its immediate objective, the work had a significant demonstration value and also resulted in the technical training of a large personnel, several of whom are still at work in this field.

As previously intimated, many communities have simultaneously prosecuted a program devised for the control of both yellow fever and malaria, or of one or the other coupled with measures directed against one or more of the noxious species of local mosquitoes. Considering the differences in the breeding places chosen by different species of mosquitoes, projects 
with such a scope are exceedingly costly. While not decrying such extensive programs where they can be afforded, their example nevertheless has. doubtless retarded the extension of simpler programs with a definite sanitary objective. Attention to the role of different species of anophelines in malarial transmission has shown that great economies can be effected by limiting a project to the actual transmitting species, a practice known as "species sanitation," exemplified by the suceessful results in the Canal Zone which were obtained by directing efforts to the control of $A$. albimanus.

Under practicable circumstances the routine distribution of substances toxic for mosquito larvae is of great value in the control of mosquitoes. Prior to the recognition of their disease conveying powers, mosquitoes attracted so little attention from scientists and the laity that a contribution which appeared in the American Daily Advertiser of Philadelphia, on August 29, 1793, when an epidemic of yellow fever was raging in that city, is remarkable. The anonymous contributor urged the householders to pour a gill of oil on the water in rain barrels and to add more to eisterns in order to kill the mosquitoes therein (Middleton 1928). It is certain that any oil which may have been used at that date was not petroleum, but more likely whale oil. In 1892 Howard was one of the earliest to employ a petroleum oil (kerosene) for the destruction of mosquito larvae (Howard 1900), and for many years thereafter various petroleum derivatives were the most commonly employed larvicides. Since stomach poisons are so widely used in the control of insect pests, it is rather remarkable that their adaptation to mosquito control came at a comparatively late date. The possibilities of the best known insecticide of this type, Paris green, were ascertained by Barber and Hayne in 1921, subsequent to which its use as a larvicide has become widespread.

The application of metallic wire cloth to doors and windows of dwellings in order to exclude mosquitoes and other noxious insects is a practice that has had its widest development in the United States. Just when it was first introduced is uncertain, although some was manufactured as early as $\mathbf{1 8 6 5}$. The amount now annually manufactured for domestic consumption and export is enormous, in 1927 exceeding 500 million square feet.

\section{The Need for Intensification of Research on Mataria}

In another connection the writer (1939) has briefly reviewed certain aspects of the present status of the malarial problem. While recognizing that present available knowledge is not being applied in some areas with adequate resources for the control of malaria, nevertheless there are many regions in the world where any attempt to control this disease based on the application of available measures is beyond local resources. Malaria will likely continue to be endemic in such regions until cheaper control methods are devised. The hope of cheaper methods depends upon the acquirement of new viewpoints to the problem, the attainment of which necessitates an extension of our knowledge. Probably no better guide to the needs and opportunities for research in the field of malaria can be secured than through an inventory of existing knowledge which this symposium is designed to supply. 


\title{
THE DISTRIBUTION OF MALARIA IN NORTH AMERICA, MEXICO, CENTRAL AMERICA AND THE WEST INDIES
}

\author{
By ERNEST CARROLL FAUST \\ DEPARTMENT OF TROPICAL MEDICINE, TULANE UNIVERSITY OF LOUISLANA, NEW ORLEANS
}

Ат the present time, as in past decades, all three cosmopolitan species of human malaria plasmodia, Plasmodium vivax, $P$. malariae and $P$. falciparum, occur as etiological agents of human malaria in the Western Hemisphere. In North America $P$. falciparum and $P$. malariae are not normally indigenous north of the Ohio River Valley in the region east of the Mississippi River, while their distribution west of the Mississippi probably extends northward only into southern Missouri and to the northern boundary of Oklahoma. Nevertheless, under favorable circumstances sporadic cases of estivo-autumnal malaria develop from time to time in regions as far north as New York State, central Ohio, central Indiana and central Illinois, due most probably to the infection of anopheline mosquitoes from human cases imported from the south, and the transfer of the parasite by the infected mosquitoes to a few individuals of the indigenous population in these more northerly climates. Typically, the infection is not transferred again to mosquitoes and dies out at this point. An exception to the limitation of the estivoautumnal parasite to a warm climate apparently exists in New York City, where this species has become artificially established in drug addicts as a result of the use of a common hypodermic needle (Most 1940b). -Even in areas most favorable for its propagation the quartan malaria plasmodium has an unexplained "spotty" distribution.

Thus, the tertian parasite has a widespread distribution in many of the cooler portions of the United States, where the other two species of malaria plasmodia are not indigenous, while in the warmer cli- mates it shares responsibility for human malaria primarily with the estivo-autumnal parasite.

The incidence of malaria in a given area may be judged either from morbidity or mortality data. Since clinical diagnosis of "chills and fever" cannot be relied on as a diagnostic criterion, a true estimate of the distribution and amount of malaria or the species of malaria plasmodia in a particular district depends on the identification of the plasmodium in stained films made from the bloods of a representative cross section of the population (i.e., surveys to detect the parasite), or on malaria spleen surveys. Unfortunately, there are several reasons why malaria incidence statistics are usually unreliable. These include the paucity of adequate malaria parasite or malaria spleen surveys, the inability of many physicians to suspect or diagnose malaria in a sick population, the danger of using clinical laboratory records as representative of a population as a whole, the common practice of self-diagnosis and self-treatment with proprietary drugs, and prescription of antimalarials by the physician without adequate diagnosis.

Mortality data on malaria are, on the whole, more likely to be dependable than are morbidity data, and are relatively reliable as an index of malaria endemicity in the Southern United States and to a lesser extent in countries of Tropical America where vital statistics have been compiled. However, since malaria deaths in warm climates result primarily from infection with the estivo-autumnal parasite, mortality statistics fail to provide information on the distribution or intensity of infection 
with the tertian or quartan parasite. In areas where the tertian parasite alone is indigenous, accurate mortality records constitute a relatively faithful picture of this infection.

Attempts to compare the number of recorded cases of malaria in a given geographical or political subdivision of a country with the number of malaria deaths have been consistently disappointing. During the period 1917-1919 American white troops stationed in camps in malarious areas in the Southern United States had a ratio of one malaria death to 415 cases (Ireland 1925). This furnished a satisfactory check on the minimum of expected mortality, since all suspected cases were hospitalized and were checked by adequate blood-film examination. By contrast, the ratio of malaria deaths to cases reported to boards of health in certain southern states in 1934 ranged from $1: 2.7$ to $1: 103.8$ (Faust and Diboll 1935). Moreover, although the ratio of reported death rates and case rates for malaria in the State of Georgia for the year 1939 was as high as 1: 336 in one county, in three counties it was only $1: 2$, in one, $2: 3$, in five, $1: 1$, and in one county two deaths were listed with but a single illness recorded (Abercrombie 1940). Since the maximum expected ratio in Tropical America is approximately 1: 100 under conditions of high endemicity, it is clear that only a fraction of malaria cases in many states is reported to the bureaus of vital statistics.

In any malarious area, the economically lower strata of the population constitute the majority of the infected population, due to greater exposure and reduced resistance to infection and financial inability to pay for adequate treatment. Life insurance companies confining their business in the South to persons able to pay for standard policies have had no evidence of actuarial loss as a result of malaria (Faust 1939a).

Infants are particularly susceptible to malaria and mortality is very high in the one-month to five-year age group. Tolerance to infection tends to develop rapidly and reaches its height at adolescence.
Later, especially after middle life, tolerance is appreciably reduced (Dauer and Faust 1936).

\section{Distribution of Maulria in the United States and Canada}

Conservative evidence indicates that malaria is today indigenous in 36 of the United States. These include all of the southeastern states (i.e., Virginia, North Carolina, South Carolina, Georgia, Florida, Kentucky, Tennessee, Alabama, Mississippi, Arkansas and Louisiana); a large portion of Oklahoma and Texas; Missouri (especially the southeastern section); several counties each in New York, Pennsylvania, New Jersey, Maryland, Ohio, Indiana, Illinois and Michigan; all three counties of Delaware; the counties in Wisconsin, Iowa and Minnesota adjacent to the Mississippi River; Southeastern Kansas and probably Gage County in Southeastern Nebraska; several counties in New Mexico, Arizona and California; two counties each in Washington and Oregon; Boise County, Idaho, and possibly Mesa County in Central Western Colorado. The states apparently free of indigenous malaria today are the New England States, West Virginia, North Dakota, South Dakota, Utah, Wyoming, Montana and Nevada.

Malaria is not, and has not been for many years, indigenous in Canada.

\section{Malaria Mortality in the United States}

While mortality rates by states are of some value, a much more accurate evaluation of malaria can be obtained from a map showing the relative mortality rates for each county. Such maps have been compiled by Maxcy (1923) for the entire United States for the period 1919-1921; by Faust (1932) for the Southern United States (1930); by Dauer and Faust (1936) for the Southern States (1929-1933); and by Faust (1940) for the Southern States (1929-1938). The accompanying map (Fig. 1) provides a 10-year average by counties for the entire United States for the period 1929-1938 and is based on sta- 


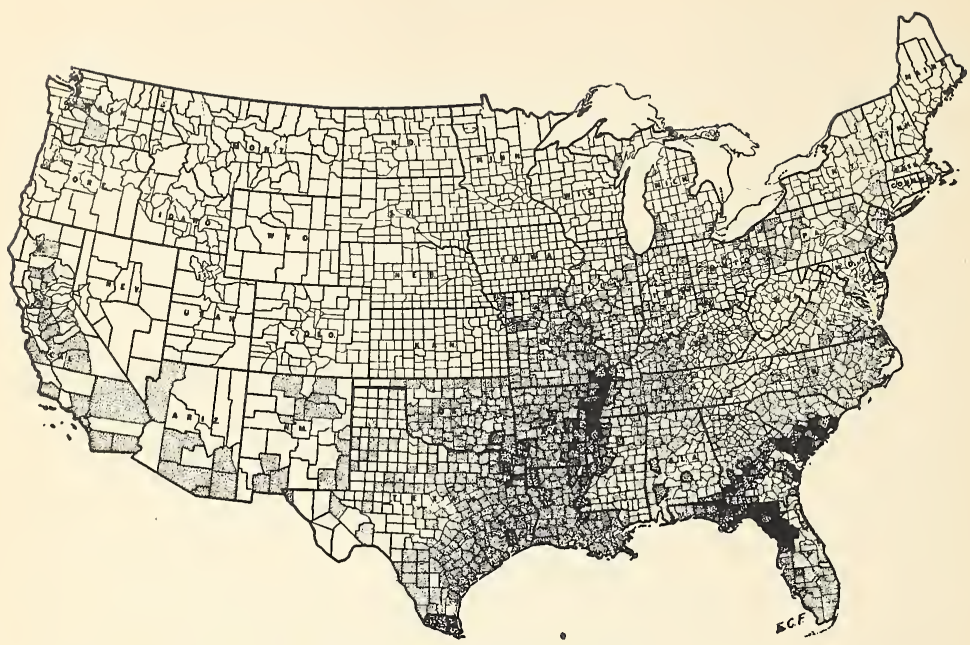

Fig. 1. Average of officially reported indigenous malaria deaths in the United States, by counties, for the period 1929-1938. The solid black indicates an average of 50 or more deaths per 100,000 population; the heavy stippling, 25 to 49.9 ; the light stippling, under 25 ; and the unshaded areas, no reported malaria deaths for the decade.

tisties provided by responsible officials of the departments of health of these states.

Heavily endemic foci that have remained relatively constant during this decade are found in (1) a wedge-shaped sector of the Southeastern States, including areas in South Carolina, Georgia, Florida and two adjacent counties in Alabama; (2) the "delta area" of the lower Mississippi Valley from Cairo, Illinois, to Natchez, Mississippi; (3) the portion of the Red River Valley near the junctions of Oklahoma, Arkansas, Louisiana and Texas; and (4) a 4-county section of Texas at the mouth of the Rio Grande. Except for the Alleghanies and Appalachian highlands, deaths from indigenous malaria have occurred within the decade surveyed in counties from northernmost New York almost without interruption down to the Gulf of Mexico (although much more consistently in the South than in the North), and with few county interruptions from Savannah to San
Diego, and from Milwaukee to El Paso. In the North and West, reports on malaria deaths have frequently been accompanied by information indicating that the infection was acquired in the highly endemic South or in Tropical America.

Within the past decade a cyclic low rate of malaria deaths was experienced in all of the Southeastern United States either during the year 1931 or 1932 (Fig. 2). However, a year or two later all of these states had a decidedly increased malaria death rate, although in. South Carolina and Georgia the peaks were not reached until 1936. There followed an unusually satisfactory decline in the rate through 1939, with an average rate-level for that year of 4.4 per 100,000 population, the lowest on record. Cyclic increases and decreases in malaria customarily recur every five to seven years, but the increase expected in 1938-1939 failed to materialize except in Alabama (1939). Whether this satisfac- 


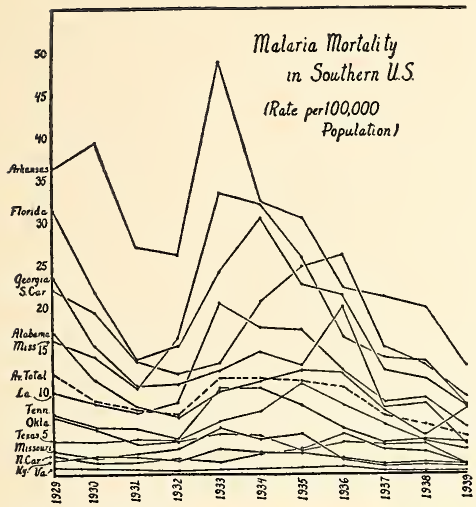

FIg. 2. Annual malaria mortality rates by states for the Southern United States and average total rates for these states for the years 1929-1939.

tory condition has resulted, at least in part, from extensive and intensive preventive measures directed against mosquito breeding and from antimalarial treatment of the human population is not known.

Viewing the United States as a whole, we find definite evidence that malaria mortality has been greatly reduced in the past five or six years and that the heavily endemic foci have shown evidence of improvement over one or two decades ago. Nevertheless, malaria is more widespread and probably more prevalent today than in 1930. In the Southern States the infection is becoming dispersed radially from the heavily endemic centers. In the North, areas of endemicity a quarter of a century ago, but presumably free a decade ago, have provided the ground for reestablishment of the disease. While it is possible that this may have resulted from the reactivation of latent autochthonous strains, it is much more likely that new strains brought into potential Northern and Western foci from the South and from Tropical America have constituted the source of the increasingly widespread distribution outside the South.

\section{Distribution of Malaria in Mexico, Cen- TRAL AMERICA AND THE WeSt INDIES}

Since no inclusive résumé of the status of malaria in the countries south of the United States has ever been published, this compilation was undertaken with considerable hesitancy. It was realized that attempts to discover the distribution and practical im-

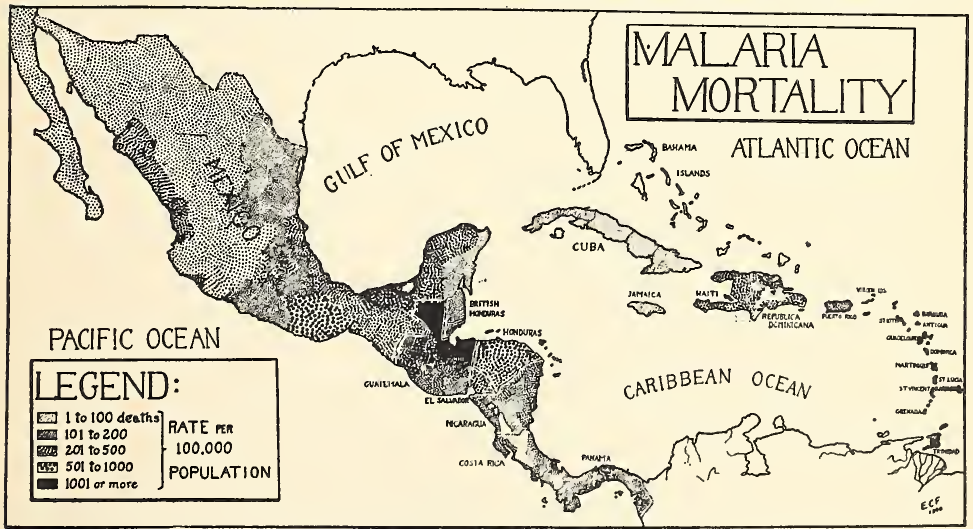

Fig. 3. Estimated average malaria mortality rates for Mexico, Central America and the West Indies for the period 1929-1938, based on official reports and other available records. 
portance of malaria differed widely in the respective countries and that some relatively uniform method of evaluating data had to be provided. To this end a questionnaire was addressed in February, 1940, to the chief of the public health service of each country in an attempt to obtain as much concrete information as possible.

In several countries the desired information was not obtainable or only partially available. On the other hand, the reports obtained from some officials were fairly complete and bear evidence of relative accuracy and reliability. In only three instances have repeated requests been ignored. It must be frankly stated, however, that much of this information is not wholly dependable, because it is based on clinical rather than survey records and is neither completely accurate nor representative.

The most essential data are summarized by countries in Table I, while the average mortality rates are shown by countries and, wherever possible, by major political or geographical subdivisions on the accompanying map (Fig. 3).

With the probable exception of the Bahama Islands, malaria is endemic throughout the entire area covered in this portion of the report. In the highlands and essentially dry areas, the disease is usually much less intense than it is along the swampy coastal areas and in the tropical rain-forests. Nevertheless, highland malaria constitutes an important problem in parts of Mexico and Central America. Official government reports indicate that the most highly malarious territory on the mainland begins in Southern Mexico and, with few interruptions, extends through Panama. The only comparably malarious countries of the West Indies are Haiti, Republica Dominicana, portions of Puerto Rico, St. Lucia and Trinidad.

The range in malaria mortality in countries with important political subdivisions may be very wide, as is seen when the rates for these subdivisions are compared with the averages of the country as a wole (Table I, Fig. 3). Thus, the official figures indicate that Mexico has an average rate of
140.7, although the Distrito Federal is stated to have the low rate of 6.16, Michoacan, 9.43 and Chihuahqa, 12.55. In contrast, the rate for Tabasco is reported to be 502.29 and that for Oax\&ca, 563.02. Guatemala, for which there is the official average rate of 414 , has reported department rates ranging from 125 to 1671 . Costa Rica, with an average rate of 152 , has department rates ranging from 25 to 348 . Cuba, with an average rate of 20.6 , has a range from 3.2 (Habana) to 88.5 (Camaguey).

Attention is directed to the relatively low ratio of recorded morbidity rates compared with the mortality rates. This is explained by some reporting government officials as due to the extensive distribution of antimalarials by local dispensaries. However, in only three of the countries surveyedNicaragua, Puerto Rico and the Virgin Islands (U. S. A.) -is malaria a reportable disease. With the exception of Guatemala, Jamaica, Puerto Rico, the Virgin Islands and possibly Nicaragua, routine diagnosis is based primarily on scanty clinical histories and on therapeutic tests, since many of the physicians and practicantes either have no immediate access to diagnostic laboratories or do not avail themselves of existing services. Thus, it is highly probable that only a small percentage of malaria cases is included in the official figures.

The computed average malaria mortality rate for all of the tropical countries considered in this paper (with a reported population of $38,500,000$ ) is 166 per 100,000 population, a figure probably far too low. This means that approximately 63,900 deaths in this area are caused each year by malaria and contrasts conspicuously with the average of 3,258 deaths (1933-1939) for the Southern United States, which has approximately the same size population group (i.e., a ratio of nearly 20 to 1 ).

In several of the reporting countries extensive malaria surveys have been conducted in recent years or are being carried out by health departments, at times in coltaboration with staff members of the International Health Division of the Rockefeller Foundation, and in Panama with the added 
DISTRIBUTION OF MALARIA

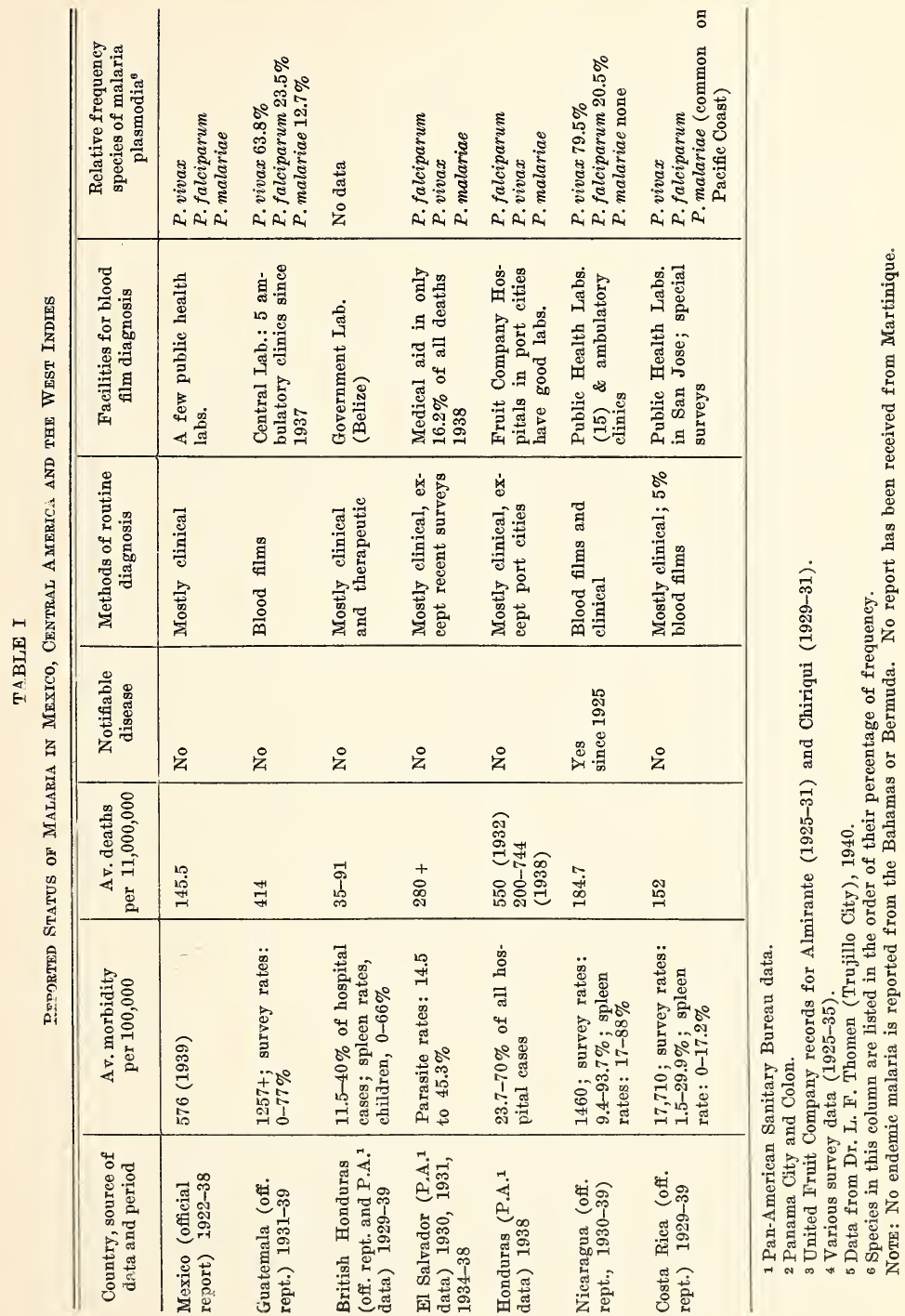




\begin{tabular}{|c|c|c|c|c|c|c|c|c|c|}
\hline 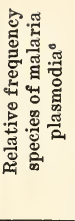 & 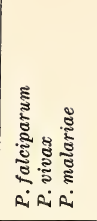 & 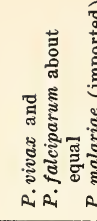 & 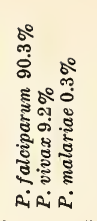 & 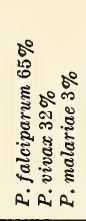 & 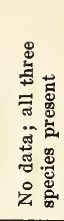 & 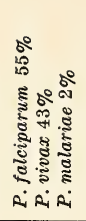 & 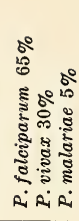 & 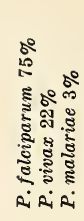 & 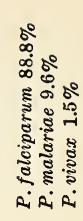 \\
\hline 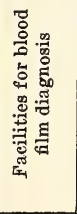 & 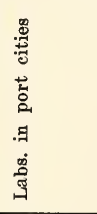 & 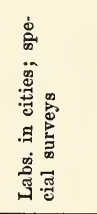 & 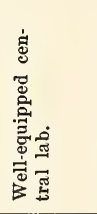 & 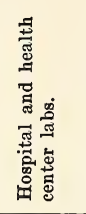 & 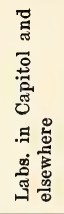 & 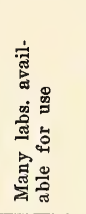 & 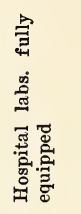 & 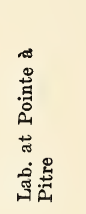 & 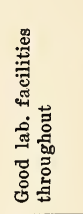 \\
\hline 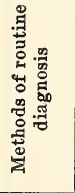 & 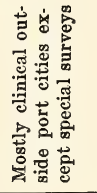 & 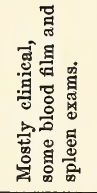 & 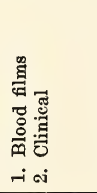 & 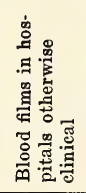 & 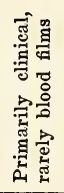 & 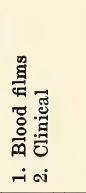 & 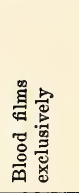 & 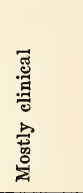 & 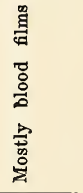 \\
\hline 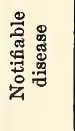 & $\ddot{z}$ & $\stackrel{\circ}{z}$ & ż & 艺 & $\stackrel{\circ}{z}$ & $\stackrel{\infty}{\pi}^{\infty}$ & 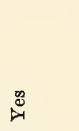 & 르 & $\stackrel{\infty}{\stackrel{\infty}{~}}$ \\
\hline 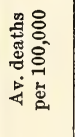 & 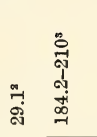 & ஷ̊. & 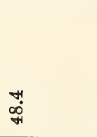 & 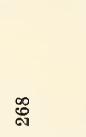 & 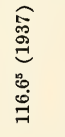 & 亲 & 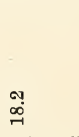 & 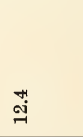 & $\stackrel{\circ}{\circ}$ \\
\hline 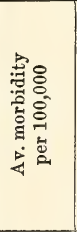 & 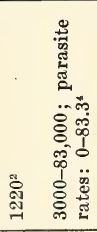 & 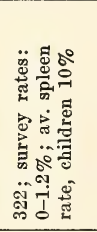 & 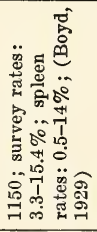 & $\underset{\leftarrow}{\stackrel{S}{S}}$ & 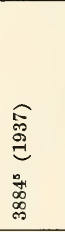 & 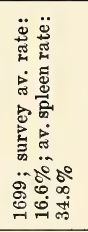 & 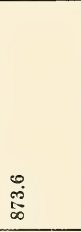 & 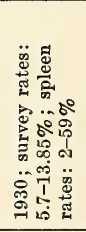 & 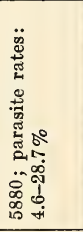 \\
\hline 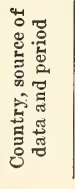 & 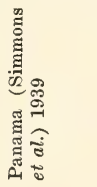 & 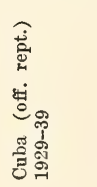 & 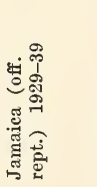 & 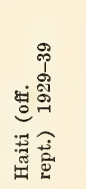 & 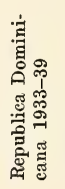 & 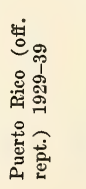 & 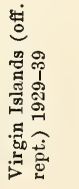 & 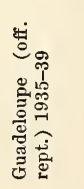 & 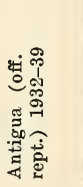 \\
\hline
\end{tabular}


DISTRIBUTION OF MALARIA

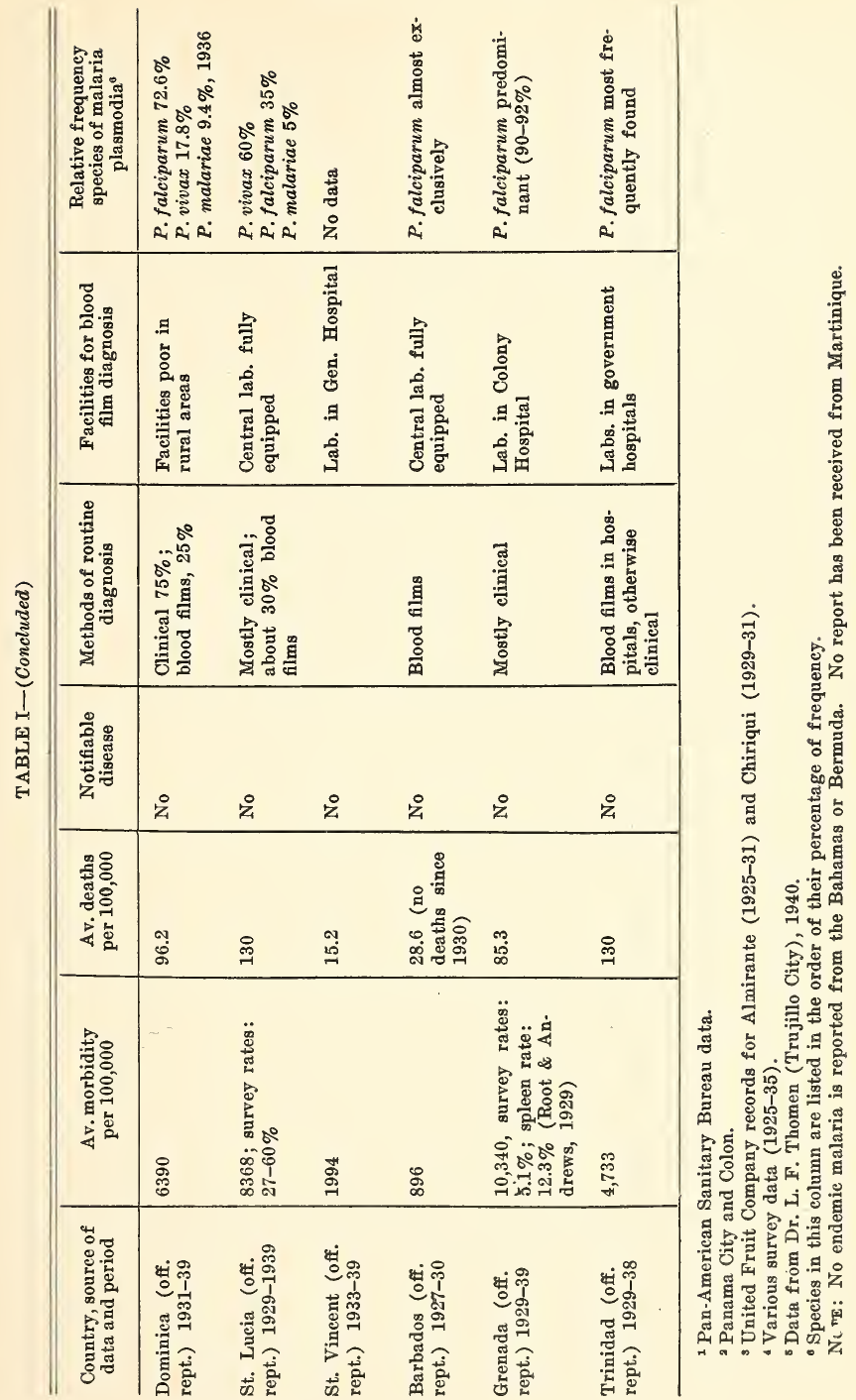


assistance of the Gorgas Memorial Laboratory and the agencies of the Canal Zone. Based on the diagnosis of malaria plasmodia in blood films or on enlarged spleens in representative groups of the population, these surveys show a latent malaria incidence many fold higher than the reported malaria morbidity, approaching at times 75-95 per cent of the entire population of certain areas. Such surveys provide a much more reliable basis for estimating the malaria incidence than do hospital records or mortality statisties.

The relative proportion of the three common species of human malaria plasmodia varies considerably. In the interior of Mexico, in Guatemala, Nicaragua, Costa Rica and Panama, Plasmodium vivax preponderates; in Nicaragua it is reported to constitute $79.5 \%$ of the total infections. In Cuba, extensive surveys indicate that $P$. vivax and $P$. falciparum are about equal. In the littoral areas of Mexico and in Yucatan, in El Salvador, Honduras, Haiti, Republica Dominicana and the Lesser Antilles, $P$. falciparum is much the more common species, or at least is the species most commonly found in blood film examinations. Although $P$. malariae ranks third in all of the countries, its proportion of the total infections varies from $12.7 \%$ in Guatemala to $0 \%$ in Nicaragua and Cuba. (In Cuba, cases of diagnosed quartan malaria are reported to have been imported.) In Costa Rica surveys show that it is relatively common on the Pacific Coast. However varied its incidence may be in the populations of the several countries, $P$. falciparum is primarily the parasite responsible for malaria mortality.

\section{General Considerations and Conclusions}

From the days when malaria was first recognized as a serious, disabling, and frequently fatal disease in the Western Hemisphere, attempts have been made to combat it. Control measures became more effective with the known relationship of anopheline mosquitoes to the life cycle. Yet, with few exceptions, the epidemiology of malaria in the Americas has been seientifically under- taken on an extensive scale only within the past two decades and in some countries is only now being initiated. Moreover, there is evidence in at least two countries of Central America that extensive antimalarial treatment is in progress without any antecedent fundamental epidemiological investigations based on surveys of the prevalence of the malaria parasites in the population. While there is some indication that the most heavily malarious areas in the United States are gradually coming under control, the much more intensely infected areas in tropical America are probably as malarious today as they were fifty years ago. In many localities the urban communities are under relative control as a result of anti-mosquito and therapeutic measures, while the rural population, with higher parasite rates and better opportunity for mosquito transmission, constitutes the essential seedbed of the infection.

The data which have been assembled vary from scant statements admittedly inaceurate to voluminously documented evidence covering practically all phases of the epidemiology of malaria in every subdivision of the country. Such data cannot be placed in the same category. They indicate public health attitudes as widely separated as apathetic tolerance and progressive realism.

In spite of the difficulties encountered in evaluating such varied evidence, the following conclusions may be tentatively drawn:

1. Malaria is widespread throughout the Southern United States, where all three common species of human malaria plasmodia are established. It also has an extensive distribution in the Eastern, Central and Western States, where $P$. vivax is the only proven autochthonous species. Malaria is probably not now endemic in Canada.

2. Except for the Bahama Islands and Barbados, malaria is a serious menace throughout practically all of Mexico, the countries of Central America and the West Indies, in which countries practically every political subdivision has an unsolved malaria problem.

3. In the United States outside of the 
South, malaria mortality is relatively low, although the morbidity may be appreciable. These inferences are in accord with the evidence that the benign malaria parasite, $P$. vivax, is the only known autochthonous species in this territory. In the Southern United States, malaria appears to be spreading radially from three or four highly endemic foci. Although the mortality rate in the South as a whole has reached a relatively satisfactory low level, certain foci have a maintained tropical malaria death rate. While there is a wide range in malaria mortality rates reported from Mexico, Central America and the West Indies, the average for these countries as a whole is at least 20 times that of the Southern United States.

4. There are adequate laboratory facilities for the accurate diagnosis of malaria films in several of the United States and in a few of the tropical countries surveyed, but diagnosis is still too commonly based on clinical evidence and therapeutic tests. Moreover, diagnosis of malaria plasmodia in the blood of clinic patients provides no real evidence of the prevalence of the infection in the population as a whole. Thus, most official records of malaria morbidity are unreliable as an index of the amount of malaria and of the relative incidence of the different species of malaria plasmodia in a given area.

5. Extensive blood film examinations of representative cross sections of the population constitute the most accurate basis for determining the malaria index of a country. Surveys of this type have been carried out or are in progress in some of the United States and in several of the other countries under consideration.

6. While splenic enlargement is probably not as accurate an index of malaria as is blood film examination, spleen surveys carried out on children are fairly reliable and offer an opportunity for determining malaria incidence in areas where facilities and training are inadequate for diagnosis of the parasites in blood films.

7. Throughout the territory favorable for the propagation of all three common species of human malaria plasmodia, the relative incidence of $P$. vivax and $P$. falciparum varies, but for any lárge area $P$. malariae is relatively unimportant.

8. In all of the warm areas surveyed $P$. falciparum is the species primarily responsible for malaria deaths.

\section{ACKNOWLEDGMENT}

Sincere thanks and appreciation are extended to the many persons who have provided the basic data from which this report has been compiled: to all officials in the bureaus of vital statistics of the departments of health of each of the United States, to Dr. J. J. Heagerty, Director of Public Health Services, the Dominion of Canada and to Professor Thomas W. M. Cameron, MeGill University, for information on malaria in Canada; to Dr. Jorge Rendon Gomez of the Federal Department of Public Health and to Professor Carlos C. Hoffman, Instituto de Biología, for Mexico; to Dr. Carlos Estévez, Director General of Public Health, for Guatemala; to Dr. Vernon F. Anderson, Acting Senior Medical Officer, for British Honduras; to Dr. Pedro H. Ordóñez Diaz, Director General of Public Health, for Honduras; to Colonel Luis Manuel Debayle, Medical Director General of Health, for Nicaragua; to Dr. A. Peña Chavarría, Secretary of Public Health, and to Dr. H. W. Kumm, International Health Division, Rockefeller Foundation, for Costa Rica; to LieutenantColonel James S. Simmons, M. C., United States Army, for Panama; to Dr. Laureano Lopez Garrido, Office of the Secretary of Health and Welfare, Professor Pedro Kourí, University of Habana, and Dr. Henry P. Carr, International Health Division, Rockefeller Foundation, for Cuba ; to the Director of Medical Services, for Jamaica ; to Dr. Rulx Léon, Undersecretary of State for Hygiene, Public and Social Welfare, for Haiti; to Dr. Luis Francisco Thomen, for Republica Dominicana; to Dr. E. Garrido Morales, Commissioner of Health, for Puerto Rico; to Dr. Meredith Hoskins, Chief Municipal Physician, De- 
partment of Health, for the Virgin Islands (U. S. A.) ; to Medical Lt.-Colonel Colin, Chief of Sanitation, for Guadeloupe ; to Dr. John E. Wright, Senior Medical Officer, for Antigua; to Dr. H. Scott Gillett, Medical Officer of Health, for Dominica; to Dr. H. D. Weatherhead, Senior Medical Officer, for St. Lucia; to Dr. Leslie Webb, Senior Medical Officer, for St. Vincent; to Dr. B. M. Bailey, Chief Medical Officer, for Barbados; to Dr. E. Cochrane, Senior Medi- cal Officer, for Grenada; to the Director of Medical Services, for Trinıdad; to Dr. L. W. Fitzmaurice, Acting Chief Medical Officer, for the Bahama Islands; to Dr. Henry Wilkinson, Medical and Health Department, for Bermuda, and to Dr. Aristídes A. Moll, Secretary, Pan American Sanitary Bureau, for British Honduras, El Salvador and Honduras. I also wish to thank Miss Lois DeBakey for valued assistance in translation. 


\title{
THE TAXONOMY OF THE HUMAN MALARIA PARASITES WITH NOTES ON THE PRIN- CIPAL AMERICAN STRAINS
}

\author{
By G. ROBERT COATNEY and MARTIN D. YOUNG
}

U. S. PUBlic HeAlTh SERVICE, COLUMBIA, s. c.

THE plasmodia as single-celled animals belong to a large and heterogeneous group, the phylum Protozoa. The presence of syngamy places them in the subphylum Plasmodroma. As spore producers, they belong to the class Sporozoa and as the spores are formed at the end of the cycle and have neither polar capsules nor polar filaments they fall in the subclass Telosporidia. Having motile zygotes and sporozoites without envelopes, they belong to the order Haemosporidia. They are in the family Plasmodiidae, which is characterized by schizogony in the peripheral blood, pigment production, and the mosquito as an invertebrate host. This family has a single genus, Plasmodium, which has the same characteristies as the family.

\section{Valid Species}

Until comparatively recently certain groups of malaria students were reluctant to accept the idea of there being more than one species of human malaria, even though Golgi (1886a) long ago carefully disinguished between tertian and quartan malaria fevers. Part of the confusion resulted from observations on mixed infections where the different morphological types encountered were erroneously thought to comprise an entity.

Specific status of a species, in line with the accepted zoological practice, is assessed on the basis of certain morphological characters which must remain constant during the life of the parasite in the human host and in the insect vector. Consequently with the advent of malaria therapy opportunities were afforded for controlled studies of both the asexual and sexual cycle under laboratory conditions. The results of these studies have confirmed that there are several species of human plasmodia and that these separate species are made up of races or strains, which, although as yet indistinguishable on morphological grounds, can be separated on the basis of antigenic properties, effect of drugs, virulence, and infectivity.

On the basis of available knowledge the accepted species of human plasmodia are: $P$. vivax, $P$. malariae, $P$. falciparum, and $P$. ovale.

There was a fair quantity of data in support of the acceptance of the validity of the three classical species prior to 1918 , but these data had been obtained from the study of natural infections or from limited observations on induced cases in volunteers. We now have extensive experimental supporting evidence from the Horton Malaria Therapy Center in England, Socola Malaria Therapy Center in Roumania, Station for Malaria Research, Tallahassee, Florida, the Williams Malaria Research Laboratory, Columbia, S. C., and many others. For $P$. ovale, however, the experimental data rest for the most part on the observations of James and his associates at the Horton Malaria Therapy Center in England, and they have presented ample evidence to justify ranking $P$. ovale as a separate species; it is specifically different in morphology from the other three species, and these differences are constant in all infections resulting from direct blood inoculation. In the mosquito, the character and arrangement of the pigment in the oocysts is decidedly characteristic and the sporozoites found in the salivary glands are much smaller than those of $P$. vivax, its nearest relative. In the infections resulting from mosquito bites the 
periodicity of the asexual cycle, the clinical course of the disease, and the characteristic morphology of the parasites are maintained. As proof of the separateness of the species, patients refractory to the other three species are not necessarily immune to $P$. ovale.

It is hoped for reasons of practicability and unity, that an agreement can be reached as regards the specific names. For the present report, all of the important papers dealing with nomenclature have been studied. The conclusions reached by other authors have been reviewed and an attempt made to bring together the correct synonomy for the different species of human plasmodia. It is not proposed to give a complete discussion on the question of specific nomenclature, but only such points as seem essential. For a more complete discussion, the reader is referred to the papers by the Sergents and Catanei (1929, 1939), Christophers and Sinton (1938), Sinton (1939a), Craig (1933) and James, Nicol and Shute (1933).

$P$. vivax. Students of the subject are agreed that the correct name for the parasite of benign tertian malaria is $P$. vivax (Grassi and Feletti, 1890).

$P$. malariae. There has been general acceptance of the name $P$. malariae for the parasite of quartan malaria, but, seemingly since Lühe (1900), this name has been credited to Laveran rather than to Grassi and Feletti. This diserepancy can only be explained by assuming that Laveran (1881) gave the name malariae to the human malaria parasites in general, which in the light of our present knowledge, he did not do. Grassi and Feletti (1890) definitely gave the name malariae to the quartan parasite under the genus Haemamoeba, a correct procedure if two genera were recognized. Celli and Sanfelice (1891) used the name $P$. malariae var. quartanae which makes $P$. quartanae Celli and Sanfelice, 1891, the de jure name for this parasite since only one genus is recognized. As is pointed out below, the adoption of this name would lead to much confusion, and it is suggested that the de facto name of $P$. malariae be con- tinued for this species and credited to Grassi and Feletti, 1890.

$P$. falciparum. The correct name for the parasite of malignant tertian malaria is not so easily determined. Laveran (1881) gave the name Oscillaria malariae to the new parasite found by him in the blood of malaria patients. The parasites he described were undoubtedly those of malignant tertian malaria. This being true, malariae, by right of priority is the correct specific name for the parasite of malignant tertian malaria and the one which should have been retained. Grassi and Feletti (1890) proposed their own name malariae within the genus Haemamoeba for the parasite of quartan malaria, retaining Laveran's malariae within their new genus Laverania for the parasite of malignant tertian malaria. Such a procedure is zoologically correct if two genera are recognized, but, since only one genus, Plasmodium Marchiafava and Celli, 1885, is now recognized (Opinion No. 104 of the International Commission on Zoological Nomenclature, 1928), it follows that the two generic names Haemamoeba and Laverania sink.

Welch, 1897, proposed the name Haematozoon falciparum for the parasite of malignant tertian malaria.

The Sergents and Catanei (1929) after an exhaustive study came to the conclusion that the correct name for the malignant tertian parasite should be $P$. praecox Grassi and Feletti, 1890. Giovannola (1935) reexamined the problem and concluded that the correct name should be $P$. immaculatum (Grassi and Feletti, 1892) ; but, as recently pointed out by Christophers and Sinton (1938), this specific name should be eredited to Grassi (1891) if it is to be recognized at all. However, they point out that malariae was the specific name applied originally to the parasite by Laveran and is the de jure name. The de facto name for this parasite is $P$. falciparum (Welch, 1897).

In considering this question, Christophers and Sinton (1938) and Sinton (1939a) drew special attention to the difference in names for the three classical species under the specific de jure and de facto nomencla- 
ture. They pointed out the almost intolerable confusion sure to result from the strict application of the rules of zoological nomenclature and proposed, in the interest of uniformity and convenience, the general adoption of the commonly used de facto names.

Following the suggestion of Christophers and Sinton, the Sergents, Parrot, and Catanei (1939) surrendered their proposal of 1929 , and also urged the general adoption of the commonly used names.

A study of the problem has brought us to the point of agreement with the English and French authors. Vast confusion could arise from an insistence to adhere to the theoretically correct (de jure) nomenclature of the malaria parasites. Therefore, the general adoption of the de facto names, P. vivax (Grassi and Feletti, 1890), P. malariae (Grassi and Feletti, 1890), and $P$. falciparum (Welch, 1897) is urged.

$P$. ovale. Malariologists are now in agreement regarding the nomenclature of the "ovale" parasite. The correct name is $P$. ovale Stephens, 1922.

There is offered below what is believed to be the correct synonymy for the four valid species of human malaria.

P. vivax (Grassi and Feletti, 1890)

Haemamoeba malariae Feletti and Grassi, 1890 , partim

Haemamoeba vivax Grassi and Feletti, 1890

Plasmodium malariae tertianae Celli and Sanfelice, 1891

Plasmodium malariae tertianae Kruse, 1892

Haemamoeba laverani var. tertiana Labbé, 1894(?)

Haemosporidium tertianae Lewkowicz, 1897

Haemamoeba malariae tertianae Laveran, 1901

Plasmodium camarense Ziemann, 1915

P. malariae (Grassi and Feletti, 1890)

Haemamoeba malariae Feletti and Grassi, 1890, partim

Haemamoeba malariae Grassi and Feletti, 1890

Plasmodium malariae var. quartanae Celli and Sanfelice, 1891
Plasmodium malariae quartanae Kruse, 1892

Haemamoeba laverani var. quartanae Labbé, 1894(?)

Haemosporidium quartanae Lewkowicz, 1897

Haemamoeba malariae var. quartanae Laveran, 1901

Plasmodium golgii Sambon, 1902

P. falciparum (Welch, 1897)

Oscillaria malariae Laveran, 1881

Laverania malariae Feletti and Grassi, 1890

Haemamoeba praecox Grassi and Feletti, 1890, partim

Plasmodium malariae quotidianae Celli and Sanfelice, 1891

Haemamoeba immaculata Grassi, 1891, partim

Haemamoeba immaculata Grassi and Feletti, 1892, partim

Laverania malariae Grassi and Feletti, 1892

Plasmodium malariae irregularis Kruse, 1892

Haemamoeba laverani var. tertiana Labbé, 1894(?)

Haematozoon falciparum Welch, 1897

Haemosporidium undecimanae Lewkowicz, 1897

Haemosporidium sedecimanae Lewkowicz, 1897

Haemosporidium vigesimo-tertianae Lewkowicz, 1897

Haemamoeba malariae var. parva Laveran, 1901

Plasmodium falciparum var. subtertianum Bates, 1913

Plasmodium falciparum var. quotidianum Bates, 1913

Plasmodium tenue Stephens, 1914

Plasmodium caucasium Marzinowsky, 1916

P. ovale Stephens, 1922

None

Plasmodia of Doubtrul Taxonomic
Status

P. vivax var. minuta Emin, 1914. Craig 
(1900) described a plasmodium of distinct morphological character which he found occurring in the blood of soldiers returning from the Philippines. Emin (1914) named a form which he found in the blood of some pilgrims in Camaran Island $P$. vivax var. minuta. Craig (1926) accepted Emin's form as the one earlier described by him but not named. Later Craig (1933), after studying slides of $P$. ovale obtained from Yorke, James, Nicol and Shute, came to the conclusion that the parasite described by him in 1900 was $P$. ovale Stephens and not $P$. vivax var. minuta Emin as he had previously thought in 1926 .

Ziemann (1915) studied the Camaran Island parasites and was so convinced of their separateness that he gave them the name $P$. camarense. Ziemann (1938), however, says that he believes Emin's parasite should be considered as a variety of $P$. vivax.

Wenyon (1926a) states that he has seen similar forms in Mesopotamia and Macedonia but regarded them as aberrant $P$. vivax or $P$. malariae.

$P$. falciparum quotidianum Craig, 1909. Craig and Faust (1940) are of the opinion that Grassi and Feletti (1890) described this form in part and that later Marchiafava and Bignami (1891-1892) called it "the quotidian aestivo-autumnal plasmodium." Craig (1909) studied several infections with this organism and became convinced of its separateness from the true $P$. falciparum. This plasmodium was said to produce a paroxysm every 24 hours; the gametocytes were crescentic but smaller than the typical $P$. falciparum. The rings were rich in chromatin and the schizonts produced 6 to 18 merozoites with an average of from 12 to 14. For this plasmodium Craig proposed the name $P$. falciparum quotidianum.

Ziemann (1938) states that he has never been able to recognize this quotidian form and, therefore, questions its validity. Giovannola (1938) believes that further study is needed before a definite decision can be given.

P. perniciosum Ziemann, 1915. Ziemann studied a malignant tertian malaria in the Cameroons and became convinced of its separateness from the typical falciparum infection in Italy and other countries. This form was said to produce only a small quantity of dark pigment. There was complete disappearance of the parasites from the peripheral blood after ring formation. The infected cells have a "brassy" tint. The schizonts occupy only one-third to one-half the diameter of the infected cell and the merozoites number 12 to 16 . The gametocytes are smaller and are not produced abundantly.

Wenyon (1926a) states that he has studied both the European and West African types of $P$. falciparum and has frequently observed the African form to behave entirely opposite to that ascribed to it by Ziemann. As a consequence, he thinks there is little reason for accepting $P$. perniciosum as a distinct species.

Ziemann (1938) still holds for the separateness of this type but now considers it a variety or subspecies of $P$. falciparum. In this later paper he lists the characteristics again but says the schizonts produce only 10 to 12 merozoites in contrast to 12 to 16 as given by him in 1915 and that sickle forms are not seen. Also, this form is more resistant to quinine than the typical $P$. falciparum.

Raffaele and Lega (1937) described the differential characteristics of parasites which they found in some malignant tertian cases from Italian East Africa, and because these forms were shorter and broader and, especially in the males, less falciform, they proposed to recognize this type as a new variety for which they proposed the name P. falciparum var. aethiopicum. Later Giovannola (1938) concluded that the Aethiopian strain is probably identical with Ziemann's perniciosum.

$P$. wilsoni Roberts, 1940 . This recently described parasite was first seen by Dr. Bagster Wilson in the blood of two African patients from Tanganyika and has since been found by Roberts (1940) amongst all races in East Africa. According to the description, the young trophozoites are very 
compact and bear a close resemblance to $P$. ovale. The nucleus and vacuole are well defined. Multiple infections of the red cells are common; band forms are absent. The older parasites are compact and exhibit a well defined vacuole. The pigment is yellow-black and is not clumped. The "quartan-like" gametocytes are pale in color with well-distributed pigment, the females being markedly different from those of $P$. vivax. There is little cell margin around the full grown parasites. The merozoites range from 10 to 12 in number. The host cells are always enlarged after the parasites are more than one-quarter grown and this enlargement may surpass anything seen in $P$. vivax infections. A faint stippling, finer than Schüffner's dots, is sometimes present. Relapses are frequent even under intensive quinine and atabrine treatment, each attack resulting in heavy infections.

Wenyon (1941) is of the opinion, in which we concur, that although this is an unusual parasite the full proof of its specificity is still to be demonstrated.

In view of the conflicting evidence and opinions, it seems that before rejecting or accepting any of the above types it would be expedient to follow the successive life cycles through both the human and the invertebrate hosts under controlled conditions. The practice of malaria therapy allows for such a procedure.

\section{Strains or Races}

It is generally recognized that within the morphological limits of the species of malaria there are numerous strains or races. These minor groups are now recognized on the basis of studies dealing with clinical virulence, infectivity to man and mosquitoes, response to antimalarial drugs, and antigenic response. With better staining technique it may be possible later to correlate these immunological differences with now unrecognized morphological characters.

The rise of malaria therapy gave opportunity for the systematic study of these strains of human malaria under controlled conditions and much data of practical importance have been accumulated both in this country and abroad.
In this country various strains have been isolated and studied for varying lengths of time. The discussion here will be limited to those that are well established.

McCoy strain of $P$. vivax. This strain of human malaria has been intensively studied by Boyd and his associates. It was secured in 1931 from a patient living near Tallahassee, Florida, and has been maintained at the Florida State Hospital since that time. Up to August 1, 1939 (Boyd 1940a), this strain had been maintained through 47 consecutive human-anopheline passages involving approximately four hundred patients. This strain produces attacks of more than average severity, is a consistent gametocyte producer, and relapses are frequent. Boyd also shows this strain to be immunologically distinct when tested against four other strains: the White, the Wilson Dam, the Mayo, and the Cuban.

Cleveland strain of $P$. vivax. To our knowledge this is the oldest strain of tertian malaria now maintained in the United States. We are indebted to Dr. H. N. Cole of the Cleveland City Hospital for information on this strain. He reports that it was obtained from a patient in 1925 and has been propagated since that time entirely by blood inoculation. The strain has been passed through from 1,500 to 1,800 patients during the 15 years without loss of virulence.

The St. Elizabeth strain of $P$. vivax. This strain was obtained from St. Elizabeth's Hospital in Washington, D. C., in the spring of 1937 and has since been kept under continuous observation at the South Carolina State Hospital. It has occasionally been passed through mosquitoes but ordinarily it is transferred by the intravenous inoculation of 5 cc. of infected blood. When this strain and the U. S. Public Health Service quartan strain were introduced simultaneously in paretics, the vivax parasites usually dominated so that the $m a$ lariae parasites disappeared from the blood stream (Mayne and Young 1938). Up to December 15, 1940, this strain had been carried through 39 consecutive serial passages involving approximately 94 patients. It ordinarily produces at least 20 parox- 
ysms, regularly produces gametocytes, and relapses are decidedly infrequent.

U. S. Public Health Service strain of $P$. malariae. This strain was isolated in May, 1932, from a young seaman, a resident of Monroe, Louisiana, who was a patient at the U. S. Marine Hospital in New Orleans. This strain has been under continuous observation at the South Carolina State Hospital where up to December 1, 1940, it has been passed through 57 continuous serial transfers and 418 patients, by direct blood inoculation. Most of these patients were colored pareties, although the strain is also used on the white service. In patients of normal temperament and habits this strain is generally synchronous (Young, Stubbs, and Coatney 1940). In colored patients it usually produces 25 to 30 paroxysms or more and relapses are infrequent. The incubation period, using 5 ec. of blood with a moderate number of parasites, is approximately three weeks. Patients infected with this strain who experience 15 to 20 paroxysms acquire a relatively high immunity to reinoculation with the same strain; to date the heterologous immunity has not been tested.

This strain was shown to produce 3 per cent infections in A. punctipennis (Mayne 1932) ; Boyd (1940b) also reports successful transmission by $A$. quadrimaculatus. During the eight years this strain has been under observation at the South Carolina State Hospital, it has continued to produce gametocytes, and there has been no apparent change in virulence. Dr. G. C. Branch of the Veterans Administration, Tuskegee, Ala., obtained this strain from our laboratory in November, 1932. In a personal communication of December 2, 1940, he states that the strain is still maintained and has been carried through 62 consecutive passages by blood inoculation involving approximately 600 patients. Branch (1940) reported that this strain was no longer producing gametocytes. However, infected blood obtained from him in the summer of 1940 and inoculated into patients at this hospital produced typical infections with gametocytes.

This strain was sent to the New Jersey State Hospital, Greystone Park, N. J., in June, 1934, where it has been used therapeutically ever since. Dr. C. M. Sagert of that institution reports in a personal communication that up to December 1, 1940, it had been grown in 355 patients.

Jones strain of $P$. malariae. This strain was isolated from a colored patient at the Florida State Hospital in the spring of 1932. Boyd (1940b) reports the successful passage of this strain through two complete human-anopheline cycles. From one patient in the first mosquito series the strain has been propagated artificially through 18 serial passages, involving 29 patients.

The Long strain of $P$. falciparum was obtained from a colored patient at the Florida State Hospital in 1935 and has been maintained in both negro and white patients at that institution since that time. Boyd and Kitchen (1937e) report that this strain has an intrinsic incubation period, after mosquito bites, of 9 to 13 days, with a special predeliction for 11 days, while his (Boyd) Coker strain had limits of 6 to 25 days. According to Boyd (1940a) the Long strain has been carried through 19 consecutive human-anopheline passages. Also, Boyd tested this strain immunologically against the Cuban, Mexican, and Panamanian strains by inoculating these strains into Long strain convalescents and the Long strain into the convalescents of the other strains; he found the Long strain to be immunologically different from any of the others tested.

The above reports show that there are several well established strains of human malaria in this country. In addition, there are certain lines under observation which, with further study, will undoubtedly prove to be definite strains. 


\title{
THE MORPHOLOGY, LIFE CYCLE AND PHYSI- OLOGY OF PLASMODIUM MALARIAE (GRASSI AND FELETTI, 1890)
}

\author{
By MARTIN D. YOUNG and G. ROBERT COATNEY
}

U. S. PUBLIC heALTH SERVICE, COLUMBIA, S. C.

\section{Morphology, Schizogonous Cycle}

Plasmodium malariae has several characteristics which sharply distinguish it from the other human malaria parasites. The length of the asexual cycle is 72 hours; a small number of merozoites are produced; it has the heaviest staining protoplasm and produces a large quantity of dark granular pigment; the movements of the living parasite are quite sluggish.

The morphology of the parasite changes as it progresses through its life cycle. The youngest stage of the parasite observed in an erythrocyte comprises a circle of cytoplasm containing a spherical bit of chromatin and enclosing a vacuole, the so-called "signet ring stage." This ring is from onefourth to one-third the size of the infected red cell. Although this parasite has denser cytoplasm and chromatin than $P$. vivax, it it quite difficult to distinguish between them at this stage as both are approximately the same size. The distinction between $P$. $m a$ lariae and $P$. falciparum is easier; in the latter the rings assume characteristic bizarre forms and are only about half the size of $P$. malariae.

$P$. malariae grows more slowly than any of the other human malaria parasites. The vacuole disappears after a few hours. In the living condition the movement is sluggish. Pigment granules begin to appear early in its growth and sometimes a granule may be found in a late ring stage. The pigment increases rapidly and half grown parasites may exhibit 30 to 50 granules. This parasite develops more pigment than any of the other human malarias. The pigment is in irregular granules, which distinguishes it from the rod-like pigment of $P$. vivax. When the cytoplasm and pigment are together, the latter appears to be brownish-black or even jet black; however, when the pigment is seen free of the parasite cytoplasm, it often appears amber or yellowishbrown. It appears that the heavy cytoplasm of the parasite might cut out some of the light and give the pigment the dark appearance when the two are seen together. In the living parasite, the coarse pigment exhibits oscillatory motion but little directional movement.

As the parasites become larger some of the forms may be found stretched out like a ribbon across the cell, the so-called "band forms." These bands are found lying in every direction as well as that in which the smear was made, and, therefore, it can hardly be said that they are the result of the directional pressure in making the smear. Furthermore, these bands are found much more frequently in $P$. malariae than in the other species so that they are considered diagnostic. The bands may be seen at any time until the parasites practically fill the host cell. Quite frequently in these band forms the chromatin will be distributed along one longitudinal or vertical border, with the pigment in a parallel distribution on the opposite border. In such cases, the chromatin often appears dispersed instead of being in a typical concentrated mass. At other times, the chromatin and pigment may be scattered indiscriminately throughout the parasite.

As the parasite matures, its size approaches that of its host erythrocyte. This species of parasite does not cause enlargement or blanching of the parasitized red cell; in fact, sometimes the parasitized red cells seem to shrink slightly.

After about the 54th hour, multiple chro- 
matin masses begin to appear (Young, Stubbs and Coatney 1940), at which time the parasite practically fills the host cell. Occasionally precocious multiplication of chromatin occurs so that quite young parasites show multiple masses.

According to Schaudinn (1902), the chromatin masses of $P$. vivax increase by nuclear division of a mitotic type. In studying the growth stages of $\boldsymbol{P}$. malariae, we have noticed several characteristics which are not compatible with the general idea of mitosis. When stained with the Giemsa polychromatic stains, the new bodies of chromatin do not resemble the original chromatin mass. The new chromatin masses are often at opposite sides of the parasite and are much smaller than the original chromatin body, which does not suggest mitotic division. Also, the new masses do not stain as deeply or distinctly as the original mass, which suggests that they do not arise by fragmentation.

Coatney and Young (1939) found that colchicine, which arrests division at the metaphase in many types of cells, had no effect on the division of $P$. relictum in pigeons, which might suggest the absence of an achromatic figure.

Pawan (1931) using a modification of the Feulgen nuclear reaction was unable to demonstrate thymonucleic acid in the nucleus of $P$. vivax and $P$. falciparum. $\mathrm{He}$ concluded that the nuclei of these malarias differ chemically from the nuclei of certain other protozoa.

From these observations it seems that the chromatin of malaria differs in chemical composition and in method of formation from the ordinary nuclear structures.

By the 65th hour the host cell is completely filled. The principal change, during this period, is the increase in the number of chromatin masses to about five. The pigment may increase and remains seattered throughout the cell.

Between the 65th and $72 \mathrm{~d}$ hours the definitive number of chromatin masses is formed. With the appearance of the final number, the cytoplasm begins to divide so that in the end each chromatin mass is sur- rounded by a small amount of cytoplasm. These new bodies are known as merozoites. During this process of segmentation, the pigment is extruded to the cleavage lines between the daughter masses of cytoplasm so that it often appears segregated in a radial fashion, or it may be clumped in a mass which is often in the center of the cell, together with the residuum of the cleavage process. A central mass of clumped pigment, surrounded by symmetrically arranged merozoites, gives rise to the "rosette" arrangement characteristic of this species. The number of merozoites formed may be any number from 6 to 12 , with an average of about 8 .

Coincident with the maturity of the asexual parasite, at approximately 72 hours, the red cell ruptures, liberating the merozoites, pigment, residual solids and other metabolic products into the blood stream. This segmentation occurs in the U. S. Public Health Service strain of quartan malaria between 9:00 A.M. and 3:00 P.M. (Young et al. 1940). In a synchronous infection all the parasites segment in about 6 hours. The period that the merozoites remain free in the blood stream seems to be very short, for smears taken at the height of sporulation seldom reveal free forms. According to Knowles and Das Gupta (1930) the free merozoite phase is one hour in length. In our opinion, this seems to be a liberal estimate.

Immediately after segmentation, ring forms can be found in the red cells. Just how the parasite enters the erythrocyte has not been described for this species. As the ring forms are about the same size and shape as the merozoites, it seems probable that the ring forms are derived from them. Hegner (1938) found that merozoites of $P$. malariae invaded reticulocytes 9.5 times as frequently as mature red cells, supposing both types were equally available. On the other hand, Kitchen (1939a) found rings in mature erythrocytes in both greater absolute and relative numbers than in reticulocytes.

As yet there are few reports of an exoerythrocytic phase of $P$. malariae. Jerace 
(1939) in a mixed infection of $P$. malariae and $P$. falciparum found an unpigmented parasite contained in a basophilic monoblast, but this was not shown definitely to be of $P$. malariae origin. Raffaele (1940) reports finding unpigmented forms, for the first time, of $P$. malariae in the bone morrow.

\section{Sporogonous Cycle}

Gametocytes. In the blood stream the young stages of the gametocytes of $P$. malariae are similar to the asexual forms. It is virtually impossible to distinguish a gametocyte from an asexual form until about the 54th hour, at which time the asexual form begins to show multiple chromatin masses.

From the 54th to the $72 d$ hour, differentiation between the sexes of the gametocytes becomes more certain. The female exhibits a heavy deeply-staining blue cytoplasm with a small, eccentric, sharply-defined and wellstaining chromatin mass. In the male gametocyte, the cytoplasm stains a light to pinkish blue. The chromatin is diffusely scattered, sometimes occupying one-half of the cell, and stains a very light pink in contrast to the deep pink or red of the female. The pigment in both types of gametocytes remains seattered as long as the parasites remain in the blood stream.

When fully grown, the gametocytes completely fill the host cell. The gametocytes mature at or shortly after the segmentation of the asexual forms. The evidence so far indicates a cyclic development parallel to that of the asexual forms.

Ordinarily more female than male gametocytes are produced. However, this may vary with different strains as de Buck (1935) believes that the Vienna strain produces more male than female gametocytes.

Many workers believe that gametocytes are rarely produced by $P$. malariae. Our studies on induced quartan malaria do not bear this out, for we have found them at all seasons and in all types of infections. However, it is true that in absolute numbers few gametocytes are found, but it must be remembered that this species has the lowest blood stream population density of the human malarias.

\section{SPOROGONy IN Mosquito}

Because of the rarity of natural infections, the scarcity of gametocytes, and the small number of mosquitoes which become infected, relatively few observations have been made on the developmental cycle of this species, and knowledge of the sporogonous cycle is far from complete.

Maturation. When the mosquito ingests blood containing mature gametocytes, micituration of the female gametocyte occurs. This process has not been adequately observed for $P$. malariae. Following the maturation, the female gamete is ready for fertilization by a male gamete.

The matured male gametocyte ejects several "flagella" which are the male gametes. Raffaele (1939) found the gamete consists of a tapering filament containing chromatin, with a long flagellum attached at the anterior end; this flagellum is more or less adherent to the filament throughout its length.

It has been found in our laboratory that varying numbers of male gametes are formed when exflagellation occurs in a moist chamber, the number varying between two and five.

In a moist chamber, Kligler and Mer (1937) found this species to be the slowest of all to "exflagellate." Mer (1933) obtained better infections in Anopheles elutus when both young and fully-grown gametocytes were present than when only fullygrown gametocytes were found, although the latter "exflagellated" well.

Fertilization, ookinete (vermicular zygote). Although the actual process of fertilization of other parasites has been witnessed, little specific information of this process in $P$. malariae is available. The same is true of the resulting mobile ookinete or vermicular stage.

Developing oocysts. The developing oocyst is first seen as a clear oval or spherical body containing pigment, located on the outer surface of the midgut. Hylkema (1920) found oocysts measuring 5 micra 
about 50 hours after the infective feeding. The pigment was coarse. De Buck (1935) found small oocysts after six days. Mayne (1932) followed the development in $A$. punctipennis (at $72^{\circ} \mathrm{F}$. with 84 per cent humidity) in which, on the fifth and sixth days, pigmented oocysts measuring from 11 to 15 micra were found.

Many workers believe that the parasite species can be differentiated by the pigment in the young oocysts. According to Stephens and Christophers (1904) the young oocysts of $P$. malariae have rather coarse pigment which tends to clump. Gibbons (1933) believed that he could identify the species of the oocysts found in naturally infected mosquitoes. According to this author, the $P$. malariae pigment was dark brown or black, coarse and clearly visible irregular rods, usually 6 to 8 in number, and occasionally up to 12 . The pigment was arranged in a straight or curved line, in a clump or rarely scattered around the oocyst. On one occasion he found the pigment to be light brown. Christophers, Sinton, and Covell (1936) state that the younger oocysts are about the size of red blood corpuscles, the pigment has a dancing movement and resembles that seen in the gametocytes. They described the pigment as coarse and clumped; it was usually black but sometimes it was as light as in $P$. vivax or might have a greenish tinge.

Mer (1933) followed $P$. malariae through A. elutus at $23-26^{\circ} \mathrm{C}$. After 12 days the oocysts measured from 8 to 15 micra; the pigment was commonly distributed on the periphery. The oocysts were transparent and easily overlooked. Mayne (1932) after 15 days found a heavily pigmented oocyst measuring 7 micra in diameter. After 12 to 13 days at $23^{\circ} \mathrm{C}$, Kligler and Mer (1937) found oocysts in A. elutus measuring 5 to 10 miera.

Stephens and Christophers (1904) say that the species of the older oocysts (40 to 60 micra) are indistinguishable. This is in agreement with Hylkema (1920) who states that as the oocysts become larger the pigment becomes finer and then disappears.

On the twenty-first day, Mayne (1932) found unpigmented matured oocysts, with sporozoites, measuring 50 to 59 micra. Mer (1933) found oocysts up to 60 micra in diameter, with active sporozoites, after 28 days. Hylkema (1920) found mature oocysts, containing sporozoites, after 10 to 11 days which measured about 50 micra. He used mosquitoes eaught in nature, and, although he seems to have eliminated the probability of natural infections, this possibility should not be overlooked.

Most workers have found relatively few oocysts developing after experimental feedings. However, de Buck (1935) found one exceptional $A$. maculipennis var. atroparvus which had about 150 oocysts.

The oocysts do not develop at a uniform rate, even in the same mosquito. It is not surprising, therefore, that various growth rates of these developmental forms were noted in different mosquitoes under varying conditions.

Sporozoites. When fully mature, the oocysts burst, releasing the sporozoites into the body cavity. These forms then shortly invade the salivary glands.

Mer (1933) gives the following comparative sizes for the sporozoites: $P$. vivax, 8 (micra ?), P. falciparum, 9, and P. malariae, 11. In the latter the extremities were more curved and the movements more active. Mayne (1932) found the uniform measurement of around 200 sporozoites, from the glands, to be 13.2 micra.

Boyd (1935a) elaimed that $P$. malariae sporozoites are the coarsest and had the most diffuse chromatin. The diameter of the chromatin was sometimes greater than that of the cytoplasin. The extreme variations in length were between 6 and 15 micra. The length was from 10 to 30 times the diameter. While he noticed vibratory movements of masses of sporozoites in fluid preparations of the stomach, he never observed any locomotive motility in unfixed preparations from the glands. Identification of the species of sporozoites on morphological grounds, he believes, would be unreliable. Christophers, Sinton and Covell (1936) concur in this opinion.

Length of Cycle in Mosquito. It is well 
established that $P$. malariae requires the longest time of any of the human parasites to complete its sporogonous cycle. As indicated in the discussion of the developmental forms, the time reported for the complete development of the extrinsic cycle in the mosquito varies widely.

Kligler and Mer (1937) found it required 27 days at $24^{\circ} \mathrm{C}$ and 24 days at $27^{\circ} \mathrm{C}$ to complete the cycle in $A$. elutus. Iyengar (1932) found sporozoites in glands after infective feedings as follows: $A$. ludlowi var. sundaica, 21 days ; $A$. stephensi, 10, 14 and 19 days.

Hylkema (1920) found the developmental period in A. ludlowi to be 11 to 13 days. De Buck (1935) found glands infected with sporozoites in 15 days at $27^{\circ} \mathrm{C}$ and a relative humidity of 90 . Mayne (1932) found sporozoites the twenty-fifth day after feeding; Marotta and Sandicehi (1939) in 24 days.

Transfer to Man. After the sporozoites of $P$. malariae pass into the tissues of man by the bite of an infected mosquito, the development of the disease usually requires a longer period than in the other species. Knowles and Senior-White (1927) give this as to 18 to 21 days. Boyd (1935, 1940) found the prepatent period of the parasites to be from 28 to 37 days, the paroxysms occurring a few days later. De Buck (1935) found the "intrinsic" incubation period to be from 24 to 25 days in several cases. Usually the symptoms occurred a few days later. Mer (1933) found that parasites appeared in the blood stream from
26 to 31 days after the bite of infected mosquitoes. Hylkema (1920) gave 12 days as the incubation period in a self-inflicted infection. However, as he was bitten on successive days, there seems to be some question as to the accuracy of this estimate. Marotta and Sandicehi (1939) found the incubation period in two experimentally infected patients to be 23 and 29 days.

\section{Physiology}

This is probably the most neglected field in human malariology, with the result that very little is known about the life functions of these important disease producers.

There are general statements that residues result from the metabolic processes of the parasites and that these, when released into the blood stream, contribute to the paroxysmal attack. There is little real evidence to support this view. There is still uncertainty whether the parasites produce toxins at all, and that if they do, these toxic products initiate the paroxysm.

Comes (1922) concludes that in P. vivax and $P$. malariae the pigment is formed by the activity of the alimentary vacuole under the influence of the adjacent nucleus. If it can be assumed that the malarial pigment of $P$. knowlesi and of the human parasites are similar, it would appear from the work of Sinton and Ghosh (1934a and 1934b), Ghosh and Sinton (1934), and Ghosh and Nath (1934) that the pigment of the $P$. malariae parasite originates from the hemoglobin of the infected red blood cell by direct metabolic action of the parasite. 


\title{
THE MORPHOLOGY, LIFE CYCLE AND PHYSI- OLOGY OF PLASMODIUM VIVAX
}

\author{
By REGINALD D. MANWELL \\ DEPARTMENT OF ZOOLOGY, SYRACUSE UNIVERSITY, SYRACUSE, N. Y.
}

OF the three generally recognized species of human malaria, Plasmodium vivax is by far the most widespread and probably accounts for the majority of cases of malaria infection. It occurs over a large part of the tropical, subtropical and temperate regions of the earth, and may be regarded as perhaps the most important of all the causes of disease to which man is heir. Thus it is this species which is largely responsible for Osler's (1923) statement that malaria would probably be voted "the greatest single destroyer of the human race" by those who know most about disease, although it is not the most death-dealing of the three. It is, however, the cause of an infection which is both ehronic and disabling, and which at any one time is responsible for millions of cases of illness. Just as this is true today, so we can be quite sure that it has been since before the dawn of recorded history, although as facilities for human travel have increased the range of malaria has no doubt inereased also.

In view of the importance of malaria it is rather surprising that the three varieties of the parasite were apparently not clearly separated until Golgi did so in 1885 . He discovered the relationship between the symptomatology and stages in the reproductive cycle of the parasites, and soon afterward (1886a) found that the parasite of tertian malaria differed from that of the quartan variety. The species name vivax was proposed by Grassi and Feletti in $\mathbf{1 8 9 0 .}$ In this paper the morphology and what is known of the physiology of the tertian malarial parasite will be discussed.

\section{Morphology, Schizogonous Cycle}

The appearance of the living parasites presents nothing of great interest, and it is no doubt for this reason that they remained so long undiscovered. The younger forms appear as small hyaline areas, or simulate vacuoles in the erythrocytes which, as growth of the parasite takes place, soon exhibit granules of yellowish-brown pigment (hemozoin). They also show a very active amoeboid movement, and this peculiarity suggested the name vivax. As the parasites increase in size however this movement becomes less perceptible, until finally it almost ceases.

Malarial parasites are, however, generally studied in stained preparations, the method used being some modification of that devised by Romanowsky. The most commonly used stains are those of Giemsa or Wright. When thin blood smears are stained by one or another of these methods, the youngest parasites appear as minute masses of rather heavily stained protoplasm (Fig. 1) in which there is relatively little differentiation of chromatin and cytoplasm. The former usually appears reddish or some shade of violet, and the latter has a bluish tint. In older parasites the differentiation is more marked. After entering another erythrocyte (apparently in this species the young parasites prefer reticulocytes-Eaton 1934; Jacobsthal 1936; Kitchen 1938) the parasite soon develops a central vacuole which gives it the appearance of a ring, on the periphery of which the chromatin appears as a small granule, or sometimes as two granules. Rings of the latter sort have been interpreted as evidence of the occasional occurrence of binary fissio (Beach 1936), not only in this but in other speciés of parasites, both human and avian. These ring-shaped parasites may take on a great variety of shapes as they increase in size, with their pseudopodial processes extended here and there throughout the cell (Figs. 
2-6). Eventually, however, if the parasite is destined to reproduce asexually, the chromatin divides twice and finally into many small masses, and in the latter condition is generally known as a segmenter. Fig. 7 shows an immature schizont, and Fig. 8 one almost completely mature. The young parasites produced as a result of the multiple division process are known as merozoites. The number produced by a single segmenter (Fig. 9) varies between 12 and 24 , with an average of about 16 . It is probable, however, that both the range and the average may vary, for it has been shown that different races of plasmodia exist within a species, and also (in avian malarias) that larger numbers of merozoites are produced by segmenters early in the course of an infection than are produced later (James and Ciuca 1938; Boyd 1940a). The schizogonous cycle requires 48 hours.

\section{Sporogonous Cycle} (In man)

Most of the merozoites enter other red cells and repeat the process of growth and reproduction. There are some, however, which grow but exhibit no evidence of nuclear division, and these are the sexual stages, or gametocytes. The forms can be distinguished from reproducing forms of similar size by the presence of a single mass of chromatin which, in the female or macrogametocyte, is compact and usually situated near the margin of the parasite. In the male, or microgametocyte, it is more diffuse and stains less intensely. The cytoplasm of the female forms takes a deeper blue color, and contains larger and more numerous granules of pigment than that of the microgametocyte. In both male and female cells the pigment granules are scattered, in contrast to the asexual parasites. The cytoplasm of the microgametocyte usually takes a relatively pale bluish tint, but it may also appear a marked purple or lilac (Craig 1928, quoting St. John). When the cytoplasm stains in this way it is quite possibly because of greater maturity. Gametocytes of both sexes are shown in Figs. 11 and 12. They are unable to de- velop further unless ingested by a female mosquito belonging to the genus Anopheles. The distinguishing characteristies of the species, and also of the different stages are tabulated in Table I.

TABLE I

Distinguishing Characteristics of Plasmodium vivax

(in Romanowsky-stained preparations)

\begin{tabular}{|c|c|}
\hline $\begin{array}{l}\text { Characters per- } \\
\text { taining to }\end{array}$ & Species character \\
\hline Rings* & $\begin{array}{l}\text { Usually larger than falcipa- } \\
\text { rum; smaller than malariae; } \\
\text { stages other than rings also } \\
\text { present. }\end{array}$ \\
\hline Trophozoites & $\begin{array}{l}\text { Markedly amoeboid in appear- } \\
\text { ance; strap-like or band- } \\
\text { shaped forms seldom seen; } \\
\text { present in peripheral blood. }\end{array}$ \\
\hline Schizont & $\begin{array}{l}\text { Larger than in other species } \\
\text { and present in peripheral } \\
\text { blood. }\end{array}$ \\
\hline $\begin{array}{l}\text { Number of mero- } \\
\text { zoites per seg- } \\
\text { menter }\end{array}$ & $12-24$; average about 16 \\
\hline \multicolumn{2}{|l|}{ Gametocytes: } \\
\hline Mierogametocyte & $\begin{array}{l}\text { Rounded; larger than in ma- } \\
\text { lariae. Smaller than female } \\
\text { form; nucleus diffuse and } \\
\text { often marginal; eytoplasm } \\
\text { takes pale-bluish stain (or } \\
\text { may appear lilac or pur- } \\
\text { ple); pigment seattered, } \\
\text { granules appear as short } \\
\text { rods. }\end{array}$ \\
\hline Macrogametocyte & $\begin{array}{l}\text { Rounded; larger than in ma- } \\
\text { lariae. Larger than male } \\
\text { form; nucleus more compact } \\
\text { and often marginal; cyto- } \\
\text { plasm stains a deeper blue; } \\
\text { pigment seattered and more } \\
\text { abundant than in micro- } \\
\text { gametocyte, granules appear } \\
\text { as longer rods. }\end{array}$ \\
\hline Effect on host cell & $\begin{array}{l}\text { Markedly enlarged; partially } \\
\text { decolorized (especially in } \\
\text { unstained preparations); } \\
\text { Schüffner's dots usually } \\
\text { present. }\end{array}$ \\
\hline
\end{tabular}

* Identification cannot usually be safely attempted from the rings alone.

At this point the question as to what determines the fate of a given merozoite may well be asked. Why do some give rise 
MALARIA

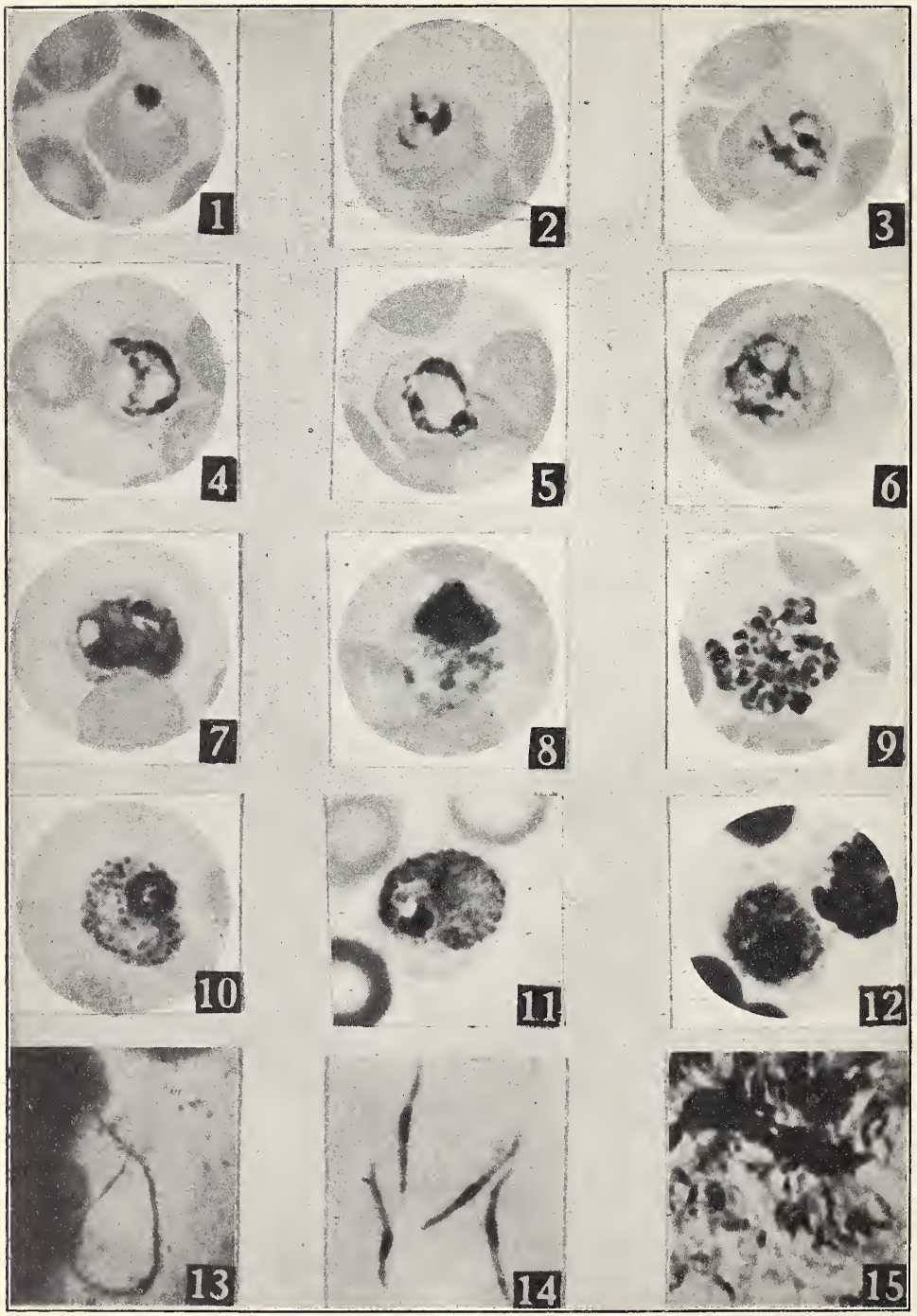


to more reproductive forms, with the result that in certain individuals the infection may persist for three years ${ }^{1}$ or even longer, when others exhibit no capacity for reproduction at all, and develop into gametocytes?

\section{Gametogeny}

This question cannot be satisfactorily answered at present, but Boyd (1935b) has made an interesting study which may throw some light on the problem. He believes that parasites occurring in the blood may be divided into five series, differing morphologically in minor ways. From one series, into which most forms fall, come merozoites of three types. One type, which is produced in the largest numbers, is destined to go on and repeat the reproductive cycle. The two other types also go through the process of schizogony, but the merozoites they produce become gametocytes-

1 It is believed that vivax infections do not persist more than three years in default of fresh infection, even when untreated. Exceptionally they may possibly persist longer. the male forms arising from one type and the female from the other. The developmental stages of each of the latter make up the other two series. Fig. 19 is taken from his paper, but with some changes to indicate the relationship of the series to one another. It may be noted that although parasites of the different series differ somewhat in morphology, all of them require 48 hours for maturation. ${ }^{2}$ The factors which cause certain merozoites to develop in one direction rather than another still remain, however, quite obscure.

2 In this regard Boyd's conclusions are not in accord with the generally accepted belief that gametocytes require 96 hours for development. Boyd also expresses the opinion that the sexual forms probably do not live more than 24 hours. It is difficult to devise experiments to determine exactly the life of the sexual forms, but it has been thought that they live considerably longer than this. It is also usually stated that the gametocytes go through a large part of their development in the eapillaries of the spleen and bone marrow ( $c f$. Wenyon 1926; Manson-Bahr 1931). The relationship of the gametocytes to forms belonging to the pigment-free eycle is also a problem which may need future investigation.

Explanation of Plate I

1. A very young parasite.

2. A somewhat older form. The characteristic pseudopodial processes are very evident.

3. A trophozoite of moderate size. The enlargement of the host-cell, which is invariable with this species, is already evident.

4. A trophozoite showing the large vacuole which very frequently characterizes growing parasites of this species.

5. Here the vacuole gives the parasite the appearance of a large ring. Smaller rings are also very often seen. In this parasite, and also in those shown in Figs. 4 and 6 , the three granulee which, according to Boyd, are characteristic of forms destined for ordinary schizogony, may be seen. (See Series A in Fig. 19, Plate II.)

6. A large trophozoite exhibiting conspicuous pseudopodial processes.

7. A young sehizont in which the nucleus has divided onee.

8. An older sehizont. The two ehromatin masses on the left are in division. In the original preparation the division figures were very clear, and resembled spindles, although nothing having the appearance of ehromosomes could be seen.

9. A mature schizont containing apparently 17 merozoites. Sixteen is generally regarded as the typical number.

10. A trophozoite probably destined to become a gametocyte. The Schüffner's dots are very conspicuous.

11. A macrogametocyte. Note the rather small and compact nucleus.

12. A microgametocyte next to a schizont. The large and relatively diffuse nueleus is very clear.

13. A microgamete.

14. Sporozoites.

15. Sporozoites within the salivary glands of a mosquito. Careful observation will show several with a divided nucleus (indicated by arrow).

Magnification in Figs. $1-10,12,15$ is 2000 diameters; in Figs. 11, 13, 14 it is 2700 diameters.

Figs. 11, 13 and 14 are from mierophotographs by Dr. Mark F. Boyd, to whom the author desires to express his appreciation for their use. Fig. 12 is copied from one of Dr. Boyd's photographs. The others are from the author's own preparations and were made by Miss Stella Zimmer of the Department of Photography, Syracuse University Medical Sehool. 

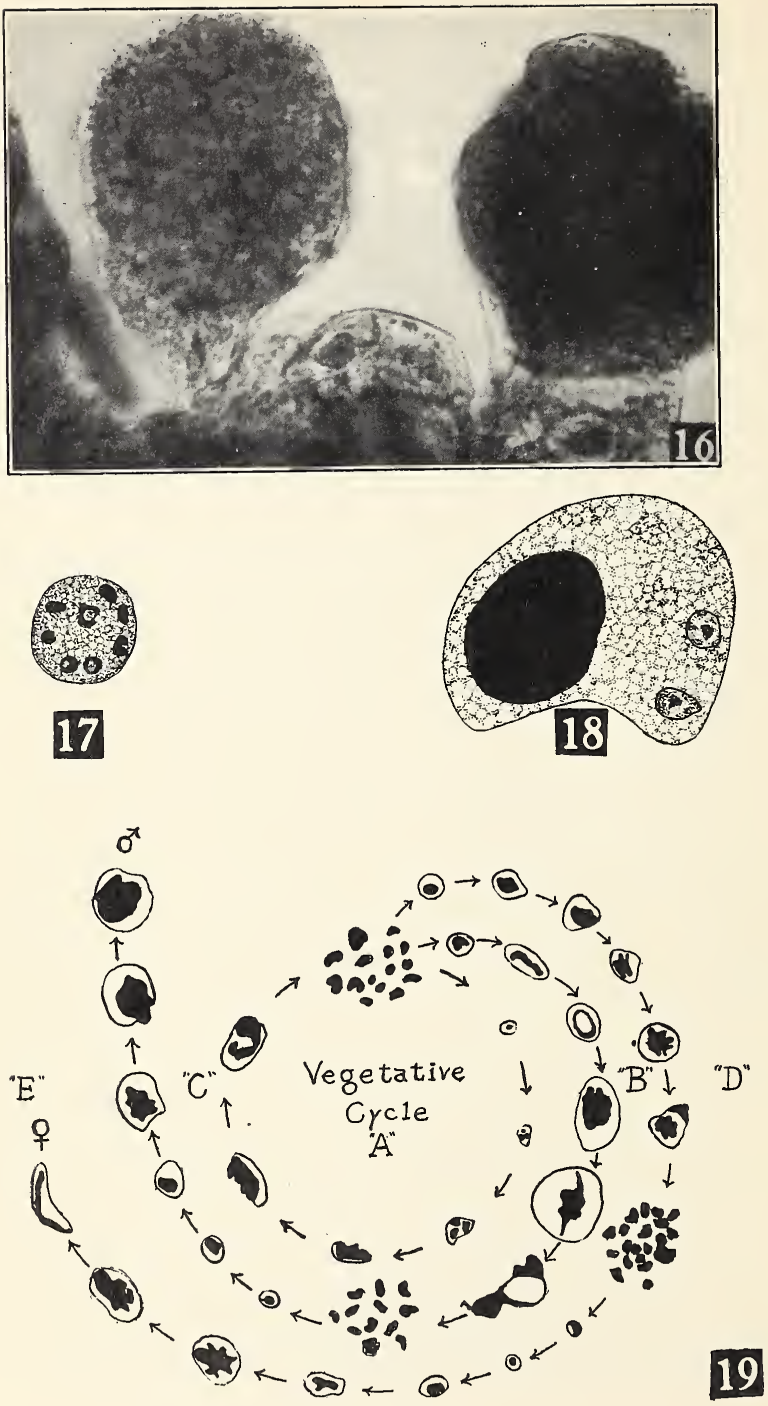


\section{Mitosis}

Another question which may be asked concerns the mechanism of division. Is it mitotic or otherwise? This is also still an unsettled problem, although Schaudinn many years ago (1902-03) deseribed a modified mitosis in $P$. vivax. Several other authors (e.g., Ivanic 1935, 1937) have made the same elaim since. Unfortunately, however, malarial parasites are difficult to stain with dyes such as haematoxylin, and the dyes ordinarily used do not give results good enough for careful cytological study, particularly when the preparations have been made from air-dried smears. Obviously the very small size of malarial parasites (especially in sections) adds to the difficulty. In this connection reference may be made to studies on the Feulgen reaction of the malarial parasites, particularly those of Pawan (1931), Jirovec and Cerny (1932), Breindl and Jirovec (1932), and Ungo-Mugdan (1938). Pawan found that thymonucleic acid (nuclein) was absent, thus differentiating the malarial parasites from most other protozoa. Breindl and Jirovec demonstrated the presence of this form of nuclear chromatin in dividing forms ( $P$. falciparum) and sporocysts and sporozoites (P. relictum), while UngoMugdan found the reaction slightly positive in schizonts and strongly positive in exoerythrocytic stages of $P$. gallinaceum. While these results do not agree, they do suggest that the nucleus of the malarial plasmodia may be different in nature from that of other protozoa during much, if not all, of the life-history, and perhaps the difficulty with which the parasites take ordinary stains is due to this fact.

\section{Effect of Parasites on Host-Cell}

There is also the matter of the way in which parasites of the species under consideration affect the host erythrocyte, and the question of whether the plasmodia are within or on the surface of the cell. It is now almost universally believed that they are intracellular, and all that is known of the behavior of the parasites is eonsistent with this view. Rateliffe (1927) showed that when very thin sections were made of clots of parasitized blood the parasites generally appeared within the cell boundaries, and this was true not only in the case of Plasmodium vivax but of $P$. falciparum and $P$. praecox (relictum) also. Additional evidence for this conclusion is the fact that the anti-malarial drugs of proved value seem to possess a favorable partition coefficient in all cases in which the problem has been studied (Hegner, Shaw and Manwell 1928; Shaw 1928). They are, in other words, substances which are capable of selective absorption by the red cell.

The effect of the parasite on the cell which it invades is pronounced and very eharacteristic in the case of $P$. vivax. Even while the plasmodium is still small there often appear the well-known Schüffner's dots in the cytoplasm of the host cell (Fig. 10). These are ehromophilic particles which take a reddish tint when stained by Giemsa, or one of the other Romanowsky stains. What the nature of these dots is remains uncertain, but it has been suggested that they are of reticular origin, perhaps arising from basophil granules. In any case they are found only in vivaxinfected erythrocytes, although they may be occasionally absent. It is said by some

\section{Explanation of Plate II}

16. Several oöcysts on the gut wall of a mosquito. Magnification 1200 diameters.

17. A multinucleated body found in a bone marrow smear of the sternum of a patient infected with Plasmodium vivax. This and the following figure are redrawn from Raffaele.(1937), who interpreted them as pigment-free stages corresponding to those previously found in various species of avian malaria. He states that they occurred in cells of the reticulo-endothelial system.

18. Two young trophozoites in a reticulo-endothelial cell.

19. This schema shows the relationship of the five series of parasites belonging to the erythrocytic cycle, according to Boyd (1935b). The individual figures have been redrawn from those of Boyd, and indicate the various types of nuclei characterizing the parasites of the different series. The arrangement and the diagrammatic figures of schizonts are the author's. 


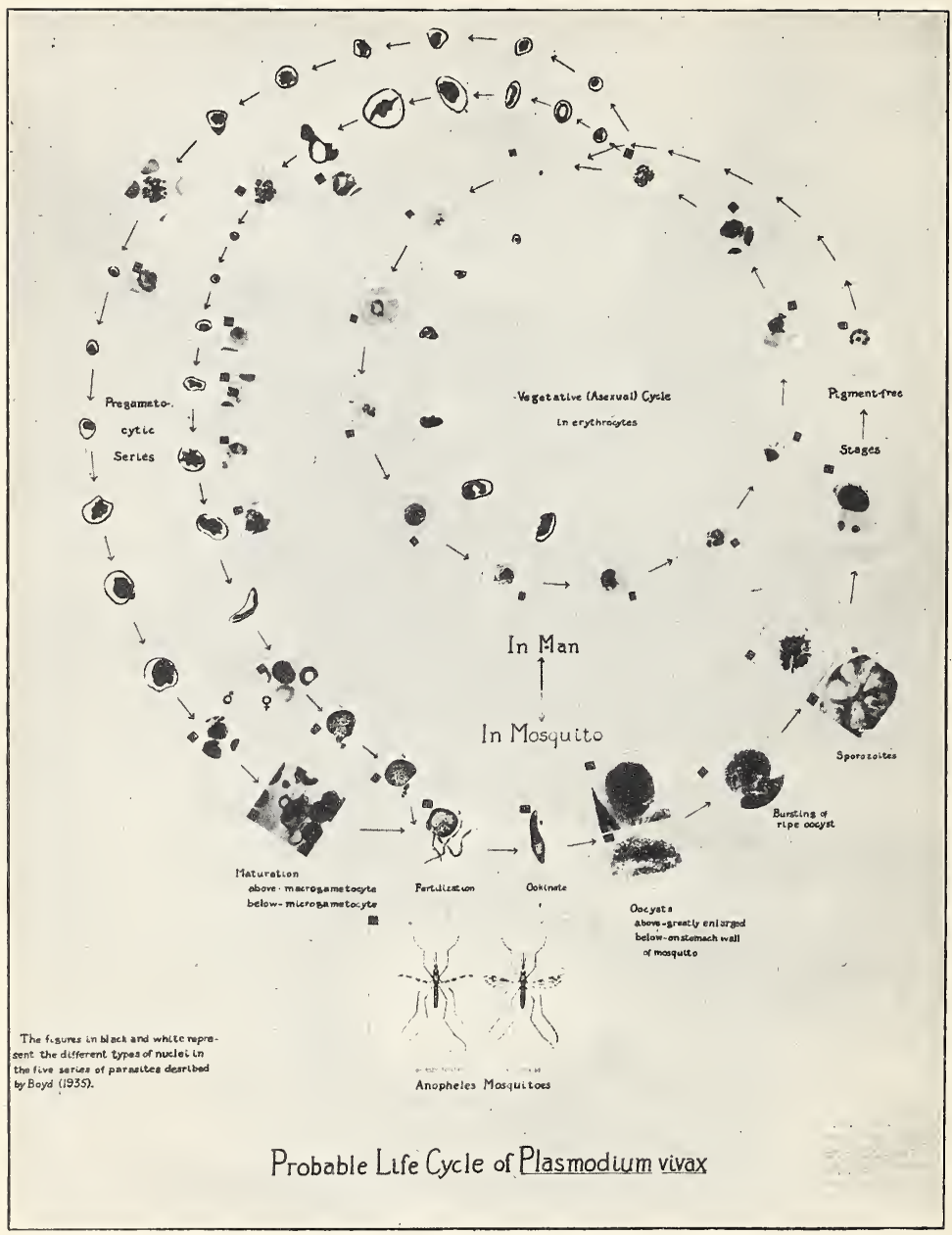

This plate is intended to show the relationships of different stages in the life-cycle of $P$. vivax. The figures are taken from various sources, especially Boyd (1935b), Schaudinn (1902) and Raffaele (1937). The schema is different from the conventional one in that it includes the five series described by Boyd (1935b) as occurring in the blood of man, and the pigment-free stages described by Raffaele (1937). 
authors that when they are not seen the fault is with the staining, but this is certainly not always true.

A second change in the host cell is a very considerable enlargement which becomes apparent even while the parasite is still relatively small, and may continue until the normal size is doubled (Fig. 3). Along with the increase in size and the appearance of the Schüffner's dots is a progressive decoloration. These three changes in the infected red cell are diagnostic of infections with $P$. vivax. A fourth peculiarity of this species is the relatively lighter shade of brown exhibited by the pigment.

\section{Sporogonous Cycle}

(In mosquito)

As in the case of the other species of human parasites the final, or definitive, host is some species of anopheline mosquito In the mosquito only the gametocytes can survive the processes of digestion, and these begin the changes known as maturation very soon after ingestion. Darling believed that at least 12 gametocytes per cubic millimeter must be present in the blood to make the patient infectious for mosquitoes, but others have found that infection may take place when there are fewer than this (Craig 1928). It is a remarkable fact that even in insusceptible species of mosquitoes maturation of the gametocytes can apparently take place, and that it will also take place in vitro, as for example under the microscope. Indeed it was the observation of the phenomenon known as " exflagellation," or microgamete formation, which convinced Laveran that the pigmented bodies he saw in the blood of malarious persons were in reality parasites. What the factors are which govern this process are still very incompletely known, but the work of Marchoux and Chorine (1931) on maturation and fertilization in Hemoproteus paddae suggests that the $\mathrm{pH}$ of the medium is the controlling factor, and that this in turn is primarily determined by the amount of carbonic acid present. The process in this parasite is stopped by a pH of 7.3 or less, but proceeds normally between a $\mathrm{pH}$ of 7.6 and 9.0 .

Maturation of the microgametocyte involves the production of from 4 to $8 \mathrm{mi}$ nute flagellated bodies which are then known as microgametes (Fig. 13). Raffaele (1939) has described the process in some detail, and states that each microgamete consists of two elements, a cytoplasmic flagellum and a thin filament containing the fertilizing chromatin. These generally adhere for their entire length. When maturation is completed, all that remains of the parent microgametocyte is a small amount of eytoplasm and chromatin, with a mass of pigment. The entire process is easily observed under the microscope, and will usually take place within a short time after the blood is drawn (often in 10 to 20 minutes). Flagellating microgame, tocytes are recognized by the violent disturbance which the developing gametes cause as they endeavor to leave the parent cell.

The changes involved in the maturation of the macrogametocyte are no doubt as profound as those just outlined, but they are less dramatic and less carefully worked out. After rupturing the wall of the host erythrocyte the gametocyte becomes rounded, and it is said to throw off one or two tiny masses of protoplasm which are generally supposed to represent polar bodies. Schaudinn studied the process in $P$. vivax, and also believed that he saw parthenogenesis of the macrogametocyte, but in this he was quite certainly mistaken.

Fertilization takes place when maturation is complete, and the zygote (or oökinete, as it is now called) makes its way by worm-like movements into the intestinal epithelium. Between the epithelium and outer wall of the gut it becomes rounded and is soon seen to be surrounded by a cyst wall. All this may require from 24 to 48 hours, the time for these and subsequent changes depending chiefly on the temperature. $^{3}$ It is said that for $P$. vivax the optimum temperature for development in the mosquito is $25^{\circ}$ C., although development 
may go on slowly even at $15.5^{\circ}$ C. ${ }^{4}$ Growth now begins, and the öocyst (Fig. 16) multiplies its size many times, reaching a diameter of 50 micra, or even more. Microscopically, the ehromatin may be seen to divide repeatedly until by the time maturity is reached there are almost countless fragments. Each of these then appropriates, so to speak, a bit of cytoplasm, and the whole oöcyst breaks up into minute elongated forms known as sporozoites. These are set free in the body cavity of the mosquito, but eventually some of them reach the salivary glands and are then ready to infect a new host. The sporozoites resemble the microgametes somewhat, but do not possess a separate flagellum, and often appear to have a nucleus made up of several masses of chromatin (Figs. 14 and 15). There may be one or many oöcysts in a single mosquito, depending on the number of gametocytes in the ingested blood, so that the number of sporozoites produced may be extremely large. Yet there is no reason to think that the malaria infection appreciably shortens the life of the infected mosquito.

The subsequent history of the sporozoite is still in doubt, for it is now quite certain that it does not enter a red cell immediately after reaching a new host. Gordon and Lumsden (1939) studied the mechanism of biting in Culex mosquitoes, and found that blood might be taken either from a pool resulting from injury to adjacent capillaries, or (more often) directly from a pierced capillary. Saliva from the mosquito was injected at intervals during the feeding, so that contained sporozoites would probably be ejected directly into the capillaries and carried to remote situations very quickly. Boyd and Kitchen (1939) confirmed this conclusion in indirect fashion when they found that even imme-

3 The time required for the completion of that part of the eycle oceurring in the mosquito is from one to two weeks in the ease of $P$. vivax.

4 Stratman-Thomas (1940) states that it will not go on at temperatures above $30^{\circ} \mathrm{C}$., and that 24 hours at $37.5^{\circ} \mathrm{C}$. will sterilize nearly all $A$ nopheles quadrimaculatus. He also says that in some regions oöeysts might survive the winter. diate excision of the tissues about the area bitten failed to prevent the development of malaria later. Further, the blood of persons bitten by vivax-infected mosquitoes has been shown by Raffaele (1937) to be non-infectious to other persons until after the fourth day, and he made similar observations in the case of $P$. relictum. That the sporozoites might go through further developmental stages was suggested by Knowles and Basu (1935), as a consequence of the observation that these forms often showed evidence of division while in the mosquito. Missiroli (1934) showed that the sporozoites of $P$. relictum may pass through several divisions after injection into the canary, and recently Schuleman and Spies (1940) have shown this to be true of $P$. gallinaceum also. Whether still another stage intervenes in the cells of the reticulo-endothelial system before the erythrocytes are invaded is still a matter of controversy, and the answer may well be different for the different species of plasmodia.

\section{The Possibility of aN Exoerythrocytic CyCle}

When pigment-free stages were first discovered in certain of the species of avian malaria it was thought that similar stages might be found to exist in some or possibly all of the other species of plasmodia, including those of man, and as a result an energetic search has been made by a number of investigators. So far, however, exoerythrocytic stages have been reported only in P. vivax (Raffaele 1937) (Figs. 17 and 18) and $P$. falciparum (Casini 1939), while their occurrence in these species has not been confirmed by others. Raffaele interpreted them as forms derived from the sporozoites and believed that they gave rise to parasites of two series, the one an unpigmented series occurring in cells of the reticulo-endothelial (lymphoid macrophage) system, and the other the familiar erythrocytic series. Casini's observations were made on a chronic case. In birds such stages are usually seen during the acute stage, but may also be seen (especially in 
certain species) after parasites have largely disappeared from the blood. We may therefore believe that the exoerythrocytic eycle may be originated from the sporozoites, ${ }^{5}$ and that it may continue, presumably at a low level, and possibly play a part in the genesis of relapse (Schuleman 1940). But there is also a strong possibility that even in those species of avian plasmodia, in the cycle of which it occurs most regularly and can be most easily demonstrated, the occurrence of exoerythrocytic schizogony may not be a usual thing but dependent on a relative failure of the immune mechanism. As such it may be chiefly significant as an expression of what might be called the biological potentialities of the parasite, and as an indication of the close relationships of the malarial plasmodia to the Hemoproteidae. If this is true we may have to look still further for stages bridging the gap between the sporozoite and the forms seen in the red cells.

\section{Physiology}

It is more difficult to give an account of the physiology of the malarial parasites than to discuss their morphology. This is largely because there is still no practicable method of cultivation, although the method devised by Bass and Johns (1912) makes it possible to maintain the parasites for a limited time in vitro. The fact that the addition of dextrose is required for such cultures suggests that the parasites need this substance for their vital processes, presumably as a source of energy. Further support for this belief may be had from the work of Christophers and Fulton (1939) and Fulton (1939), in which it is shown that the addition of dextrose to the medium causes an increase in the oxygen consumption of $P$. knowlesi. They also found that a number of other sugars (including laevulose, mannose, maltose, and glycerol) were

5 Although it is of course necessary to suppose that when exoerythrocytic stages occur they must arise directly or indirectly from the sporozoites, yet it is now quite certain that they may also originate from the pigmented forms occurring in the erythrocytes (Coulston and Manwell, in press). utilized by the parasites, and they remark that all of these have a "common chemical grouping in their molecule which does not include a ketonic or aldehydic group." They found no clear evidence of the phosphorylation of dextrose in knowlesi infections in monkeys. Whether any conclusions applying to the parasites of human malaria can be drawn from such experiments is somewhat doubtful. We can be quite sure that with the factors which make the different species distinctive in morphology and host go others, no less real, making for similar differences in physiology. An example is the demonstration by Coggeshall (1940a) that the oxygen consumption of $P$. knowlesi is six times as great as that of $P$. inui. Such characteristics as these are probably important in interpreting the effects of therapeutic drugs.

Apparently the globin fraction of the hemoglobin molecule is also utilized by the parasites, for hemozoin, or malarial pigment, seems to be derived from what remains. This substance seems to be very similar to, or perhaps identical with, haematin (Sinton and Ghosh 1934b; Ghosh and Nath 1934). The formula of haematin is variously given but $\mathrm{C}_{33} \mathrm{H}_{32} \mathrm{~N}_{4} \mathrm{O}_{4} \mathrm{FeOH}$ may be taken as at least a close approximation. Since, however, the color and form of the pigment produced by the different species vary somewhat, it is probable that the composition is not always the same. The work cited above was done with $P$. knowlesi, a species of monkey plasmodium, and is probably more significant than most such investigations previously made because the pigment was extracted directly from the parasites rather than from tissues, and because it was not previously exposed to the action of fixatives.

There is as yet no agreement as to whether there is anything in the nature of a toxin produced by any of the species of malaria parasites. The pathological changes characteristic of malaria can for the most part be quite easily explained on other grounds.

The problem of the effect of the parasite on the host is to a certain extent tied up 
with the effect of the host on the parasite. The immunology of the malarial organisms will be discussed elsewhere, but it may be pointed out here that so far only man has been found susceptible to $P$. vivax, although there are very similar species occurring in monkeys and some other mammals. What the factors are in the physiology of host and parasite which make for so strict a host-specificity would make a very interesting study, but are so far quite unknown.

Some of the most significant of the recent work on malaria has been concerned with the factors responsible for the remarkable periodicity exhibited in the asexual cycle by many of the species of malarial plasmodia. It was shown first from work on birds and later by similar experiments on monkeys (L. G. Taliaferro 1928; G. H. Boyd 1929; L. G. and W. H. Taliaferro 1934) that diurnal variations in the physiology of the host somehow cause the parasite to sporulate at certain definite times. When the length of the day is artificially changed the length of the cycle of the parasite. soon exhibits a corresponding change. It is of course obvious that there is some- thing in the genetic constitution of a given species of plasmodium which determines the way in which it will be affected by variations in its environment caused by the metabolic activities of the host, and thus we have illustrated the very delicate and intricate nature of the relationship which exists between host and parasite. We may feel fairly confident, though of course we cannot be sure, that the human species of parasites, including $P$. vivax, would be found to exhibit similar changes in periodicity with a similar alteration in conditions. It is stated by Hewitt (1940) in his recent monograph on bird malaria that Young, Coatney and Stubbs (unpublished) have found that periodicity may be reversed in $P$. malariae infections by reversing the host's period of rest and activity. On this point, as on many others, we see that more research is needed. It is indeed a rather remarkable fact that so many problems of fundamental importance relating to the malarial parasites of man remain unsolved, especially when we recall the place of leadership among human diseases which malaria holds. 


\title{
THE MORPHOLOGY, LIFE CYCLE AND PHYSI- OLOGY OF PLASMODIUM FALCIPARUM
}

\author{
By S. F. KITCHEN \\ INTERNATIONAL HEALTH DIVISION, ROCKEFELLER FOUNDATION, TALLAHASSEE, FLA.
}

\section{MORPHOLOGY}

The schizogonous and sporogonous eycles of this parasite are depicted in Fig. 1.

Reproduction of this parasite is accomplished in two ways, either by an asexual or by a sexual process. There is no acceptable proof of the occurrence of binary fission or parthenogenesis among the malaria plasmodia.

\section{Schizogonous Cycle}

Asexual reproduction of the plasmodium occurs through schizogony in the intermediate host, man. This type of multiplication involves repeated division of the nucleus, with division of the eytoplasm about each daughter nucleus. Following release of the merozoites, or daughter cells, those which survive attach themselves to erythrocytes and develop in the peripheral circulation until they are somewhat more than half grown. They then accumulate in the capillaries of the viscera, and here undergo schizogony. Thus, less than half of the schizogonous eycle is spent in the peripheral circulation and the parasite may go through an indefinite number of such asexual cycles.

Trophozoites. The merozoites, the youngest forms of this series, seldom in our experience, find their way into the peripheral circulation. They are smaller than in the other species and usually exhibit no vacuole; their diameter hardly ever exceeds one micron and generally it is less than that. Young "ring forms" are usually the first to be found, and it is these that are frequently characteristic of this species of plasmodium. They are often quite delicate in appearance, the cytoplasm consisting of a slender circle of blue cytoplasm (Wright or Giemsa stain), sometimes so fine that it can barely be seen, enclosing a vacuole. The chromatin, too, is commonly characteristic.
It may be present as a small red dot which appears to be placed upon the outer surface of the ring. On the other hand it may take the shape of a small red bar, bent or straight, the thickness of which is greater than that of the thread-like eytoplasm. Not infrequently the size of the dot, however, appears quite out of proportion to the slenderness of the band of cytoplasm. Often (and practically diagnostic of this species when present) the chromatin is multiply represented by two or more small dots which may lie closely together or on opposite sides of the small, fine eytoplasmic ring. Characteristically, too, is the common observation of two or more, possibly as many as five or six, rings in one erythrocyte. Not infrequently these young forms are found, as it seems, "applied" to the circumference of the erythrocyte so that one may see in the stained smears only a short, thin streak of blue cytoplasm seemingly lying on the periphery of the red cell and surmounted by a red dot of chromatin, hence the terms, "accolé" or "appliqué." Occasionally the thread of cytoplasm will be seen to be looped over an indentation in the outline of the erythrocyte. Such appearances naturally raise the perplexing question (a long standing but still unsettled one) as to whether the young parasites are intra- or extracorpuscular. A vacuole is not evident in these forms. As the parasite grows, one notices a thickening of the cytoplasm, which usually takes place first almost opposite the position of the chromatin dot. With the increase in size of the parasite, the amount of cytoplasm increases and the whole ring gradually thickens, though not necessarily symmetrically. Too, with the increase in amount of cytoplasm, irregularities in the shape of the parasite become apparent. The originally almost perfectly spherical 


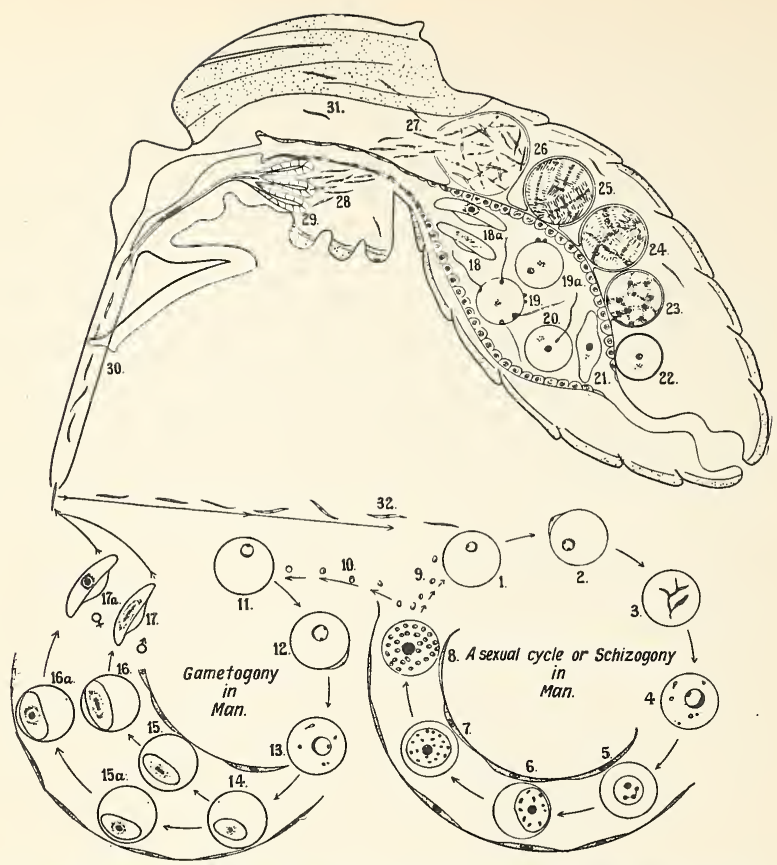

FIG. 1. The sporogonous and schizogonous eycles of Plasmodium falciparum. (From Protozoology by Thomson and Robertson through courtesy of Williams and Wilkins Company, publishers.)

shape is now altered by indentations or outpouchings, and pigment granules appear.

The erythrocytes infected with this parasite, unlike vivax-infected red cells, do not enlarge as the parasite grows; if anything, they tend to shrink in size. Neither do they show the same alterations, viz., Schüffner's granules. They do, however, exhibit another type of stippling known as Maurer's dots or Maurer's elefts. These differ from Schüffner's granules in that their incidence is usually not so common, they are generally darker, more of a brick red shade, are not usually as numerous, are quite irregular in size and shape, and are often larger. It frequently requires a somewhat longer staining period or stronger staining solution than usual to bring them out. They are said to be more commonly present during the afebrile stage of the infection, which would suggest their association with older parasites. Undoubtedly they represent a change in the erythrocyte effected by the growing parasite but the mechanism of this is not yet understood. The infected cell is often observed to stain more intensely.

Actually these larger and irregular socalled "ring" forms are probably equivalent in age to the somewhat less than halfgrown vivax parasites which we term amoeboid forms. Thomson and Robertson (1929) state that the trophozoites remain "in the peripheral blood in the ring form for at least 18-24 hours, possibly longer." Prior to schizogony most of the tropho- 
zoites withdraw from the peripheral circulation. Marchiafava and Bignami (1894) remarked on the tendency of the adult forms to aceumulate in the internal organs. Some authors have observed a tendency of the infected erythroeytes, particularly those containing older "ring" forms, to adhere both to one another and to the vaseular endothelium. To this characteristic Thomson and Robertson (1935) attributed the collection of the infected cells in the internal organs. Marchiafava and Bignami (1901) considered that such aggregation was due to loss of elasticity of the infected red cell with eonsequent irregularity of the surface resulting in difficulty in traversing the capillary bed. On the other hand, infected erythrocytes do not get held up in the eutaneons capillaries so that there may well be another factor involved of which we are not yet aware. At any rate it is presumably in the visceral circulation that schizogony occurs, as we rarely see evidence of it in the peripheral circulation. In fatal pernicious forms of falciparum malaria with, for example, cerebral involvement, it is common to find all stages of schizogony in the cerebral capillaries, which may be blocked by infected erythrocytes.

With further development, the parasite tends to become more compact and the chromatin becomes more centrally located, although the latter is usually not in a compact mass, but on the other hand assumes very irregular shapes. Much less of the vacuole is evident and soon none remains.

In smears from a falciparum-infected placenta we have observed a very compactly formed stage evidently just prior to the onset of schizogony. This form is no larger than the oldest rings one sees in the peripheral circulation. It has no vacuole and its more or less spherical area appears about equally divided between chromatin and cytoplasm, yet it contains a mass of pigment, the area of which may be a third as large as that of the cytoplasm.

Typical of the schizogonous stage in this species is the tendency of the pigment to form one compact mass. This is evident when only one division of the ehromatin has taken place, and such a block of pigment is most usually situated eccentrically even though schizogony may be complete. The chromatin masses of the very young schizonts are notably irregular in shape and size and eytoplasmic divisions are not evident. It is not until sehizogony nears completion that some semblance of uniformity appears. The mature schizonts of $P$. falciparum are characteristically smaller than those of $P$. malariae; some have been observed that did not occupy more than a quarter of the area of an erythroeyte. Oceasionally more than one segmenter may be found in a single red cell.

Gametocytes. Although a variety of shapes may be met with, the falciparum gametocyte most frequently has the contour of a sausage and it is unfortunate that it was given the name "crescent." As a matter of fact a crescent-shaped gametocyte is relatively uncommon and the shape of the mature stage is rarely rounded as is the case in the other species. This latter fact convinced some observers that $P$. falciparum ought to be placed in a different genus designated Laverania. Majority opinion, however, is probably against its segregation on the basis of a differently shaped gametocyte.

Characteristic of this species of plasmodium is the fact that not only does it undergo schizogony and sporulation in the internal organs but also, for the most part, the early stages of gametogeny.

Because of the wave-like release of gametocytes into the peripheral circulation, usually about the end of a period of clinical activity and when the trophozoites are very low in number, one might get the impression that practically all parasite production had been given over to gametogeny. Thomson (1914), and Thomson and Robertson (1935), however, felt that the evidence is in favor of their requiring about ten days for development from the ring form. These authors also feel that the gametocytes have a restricted life span, perhaps only a few days, in the peripheral blood and that their appearance over a long period means con- 
tinued development from the asexual forms. On the other hand, between the gametocyte waves we have observed these forms to disappear entirely and to reappear only after a certain number of trophozoite cycles had occurred (note charts in article by Boyd and Kitchen 1937f).

Thomson (1914) found that gametocytes originated from the asexual forms and chiefly in the spleen and bone marrow. Apparently he did not observe the spindleshaped forms originally described by Watson (1903). Aragão (1930), studying the development of gametocytes in splenic puncture smears, noted that the earliest male forms tend to be rounded, about two microns in diameter and, growing older, become ovoid or elliptical in shape, finally assuming the adult form. The females, on the other hand, are first more band-like, stretching across the red cell (Thomson and Robertson, 1935, could not confirm this observation). As these cells grow they assume a spindle shape or in some instances a plano-convex form. Later cigar-shaped gametocytes may be seen and, finally, the mature sausage-like form appears. In the earliest stage pigment is not found.

The present writer observed in peripheral blood smears from one patient infected with a local strain of $P$. falciparum, numerous spindle-shaped, oval-shaped, and other immature forms (such immature forms were not encountered with three other strains obtained from different points outside this country). This person had not had a severe course and these forms appeared on the eighth and ninth days after first detection of trophozoites. Diagnosis of the sex of these parasites could not be made by reason of their shape but had to be made on the characteristies of the chromatin and pigment.

While as a rule the adult male and female gametocytes possess morphological characteristics which serve to differentiate them, occasionally a little overlapping appears and raises doubt. This probably occurs chiefly in the case of forms which, while they appear to be mature in shape, have probably been in the circulation only a short while and the diagnostic characteristies have not yet settled into the individual sexual type.

The characteristies of the two sexes briefly are: (a) pigment. In the male the pigment is loosely knit, golden brown in color, and may be in the form of fine or coarse granules, or coarse rods. The female pigment is darker, often of a greenish black color. The granules tend to be closely grouped, even though it is not always in a compact mass. Most often it adopts an annular arrangement or that of a compact mass, neither of which is necessarily symmetrical. In both male and female gametocytes the pigment is in close association with the nucleus. They occupy a larger area in the case of the male, in which the position is more frequently essentially in the central zone of the parasite, whereas in the female they are often situated toward one or other pole. (b) chromatin. The female chromatin is almost always in a compact mass. There may be two such but this has not been usual in the writer's experience. Thomson (1932) found binucleated female forms to be common and he noted that these were vesicular. We have frequently observed vesicular nuclei in the female when the nucleus has not been obscured by the pigment mass. In the case of a single chromatin body it is usually partly or completely surrounded by pigment. The color of the chromatin commonly appears a little darker in the female, more nearly a lavender shade (Giemsa stain). Male chromatin is more pinkish and quite diffuse. It may be in the form of granules or rods and is found intermingled with the pigment and usually extending beyond it into an adjacent, almost colorless, semilunar shaped area of the cytoplasm. One often notes colorless areas between the pigment granules and it is assumed that these are unstained portions of the nucleus. (c) cytoplasm. In well stained preparations the cytoplasm of the female is usually of a blue color the depth of which varies. In the male, on the other hand, the color of the cytoplasm varies from a pale to a distinct pink shade. 
Regarding the question of a capsule in the case of gametocytes, our experience has been similar to that of Thomson (1932) who found that evidence of a periplast was not constant.

\section{Sporogonous Cycle}

Most of the sexual cycle of $P$. falciparum occurs in the definitive host, some member of the gemus Anopheles. We have noted that after a certain number of asexual cycles in a falciparum-infected patient, on the average about the ninth or tenth day after the first detection of parasites, gametocytes commence to appear in the peripheral circulation; Thomson (1914) observed a ten-day interval. As a rule the female forms predominate numerically. The gametocyte production in falciparum infections usually is relatively slow, and by no means in every instance are equal or even appreciable densities observed. Nevertheless greater densities are commonly encountered and they persist for much longer periods than with the other plasmodia of man. At the time of first appearance of the gametocytes in the peripheral circulation the trophozoite count has most often dropped to negligible numbers, clinical activity has usually ceased and some days later it is not uncommon to see the parasites represented by gametocytes alone. There would appear to be a cause and effect relationship between a wave of trophozoite production and the succeeding wave of gametocyte production since this phenomenon may oceur two or even three times in close succession during a falciparum attack. Thomson and Robertson (1935) stated that "experimental evidence, therefore, is lacking which would associate the wave of crescent increase-and decrease-with any reaction of the host to the parasite." Generally speaking, it requires upwards of a week or so after their first appearance before the gametocytes acquire sufficient densities or are sufficiently mature to infect mosquitoes. The preparation and examination of stained exflagellation smears will serve as a guide to the maturity of the gametocytes. Exflagellation can also be demonstrated in fresh prep- arations which are kept from drying out. When the anopheline mosquito ingests mature microgametocytes (male) this process of exflagellation occurs and from four to eight highly motile, thread-like microgametes are released. In the stomach of the mosquito the microgamete penetrates the macrogamete (female), a finsion of their chromatin oceurs and the zygote is formed. The zygote, which early has a rounded shape, becomes elongated and motile; known then as an oökinete, it finally penetrates the stomach wall and forms a cyst (oöcyst) subjacent to the outer surface. The oökinetes may be found in smears made with the stomach contents during the twenty-four hours following an infective feeding. They may be as long as 20 or more microns and as wide as 5 microns but they are frequently not symmetrical. They retain some pigment and the chromatin is usually centrally located.

In preparations made after about two weeks incubation (at 20-21 ${ }^{\circ} \mathrm{C}$ ) following the infective feeding, well developed, but as yet immature, eysts may be seen on the stomach wall. After three weeks' incubation they may range from 46 to 54 microns in diameter and are commonly about 48 microns. Falciparum cysts are characterized by two or three small dark masses of pigment which are usually located close to one another. Within the cysts the masses of sporozoites are arranged in a radiating fashion having roughly the appearance of a rosette.

On maturity the oöcysts release the soporzoites into the body cavity of the mosquito. These may be found in most of the insect's tissues but of chief importance are those which find their way to the salivary glands. These glands usually are sporozoite positive about three weeks after the infective feeding, if incubated at $20-21^{\circ} \mathrm{C}$. It has been our experience that mosquitoes with falciparum sporozoites are more surely infective if they are utilized within the first ten days after the salivary glands become sporozoitepositive.

When the proboscis of an infected anopheline penetrates the skin of a person, sali- 
vary secretion is delivered through a duct in a structure adjacent to the sucking tube. It is assumed that at least some of the sporo. zoites so injected find their way into the blood stream. Concerning what takes place between the time of injection of the sporozoites and the first appearance of trophozoites in the inoculated person's peripheral circulation, there is a gap in our knowledge of the pathogenesis which is not satisfactorily explained on the basis of time needed for the parasites to reach a detectable density. Schaudinn (1902) claimed to have observed the penetration of an erythrocyte by a sporozoite, but no one has been able to support this observation and it is not generally accepted as the usual course of events. Recently considerable work has been done on a fixed tissue stage of the asexual cycle in bird malaria. If such a stage were proved to exist between the sporozoite and merozoite in human infections it might explain several vexing problems.

\section{Physiology}

Relatively little is known regarding the physiology of the plasmodia in general. Presumably the trophozoite obtains all its nourishment from the erythrocyte it oceupies. Whether a parasite exhausts the available food of more than one red cell during its growth is not known, although it seems unlikely that migration from one erythrocyte to another takes place. Of interest is the tendency of $P$. vivax and $P$. malariae to invade erythrocytes of a certain age, in comparison with the indifference exhibited by $P$. falciparum.

The growing parasite utilizes the hemoglobin of the red cell and deposits the characteristic pigment, hemozoin, in its own cytoplasm. The pigment in this species is much darker than that of $P$. vivax. It is at first deposited in the form of fine, dust-like granules which, as they are added to, be- come coarse clumps or blocks. Though the pigment was called melanin for some years, it is actually more closely related to hematin. It is soluble in alkalies, and iron has not been satisfactorily demonstrated in it. In $P$. falciparum infections it is commonly seen in the monocytes of peripheral blood smears and at autopsy in the fixed tissue phagocytes, sometimes in large quantities so that it may impart a characteristic slate grey appearance to certain organs.

In its early stages the unstained, noncontractile vacuole that is enclosed by the ring of eytoplasm is considered to be of importance in the matter of the parasite's nutrition. This function was attributed to it by Marchiafava and Bignami (1901). The vacuole appears to be of less consequence to the older forms, however, since it gradually disappears with the increase in size of the parasite. The amoeboid stage, even with its limited activity, doubtlessly facilitates the nourishment of the organism. Presumably hemoglobin is taken in through the pseudopodia to be broken down subsequently by enzymic action.

Some writers have hypothesized the production of a hemolytic toxin by this parasite. They feel that the blood destruction is too great to be accounted for by multiplication of the parasite alone. We do not, however, have any proof of such a hemolytic toxin. It has been assumed, furthermore, that the parasite secretes a specific toxin which is released at the time of sporulation and which is responsible for the paroxysm. Of this we do not have any proof either. The paroxysm may be due wholly to the release of the foreign protein into the host's circulation at the time of sporulation. It has been evident, according to our observations, that the toxic effect of this species per se is not particularly marked; the high parasite densities attained are probably the most important factor. 


\title{
THE DETECTION AND DIFFERENTIAL DIAGNOSIS OF MALARIAL PARASITES IN THE SCHIZOG- ONOUS AND SPOROGONOUS CYCLES
}

\author{
By AIMEE WILCOX \\ NATIONAL INSTITUTE OF HEALTH, WASHINGTON, D. C. \\ LUCILE LOGAN \\ STATION FOR MALARIA RESEARCH, TALLAHASSEE, FLA.
}

\section{DETECTION OF PARASITES Schizogonous Cycle}

THE microseopical detection of malarial parasites in stained blood films is the most reliable and accurate method of laboratory diagnosis thus far devised. For accurate results with either the thin or thick film techniques, special materials and procedures have been found highly desirable, since the best efforts of a qualified microscopist may be frustrated by poor condition of the specimen.

Slides. For the preparation of blood films new slides are preferable and these should be thoroughly cleaned. In order to avoid touching the surface of the clean slide in handling, it should be grasped by the edges. To prevent annoyance and loss of time in field surveys and to facilitate filing of slides in the laboratory, the slides should be of a length and thickness to fit easily into regular or special slide boxes (Barber and Komp 1929b). The slides used should be clear, unscratched, non-corrosive, and above all meticulously clean, i.e., free from grease, dust, acid or alkali, with measurements of $75 \mathrm{~mm} \times 25 \mathrm{~mm} \times 1.25 \mathrm{~mm}$. These requirements are met in a slide the equivalent of No. 7030-B of A. H. Thomas Company.

Other materials. For field work, in addition to clean slides, the materials necessary are a supply of small gauze squares or a roll of absorbent cotton, alcohol for cleansing, a needle for pricking (preferably with a pyramidal point), wax pencils for numbering the slides, a supply of wooden slide boxes with capacity of 25 each or special field boxes holding 100 slides horizontally, and record forms or eards (Komp 1933). An inverted "T" block, against which two of the small slide boxes may be held upright with a rubber band while blood smears are being made, is a great convenience in supporting and preventing the tipping of boxes of wet films. This block may be made of two pieces of soft wood $3 \frac{12^{\prime \prime}}{2}$ wide and $1^{\prime \prime}$ thick; the upright $6^{\prime \prime}$ long fastened at right angles in the center of the horizontal piece which is about $8^{\prime \prime}$ long. A carrying kit for this equipment is convenient.

Making smear. The skin surface to be pricked, preferably of the finger, should be well cleansed by a pledget of cotton or small piece of gauze moistened with alcohol to remove grease, perspiration or dirt, and then should be dried with a fresh piece of cotton or gauze so that residual alcohol will not mix with the blood drop. The puncture should be deep enough to allow a free flow of blood under gentle pressure. The first blood to exude should be wiped off.

Thin smear. To make a thin film, a small drop of blood is collected on the face of a slide near one end. The end of a second, or "spreader," slide (preferably with a narrower end), is lowered onto the face of the first, in an inclined position slightly in advance of the drop so as to make an angle of about $30^{\circ}$, and is then drawn back against the blood which spreads out at the line of contact. Then with a rapid even motion the film is made by pushing the spreader toward the other end of the slide. A good thin film should show the erythrocytes well separated with no overlapping or massing.

Thick film. The thick film was devised 
by Ross (1903). It is placed near one end of the slide and may be made in either of two ways. The first method is to touch the under surface of the slide to a large, rotund drop of blood and, without losing contact with the drop or touching the slide to the finger, move the slide in narrow circles until the blood on the slide covers an area about the size of a dime. Such a smear holds an amount of blood contained in 3 to 5 average drops. The second method is to place several average drops of blood quite near each other and then, with the needle or the corner of a clean slide, quickly puddle these into one fairly homogeneous drop about the size of a dime. Practice soon teaches one the amount of blood to take to assure suffcient thickness and yet prevent crackling and peeling of the blood when dry. A satisfactory thick film is several layers of erythrocytes thick in the middle and thins at the extreme edge to a one layer thickness.

Thick and thin smears on the same slide. It is often and perhaps usually desirable to have a thick and a thin film on opposite ends of the same slide. This permits the number or identifying character to be written with pencil in a part of the thin film. If the thick only is taken, a wax pencil may be used to make the label on the end of the slide opposite the blood film.

Drying. Thick films should always be dried in a horizontal position to assure even distribution of the blood. All blood films should be protected from dust and insects, especially house flies. Slides taken in the field should be placed film side down in a slide box (held vertically as previously described) to prevent dust from falling into the wet film. They should be sent as soon as visibly dry to the laboratory for staining, for age or summer heat will prevent perfect staining of thick films. If thick films are air-dried, several hours' desiccation seems necessary to effect complete adherence of the smear to the slide. Drying may be hastened by placing in an incubator at $37^{\circ}$ $C$ for a short time, or by a blast of warm air from an electric hand hair dryer held not too close to the wet films (Young 1938). Direct heat must be avoided, for like alcohol it "fixes" the red blood cells and prevents dehemoglobinization, so necessary in thick film staining.

Stains. The most dependable stain, particularly for thick films, is obtained with a good quality of Giemsa stain diluted with distilled water of a $\mathrm{pH}$ from 7.0 to 7.2. Grübler's dyes have long given complete satisfaction in this work (Beck 1911). The imminence of a European war and then its actual existence gave rise recently to the study of American dyes in order to produce stains consistently satisfactory on malarial parasites, particularly in thick films. In this study, it was found that Azure B, not Azure $A$, is the principal ingredient of German Azure I. With this knowledge as a basis, formulae were devised for the compounding of satisfactory Giemsa solutions (formula "A" below) (Roe, Lillie and Wilcox 1940). To make these entirely reliable, however, Azure B should be added to the list of certified dyes, with its dye content determined. It is hoped and expected that this step will soon be taken. In the absence of certified Azure B, and in order to facilitate the manufacture of Giemsa stain from American dyes, another study was made to devise a Giemsa stain from the eosinates of Azure A, Azure B, and Methylene Blue, since the eosinates are easily made and are of uniform composition and dye content (Roe, Wilcox and Lillie, in

Plate I-Phases of Malarial Parasites in the Mosquito Host

1. P. vivax. Stomach of A. quadrimaculatus with heavy eyst infection. Cysts about 35 miera in diameter, 15 days old.

2. P. vivax eyst about 4 micra in diameter, 3 days old. Pigment clearly discernible.

3. P. vivax cysts 6 to 8 micra in diameter, 7 days old. Pigment clearly discernible.

4. $P$. vivax eysts 13 days old. Sporoblast formation well advaneed, some pigment still visible.

5. P. vivax. Optical equatorial section of nearly mature cyst, showing developing sporozoites projecting from sporoblasts. Same speeimen as Fig. 1.

6. $P$. vivax. Nearly mature eyst in higher foeus. Rosette arrangement of developing sporozoites characteristic. Same specimen as Fig. 1. 


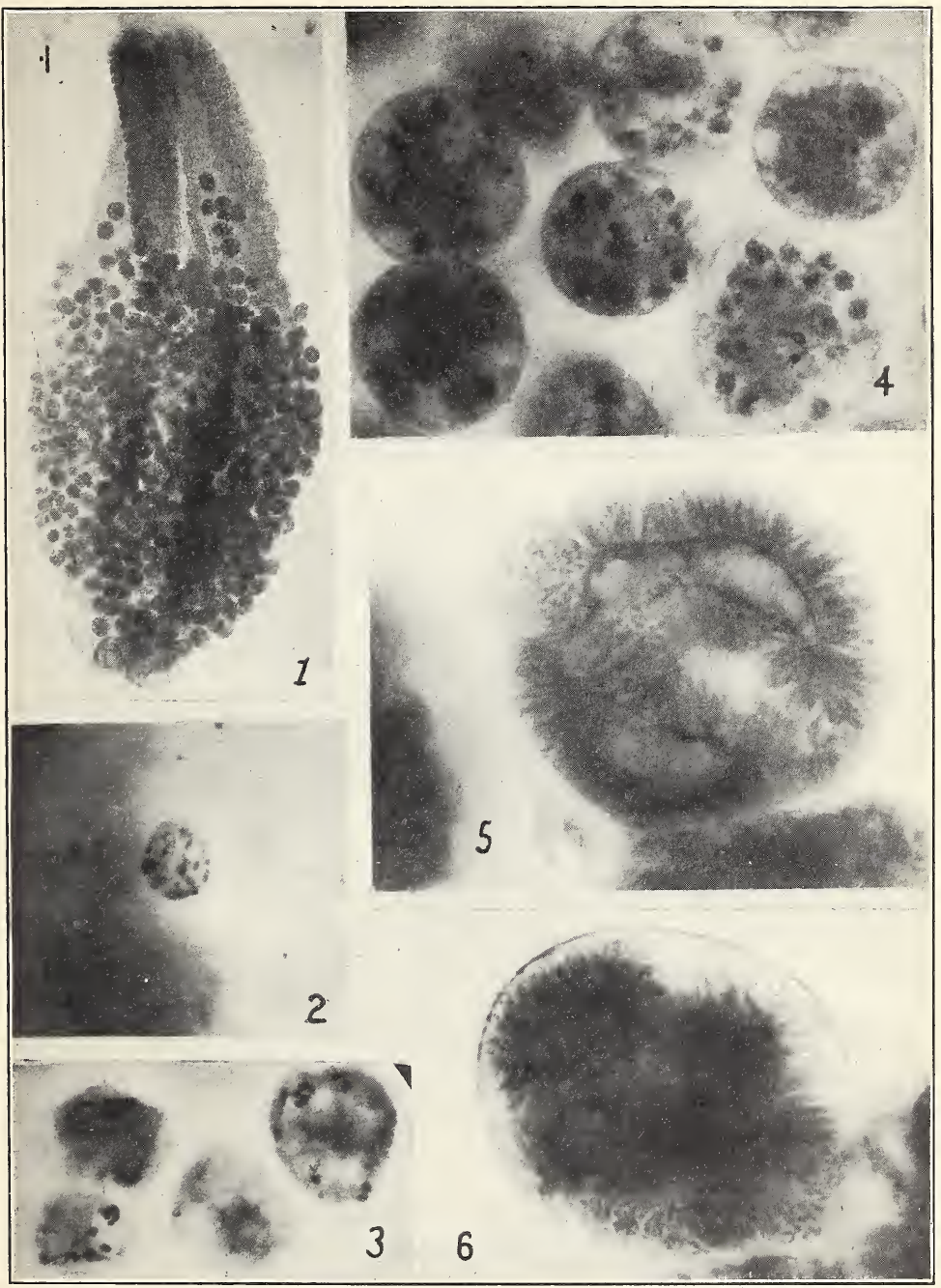


press). It is believed that stains based on this second study will soon be on the market (formula "M" below) : set of brom-thymol blue color standards are used for testing.

Staining. For the staining of thin films

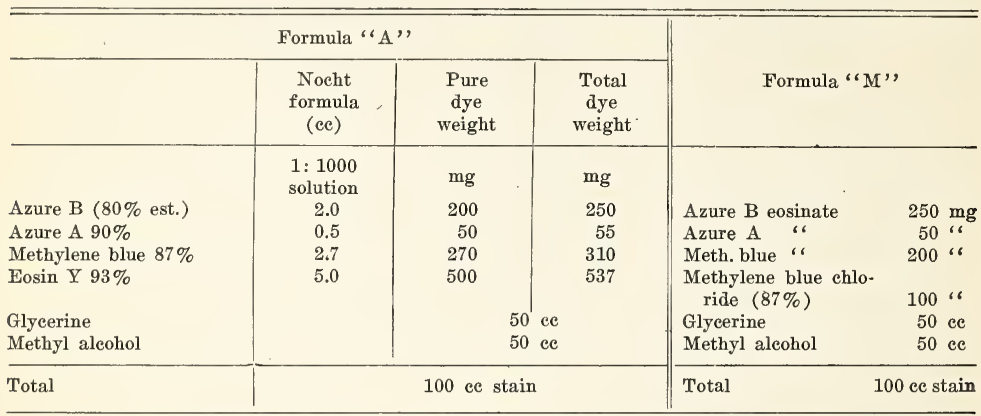

For thin films Wright's and Leishman's stains made from standard formulae will also give satisfactory results.

Buffer solutions. To obtain a $\mathrm{pH}$ of from 7.0 to 7.2 in the water used for the dilution of the stock stain, as well as for washing the stained slides, buffer solutions should be added to the distilled water. Disodium phosphate and either sodium or potassium acid phosphate in $\mathrm{M} / \mathbf{1 5}$ solutions are used and are prepared by dissolving the salts as follows : $\mathrm{Na}_{2} \mathrm{HPO}_{4}$ (anhydrous), $\mathrm{M} / \mathbf{1 5}=9.5$ grams per liter; $\mathrm{NaH}_{2} \mathrm{PO}_{4} \cdot \mathrm{H}_{2} \mathrm{O}, \mathrm{M} / \mathbf{1 5}=9.2$ grams per liter; and $\mathrm{KH}_{2} \mathrm{PO}_{4}, \mathrm{M} / \mathbf{1 5}=9.07$ grams per liter. The stock solutions are kept in separate glass-stoppered pyrex bottles from which are removed the following quantities to make the indicated amount of the buffered water:

\begin{tabular}{lc|cc|c}
\hline \hline $\mathrm{pH}$ & $\frac{\mathrm{M}}{15} \mathrm{Na}_{2} \mathrm{HPO}_{4}$ & $\frac{\mathrm{M}}{15}$ & $\mathrm{NaH}_{2} \mathrm{PO}_{4} \cdot \mathrm{H}_{2} \mathrm{O}$ & Distilled \\
& & $\frac{\mathrm{M}}{15}$ & $\mathrm{KH}_{2} \mathrm{PO}_{4}$ & \\
& & & $\mathrm{H}_{2} \mathrm{O}$ \\
7.0 & $61.1 \mathrm{ce}$ & & $38.9 \mathrm{ec}$ & $900 \mathrm{ce}$ \\
7.2 & $72.0 \mathrm{ce}$ & & $28.0 \mathrm{ec}$ & $900 \mathrm{ce}$ \\
\hline
\end{tabular}

The $\mathrm{pH}$ of the buffered water is then tested and adjustments made if necessary. Brom-thymol blue indicator solution and a with Wright's or Leishman's stains, which are alcoholic solutions, the red cells are fixed by placing the undiluted stain directly on the blood films for a given period of time. Then the stain is diluted with the amount of buffered water found to give best staining results, and the slide washed by flushing away the stain with additional water. The amounts and time for each step must be ascertained for different lots of dye. To use Giemsa stain on thin films, the blood smears must be previously fixed with acetone-free, absolute methyl alcohol, then stained in the manner described below for thick films, except that they are washed by dipping quickly in clear, buffered distilled water.

Thick films should never be fixed by chemical or other means, since lysis of the erythrocytes is necessary to make such smears transparent. The portion of the slides containing the smears is vertically immersed. in a solution of one part stock Giemsa stain solution diluted with 50 parts of neutral distilled water for 45 minutes. Then they are removed and washed for a sufficient time to clear the background and differentiate the parasite colors. This varies with the age and thickness of smears-from several dips in neutral distilled water to a five minutes' immersion. Older and thicker 
smears require longer washing. The slides are stood on end to dry, never blotted. An electric fan may speed the drying, but direct heat is not recommended, since some of ${ }^{*}$ the excess stain which might flow from the smear, may be dried upon it and eloud this background.

Rapid staining. The time consumed in staining a thick film for diagnosis has been a drawback to its general use, but a combination of Wright and Giemsa stains has been devised recently, which reduces staining time to ten minutes, without detracting from the desired coloration (Michelson and Wilcox 1940). The stain is prepared by dissolving $1.515 \mathrm{gm}$ of Giemsa powder (National Aniline and Chemical Company, Inc., N. Y.) in 100 ec of glyeerine (using heat and avoiding absorption of $\mathrm{H}_{2} \mathrm{O}$ ). When this is dissolved, $100 \mathrm{ec}$ of Wright's stain solution, previously made by adding $2 \mathrm{gm}$ of Wright's powder (National Aniline and Chemical) to $1000 \mathrm{ec}$ methyl alcohol and aging for at least one month, is added and the mixture is allowed to stand over night. The next morning an additional $800 \mathrm{ec}$ of the aged Wright stain solution is added. The amount of stain needed for a few days is filtered into a small bottle and is ready for immediate use.

For staining the solution is diluted 1 part to 10 parts of neutral distilled water and poured over the slides in a staining dish. Ten minutes are allowed for staining, then the stain is flushed from the dish and the slides washed for one minute with neutral distilled water, after which they are airdried and examined.

Staining smears in bulk. Where large numbers of slides earrying thick smears are to be stained, time may be saved and uniformity in the quality of staining assured by a simple method deseribed by Barber and Komp (1929b). The slides are made into blocks of as many as 25 , by placing pieces of eardboard an inch square and about $1.2 \mathrm{~mm}$ thick between the slides at the ends opposite the thick films, compressing the alternating slides and squares, and winding around that end of the block an inch wide strip of heavy paper which is se- eured with a strong rubber band (Komp 1933). The outer slides in the block should be reversed with the film side "in" to prevent the wet smears from being scraped off in the staining process. By this method hundreds of slides may be stained in bulk and consumption of stain solution diminished by using a container of the exact size necessary to hold the number of blocks to be stained.

Examining smears. For examining blood films the oil immersion lens is used. Thick films are searched with a low power (preferably $5 \times$ or $6 \times$ ) ocular, while any uncertain object may be serutinized with the $10 \times$ ocular. The low power eye-piece gives a larger field, greater illumination and clarity of color and outline. It lessens eye strain by giving a feeling of distance. The $10 \times$ ocular enlarges the object and brings the field closer.

Use and advantages of both thin and thick films. The thin film is ideal for the study of the morphology of the individual parasite, for identification of stages and species and suffices for diagnosis if the infection is heavy. It also gives the accompanying blood pieture. It dries quickly, can be stained in from 5 to 10 minutes, and is ready for immediate examination. However, it has the great disadvantage of failing to reveal parasites when density is low. Parasites are found most easily along the outer edges and in the tails of the smears.

The thick film is a concentration method, particularly valuable for diagnosis and, where large numbers of slides are to be examined, is a method of necessity rather than of choice (Green 1931). It quickly reveals the sparse or seanty parasites of new or chronic cases for the practitioner and gives a more accurate idea of the malaria incidence in a survey by greatly increasing the number of positives found in single slide examinations. It has been estimated that the amount of blood covered per microscopic field is increased from 10 to 50 times over that in a thin film field, hence examination time is cut drastically (Sinton and Banerjea 1925). Practice and experience are necessary to become proficient in 
examining thick films because of the loss of erythrocyte outlines and a slight change in parasite contour and size. The advantages of the technique, however, fully recompense one for the time spent in becoming proficient.

Enumeration of parasites. To obtain a definite idea of the degree of infection a quantitative thick smear may be made and examined by the following modification of Earle's method (Earle and Perez 1932). Five cubic millimeters of blood are taken in a special pipette and spread evenly over an accurately measured $3 \times 15 \mathrm{~mm}$ area on a clean slide. (This may be marked on the slide by ruling instruments having diamond points set at the exact distances.) When dry the smear is stained like any other thick film. Preliminary to enumeration the microscope is calibrated as follows : A Howard dise with a ruled square is inserted in the ocular. One side of the large square is measured with a slide micrometer and the area of the field covered by the square computed in square millimeters. The number of fields to be counted in order to cover one square millimeter is ascertained by dividing one square millimeter by the area of one square microscopic field. For example, since 5 cubic millimeters of blood are deposited on 45 square millimeters of space, $5 \div 45=0.11$, which is the portion of one cubic millimeter of blood spread over one square millimeter. Then also since $1 \div 0.11$ cubic millimeter $=9$ (the number of square millimeters carrying a blood volume equal to one cubic millimeter), the number of parasites counted in the required number of microseopic fields to equal one square millimeter is multiplied by 9 to obtain the number of parasites per eubic millimeter. The requisite number of fields is selected from different parts of the smear to make the count as representative as possible. If counts run high, fewer fields are examined and an estimation made for the required number of fields.

\section{Sporogonous Cycle}

\section{Experimental Infection}

In the experimental infection of mosquitoes the maturity of the gametocytes should be ascertained prior to the application of the mosquitoes to the patient, assuming that satisfactory densities of these cells are present. While mosquitoes may be lightly infected when fed on vivax patients with submicroscopic densities of gametoeytes, it is desirable to limit their application to patients who, in the case of vivax, have counts of not less than 5 males and 5 females per emm. In falciparum patients a much higher density of gametocytes is necessary, approximately 100 of each sex to secure reasonably adequate infections. An exflagellation smear should also be made at this time. For this purpose a rather thick "tlin" smear is prepared, over which the breath is exhaled. Before desiccation begins this smear is placed in the saturated air of a Petri dish having moistened filter paper in the bottom, and allowed to stay for about 30 minutes at a cool temperature. At the end of this time the smear is removed, dried and stained as are ordinary smears. If exflagellating microgametocytes are found one can be sure of the maturity of the gametocytes present in the donor. By treating a preparation in this manner the same development can be initiated which normally takes place in the stomach of the mosquito.

The mosquitoes were previously placed

Plate II-Phases of Malarial Parasites in the Mosquito Host (Continued)

7. P. vivax. Cross section of lobe of salivary gland of A. quadrimaculatus, showing sporozoites within secretory cells of gland. Hematoxylin stain.

8. P. vivax. Individual sporozoites extruded from crushed gland. Wet fixation, Giemsa stain.

9. P. malariae. A 12 day old eyst, 8.4 micra diameter.

10. P. malariae. A 23 day old cyst. Beginning formation of sporoblasts.

11. P. malariae. Sporozoites extruded from crushed gland. Wright stain.

12. P. falciparum. Cyst showing early stage of sporoblasts. Nine days old.

13. P. falciparum. A cyst 17 days old showing sporozoites budding from sporoblasts.

14. P. falciparum. Group of sporozoites. Wet fixation, iron-hematoxylin stain. 


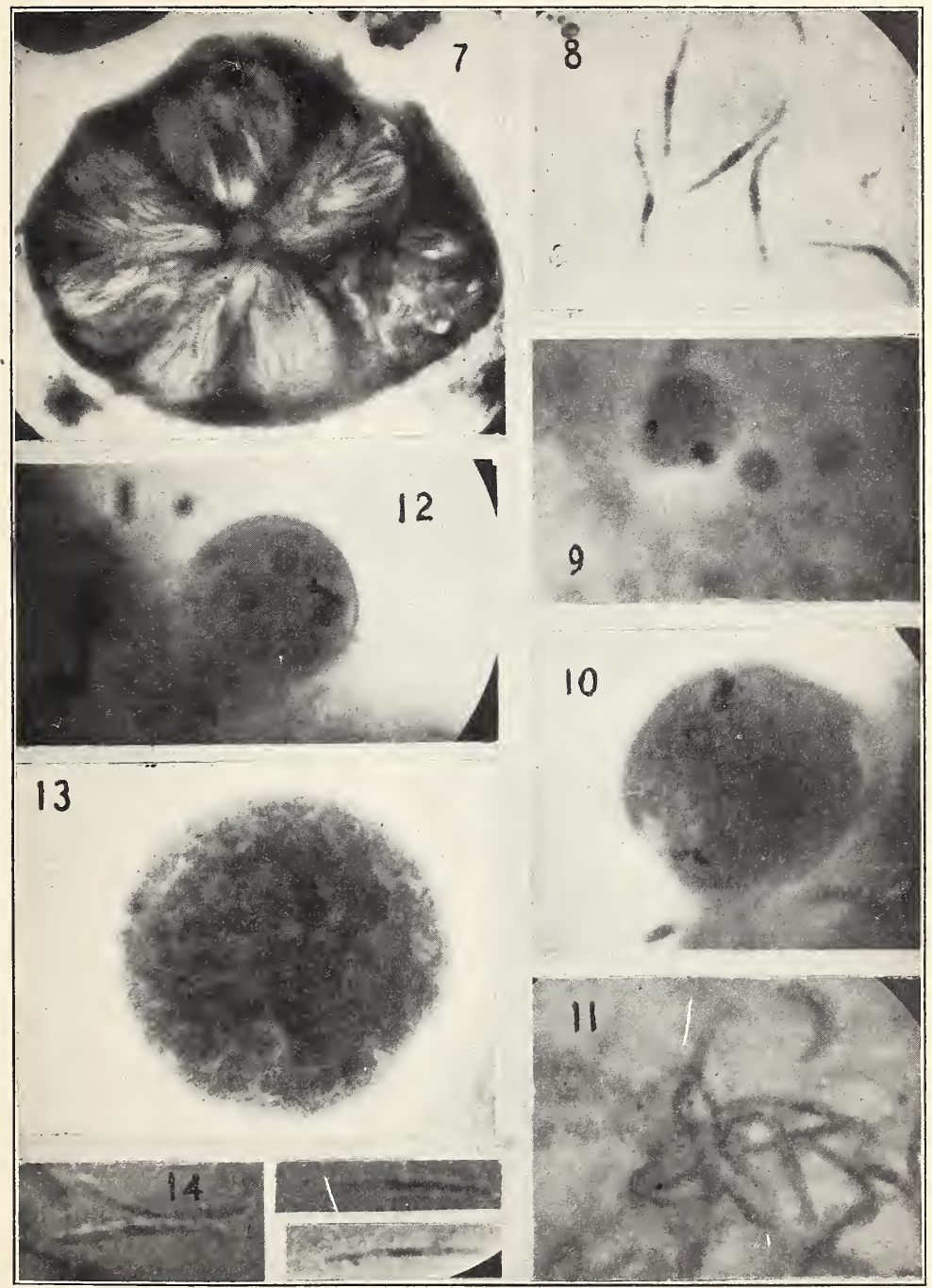


in bobbinet cages containing not more than 20 or 25 insects to a cage. If one end of the cage is covered with black bobbinet the mosquitoes are more easily seen. Previous to application the mosquitoes are chilled in an ice box, and in the meantime a hyperemia of the donor's skin has been produced by a hot water bottle placed on that part of the body to which the caged mosquitoes are to be applied. When the eages are applied to the skin the chilled mosquitoes are attracted to the donor by the heat. The arms or thiglus are convenient sites for application. Subsequently the fed mosquitoes are separated from the unfed, and the former are stored in a $20^{\circ} \mathrm{C}$ incubator. Every two days they are given an opportunity to feed on an immobilized rabbit. Those dying naturally are removed daily and dissected if their condition permits, and examined for the current stages of the parasites. If at the end of ten days none have died, 8 or 10 should be killed for dissection. After parasites have migrated to the gland they are stored at a lower temperature and, until used for inoculation purposes, are given an opportunity to feed on an animal but once a week. When required for inoculation they are previously starved for several days, then transferred to individual cages for application to the patient. The same technique of application is followed as previously described although more patience is necessary. Those feeding are killed and their salivary glands removed and examined for the presence of sporozoites.

\section{Dissection}

Engorged wild mosquitoes should be kept in a cool incubator for several days prior to dissection, until the stomach is emptied from digestion. Kill not more than 2 or 3 mosquitoes at a time with chloroform. If several are killed at once, place those not immediately required in a moist chamber. After identification eut off the wings and legs. Place the mosquito on a slide on the stage of a dissecting microscope with the abdomen pointing into a drop of saline. Secure the mosquito on its back or side by piercing the thorax with the point of a straight needle held in the left hand. With a curved needle, held in the right hand, nick the seventh or eighth segment above and below, then insert the needle point in the last segment and pull gently, drawing out the intestinal tract and appendages into the saline solution. Trim off the Malpighian tubes leaving enough to identify the posterior end, and cut through the fore and hind guts close to the stomach. Transfer stomach, on the needle tip, to a clean drop of saline-straighten it out and cover with a cover slip for examination in a fresh condition. If excessively contracted, the stomach may be expanded by cautiously warming the slide.

In the dissection of gravid mosquitoes it may be difficult to remove the stomach in the manner described, since the ovaries block the opening and the tissues may break. In this case tear the membranous sides of the abdomen with the needle points and dissect out the stomach.

Salivary glands. Various techniques have been described, some of which may be better adapted to certain technicians than others. Practice is more important than method. Place the remainder of the mosquito body in a drop of saline tinted with methylene

Plate III-Malarial Parasites in a Thin Blood Film

1. P. falciparum. Small trophozeites, illustrating a doubly infected cell with one marginal parasite, a parasite with double chromatin dot, and an uninfected cell showing basophilie stippling.

2. P. falciparum. Microgametocyte.

3. P. falciparum. Macrogametocyte.

4. P. vivax, Growing ameboid trophozoite, Sehüffner's dots clearly discernible.

5. P. vivax. Presegmenting schizont. Another parasite in close proximity.

6. P. vivax. Microgametocyte. Chromatin mass surrounded by vesicular area.

7. P. vivax. Macrogametocyte.

8. P. malariae. Band forms, two stages of growing trophozoite. Concentration of pigment along periphery opposite the nucleus is apparent.

9. $P$. malariae. Segmenting schizont and large trophozoite. 


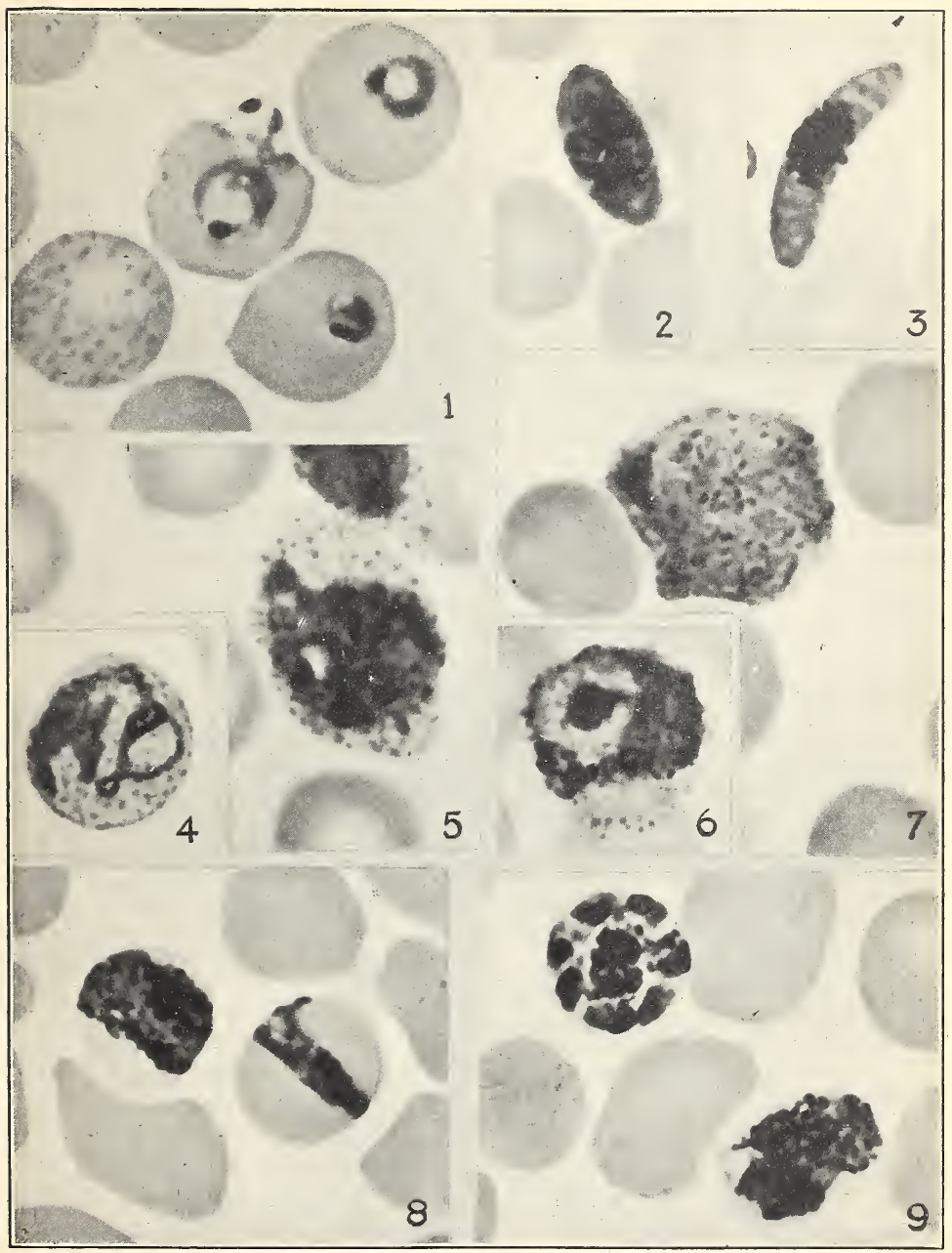


blue. With the head and thorax pointing to the right, hold the mosquito in place with the needle in the left hand. Cut off the head with the curved needle held in the right hand. Press the thorax gently with the side of the curved needle, forcing a small mass of tissue from the neck opening. The tips of the glands may be seen protruding from this mass and are gently teased out. If they are not seen, tear the greenish-colored mass apart in search of the two glistening, blue-stained bodies. Transfer the glands, free of other tissues, by the curved needle tip to a clear drop of saline and cover with a cover slip.

\section{Examination of Fresh Preparations}

The preparations are examined with a compound microscope equipped with a mechanical stage.

Stomach. Focus a two-thirds objective on the stomach and move it to center of the field. Place the tip of a needle held in the left hand against the edge of the cover slip. Move the slide by means of the mechanical stage against the needle point, so that the cover slip is held fixed. This causes the stomach to roll, so that all parts of its surface may be examined for projecting eysts. If suspicious bodies are found these are examined under a higher magnification.

Small cysts are clear, round or oval bodies, without a refractile wall but with unmistakable pigment granules. Large eysts show a distinct wall and only rarely is pigment visible. Mature cysts are finely striated, owing to the myriads of sporozoites which they contain. When only a few cysts are found they are usually in the posterior end of the stomach.

The contraction of some of the circular muscle fibers in the central part of the stomach may produce externally extruding sacs of the stomach membrane that may cause confusion. Some of the fat cells containing fat globules might also be confused with cysts. Small cysts should never be diagnosed unless pigment is seen; large cysts are unmistakable.

Salivary glands. Move the glands to center of the field of a two-thirds objective.
With the tip of a needle press gently on the cover slip above so as to erush them. Then the fluid about the crushed glands is examined under a one-sixth objective for sporozoites. These are slender, glistening rods, straight or slightly curved and about 12 to 14 micra in length. Motility, at least in the sense of a translation of position, is questionable.

\section{Staining of Preparations}

Stomach. If a high incidence of infection in the mosquitoes is expected, it is preferable to fix and stain the stomachs before examination. Fixation and staining of specimens which have been for some time under a cover slip in saline is unsatisfactory. By staining freshly dissected stomachs in Mayer's acid haemalum (formula follows) before examination, infections may be found which would be missed if examination is limited to the fresh preparation. Cysts, while small, are detectable as early as the third day after an infective feeding in stained specimens, while such small cysts are very difficult to detect in unstained preparations.

After the stomach is removed and the Malpighian tubes trimmed off, it is covered with a large drop of Bouin's fixative. Then cut off the fore gut and hind gut. Allow the fixative to act for 5 minutes. The specimen is transferred with a pipette throughout the different steps of the staining process. After fixation the preparation is rinsed in distilled water to remove any excess of the fixative and is allowed to remain in distilled water until all traces of the yellow color of the picric acid are gone. This requires several hours or preferably over night. From the distilled water the stomach is transferred to a $1: 10$ dilution of Mayer's acid haemalum for an hour. The stomach is next washed to remove excess of stain in a one per cent solution of acetic acid for 3 to 5 minutes, then transferred to a one per cent solution of sodium carbonate to neutralize (the color changes to blue). For dehydration it is passed successively through $50,70,85,95$ and 99 per cent solutions of alcohol, remaining from 3 


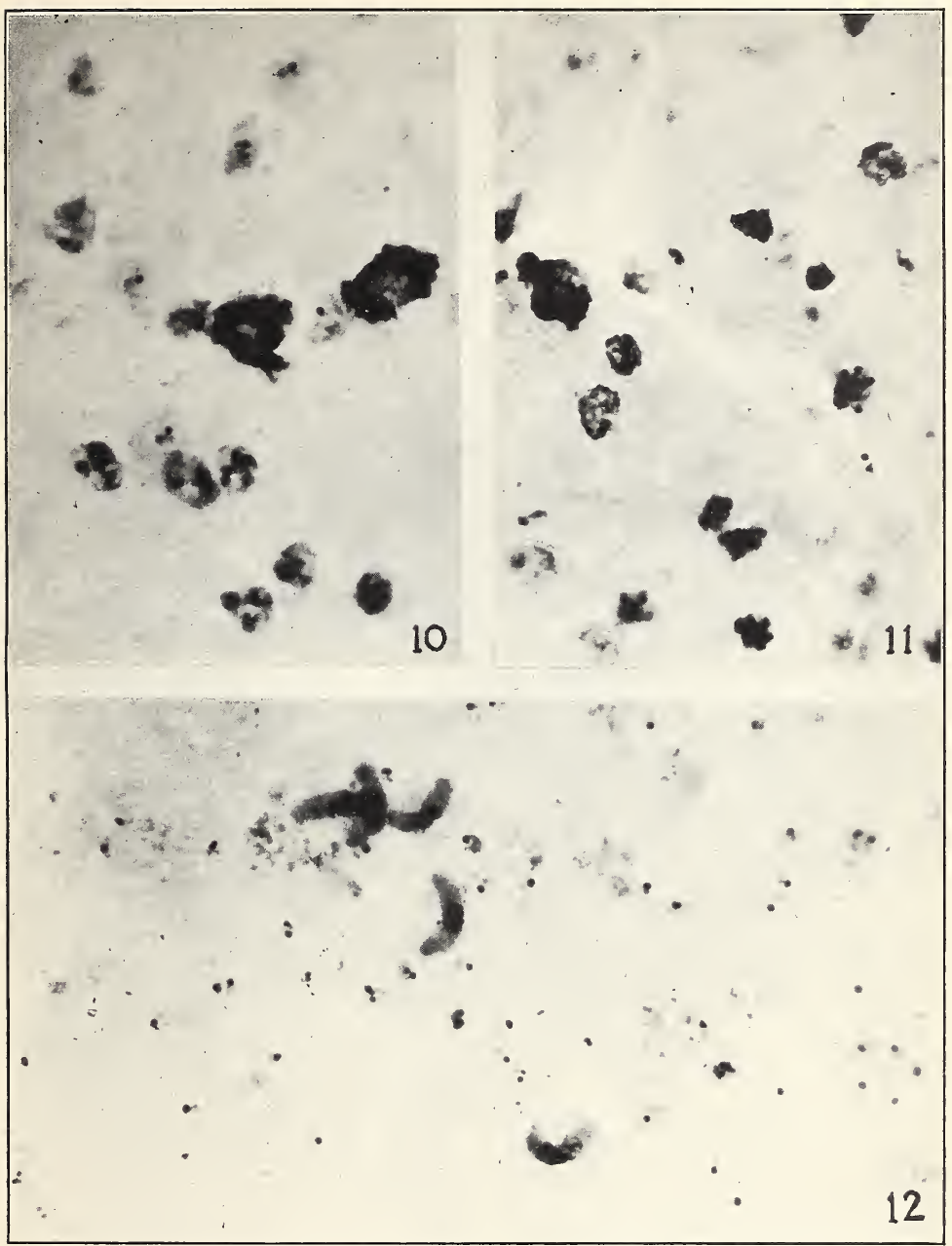

Plate IV-Malarial Parasites in a Thick Blood Film

10. P. vivax. Small and growing trophozoites showing fragmented appearance of cytoplasm. Outlines: of infected cells visible. Nuclei of two white eells as they appear in thick films.

11. $P$. malariae. Small and large trophozoites and segmenting schizonts.

12. P. falciparum. Young trophozoites and gametocytes. Background clouded with remains of young red cells. 
to 5 minutes in each. It is then cleared in carbol-xylol and mounted in balsam underneath a cover slip.

Formulae for the stains mentioned are as follows :

\section{Mayer's Acid Haemalum}

Dissolve 1 gram of haematin in 50 ce of 90 per eent aleohol and warm. Add this solution to a solution of 50 grams of aluminum potassium sulphate in 1000 ee of distilled water dissolved by heating. Mix warm, cool, and filter. Add 2 ec of glacial acetic acid to 100 ec of haemalum solution.

\section{Bouin's Fixative}

Picric acid, saturated aqueous solution ..... 30 parts Formalin (40 per cent) ............................... 10 parts Acetic acid

This should be prepared daily.

2 parts

Salivary glands. The cover slip is gently raised from the crushed gland and inverted. The material on both the slide and the cover slip is allowed to dry, is then fixed by flooding with absolute methyl alcohol, and is stained with Giemsa stain exactly as a blood smear. Forceps for holding the eover slips are very convenient in staining. The surface of both the slide and cover slip is examined with a low power lens and the one to which the glands have adhered is saved. If on the cover slip, the slip is attached, gland side upward, to a slide by a drop of balsam (Boyd 1932).

\section{Examination of Stained Preparations}

A well-stained stomach preparation shows the cysts slightly darker than the stomach tissue, with the pigment showing as in a fresh preparation. Large or mature eysts are readily recognized by their internal content of sporoblasts or sporozoites. Small cysts are identifiable by their content of pigment, the recognition of which is indispensable for diagnosis of the body as a cyst. The only criterion for the specific identification of the eysts is by the characteristies of the pigment, which is retained from the macrogametocytes from which it developed. The characteristics of the pigment in the different species are shown in the accompanying tabulation (Table I). Pigment is rarely observed in the large eysts. It is not certain whether its disappearance is apparent or real, i.e., whether it is masked by the increased internal structure of the cyst or has been metabolized. The stained sporozoites exhibit the characteristic colors of a malarial parasite. The reddish chromatin is usually a central oval mass or may appear as three or four small, round granules. The cytoplasm has the familiar blue of a parasite. The sporozoites of $P$.vivax and $P$. falciparum are morphologically indistinguishable, but one could probably differentiate the sporozoites of $P$. malariae, as these are definitely larger and coarser, with splotchy chromatin. Sporozoites from different mosquitoes vary greatly in their size, although those derived from any particular insect show relatively slight difference (Boyd 1935a).

TABLE I

Differential Diagnosis in Sporogonous Cycle

\begin{tabular}{|c|c|c|c|c|}
\hline \multirow{3}{*}{ Cysts } & \multirow{2}{*}{$\begin{array}{l}\text { Produce } \\
\text { sporozoites at } \\
20^{\circ} \mathrm{C} \text { in }\end{array}$} & P.vivax & P. malariae & $P$. falciparum \\
\hline & & 16-17 days & 30-35 days & 22-23 days \\
\hline & $\begin{array}{l}\text { Pigment in small } \\
\text { cysts }\end{array}$ & $\begin{array}{l}\text { Fusiform crystals } \\
\text { frequently in a } \\
\text { chain }\end{array}$ & $\begin{array}{l}\text { Rounded, angular } \\
\text { masses, golden } \\
\text { brown }\end{array}$ & $\begin{array}{l}\text { Rectangular blocks } \\
\text { dense black }\end{array}$ \\
\hline \multirow{2}{*}{ Sporozoites } & Size & $\begin{array}{l}\text { Variable, probably } \\
\text { dependent on de- } \\
\text { gree of infection } \\
\text { in mosquito }\end{array}$ & $\begin{array}{l}\text { Definitely largest } \\
\text { and coarsest }\end{array}$ & $\begin{array}{l}\text { Variable, probably } \\
\text { dependent on de- } \\
\text { gree of infection } \\
\text { in mosquito }\end{array}$ \\
\hline & $\begin{array}{l}\text { Romanowsky } \\
\text { staining }\end{array}$ & $\begin{array}{l}\text { Indistinguishable } \\
\text { from } \\
P . \text { falciparum }\end{array}$ & $\begin{array}{l}\text { Chromatin displays } \\
\text { the diffuse char- } \\
\text { acter typical of } \\
P \text {. malariae }\end{array}$ & $\begin{array}{l}\text { Indistinguishable } \\
\text { from } P \text {. vivax }\end{array}$ \\
\hline
\end{tabular}




\section{DIFFERENTIAL DIAGNOSIS}

\section{Schizogonous Cycle}

\section{General Appearance of Thin and Thick Blood Films}

The blood picture presented by the thin film fixed with methyl alcohol and stained by one of the stains mentioned is too familiar. to warrant discussion. The descriptions of the morphology of malarial parasites are based on their appearance in films of this type. They lie within or on the erythrocytes, show red to purplish-red chromatin, blue cytoplasm, and pigment granules varying from a yellow tinge to almost black. Differentiation by stages and species in the thin film is given in the accompanying Table II. Further detail may be found in James, Nicol and Shute (1933); Nocht and Mayer (1937); Stitt, Clough and Clough (1938); Thompson and Woodeock (1922), and Wenyon (1926b). A working knowledge of the morphology of the malarial parasites in the thin film is absolutely necessary before attempting their diagnosis in the thick film.

TABLE II

Summary of Parasite Differentiation (Stained thin films)

\begin{tabular}{|c|c|c|c|c|}
\hline & $\begin{array}{c}P . \\
\text { vivax }\end{array}$ & $\begin{array}{c}P . \\
\text { falci- } \\
\text { parum }\end{array}$ & $\begin{array}{c}P . \\
\text { ma- } \\
\text { lariae }\end{array}$ & $\begin{array}{c}P . \\
\text { ovale }\end{array}$ \\
\hline Infected cell ....... & (a) & (b) & (c) & (d) \\
\hline $\begin{array}{l}\text { Small tropho- } \\
\text { zoite (early } \\
\text { rings) }\end{array}$ & (a) & (b) & (c) & (d) \\
\hline $\begin{array}{l}\text { Growing } \\
\text { trophozoite }\end{array}$ & (a) & (b) & (c) & (d) \\
\hline $\begin{array}{l}\text { Large } \\
\text { trophozoite } . . . .\end{array}$ & (a) & (b) & (c) & (d) \\
\hline $\begin{array}{l}\text { Presegmenting } \\
\text { schizont }\end{array}$ & (a) & (b) & (c) & (d) \\
\hline $\begin{array}{l}\text { Segmenting } \\
\text { schizont }\end{array}$ & (a) & (b) & (c) & (d) \\
\hline $\begin{array}{l}\text { Macro- } \\
\text { gametocyte } \\
\text { Micro-... }\end{array}$ & (a) & (b) & (c) & (d) \\
\hline $\begin{array}{l}\text { gametocyte } \\
\text { Length of asex- } \\
\text { ual eycle }\end{array}$ & (a) & (b) & (c) & (d) \\
\hline $\begin{array}{l}\text { Stages in } \\
\text { peripheral } \\
\text { blood ............ }\end{array}$ & All & (b) & All & All \\
\hline 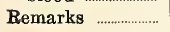 & (a) & (b) & (c) & (d) \\
\hline
\end{tabular}

Note. The above table is a guide to differentiating the species of Plasmodia. The first column lists the chief stages of the parasites as found in thin smears. It is from differences in the characteristics of these stages that the species may be determined. In the text below the stages appear at the beginning of paragraphs in the same order as in the table above. In each paragraph the four species are given in the same order and following the same letters as in the table. To read down through a paragraph below is equivalent to reading across the corresponding line in the table above.

Infected cell. (a) Larger than normal, pale, often bizarre in shape. Schüffner's dots very often present. Multiple infection of erythrocyte not un. common. (b) Normal in size. Multiple infection of erythrocytes more frequent than in the other species. Maurer's dots sometimes seen (in overstained smears or when $\mathrm{pH}$ of $\mathrm{H}_{2} \mathrm{O}$ is on alkaline side). (c) About normal or slightly smaller. Sometimes darker in early stages. Ziemann's dots rarely seen. Multiple infection of erythroeyte rare. (d) Somewhat larger than normal, often with fringed or irregular edge and oval in shape. Schüffner's dots appear even with younger stages, stain more readily and more deeply than in vivax.

Small trophozoite (early rings). (a) Signet ring form with heavy chromatin dot and large eytoplasmic eirele, possibly with fine pseudopodia. (b) Small threadlike eytoplasmie eirele, and one or two small chromatin dots. (Double ehromatin dots more frequent than in other species.) Marginal and bridge forms are frequent. May disappear in this stage from peripheral eirculation and return to internal organs for development. (c) Signet ring form with heavy chromatin dot and eytoplasmic circle which is of ten smaller, thicker, and heavier than that of vivax. (d) Small, darker in color and more solid, as a rule, than those of falciparum. Sehüffner's dots regularly present in almost $100 \%$ of infected cells.

Growing trophozoite. (a) Same as above with gradual increase in amount of cytoplasm and ehromatin. Often with more distinet pseudopodial processes. Small yellowish-brown pigment granules in eytoplasm, number inereasing with age of parasite. (b) This stage remains in the ring form but chromatin and eytoplasm increase to the extent that in size the parasite resembles closely the small trophozoite of vivax. A few pigment granules give a yellowish tinge to the cytoplasm. This is usually the oldest asexual stage seen in peripheral cireulation. (c) Chromatin rounded or elongated, cytoplasm in a compact form with little or no vacuole or in a narrow band form across the cell. Dark brown pigment granules-may have peripheral arrangement. (d) Resembles elosely same stage of $P$. malariae, but is considerably larger. Pigment is lighter in color and less conspicuous.

Large trophozoite. (a) One abundant mass of chromatin, loose, irregular or elose compact eytoplasm, with increasing amounts of fine brown pigment. Parasite practically fills enlarged cell by end 
of 36 to 40 hours. (b) Stage seldom seen in peripheral blood. Very small, solid, with one small mass of ehromatin; lightly staining, compact cytoplasm; and with the very dark pigment usually collected in one small, dense block. (c) One mass of ehromatin, often elongated, frequently less definite in outline than that of vivax. Cytoplasm dense, compact with few irregularities of outline; in rounded, oblong or sometimes band shape. Pigment granules larger, darker than in vivax with great tendency toward peripheral arrangement. Fills or almost fills normal cell. (d) Resembles closely same stage of $P$. malariae, but is considerably larger. Pigment is lighter in color and less conspicuous.

Presegmenting schizont. (a) Chromatin divided into a number of masses; cytoplasm shows varying degrees of separation into strands and particles; pigment shows tendency to collect in parts of the parasite. (b) When found in peripheral blood this stage resembles the same stage of $P$. malariae but is smaller and the pigment is likely to be completely clumped in one small dark mass. (c) Same as vivax except that the parasite is smaller and shows fewer divisions of chromatin, as it approaches segmentation. (d) About $25 \%$ of infected cells are definitely of oval shape. Usual pieture is that of a round parasite in center of an oval cell. Many cells with indefinite fringed outline. Pigment lighter in color and less coarse than in $P$. malariae. Segmenting schizont. (a) 12 to 24 divisions or merozoites, composed of a dot of chromatin and a portion of cytoplasm. The pigment is in one or two clumps. Parasite practically fills enlarged cell. (b) 8 to 24 merozoites, which are very small compared to those of other species. Rarely found in peripheral blood. Fills about two-thirds of normalsized cell. (c) 6 to 12 , usually 8 or 10 merozoites in a rosette or irregular cluster. Practically fills normal-sized cell. (d) Usually 8 merozoites arranged around a central block of pigment.

Macrogametocyte. (a) Dark blue, homogeneous eytoplasm with no vacuoles; small, compact, dark red, usually eccentric ehromatin; abundant dark brown pigment seattered through cytoplasm. When grown usually fills or nearly fills enlarged cell. The outline is circular or ovoid and regular. (b) Cytoplasm possibly a deeper blue than in microgametocyte. Usually single dark red chromatin mass near center associated with concentrated aggregation of pigment, darker than in microgametocyte. Crescentic or sausage-shaped about $1 \frac{1}{2}$ times diameter of erythrocyte in length, possibly longer and more slender than microgametocyte. (c) Cytoplasm and chromatin same as vivax. Pigment is abundant, dark brown, coarser than in vivax. When grown usually fills the normal-sized cell. Outline circular or ovoid. (d) Distinguished from $P$. malariae by size of infected cells and by Schüffner's dots. Less easy to differentiate from vivax. Seldom or never contained in an oval erythrocyte.

Microgametocyte. (a) Light blue, gray, pink or almost colorless eytoplasm; large diffuse light red or pink chromatin-usually centrally placed, often with vesicular area around chromatin mass. Abundant yellowish-brown pigment throughout cytoplasm. When grown, about size of a normal cell. Usually circular in outline. (b) Often the cytoplasm is paler than in macrogametocyte-grayish blue or pink. Loose, diffuse, light staining granules or threads of chromatin scattered with numerous granules of pigment throughout central half or more of parasite. Parasite possibly broader, shorter, and with more rounded ends than those of macrogametocyte. (c) Same as vivax except in size. When grown, fills or almost fills normal-sized cell. (d) Distinguished from $P$. malariae by size of infected cells and by Schüffner's dots. Less easy to differentiate from vivax. Seldom or never contained in oval erythrocyte.

Stages in peripheral blood. (b) Usually ring form trophozoites and gametocytes. Other stages rarely found except in severe cases.

Remarks. (a) More stages of growth likely to be seen in one film than in other species. Gametocytes appear early in cycle. (b) Parasites frequently more numerous than in other infections. Unlike other species-growth of asexual forms, following the ring stage, take place in internal organs. (c) Parasites are usually more compact and hence appear more intensely stained than those of other common species. Gametoeytes rarer than in other species, appear late. Least often found of 3 species in United States. (d) Species not found to date in United States. Differentiation not possible in thick films.

In thick films, stained as recommended, the erythrocyte outlines are destroyed by lysis, leaving a background which varies from clear light blue to mottled gray-blue, often in anemic bloods clouded with nuclear and reticular remains of immature erythrocytes. Against this background is seen the familiar purple or violet nuclei of the leukocytes, sometimes with ragged cytoplasm. The neutrophilic granules are indistinet or absent, eosinophilic granules are usually retained with characteristic color, and platelets appear singly or in groups with distinctive texture and more or less hazy outline. White cells containing malarial pigment are much more easily found in the thick film than in the thin film.

In the thick film, the parasite, minus the cell wall, has much the same appearance as in the thin film, except that it may seem smaller and more shrunken in the thicker portion of the smear. The thin edge of the thick film resembles the thin film so elosely that it is very valuable to the technician 
(particularly the less experienced) for study and for classification of species and determination of mixed infections. Schüffner's dots frequently show quite plainly here, whereas in the thicker portion they may be lacking entirely or may show only as a delicate pink area around the parasite.

\section{Parasites in the Thick Film}

Young trophozoites. In the thick film the young parasites of all species often appear, not as complete ring forms, but as chromatin dots associated with only a portion of the cytoplasmic circle. These forms have been described aptly, according to their appearance, as "interrupted rings, exclamation marks, and swallow forms" (Field and LeFleming 1939). When only this stage of the parasite is present in the blood, difficulty is encountered in differentiating the species. However, if the rings are very small and delicate, or if there are many ring forms and no older stages of the parasite, the species is very likely $P$. falciparum.

Half grown and old trophozoites. The older trophozoites of $P$. vivax exhibit a decided tendency for the cytoplasm to be fragmented and arranged in a cluster of varying-sized particles often with no visible connection. This eytoplasm is associated with a round or irregularly shaped red or magenta mass of chromatin. The pigment appears as a yellowish haze or as small light brown grains on the eytoplasm. Some of the older trophozoites, with one chromatin mass, are rather solid and regular in outline. Those of $P$. malariae exhibit a heavy dark pigment in the compact cytoplasm which gives a dense appearance to many of the parasites of this stage. The chromatin is often not conspicuous, though it may be found in an elongated mass so characteristic of this stage in the thin film. The older trophozoite of $\boldsymbol{P}$. falciparum is very small, often non-vacuolated, the cytoplasm is a lighter color than that of $P$. malariae, and the pigment, even at this stage, is usually clumped in one or two very small masses.

Presegmenting schizont. In the presegmenting stage of $P$. vivax the cytoplasm is less tenuous than in the trophozoite and the parasite is generally more compact in appearance. The chromatin is divided into several more or less irregular masses, and as the segmenting stage approaches these appear more rounded, and are individually associated with smaller amounts of the cytoplasm. The pigment meanwhile is gradually collected into one or more definite clumps. This stage of $P$. malariae is very similar to the corresponding one of $P$. vivax and often is difficult to classify in a thick film unless more readily recognized stages are present also. When this stage of $P$. falciparum is found in the peripheral blood, it is small, compact, irregularly shaped, with deeply staining cytoplasm in which are embedded the vague magenta chromatin divisions, and a small dense mass of dark pigment.

Segmenting schizont. The segmenting schizonts of the three species resemble very closely the same stages in the thin film, practically the only difference being the absence of the cell outline and a possible shrinkage of the parasite. Species may be differentiated by the comparative size of the parasites and by the number of daughter cells. It is unusual to find this stage of $P$. falciparum except in severe cases.

Gametocytes. In the thick film it is impossible to distinguish the macrogametocytes of either $P$. vivax or $P$. malariae from the compact, rounded or oval mature trophozoites. Microgametecytes of these two species are more easily determined because of their large, usually rounded nucleus surrounded by a small amount of very light staining or colorless eytoplasm containing numerous grains of prominent pigment. The nucleus stains more deeply, comparatively speaking, than in the thin film. There is no other stage that resembles this closely. In thick films which have dried slowly, exflagellation of fully matured microgametocytes may occasionally be observed.

The gametocytes of $P$. falciparum are easily determined by their characteristic elongate or sausage-like shape, though it is often difficult to differentiate the sexes in 
thick films. In heavier portions of thick films, particularly when the blood dries slowly, mature gametocytes assume a rounded shape, a change which would take place normally in the mosquito during the early stage of maturation. Usually typical forms will be found in the thinner edge of such films, however.

Mixed infections. Mixed infections are undoubtedly more prevalent than reports would indicate, since the parasites of one species usually predominate and the other species is overlooked or not recognized in examinations.

\section{Fallacies and Puzzles in Thick Film Examinations}

The inexperienced microscopist may be confused in thick film examination by dirt or bacteria from the skin; by dust particles on the slide; by vegetable spores, yeast cells or fungi from the air; and by bacteria, molds and protozoa, or other contaminants from distilled water used in staining. Hence great cleanliness should be exercised to reduce these artefacts to a minimum. Artefacts which may deceive the inexperienced will be found frequently to lie above the blood plane, or they may be refractile and focus out of the field unevenly. Cocei from the skin may stain like chromatin dots and may resemble parasites if adjacent to blue-stained particles of fibrin or cellular substance. This rarely occurs more than once or twice on a single slide and numbers of free cocci will usually be found also ; whereas in well stained smears free chromatin dots are rarely found. Thick films should not be diagnosed as positive on what appears to be only one parasite. The parasites should be unmistakable, or search should be continued until others are found. If this is impossible, later smears should be made.

\section{Possibility of Detection of Parasites by the Thick Film}

Many malariologists believe that the number of parasites necessary to produce symptoms of malaria as a general rule is more than the minimum which can ordinarily be detected in thick films, unless the patient has been recently taking antimalarial treatment. However, in persons with extreme susceptibility symptoms occur before parasites can be found in the blood stream. In these cases further subsequent smears should be made on successive days. 


\title{
GENERAL MORPHOLOGY OF ANOPHELES AND CLASSIFICATION OF THE NEARCTIC SPECIES
}

\author{
By W. V. KING and G. H. BRADLEY \\ BUREAU OF ENTOMOLOGY AND PLANT QUARANTINE, UNITED STATES DEPARTMENT OF AGRICULTURE, \\ WASHINGTON, D. C.
}

Morphologically the tribe Anophelini is an unusually homogeneous group of insects and for the most part is rather sharply differentiated from other tribes of mosquitoes, particularly in the larval stage.

Adult anophelines are characterized principally by the long palpi in the female, club-shaped palpi in the male, a vestiture of hair, rather than scales, on the body, and a, rounded scutellum. The last also occurs, however, in Megarhinus. Absence of scales on the body is usually, or frequently, complete, but a number of species have some scaling toward the posterior end of the abdomen and a few have scale tufts on the abdominal segments. The palpi in both sexes are of about the same length as the proboscis. Nearly all anophelines have spotted wings, produced either by a clumping of dark scales, or more usually by alternating spots of black and white. Wing spotting, however, also occurs in other groups of mosquitoes.

The mouthparts of anophelines are similar to those of other mosquitoes, but the number of maxillary teeth (consisting of fine serrations at the tip of the maxillae) has been given considerable attention in taxonomic work. Internally, the number of teeth and shape of the sclerotized plates in the pharynx serve to differentiate subgenera and other species groups in the tribe. The principal characters are found in the toothed portion known as the pharyngeal armature (Christophers 1933).

Among the characters of the male genitalia are the elongated, sclerotized mesosome, or phallosome, which, in the great majority of species, is surmounted by one or more pairs of small leaflets. The latter frequently vary in number and shape in different species. Below the mesosome and connecting the bases of the sidepieces is a membrane, which in most of the nearctic species is expanded on each side into a lobe having several spinelike hairs on the posterior margin. These lobes are called the claspettes, or basal membranous lobes, and the shape or arrangement of their spines is important in the classification of species. The modified hairs on the sidepieces also show differences of subgeneric value.

In the larvae the absence of an elongated breathing tube distinguishes anophelines at once from other mosquitoes. The paired spiracles open into a large sclerotized plate on the eighth and ninth abdominal segments. The larvae are also characterized by the presence of palmate float hairs on the dorsum of some or all of the abdominal segments and sometimes on the thorax. These structures, together with a peculiar pair of membranous, retractile rrgans on the anterior edge of the thorax icalled the notched organs or retractile appendages) serve to suspend the larva in its habitual position in the water just below the surface film. Below the spiracular apsaratus, on each side, is a sclerotized plate vith a row of teeth, usually long and short ones more or less alternating, on the posterior margin. These plates have usually been referred to as the combs but probably zorrespond to the pecten in other Culicidae, the true comb scales being absent in anopheline larvae except in the first instar.

The larval pilotaxy is notably consistent within the group in the number and disposition of hairs but differs considerably from that of other kinds of mosquitoes. Of 
special note are the frontal head hairs, which consist of three pairs, usually large and plumose, placed in a straight line across the middle of the head capsule, or frontoclypeus. Large plumose lateral hairs occur on the thoracic segments and on the first three abdominal segments. Toward the front of the head there are three pairs of so-called clypeal hairs, the two anterior pairs especially being used extensively in identification. On the ventral side of the thorax are three paired groups, known as the pleural hairs, the points of origin of which correspond to structures found on other dipterous larvae and are believed to be the remains of larval legs in this order. The character of these hairs tends to be correlated with other characters that differentiate subgenera and other species groups.

A system of numbers has been developed for all the hairs of the fourth instar and is convenient for taxonomic work. Perhaps the most complete of the several lists published is that of Puri (1931).

In the younger instars a broad collar of dark chitin is present at the base of the head, decreasing in comparative width with each succeeding molt. This collar is useful in distinguishing fourth-instars from earlier stages. Third-instars, so far as known, have the same number of hairs as in the fourth instar but the number of branches of the branched hairs is usually less. A detailed study of the pilotaxy of the different instars of Anopheles walkeri has been presented by Hurlbut (1938a). He states that the definitive form and position of the hairs are already evident in the second instar but that certain hairs are lacking and the number of branches of other hairs is still further reduced. In the first instar he finds that many of the hairs differ strikingly in form and position from those in the next instar. This author has also made a study of the early instars of other species and has prepared a key (in press) for the identification of first-instars of the common Southern species.

In the pupa of anophelines the air trumpets invariably have a short base and a wide opening, usually decidedly flared in appearance. This has proved to be a very useful character for recognizing Anopheles pupae in field collecting. There is also a stout, black spine on the posterior corners of abdominal segments 3 to 7 , while the eighth segment has a similar stout spine usually with plumose lateral branches. Edwards (1932) separates the pupae of this tribe by the fact that the apical lateral hair of the abdominal segments is placed at the corner of the segments, whereas it is well away from the corner in other mosquitoes. As a rule, the number of branches of the pupal hairs is subject to considerable variation, but specific differences have been found in some cases. The pupal characters, however, do not appear to be of much value for the separation of higher groups.

The eggs of anophelines are boat-shaped in outline and their most distinctive feature is the presence of floats, or hydrostatic organs, on each side. These floats are divided into a number of small compartments, filled with air. The surface of the egg is finely reticulated and the outer membrane, or exochorion, on the dorsal surface varies considerably in pattern. The egg characters, principally those of the floats and dorsal pattern, have been studied extensively in recent years and have been employed for the separation of closely related species and subspecies, particularly those of the maculipennis group.

\section{Classification}

The anophelines of the world are divided by Edwards (1932) into three generaAnopheles, Chagasia, and Bironella; and the genus Anopheles into four subgeneraAnopheles, Myzomyia, Nyssorhynchus, and Stethomyia. Dyar (1928) recognized only the single genus Anopheles in the Americas, with Anopheles, Chagasia, Kerteszia, and Nyssorhynchus as subgenera (Bironella and Myzomyia being limited to the old World). Except for Anopheles albimanus, which is found in the lower Rio Grande Valley, all the anophelines of the United States and Canada belong to the subgenus Anopheles and also to the group Anopheles as defined by Edwards. 
The following 12 species and subspecies of the subgenus Anopheles may now be recognized in this territory, with one additional variety (pseudopunctipennis boydi). whose status is not entirely clear. The type locality of each is shown following the date of publication.

Anopheles atropos D. and K., 1906; Florida Keys. Anopheles barberi Coq., 1903; Plummers Island, Md.

Anopheles crucians Wied., 1828; Pennsylvania and New Orleans.

Anopheles bradleyi King, 1939; Brevard County, Fla. (crucians bradleyi).

Anopheles georgianus King, 1939; Brooks County, Ga. (crucians georgianus).

Anopheles occidentalis D. and K., 1906; Stanford University, Calif. (maculipennis auet., in part). Anopheles freeborni Aitken, 1939; Davis, Calif. (maculipennis freeborni).

Anopheles punctipennis (Say), 1823; United States.

Anopheles pseudopunctipennis pseudopunctipennis Theob., 1901; Grenada.

Anopheles pseudopunctipennis franciscanus McC., 1904; California (Stanford University).

Anopheles ? pseudopunctipennis boydi Vargas, 1939; California.

Anopheles quadrimaculatus Say, 1824; Northwest Territory.

Anopheles walkeri Theob., 1901; Lake Simeoe, Ontario, Canada.

Another species, or subspecies, belonging to the maculipennis series, Anopheles aztecus Hoffmann (1935) (maculipennis aztecus), occurs in the highlands of Mexico.

With reference to $A$. occidentalis, this species has usually been referred to in North America as A. maculipennis and was so treated by Dyar (1928) and by Matheson (1929). Edwards (1932) listed it as a questionable variety of the European form, while Dampf $(1935,1936)$ and Bates (1940) thought it could be regarded as a separate species. Martini (1933) and Dampf pointed out that the male genitalia differ from those of the typical $A$. maculipennis of Europe and are more nearly related to $A$. labranchiae and $A$. atroparvus.

The species was originally described on the basis of the area of pale fringe scales at the tip of the wing. Freeborn (1926), however, stated that this form in California seemed to be limited to a narrow strip along the coast, whereas material from in- land localities did not have a distinct spot. Aitken (1939) proposed a new name, $\boldsymbol{A}$. maculipennis freeborni, for the dark-tipped form, finding additional difference in the eggs, larvae and pupae but not in the male terminalia. He considered that typical $A$. maculipennis does not occur in this country. Dampf stated that $A$. aztecus Hoffmann does not differ in male genitalia from A. occidentalis but does in the number of compartments of the egg floats. One of the present writers (King) has noted that larval specimens of aztecus (obtained in $\mathbf{1 9 3 0}$ from Xochimilcho and from the Lerma River in Mexico, D. F.) differ rather markedly in the inner anterior clypeal hairs, which are widely separated and very long and stout in comparison with $A$. occidentalis or $A$. freeborni.

Dr. S. B. Freeborn, in a personal communication, indicates that he is in favor of retaining all three as subspecies of $A$. maculipennis (in order to show relationship), and this has been done by Aitken in a valuable fortheoming monograph, "Studies on the Anopheline Complex of Western America." ${ }_{1}$ Since there are appreciable differences in the spines of the claspette, however, it is believed that the American forms should be separated specifically from A. maculipennis s.s., and this opinion is concurred in by Dr. Alan Stone of the National Museum (personal communication). The writers are also of the opinion that these three forms, as well as those of the crucians group, represent distinct species and could well be raised to a specific status. This has the advantage of eliminating the use of triple names.

Two larval forms of $A$. walkeri have been described by Bradley (1936), but Matheson and Hurlbut (1937) were of the opinion that the differences were merely variations. The characters are entirely similar, however, to those that have been

1 Through the kindness of the author a copy of the manuseript of this work has been made available to the present writers, which has made it possible to incorporate some of its information in the present review. 


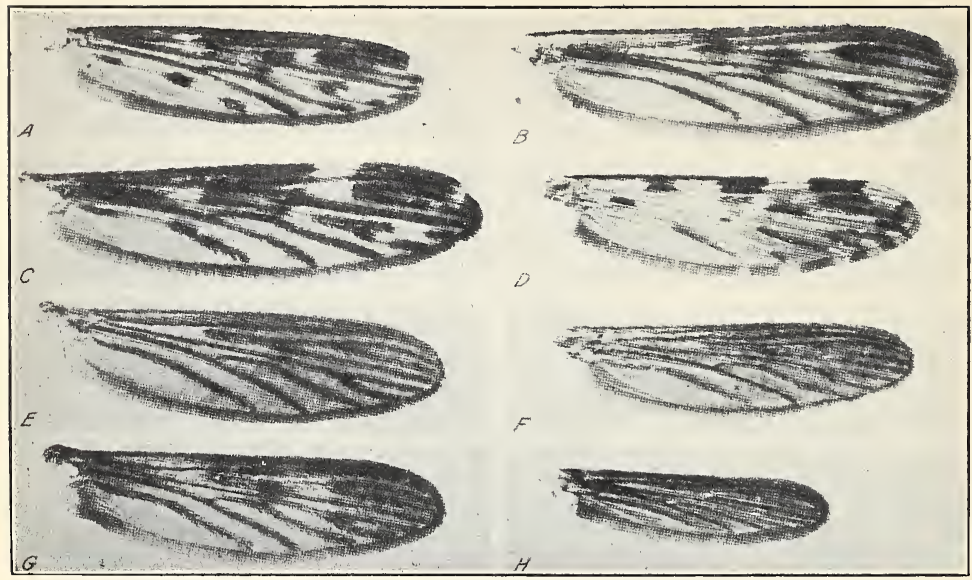

Plate I. Wings of nearetic Anopheles: A, crucians; B, maculipennis; C, punctipennis; D, pseudopunctipennis; E, walkeri; F, atropos; G, quadrimac llatus; $\mathrm{H}$, barberi (from King, Bradley and MeNeel 1939).

found reliable in some of the oriental species, so it would appear desirable to reexamine the question in larger series of specimens.

As might be expected, in view of its wide range, $A$. punctipennis shows a great deal of variation in the wing markings, and to a lesser degree in larval characters. The wings usually have large and very distinet white spots, but the white may be greatly reduced or even practically lacking in some individuals. A series of specimens from central Florida placed beside a series from the Northwest would almost certainly be taken for different species at first glance. The dark form has in fact been deseribed as another species (Anopheles perplexans Ludlow 1907), which was placed in synonymy, as all intergradations appear to occur.

The taxonomic status of Anopheles pseudopunctipennis in the United States is somewhat confusing at the present time. Anopheles franciscanus was described from California by McCracken in 1904 but later placed as a synonym of $A$. pseudopunctipennis. Freeborn (1926) noted that the mesosome of male specimens in California were without leaflets although these had been recorded as present in material from Mexico. Vargas (1939b) described Anopheles boydi as a California species differing from the typical pseudopunctipennis in certain egg characters. Aitken (1941) after an extended study has come to the conclusion that typical pseudopunctipennis does not oceur in California and and that the common form in that region is franciscanus, which he treats as a subspecies of pseudopunctipennis. This form has very delicate mesosomal leaflets. $\mathrm{He}$ also thinks that boydi may be a variety of franciscanus, represented by the material in which the leaflets are lacking.

One of the distinguishing characters given by Aitken for the California material (both forms) is the absence of tails on the larval spiracular plate. These have been described from tropical material and were noted by Aitken on specimens from New 


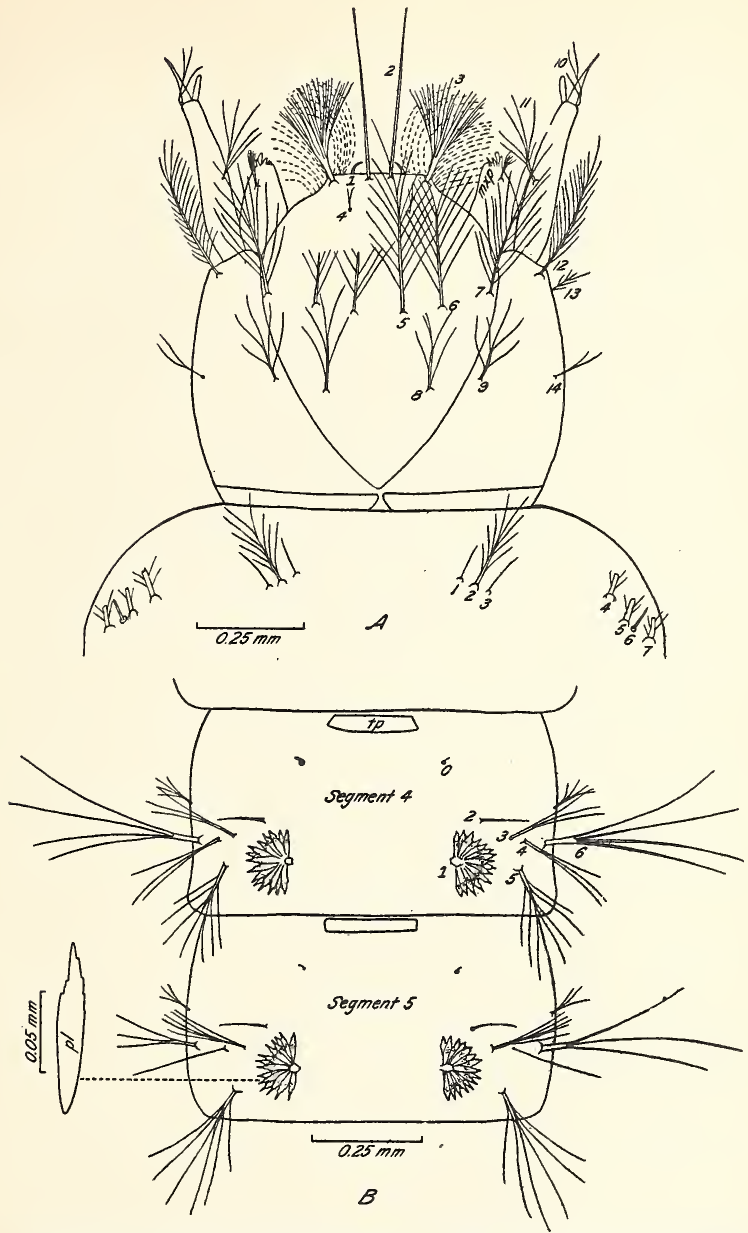

Fic. 1. Dorsal hairs of larva of Anopheles quadrimaculatus: A, head and part of prothorax; B, abdominal segments 4 and 5 (after King, Bradley and MeNeel 1939).

Mexico and Texas. Among a few larvae from these states examined by one of the present writers (King), the tails are pres- ent in some but lacking in others. Further study of adequate material will be required to determine whether both franciscanus 


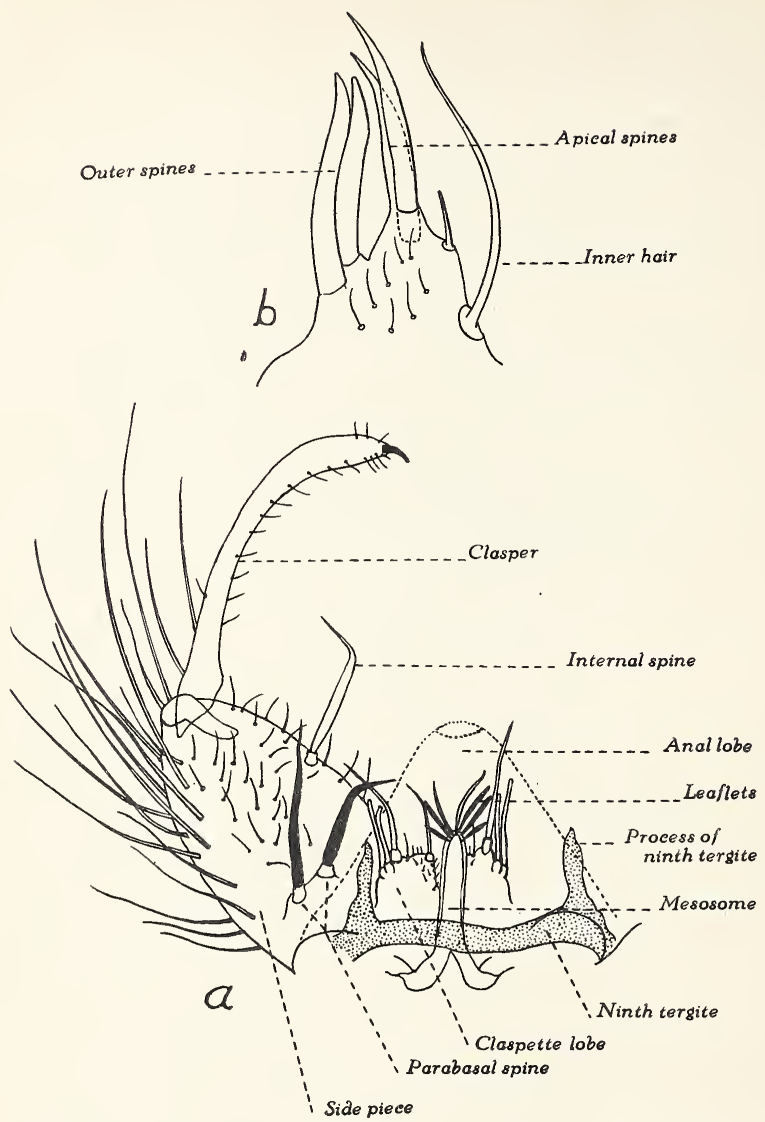

F'IG. 2a. Male terminalia of Anopheles punctipennis (after Root, Amer. Jour. Hyg., 3: 264); b. claspette spines of Anopheles crucianus (after King, Amer. Jour. Trop. Med., 19: 461, 1939.)

and the type form are present in these areas.

\section{IDENTIFICATION}

Brief keys are appended for the identification of the nearctic species by adult coloration, male terminalia, and larval pi- lotaxy. A. albımanus, although occurring in extreme southern Texas, has not been included, as it is chiefly a tropical species and will be treated by other authors in the section covering tropical America. Both the larvae and adults are easily distin- 
guished from the nearctic species. Illustrations of the wings of the nearetic species are given in Plate 1 , the general larval characters in Fig. 1, and the male terminalia in Fig. 2.

\section{Kex to AduLts}

1. Wings with areas of white seales on the veins .....2 Wing veins entirely dark-sealed ................................... 4

2. Costa with a white spot at the outer third (opposite tip of subcostal vein); anal vein with one or two areas of dark scales.

Costa dark except at extreme tip of wing; anal vein with three dark spots separated by white; apical segment of palpi pale and segments 3 and 4 with narrow pale bands or a few pale scales at base and apex.

\section{Anopheles crucians group}

3. Veins 3 and 5 entirely dark-scaled; wing fringe without pale spots at tips of veins; apical half and basal one-fourth of anal vein dark-scaled; palpi dark, unbanded...Anopheles punctipennis

Veins 3 and 5 with long pale areas centrally; wing fringe with pale spots at tips of veins; apical half of anal vein dark, basal half white; apical palpal segment of female either entirely pale or dark tipped, a narrow ring at base of fourth segment.

Anopheles pseudopunctipennis

4. Wings unspotted; palpi and legs dark-scaled; mesonotal bristles very long, the average length about one-half the width of mesonotum. A small species. Anopheles barberi

Wings with spots of dark seales, more or less distinct, or palpi with rings of white seales: mesonotal hairs comparatively short.....................5

5. Palpi narrowly but usually distinetly ringed at apex of the apical segments; knee spots present …............................................

Palpi dark scaled (faint white rings sometimes present at base of apical segment in atropos)

6. Wing spots indistinct; knee spots absent; general coloration very dark. Gulf and Atlantic Coasts Anopheles atropos

Wing spots usually distinet; color brown ........... 7

7. Tip of wing with a patch of silvery or golden fringe seales; dark wing spots very pronounced. Northern and Western distribution. Anopheles occidentalis

Wing fringe dark.

8. Occurs east of the Rocky Mountains.

Anopheles quadrimaculatus

Occurs west of the Rocky Mountains; wing spots usually more distinct than in quadrimaculatus Anopheles freeborni

\section{Kex to Male Terminalia}

1. Mesosome (phallosome) without leaflets; claspette bilobed, the outer lobe prominent, oval with two or three flattened spines at apex, the inner with two slender spines.

Anopheles barberi

Mesosome with leaflets or claspette not as above

2. Leaflets of mesosome serrated (lacking in some California specimens, possibly variety boydi) claspette bilobed, with two or three spines at the apex of each lobe, those on the dorsal lobe short and flattened; ninth tergite without processes...............Anopheles pseudopunctipennis Leaflets stout, nonserrated

3. Outer (dorsal) spines of elaspette stout, bluntly rounded sometimes expanded at tip or partially fused

Spines of claspette not as above $\quad 6$

4. Processes, or arms of ninth tergite short, stout, usually expanded at apex; outer spines of claspette frequently fused at apex.

Anopheles quadrimaculatus

Processes comparatively slender and pointed or slightly rounded

5. Mesosome with second pair of leaflets from apex more than half the length of the first pair. Anopheles walkeri

Second pair of leaflets no more than half the length of the first pair ......... Anopheles atropos

6. Claspette with one large apical spine, tapered to a sharp point, and one or two slender external spines, rounded or slightly tapered at tip; arms of ninth tergite short, usually stout, expanded or obliquely truncate at apex.

Anopheles punctipennis

Claspette usually with three or four apical and external spines, all acute

7. Sidepiece with scales, usually numerous; clasper without vestiture of fine hairs; claspette lobe triangular in shape.

Anopheles crucians group

Sidepieces with few if any seales; basal third of clasper with numerous fine nonpapillated hairs (Aitken); elaspette usually rounded ....8

8. Arms of ninth tergite short, stout.

Anopheles occidentalis

Arms of ninth tergite comparatively long and slender. Anopheles freeborni

\section{KEY TO LARVAE (FouRTH INSTAR)}

1. Abdomen with plumose lateral hairs on first six segments; frontal head hairs (hairs 6-8) minute, simple ……………........ Anopheles barberi

Abdomen with plumose lateral hairs on first three segments only; head with large plumose frontal hairs

2. Outer elypeal hair (hair 3) branched ….......... 3 All elypeal hairs simple, the inner pair (hair 2) well separated; postspiracular plate with or without a slender, blackish tail posteriorily on each side; leaflets of palmate hairs ending in long, slender filaments.

Anopheles pseudopunctipennis 
3. Outer clypeal hairs sparsely feathered or branched ( 5 to 10 short branches) on apical half; inner elypeal hairs sparsely feathered at tip ... Anopheles atropos

Outer elypeal hairs thickly branched, the branching dichotomus.

4. Inner elypeal hairs with sparse, minute feathering toward tip; hair 1 of prothorax with three to five strong branches from near base; hair 0 of abdominal segments comparatively large, with three to seven branches. (Specimens from New York State have been observed with these last two hairs undifferentiated.) ……............................Anopheles walkeri

Inner elypeal hairs simple (or sometimes forked toward tip); hair 1 of prothorax short, single or weakly branched at tip

5. Abdominal segments with two conspicuous tufted hairs (hairs 2 and 0 ) anterior to palmate hair; these hairs usually approximately equal in size and with four to nine branches on segments 4 and 5 ; lateral hairs (hair 6) of segments 4 and 5 usually branched at about basal third ..............................Anopheles crucians

Anterior submedian hair (hair 0 ) vestigial; antepalmate hair (hair 2) on segments 4 and 5 , usually with no more than 3 branches........... 6

6. Palmate hairs on segments 3 and 7 well de- veloped, the leaflets broad and mostly serrated toward tip. 7

Palmate hairs on segments 3 and 7 less developed than those on segments 4 to 6 , the leaflets slender and mostly nonserrated, or rudimentary

8

7. Basal tubercles of inner anterior elypeal hairs separated by at least the diameter of one of the tubercles; antepalmate hairs on segments 4 and 5 usually single; leaflets of palmate hairs sometimes developed on segment 2 .

Anopheles quadrimaculatus

Basal tubercles of inner anterior elypeal hairs separated by less than their diameter; antepalmate hairs on segments 4 and 5 usually double Anopheles punctipennis, Anopheles freeborni and Anopheles occidentalis

8. Five pairs of functional palmate hairs (segments 3 to 7 ); antepalmate hairs an segments 4 and 5 single or double. Gulf and Atlantic Coasts.. Anopheles bradleyi

Three pairs of functional palmate hairs (segments 4 to 6 ), those on segments 3 and 7 rudimentary or only slightly developed; antepalmate hairs on segments 4 and 5 three to five branched. Inland Georgia.

Anopheles georgianus 


\title{
DISTRIBUTION OF THE NEARCTIC SPECIES OF ANOPHELES
}

\author{
By W. V. KING and G. H. BRADLEY \\ BUREAU OF ENTOMOLOGY AND PLANT QUARANTINE, UNITED STATES DEPARTMENT OF AGRICULTURE, \\ WASHINGTON, D. C.
}

A CONSIDERABLe list of locality records of North American Anopheles was published by Howard, Dyar, and Knab (1917a) and Dyar (1922), to which many others have been added. Kumm (1929b), in recording the distribution of malaria-carrying mosquitoes of the world, assembled most of the available records for six of the species of Anopheles covered in the present article, quadrimaculatus, maculipennis, punctipennis, crucians, pseudopunctipennis, and albimanus. Small-seale maps showing their distribution were included. Since Kumm's publication, additional records have become available, and maps showing State-wide distributions of anophelines have been published for Mississippi by Perez (1930), for
California by Herms (1929), for New Mexico by Barber and Forbrich (1933), and for Minnesota by Owen (1937).

The recognition and classification of mosquitoes have had a gradual development since about 1900 , but some of the earlier identifications are questionable. During recent years there has been some activity in the separation of closely related species or subspecies, which also complicates the matter of previous records.

For the present article small-scale maps (Figs. 1 to 4 ) have been prepared to show the distribution of the species in the United States and southern Canada. For the commoner species the areas in which they are known to have a more or less general dis-

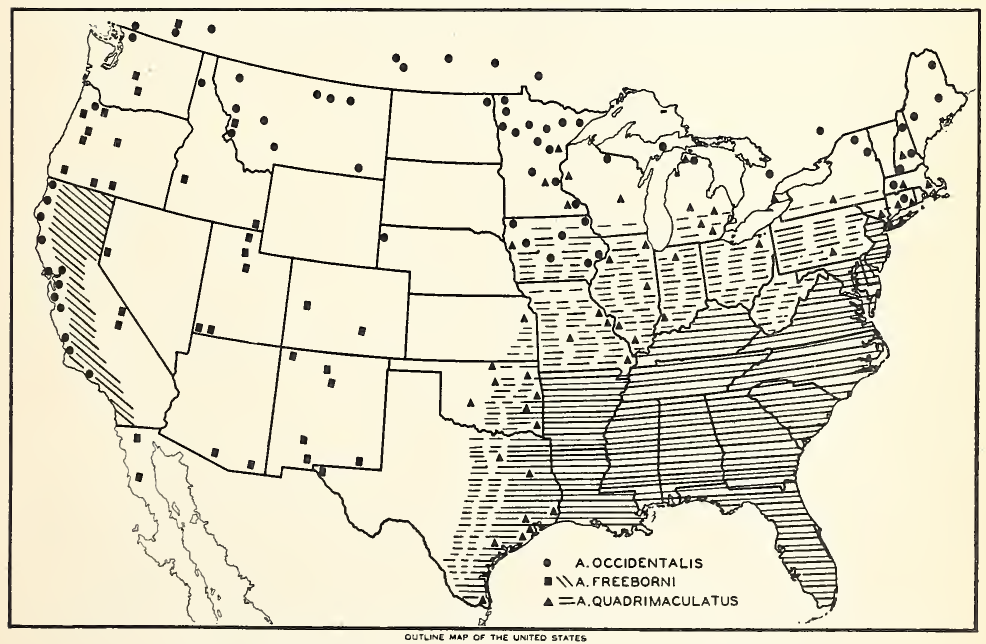

FiG. 1. Anopheles quadrimaculatus, A. occidentalis, and A. freeborni. 


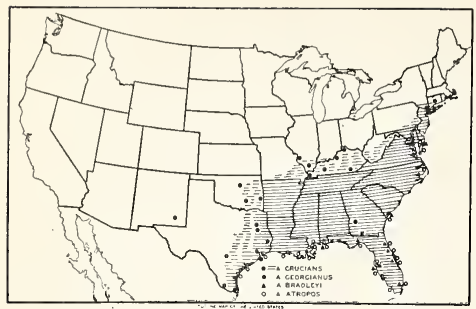

FIg. 2. Anopheles crucians, A. bradleyi, A. georgianus, and $A$. atropos.

tribution are indicated by shading with solid lines, while broken lines are used to indicate areas of probable or seattered occurrence. Individual locality records are shown approximately by spot-marking around the periphery of the shaded areas to indicate the present available information as to the limits of range. For the less common species all the locality records have been spotted in, except that in the case of closely grouped localities, a single spot may represent several records. The specific localities represented by the spotting and the sources of the records (many unpublished) are given in the locality records, but for lack of space the complete literature citations have had to be limited to the general mention of the works in other parts of the text. For the same reason locality records for most of the shaded areas have been omitted. In eiting the authorities for the records given, the names of Howard, Dyar and Knab (1917a) have been abbreviated to $H$. D. and $K$.

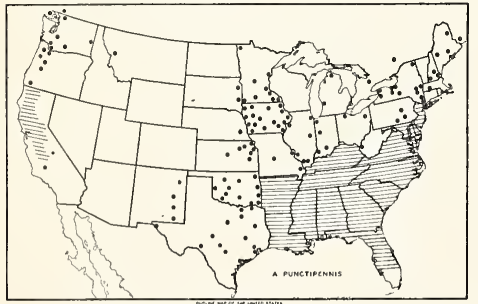

FIG. 3. Anopheles punctipennis.
An unexpectedly small number of records is to be found in these lists for such states as Pennsylvania, Ohio, Indiana, Missouri, Iowa, Kansas, and Oklahoma. Since endemic malaria is known to occur in portions of these states, the paucity of records may be attributed in part to lack of collections or publication. There are almost no published records for some of the region east of the Rocky Mountains.

While it is possible that a part of this region is free of anophelines, recent correspondence with workers in these states has brought out new records; included here, for certain parts of Nebraska, the Dakotas and Iowa. Of special interest is the discov-

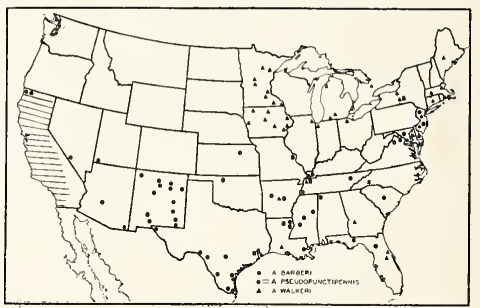

FIG. 4. Anopheles pseudopunctipennis, A. barberi, and $A$. walkeri.

ery of Anopheles occidentalis in Iowa and northwestern Nebraska.

\section{Anopheles albimanus Wied.}

Anopheles albimanus Wied. is principally of tropical distribution and is known to occur at present in the United States only in the lower Rio Grande Valley of Texas. It was discovered in Key West, Fla., in 1904, but apparently was eliminated from that locality. Locality records are available as follows :

Texas: Brownsville (Dyar 1928). Limited to Cameron and Hidalgo Counties (Kumm 1929). Cameron County and Brownsville (King 1937). Donna (P. T. Riherd coll. 12/24/39). Cameron, Hidalgo, Willacy, and Jim Wells Counties (notes from T. E. MeGregor to F. C. Bishopp 1940).

Florida: Key West (Gardner 1904; G. N. MacDonell; H. D. and K. 1918). Not found on the Florida Keys since 1904 (King 1937). 


\section{Anopheles atropos D. and K.}

Anopheles atropos $\mathrm{D}$. and $\mathrm{K}$. breeds in salt marshes and occurs on the Gulf and Atlantic coasts from Texas to Maryland. Adults have occasionally been encountered in large numbers. Locality records are available as follows :

Maryland: Chesapeake Beach and Crisfield (Bishopp, Cory and Stone 1933).

Virginia: Onley (Bishopp, Cory and Stone 1933).

South Carolina: Parris Island (King, Bradley and MeNeal 1939).

Florida: Florida Keys (type locality, Dyar and Knab 1906). New Smyrna; Lostmans River; Sebastian Inlet; Atlantic coast in southern Florida (King, Bradley and MeNeel 1939). Key West (Fisk 1939).

Alabama: Bayou Labatre, Mobile County (Griffitts 1927).

Mississippi: Gulfport (Beyer 1923). Cat Island; Ship Island; Gulfport (Komp 1926). Lake Bourne Lighthouse; Pointe aux Chenes; Biloxi (Griffitts 1927).

Louisiana: Buras (Dyar 1922). Terrebonne (Dyar 1922, identified as walkeri; cf. Hinman 1932). Freshwater Bayou, Vermilion Parish; Bayou Terrebonne; Cocodrie (Beyer 1923 and 1926). Buras (Griffitts 1927). Buras and Grand Bayou (Hinman 1932).

Texas: Corpus Christi (2/1/34), Port Lavaca (2/6/34) (CWA collections, determined by Alan Stone). Galveston (Dr. Mattes coll., 1938).

Mexico: Provisionally identified by Martini (1935), but the record reported as in error by Hoffmann (1936).

Cuba: Oriente Province (Carr, Melendez and Ros 1940).

\section{Anopheles barberi Coq.}

Anopheles barberi Coq., a tree-hole breeder, is quite widely distributed in the Southern and Eastern States, but because of its limited habitat is not commonly found. Matheson (1929) states that the most northern record of its occurrence is Ithaca, N. Y. In its western range it has been recorded from eastern Missouri to southeastern Texas. Locality records are available as follows :

New York: Ithaca, most northern point (Matheson 1929).

New Jersey: Bordentown and Chester (J. B. Smith, quoted by Headlee 1921). Morris and Union Counties (Mulhern 1939). Passaic, Bergen, Middlesex and Camden Counties (Mulhern 1940).

Maryland: Plummers Island and Cabin John (H. D. and K.).
Virginia: Bluemont and Woodstock (H. D. and K.).

Kentucky: Paducah (G. E. Quinby coll., 6/10/39). Wickliffe; Holloway Landing; Murry; Fulton; Arlington (G. E. Quinby eoll.).

Missouri: Saint Louis (H. D. and K.).

North Carolina: Tryon (H. D. and K.).

South Carolina: Columbia (H. D. and K.).

Florida: Tallahassee; Gainesville (King, Bradley and McNeel 1939).

Alabama: Colbert County (Shields and Miles 1937; Shields and Lackey 1938; Shields 1938).

Tennessee: Reelfoot Lake (Brown and Pearson 1938). Norris (Shields 1938).

Mississippi: Agrieultural College (H. D. and K.).

Arkansas: Lonoke County; Mt. Nebo (Thibaulti 1910). Scott (H. D. and K.). Southeastern Arkansas (Horsfall 1937). Little Rock (Carpenter 1939).

Louisiana: Mound (King, Bradley and McNeel 1939). Ouchita Parish (E. B. Johnson coll., 1938).

Texas: Houston (M. Martin coll., 1938).

\section{Anopheles crucians Wied.}

The range of Anopheles crucians Wied. is nearly as wide as that of $\boldsymbol{A}$. quadrimaculatus in the Eastern and Southern States, but it is primarily a species of the coastal plain and inland is almost always much more restricted, both in abundance and in local occurrence, than is quadrimaculatus. Toward the coast it is frequently the predominant anopheline and probably reaches its greatest abundance in the southern half of Florida, where the larvae are commonly found in pure culture in the cypress swamps and other types of breeding places. In the vicinity of salt marshes, unless the identifications have been based on larval specimens, records may include the similar coastal species bradleyi.

The general distribution of crucians may be described as follows: From southern New York, Connecticut, and New Jersey, through the Southeastern States to central Texas and Oklahoma, and northward to southern Illinois and Kentucky. It has also been recorded recently from one isolated locality in New Mexico. No records are at hand from Missouri, Indiana, Ohio, or West Virginia, although it may occur in some of these areas. Outside of the United States it is found in Cuba and other islands in the Caribbean area, and. on the east coast of Mexico. Locality records for the north- 
ern and western portions of its range are as follows:

New York: Bellport (H. D. and K.). Southern New York (Matheson 1929). Amaganset; Ft. Hamilton (quoted by Kumm 1929). New York City ("M.B." 1939).

Massachusetts: Orleans (Tulloch 1939).

Connecticut: Reported in State (Botsford and Turner 1933).

Illinois: Herrin (Kumm). East Saint Louis (collected, 1937 ; notes from F. C. Bishopp).

Kentucky: Mercer; MeGee Springs; Louisville; Bardwell; East Bernstadt; Kent and Whiteley Counties (notes from Go E. Quinby 1940).

Oklahoma: Stillwater (Gaines Eddy coll., 7/4/37). Muse (D. E. Howell 1940). Colgate (L. I: Rozeboom 1940).

Texas: Buena; Galveston; Tyler (H. D. and K.). Ft. Ringold; Leon Springs (Ludlow 1914). Dialville; Ellington Field; Ft. Crockett (quoted by Kumm 1929). Houston (Matthes 1935). Brownsville (Barber, Komp and King 1929): Seabrook (CWA coll. 1934).

New Mexico: Artesia, Pecos Valley (Barber 1939).

\section{Anopheles bradleyi King}

Anopheles bradleyi King breeds in salt marshes and has almost the same distribution as A. atropos along the Gulf and Atlantic coasts. The two species apparently do not often breed together, however, as bradleyi occurs in brackish water while atropos seems to prefer water of higher salt content.

Locality records: King (1939) gave the known localities from which larvae of this form had been identified. These are as follows: Ocean City, Md.; Parris Island, S. C.; St. Johns River (Brevard County), Apalachee Bay, and various coastal localities in Florida; Coden, Ala.; St. Bernard Parish, Buras, and Grand Bayou, La. Additional specific localities in Florida are the counties of St. Johns, Volusia, Brevard, Indian River, St. Lucie, Broward, Dade, Manatee, and Hillsboro (collections by the writers). Vargas (1940) states that larvae of this form have been identified from saline pools in the State of Vera Cruz, Mexico.

\section{Anopheles georgianus King}

Anopheles georgianus King was first found by R. E. Bellamy in fresh-water pools in Brooks County, Ga., and subsequently was collected by him in Sumter, Terrell, and Thomas Counties (Bellamy 1939). Material from several of these areas was examined by King (1939) in describing the variety. No other records of its occurrence have been reported.
Anopheles occidentalis D. and $\mathrm{K}$. and $\boldsymbol{A}$. freeborni Aitken (maculipennis auct.)

Anopheles maculipennis has been recorded from southern Canada and the northern part of the United States from Quebec, Maine, and Massachusetts westward, the Rocky Mountain region from southern New Mexico northward, and the Pacific Coast from Alaska, Yukon Territory, and the Fraser Valley in British Columbia to Baja California in Mexico. In California and Oregon freeborni, with the dark-tipped wing, is said to have the wider distribution, while occidentalis is said to be limited to a narrow strip along the coast from San Luis Obispo northward. The species occidentalis is the one that extends across southern Canada and the northern part of the United States, including also Nebraska and Iowa; the species freeborni extends into New Mexico and, according to Aitken (1941), occurs throughout the Rocky Mountain region west of the Divide as far north as Montana and possibly southern British Columbia, where the two are thought to overlap. Another species, aztecus Hoffmann, is found in the high table lands of the Valley of Mexico.

In some of the earlier work with Anopheles maculipennis, specimens having a dark wing tip were identified as quadrimacula tus, so the latter was probably credited erroneously to Utah, Montana, and Colorado (Howard, Dyar, and Knab 1917a).

Locality records for Anopheles occidentalis (based principally on the distributional data or confirmed identifications, indicated by asterisks, as given by Aitken (1941). Most of the records, except those of Howard, Dyar, and Knab (1917a) and Aitken, were reported as maculipennis).

Alaska: Ft. Gibbon" (H. D. and K.). "Alaska", (Tulloch 1934). Gilmore (Tulloch specimens) and Anchorage (Aitken 1941).

Yukon Territory: Hootalingua* (Dyar 1920). Valley of the Mayo River* (H. D. and K.).

Northwest Territory: Aklavik and Fort MePherson (Aitken 1941).

British Columbia: Revelstoke* (H. D. and K.). Between Chilliwack and Mission, probably generally distributed in the lower Fraser Valley; southern Okanagan Valley (Hearle 1926, 1928). Sun- 
derland (notes from R. Matheson 1940). A. occidentalis and $A$. freeborni apparently overlap in the Okanagan Valley but occidentalis is the dominant form (Aitken 1941).

Alberta: Bilby and Banff (Aitken 1941).

Saskatchewan: Indian Head and Moose Mountains (Aitken 1941).

Manitoba: Riding Mt. National Park (Gibson 1940). Aweme* (H. D. and K.). Saint Boniface (Ludlow 1913). Winnipeg; Whitemouth (Aitken 1941).

Ontario: Lake Simeoe (Ludlow 1913). Little Current River*; Nagami River*; Ottawa* (H. D. and K.). Ottawa* (Twinn, 1931). Kenora (Aitken 1941).

Quebec: Mirrigan River; Gatineau Point (Aitken 1941).

Maine: Noreross*; Weld* (H. D. and K.). Aroostook County* (Lathrop 1939).

New Hampshire: Berlin Falls and Center Harbor $^{*}$ (H. D. and K. "quadrimaculatus," probably maculipennis in part; see note under locality records for the former species). East Jaffery; Dublin (Aitken 1941).

Massachusetts: Leverett Pond, near Amherst

(Freeborn 1923, quoted by Kumm 1929).

Connecticut: Stafford Springs (Aitken 1941).

New York: Racquette River; Raybrook (Matheson and Shannon 1923).

Michigan: Douglas Lake (Matheson and Shannon 1923). Germfast (Aitken 1941).

Wisconsin: Starlake (Aitken 1941).

Minnesota: Basswood Lake; Saint Anthony Park (Matheson and Shannon 1923). Common over much of the State east of the prairie country; map shows numerous localities, one in the southeastern corner (Owen 1937; list of the localities is given by Aitken 1941). Crystal Bay; Cedar Lake; Lake Nocomis (Riley and Chalgren (1939 १)).

Iowa: Wexford; LeClaire; Allendorf; Blackhawk Lake; Des Moines; South Fruitland; New

Hartford (J. A. Rowe survey 1940).

Nebraska: Glen, Sioux County, August 1906 (notes from M. H. Swenk 1940, as quadrimaculatus; two females from this collection identified by W. V. King as occidentalis).

North Dakota: Pembina (Aitken 1941).

Montana: Glacier Park*; Victor* (Dyar 1929). Manhattan* (Mail 1934). Florence (W. V. King coll., March 1914). Florence; Missoula; Helena; Chinook; Glasgow; Powderville; and Phillips County (Aitken 1941).

Idaho: Riverdale (Knowlton and Rowe 1935). Coeur d'Alene (Aitken 1941).

Wyoming: No records of Anopheles (notes from J. W. Seott 1940).

Washington: Lake Whatcom (Aitken 1941).

Oregon: Portland* (H. D. and K.).

California: Restricted to a narrow strip along the coast from San Luis Obispo north to Canadian Northwest Territory (Aitken 1941; locality records given; previous records for " maculipennis," by Herms and others, listed by Kumm 1929)

\section{Anopheles freeborni}

British Columbia: Vernon; ? Pemberton (Aitken 1941). ? Oliver and Nicola Lake ("quadrimaculatus," Hearle 1927, quoted by Aitken).

Montana: Hamilton (Aitken 1941).

Idaho: Boise" (H. D. and K., "occidentalis",). Fish Haven* (Knowlton and Rowe 1936).

Nevada: Reno; Steamboat Springs (H. D. and K., "occidentalis"').

Utah: Logan; Utah County ("quadrimaculatus"); Lehi ("occidentalis") (H. D. and K.). Murray; Salt Lake City; Holiday; Bear Lake; Ibapah; Zion Nat. Park (Rees 1934). Benson; River Heights; Appledale; Amalga; Enterprise; Geneva; Logan; Murray; Payson; Salt Lake City; Public Shooting Grounds (Knowlton and Rowe 1935 and 1936). Lehi* ; Saint George (Aitken 1941).

Colorado: Hotchkiss; Delta (H. D. and K., "quadrimaculatus"). Appleton ("quadrimaculatus,"' Walker 1930). Delta (Aitken 1941).

New Mexico: Near Farmington, San Juan County, in San Juan River Valley; Arriba (Espanola); Santa Fe, Sierra, and Dona Ana Counties in the Rio Grande Valley (Barber, Komp and King 1929; Barber and Forbrich 1933). Rattlesnake Springs on the Black River in the Pecos Valley (Barber 1939).

Texas: North of El Paso (Barber and Forbrich 1933). Western tip, ehiefly in region between El Paso, Val Verde, and Gaines Counties (C. P. Coogle, quoted by Aitken 1941).

Arizona: Arivaca; San Bernardino Ranch near Douglas (Aitken 1941).

California: Anopheles "maculipennis" oceurs practically everywhere except in desert areas and along the Sierra crest; the fringe spot present in specimens from the coastal strip but almost impossible to distinguish elsewhere (abstract from Freeborn 1926). A. freeborni occurs throughout the inland regions and reaches the coast in southern California from San Luis Obispo into Baja California, Mexico (Aitken 1939 and 1941).

Oregon: Klamath Falls (H. D. and K. "occidentalis"'). Willamette Valley; South Warner Valley, Lake County; taken both east and west of the Cascades (Stage and Gjullin 1935; Stage 1938). Portland; Junetion City; Klamath Falls; Prineville; Grants Pass; Independence; John Day River; Harrisburg; and Eugene (notes from C. M. Gjullin and H. H. Stage 1940).

Washington: A. "maculipennis" taken in State (Stage and Gjullin 1935). Yakima; Colville; Cle Elum (notes from C. M. Gjullin and H. H. Stage 1940).

Anopheles pseudopunctipennis pseudopunctipennis Theob. and A. $p$. franciscanus $\mathrm{McC}$.

Anopheles pseudopunctipennis Theob. is primarily a species of the arid regions of the Southwestern States but is found as a 
rarity as far east as the Mississippi River from Louisiana to Tennessee. It is reported as being very abundant in some of the river valleys in New Mexico, western Texas, and California. South of the United States it occurs through Central and South America into Argentina, and in some of the islands of the Caribbean.

As mentioned in the preceding paper, Aitken (1941) believes that the type form of this species does not occur in California, being represented there by the subspecies franciscanus and its variety boydi.

Tennessee: Reported for the State by Dyar (1928). Memphis (Aitken 1941).

Mississippi: Counties of Attola, Hinds, Holmes, Leake, Madison, and Yazoo (Balfour, Carley and Parker, quoted by Kumm 1929).

Louisiana: Mound; New. Orleans (King, Bradley and MeNeel 1939).

Kansas: Manhattan (Hill 1939).

Oklahoma: Alva (record from L. E. Rozeboom 1940).

Texas: Brownsville; Devils River (H. D. and K.). Fort Brown; Fort Clark; Fort Sam Houston; Leon Springs (Ludlow 1914, “franciscanus"). Camp Travis; Corpus Christi; Del Rio ; Laredo; San Antonio (quoted by Kumm 1929). Mitchell Lake (Howard 1922; also collected by H. O. Schroeder 1937). Cameron County; Olmito (CWA coll., 1934). Brownsville; Laredo; Del Rio; El Paso (Barber, Komp and King 1929). Houston (Matthes 1935). Below El Paso (Barber 1939). Donna (P. T. Riherd coll., 12/24/39). Brownsville (Aitken 1941).

New Mexico: Las Vegas (H. D. and K.). Various localities in the San Juan, Pecos, and Canadian River Valleys (Barber, Komp and King 1929; Barber and Forbrich 1933; Barber 1929). Leasburg and Carlsbad (Aitken 1941).

Arizona: Bill Williams Fork; Oak Creek Canyon (H. D. and K.). Tempe (Barber, Komp and King 1929). Wilcox (Aitken 1941).

Nevada: Beatty, Indian Springs, and Tule Springs (Aitken 1941).

Utah: Saint George (Aitken 1941).

California: Occurs southward along the coast from Sonoma County and the central valleys from Red Bluff, Tehama County, into the foothills of the San Joaquin, and the most abundant anopheline south of the Tehachapi (Freeborn 1926). Various localities (Herms 1917, and others, quoted by Kumm 1929). Only two counties in the State (Mono and Imperial) where the species has not been recorded (Aitken 1939). Most northern records at Wonderland Park, Del Norte County, and Yreka, Siskiyou County; form with delicate leaflets on the mesosome (franciscanus) of wide occurrence in the State; those without leaflets
( $\$$ boydi) recorded in several counties and both forms occasionally found in the same locality (Aitken 1941).

Oregon: ( 8 ) Corvallis and Forest Grove (Cole and Lovett 1921, quoted by Aitken 1941; records this far north considered doubtful by Freeborn 1926).

\section{Anopheles punctipennis Say}

As pointed out by Matheson (1929), Anopheles punctipennis Say has the greatest range of any nearctic anopheline. It occurs in southern Canada and throughout the Eastern, Central, and Southern States to New Mexico, and from the Pacific States into western Canada. Except for one record in western Montana, however, it seems not to have been reported from the Rocky Mountain region between Canada and New Mexico. Its range extends into the tablelands of Mexico. In the Eastern and Southern States its breeding habitats are usually more restricted than those of quadrimaculatus. Locality records (except the southeastern states) are as follows:

Canada: New Brunswiek: Saint John (H.D. and K.). Ontario: Ottawa (H. D. and K.). Lake Simco (quoted by Kumm). Ottawa (Twinn 1931). Eastern Ontario (Gibson 1938). Manitoba: Brandon (Gibson 1940). British Columbia: Duncans; Nanaimo; Wellington (H. D. and K.). Fraser Valley from Hope to coast, etc. (Hearle 1926). Maine: Weld (H. D. and K.). Orono and Presque Isle (Lathrop 1939).

New Hampshire: Center Harbor; Dublin; Durham; Monadnock (H. D. and K.). Intervale and Hampton; most common anopheline in State (Lowry 1929).

Vermont: Castleton (Howard 1900).

Massachusetts: West Springfield; Granby; Chicopee (H. D. and K.).

Connecticut: Reported in State (Botsford and Turner 1933).

New York: Various localities (quoted by Kumm 1929).

Pennsylvania: Danville; West Fairview (H. D. and K.). Camp Roosevelt, Mt. Gretna ("perplexans,', Ludlow 1907; quoted by H. D. and K.). West Virginia: Huntington (H. D. and K.).

Ohio: Westlawn (quoted by Kumm). Aurora (Hoyt 1935). Toledo (G. H. Bradley coll., $7 / 26 / 38$ ). Columbus; Medina (records from D. M. DeLong 1940).

Indiana: Hessville (Gerhard 1910). Lafayette; Lawrence and Knox Counties (notes from J. J. Davis 1940, J. M. Amos coll.).

Michigan: Eastmanville (H. D. and K.). Douglas Lake (Matheson and Shannon 1923). 
Illinois: Chicago; Lemont; Beverly Hills; Palos Park (Gerhard 1910). Chicago; Urbana (H. D. and K.). Carbondale; Herrin; Murphysboro; Rock Island (quoted by Kumm). East Saint Louis (collected in 1937, notes from F. C. Bishopp). Des Plaines Valley (G. H. Bradley coll., July 1938).

Minnesota: St. Paul and Savage (Howard, C. W. 1916). Crystal Bay and Cedar Lake (Riley and Chalgren 1939 १). Common in south half but also found in northern plains section; not in coniferous forest (Owen 1937).

North Dakota: No anophelines recorded in list of mosquitoes taken at Fargo, Mandan, and Minot (Munro and Sangston 1939).

South Dakota: Taken very frequently in eastern part of state (notes from H. C. Severin, 9/11/40).

Iowa: Various localities in state (notes from C. J. Drake 1940, J. A. Rowe coll.).

Missouri: Saint Louis (H. D. and K.). Pretty generally distributed over state (notes from L. Haseman 1940). Sikeston area (Ziegler and Maxey 1923). Lake of Ozarks (Johnson 1932).

Kansas: Onaga (H. D. and K.). Fort Leavenworth (quoted by Kumm). Burlington (Barber, Komp and Hayne). Arcola ; counties of Douglas, Doniphan, Riley and Pottawatomie (Hill 1939).

Nebraska: Lincoln, 1903; Salt Creek, near Waverly, 1904 (notes from M. H. Swenk 1940).

Oklahoma: Fort Gill (iSill) and Fort Reno (quoted by Kumm). Stillwater (H. O. Schroeder coll., 1938). Page; Grove; Broken Bow; Flint; Sherwood; Hinton; Eagletown; Roff; Idabel; Sulphur; Sallisaw; Gore; Sayre; Grandfield; Spavinaw; Grant; Cheyenne; Cleo Springs; Muse; Wyandotte (notes from D. C. Howell 1940):

Texas: Brazos River (Howard 1900). Dallas; Denison; Devils River; Kerrville; Paris (H. D. and K.). Galveston (Dyar 1922). Dialville; Leon Springs (quoted by Kumm). Del Rio (Barber, Komp and King 1929). Port Lavaca (CWA coll., 1934, det. Stone). Menard (H. C. Parish coll., 1934, "'abundant and annoying," notes from F. C. Bishopp). Houston (Matthes 1935). Brazos County (Cushing 1936). Stephensville (P. T. Riherd coll., 5/30/39). South of El Paso (Barber 1939). Dallas (W. G. Bruce and E. W. Laake coll., 1940).

New Mexico: Several localities in Pecos and Canadian River Valleys. (Barber, Komp and King 1929 ; Barber and Forbrich 1933).

Montana: Lolo (Mail 1934).

California: Rarely taken south of the Tehachapi but is the predominating anopheline in the foothill sections and in limited foci along the Sacramento River (Freeborn 1926). Various localities (quoted by Kumm 1929). Inyo County (Aitken 1939).

Oregon: Portland (H. D. and K.). Same distribution as maculipennis (Stage and Gjullin 1935). Portland; Junction City; Buckeye Lake; Grants Pass; Sherwood; Independence; Harrisburg; Lebanon (notes from C. M. Gjullin and H. H. Stage 1940).
Washington: Ashford (H. D. and K.). Taken in state (Stage and Gjullin 1935). Lake Tapps; Toppenish; Plaza; Vancouver; Yakima; Deming; Kalama (notes from C. M. Gjullin and H. H. Stage 1940).

\section{Anopheles quadrimaculatus Say}

Anopheles quadrimaculatus Say ranges from New Hampshire, Massachusetts, and southern New York westward to southern Ontario, Minnesota, and Iowa, and throughout most of the southern territory to central Texas and Oklahoma. In the Southern States it is usually the predominant anopheline. Few records are available as to its occurrence in most of the North-Central and Midwestern States, although its presence may be assumed in areas where endemic malaria is still found.

Records for states where it is less common or near the limits of its range are given below. In Massachusetts, Minnesota, Michigan, and Iowa ${ }^{1}$ the species has been definitely shown (by identification based on male terminalic characters) to overlap the range of occidentalis. Overlapping probably occurs also in other areas. Locality records for the northern and western parts of its range are as follows :

New Hampshire: Center Harbor and Berlin Falls (H. D. and K.; probably occidentalis, in part). A male specimen from Center Harbor ("Iss. Sept. 6 \#114. H. G. Dyar Coll.') has been identified recently by W. V. King as quadrimaculatus. Other specimens from this collection are listed as occidentalis by Aitken (1941).

Massachusetts: Westfield and West Springfield (H. D. and K., possibly occidentalis). Found overlapping quadrimaculatus in State (Freeborn 1923). Scituate (F. C. Bishopp and G. H. Bradley coll., 9/12/32).

Connecticut: Pine Orchard (H. D. and K.). Mystic (F. C. Bishopp and G. H. Bradley coll., 9/12/32).

New York: Ithaca; Bellport; Bayside, Long Island (H. D. and K.). Central New York (Matheson and Shannon 1923; Matheson 1929). New York City. ("M.B."' 1939). (Various other localities listed by Kumm, Felt, etc., questionable as to exact identification).

Ontario: Cayuga (notes from R. M. Matheson, also E. H. Hinman 1940).

Pennsylvania: West Fairview (H. D. and K.).

1 Through the kindness of C. J. Drake, records from a recent survey of Iowa made by J. A. Rowe were furnished the writers in advance of publication. 
Ohio: Aurora (Hoyt and Worden 1935). Cedar Point; Sandusky (records from D. M. De Long 1940).

Indiana: Lake Maxinkuckee (H. D. and K.). Wabash River, Knox County (notes from J. J. Davis 1940, J. M. Amos coll., 9/14/37).

Illinois: Chicago and Willow Spring (Gerhard 1910). Urbana (H. D. and K.). Carbondale; Herrin; Murphysboro; Rock Island (quoted iy Kumm). East Saint Louis (coll. 1937, notes from F. C. Bishopp). Des Plaines Valley (Dept. Pub. Health, (1939 \&); also coll. by G. H. Bradley, July 1938).

Kentucky: Taken at various places in western and northern parts of State (notes from G. E. Quinby 1940).

Michigan: East Lansing (record from C. Sabrosky 1940). Cheboygan, Emmet, Genesee, Ingham, Montcalm, and Washtenaw Counties (Irwin 1941).

Wisconsin: Osceola and Saxeville (H. D. and K.). Minnesota: Saint Anthony Park and Grand Rapids (Howard, C. W. 1916; may have been occidentalis). Fort Snelling (quoted by Kumm). Present over much of southern and eastern half of state but not common; Carlton County, most northern record; four localities shown on map (Owen 1937). Crystal Bay (Riley and Chalgren 1938).

Iowa: Collected in the state (notes by $\mathrm{R}$. W. Wells 1938). Scattered through state to Western border (J. A. Rowe survey, 1940).

Missouri: Saint Louis (H. D. and K.). Sikeston area (Ziegler and Maxey 1923). Jefferson Barracks (quoted by Kumm). Ellsberry (Barber 1930). Lake of Ozarks (Johnson 1932). Pretty generally distributed over State (notes from L. Haseman 1940).

Kansas: Lawrence (Hill 1939).

Oklahoma: Stillwater (coll. notes, H. O. Schroeder 1938). Gore; Broken Bow; Sallisaw; Idabel; Spavinaw; Eagletown; Hinton; Millerton; Wilberton (notes from D. C. Howell 1940).

Texas: Dallas and Victoria (H. D. and K.). Dialville (Parker 1922). Ellington Field; Fort Crockett (Borden 1926, quoted by Kumm). Brownsville (Barber, Komp and King 1929). Orange (CWA coll., 1/2/34, Alan Stone ident.). Houston (Matthes 1935). Brazos County (Cushing 1936). Dallas (W. G. Bruce coll., 1937 and 1940). Brownsville area (P. T. Riherd coll., 1939).

\section{Anopheles walkeri Theob.}

Anopheles walkeri Theob. has a wide distribution in eastern America from southeastern Canada to the Gulf and westward to southwestern Louisiana, central Arkansas, and Minnesota. Matheson and Hurlbut (1937) have given the known distribution of the species, to which a few additional records have been added. The recorded localities are very scattered, but the species has been taken in considerable abundance in certain areas as, for example, near Lake Apopka in central Florida and at Reelfoot Lake in northwestern Tennessee. Locality records are available as follows:

Canada: Ontario: Lake Simcoe (type locality, Theobald). Ottawa (H. D. and K, ; Twinn 1931). Maberly and Nation Rivers (Gibson 1940).

Maine: Found in state (Alan Stone, quoted by Matheson and Hurlbut 1937).

Massachusetts: Westfield; Auburndale (H. D. and K.).

Connecticut: Reported in state (Botsford and Turner 1933; Matheson and Hurlbut 1937).

New York: North Fair Haven (Matheson and Shannon 1923). Ithaca (Matheson and Hurlbut 1937).

New Jersey: Collected in light traps in five counties in 1938 and in four more in 1939 (Mulhern 1939 and 1940; also previous records).

Delaware: Delaware City (Bishopp, Cory and Stone 1933). Newark (Stearns et al. 1933; MacCreary 1939). Lewes (Stearns 1940).

Maryland: College Park; Princess Ann; Chestertown (Bishopp, Cory and Stone 1933).

District of Columbia: Washington; Chain Bridge (Dyar 1922).

Virginia: Addison (H. D. and K.). Long Bridge (Dyar 1922). South End (A. H. Jennings coll., 1916).

Kentucky: Bondurant, Reelfoot Lake area (Bang, Quinby and Simpson 1940).

Ohio: Toledo (G. H. Bradley coll., 7/26/38).

Indiana: Hessville (Gerhard 1910).

Michigan: Camp Custer; Battle Creek (Dyar 1922). Nottawa, Saint Joseph County (record. from C. Sabrosky 1940).

Minnesota: Grand Rapids (Matheson and Shannon 1923). Probably confined to part of State east of prairies, eight localities shown on map (Owen 1937). Crystal Bay; Cedar Lake; Lake Nocomis; University Farm (Riley and Chalgren 1939 ?).

Iowa: Collected in state (notes by R. W. Wells 1938). Brooklyn; Onawa; South Fruitland; Dubuque; Clear Lake; Little Wall Lake; Lake Park; Nichols (J. A. Rowe survey, 1940).

Illinois: Beach and Palo Park, near Chicago (Gerhard 1910).

Georgia: Sumter County (Bellamy and Andrews 1938).

Florida: Zellwood (Bradley and MeNeel 1935; Bradley 1936). Zellwood; Orlando (King, Bradley and MeNeel 1939). Volusia County (G. H. Bradley coll., 1939 and 1940).

Tennessee: Reelfoot Lake (Johnson 1936; Brown and Pearson 1938).

Louisiana: Crowley (Komp 1926; Barber, KomI and Hayne 1926).

Arkansas: Lonoke County (Thibault 1910). Little Rock (H. D. and K.). 


\title{
BIONOMICS AND ECOLOGY OF NEARCTIC ANOPHELES
}

\author{
By G. H. BRADLEY and W. V. KING \\ BUREAU OF ENTOMOLOGY AND PLANT QUARANTINE, UNITED STATES DEPARTMENT OF AGRICULTURE, \\ WASHINGTON, D. C.
}

IN common with all mosquitoes, anophelines have four stages in their life cyclethe egg, the larva, the pupa, and the adult. The life history is briefly as follows: The eggs, which are laid singly on the water surface, hatch in from 2 to 3 days, under favorable conditions, into larvae, or wigglers. These grow slowly or rapidly, depending on temperature and other factors, spending from a week or so to several months in this stage. The larva molts four times, the final molt being into the pupal, or tumbler, stage. During the pupal stage changes take place within the body which transform it into the adult mosquito. This stage is usually completed in from 2 to 3 days, and after the adult emerges from the pupal case it rests for a short time on the surface of the water before taking flight.

\section{Adult Behavior}

Mating. Anopheline adults are usually produced in approximately equal numbers of males and females, although a preponderance of females sometimes occurs (Bradley 1926; Boyd 1930a). For the species that have been observed, mating occurs about sundown during swarming of the males. In the case of $A$. punctipennis the male swarm consists of about 100 individuals flying up and down in corkscrew fashion from 7 to 12 feet above the ground (Howard, Dyar, and Knab, 1912-17a, v. 1, p. 127). Freeborn (1923) observed swarms of A. maculipennis (occidentalis) and of A. quadrimaculatus in close proximity to one another near a pond in Massachusetts, one of which was above a small pine tree, the other in the open.

Longevity and number of generations. The duration of adult life varies greatly, being shortest during the hot, dry weather of the summer and longest during the fall, particularly with those females that go into hibernation. Humidity, as well as temperature, is an important factor in longevity. The life of the male is probably shorter in all cases than that of the female, and they are not known to hibernate. The appearance of males in the spring is usually taken as indicating the emergence of the first brood of the season.

The number of generations of Anopheles in any region probably depends largely on the climate, inasmuch as it is known that high temperatures accelerate and low temperatures retard development. The number of generations has been studied for $A$. quadrimaculatus by Boyd (1927), who believes that this species may have from 8 to 10 generations annually in southwestern Georgia, while in northeastern North Carolina the number is 7 or probably 8 . Freeborn (1932) states that in California there are 6 generations of $A$. maculipennis ${ }^{1}$ annually.

Biting activity. Female anophelines seek blood meals within a day or two after emergence when the mouthparts are sufficiently hardened and conditions for flight are favorable. For most of the species feeding activity is probably greater soon after dusk, but it may continue throughout the night. Most of the species will bite during the daytime on dark days or in shelters, particularly if disturbed. A. atropos provides a striking exception to this generalization, however, for in addition to being active during the night, it has been observed at times to attack in swarms in bright sunlight. A. crucians (the common

\footnotetext{
1 Probably $A$. freeborni in this and other references to maculipennis in California, except where the coastal form with pale wing tips is referred to.
} 
inland form) has also been reported as attacking in sunlight as well as in shade, the attacks beginning as early as 7:15 A.M. (Mayne 1926b). The species is, in fact, referred to in the Middle Atlantic States as the daylight anopheline. A. maculipennis is said by Freeborn (1926) to bite viciously in bright sunlight on becoming active in the spring.

Adult activities are dependent on temperature probably more than on any other factor. The optimum biting temperature for anophelines was found by Mitzmain (1919) to be between 28 and $30^{\circ} \mathrm{C}$ and the minimum between 16 and $17^{\circ} \mathrm{C}$. A sudden rise in temperature is said by Mayne (1926a) to stimulate the blood-seeking impulse. Boyd (1930a) states that on moonlight nights the period of activity of anophelines is extended. Before heavy rains they are particularly voracious and some species enter houses at night in larger numbers than usual at such times. Heavy rains also may cause fewer mosquitoes to be on the wing, but they become active again soon after the rains cease.

Rudolfs (1923) found that mosquito activity, as determined by the numbers alighting to bite, increased with the relative humidity up to 85 per cent, above which no change was noted in mosquito behavior. Freeborn (1932) states that there is a midsummer depression in the abundance of A. maculipennis in California due to high temperatures and low humidities. During this period the females of the second and third generations live only long enough to deposit eggs but not long enough to permit malaria transmission. Maximum daily temperatures in excess of $33^{\circ} \mathrm{C}$ and evaporation from a white ball atmometer in excess of 70 ec occur during this season.

Blood feeding. So far as known the food of female anophelines primarily is blood, and a blood meal appears necessary for the maturation of eggs. The females, however, may subsist for long periods in eaptivity on fruit juices or sirup, and it has been concluded by Boyd (1930a) that the pursuit of blood not only satisfies a whim of appetite but arouses the mating instinct as well.
While male mosquitoes often alight on warm-blooded animals and probe around with the proboscis, they are unable to pierce the skin. They subsist on water, nectar, and fruit juices.

The different species vary considerably in their host preferences. Bull and Reynolds (1924) and Bull and Root (1923), in comparing horses and cows as hosts for $A$. quadrimaculatus, showed experimentally that attractiveness in the hosts varied more among individuals than among species. Humans, although varying individually also, received on the average only one-sixth as many bites as horses or cows. Sheep, goats, dogs, and pigs appeared to be less attractive in the order given, while rabbits and chickens were very poor hosts even in the absence of other animals.

King and Bull (1923) determined the blood-feeding habits of A. quadrimaculatus in nature by identifying the blood meals from a large series of females caught in various locations. They found that an average of 38 per cent of those taken inside houses had fed on man, but only 2 per cent of those taken in outbuildings. The weighted average was 4.3 per cent for the entire female $\boldsymbol{A}$. quadrimaculatus population, being 6 to 8 per cent when the average number of females per location was about 200 to 500 and decreasing to 3 per cent or less when the average reached 1,500 or more. The average percentages for the other hosts for which precipitin tests were made were as follows : Cow 36 , horse 33 , pig $16, \operatorname{dog} 8$, and chicken and eat 3 . These observations indicate quite definitely that certain animals, horses and cows particularly, have a greater attraction for $A$. quadrimaculatus than has man, so some degree of protection from bites must be afforded when such animals are available. In these experiments the blood meals of a few $A$. punctipennis and A. crucians were also tested. Of 79 A. punctipennis none had fed on man, and of 125 A. crucians 6 contained human blood.

Attraction to lights. Anophelines are attracted to artificial lights, and most of our species have been taken in light-trap 
collections. Judging by the reports of such collections, as well as the writers' own experience, $A$. crucians, $A$. walkeri, and $A$. atropos are more readily attracted to lights than is A. quadrimaculatus. Bradley and McNeel (1935), for instance, report that in central Florida, where $A$. crucians was the predominant anopheline, larval examinations made in the vicinity of a trap location gave a much higher relative incidence of A. quadrimaculatus over $A$. crucians than was shown by the trap collections.

Resting places. The daytime resting places of the various species is subject to some variation, depending probably on a preference for different temperatures, light intensities, and humidities. Inside houses they are to be found behind pictures and curtains, underneath furniture, behind dark clothing, and in the darker nooks and corners generally. They are found in large numbers underneath houses and other raised buildings, in dark corners in stables, porches of all sorts, chicken houses and outbuildings, especially privies, culverts, under bridges, beneath overhanging vegetation on stream banks, in caves, and in hollow trees. Abandoned spider webs in such places are favorite roosting places. In general, anophelines probably prefer to rest on dark-colored surfaces. It has been pointed out, however, by Boyd (1930a, quoting Nuttall and Shipley) that certain colors such as navy blue, dark red, reddish brown, and scarlet are preferred to black. Soot-covered walls and draughty places seem to be avoided. Anopheles punctipen$n$ is is said to tolerate more illumination in its daytime shelters than does quadrimaculatus. A. crucians has been reported by Metz (1918) to prefer stables and pigpens to houses. Locations underneath houses, bridges, etc., in damp places near the ground have been found to be favored shelters for most of the common species. In California A. maculipennis is reported by Freeborn (1926) to be addicted to manmade shelters, particularly dwellings, whereas $A$. punctipennis and A. pseudopunctipennis are rarely found inside houses. In central New York Matheson
(1932) found that $A$. punctipennis readily entered houses, and large numbers were taken in cellars, houses, and stables and other outbuildings. Barber and Forbrich (1933) report A. pseudopunctipennis as frequenting houses in New Mexico, and that this is usually for shelter was attested by the fact that a high percentage of males and a low percentage of engorged females were found. They also observed that occupied houses usually harbored $A$. maculipennis (freeborni) while in unoccupied houses A. pseudopunctipennis predominated. $A$. atropos readily enters houses and utilizes man-made shelters as daytime resting places (Bishopp, Cory and Stone 1933). The adults of $A$. walkeri have been found resting in barns and under houses (Komp 1926) and also under overhanging grass on the margins of a marsh, whence they flew into bright daylight to bite when disturbed (Bradley 1936). This species has a tendency to be secretive and its shelters are often difficult to locate. Johnson (1936) reported that, although $A$. walkeri adults were being taken in numbers in a light trap near Reelfoot Lake in Tennessee, daytime shelters could not be located. Cowsheds, stables, chicken houses, places under houses, open woods, tree trunks, stumps, and dense weeds were searched, and 2,510 anophelines were collected and examined without a single one of $A$. walkeri being found.

Flight and migration. Our anophelines are considered to be comparatively weak fliers. In the case of $A$. quadrimaculatus it is a common practice to limit control measures to a radius of from one-half to 1 mile from habitations, depending on intensity of breeding, as the consensus is that few migrate farther than a mile from their breeding grounds (Barber and Hayne 1924) (LePrince and Griffitts 1917). However, Geiger, Purdy and Tarbett (1919) have reported that a control area in Arkansas was invaded by flights of A. quadrimaculatus apparently from a swamp 1.7 miles distant, and other important flights of more than a mile have been reported for this species. Kumm (1929a) observed a maximum migration of only 0.4 of a mile 
for A. quadrimaculatus and found marked daily migration among shelters. A. crucians (possibly bradleyi) was reported by King, Bradley and MacNeel (1939) as migrating several miles when intense breeding occurred. On the Mississippi coast, flights of $A$. crucians from islands situated from 3 to 12 miles off shore were reported by Barber, Komp, and Hayne (1924), and MacCreary and Stearns (1937) have recorded flights of this species to two lighthouses situated 3.2 and 5.5 miles, respectively, from shore. Freeborn (1932) states that in California "prehibernation" forms of $A$. maculipennis appear during October and early in November, which are darker and more robust than the summer forms and migrate long distances from their breeding grounds before entering shelters. Although they become active on warm days in the winter, they do not develop eggs until after an "emergence" flight during the middle of February. At this time they infest areas both suitable and unsuitable for breeding. From studies on overwintering individuals of $A$. quadrimaculatus made at Fort Jackson, La., Hinman (1934c) concluded that the enormous number of females which congregate in the old fort in November probably migrated there from considerable distances, since there was a scarcity of breeding places nearby, and that they undergo a sort of pseudohibernating period of 2 to 3 months. It may be significant that very few of $A$. crucians and none of $A$. atropos were noted in this winter retreat even though these species breed abundantly in the vicinity.

Hibernation. There is probably no true hibernation of Anopheles quadrimaculatus, A. punctipennis, or A. crucians in the more southern part of their range, as numerous authors have reported that these species become active during warm periods in the winter. In cold weather adults seek shelter in unoccupied houses, caves, hollow trees, and other such places, where they become inactive. Larvae and pupae are also to be found throughout the winter, and it is probable that these species pass the winter to some extent in the aquatic stages, as well as by overwintering females. Balfour (1928) found that some larvae of $A$. crucians passed the winter in North Carolina during the winter of 1926-27 when the temperature over a 10-day period had a mean minimum of $24^{\circ} \mathrm{F}$ and an absolute minimum of $12.5^{\circ} \mathrm{F}$. Larvae of $A$. quadrimaculatus and $A$. crucians were collected in every month except January, but no $A$. punctipennis were found in January or February. Hinman and Hurlbut (1940) stated that in the Tennessee valley $\boldsymbol{A}$. quadrimaculatus passed the winter chiefly as inseminated females. Hibernating females in caves survived periods up to 69 days without food.

Matheson and Hurlbut (1937) discovered that two forms of eggs are laid by $A$. walkeri, which they called summer eggs and winter eggs. In New York State the former hatched normally soon after oviposition and could not withstand freezing temperatures, but the latter did not hatch unless subjected to freezing temperatures. It is believed that the winter eggs are the only means by which this species survives the winter in New York. Winter eggs of $A$. walkeri from Tennessee showed a marked instability and the greater part of those obtained hatched soon after oviposition (Hurlbut 1938b).

Owen (1937) states that in Minnesota the females of A. maculipennis (occidentalis) hibernate. He found that in two out of four caves under observation the places selected for hibernation did not afford adequate protection, as the adults were killed by becoming frozen in ice on the walls. In the anteroom of a larger cave all died. Apparently he assumes that those in the deeper recesses of the large caves successfully passed the winter, although they were not located.

Enemies of adults. Birds, bats, toads, frogs, and lizards, as well as insects and spiders, are included among adult predators of anophelines, and protozoans, filaria embryos, other nematodes, and trematodes among the parasites. Hinman (1934b) has prepared a summary of the available literature on the predators and Boyd (1930a) 
briefly discusses both predators and parasites. Boyd (1930a) credits the nighthawks (Caprimulgidae) as being probably the most important bird enemies of anophelines. It is common knowledge that dragonflies eatch mosquitoes while in flight, and that spiders take a heavy toll of resting mosquitoes. In former years considerable publicity was given to the reputed value of bats as mosquito destroyers, but, although they undoubtedly eatch many anophelines, observations in places where bats are numerous have shown that in reality they have little effect in reducing the mosquito population (Howard 1916).

Egg laying. Female anophelines deposit their eggs singly on the water surface during the night. Whether a selection of favorable breeding waters is made before oviposition is not known with certainty, although it can be inferred from the fact that even the more general breeders show a certain amount of segregation in different types of breeding places. It is, of course, plainly evident in the case of the specialized forms such as $A$. barberi and $A$. atropos. Certain waters often contain only the first or second instars, indicating conditions unfavorable for their maturation. These have been termed incomplete breeding places in contrast to those where all immature stages occur regularly. In these cases, of course, failure to mature may be due to the activities of predators rather than to lack of adequate nutritional or other factors.

From about 100 to 300 eggs are usually laid singly at one oviposition time and there may be several oviposition periods for a single female. The eggs are white at first but soon turn to a dull black. The incubation period is usually about 2 days, although at high temperatures eggs may hatch in 24 hours (Mayne 1926a). The same author reports that oviposition did not occur below $55^{\circ} \mathrm{F}$, and that eggs of A. quadrimaculatus would not hatch at temperatures of 58 to $59^{\circ} \mathrm{F}$, but that hatehing occurred at 66 to $70^{\circ} \mathrm{F}$.

Anopheline eggs will resist a certain amount of desiccation after the embryo is developed. Herms and Freeborn (1920) found that eggs of $A$. freeborni ("quadrimaculatus") would stand drying for 72 hours, whereas drying for 24 hours was fatal to those of A. punctipennis. Mayne (1926a) found that eggs of A. quadrimaculatus were viable after being left on drying mud for periods of from 42 hours to 16 days. Eggs of A. crucians similarly exposed survived drying for periods of 10 to 21 days. Larvae hatched and developed normally from these dried eggs. Control eggs in these tests hatched within 48 hours on the surface of water at the same temperature. Other observers apparently have not been able to duplicate these results.

\section{LARVAL ECOLOGY}

Of the 13 species and subspecies of Anopheles that occur in the United States and Canada, 11 breed normally in fresh water and $\mathbf{2}$ in brackish or salt water. Both permanent and temporary, ponded and slowly moving waters are utilized. The most general characterization of anopheline breeding waters is that they are clean, foul or contaminated waters being seldom inhabited. Of the fresh-water species, $A$. quadrimaculatus, A. occidentalis, A. freeborni, A. crucians, A. pseudopunctipennis, and $A$. walkeri are what may be termed chiefly pool and pond breeders, $A$. freeborni and A. pseudopunctipennis being especially associated with pools in irrigated areas. A. punctipennis may occur in similar locations to the foregoing but frequently also is found in moving water, particularly along the margins of streams. The larvae of $A$. barberi occur almost exclusively in water held in tree holes. They have been taken rarely in artificial wooden receptacles in shaded locations where accumulations of leaves and other decaying matter in the water make for conditions approximating those of tree holes. Of the two species inhabiting the salt marshes, A. atropos appears to be strictly a salt-water bieeder, as it has not been found in fresh water. A. bradleyi (crucians bradleyi) breeds in waters of lower salt concentrations as a rule and also has been found in nearly fresh 
waters near the coast. The tropical species A. albimanus, whose range extends into the United States in southern Texas, is said to occur at times in salt marshes in the tropies.

Attempts at a specific classification of the breeding places of Anopheles in this region have been made chiefly for the three common species occurring in the Southeastern States, A. quadrimaculatus, A. punctipennis, and $A$. crucians. Owing to the paramount importance of A. quadrimaculatus as a malaria vector, most of these studies have been made to determine the factors correlated with the presence or absence of this species in various environments in order that such factors may be evaluated for control purposes.

Temperature. Temperatures of anopheline breeding waters in North Carolina were analyzed by Boyd (1929b), who concluded that $A$. quadrimaculatus breeds in the warmer waters having a rather limited diurnal range, whereas A. punctipennis chooses waters that either are distinctly cooler or have a wide variation in diurnal range. These differences were especially noted in the fall when breeding of $A$. quadrimaculatus was definitely limited to the warmest places. As the temperatures decreased, this species was replaced by $A$. punctipennis in many locations. A. crucians was found to require water as warm or warmer than those required by $A$. quadrimaculatus, and this is evidently correlated with the more southerly range of the species. Boyd also states that a mean temperature of $70^{\circ} \mathrm{F}$ or higher appears to be essential for the rapid evolution of the stages of A. quadrimaculatus, and notes that the northern limit of the distribution of this species coincides closely with the July isotherm of $70^{\circ} \mathrm{F}$. The range of $A$. punctipennis extends much farther north. Barber and Komp (1929c) found the latter species in waters in which the temperatures went well above $100^{\circ} \mathrm{F}$ during the daytime, although the fact that it is commonly found in springs and shaded pools and has a wider distribution in the cooler seasons indicates a preference for lower temperatures. Barber and Forbrich (1933) distin- guish two extreme types of anopheline breeding waters in northern New Mexico, those having cool waters at all times of the day, which produced $A$. freeborni exclusively, and those wholly exposed to the sun, in which A. pseudopunctipennis usually occurred in "pure culture." They conclude that day-time temperatures seem to be the determining factor in these cases, although cold springs far up in the mountains if exposed to the sun were found to contain A. pseudopunctipennis.

Hydrogen-ion concentration. The preference of $A$. quadrimaculatus and A. punctipennis for alkaline breeding waters and of $A$. crucians for acid waters is quite well established. Boyd (1929b) reported that larvae of $A$. quadrimaculatus and A. punctipennis in both Georgia and North Carolina have but rarely been found in waters having a $\mathrm{pH}$ lower than 6.1 and never in those below $\mathrm{pH}$ 5.1. Breeding waters of $A$. crucians were usually on the acid side of neutrality, but never below 4.6 or more alkaline than 8.0. The mean $\mathrm{pH}$ values for the breeding waters in North Carolina were found to be approximately 7.06, 7.10, and 5.24 and in Georgia 7.46, 7.32, and 6.99 for A. quadrimaculatus, A. punctipennis, and A. crucians, respectively. The Georgia records show breeding waters there to be generally alkaline, but the bulk of breeding waters of $A$. crucians was slightly acid. In northeastern Louisiana, where $A$. quadrimaculatus was the predominant anopheline, Bradley (1932) found the breeding waters to range from $\mathrm{pH} 6.8$ to 9.2. Watson and Spain (1937) state that spring-fed lime sink ponds having a mean $\mathrm{pH}$ of 7.2 and a range of 6.5 to 7.8 more nearly approximate the ideal breeding place of A. quadrimaculatus than do any other waters in northern Alabama. An extensive series of observations has been made by Legwen and Kirby (1939) to determine the feasibility of employing hydrogen-ion determination as an index to the breeding of $A$. quadrimaculatus in connection with larvicidal control operations. In most of the work reported it is not stated whether the determinations were made in 
the larval microhabitat itself or in the general area. It is now known that the readings may vary considerably in different types of vegetation in the same pond, and also at different times of the day.

Dissolved gases. Boyd (1929b) reported a considerable variation in the amount of dissolved oxygen in various anophelinebreeding waters in North Carolina, the range being from 0.1 to 14.0 p.p.m. No larvae were found in waters devoid of this gas. Fairly high concentrations of carbon dioxide appeared to favor breeding of $A$. quadrimaculatus whereas waters with lower concentrations favored A. punctipennis, although breeding of both species occurred in the absence of this gas. Studies on the effect of ammonia in breeding waters in this country are lacking. In India, SeniorWhite (1928) found saline ammonia in excess of one part per million usually inhibitory to anopheline breeding, and Beattie (1932) in Trinidad found that larvae of $A$. tarsimaculatus were more numerous when the ammonia nitrogen content was low.

Specific aquatic environments. A. quadrimaculatus breeds chiefly in ponds, pools, lake margins, swamps, and miscellaneous water collections of a more or less permanent nature where aquatic vegetation or surface debris are present in abundance. It usually occurs more intensively in open, sunlit waters than in shady places. This apparently is not due to any inhibitory effect of shade but to the fact that vegetation on which the larvae depend for food and shelter becomes scarce in absence of sunlight.

A. punctipennis is the most widely distributed of our anophelines and breeds in a great variety of places. Margins of flowing streams, pools in intermittent stream beds, springs, ponds, and pools, artesian wells, artificial water receptacles, and new borrow pits and other excavations are noted as breeding places of this species. It is often associated with $A$. quadrimaculatus in the more permanent waters. It has been noted as the first anopheline to occur in certain rain or flood pools when micro- organisms are scarce, and to be replaced by $A$. quadrimaculatus as a richer flora and fauna develop. Cool, clean water free from the products of organic decay is, in the opinion of most writers, most suited to the species. Carpenter (1939), however thought that they tolerate more filth in their breeding places than other anophelines in Arkansas.

A. crucians breeds in ponds, lake margins, swamps, and pools of both an intermittent and permanent character and is often associated with $A$. quadrimaculatus and $A$. punctipennis in such places. It is often the only anopheline present, however, in acid waters such as occur in cypress swamps in Florida and southern Georgia. Metz (1918) has reported that the species apparently can subsist in nature on a diet primarily of decaying vegetable matter, as the larvae were found by him in enormous numbers in water contaminated by sulfuric acid wastes, having scanty microscopic flora and fauna but rich in small particles of disintegrated plant tissue.

Larvae of the A. maculipennis group breed in a variety of situations. In California Freeborn (1926) reports them as occurring in shallow sunlit pools of clear water, preferring those having mats of green algae, and states that hoof prints, wayside pools, neglected irrigation and drainage ditches, and seepage areas furnish the most favorable locations. In New Mexico Barber and Forbrich (1933) found the species to prefer completely shaded situations and to occur with increasing scarcity as illumination increased. In Minnesota Owen (1937) found A. occidentalis more often in semipermanent and permanent ponds along the shoreline in the presence of vegetation, although it also occurred in a variety of other locations. He reports it as breeding in association with $A$. punctipennis and $A$. quadrimaculatus.

A. walkeri breeds in swamps having a thick growth of emergent aquatic vegetation, cattail ponds and lake margins apparently being the most favored habitats. It has also been reported by Komp (1926) from rice fields and from water covered by 
water hyacinth. In Georgia it was found by Bellamy and Andrews (1938) in shallow ponds with heavy growths of emergent vegetation, the water being slightly acid in reaction.

In northern New Mexico Barber and Forbrich (1933) found that waters wholly exposed to the sun and flled with aquatic vegetation were usually occupied exclusively by $A$. pseudopunctipennis, while those completely shaded and containing cool water at all times were occupied exclusively by $A$. maculipennis (freeborni). Between these two extremes of illumination the species bred together. Barber and Forbrich considered daytime temperature to be the determining factor in this selection of breeding places, although A. pseudopunctipennis was also found in sunlit cold springs at high elevations. It was also noted in southern New Mexico that A. pseudopunctipennis bred in a wider range of environments. Freeborn (1926) states that wayside sunlit pools are breeding places of $A$. pseudopunctipennis, in which it is found with $A$. maculipennis, and that breeding continued in such places after they had become too foul for the latter species.

A. georgianus (crucians georgianus), a species recently discovered by Bellamy (1939), was described from larvae taken in hoof prints and pot holes in a seepage area at the head of a small stream in Georgia (King 1939). Further observations by Bellamy point to pastureland seepage areas ("helocrene springs") with acid waters (pH 5.0 to 5.8 ) as the typical habitat. $A$. punctipennis and $A$. crucians were occasionally found in association with it in such places. A few of the larvae were taken in a swampy, sluggish stream, and once in a shallow pond having a $\mathrm{pH}$ of 6.6 , in association with A. punctipennis, A. walkeri, and $A$. crucians.

A. atropos breeds in water on coastal marshes, where it is found in pools and around pond margins and on open flooded marshes, often in very shallow water. It has been found in waters varying from brakish to full strength sea water and ap- parently prefers those having the higher salt concentrations.

A. bradleyi breeds in brackish to fresh waters along the coast, usually in waters having a salts content of 1.5 per cent or less. It may be associated with larvae of A. atropos at the higher concentrations or with $A$. crucians at low concentrations. It occurs in pools and around pond margins and on flooded marshes covered by vegetation.

The breeding places of $A$. albimanus in the United States have not been reported, although the species appears to be of fairly common occurrence in the lower Rio Grande Valley in Texas, and larvae have been collected there.

Larval food. Anopheline larvae feed chiefly while at the surface of the water, and their food consists of microscopic plant and animal matter, both living and decomposed, which they filter from the water by the action of their mouth brushes. It has been computed by Hinman (1932) from data given by foreign writers that a volume of water at least 20 times that displaced by the body of a larva may be filtered by it during each 24-hour period. Studies on the food of anophelines by various workers (Metz 1919a; Boyd and Foote 1928; Bradley 1932; Coggeshall 1926) have not shown that a selection of food particles is made, the larvae ingesting anything of suitable size which comes within reach. Barber (1927) reared larvae of $A$. quadrimaculatus and $A$. crucians to maturity on cultures of single organisms (algae, bacteria, and protozoa), and Hinman (1932) found that both these species were able to make a significant growth on colloids in suspension and material in solution. It would therefore appear that the food available in most waters, provided it is sufficient in quantity, is not a prime factor in influencing specific breeding of Anopheles in various places. The presence or absence of different kinds of organisms may, however, provide an index to the suitability of water for breeding, as has been discussed by Frohne (1939).

Enemies of larvae. Anophelines are 
preyed upon by a great variety of predaceous and parasitic forms of animal and plant life, which in the aggregate take a large toll of these and other mosquitoes. Certain aquatic plants have also been credited with deterrent as well as larvicidal properties. For summaries dealing with these, the reader is referred to works of Howard, Dyar, and Knab (1912-17a), Boyd (1930a), Matheson (1930), and Hinman (1934a). The most important enemies of the larvae are the small insectivorous fishes of the family Poecilidae, which actively search for their prey in shallow water amongst vegetation and debris (Rockefeller Foundation 1924). In the Southern States the most important species is Gambusia patruelis (affinis). There can be no doubt about the influence of these minnows in limiting mosquito breeding in areas where they abound, and ecological studies on the occurrence and abundance of mosquito larvae in various locations must always take into consideration their presence or absence, as well as the amount of protection against them afforded by plants and debris. A review and discussion of the use of fishes in mosquito control has been published by the International Health Board (Rockefeller Foundation 1924). Of the aquatic insects, the nymphs of damselflies and dragonflies (Odonata), water scorpions (Ranatra spp.), backswimmers (Notonectidae), and the larvae of water beetles, particularly those of the family Dytiscidae, are frequently noted as preying on anopheline larvae.

Aquatic plants of the genus Utricularia trap mosquito larvae by means of small bladders and have been observed to be rather efficient in reducing a larval population, particularly in aquaria (Matheson 1930).

While these predaceous forms must destroy countless numbers of larvae, nevertheless it is true that they may all be found in association in even the heaviest of anopheline breeding areas, so the final result appears to be merely a natural balance. Certain types of waters are undoubtedly eliminated as breeding places for anopheline larvae because of the presence of their natural enemies, and control efforts can sometimes be aided materially by changing conditions in favor of these predators. 


\title{
THE CLASSIFICATION AND IDENTIFICATION OF THE ANOPHELES MOSQUITOES OF MEXICO, CENTRAL AMERICA, AND THE WEST INDIES
}

\author{
By W. H. W. KOMP

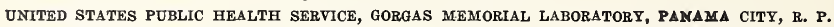

THE systematist finds in his material "certain regular correlations, associations of a limited number of eharacters that occur regularly in individuals, amid a very great amount of individual variation. It is this correlation that constitutes the basis of species-diagnosis" (Robson, G. C. and Richards, O. W. 1936). Species, the unit of classification, is a concept, existing only in the mind of the taxonomist, and not corresponding to any sharp separation recognizable in nature. Minor variations are present which must be recognized and given a place in the scheme of classification. These minor variations, below the rank of what are usually considered species, are called varieties, subspecies, or races. The usual connotation of variety is geographical; that of race is physiological, although here no uniformity of usage exists.

For the purposes of this paper, it seems advisable to adopt the following definitions: A taxonomic species is an assemblage of individuals in which certain regular assoeiations of structural or colorational characters are found. In the Anophelini, the most useful structural characters are found in the male sexual organs, the terminalia. A variety is a subdivision of a species occurring in one part of the range of the species, and set off from it by slight but constant differences in color-markings, but showing no constant differences in the strueture of the male sexual organs. A race is a purely physiological concept, based upon differences in behavior, which may or may not be reflected in differences in structure. As instances of these categories, the concept of a species may be represented by A. albimanus, which agrees throughout its range in possessing certain association of characters in all its stages, so that it ean be recognized by the trained entomologist by examination of the larvae and the male terminalia. The concept of a variety may be represented by C. C. Hoffmann's two forms of A. albimanus, A. bisignatus and A. trisignatus, found in southern Mexico (Hoffmann 1938a); these forms have male terminalia indistinguishable from those of A. albimanus as found elsewhere, but have extra black bands on the hind tarsál segments. The physiological race is exemplified by the two kinds of $A$. albimanus found respectively in Panama and in Venezuela. These are indistinguishable from color or structural characters, so far as now known, but the Panama race will mate in small cages, while Gabaldon tells me he has failed to obtain mating under such conditions.

Something must be said concerning the recent use of egg-characters in taxonomy. The classic example is of course the separation of the European A. maculipennis into six or more forms on the basis of differences in the eggs. Under the classification indieated above, all these forms of $A$. maculipennis are included in a single taxonomic species; opinion is at present divided as to whether these six forms shall be considered varieties or races. Apart from the eggs, apparently only two morphological differences have been found to separate these forms : variations in the form of a hair of the larva and in the shape of the spine on the claspette lobes of the male terminalia; but Hackett states that these differences are so small and overlap so much that they are "not sufficient in themselves for the classification of any given specimen." So by 
definition these forms would be regarded as physiological races, unless we admit that the characters of the eggs differentiate higher categories than the race.

Marston Bates (1940), in a closely reasoned paper, makes out a strong case for regarding the forms of $A$. maculipennis found in Europe as distinct species. Much of his argument is based on the varying sexual behavior of the forms; he has found that the males of some forms mate readily with those of others, but in few instances are fertile eggs obtained. Some of his argument is based on egg-characters, it apparently being true that certain forms which can be distinguished by distinct sexual behavior lay eggs which are easily differentiated from those of other forms. Probably when as much study has been applied to some of our tropical forms as has been given to the European $A$. maculipennis, similar differences, possibly correlated with observable differences in egg-structure, will be found. At present we are not in a position to state that such differences occur, as very little study has been given to this aspect of taxonomy, in American mosquitoes.

Some work has been done in Brazil and Panama on the egg-characters of the species of Nyssorhynchus, among which are the principal vectors of malaria in the New World. Ayroza Galvão and Barretto (1938) have shown that the eggs of $A$. darlingi exhibit great variations in the form of the floats, frill and terminal collar of the egg. Rozeboom in Panama (1938b) and Ayroza Galvão (1940) in Brazil, have shown that the eggs of $A$. strodei are exceedingly variable; Rozeboom distinguishes three types from his relatively scanty material; Ayroza Galvão also distinguishes three types, and gives photomicrographs of other anomalies which bear no resemblance to these types.

In Panama, at least, the various types of A. strodei eggs apparently do not indicate physiological races, as they were all obtained from the same locality at approximately the same time. Ayroza Galvão has not studied the possibility that his types represent true physiological "races," but hints that this may be the case.
So it will be seen that considerable study must be made of the eggs of the species of the subgenus Nyssorhynchus before it can be decided whether their characteristics denote physiological races, or are possibly valid indicators of taxonomic "species."

With these preliminary remarks, it should be evident that the taxonomy of the Anophelini is not static but in a state of flux. However, the broad outlines of generic classification are fixed. It is.very likely that the number of good taxonomic "species" will be materially increased when adequate collections are made in Central and South America, and even more probable that many so-called species will have to be split up into varieties and physiological races.

For the purposes of this paper, only taxonomic species will be considered, that is, species which can be recognized by some outward and visible structural or color character, or lacking this, by the characters of the larvae and male terminalia.

\section{Cluassification}

In 1924 the late F. M. Root (1924a) listed 34 species found in North and South America, of which three are now known to be synonyms, making a total of 31 species. During the past 16 years, our knowledge of the Anophelini of the American tropics has increased very materially, not only as to the number of species, but in regard to their eggs, larvae, the characters of the male terminalia, their distribution, and their ability to transmit malaria. For instance, the list of species found in North and South America, as of January 1, 1940, stands more or less at 64 species, of which 27 are found north of South America and south of the southern boundary of the United States, and including the Caribbean islands, the region with which we have to deal.

The list of these 27 species, and the manner in which they are classified, is given in the following table:

\footnotetext{
Family Culicidae

Subfamily Culicinae

Tribe Anophelini

Genus chagasia
}

bathanus Dyar 1 


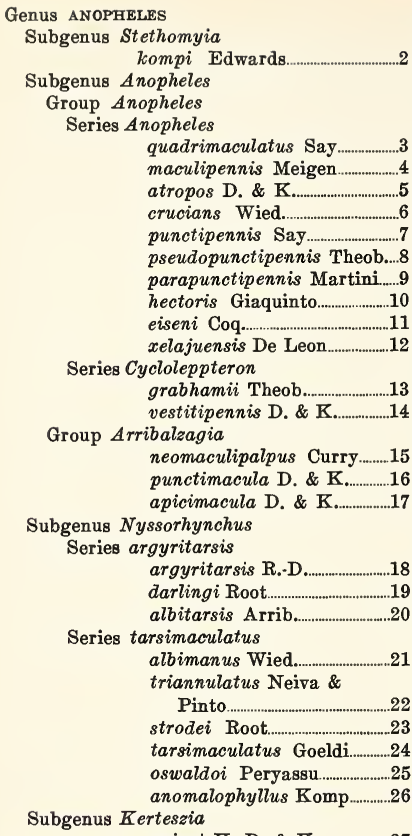

This classification is adapted from Edwards (1932), and differs from his in one minor modification. The writer believes that Edwards' group Kerteszia should be elevated to a subgeneric rank, co-equal with subgenus Nyssorhynchus. Edwards, in a personal communication, has agreed with this contention.

Most of these species are strictly neotropical, but a few species penetrate the area from the north and are found mostly at higher elevations on the Mexican plateau. These species are A. punctipennis, A. maculipennis, A. quadrimaculatus, $A$. crucians, and $A$. atropos. One neotropical species, $A$. albimanus, penetrates to the northern limit of the area, being found in the lower Rio Grande valley.

The characters of the male terminalia are the basis of classification of the Anophelines into genera and subgenera. It is possible to classify all the 27 species of the area under consideration into these categories by noting the arrangement of the spines on the side-piece of the terminalia. The following table shows how this is done:

\section{Characters of Male Terminalia}

Of the genera and subgenera noted above, Chagasia, Stethomyia, Nyssorhynchus and Kerteszia are easily recognizable by the characters of the spines on the sidepieces of the male terminalia. But the subgenus Anopheles is divided into a number of groups and series, which are rather more difficult to separate on male terminalia characters. The group Anopheles contains two series, Anopheles and Cycloleppteron. In the series Anopheles, the leaflets of the mesosome may be variable in number of pairs, but all the leaflets are alike in form. In the series Cycloleppteron, a single pair

Classification of the Anophelini on Basis of Spines of Side-PIEce

\begin{tabular}{c|c|c|c|c}
\hline Division & $\begin{array}{c}\text { Basal } \\
\text { lobe }\end{array}$ & $\begin{array}{c}\text { Parabasal } \\
\text { spines }\end{array}$ & $\begin{array}{c}\text { Accessory } \\
\text { spines }\end{array}$ & $\begin{array}{c}\text { Internal } \\
\text { spine }\end{array}$ \\
\hline $\begin{array}{c}\text { Genus CHAGASIA } \\
\text { Genus ANOPHELES } \\
\text { Subgenus Stethomyia } \\
\begin{array}{c}\text { Anopheles } \\
\text { Nyssorhynchus }\end{array}\end{array}$ & $\begin{array}{c}\text { Present } \\
\text { Absent }\end{array}$ & $\begin{array}{c}\text { Undifferentiated } \\
\text { (See below) } \\
\text { One, large } \\
\text { Two }\end{array}$ & $\begin{array}{c}\text { Undifferentiated } \\
\text { (See below) } \\
\text { Absent } \\
\text { One } \\
\text { Two }\end{array}$ & $\begin{array}{l}\text { None } \\
\text { (See below) } \\
\text { One, large } \\
\text { One } \\
\text { One, between ac- } \\
\text { cessory spines and } \\
\text { tip of side-piece } \\
\text { One, between para- } \\
\text { basal spine and } \\
\text { accessory spines }\end{array}$ \\
\hline
\end{tabular}


of very large leaflets is present, with or without a smaller pair. In the group Arribalzagia, the number of pairs of leaflets is variable, but the largest pair differs in shape and size from the smaller pairs. Subgenus Nyssorhynchus is easily recognized because the dorsal lobes of the claspette are fused to form a single median structure, the form of which is characteristic in most of the species. In subgenus Kerteszia, the position of the internal spine, lying as it does between the parabasal and the two accessory spines, is characteristic.

A rather complete knowledge of the characters of the male terminalia is required for the separation of the species of the subgenera Nyssorhynchus and Kerteszia. As Root has well said, "The adult markings of species of this subgenus (Nyssorhynchus) are unusually variable, and in working out the fauna of a region which has not been carefully studied previously, it is essential to base identifications on larval and hypopygial characters, which are comparatively stable, instead of on the variable characters of the adult coloration." Because of limitations of space, it is impossible to do more than to indicate the characters by which species may be identified. Students desiring to obtain further information on the use of the characters of the male terminalia are referred to the separate works listed in the bibliography.

\section{Larval Characters}

In many instances, particularly among the neotropical species, the larvae show better differential characters than the adults. This is particularly true of the subgenus Nyssorhynchus. The principal characters used in differentiating species are: the form and spacing of the anterior clypeal hairs of the head; the shape of the inner hair of the prothoracic submedian hairgroup; the shape of the pleural hairs; the shape and number of the lateral abdominal hairs ; the number of pairs of palmate hairs of the abdomen and the form of the individual leaflets; the shape and arrangement of teeth of the comb of the eighth abdominal segment; and in a few species, the peculiarities of the form of the respiratory apparatus. A number of other characters are of use in differentiating certain species, but most of the larger groups may be separated on the characters enumerated above.

\section{Pupal Characters}

Very little attention has been paid to the pupal characters of the Neotropical Anophelines, as in most cases the differences are very slight and variable. In genus Chagasia, series Cycloleppteron, and group Arribalzagia, however, the pupal trumpets have characteristic shapes, and afford better means of separation of species than either the larvae or the female adults. In one important species of subgenus Nyssorhynchus, $A$. darlingi, the pupal trumpets are unique in form, and afford. excellent characters for identification.

\section{Characters of the Female adults}

A large number of characters has been used to define the various genera and subgenera, and smaller divisions. Probably one of the most useful gross characters is the color of the legs. The tarsi of all the legs are black in Stethomyia kompi, and in the 10 species of the series Anopheles. In the series Cycloleppteron and group Arribalzagia, the legs are speckled and banded irregularly with spots of white or yellow. In the subgenus Nyssorhynchus, the hind tarsi have the first segment black, the base of the second segment black, the apex white, while all the remaining three segments are either pure white, or have a narrow black basal band on the fifth segment.

The scaling of the body is another important group character. The species of the series Anopheles have no scales on the dorsum of the abdomen. Those of the group Arribalzagia have prominent lateral scale tufts on the abdominal segments, as do the species of subgenus Nyssorhynchus. Kerteszia lacks these scale tufts, no dorsal abdominal scales being present except in one species, which is not found in our area.

The wing-pattern, composed of aggregations of scales of different colors, disposed along the wing-veins, is of use in determin- 
ing species, but because of the great variations which may occur, it is less useful than might at first appear. The principal regions in which spots of definite value are found are the costa, the leading edge of the wing; the third vein; and the sixth vein. All the species of the subgenus Nyssorhynchus have very similar wing-patterns, and there are great variations within the species, making it impossible in many instances to identify females by these patterns. In the series Anopheles, the wingpattern of each species is distinctive, and usually will serve to distinguish species readily. In the Arribalzagia group, the pattern is very similar in most of the species, and other characters, such as the shape and color of the wing-scales, offer better means of separation.

The color-markings of the palpi are also important characters used in distinguishing species. Usually the color of the last two segments is of some value in separating related species.

In the following section, keys to the female adults, larvae, and male terminalia of the 27 species of the Anopheline species found in the region are given. The key to the female adults includes all the species found in the region.

That portion of the key to the female adults which includes the subgenus Nyssorhynchus must be used with caution, for the species in this subgenus resemble one another closely and are extremely variable in color-characters. This is particularly true of the species of the tarsimaculatus series, in which are the most dangerous vectors of malaria.

\section{Key to Adult Females of-Tribe Anophelini, Found in the NeOtropical Region NORTH OF SOUTH AMERICA}

1. Seutellum trilobed; a medium-sized shaggy brown species, with dark shaggy palpi; mesonotum with erect black scales before wing-bases; wings heavily elothed with broad ovate seales, mixed dark and light, not forming definite spots; second to fifth hind tarsal segments broadly white basally, black apically, with a narrow black ring near base of each segment (Atlantic coast of Costa Rica and Panama) (Genus CHAgASIA) BATHANUS
Scutellum not trilobed, crescent-shaped; mesonotum without erect scales before wingbases; legs not marked as above .......................2

2. Slender black species, without scales on body; mesonotum dark brown, with a narrow white median line in integument; wingscales all black; legs very long and slender, all black (Atlantic coast of Costa Rica, and Panama) (Subgenus STETHoMrIA) ......KOMPI

Body with scales, at least on anterior promontory of thorax; mesonotum without median white line

3

3. Hind tarsi all dark ............................................................4

Hind tarsi with some or all of the terminal segments white; or variously spotted, speckled or banded with white …………............................12

4. Seales on wings all dark; thorax without median gray stripe ................................................................5

Scales forming wing-markings white and black; thorax with broad median gray stripe

5. Wings very dark, the scales aggregated into four indistinct patches on origin of second vein, on cross-veins, and at bases of forks of 2nd and 4th veins; palpi with white rings at bases of terminal segments (rarely absent) (southwest Cuba) ............................ATROPOS

Wings lighter, the seales forming four distinct darker spots on origin of second vein, on cross-veins, and at bases of forks of 2 nd and 4 th veins; palpi without white rings

MACULIPENNIS QUADRIMACULATUS

6. Hind tibia with broad white apical band; wing-scales dark, except for a small white spot at basal third of first vein, and one large and one small white spot at apex of wing (Panama to Mexico) ..........................ISENI

Hind tibia all dark; white wing-spots not as above

\section{7}

7. Hind femur with narrow white apical band; wing-scales dark, except for white areas at apex of wing on tip of costa and on tip of first vein; wing-fringe white at this point (Guatemala) ……..................................

Hind femur and all other segments of leg dark; wings with many areas of black and white scales

8. Palpi all dark, without white rings; a broad white spot on costa, also involving first vein and base of second vein; another at tip of first vein, remainder of costa dark; sixth vein dark at both ends, white in middle

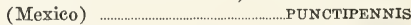

Palpi with white rings on some of the segments; wings variously marked, but sixth vein not as above ...................................................

9. Palpi marked with white on terminal segment and on two preceding segments; costa of 
wing all dark, except for a white spot at apex, involving also the tips of first vein and upper branch of second vein; sixth vein with three dark spots (Antilles; Yucatan to Honduras) CRUCLANS

Palpi white-marked; costa of wing not as above; sixth vein variously marked .................10

10. Costa of wing with two white spots, one at junction of subcosta, and another at apex of wing, both also involving areas opposite on first vein; sixth vein basally white, apically dark ..................................

Sixth vein not basally white, apically dark .....11

11. Costa of wing with three large white spots, one at junction of costa and subcosta, another between this and base of wing, and a third at apex of wing, involving also tip of first vein; sixth vein white, with a central dark area and one at apex

PARAPUNCTIPENNIS

Costa of wing with two white spots, one at junction of costa and subcosta, broadly involving first vein, and another at apex of wing, involving tip of first vein also; three dark spots on sixth vein, one at base and one in middle small, the spot at apex of vein larger; another small black spot near origin of fourth vein (Mexico, Guatemala)

HECTORIS

12. Hind tarsi with apical portion of second segment white, all of third, fourth and fifth segments white, with or without a narrow black basal ring on fifth segment (subgenus Nyssorhynchus) 13

Hind tarsi not so marked 20

13. Hind tarsi with fifth segment all white ............14 Hind tarsi with narrow black basal ring on fifth segment

14. First abdominal sternite with two parallel lines of white scales; mid tarsal segments with white rings; second hind tarsal segment nearly half black, half white (Panama, Costa Rica) ...........................................ALITARSIs

First abdominal sternite bare, without lines of white scales

15. Mid tarsal segments with narrow white rings; next to last segment of palpi with many white scales, terminal segment white; second hind tarsal segment usually half black (sometimes more); a narrow white ring at apex of first hind tarsal segment (British Honduras and Guatemala) ................ DARLINGI

Mid tarsal segments without white rings; next to last segment of palpi all black, terminal segment only white; second hind tarsal seg ment about one-third black; first hind tarsal segment without apical white ring (Panama to Mexico) ARGYRITARSIS
16. Second hind tarsal segment about one-sixth black at base (Panama, Costa Rica)

OSWALDOI

Second hind tarsal segment more than onesixth black at base

17. Last two segments of palpi white ……................18

Terminal segment of palpi white; preceding segment all black, or with few white scales

18. Second segment of hind tarsi about half black, half white (breeds in salt water, Atlantic coast of Panama) .......................TARSIMAcULATUS Second segment of hind tarsi less than half black (strodei in fresh-water ponds, Panama to Mexico, anomalophyllus in running streams, Atlantic coast of Panama and southern Costa Rica) STRODEI

ANOMALOPHYLLUS

19. Large species, the white spots on costa of wing broad; spot B2 (the costal white spot second from base) larger than preceding black spot (Panama to Brownsville, Texas; Greater Antilles) ..................................................ABIMANUS

Smaller species, the white spots on costa of wing reduced; spot $\mathrm{B} 2$ usually smaller than preceding black spot (Panama, Costa Rica). .TRIANNULATUB

(Formerly BACHMANNI)

20. Mesonotum gray, with four bare black lines; costa of wing with four or five alternating subequal black and white spots; hind tarsal segments narrowly black basally, broadly white apically; abdomen without scales (subgenus Kerteszia; Panama northward).

NEIVAT

Mesonotum, wings, and legs not as above; femora and tibiae spotted

21

21. Costa of wing with a prominent bend or " kink" at junction of subcosta; lateral abdominal scale-tufts present (group Arribalzagia) 22

Costa of wing straight, without such a kink; hind tarsi with narrow light rings at the joints; lateral abdominal scale-tufts absent (series Cycloleppteron) ...24

22. Costa of wing with only two large jark spots; black spot at apex of wing usually large, distinct; fifth vein speckled with light and dark scales (Panama, Costa Rica, probably northward) ....................................NEOMACULIPALPUS

Costa of wing with three large dark spots; brownish or blackish species, with pale scales of wing either white or yellow 23

23. Large brownish species; wings with brown, white, and yellow scales; fifth vein speckled with dark and pale scales; dark spot at apex of wing diffuse, about the same size as the 
dark spot between it and the third large costal spot (Panama to Mexico)

PUNCTIMACULA

Smaller black species; wings with black and white scales only; fifth vein black on stem and lower fork; black spot at apex of wing prominent, larger than black spot between it and the third large costal spot (Panama northward) APICIMACULA

24. A small species, wings mostly white-scaled; edge of costa black; with a conspicuous dark spot at middle; dark wing-seales large, some nearly circular (West Indian islands)

GRABHAMII

A large brown species, wings mostly darkscaled, scales narrow; a few small yellow spots on costa and apex of wing; hind tarsi usually with narrow yellow rings on both ends of segments (sometimes absent) (Greater Antilles, Dominica, southern Mexico, British Honduras southward along Atlantic coast to northern Panama)

VESTITIPENNIS

Key to the Larvae of the Anophelini Found in THE NeOTrOpical REgION NORTH OF SOUTH AMERICA

1. Without apparent and functional palmate hairs; a long annulated process ending in a split bristle arises from each lateral flap of respiratory apparatus (Panama, Costa Rica) (subgenus Stethomyia) KOMPI

With three or more pairs of well-developed abdominal palmate hairs; lateral flaps of respiratory apparatus not as above .................2

2. Integument very hairy; palmate hairs with racket-shaped elements present on third, fourth and fifth abdominal segments; a single long filament arises from anterior plate of respiratory apparatus (streambreeder, Panama, Costa Rica, British Honduras) (Genus CHAGASIA) ...............BATHANUS

Integument not hairy; with more than three pairs of well-developed abdominal palmate hairs ; respiratory apparatus not as above ......3

3. Lateral abdominal hairs on segments 4,5 and 6 with coarse lateral branches

Lateral abdominal hairs on segments 4, 5, and on 6, if present, without coarse lateral branches

. .5

4. Outer and inner anterior, and posterior clypeal hairs long, simple, subequal; mesothoracic and metathoracic pleural hairs much thickened, spine-like; tips of posterior plates of respiratory apparatus produced into two long black upcurving tails .... PSEUDOPUNCTIPENNIS

Anterior internal clypeal hairs notably longer and stronger than external hairs; meso- and metathoracic pleural hairs slender, normal, not thickened; tips of posterior plates of respiratory apparatus rounded (Guatemala, at high altitudes) HECTORIS

5. Outer anterior clypeal hairs usually forming a fan-shaped tuft; inner clypeal hairs simple, set close together; well-developed palmate hairs on third to seventh segments

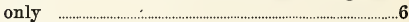

Outer anterior elypeal hairs never forming a fan-shaped tuft; inner clypeal hairs simple, set close together or far apart; palmate hairs,present or absent on abdominal segments 1 and 2 ; present on segments 3 to 7 .....9

6. Anterior dorsal hair of prothoracic pleural hair-group with a few lateral branches; lateral hairs of abdominal segments 4 and 5 normally double, sometimes triple; ventral sabre of antenna with truncate, frayed tip. VESTITIPENNIS

All long hairs of prothoracic pleural hairgroup simple, unbranched; lateral hairs of abdominal segments 4 and 5 single or double . .7

7. Ventral sabre of antenna squarely truncate, the tip frayed; outer anterior elypeal hairs normally forming a long tuft with many ultimate branches; lateral hairs of abdominal segments 4 and 5 single (Central and South American mainland, Trinidad) PUNCTIMSACULA

Ventral sabre of antenna pointed; outer anterior elypeal hairs forming a dense flattened tuft, shorter than inner clypeal hairs; lateral abdominal hairs of 4 th and 5 th seg. ments branched

$$
8
$$

8. Branching of outer anterior clypeal hairs dichotomous, without additional fine branches; lateral hairs of abdominal segments 4 and 5 usually double from base (Caribbean Islands) .....................................................ABHAMII

Branching of outer anterior clypeal hairs falsely dichotomous, with many fine simple elements in addition to dichotomous branches; lateral hairs of abdominal segments 4 and 5 variable, usually triply branched (sometimes more) from basal third (Caribbean coast from Yucatan to Spanish Honduras; Greater Antilles) .CRUCLANS

9. Frontal hairs of head simple, at most finely frayed; lateral abdominal hairs of segments 4,5 , and 6 finely feathered; teeth of pecten all long, subequal; palmate hairs absent on first abdominal segment; leaflets straplike, with truncate tips (subgenus Kerteszia, bromeliad-inhabiting, Central America) ............. NEIVAI

Frontal hairs of head plumose; lateral hairs of abdominal segments 4 and 5 , and on 6 , if presont, simple, not feathered; pecten teeth 
irregular in length; leaflets of palmate hairs always pointed

10. Well-developed palmate hairs present on all abdominal segments ( 1 to 7 ); lateral abdominal hairs present on segments 4,5 , and 6 ; clypeal hairs variously arranged (subgenus Nyssorhynchus) ……….......................................

Well-developed palmate hairs present on abdominal segments 2 to 7 only; lateral abdominal hairs present or absent on segment 6 ; inner clypeal hairs close together; outer clypeal hairs simple 11

11. Well-developed palmate hairs present on abdominal segments 2 to 7 only; lateral abdominal hairs present or absent on segment 6

Well-developed palmate hairs present on abdominal segments 3 to 7 only; lateral abdominal hairs present on segments 4 and 5 only

.. .14

12. Lateral abdominal hairs present on segments 4, 5 and 6 single; one of the two long metathoracic pleural hairs branched at tip; palmate hairs with narrow, lanceolate, smooth leaflets ARGYRITARSIS

Lateral abdominal hairs present on segments 4 and 5 only, absent on 6 ; both long metathoracic pleural hairs simple 13

13. Lateral hair of third abdominal segment stout, plumose, normal; lateral abdominal hairs of segments 4 and 5 normally double; elements of palmate hairs wide, with serrations beyond middle; usually a small, transparent larva (Central and South American mainland)

EISENI

Lateral hair of third abdominal segment with slender, straight central shaft, with fine lateral branches difficult to see; lateral abdominal hairs of segments 4 and 5 simple, short, slender; elements of palmate hairs long, lanceolate, with faint serrations; a very large dark larva (Central America at high altitudes, syn. chiriquiensis)

PARAPUNCTIPENNIS

14. Inner anterior clypeal hairs notably thicker and stronger than outer elypeal hairs; all the long hairs of the prothoracic pleural hairgroup simple; lateral hairs of abdominal segments 4 and 5 usually single; a dorsal longitudinal white line usually present on thorax and abdomen ............EOMACULIPALPUS

Inner anterior clypeal hairs not notably thicker and stronger than outer clypeal hairs; anterior dorsal hair of the prothoracic pleural hair-group with a few lateral branches; lateral hairs of abdominal segments 4 and 5 usually double; dorsum of thorax and abdomen variously marked ...........APICIMACULA

15. Posterior plates of spiracular apparatus with two long filaments arising from strong tubercles about midway of length of plate; spurs at bases of pleural hairs very long, strong; inner prothoracic submedian hair with fine branches from slender shaft

DARLINGI

Posterior plates of spiracular apparatus not as above, without long flaments; spurs at bases of pleural hairs moderate; inner prothoracic submedian hair not with fine branches from slender central shaft .............16

16. Inner prothoracic submedian hair with shaft thickened and widened, with many hairlike lateral branches; anterior dorsal hair of prothoracic pleural hair-group with many fine lateral branches ..................................LBIMANUS

Inner prothoracic submedian hair palmate; all the long hairs of the prothoracic pleural

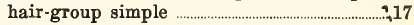

17. Inner anterior elypeal hairs so spaced that distance between them is more than onethird of the distance between the inner and the outer clypeal hairs ……..................................18

Inner anterior clypeal hairs so spaced that distance between them is less than one-third of the distance between the inner and the outer clypeal hairs; elements of abdominal palmate hairs notably very long, smooth, pointed

18. Anterior clypeal hairs nearly evenly spaced, with few fine lateral branches .......................19

Anterior internal clypeal hairs closer than distance between inner and outer elypeal hairs, and with many coarse lateral branches........20

19. Inner submedian prothoracic hair small, palmate, about one-third the length of the middle hair, with about 15 to 18 very fine, hairlike leaflets; larva small, usually dark green with white spots ..............................BACHMANNI

Inner submedian prothoracic hair very large, about half the length of the middle hair, palmate, with about 15 narrow lanceolate leaflets, usually heavily infuscated; larva large, variously colored ………..........ALBITARSIS

20. Outer anterior clypeal hairs with many lateral branches from shaft; inner submedian prothoracic hair palmate, with about 12 broad lanceolate blunt-tipped leafiets; outer pair of frontal hairs of head not notably longer and stronger than other frontal hairs (brackish water breeder).....TARSIMACULATUS

Outer anterior clypeal hairs with few lateral branches from shaft; inner submedian prothoracic hair palmate, with about 10 broad lanceolate leaflets; outer pair of frontal hairs of head notably longer and stronger than other frontal hairs (fresh water breeder) OSWALDOI

21. Inner anterior clypeal hairs very close to- 
gether, less than one-third the distance between inner and outer elypeal hairs; inner submedian prothoracic hair palmate, with about 15 narrow lanceolate leaflets ..... STRODEI

Inner anterior clypeal hairs not so close together, about one-third the distance between inner and outer clypeal hairs; inner submedian prothoracie hair palmate, with about 10 narrow lanceolate leaflets (rapid stream breeder, Costa Rica) ............ANOMALOPHYLLUS

Key to the Male Terminalia of the Neotropical SPECIES OF THE ANOPHELIN ENCOUNTERED

\section{NORTH OF SOUTH AMERICA}

1. Side-piece without parabasal spine; a large spinose sub-basal lobe present (genus СHAGASIA) BATHANUS

Side-piece with parabasal spine or spines (genus ANOPHELES)

2. Side-piece with two spines, one very large parabasal, one internal (subgenus Stethomyia) KOMPI

Side-piece with three spines, two parabasal, one internal (subgenus Anopheles) ....................3

Side-piece with four spines, one parabasal, two accessory, one internal (subgenera Nyssorhynchus and Kerteszia) .........................12

3. Mesosome without leaflets; outer lobe of claspette with three long filaments, the tips much broadened and bent inwards, pipeshaped; inner lobe with two or more narrow, flattened, pointed filaments, the outermost the longest (Guatemalan highlands) ......

XELAJUENSIS

Mesosome with leaflets; lobes of claspette not as above

4. Mesosome with leaflets all of the same general shape (series ANOPHELES)

Terminal leaflets of mesosome always longer and broader than others (group Arribalzagia and series Cycloleppteron)

\section{8}

5. Mesosome with a single pair of long, black, serrate leaflets; mesosome long, slender, normal EISENI

Mesosome with more than a single pair of leaflets; these leaflets small, transparent, difficultly visible; mesosome short, curved.....6

6. Claspette lobes with two or three very long, inwardly curving setae at apex ............................7 Claspette lobes with one or two fused bladelike filaments on outer lobe; inner lobe with a single long, pointed seta at apex, and a shorter slender seta on internal aspect at base; mesosome with one or two pairs of small, broad, shallowly serrate leaflets

PARAPUNCTIPENNIS

7. Mesosome with two or three pairs of long, serrate leaflets; if three pairs, the middle pair the longest; outer lobe of claspette with three somewhat fused, bladelike, pointed filaments .......................................................

Mesosome with one to four pairs of small, delicate, deeply serrate leaflets; outer lobe of claspette with two or three somewhat fused, bladelike, rounded filaments

PSEUDOPUNCTIPENNIS

8. Mesosome with a single pair of very long, lanceolate leaflets, three-quarters the length of the mesosome ............................VESTITIPENNIS

Mesosome with more than a single pair of leaflets

9. Mesosome with two pairs of leaflets, the terminal pair large, the smaller pair less than half the length of the larger (Antilles) ............ GRABHAMU

Mesosome with more than two pairs of leaflets

10. Terminal leaflets much larger than the other pairs, elongate diamond-shaped, with central rib and hyaline margins ...........PUNCTIMACULA Terminal leaflets without hyaline margins.....11

11. Terminal leaflets about twice the length of the next smaller pair, saber-shaped; ventral claspette lobes very hairy ...............APICIMAcula

Terminal leaflets very wide, the other pairs, usually four, all very slender and nearly as long as the terminal pair; claspette lobes searcely hairy, with one stout apical hair and two smaller accessory hairs

NEOMACULIPALPUS

12. Side-piece with internal spine between accessory spines and parabasal spine; mesosome slender, tapering, without terminal leaflets (subgenus Kerteszia) .......................................EIVAI

Side-piece with internal spine between accessory spines and apex of side-piece (subgenus Nyssorhynchus)

13. Mesosome with a pair of leaflets .13 Mesosome without leaflets .14

14. Fused ventral lobes of claspette with long, hairy basal lobules, as in oswaldoi; mesosome with two long, straight, thick, tapering leaflets, coarsely serrate on outer terminal half ...............................................ANOMALOPHYLLUS

Fused ventral lobes of claspette low, without long, hairy basal lobules ..........................................15

15. Tip of mesosome long, scoop-shaped; a pair of leaflets from slight offsets on sides; leaflets long, straight, somewhat widened at middle, deeply serrate on terminal half; fused ventral lobes of claspette with rounded apex, with microtrichia, and two small, hairless, wrinkled, pouchlike ventral projections basally DARLINGI

Tip of mesosome very short, rounded; the pair of leaflets not from offsets, short, curved, serrate; fused ventral lobes of claspette low, 
without pouchlike projections, but with curved ridges from apex ARG YRITARSIS

16. Fused ventral lobes of claspette with columnar truncate apex, with distinet median emargination; the membrane below expanded into a pair of prominent, hairless, ovoid lobes .....

ALBIMANUS

Fused ventral lobes not as above

17

17. Apex of fused ventral lobes of elaspette notably modified, with raised striations or projeetions . .18

Apex of fused ventral lobes of claspette rounded, or slightly emarginate, not notably modified ...19

18. Apex of fused ventral lobes of claspette with lateral ear-like expansions; basal lobules small, with fine hairs; filaments of dorsal claspette lobes short, with rounded tips (syn. BACHMANNI) .TRIANNULATUS
Apex of fused ventral lobes of claspette with erect, rugose lateral expansions; basal lobules large, with long hairs from free margin; filaments of dorsal lobes long, pointed ……................................................

19. Fused ventral lobes of elaspette with long, apron-like hairy basal lobules ………............ 20

Fused ventral lobes of claspette without such basal lobules, low, rounded, obsoletely striate ALBITARSIS

20. A crescent-shaped chitinized area above basal supporting strip, below apex of ventral lobes; long, apron-like basal lobules with distal free margins fringed with very long, reflexed hairs OSWALDOI

A round chitinized spot just above supporting basal strip, below apex of ventral lobes; long, apron-like basal lobules with short, radiating hairs (Aquasalis)

TARSIMACULATUS 


\title{
DISTRIBUTION AND ECOLOGY OF THE ANOPH- ELES MOSQUITOES OF THE CARIBBEAN REGION
}

\author{
By L. E. ROZEBOOM \\ DIVISION OF MEDICAL ENTOMOLOGY, JOHNS HOPKINS UNIVERSITY SCHOOL OF HYGIENE AND \\ PUBLIC HEALTH, BALTIMORE, MD.
}

THE biological characteristics that determine whether an Anopheles mosquito is harmless or a dangerous malaria vector are its susceptibility to malaria parasites, blood preferences, attraction to human habitations, range of flight, longevity, and abundance. To be abundant enough to be dangerous, usually a wide range of breeding places must be available to the mosquito; ordinarily those species that are restricted to a special type of water are too scarce to play an important role in malaria transmission. With our present knowledge it is impossible to outline the characteristies that make various types of water favorable or unfavorable as breeding places for the Caribbean Anophelines. Beattie (1932) made a study of the physico-chemical factors of $\boldsymbol{A}$. tarsimaculatus (=aquasalis?) breeding waters in Trinidad, but could find no correlation between larval incidence and $\mathrm{pH}$, carbon dioxide, organic nitrogen, dissolved oxygen, nitrites, nitrates, or phosphates, although ammonia nitrogen repelled the ovipositing females. In general, the Caribbean anophelines can be divided into two groups: those that require sunlight for breeding, and those that require shade. Some of the former are apt to be more closely associated with man, as they are often abundant in cleared areas near human habitations; the latter are typically forest species. Most species are found in fresh water, one prefers brackish water, while others will breed in both fresh and brackish water. Some species are rather closely associated with certain kinds of aquatic vegetation, and often this association seems to be more than a mere concentration of larvae in protected places.
Twenty-nine species of anophelines have been found in the Caribbean region, from Panama to the southern border of the United States. Several of them really belong to the temperate fauna, and to avoid duplication, little mention will be made of them in this paper.

\section{Notes ON THE SPECIES}

Chagasia bathanus was described by Dyar from specimens collected near Gatun, Canal Zone, by Mr. C. H. Bath (Curry 1928; Dyar 1928). It has been recorded from western Panama (Komp 1929a), Costa Rica (Kumm, Komp and Ruiz 1940), British Honduras (Kumm 1940b), and Venezuela (Gabaldon, Herrera and Perez-Vivas 1940). Martini (1935) says that a single specimen was captured by Dampf in the State of Chiapas, Mexico. The larvae prefer shaded water along the edges of streams; they have a predilection for shady pools in the streams (Kumm, Komp and Ruiz 1940), but are also found in running water. The species may be abundant in certain restricted localities, but it is a rare mosquito. Adult females have been captured while they were feeding on horses (Kumm, Komp and Ruiz 1940), but apparently they seldom feed on man.

A. (Stethomyia) kompi is rather rare, but has been recorded from Brazil (Shannon 1933), Venezuela (Gabaldon 1939a), Panama (Curry 1931b; Edwards 1930), and Costo Rica (Kumm, Komp and Ruiz 1940). Komp (1940b) states that in Panama the larvae are found in stream pools at the end of the rainy season, and are rarely abundant. The adults will bite man.

A. albimanus, the most important malaria vector of the Caribbean region, is dis- 
tributed from Brownsville, Texas (King 1937), to the northern part of South America. King doubts the identity of specimens taken in New Orleans, and although a number of adults were captured by MacDonell in Key West in 1904, the species did not become established there (King 1937). According to Hoffmann (1932, 1938a), its range in Mexico includes the Gulf Coast from Brownsville to the State of Quintana Roo, but north of Tampico it is rather scarce. South of Tampico it is the predominant Anopheline, and breeds not only in the littoral, but extends inland to an altitude of 400 meters along the river valleys. The northern part of the Peninsula of Yucatan is very dry, and here A. albimanus is found near villages and other human habitations where water is stored in artificial reservoirs. Swamps that form in isolated areas along the coast during the rainy season are inhabited by the species. In the southern part of the Peninsula there are more "natural" breeding places; here Hoffmann also took the species inland in uninhabited forest areas. On the Pacific coast of Mexico it is distributed from Guatemala to the northern part of the State of Sinaloa. This region is much drier than is the Gulf Coast; thus $\boldsymbol{A}$. albimanus is not so abundant here. Although the natural range of $\boldsymbol{A}$. albimanus in Mexico is limited to the coastal areas, occasionally it is found farther inland at higher altitudes, especially during the rainy season. Hoffmann records it from Monterrey, Nuevo Leon, 545 meters above sea level, and from the village of Autlan, in the State of Jalisco, 1,003 meters above sea level.

Molloy (1932) says that A. albimanus is "constantly encroaching on the highlands of Central America," and de Leon's (1933b) map shows it to be not only along the Atlantic and Pacific coasts of Guatemala, but inland as far as Guatemala City, at an altitude of 4,500 feet. It has been taken in a number of localities in El Salvador (Larde Arthes 1921; Sutter 1939). Nicaragua (Kumm 1929b), Spanish Honduras (Whitmore, Roberts and Jantzen 1929), and British Honduras (Kumm 1940b). In Costa Rica it is the predominant species
(Barber and Komp 1927; Kumm, Komp and Ruiz 1940; Kumm and Ruiz 1939b; Root 1924c), especially in the lowlands, but breeding may be heavy in drying river beds at elevations of 2,500 feet (Salisbury and Corrigan 1927). Kumm and Ruiz (1939b) found it in 85 localities at less than 1,000 feet elevation, in six from 1,000 to 3,000 feet, and not at all above 3,000 feet.

Published records from Panama, excepting those from the Canal Zone, seem to be rather meagre, but there are enough to show that the species is present throughout the country, at least in the coastal areas (Barber, Komp and Newman 1929a; Dunn 1934; Komp 1929a; Komp 1929b; Kumm 1929b).

A. albimanus is found in many of the West Indian Islands. It appears to inhabit almost all of Cuba (Carr, Melendez and Ros 1940; Kumm 1929b; Malaret 1929), but Carr, Melendez and Ros (1940) state that in the Province of Oriente it is most abundant in areas less than 1,000 feet above sea level. Hoffman (1926), Root (1927) took it in a number of localities in Haiti, including La Vanneau with an elevation of 2500 feet, but it is more prevalent at lower altitudes (Mink 1933). It is distributed throughout Puerto Rico, from the mountains to the seashore (Earle 1930b; Root 1922; Tulloch 1937), and although it may be taken in most parts of Jamaica, it seems to be scarce in those areas of the Island that have an elevation of 500 feet or more, while it is especially abundant in the lowlands (Boyd and Aris 1929). Russo (1927) says that it is the most important anopheline in Santo Domingo. It has been reported from St. Thomas (Hoffman 1930), St. Croix (Kumm 1929b), Culebra (Hofiman 1940), Vieques (Howard, Dyar and Knab 1917b), Tortola (Hoffman 1930), Nevis (Hoffman 1930), Montserrat (Hoffman 1930), Antigua (Edwards and Box 1940), St. Vincent (Senevet 1936), Dominica (Senevet 1936), Guadeloupe (Francois-Julien 1930; Senevet 1938), and Barbados (Kumm 1940b; Seagar 1928). It is absent from Grenada (Earle 1936b; Root and Andrews 1938), St. Lucia (Earle 1936b), Martinique (Senevet 1936), 
and although Beattie (1932) records it from Trinidad, Komp showed that this record is probably erroneous, in which F. W. Edwards concurs (Komp 1937b, 1940b).

As King (1937) has pointed out, its distribution shows that $A$. albimanus is essentially a mosquito of hot, humid climates. Although outside of the area covered by this Symposium, it is of interest to consider briefly the distribution of A. albimanus in northern South America, where, for no apparent reason, we find the southern limit of its range. Campos' record from Guayaquil, Ecuador, has been confirmed by King and Stone, who examined the male terminalia of Campos' specimens (King 1937). Komp (1937b) says it occurs for some distance up the Magdalena River in Colombia, but Antunes (1937) did not include it in his list of species from the interior of Colombia (Intendancia of Meta). In these western countries the mountains and climate may form a natural barrier to a southward extension of the $A$. albimanus range. In Venezuela, Gabaldon (1938, 1939a) has taken $A$. albimanus in large numbers in certain localities on or near the coast, but not in others farther inland. Gabaldon states that the eastern limit of the range is Carupano; Komp (1937b) could not find it near the mouth of the San Juan River, in the Orinoco drainage.

A. albimanus breeds in a great variety of water collections, either fresh or brackish, and the chief requisite for breeding seems to be an abundance of sunlight. During the rainy season it is found in rain pools; other favored breeding places are seepages, irrigation ditches (Earle 1930b), and quiet, sunlit waters of lakes, backwashes of streams, swamps, and ponds. Hoffmann (1938c) says that the breeding water must be in a biological equilibrium, exposed to sun, and rich in microorganisms but without putrefaction; as these conditions are more apt to exist in permanent and semi-permanent water collections, temporary rain pools are not suitable for breeding at the beginning of the rainy season, but require time to develop a biological equilibrium. In larger bodies of water $A$. albimanus larvae must find protection from fish in various types of floating aquatic vegetation. Of these the Characeae, Utricularia, and Naias are especially favorable, when the water level is such that these plants can form thick mats on the surface. In these mats A. albimanus larvae not only are protected from their enemies, but find enough food to enable them to breed in tremendous numbers. In the Canal Zone, Curry (1934) has described the great abundance of the larvae among these plants during the dry season, when the level of Gatun Lake drops several feet, allowing the plants to reach the surface of the water and form extensive mats. Hoffman $(1930,1940)$ collected larvae in crab holes in Haiti, Nevis, and Montserrat; the water in these crab holes was as low as two feet below the surface of the ground. Perhaps the species becomes adapted to these unusual breeding places during periods of drought. Certain artificial water collections may become favorable breeding places; thus in the dry coastal areas of the northern part of the Peninsula of Yucatan, A. albimanus is dependent for its existence upon the tanks, water troughs, and similar artificial containers that are used for the storage of water near the villages (Hoffmann 1938a), while Clark (1932) found larvae in barrels and troughs in which algae were growing on the bottom and sides. In Cuba, water is piped from the hills to all parts of the Preston Division of the United Fruit Company, and excess water from the terminal pipes forms "water-faucet" drains, which were ideal for $A$. albimanus breeding (Malaret 1929). Kumm, Komp and Ruiz (1940) call attention to their discovery of larvae in an iron drum full of rain water.

The adult is a strong flier. In Panama, towards the close of the dry season, a sudden rise occurs in the number of adults found in sanitated areas. Curry (1934) believes that these mosquitoes originate in the mats of aquatic plants far away in Gatun Lake; therefore they must be able to fly 12 miles or more. Curry does not consider this to be a single, sustained flight, 
but that the distance is covered in a series of short flights, during which the mosquitoes feed on gametocyte carriers outside of the sanitated areas, causing many of the mosquitoes to become infected with malaria, thus carrying the disease to inhabitants of the sanitated areas. According to Le Prince and Orenstein (1916), who observed flights of large numbers of $A$. albimanus and $A$. aquasalis in the Canal Zone, the mosquitoes become active during twilight; in the evening they flew from a brackish water area to Gatun, and in the early morning they returned to the breeding places. These mosquitoes flew from a half mile to a mile at right angles to the strong dry season winds, above ground that had very little protection in the form of bush or grass. Judging from the actions of birds feeding on the flying insects, flight took place from less than six feet to 30 or 40 feet above the surface of the ground. Jobbins (1940) in Panama also observed flight of $A$. albimanus between 6:30 and 7:30 P.M. Apparently copulation takes place only during twilight hours (Rozeboom 1936).

The seasonal abundance of $A$. albimanus seems to be directly related to rainfall. Gabaldon (1939a) shows this in his Venezuelan records. Nevertheless, in some areas where breeding is largely confined to impounded waters or rivers, the dry season favors the appearance of certain types of aquatic vegetation in which breeding is especially heavy. This is the case in Gatun Lake in the Canal Zone (Curry 1934), and in the Chagres River, where Jobbins (1940) and Clark, Komp and Jobbins (1940) noted the peak of emergence in 1939 to be in April, several weeks before the onset of the rainy season. In Mexico there is a marked reduction of $A$. albimanus during the dry winter season, and in some areas the larvae disappear, while the females pass through the winter in a state of inactivity (Hoffmann 1938a). Giaquinto Mira (1936) noted that during the long dry season at Champerico, Guatemala, the females remained in crab holes containing water and did not mature their eggs until the coming of the rainy season.
The females feed readily on man, but also attack animals; in fact, the observations of Earle and Howard (1936) in Puerto Rico indicate a marked preference by $A$. albimanus for horses and oxen, and while goats and pigs were not so attractive as horses and oxen, the mosquitoes fed on them as readily as they did on man. Le Prince and Orenstein (1916) noticed that a horse was more attractive than men. In the villages of Las Guacas and Santa Rosa, in Panama, Rozeboom (1938a) caught 578 Anopheles in native huts, of which 472 were $A$. albimanus, but of 349 female anophelines taken while they were feeding on pigs near these villages, only four were $A$. albimanus.

The females are very domestic, and within most of its range $A$. albimanus is the most common anopheline found in houses. But the females do not remain long in houses; most of them return to the jungle or to their breeding places, soon after feeding or early in the morning (Barber and Komp 1927; Green 1922). They do not seem to congregate in preferred daytime resting places (Earle and Howard 1936; Rozeboom 1938a), although Le Prince and Orenstein (1916) saw large numbers on the leeward side of trees near extensive breeding places.

A. triannulatus was described by Neiva and Pinto (1922) ; unfortunately this misleading name has priority over $A$. bachmanni Petrocchi, 1925 (Galvão and Barretto 1938). The species was described from Brazil, but it has been collected as far north as Puntarenas, Costa Rica (Kumm, Komp and Ruiz 1940). In Panama the larvae are found within the cup-shaded enclosure formed by the crown of leaves of Pistia (water-lettuce), and where extensive areas of these plants exist, triannulatus may be very abundant. In other countries, the larvae may breed among other aquatic plants; even in Panama they are often found among the floating stems of Jussiaea natans (Curry 1931b; Rozeboom 1935). Adults of triannulatus have been observed attacking man outdoors (Rozeboom 1935), but they do not seem to enter human dwellings readily, even in the immediate vicinity 
of the breeding places; on the other hand large numbers have been observed feeding on pigs (Rozeboom 1938a). Hill (1934) concluded that this mosquito definitely prefers animal blood.

$A$. strodei was described from Brazil, but in the Caribbean region it has been reported from Panama (Curry 1932), Costa Rica (Kumm, Komp and Ruiz 1940), and recently from Veracruz, Mexico, by Vargas (1940b). This species breeds in a variety of water collections. During the rainy season it may be found in rain pools, but in Panama its chief breeding places are clear, cool pools in drying creeks. Kumm, Komp and Ruiz (1940) state that in Costa Rica the larvae were taken from slowly running streams in the sunshine; however, the larvae may also be abundant in partially shaded waters. In Panama the larvae are most abundant at the end of the rains, in January. Little is known concerning the habits of the adults. Occasionally the females may be captured in houses, but they seem to be attracted to animals more than to man (Kumm, Komp and Ruiz 1940); however, in a locality in São Paulo, Brazil, Correa (1938) found that 95.3 per cent of the anophelines he captured in houses were $A$. strodei, and two of 163 females dissected showed oocysts.

A. aquasalis was considered to be a variety of $A$. tarsimaculatus by Curry (1932), who distinguished it from var. aquacaelestis (=oswaldoi) on morphological characters and by its breeding habits. Rozeboom and Gabaldon (1941) consider A. tarsimaculatus to be a synonym of $A$. albimanus, while aquasalis, a member of a group of mosquitoes lumped under the name "tarsimaculatus," was raised to specific rank by these authors. In Panama the species is found only along the Atlantic coast. The brackish water "tarsimaculatus" listed from Costa Rica by Kumm, Komp and Ruiz (1940) is also this species. Hoffmann (1929b) discovered a few specimens of "tarsimaculatus" (=strodei ?) in Veracruz (Panuco), but considered that they were accidentally introduced. The "tarsimaculatus" that Root and Andrews (1938) concluded was the malaria vector in Grenada is really aquasalis, and perhaps so is the brackish water "tarsimaculatus" of Trinidad. Senevet $(1936,1938)$ reports "tarsimaculatus" from Martinique and Guadeloupe, and Edwards and Box (1940) include it in their list of mosquitoes from Antigua. The larvae breed in brackish water along the seacoast, in swamps, ditches, and similar situations. Occasionally they will breed in fresh water; in Costa Rica, Kumm, Komp and Ruiz (1940) found them once in a fresh-water stream about fifty yards from the sea, while in Trinidad, in times of great abundance, they develop temporarily several miles inland in rice fields, drains, and other collections of fresh water (de Verteuil 1933; de Verteuil and Spence 1937). Beattie (1932) states that A. aquasalis has no apparent preference for light or shade; Curry (1932) believes it prefers fairly well shaded places; Kumm, Komp and Ruiz (1940) made three collections in Costa Rica, all of them in water exposed to sunlight. Earle (1936b) noted that in Grenada it would breed in densely shaded mangrove swamps. The adults are strong fliers; Curry (1932) speaks of the huge swarms that used to fly into Colon and Cristobal, in Panama, from swamps located several miles from these cities, and in Trinidad adults migrate inland from the coastal swamps at least three miles away (de Verteuil and Spence 1937). It is puzzling that this mosquito, considered to be a vector of malaria in some of the Lesser Antilles, is not attracted to man in Panama, where, in former years, the appearance of great numbers of $A$. aquasalis in the cities was not followed by a rise in the malaria rate (Curry 1932). This is a marked contrast to the observations of Earle $(1936 \mathrm{~b})$, who had no difficulty in finding $A$. aquasalis adults in houses in Grenada and St. Lucia. The biting and trapping experiments of Earle and Howard (1936) showed that in these islands $A$. aquasalis was attracted to man much more readily than to a calf or a donkey.

A. oswaldoi has been reported from Panama (Curry 1932), Trinidad (de Verteuil 
1933), and from several South American countries, but it has not been found as far north as Costa Rica (Kumm, Komp and Ruiz 1940). It breeds in fresh, well shaded water in pools or swamps. Being a "jungle" mosquito, it is not closely associated with man, and although it will attack man when its haunts are invaded, it does not seem to be attracted to man's habitations (Curry 1932; Rozeboom 1938a).

A. rangeli is a species recently described by Gabaldon, Cova-Garcia and Lopez (1940). Perhaps this species should not be included with the Caribbean anophelines, but it is mentioned because we know that it is present in Trinidad, and because it has been confused with $A$. aquasalis. Rozeboom and Gabaldon (1941) have found specimens of $A$. rangeli among material collected by Root in Venezuela and Trinidad; the species must be quite common in these countries. It breeds in fresh, shaded water that contains an abundance of vegetation (Gabaldon, Cova-Garcia and Arevalo 1940).

$A$. anomalophyllus is a rare mosquito. The type specimens were collected as larvae from the running water of a small, shaded stream near Almirante, Panama (Komp 1936b). It was found recently in Costa Rica by Kumm, Komp and Ruiz (1940).

A. albitarsis is widespread in South America; the northern limit of its range seems to be Costa Rica (Kumm, Komp and Ruiz 1940). It is evident that the species is composed of several races or subspecies (Galvão and Lane 1937b ; Root 1926, Rozeboom 1937a), although the taxonomic relationships between the races are not yet entirely clear. In Panama the larvae breed in a rather restricted area of Gatun Lake, in the mats of Chara, Naias, and Utricularia that produce such large numbers of $A$. albimanus; these mats are exposed to full sunlight (Curry 1934). In Costa Rica the larvae were taken from a sunny pond with algae, and vertical and horizontal vegetation (Kumm, Komp and Ruiz 1940). The rice fields of Trinidad furnish suitable water for larval development (de Verteuil 1933), while Gabaldon (1939a) says that the most important breed- ing places in Venezuela are lakes full of Pistia and Eicchornia. Although it is a dangerous mosquito in parts of South America, in Panama it does not attack man and does not enter man's habitations, even in the immediate vicinity of the breeding places (Curry 1934).

A. argyritarsis is distributed throughout the Caribbean region, from tropical Mexico (Hoffmann 1929b) to South America (Clark 1926; Curry 1925; Giaquinto Mira 1936; Hoffmann 1932; Komp 1937a ; Kumm, Komp and Ruiz 1940; Kumm and Ruiz 1939b; de Leon 1936b; Martini 1935; Sutter 1939); it also inhabits much of South America, and some of the Caribbean islands: Trinidad (Beattie 1932; Howard, Dyar and Knab 1917b), Grenada (Earle 1936b; Howard, Dyar and Knab 1917b; Root and Andrews 1938), St. Lucia (Earle 1936b; Howard, Dyar and Knab 1917b; Senevet 1936), Guadeloupe (FrancoisJulien 1930; Senevet 1938), Dominica (Hoffman 1930; Howard, Dyar and Knab 1917b ; Senevet 1936), Martinique (Howard, Dyar and Knab 1917b; Montestrue 1936; Senevet 1936), St. Vincent (Howard, Dyar and Knab 1917b; Senevet 1936), and Antigua (Edwards and Box 1940; Howard, Dyar and Knab 1917b). Theobald's records of the species from the Greater Antilles probably concern A. albimanus (Boyd and Aris 1929; Howard, Dyar and Knab 1917b). A. argyritarsis breeds in clear waters of small, partly shaded or sunlit streams; it is found along the grassy edges of running streams, and it also breeds in quiet pools in streams, and in pools in drying stream beds, as well as in grassy rain pools. The females do not seem to attack man readily, and are not often found in man's habitations (Earle 1936b ; Hoffmann 1929b ; Kumm, Komp and Ruiz 1940).

A. darlingi was described by Root (1926) from specimens collected in Brazil. It is widespread, in South America, and for some time it was thought that records from Venezuela and Colombia represented its most northern distribution, but in $1940 \mathrm{Komp}$ proved that it is also present in Guatemala and British Honduras. Shannon (1933) 
considers that in the Amazon Valley the larvae are especially adapted to flood-water. In Brazil, Barretto (1938) could find few A. darlingi larvae in ditches, small pools, and small swamps, but obtained many larvae from the edges of an impoundment, where the water was deep, without current, and well shaded by trees with branches reaching down into the water; these branches prevented wave action in the water underneath them, and in this pooled water, where there was some floatage but little vegetation, the larvae were concentrated. In British Honduras, Kumm (1940b) collected larvae along the edges of streams, in deeply shaded water. The adults feed readily on man, and can be taken in large numbers in man's habitations (Davis 1931; Gabaldon 1938; Giglioli 1940; Shannon 1933).

A. eiseni has a range which includes much of South America and extends northward through Central America as far as tropical Mexico (Aguilar 1931; Curry 1931b; Dyar 1928; Giaquinto Mira 1936; Howard, Dyar and Knab 1917b; Kumm, Komp and Ruiz 1940; Kumm and Ruiz 1939b; de Leon $1936 \mathrm{~b}$; Martini 1935), but it seems to have been found on none of the islands, except Trinidad (Beattie 1932). It is another "jungle" mosquito, breeding in densely shaded, clear, fresh waters of small streams, pools, and swamps. Simmons (1939) collected many larvae from coconut husks, while Kumm, Komp and Ruiz (1940) found them even in tree holes and bromeliads. Locally the species may be very abundant; for example, Simmons (1939) says that it was one of the most common species on the Fort Sherman Reservation in the Canal Zone during the fall and winter of 1935 and 1936. Very little is known concerning the biology of the adults, but the females do not seem to be attracted to man and seldom enter houses (Gabaldon 1939a; Simmons 1939).

A. grabhami has been reported from Cuba (Carr, Melendez and Ros 1940), Jamaica (Boyd and Aris 1929; Howard, et al. 1917b; Theobald and Grabham 1905), Haiti (Clark 1926; Mink 1933), Santo Domingo
(Howard, Dyar and Knab 1917b), Puerto Rico (Earle, 1936a; Howard, Dyar and Knab 1917b; Tulloch 1937; Wells 1930), and St. Thomas (Hoffman 1930). Theobald and Grabham (1905) noted that in Jamaica the larvae would develop in any stagnant water, and Boyd and Aris (1929) also found them in all types of breeding water on the island, including upland streams with swift currents, and brackish water along the coast, where they were associated with the larvae of A. albimanus. Earle (1936a) showed that in Puerto Rico the larvae are much less tolerant of salt than are the larvae of $A$. albimanus, and that they will occur in densely shaded waters, including "pastures where the growth of grass is so dense that one's weight is almost supported on it as it overgrows water courses." The adults will attack man; some authors (Root 1922; Theobald and Grabham 1905) consider the species to be an ardent blood sucker that feeds on man readily in the evening, but others (Boyd and Aris 1929; Earle 1936a) conclude that it prefers the blood of animals. It is not often found in houses (Earle and Howard 1936), and is not especially abundant.

A. crucians has been found in Cuba (Carr, Melendez, and Ros 1940; Kumm 1929b), Jamaica (Boyd and Aris 1929; Kumm 1929b), along the Caribbean littoral of Mexico (Hoffmann 1932), and British and Spanish Honduras (Clark 1926; Kumm 1929b, 1940b ; Martini 1935; Whitmore, Roberts and Jantzen 1929). Martini (1935) gives a record from Iguala, State of Guerrero, Mexico, and another from Lago Peten, Guatemala. Tulloch (1937) includes $\boldsymbol{A}$. crucians in his list of brackish-water mosquitoes from Puerto Rico. In Jamaica, Boyd and Aris (1929) collected larvae from streams, seepages, and ponds, in sunlit or slightly shaded water; no larvae were found in brackish water. According to Vargas (1940a), both A. crucians var. crucians and var. bradleyi are present in Mexico, in the states of Tamaulipas and Veracruz.

A. punctipennis was reported from Jamaica by Theobald and Grabham (1905); however, Boyd and Aris (1929) point out 
that this record is doubtful; A. punctipennis has never been taken in Jamaica since Grabham's time. Hoffmann (1932) speaks of its presence in the littoral zone of the Gulf of Mexico and in the "northern zone" of Mexico; Martini (1935) gives records from Sonora, Tamaulipas (Tampico), and Veracruz (Pueblo Viejo). Hoffmann (1937) collected larvae from a shaded pool near Ixmiquilpan, in the state of Guanajuato, Mexico.

A. pseudopunctipennis is the most widespread of the New World Anophelini, ranging from Oklahoma and California to Argentina. Shannon, Davis, and del Ponte (1927) show that its distribution coincides to a great extent with that of the western mountains, and that apparently it is essentially a mountain species, occurring in the lowlands only where special conditions permit its existence. According to Hoffmann (1932) it inhabits almost all of Mexico between the eastern and western mountain ranges; only areas that are six or seven thousand feet above sea level are free from it. It extends along the entire Mexican Pacific littoral, from California to Guatemala, but does not seem to be so abundant along the Caribbean coast, although it is present in the littoral zone north of Veracruz. South of Veracruz it appears only in the winter. It is found in the villages of Yucatan. It has been collected in all of the Central American countries (Aguilar 1931; Barber and Komp 1927; Clark 1926; Giaquinto Mira 1936; Howard, Dyar and Knab 1917b; Komp 1929b; Kumm, Komp and Ruiz 1940; Kumm and Ruiz 1939b; Larde Arthes 1921; Martini 1935; Molloy 1932; Sutter 1939), and also in Trinidad (Kumm 1929b) and Grenada (Earle 1936b; Root and Andrews 1938).

The larvae of $A$. pseudopunctipennis require a great deal of sunlight for their development, and the preferred breeding places are pools and eddies in shallow or drying streams, especially those containing mats of green algae. In streams the larvae can be found not only in such mats of algae, but also in shallow, quiet or running water, where they cling to leaves or other bits of floatage dammed behind rocks or sand bars. Other breeding places are seepages, especially those in dry beds of subterranean streams (Hoffmann 1932), ground pools, and even artificial water containers such as fountains and tanks (Hoffmann 1932). Because the preferred breeding places are shallow, drying streams, this species is especially abundant in mountainous areas, and at the time of the year when the streams are not flushed out by heavy rains. Thus, in Panama and in parts of Central America, A. pseudopunctipennis is most prevalent during the dry season (Clark 1932; Molloy 1932; Simmons 1939). However, in some regions the dry season causes a diminution in the number of favorable breeding places, so that the numbers of the mosquito decrease. In northwestern Argentina the lowland breeding places disappear during the prolonged dry season, and A. pseudopunctipennis must maintain itself during this unfavorable period in springs and other fresh water collections in the mountains (Shannon 1930). Rainfall is the most important climatic factor in determining abundance in the Rimac Valley of Peru, and here too the mosquito survives the dry season by breeding in the restricted fresh water collections in the mountains, reestablishing itself in the more numerous lowland breeding places after the onset of the rains in the higher altitudes results in the formation of pools along the margins of rivers and streams. Here the peak of abundance occurs between January and June, the rainy season of the highlands (Shannon 1930). Hoffmann (1938b) calls the typical streampools "winter breeding places of concentration"; this author says that when the rains come the larvae are washed from these pools and are distributed over wide areas. In the Valley of Mexico the species is less abundant during the dry winter months; during December, January, and February, when the temperature drops below the freezing point at night, only large larvae are found in the breeding places, while small larvae appear about the middle of March. During the cold weather the males 
disappear, and only the females are found in the resting places (Hoffmann 1929a).

The habits of the adults seem to differ in various parts of the $A$. pseudopunctipennis range. In the highlands of Mexico the females will fly for considerable distances to enter houses and feed on man (Hoffmann $1929 \mathrm{~b}$; Vargas 1938). The species is considered to be a dangerous malaria carrier in the highlands of Guatemala (Giaquinto Mira 1936; Molloy 1932), but in Costa Rica, where Kumm, Komp and Ruiz (1940) collected many larvae, only a few adults were captured in houses by these workers. The females do not fly far, and are not attracted to houses, in the Canal Zone (Curry 1925) ; they are not attracted to man in Grenada (Earle 1936b), although in this island, during July and August at least, A. pseudopunctipennis is the most common Anopheline (Root and Andrews 1938). In Argentina it is a dangerous blood-sucker (Davis 1927). The suspicion that the species is divided into geographical races or subspecies has not been confirmed on morphological grounds, although there are two kinds of $A$. pseudopunctipennis eggs in California (Herms and Freeborn 1920; Herms and Frost 1932), both of which are unlike the eggs from Panama (Rozeboom 1937b), Costa Rica (Kumm 1940a), and Mexico (Vargas 1939b). The writer has examined $A$. pseudopunctipennis eggs from western Oklahoma which were identical in morphology with those he described from Panama. Vargas (1939b) considers the form in California, the egg of which was described by Herms and Frost (1932), to be different enough to merit specific rank; he calls this mosquito $A$. boydi.

A. parapunctipennis ranges through the highlands from Mexico to Panama (Kumm and Ruiz 1939b; Vargas 1940a). Komp (1936a) collected larvae in a large cold spring on the slopes of Chiriqui Volcano in western Panama. This rare species was first found by Dampf (Martini 1935) in the state of Chiapas.

$A$. hectoris was described from Guatemala by Giaquinto Mira (1931); it is also found in the mountains of Mexico. Dampf
(Martini 1935) took it near San Cristobal, Chiapas, 2,000 meters above sea level. Apparently only the adult had been captured in Mexico until April, 1940, when Mazzotti discovered larvae in Ciudad de las Casas, Chiapas (Vargas 1940a, 1940b). According to de Leon $(1936 \mathrm{a}, 1936 \mathrm{~b})$ it is the characteristic anopheline species in the higher mountain regions of Guatemala, and breeds in small, shallow, slowly-flowing streams filled with algae. The adults are said to attack man (Molloy 1932).

$\therefore$ A. vestitipennis is comparatively rare, but it ranges from the tropical regions of Mexico to South America (Aguilar 1931; Barber and Komp 1927; Clark 1926; Curry 1931a; Dyar 1928; Giaquinto Mira 1936; Hoffmann 1929b, 1932; Kumm 1940b; Kumm, Komp and Ruiz 1940; de Leon $1936 \mathrm{~b}$; Martini 1935), and is also present in Cuba (Carr, Melendez and Ros 1940), Puerto Rico (Wells 1930), Jamaica (Boyd and Aris 1929), and Dominica (Dyar 1928). The larvae develop in shaded, fresh-water streams, seepages, ponds, and rain pools (Boyd and Aris 1929; Kumm, Komp and Ruiz 1940). The adult females will attack man, and Hoffmann (1929b) has found adults in houses.

A. punctimacula has a wide distribution in the New World tropics, including all of the Central American countries (Aguilar 1931; Barber and Komp 1927; Clark 1926; Curry 1931a, 1931b; Howard, Dyar and Knab 1917b; Kumm 1940b; Kumm, Komp and Ruiz 1940; Kumm and Ruiz 1939b; Simmons 1939; Sutter 1939) and tropical Mexico (Hoffmann 1932; Martini 1935). It breeds in clear, cool, densely shaded waters in small streams, ponds, and swamps, where it may be associated with larvae of $A$. eiseni, $A$. oswaldoi, and A. apicimacula. The adults will feed on man as well as on animals, and will visit human habitations in search of blood (Kumm, Komp and Ruiz 1940; Rozeboom 1938a; Simmons 1936a, 1939).

A. apicimacula has been reported from Mexico (Hoffmann 1932; Howard, Dyar and Knab 1917b; Martini 1935), all of the Central American countries (Aguilar 1931; 
Clark 1926; Curry 1931a, 1931b; Giaquinto Mira 1936 ; Howard, Dyar and Knab 1917b; Kumm, 1940b; Kumm, Komp and Ruiz 1940; Kumm and Ruiz 1939b; de Leon 1936a, 1936b; Martini 1935; Sutter 1939), Trinidad (Beattie 1932), and several South American countries. Its breeding places are similar to those of $A$. punctimacula, $A$. eiseni, and $A$. oswaldoi. Only a few adults have been taken inside houses, and the females seem to feed on animals much more readily than they do on man (Kumm, Komp and Ruiz 1940; Simmons 1939).

Martini (1935) identified specimens collected by Dampf in Yucatan, British Honduras (Blue Creek, Rio Cacao) and Guatemala (San Miguel, Lago Peten), as A. intermedius; this species has not been found since in Mexico (Vargas 1940a).

A. quadrimaculatus inhabits the Gulf coast of Mexico as far south as Tuxpan, Veracruz, but records from the interior of Mexico actually refer to a race of $A$. maculipennis (Dampf 1935; Hoffmann 1935).

A. maculipennis var. aztecus was described by Hoffmann (1935) from the states of Guanajuato and Michoacan, Mexico. The larvae breed in canals, irrigation channels, and clear water pools in which algae and protozoa are present. This form is not so dependent on clean water as is $A$. pseudopunctipennis, so that it is replacing the latter species in the outskirts of Mexico City, where urbanization results in a pollution of the water available to the mosquitoes. The larvae are encountered in the breeding places throughout the year, even when a layer of ice forms over the surface of the water (Hoffmann 1935). The females do not seem to be attracted to man, and Vargas (1939a) has described a condition of anophelism without malaria in a locality in the State of Mexico, in which this form was the only anopheline present.

Vargas (1940b) reports the finding of A. maculipennis var. freeborni at Imuris, Sonora.

A. atropos is included in this list because Carr, Melendez and Ros (1940) discovered it recently in Cuba. It was found once, in Havana Province, in an animal-baited trap. Martini (1935) reported it from the interior of Mexico, but Hoffmann (1936) points out that Martini's specimens were really $A$. aztecus.

A male of $A$. walkeri has been taken in Tuxpan, Veracruz, Mexico (Vargas 1940a).

A. barberi was discovered by Sr. A. Martinez Palacios at Imuris, Sonora, Mexico (Vargas 1940c).

A. neivai breeds only in water caught in the leaf-bases of certain water-holding plants, such as the epiphytic and terrestrial bromeliads. The species ranges from tropical Mexico to Panama, and southwards into Ecuador; records of cruzii, from Panama northwards, actually refer to $A$. neivai (Komp 1937a). It may be quite abundant and may bite man freely, in the neighborhood of its breeding places (Komp 1937a).

A. xelajuensis was described from Guatemala by de Leon (1938). 


\title{
FACTORS INFLUENCING INFECTION OF ANOPH- ELES WITH MALARIAL PARASITES
}

\author{
By CLAY G. HUFF \\ DEPARTMENT OF BACTERIOLOGY AND PARASITOLOGY, UNIVERSITY OF CHICAGo, CHICAGo, ILL.
}

To BE an effective transmitting host of malaria an anopheline must fulfill certain exacting requirements. Its choice of breeding place and its flight range must make it a frequenter of human habitations. It must breed in sufficiently large numbers to offset the natural hazards connected with the transmission of the malarial parasites. It must have the proper tropisms for bringing it to feed upon man. It must be susceptible to the parasites ingested along with its blood meal. Its length of life must be sufficient to permit the complete development of the sporozoites, and it must live in an environment suitable to this development of the parasites. It will be convenient to classify all of the factors which contribute to determining whether the anopheline will be an effective vector into two eategories: (1) those responsible for bringing the mosquito to the act of ingesting the blood of malarial patients, and (2) those responsible for the successful completion of the extrinsic malarial life-cycle within the mosquito. Into the first eategory fall the many behavioristic characters of the mosquito conducive to the contacts between it and man necessary to successful transmission. Into the second eategory fall the physiological and environmental influences acting directly or indirectly upon the parasites while they are within the mosquito. The following discussion will be concerned primarily with the second eategory of factors, with only a brief consideration of the factors in the first eategory. Many of the factors responsible for bringing the mosquito to and causing it to bite man will be adequately discussed by others in connection with other problems in malariology considered in this symposium.

Without eiting specific literature dealing with this first eategory of factors, many statements about them can be made almost axiomatically. It is well established that great differences do exist between species (and to a lesser extent between varieties or races of species) in respect to their choice of breeding places, the density of their breeding, the food preferences of the females, and the ability to withstand unfavorable environmental conditions. Examples might be multiplied illustrating the importance of any of these factors or combinations of them in making of a particular anopheline a dangerous vector, a less important one, or an innocuous species. The chief concern, however, in this discussion will be with those factors which influence the infection of the anopheline, assuming that the latter has had a blood meal containing viable gametocytes.

From the time that viable gametocytes are ingested by an anopheline mosquito until the moment of delivery of viable sporozoites to a new host a complicated sequence of events occurs. These events include very remarkable changes in the organism itself as well as migrations within the mosquito. It will be helpful to think of the factors which influence the development of the parasite-both intrinsic and extrinsic-as acting continuously upon the parasite throughout this period of development.

It is desirable at this point to enumerate the more important events in the life cycle of the parasite within the mosquito in relation to the possible effects of these intrinsic and extrinsic factors. Gametogenesis is the first important happening after the ingestion of the parasites in the infected blood. Then comes fertilization of the macrogametes. We shall soon review some of the factors which may influence these two processes. After fertilization of the macro- 
gametes, the zygotes must next penetrate the stomach wall. This is a critical period in the life of the zygote, or oökinete as it is now called. While as yet we do not know the relative importance of the factors operating against the penetration of the stomach wall by the oökinete, we can be fairly sure that the most important factors are intrinsic, that is, are connected with the chemical composition of the stomach wall or with the physiological processes therein. After penetration of the stomach wall the oökinete now proceeds to develop into an oöcyst on the outside of the mosquito's stomach. Both extrinsic and intrinsic factors may conceivably operate in favor of or against development in this location. Upon maturation of the oöcyst and liberation of the sporozoites through bursting of the former, the parasite now runs the additional hazards of migration through the hemocoele to the salivary glands, and of penetration of the glands. Once the glands are infected with viable sporozoites certain factors, for the most part yet unknown, may operate for or against the preservation of the sporozoite in a viable condition. It will thus be seen that the hazards encountered by the parasite during its lifetime in the mosquito are, in complexity and importance, comparable to if not greater than those encountered in man. While the mosquito may be thought of as a somewhat simpler organism, it must be remembered that such environmental factors as temperature may have greater effect upon the parasite in it than in man. It should be noted that greater changes take place in the structure and biology of the parasite while in the mosquito than while it is in man.

As soon as the mosquito theory of malarial transmission had been established by Manson, Ross and Grassi, there arose the task of determining what mosquitoes are responsible for transmission. As already indicated, many factors play parts in contributing to the success of a given mosquito in transmitting the malarial parasites. Let us assume for the moment that all other factors are conducive to transmission pro- viding the mosquito is susceptible to infection. Susceptibility-or the lack of itmight then play the all-important part in determining whether a given mosquito may act as a vector. A great deal of effort has been spent in testing various species and varieties of Anopheles to discover whether they are susceptible to one or another of the malarial parasites, and it is clear that a great deal still needs to be done in this direction.' Species sanitation as a technique for control has arisen from the recognition that species differ in their abilities to transmit malaria, and at least some of these differences are known to be attributable to differences in the susceptibility of the mosquitoes to the parasites. Very little attention has been directed to the underlying causes of susceptibility or non-susceptibility, especially in Anopheles.

While it was early known that species differed greatly in their susceptibilities, Darling. (1910) clearly showed that individuals within a susceptible species vary greatly in their capacities for becoming infected. When uninfected individuals had previously been discovered within lots of mosquitoes fed on malarial patients, it was often assumed that the failure to become infected is to be explained on the basis of an insufficiency of parasites in the infecting meal. When the heavy infections obtainable in avian malaria were used (Huff 1927), however, the fact was inescapable that some individuals are refractory to infection. Double feedings of the same mosquitoes on the same strain of malaria resulted, with few exceptions, either in failure to become infected at all or in infection with two broods of parasites (Huff 1930). Many of the malariologists who have tested species of Anopheles for susceptibility to human malaria have failed to indicate the degree of infection in the patient. Therefore, one cannot be sure that their lack of infections in mosquitoes was not the result of an insufficient dosage of gametocytes in the blood meals. However, King's (1929) results and a large mass of data published by M. F. Boyd and his coworkers from the Tallahassee station 
have included the numbers of gametocytes in the patient's blood at the time of its ingestion by the mosquito. There are evident examples in these data, as well as in the work of malariologists in other parts of the world, of individual mosquitoes which remained refractory to huge doses of infectious gametocytes.

The fact that most species of Anopheles cannot be bred in small space has prevented their use in breeding experiments. The culicine mosquitoes have been more suitable for this kind of study. It has been possible in mass selection experiments with Culex pipiens and Plasmodium cathemerium to lower or raise the percentage of susceptible mosquitoes in a stock by selecting from uninfected or infected individuals respectively (Huff 1929). The characters responsible for susceptibility have been shown to be recessive and to be inherited in the 1:3 ratio (Huff 1931).

Fertilization of the macrogamete and the formation of oökinetes apparently take place in a species of mosquito regardless of whether the latter is susceptible to infection by the parasite. This has been shown in my experiments with avian malaria (1927) and by Nicolaew and Yakowlewa (1929) for $P$. vivax and three species of culicine mosquitoes. Oökinete formation may even occur in vitro. The success or failure on the part of the oökinete in penetrating the stomach wall is to a greater extent than any other incident responsible for infection or escape from infection by the mosquito. We know (in Culex pipiens) that by no means all of the oökinetes succeed in getting through the stomach wall of a susceptible mosquito (Huff 1934). That some mechanism is responsible for permitting only a certain number to get through is shown by the high correlation between the numbers of parasites from two infectious feedings which, in the first brood, get through the wall and which, in the second brood, appear to be normal and capable of continuing their migration through the wall. However, histological studies of the gut wall and of the cellular elements in the blood meals of susceptible and insusceptible individuals have failed to show any difference in appearance between the two individuals (Huff 1934).

Once the oökinete has penetrated the gut wall and become an oöcyst it apparently runs relatively little chance of failure to develop if environmental conditions are favorable. A good many malariologists feel, however, that stomach infections do not constitute as good an index of infectiousness as salivary gland infections. We have, of course, evidence in favor of this belief. Chitinization of the oöcysts may result in black spores and such oöcysts do not usually produce normal sporozoites. Barber (1936b) studied the question of degeneration of sporozoites and was inclined to the belief that some of the degeneration within the glands began so early that it must have been initiated in the oöcyst. If an inimical, humoral principle exists in the blood of the mosquito the sporozoites would be exposed directly to it during their passage from oöcyst to salivary gland. Barber found degeneration to be more frequent in some than in other species of Anopheles, which might possibly be interpreted as evidence of some inimical principle in certain mosquitoes. Boyd (1940c) found very good agreement between the incidence of stomach and gland infection in A. quadrimaculatus infected with $P$. vivax.

Recent quantitative studies on the oöcysts of avain malaria in Culex pipiens show differences in average size in different individuals (Huff 1940). Since these differences exist between individuals having exactly the same degree of infection and since the same mean oöcyst size may be found in individuals with widely different degrees of infection, it would seem to follow that there must be at least two factors present in the mosquito affecting the growth of the oöcyst. One of these regulates the degree of infection and the other influences the rate or extent of growth of the oöcysts.

Of the environmental factors influencing infection in Anopheles, temperature has been given the closest study. Grassi 
(1901) demonstrated that the early development of $P$. vivax and $P$. falciparum could not take place in mosquitoes at temperatures between $15.5^{\circ}$ to $17.5^{\circ} \mathrm{C}$, but that development could proceed at lower temperatures $\left(9^{\circ} \mathrm{C}\right)$ after the midgut infection had been established. Janscó (1904) found that the anophelines become infected with these same parasites when kept for as much as 24 hours at temperatures between $11^{\circ}$ and $13^{\circ} \mathrm{C}$ and then brought back into temperatures of $20^{\circ}$ to $30^{\circ}$ C. Stratman-Thomas's recent (1940) excellent studies, which have been done over the whole temperature range and at all stages in the extrinsic development of the parasite, show that two and one-half days are necessary to prevent development at $1^{\circ}$ to $10^{\circ} \mathrm{C}$. Grassi and Janseó did not agree in the interpretations of their results, the former believing that low temperatures inhibited exflagellation and fertilization while the latter believed that they inhibited the development of oökintes. Indeed, it is not clear yet at what point in the early development of the parasite low temperatures have their effective inhibiting influence.

Oöcysts have been shown to develop at $30^{\circ} \mathrm{C}$ by Janseó and at $32^{\circ} \mathrm{C}$ by Stratman-Thomas, but the latter found that temperatures of $37.5^{\circ} \mathrm{C}$ for 2 to 3 hours were sufficient to prevent oöcyst formation. In this case also it is not known whether high temperatures inhibit exflagellation or fertilization, although Stratman-Thomas's results would indicate that the effect is produced at one of those points rather than against penetration of the gut wall by the oökinete. It is clearly indicated by the observations of Mitzmain (1917b), King (1917), Wenyon (1921) and StratmanThomas (1940) that low temperatures are not as inimical to the oöcysts as to the earlier stages. King found that $P$. vivax could survive exposure of the mosquito to $-1.2^{\circ} \mathrm{C}$ for 2 days and to $-0.6^{\circ} \mathrm{C}$ for 4 days and that $P$. falciparum could survive at $1.7^{\circ} \mathrm{C}$ for 24 hours. Stratman-Thomas (1940) showed that high temperatures of $37.5^{\circ} \mathrm{C}$ for 18-24 hours aborted oöcyst development and decreased the number of oöcysts showing sporozoite formation. $\mathrm{He}$ also showed that intermittent exposures of the mosquitoes to high temperatures prolonged the development of oöcysts and decreased the number of infected salivary glands. The earlier malariologists were agreed upon the deleterious effects of winter temperatures upon the mature sporozoites. (See Martirano 1902; Sehoo 1903; Cardamatis 1919; and Grassi and Sella 1920). This is confirmed by the experiments of Boyd and Stratman-Thomas (1934a) and of Stratman-Thomas (1940), who were unable to obtain infections from anophelines infected with $P$. vivax after 50 days' exposure to temperatures of $1-7^{\circ} \mathrm{C}$. The latter has shown also that exposure to $37.5^{\circ} \mathrm{C}$ for 24 hours has a marked inhibitory effect on the infectivity of sporozoites for man.

After the salivary glands have been infected with sporozoites the length of life of the mosquito and the length of time the sporozoites will remain infective are important factors in limiting the transmission of malaria. While the longevity of the anopheline imago varies greatly with the environmental factors, with its food, and probably with a number of physiological factors, it may be as long as 231 days (Mayne 1922) when these factors are favorable. It is, therefore, fairly safe to say that the inherent capacities for long life are probably sufficient to keep most mosquitoes alive a long time, providing all other conditions are favorable.

It is generally agreed that sporozoites tend to lose their infectiousness with age (Mayne 1922; James and Shute 1926; Boyd and Stratman-Thomas 1934a; and Boyd, Stratman-Thomas and Kitchen 1936b. Since the anophelines are usually kept at low temperatures after infections are established, in them in order to prolong their lives, it is not possible to determine from available reports whether loss of viability by the sporozoites is due to the effect of the low temperature or whether other factors take part in the process. In Boyd's laboratory the Anopheles are rou- 
tinely kept at $4^{\circ} \mathrm{C}$, at which temperature vivax sporozoites remain viable only 40 to 50 days while falciparum sporozoites do not live longer than 30 days. James and Shute (1926) record one example of A. maculipennis which retained viable sporozoites for 92 days. Barber (1936b) could find no single factor which seemed to be responsible for sporozoite degeneration. He saw none in West Africa (1200 specimens) but found it very commonly in Greek Macedonia. It was not associated with any degree of humidity nor with the food of the mosquitoes. He did not believe that age or low temperatures were necessary factors in the cause of degeneration of sporozoites in any of the species he studied. It is obvious that the problem of analyzing the causes of loss of viability of sporozoites is extremely complex.

It has been acknowledged for a long time that the relative humidity of the air surrounding the mosquito has little or no direct effect on the development of the parasites. Mayne (1930a) found no direct effect of humidity upon the numbers of mosquitoes of the species Culex fatigans and Anopheles stephensi infected with bird malaria and $P$. vivax respectively. In my studies with Culex pipiens and avian malaria (1941) there was also no measurable effect of different degrees of humidity upon the numbers or size of oöcysts which grew in susceptible individuals.

No extensive investigations have been made of the effect of various food substances upon infection in the mosquito. It has been conjectured that alfalfa had an antagonistic effect, but Stratman-Thomas (1931) has brought epidemiological evidence against this hypothesis, and Mayne (1930a) has shown that coumarin from alfalfa in a wide range of dilutions did not prevent infection in C. fatigans. Russell and Mohan (1939a, 1939b) have shown that larvae of A. stephensi grown in contrasting environments grew into adults which showed little or no difference in susceptibility to $P$. falciparum. Some of the larvae were grown in tap water and others in water to which cow dung had been added.

Since temperature has such a marked influence on the rate of growth of malarial oöcysts, the question arises as to whether the mean temperatures of different individual mosquitoes may differ enough to account for the large differences in mean size of the oöcysts in different mosquitoes. Although these temperatures have not been measured directly, it has been shown (Huff 1941) that the rates of growth are not affected by activity of the infected mosquitoes. Dealated mosquitoes and mosquitoes kept in light were infected and compared with their respective controls, winged mosquitoes and those kept in the dark. In neither case were there significant differences in mean sizes of oöcysts in the different lots of mosquitoes.

By way of summary, it can probably be safely conjectured that of the factors influencing infection of Anopheles with malarial parasites none seems to be more important than those present in the inherent make-up of the individual mosquito, and it can be stated that, of the environmental factors so far studied, none seems to play any appreciable role except temperature which, indeed, plays a very important part in determining whether or not a mosquito may be a good vector of malaria. Hardly any other phase of malariology has been so badly neglected as the study of all factors influencing infection in the mosquito. 


\title{
THE TRANSMISSION OF MALARIA BY THE ANOPHELES MOSQUITOES OF NORTH AMERICA
}

\author{
By JAMES STEVENS SIMMONS \\ CHIEF, DIVISION OF PREVENTIVE MEDICINE, OFFICE OF THE SURGEON GENERAL, \\ U. S. ARMY, WASHINGTON, D. C.
}

IN this chapter it is proposed to summarize briefly some of the available published information concerning the spread of human malaria by the various anopheline mosquitoes indigenous to the northern half of the western hemisphere. At intervals during the past, similar reviews have been prepared by various individuals and the present summary represents an attempt to bring up to date information obtained from such sources. The anophelines are arranged in two main groups, those of the Nearctic Region, and those of the Neotropical Region. Each species will be considered briefly from the viewpoint of its relation to malaria. The geographical distribution, breeding and habits of each species are given in the references cited.

\section{ANOPHELINE Mosquitoes OF THE NeARCtic REgion}

The species of genus anopheles considered in this section are listed in Table I.

TABLE I

\begin{tabular}{c|l}
\hline \multicolumn{1}{c|}{ Subgenus } & \multicolumn{1}{c}{ Species } \\
\hline Anopheles & 1. A. atropos Dyar \& Knab 1906* \\
" & 2. A. crucians Wiedemann 1828* \\
" & 3. A. maculipennis Meigen 1818 \\
" & 4. A. punctipennis Say 1823 \\
" & 5. A. quadrimaculatus Say 1824 \\
Coelodiazesis & 6. A. A. Aalkeri Theobald 1901 \\
\hline
\end{tabular}

* Also found in neotropical regions.

\section{Anopheles (Anopheles) atropos Dyar and Knab 1906}

a. Experimental infection. $-P$. vivax. Mayne and Griffitts (1931) reported com- parative experiments in which 85.7 per cent of the specimens of $A$. atropos fed on suitable carriers of $P$. vivax became infective. There are no data for $P$. falciparum or $P$. malariae.

b. Infection in nature. No data.

c. Epidemiological. Beyer (1923) stated that $A$. atropos was suspected as a vector of malaria, but according to Covell (1927) this suspicion was not supported by direct evidence. Hanson, Boyd and Griffitts (1935) remarked that, while $A$. atropos is susceptible, they regarded it as epidemiologically unimportant. Williams (1937a) stated that $A$. atropos is of no importance because it does not breed near man, being confined wholly to salt marsh areas of the Gulf and South Atlantic states.

It has been shown that atropos engages in flights, enters dwellings at night and feeds on man; also that it is susceptible to infection with $P$. vivax. Its distribution is restricted by its breeding habits, thus rendering it relatively unimportant as a national problem. However, this species must be considered of potential importance in situations where human dwellings are located within its flight range and. it deserves further study.

\section{Anopheles (Anopheles) crucians Wiedemann 1828}

a. Experimental infection. $-P$. vivax. Mayne $^{1}$ (1916a) first reported the experimental infection of $A$. crucians with $P$. vivax. Other reports of tests with this species have been made by Root (1924b), King (1921) and Barber, Komp and Hayne (1927).

P. falciparum. Mayne (1916a) also was 1 Earlier written "Mitzmain." 
the first to report the infection of $A$. crucians with $P$. falciparum, a fact which was confirmed by King (1916b) and Root (1924b).

P. malariae. Several investigators (Beyer, Pothier, Couret and Lemann 1902b ; Root 1924b; Mayne 1932; Boyd and Stratman-Thomas 1933a) have attempted, with negative results, to infect $A$. crucians with $P$. malariae.

In 1927 Barber, Komp and Hayne reviewed the data available on infectivity experiments with $A$. crucians, in which $A$. quadrimaculatus and $A$. punctipennis were used as controls, and concluded that the evidence did not indicate that "a given anopheles is more susceptible to one type of malaria parasite than another." In 1934b, Boyd and Stratman-Thomas reported experiments in which susceptibility of $A$. crucians (inland variety) to $P$. vivax, $P$. falciparum and $P$. malariae was compared with that of $A$. quadrimaculatus. They concluded that there was a difference in the susceptibility of the mosquitoes to all three species of parasites, that of $A$. quadrimaculatus being high even when the gametocyte density is low. A. crucians, on the other hand, was not infected when the gametocyte density was low; but, after the ingestion of larger numbers of gametocytes, it was infected with $P$. vivax and $P$. falciparum. None of the $A$. crucians was infected with $P$. malariae. Later Boyd, Kitchen and Mulrennan (1936) compared the susceptibility to $P$. falciparum of $A$. crucians, of the inland and coastal types, using A. quadrimaculatus as a control. No significant difference was noted in the susceptibility of the two $A$. crucians varieties and both were relatively poorer hosts than A. quadriniaculatus.

b. Infection in nature. In 1919 Mayne, in Louisiana, demonstrated infection in a wild specimen of $A$. crucians, and since that time various observers have reported other natural infections. In 1927 Barber, Komp and Hayne summarized as follows the results of the reported dissections in which the species of Anopheles were distinguished and the stomach infections were recorded: A. crucians. 1446 dissected, 0.02 per cent infected; A. punctipennis. 130 dissected, none infected; $A$. quadrimaculatus. 10,641 dissected, 1.1 per cent infected.

Sporozoites have been found in the salivary glands of naturally infected $A$. crucians.

c. Epidemiological. The epidemiological reports also are contradictory. According to Covell (1927) A. crucians "is generally considered not to play an important part in the transmission of malaria, although Carter (1924) stated that it conveyed the disease effectually in the tidewater section of Virginia where malaria is bad, and this species is practically the only anopheline present.' Barber, Komp and Hayne (1927) also referred to similar observations. Beyer and others (1902b) suspected A. crucians as being a vector of estivo-autumnal malaria, on epidemiological grounds, and Dyar (1922) stated that it is a dangerous carrier of malaria.

Root (1924b) suggested that the brackish water strain may be an efficient carrier. Boyd and Stratman-Thomas in their report on the comparative susceptibility of the inland variety of $A$. crucians to infection stated, "A. quadrimaculatus is more susceptible than $A$. crucians to the parasites of all three species of human malaria. They remarked that the greater epidemiological importance of $A$. quadrimaculatus in the propagation of malaria in the southeastern United States can be attributed to: (1) appreciable preference for human blood, (2) house frequency habits, and (3) high susceptibility to the malaria parasites. Contrasting differences on the part of $A$. crucians explain its relative unimportance." However, it is believed that the final decision as to its relative importance in all localities must be delayed until more exact information has been obtained concerning the characteristics of the different subspecies or varieties.

\section{Anopheles (Anopheles) Maculipennis Meigen 1818}

Dyar (1922) stated that A. maculipennis 
is evidently a good malarial carrier, though there is no record of experiments conducted with this form under the American name of "occidentalis."

a. Experimental infection. $-P$. vivax. Barber, Komp and King (1929) infected specimens of $A$. maculipennis collected in Dona Anna Co., New Mexico, with $P$. vivax. There are no adequate data for $P$. falciparum and $P$. malariae.

$b$. Infection in nature. Barber, Komp and King (1929), in northern New Mexico, dissected 669 A. maculipennis and found 2 specimens, or 0.3 per cent, with oocysts in the mid gut. Barber and Forbrick (1933), in New Mexico, dissected 868 maculipennis and found 1.4 per cent infected.

c. Epidemiological. Barber, Komp and King (1929) observed that in New Mexico A. maculipennis is undoubtedly an important vector of malaria. "It is one of the most common carriers of Europe and is considered an important vector in California." Twinn (1931) listed A. maculipennis $\mathrm{Mg}$. among the principal troublesome mosquitoes of eastern Canada and stated that it is a dangerous vector in many countries, but there is little evidence of the occurrence of this disease at present in eastern Canada. Williams (1937a) stated that "maculipennis is a good vector of malaria but occurs in sufficient quantities only in New Mexico, California and Oregon where the malarious areas are comparatively small."

In view of the confusion as to the varieties which are included under the name A. maculipennis in North America it appears that the mosquitoes of this group will require considerable entomological study before their relation to malaria can be determined.

\section{Anopheles (Anopheles) Punctipennis Say 1823}

a. Experimental infections. $-P$. vivax. In 1915 King (1916a) infected A. punctipennis with $P$. vivax. This work was confirmed by Mayne (Mitzmain) (1916b) who used this mosquito for the experimental infection of human volunteers.
Similar results were obtained by King (1916a), Root (1924b), Barber, Komp and Hayne (1927) and Boyd and Kitchen (1936a). In the past decade this species has been used extensively for the transmission of therapeutic tertian malaria to patients with paresis (St. John 1928).

P. falciparum. King (1916b) infected A. punctipennis with $P$. falciparum and Mitzmain (1917b) and others confirmed this observation.

$P$. malariae. Mayne (1932) reported the infection of 6 of 157 A. punctipennis with $P$. malariae.

Studies have been made to determine the relative susceptibility of $A$. punctipennis to infection with malarial parasites. Boyd and Kitchen (1936a), who compared it with A. quadrimaculatus, concluded that both mosquitoes were about equally susceptible to the strains of $P$. vivax used; and that A. punctipennis varied from a high susceptibility to a probable refractoriness to different strains of $P$. falciparum. Later Boyd, Carr and Rozeboom (1938) reported other comparative tests in which Florida strains of $A$. punctipennis and A. quadrimaculatus fed on blood of patients infected with nearctic (Florida) and neotropical (Cuba) strains of $P$. vivax and $P$. falciparum. Both of the mosquito species were susceptible to infection with all of the strains of plasmodia used, but $A$. quadrimaculatus was more consistently susceptible to the Cuban parasites than was $A$. punctipennis.

b. Infection in nature. In view of the demonstrated susceptibility of $A$. punctipennis to experimental infection with $P$. vivax, $P$. falciparum and $P$. malariae, it seems remarkable that so little information is available about its infection in nature. Boyd and Kitchen (1936a) stated that "no one has ever reported the capture of a wild punctipennis with gland infection, and only a single instance of a stomach infection has come to notice." The latter was reported by Mayne (1917b), but according to Williams (1940) the author has since expressed some doubt concerning the accuracy of the observation. 
c. Epidemiological. Williams (1937a) stated, "Punctipennis, although capable of transmitting the disease and occurring in considerable quantities in all parts of the United States is wholly an outdoor biter and enters houses only in the late fall and then apparently for the purpose of hibernation.",

The available data indicate that $A$. punctipennis is susceptible to infection with the three main species of plasmodia, but that it is a less effective vector than $A$. quadrimaculatus. Further studies will be required to determine more exactly its relative importance in the different regions where it exists.

\section{Anopheles (Anopheles) Quadri- maculatus Say 1824}

a. Experimental infection. The susceptibility of $A$. quadrimaculatus to experimental infection with the three main species of malaria plasmodia has been well established and because of the ease with which this mosquito can be reared in captivity it has been used extensively for the routine transmission of malaria in the treatment of paresis. This use has afforded an unusual opportunity to study its characteristics as a vector in great detail and thanks to the extensive experiments of Boyd and his associates and of others, a fund of valuable fundamental information is now available concerning the development of the different plasmodia in A. quadrimaculatus under various conditions. Since the literature on this subject is too extensive to be reviewed here, the reader is referred to the original publications for complete details. The data given below are limited to information summarized by Covell $(1927 ; 1931 b)$ and other earlier investigators and to a few of the more recent observations made by Boyd and his co-workers.

$P$. vivax. In 1900 Thayer reported the experimental infection of $A$. quadrimaculatus with $P$. vivax and this observation was soon confirmed by Berkeley (1901), King (1916a, b) Mitzmain (1916b) and others. More recently Boyd and his associates have shown that $A$. quadrimaculatus is an effective experimental vector of $\boldsymbol{P}$. vivax. The minimal parasite density required to infect $A$. quadrimaculatus is about one male and one female gametocyte per 100 leukocytes (Boyd, Stratman-Thomas and Kitchen 1935), and the intrinsic incubation period at $20^{\circ} \mathrm{C}$ is about 17 days (Boyd 1934). In a study of the influence of temperature on the sporogenous cycle of $P$. vivax, StratmanThomas (1940) found that the cycle was completed within the temperature range of $15-17^{\circ}$ to $30^{\circ} \mathrm{C}$; the shortest time after the infective feeding was 8 days and the longest 38 days. The optimum temperature was $28^{\circ}$ C. Shortly after feeding on a gametocyte carrier, A. quadrimaculatus was rendered non-infective by 2 to 3 hours' exposure to $37.5^{\circ} \mathrm{C}, 2 \frac{1}{2}$ days' exposure to $1^{\circ}-10^{\circ} \mathrm{C}$. Seven to 13 days after feeding the development of oocysts was aborted by exposure to $37.5^{\circ} \mathrm{C}$ for 18 to 24 hours, or to $1^{\circ}-10^{\circ} \mathrm{C}$ for 24 days. After completion of the sporogenous cycle the infectivity of the sporozoites in the salivary glands was markedly inhibited by exposure to $37.5^{\circ} \mathrm{C}$ for 24 hours or to $1^{\circ}-7^{\circ} \mathrm{C}$ for 50 days. The author remarked that "The fact that $P$. vivax will not develop at a constant temperature above $30^{\circ} \mathrm{C}$, and the fact that a period of 24 hours at $37.5^{\circ} \mathrm{C}$ will sterilize all but a very small per cent of $A$. quadrimaculatus of their $P$. vivax infection, have a bearing on the transmission of $P$. vivax infection during the summer months. The data presented show that in certain localities the oocyst may survive the winter and complete its development the following spring." A study of the per cent of $A$. quadrimaculatus infected experimentally with $P$. vivax in 230 batches (Boyd and Kitchen 1938b) showed that a higher proportion of mosquitoes were infected in the batches applied during the spring and autumn.

Boyd, Kitchen and Kupper (1937) reported that of $3,574 A$. quadrimaculatus fed on $P$. vivax gametocytes, 2,288, or 64.2 per cent, were infected. Boyd (1937) compared the susceptibility to $P$. vivax of a certain strain of $A$. quadrimaculatus 
maintained for 7 years in an insectary and another strain collected out of doors. Infection occurred in 63 per cent of the insectary-bred mosquitoes and in 73.4 per cent of the wild ones. Boyd (1940c) observed that with large numbers of mosquitoes the incidence of $\boldsymbol{P}$. vivax infections revealed by stomach or gland dissections will be in substantial agreement.

Boyd and Kitchen observed that in $\mathbf{1 8 0}$ successful inoculations of $P$. vivax malaria out of 182 from 8 to 23 days elapsed before the first microscopic detection of parasites. Apparently the incubation period was not influenced by the period of intrinsic incubation in the source of infection, or the period of extrinsic incubation in the mosquitoes. It was influenced inversely by the number of mosquitoes used. They concluded that the varying susceptibility of human hosts was more important in determining the length of the incubation period than any discernible factors modifying the sporozoites.

$P$. falciparum. Thayer (1900) was also the first to report infection of $A$. quadrimaculatus with $P$. falciparum. Boyd (1934) observed that the incubation period in A. quadrimaculatus, at $20^{\circ} \mathrm{C}$, is 23 days. The minimal parasite density required to infect this mosquito is about 11 males and 11 females per 100 leucocytes (Boyd, Stratman-Thomas and Kitchen 1935). Boyd, Kitchen and Kupper (1937) reported the infection of 923 , or 46.4 per cent, of 1,992 insectary-bred $A$. quadrimaculatus. They concluded that "While the results obtained appeared to indicate that $A$. quadrimaculatus is a less efficient host to $P$. falciparum than to $P$. vivax, and while a definite proportion of a large series of mosquitoes appear to be refractory to each species, yet in view of the fact that given a sufficiently high gametocyte density $A$. quadrimaculatus can be infected in approximately the same degree with $P$. falciparum as with $P$. vivax, we incline to the opinion that the differences noted may more properly be attributed to characteristics of the parasites themselves than to a differential susceptibility on the part of $A$. quadrimaculatus." Boyd and Kitchen (1938b), from a study of 230 lots of $A$. quadrimaculatus infected with $P$. vivax and 166 lots infected with $P$. falciparum, noted that there was a higher percentage of $P$. vivax infections in mosquitoes that fed when exflagellation was demonstrable, but that no such difference occurred with P. falciparum. Boyd and Kitchen (1937b) determined that in the experimental infection of $A$. quadrimaculatus, $P$. vivax gametocytes are about 10 times as effective as are $P$. falciparum gametocytes. After the inoculation of patients with $P$. vivax, fully matured infectious gametocytes are present within 5 days of the appearance of parasites and they are present for some time after the end of the clinical attack. Some of the mosquitoes were infected when the gametocyte density was less than 10 per cmm. $P$. falciparum gametocytes were not found until 10 days after the appearance of parasites and in some instances not before the end of the primary attack. Submicroscopic densities of gametocytes did not cause infection, and densities less than 100 per $\mathrm{cmm}$ generally failed to infect. Boyd, Kitchen and Kupper (1937) have shown experimentally that $A$. quadrimaculatus can be infected simultaneously with two different species of malarial parasites and that within the mosquito neither species appears to injure or inhibit the other. Such mosquitoes can transmit both parasites to man and produce infection. Boyd and Jobbins (1940) compared the susceptibility of $A$. quadrimaculatus from Florida and A. albimanus from Panama to strains of $P$. falciparum from Florida, Mexico and Panama. The infection rates were (a) A. albimanus, to Florida parasites 7.5 per cent, to Mexican parasites 13.5 per cent and to Panaman strain 13.6 per cent; (b) A. quadrimaculatus, to Florida strain 48.9 per cent, to Mexican strain 57.1 per cent, to Panaman strain 17.6 per cent.

P. malariae. Beyer, Pothier, Couret and Lemann (1902b) reported the experimental infection of 2 of 5 A. quadrimaculatus with $P$. malariae. Boyd and Stratman. 
Thomas (1933a) reported the first recorded experimental transmission of $P$. malariae by anopheles mosquitoes. In four lots of $40,63,18$ and 42, A. quadrimaculatus the per cent infected were 45, 32, 63.4 and 9.4 , respectively. The extrinsic incubation period at $20^{\circ} \mathrm{C}$ was 30 to 35 days. In 1936 these authors reported the transmission of quartan malaria to two additional cases.

b. Infection in nature. Hirshberg (1904) reported the finding of "naturally infected" A. quadrimaculatus and since that time Mitzmain (1916e, 1919), Metz (1919c), King (1921), Root (1924b) and Darling (1925) have reported infections in nature ranging from 0.3 to 19.0 per cent.

c. Epidemiological. A. quadrimaculatus is universally regarded as the most important malarial vector in the United States, because of its breeding and feeding habits, its susceptibility to infection and its distribution in malarious regions. According to Williams (1937a), it is the chief vector, a conclusion that may be accepted for the United States. It breeds almost wholly in still water that is relatively clear. It requires some sunshine, never being found in dense shade. However, it requires some darkness, never being found in waters which are wholly unshaded, unless they have a type of flotage which casts narrow strips of shade where the mosquito larvae may lie during a portion of the daylight hours. Although A. quadrimaculatus will feed on animals, as does A. punctipennis, it will also feed voraciously on human beings and frequents human habitations.

\section{Anopheles (Anopheles) walkeri Theobald 1901}

a. Experimental infection.-P. vivax. In August 1932 Matheson, Boyd and Stratman-Thomas (1933) reported experiments performed in Florida with 8 imagines of A. walkeri from larvae collected in New York. Six of them fed on a tertian patient whose blood had a gametocyte density of 16 males and 3 females per 100 leukocytes and exflagellation of the males. The next day one other mosquito fed. The mosqui- toes were kept at a temperature of $19^{\circ}-$ $22.8^{\circ} \mathrm{C}$. Six specimens were examined and 4 were infected, the extrinsic incubation period being estimated at about 17 days. One specimen transmitted the infection to a human patient.

$P$. falciparum. During 1926 Komp in Louisiana attempted to infect $A$. walkeri with $P$. falciparum with negative results. In October 1935 Kitchen and Bradley (1936a) applied groups of $A$. walkeri, collected in Florida, and control groups of A. quadrimaculatus to a patient with $P$. falciparum malaria. On the days of these feedings the gametocyte densities were: male, 540 to 580 ; female, 250 to 340 per $\mathrm{cmm}$. All dissections were made 12 days later with the following results : $A$. quadrimaculatus, total fed 26 , positive 20 , or 77 per cent; $\boldsymbol{A}$. walkeri total fed 9, positive 1 (1 cyst), or 11.1 per cent. It was concluded that the southern form of $A$. walkeri is not highly susceptible to infection by $P$. falciparum. There are no data for $P$. malariae.

b. Infection in nature. From July 11 to 29, 1939, Bang et al. (1940), using a light trap, collected mosquitoes from the environs of Bondurant, Kentucky. The 231st specimen of $A$. walkeri dissected had oocysts on the stomach and a heavy sporozoite infection of the salivary glands. The authors remarked that the human origin of the parasites was probably indicated by the fact that $A$. walkeri prefers to feed on mammals, including man, rather than birds.

c. Epidemiological. Johnson (1936) remarked that "Possibly due to its willingness to bite humans, its unusual daytime resting habits, and its presumably unrecognized prevalence, $A$. walkeri may be shown to be of importance from the malaria standpoint. This of course is dependent on its being an efficient harborer of the malaria organism." According to Williams (1937a), "The place of $A$. walkeri as a malarial vector is in some doubt. Until two years ago it was considered a very rare mosquito. It was found breeding in only a few places in the grassy edges of swamps. So few specimens were encountered that it was 
assumed to be too rare a mosquito to play any part in disease transmission. With latter-day use of the light trap with its suction apparatus, $A$. walkeri has been found in some sections to be present in considerable numbers. Observations in the year past have shown that it enters houses freely at night, feeds on human beings, and then disappears to unknown daytime roosting places. No outbreaks of malaria have yet been found ascribed to this species of Anopheles."

From the foregoing evidence it appears that $A$. walkeri is a possible vector, and that additional investigations will be required to determine its relative importance in the transmission of malaria.

\section{Anopheles (Coelodiazesis) barberi Coquillet 1903}

a. Experimental infection. $-P$. vivax. Stratman-Thomas and Baker (1936) reported the infection of a specimen of $A$. barberi by feeding on a tertian patient who had 3 micro and 11 macrogametocytes per 100 leukocytes, with demonstrable exflagellation of the former. This infected mosquito transmitted malaria to another patient. There are no data for $P$. falciparum and $P$. malariae.

b. Infection in nature. No data.

c. Epidemiological. Data are inadequate, but the mosquito is relatively rare and has not been suspected as an important vector on epidemiological grounds.

The evidence available indicates that $A$. barberi is relatively unimportant as a vector of malaria (Williams 1937a; Herms 1939), but the species should be investigated further.

\section{ANopHeline Mosquitoes of THE Neotropical Region}

The anophelines to be considered in this section are indicated in Table II, which is based on information furnished by Komp (1940b).

\section{Chagasia bathanus Dyar 1928}

a. Experimental infection. According to Komp (1940b), Barber and Komp in 1927
TABLE II

ANopheline Mosquitoes of the Neotropical Region Exclusive of South AMerica. (According to Komp 1940)

\begin{tabular}{|c|c|}
\hline Subgenus & Species \\
\hline & 1. C. bathanus Dyar 19281 \\
\hline Stethomyia & 2. A. kompi Edwards $1930^{2}$ \\
\hline Anopheles & 3. A.eiseni Coquillet 1908 \\
\hline "16 & 4. A. hectoris Mira 1932 \\
\hline “ & $\begin{array}{l}\text { 5. A. parapunctipennis Martini } \\
1932\end{array}$ \\
\hline "6 & $\begin{array}{l}\text { 6. * } A \text {. pseudopunctipennis Theo- } \\
\text { bald } 1901\end{array}$ \\
\hline 6 & 7. A.xelajuensis De Leon \\
\hline Arribalzagia & $\begin{array}{l}\text { 8. A. apicimacula Dyar and } \\
\text { Knab } 1906\end{array}$ \\
\hline “ & $\begin{array}{l}\text { 9. A. neamaculipalpus Curry } \\
1931\end{array}$ \\
\hline "6 & $\begin{array}{l}\text { 10. A. punctimacula Dyar and } \\
\text { Knab } 1906\end{array}$ \\
\hline Cycloleppteron & 11. A. grabhami Theobald 1901 \\
\hline 6 & $\begin{array}{l}\text { 12. A. vestitipennis Dyar and } \\
\text { Knab } 1906\end{array}$ \\
\hline Nyssorhynchus & $\begin{array}{l}\text { 13. *A. albimanus Wiedemann } \\
1821\end{array}$ \\
\hline "6 & $\begin{array}{l}\text { 14. A. albitarsis Lynch Arribal- } \\
\text { zaga } 1878\end{array}$ \\
\hline “ & $\begin{array}{l}\text { 15. A. anomalophyllus Komp } \\
1936\end{array}$ \\
\hline 66 & $\begin{array}{l}\text { 16. A. argyritarsis Robineau-Des- } \\
\text { voidy } 1827\end{array}$ \\
\hline “ & 17. A. bachmanni Petrocchi 1925 \\
\hline “ & 18. A. darlingi Root 1926 \\
\hline 66 & 19. A. oswaldoi Peryassu 1922 \\
\hline 66 & 20. A. strodei Root 1926 \\
\hline “ & 21. A.tarsimaculatus Goeldi 1906 \\
\hline Kerteszia & $\begin{array}{l}\text { 22. A. bellator Dyar and Knab } \\
1906\end{array}$ \\
\hline 66 & $\begin{array}{l}\text { 23. A. neivai Dyar and Knab } \\
1917\end{array}$ \\
\hline
\end{tabular}

* These species are also found in the nearctic region.

1 This species is genus Chagasia.

2 This and all following species are genus Anopheles.

attempted, with negative results, to infect a few specimens with $P$. falciparum.

b. Infection in nature. No data.

c. Epidemiological. No data.

This mosquito appears to be relatively rare in the localities where it has been identified, and it has not been incriminated as a malarial vector. Additional studies will be required to determine its possible relation to malaria. 


\section{Anopheles (Stethomyia) kompi} Edwards 1930

There are no data of infections of $A$. kompi with malarial plasmodia. The relative infrequency of $A$. kompi in Panama suggests that it is not an important vector there. Additional studies will be required to determine its possible relation to malaria.

\section{Anopheles (Anopheles) eiseni Coquillet 1908}

a. Experimental infection. $-P$. vivax. According to Covell (1927), Davis (1926) failed to demonstrate infection in four mosquitoes which had fed on a $P$. vivax gametocyte carrier. In Panama, Simmons (1936d) found a single oocyst containing sporozoites on the stomach of a specimen of $A$. eiseni which had fed on a carrier of $P$. vivax gametocytes. There are no data relative to its infection with $P$. falciparum and $P$. malariae.

b. Infection in nature. No data.

c. Epidemiological. Data inadequate.

Additional studies will be required to determine the possible susceptibility of $A$. eiseni to malaria and its importance as a vector.

\section{Anopheles (Anopheles) hectoris Giaquinto Mira 1931}

a. Experimental infection. $-P$. vivax. De Leon (1933) reported the infection of 1 to 5 mosquitoes, with the formation of oocysts.

$P$. falciparum. De Leon (1933) also dissected 41 mosquitoes fed on $P$. falciparum and found oocysts in 3. P. malariae. No data.

b. Infection in nature. No data available.

c. Epidemiological. According to Giaquinto Mira (1936), A. hectoris must be dangerous in its restricted area of distribution, for 'it is the only species found in a place where an epidemic of malaria occurred, and it has been infected experimentally.

The evidence indicates that $A$. hectoris is a potential vector of malaria, but additional studies will be required to determine its importance in this respect.

\section{Anopheles (Anopheles) parapunc- tipennis Martini 1932}

This is a rare highland species found in Mexico and Central America. The data of its relation to malaria are inadequate (Komp 1940b).

\section{Anopheles (Anopheles) pseudo- punctipennis Theobald 1901}

a. Experimental infection.-P. vivax. Darling (1910) in Panama failed to find infection in 4 mosquitoes. Barber, Komp and Hayne (1927), in New Mexico, reported the experimental infection of 1 of 12 mosquitoes, and Shannon and Davis (1930), in Argentina, reported negative results with 6 mosquitoes. Simmons (1939b) fed 6 mosquitoes on a tertian patient with an undetermined gametocyte density and found 1 infected.

$P$. falciparum. Darling (1910), in Panama, found 4 of 27 mosquitoes infected; Shannon and Davis (1930), in Argentina, reported the infection of 3 of 8 mosquitoes which had fed on patients treated with quinine. Simmons (1939b), in Panama, during 1935-36 fed 84 A. pseudopunctipennis on carriers and found 7 to be infected. Forty-nine of the latter fed on patients with low gametocyte densities ( 0.4 to 3 per 100 leukocytes), and the others on patients with densities from 2.8 to 12. Earle (1936b), in Granada, reported the infection of 4 of 21 mosquitoes.

Boyd and Earle (1939), comparing the susceptibility of a Mexican strain of $A$. pseudopunctipennis to infection with strains of $\boldsymbol{P}$. falciparum from Mexico and Florida, reported the infection of 7.1 per cent of 28 mosquitoes with the Florida parasite and 4.1 per cent of 24 mosquitoes with the Mexican parasite. In these experiments the susceptibility of insectaryraised imagines of $A$. pseudopunctipennis from the Mexican highlands to Mexican and Floridan strains of $P$. falciparum was not significantly different, and it was distinctly lower than that of $A$. quadrimaculatus controls.

$P$. malariae. A few negative attempts to infect this mosquito with $P$. malariae have 
been reported as follows: Darling (1910), in Panama, 1 mosquito ; Davis and Shannon (1928), in Argentine, 3 mosquitoes.

b. Infection in nature. Covell (1927) listed the reports of natural infections in northwestern Argentina as follows: "Paterson (1911), 16 out of 1549; Mühlens et al. (1925), 2 out of 62; Maza and Gonzales (1926), 2 out of 28; Davis (1927), 12 out of 435." Later Covell (1931b) recorded two additional reports, namely, one by Benarroch (1928), in Venezuela, who dissected 103 mosquitoes with negative results; and another by Davis and Shannon (1928), in Argentina, who dissected 369 specimens and found 8 infected. Kumm and Ruiz (1928), in Costa Rica, dissected 7 specimens with negative results. Wille (1933) found naturally infected mosquitoes in Peru. Vargas (1938) in Mexico reported that none of 801 salivary glands, but 2.28 per cent of 526 stomachs, were infected.

c. Epidemiological. Dyar (1928) concluded that it is probable that " $A$. pseudopunctipennis is the principal vector of malaria in the drier regions." According to Root and Andrews (1938), "it is known to be the main vector of northwestern Argentina, but elsewhere it is believed to be of little or no importance. Careful workers in California, Panama and Venezuela have all concluded that in these regions this species is not a vector of sanitary importance." Herms (1919) stated that in California A. pseudopunctipennis is believed to be negligible as a factor in malaria. Williams (1937a) observed that although found in West Texas and Pecos Valley of New Mexico, it does not transmit malaria in our country. Earle (1936b), largely on epidemiological grounds, did not consider this species an important vector in Granada.

On the other hand Shannon (1933) stated that it appears that $A$. pseudopunctipennis Theobald is the only anopheline in the Runac Valley, Peru, and the only one in any abundance on the entire western slope of the Peruvian Andes. It is often numerous in houses and is considered to be a vector. Hoffmann (1932) concluded that A. pseudopunctipennis is responsible for endemic malaria over an enormous part of the center of Mexico, which is characterized by a more or less dry climate and includes high valleys 6000-7000 feet above sea level. He believed it to be the principal cause of winter malaria, particularly in the southern and western parts of the country. Hoffmann and Samano (1938) concluded that in all dry regions of Mexico A. pseudopunctipennis is not only the most important but almost always the sole vector of malaria. Giaquinto Mira (1936), in Guatemala, also believed that this species is the chief vector at high altitudes and that it seems to be able to transmit $P$. vivax better than $P$. falciparum.

Because of the contradictory nature of the reports on the habits and the susceptibility to infection of $A$. pseudopunctipennis in various regions, perhaps attributable to varietal differences, it appears obvious that this mosquito will require additional investigation to determine its exact status as a malarial vector throughout the wide range of its geographical distribution.

\section{Anopheles (Anopheles) xelajuensis De Leon}

There are no data available for this species, which is distributed in the highlands of Guatemala, but according to Komp (1940b) it is so rare that it probably is of no importance as a vector of malaria.

\section{Anopheles (Arribalzagia) apicimacula Dyar and Knab 1906}

a. Experimental infection. Benarroch (1928), in Venezuela, reported the feeding of 22 A. apicimacula on a patient with malaria of unkown type. The results were negative.

$P$. falciparum. In 1936 a few A. apicimacula reared from larvae collected in Panama were examined after feeding on human carriers of $P$. falciparum gametocytes. One mosquito which took blood containing 2.8 gametocytes per 100 leukocytes was infected and had sporozoites in the salivary glands (Simmons 1937). There are no data for $P$. vivax and $P$. malariae. 
b. Infection in nature. Benarroch (1928), in Venezuela, dissected 168 specimens and failed to find infection.

c. Epidemiological. Data inadequate.

Additional studies will be required to determine the relative susceptibility of $A$. apicimacula to infection and its importance as a malarial vector.

\section{Anopheles (Arribalzagia) neomaculi- palpus Curry 1931}

a. Experimental infection.-P. vivax. During November and December, 1935, Simmons (1936d), in Panama, used 31 adult $A$. neomaculipalpus, reared from larvae collected in the Canal Zone, in feeding experiments on tertian malarial patients. Seven, or 22 per cent, of these mosquitoes were infected, compared with 3 , or 14 per cent, infected in $21 \mathrm{~A}$. albimanus controls. In the first group, which included $15 \mathrm{~A}$. neomaculipalpus and 7 A. albimanus, fed on blood containing an unknown number of gametocytes, two of the former and none of the latter were infected. In group two, which included 8 A. neomaculipalpus and $11 \mathrm{~A}$. albimanus that fed on blood containing 4 to 7.2 gametocytes per 100 leukocytes, one mosquito of each species was infected. In the third group, which took blood with gametocyte densities as high as 13.4, four of $8 \mathrm{~A}$. neomaculipalpus and 2 of $3 \mathrm{~A}$. albimanus were infected. P. falciparum and $P$. malariae, no data.

b. Infection in nature. No data.

c. Epidemiological. Data inadequate.

It has been shown that $A$. neomaculipalpus is susceptible to infection with $P$. vivax, but additional studies will be required to determine its relative susceptibility to this and other species of plasmodia, and its importance as a malarial vector.

\section{Anopheles (Arribalzagia) puncti-} macula Dyar and Knab 1906

a. Experimental infection. Darling (1910), in Panama, reported negative experiments with 17 mosquitoes from which he concluded that $A$. malefactor ( $A$. punctimacula), in spite of its name, does not transmit malarial fevers. Bennaroch (1928) failed to find infection in one specimen of A. punctimacula which had fed on a patient with malaria of unknown type. In 1936 it was shown experimentally by Simmons (1936b) that A. punctimacula is susceptible to infections with both $P$. vivax and $P$. falciparum.

$P$. vivax. Simmons (1936b), in Panama, dissected 44 mosquitoes fed on carriers of tertian parasites and found 17 , or 39 per cent, to be infected. Among 18 of these mosquitoes which fed on blood with unknown gametocyte densities, 3, or 17 per cent, were infected. The remaining 26 mosquitoes took blood containing from 6 to 7.4 gametocytes per 100 leukocytes and 14 to 50 per cent of these were found to be infected.

P. falciparum. Darling (1910), in Panama, failed to find parasites in 16 mosquitoes which fed on infected persons, 12 on a patient with gametocyte count of 6 to 10 per 100 leukocytes, and 3 on a patient with a gametocyte density of 16 . During 1935 and 1936 Simmons (1939b), in Panama, conducted a series of experiments during which a total of $545 \mathrm{~A}$. punctimacula were dissected after feeding on different carriers of $P$. falciparum malaria. The results which were summarized as follows indicate that A. punctimacula was highly susceptible. The development of oocysts and the infection of salivary glands with sporozoites occurred in A. punctimacula as in A. albimanus used as controls.

\begin{tabular}{c|c|c|c}
\hline $\begin{array}{c}\text { Gametocytes } \\
\text { per } 100 \\
\text { leukoeytes }\end{array}$ & $\begin{array}{c}\text { Mosquitoes } \\
\text { dissected }\end{array}$ & $\begin{array}{c}\text { Mosquitoes } \\
\text { infected }\end{array}$ & Infeeted \\
\cline { 1 - 3 } & & & per cent \\
$0.4-3$ & 6 & 0 & 0 \\
$2.8-5$ & 375 & 41 & 11 \\
$6-12$ & 52 & 13 & 25 \\
13.2 & 90 & 31 & 34 \\
Total & 22 & 19 & 86 \\
& 545 & 104 & 19 \\
\hline
\end{tabular}

P. malariae. Darling (1910), in Panama, reported that he failed to find infection in one mosquito that fed on a carrier of quar$\tan$ malariae with a gametocyte density of 
5 per 100 leukocytes. In view of Darling's other negative results with $A$. punctimacula, this observation is not considered significant.

b. Infection in nature. Bennaroch (1928), in Venezuela, reported the dissection of 58 specimens, with negative results. One of 6 specimens of $A$. punctimacula caught November, 1935, in the sleeping quarters of two soldiers at an outpost of the Fort Sherman reservation in Panama was found to contain oocysts filled with sporozoites (Simmons 1936a). Previously malaria had occurred in former occupants of this house and of a nearby hut. At the time of this observation, $101 \mathrm{~A}$. albimanus caught in dwellings in the same area were examined with negative results. Rozeboom (1938a) during his studies in Panama referred to above, dissected the anophelines caught in houses with the following results : A. albimanus, dissected 472 and 4 , or 1.1 per cent, were infected; $A$. punctimacula, dissected 103 and 1 , or 0.97 per cent were infected; $A$. bachmanni, dissected 3 from houses and 320 from pig stys and none was infected. Kumm and Ruiz (1939a), in Costa Rica, dissected 7 A. punctimacula, with negative results, and 559 A. albimanus, with one specimen infected.

c. Epidemiological. Prior to 1910, when Darling published the results of his negative infection experiments, $A$. malefactor (punctimacula), was suspected as an important vector of malaria in Panama, because of its great prevalence in certain seasons and locations, and because of its habit of invading dwellings and feeding avidly on man. A. punctimacula is still a common mosquito in unsanitated regions in Panama, and its feeding habits still suggest that it may be an important factor in the transmission of malaria to troops and others exposed in such regions. It has been found naturally infected and its susceptibility to experimental infection is high.

The evidence supports the conclusion that A. punctimacula is an efficient vector of malaria at least in unsanitated regions in Panama. Additional studies should be made to determine its relative importance there and in other countries where it is indigenous.

\section{Anopheles (Cycloleppteron) grab- hami Theobald 1901}

a. Experimental infection. In Jamaica, Boyd and Aris (1929) reported negative experimental results with $4 \mathrm{~A}$. grabhami.

P. falciparum. Earle (1936a), in Puerto Rico, using wild mosquitoes, infected 8 , or 20.5 per cent of $39 \mathrm{~A}$. grabhami fed on carriers of $P$. falciparum. There were $76 \mathrm{~A}$. albimanus controls of which 14 , or 18.4 per cent, were infected. For $P$. vivax and $P$. malariae there are no data.

b. Infection in nature. Green (1921, 1922), in Puerto Rico, during 1921 dissected $402 A$. grabhami caught under houses and found 3 infected, one with 4 oocysts and 2 with one oocyst each. In 1922 he dissected 351 A. grabhami and found two positive stomachs, one with 10 oocysts and the number not stated in the other. The rate of infection was about the same in 459 and 790 A. albimanus dissected in the same periods (Earle 1936a). In Jamaica, Carley (1931) examined $125 A$. grabhami caught in nature and found one infected specimen with a mature oocyst but no gland infection. Among $717 \mathrm{~A}$. albimanus dissected at the same time, he found one with a stomach infection and one with infected glands.

c. Epidemiological. According to Earle (1936a), the low density of this species in Puerto Rico and its indifference to human blood suggest that it is not an important vector there.

A. grabhami has been infected experimentally with $P$. falciparum and has been found infected in nature. Additional studies will be required to determine its relative importance as a vector of malaria.

\section{Anopheles (Cycloleppteron) vestiti- pennis Dyar and Knab 1906}

a. Experimental infection. Covell (1927) stated that Johnson (1926) attempted, without success, to infect this species with malarial parasites in Puerto Rico. He does not say which form was used in these experiments. 
P. falciparum. Earle (1936a) reported negative experimental results with 6 mosquitoes in Puerto Rico. There are no data for $P$. vivax and $P$. malariae.

b. Infection in nature. In 1927, Covell stated that there was no evidence that this species causes malaria in nature. Kumm and Ruiz (1928), in Costa Rica, dissected 20 specimens with negative results. In 1939 Kumm (1940a), in British Honduras, found a specimen of $A$. vestitipennis with a naturally acquired salivary gland infection.

c. Epidemiological. Data inadequate.

Additional investigation of this species will be required to determine its relation to malaria.

\section{Anopheles (Nyssorhynchus) albimanus Wiedemann 1821}

a. Experimental infection.-P. vivax. Darling (1910), in Panama, reported the infection of 6 , or 85 per cent, of 7 specimens fed on carriers of tertian parasites. Among others who have reported the results of experimental infections are Earle (1930a), Komp (1940) and Rozeboom (1938a).

P. falciparum. Darling (1910), in Panama, also reported the infection of 31 , or 72 per cent, of 43 mosquitoes fed on carriers of estivo-autumnal parasites. Others reporting infections with $P$. falciparum are Earle (1930a), Roseboom (1935, 1938a), Simmons (1936) and Boyd and Jobbins (1940).

P. malariae. According to Covell (1927), "Godoy and Pinto (1923) recorded that $A$. albimanus had been experimentally infected with quartan parasites by them in Brazil. Root (1926), however, says that he has never seen this species in Brazil and considers that the species so recorded are probably aberrant females of $A$. tarsimaculatus."

Studies were carried out by Boyd, Carr and Rozeboom (1938) to compare the susceptibility of $A$. albimanus from Cuba and Panama with that of A. quadrimaculatus and $A$. punctipennis from Florida, when paired lots were simultaneously infected with strains of $P$. vivax and $P$. falciparum, derived from Florida and Cuba. "The $A$. quadrimaculatus displayed a high susceptibility to both the indigenous and exotic strains of $P$. vivax and $P$. falciparum, while $A$. punctipennis was definitely less susceptible to the exotic strains of the parasites. A. albimanus was invariably inferior to A. quadrimaculatus in susceptibility to strains of parasites from its own region and it was nearly non-infective with the nearctic strains of these parasites." Boyd and Jobbins (1940) reported that, " $A$. albimanus from Panama exhibits a susceptibility to a coindigenous strain of $P$. falciparum similar to that observed in a Florida strain of A. quadrimaculatus when infected with the same strain. The relative susceptibility of these anophelines to coindigenous and exotic strains is essentially similar to those previously noted by Boyd, Carr and Rozeboom (1938)."

b. Infection in nature. A. albimanus has been found infected in nature in Venezuela, Panama, Puerto Rico, Jamaica and Costa Rica.

c. Epidemiological. This hardy species invades dwellings and feeds avidly on man and it also may show a preference for horses (LePrince and Orenstein 1916), or oxen, goats or pigs (Earle and Howard). Observations made by Rozeboom (1938a) in Panama indicate that it may prefer man to the pig. King (1937) stated that A. albimanus "is considered to be by far the most important anopheline species in the Caribbean region being the predominant form in many parts of the area and a highly effective vector of malaria. While preferring fresh water breeding places, open to the sunlight, it is also versatile in its habits since it may develop freely in brackish or salt water and in quite a variety of situations. The unfortunate results of its introduction into new territory have been demonstrated in Barbados, where as reported by Seagar (1928) it was found for the first time in 1927, accompanied by a severe outbreak of malaria. According to this report, the island had previously been entirely free of anophelines and of malaria infections." As indicated by Kumm in 
another chapter, A. albimanus is considered as the principal vector in 17 countries. Hoffmann (1938a) observed that A. albimanus is the chief vector in the tropical regions of Mexico that are humid, including both coasts but especially the Gulf coast, where it is abundant during the rainy season.

There seems to be no doubt as to the fact that $A$. albimanus is an important transmitter of malaria in Central America and the West Indies, but in view of its apparent relative unsusceptibility to exotic strains of parasites in the experiments by Boyd, Carr and Rozeboom (1938), it appears that studies to determine its relative susceptibility should be made in the different regions where it is found.

\section{Anopheles (Nyssorhynchus) albi- tarsis Lynch-Arribalzaga 1878}

Acording to Covell (1927), "Root (1926) considers that the species referred to by Brazilian entomologists as 'braziliensis' and 'argyritarsis' are really albitarsis, and further states that as far as he knows no dissections of the true braziliensis are on record. All their dissections for the above species are therefore given here."

a. Experimental infection. Covell (1927) stated, "Experimentally, Godoy and Pinto (1923) record having infected $A$. braziliensis but gave no details of their experiments, beyond stating that sporozoites were found. In nature they record the same species found infected, and Boyd records natural infections of 'argyritarsis.' "'

P. vivax. Ayrozo Galvão (1938), in Brazil, dissected 5 A. albitarsis which had fed 10-19 days previously on a carrier of $P$. vivax gametocytes and found none infected.

$P$. falciparum. Rozeboom (1938a), in Panama, reported experiments in which 100 A. albitarsis and 113 A. albimanus controls fed on human carriers of $P$. falciparum gametocytes. Four of the $A$. albitarsis and 37 A. albimanus were found to be infected, showing oocysts in the stomach. There are no data for P. malariae.

b. Infection in nature. Reports of nata- ral infections by various author's, as summarized by Covell (1927), have been made by Stephens (1921), Godoy and Pinto (1923), Boyd (1926) and Kumm (1932).

c. Epidemiological. Among observers in Panama it is the consensus that there are no epidemiological data to indicate that $A$. albitarsis may be of importance there; and Giglioli (1938a, b) believed it unimportant in British Guiana. However, in other locations, particularly Brazil (Root 1926; Kumm 1932; Townsend 1934) and in Venezuela, it is considered an important vector of malaria.

Additional studies will be required to distinguish the varieties of $A$. albitarsis and to determine their relative importance in the transmission of malaria.

\section{Anopheles (Nyssorhynchus) ano- malophyllus Komp 1936}

There are no data regarding infection of this rare mosquito by malarial plasmodia. Because of the apparent rarity of this species, it seems probable that it is of no importance as a malarial vector.

\section{Anopheles (Nyssorhynchus) argyri- tarsis Robineau-Desvoidy 1827}

Covell (1927) commented on the reports dealing with $A$. argyritarsis as follows: "Owing to the confusion which exists with regard to the correct nomenclature of this species, it is extremely difficult to be certain whether the records relating to infection are accurate. Root (1926) holds that the 'Argyritarsis' of Brazilian entomologists is really $A$. albitarsis Lynch Arribalzaga, and the records of Godoy and Pinto, Boyd and Davis are therefore given under the latter species."

a. Experimental infection.-P. vivax. Darling (1910), in Panama, failed to infect one specimen of $A$. argyritarsis fed on a patient with tertian malaria.

P. falciparum. Darling (1910), in Panama, failed to find infection in three mosquitoes fed on patients with estivo-autumnal malaria. Bennaroch (1928), in Venezuela, reported negative results with 6 mosquitoes. Earle (1936b), in Grenada, reported 
the finding of oocysts in 6 of $27 \mathrm{~A}$. argyritarsis which had fed on a carrier of $P$. falciparum gametocytes. There are no data for $P$. malariae.

b. Infection in nature. Covell (1927) doubts the record of Chagas (1904) "naturally infected" $A$. argyritarsis quoted by Boyd, "as the identity of the species found infected by him is uncertain." Darling (1910) reported the finding of a single infected specimen in Panama with sporozoites in the salivary glands. According to Earle (1936b), negative results have been reported by Stephens (1921) in Venezuela, Boyd (1926) in Brazil, Davis (1927) in Argentina and Bennaroch (1928) in Venezuela.

c. Epidemiological. According to Covell (1927), de Verteuil (1925) suspected $A$. argyritarsis to be an important carrier in Trinidad on epidemiological grounds. Root (1926) notes that in Brazil this species is abundant in the plateau region, where there is little if any malaria, and that presumably it is not a very dangerous carrier of the disease. Davis (1926), however, considers it to be the principal vector in the construction camps in the mountains of the state of Rio. Earle (1936b), commenting on $A$. argyritarsis in Grenada, stated, "Nichols working in St. Lucia reported in 1912 that he found both $A$. argyritarsis and $A$. tarsimaculatus (he called the latter $A$. albimanus) infected in nature and considered them of equal importance. While most of the other evidence is to the contrary, the results reported here from Grenada indicate that $A$. argyritarsis might be a malaria vector. A small outbreak of malaria occurred at Douglaston, Grenada, just before one of my visits, and I was not able to find any $A$. tarsimaculatus when I arrived. Argyritarsis was present in moderate numbers. There are certain hill sections such as that back of Santeurs which have been rather malarious, and while $A$. tarsimaculatus has been occasionally found there, $A$. argyritarsis has been the predominant species. As reported in this paper, I was able to infect $A$. argyritarsis readily in the laboratory. The fact, however, that it does not show a high preference for human blood and has never become abundant in these islands, probably renders it unimportant as a malaria vector." Giaquinto Mira (1936), in Guatemala, observed that $A$. argyritarsis is found in all locations where A. albimanus exists but is less abundant in the lowest altitudes. He suggested that its importance as a vector probably is greater in the higher locations.

The information concerning $A$. argyritarsis appears to be inadequate to determine its relative importance as a malarial vector.

\section{Anopheles (Nyssorhynchus) bach- manni ${ }^{2}$ Petrocchi 1925}

a. Experimental infection. Bennaroch (1928), in Venezuela, dissected 2 specimens after they had fed on a patient with an unknown type of malaria, with negative results.

$P$. vivax. Rozeboom (1935), in Panama, reported the infection of two groups of $A$. bachmanni with $P$. vivax. In the first group 3 of 7 mosquitoes were infected, with oocysts, and in the other 3 of 11 had either oocysts or sporozoites. It was concluded that this species was less susceptible than the $A$. albimanus used as controls.

$P$. falciparum. Rozeboom (1935) also reported experiments with mosquitoes which undoubtedly were $A$. bachmanni, and after feeding them on $P$. falciparum gametocytes, 2 of 13 specimens were infected, with oocysts in the stomach. Seven of $14 A$. albimanus controls were also infected. There are no data for $P$. malariae.

$b$. Infection in nature. Bennaroch (1928), in Venezuela, dissected 75 A. bachmanni with negative results. Rozeboom (1938a), in Panama, examined 3 specimens obtained from human dwellings and 320 collected from pigs, and found none infected.

c. Epidemiological. This species has been suspected as a malarial vector in Venezuela on epidemiological grounds by Bennaroch (1931); and precipitin tests done there show that while it prefers animal blood, it

2 Name changed to $A$. triannulatus by Neiva and Pinto. 
also feeds on man (Hill 1934). Townsend (1934) thought it was probably unimportant as a vector in Brazil. From his studies in Panama, Rozeboom (1938a) concluded, "A. bachmanni can be infected with human malarial parasites, but its preference for animal blood renders it harmless so far as malarial transmission in Panama is concerned."

Further studies of $A$. bachmanni will be required before its relative importance can be determined in the different regions of its distribution.

\section{Anopheles (Nyssorhynchus) darlingi Root 1926}

a. Experimental infection. Bennaroch (1928), in Venezuela, dissected $83 \mathrm{~A}$. darling $i$ that had fed on a malarial patient of unknown type, with negative results. There are no records of its experimental infection with $P$. vivax, $P$. falciparum or $P$. malariae.

b. Infection in nature. The high susceptibility of $A$. darling $i$ to natural infection is indicated by the following results of dissection of wild mosquitoes caught in different regions :

NATURAL INFECTIONS

\begin{tabular}{l|l|c|c}
\hline \multicolumn{1}{c|}{ Authorities } & Place & $\begin{array}{c}\text { Mosqui- } \\
\text { toes }\end{array}$ & Infected \\
\hline & & & per cent \\
Root (1926) & $\ldots \ldots \ldots \ldots . . .$. & $\ldots \ldots .$. & $\ldots .$. \\
Bennaroch & Venezuela & 114 & 10.5 \\
$\quad$ (1931) & Brazil & 220 & 22 \\
Davis \& Kumm & Brazil & 240 & 28.7 \\
$\quad(1932)$ & Brazil & 5 & 60 \\
Kumm (1932) & Brazil & $\ldots \ldots . .$. & 9 \\
$\quad \begin{array}{l}\text { Shannon } \\
\text { Kumm (1933) }\end{array}$ & British & & \\
& Honduras & 32 & 31 \\
\hline
\end{tabular}

c. Epidemiological. Bennaroch (1931) reported that $A$. darling $i$ was the most prevalent anopheline during outbreaks of malaria in Venezuela from May to December, 1930. It is considered as one of the most important vectors in several countries, including Brazil (Townsend 1934; De Bezerra 1936; Pereira Barretto 1938) and
British Guiana Giglioli 1938a, b). Komp (1940a) stated, " $A$. darlingi is the most dangerous vector of malaria in Brazil except the imported A. gambiae, and in British Guiana and Venezuela."

Ayroza Galvão and Pereira Barretta (1938), in Brazil, described 4 types of eggs of $A$. darlingi var. paulistensis.

The conclusion is that $A$. darlingi is apparently an important and dangerous vector, but it should be studied for the identification of races and subspecies to determine more exactly its susceptibility to different species and strains of plasmodia. In this connection the recent discovery of this species in Guatemala and British Honduras is of special interest.

\section{Anopheles (Nyssorhynchus) oswaldoi Peryassu 1922}

There are no data on experimental infections and only inadequate epidemiological data. The natural infections reported on A. oswaldoi, in Brazil, by Boyd (1926) were later referred to by him (1930e) as in $A$. tarsimaculatus. The conclusion is that the relation of $A$. oswaldo $i$ to malaria has not been determined and should be investigated further.

\section{Anopheles (Nyssorhynchus) strodei (Root 1926)}

a. Experimental infection. Bennaroch (1928), in Venezuela, reported failure to find infection in 5 specimens fed on malarial parasites of undetermined type.

$P$. vivax. Ayroza Galvão (1938), in Brazil, reported the feeding of $4 \mathrm{~A}$. strodei on a carrier of $P$. vivax gametocytes, and when dissected after 19 days at $19-24^{\circ} \mathrm{C}$ and $67-88$ per cent relative humidity, two mosquitoes contained numerous mature oocytes in the stomach and sporozoites in the salivary glands. There are no data for $P$. falciparum and $P$. malariae.

b. Infection in nature. Bennaroch (1928) failed to find natural infection in $22 \mathrm{~A}$. strodei dissected in Venezuela. Ayroza Galvão (1938) reported that R. Correa examined 174 female $A$. strodei taken in houses in São Paulo, Brazil, and found 2 infeeted with oocysts in the stomach. 
The conclusion is that $A$. strodei will require further study to determine its relative importance in the transmission of malaria.

\section{Anopheles (Nyssorhynchus) tarsi- maculatus $^{3}$ Goeldi 1906}

The confusion in the classification of $\boldsymbol{A}$. tarsimaculatus naturally creates doubt concerning the earlier reports as to the susceptibility of this mosquito to infection with malaria.

a. Experimental infection.-P. vivax. According to the annual report of the Surgeon General of Trinidad (1934), specimens of $A$. tarsimaculatus previously sent to S. P. James in England were able to carry a Roumanian strain of $P$. vivax and were proved to be a "possible carrier" of $P$. ovale.

P. falciparum. Darling (1910), in Panama, fed 5 A. tarsimaculata on blood containing $P$. falciparum and found 3 infected. Bennaroch (1928), in Venezuela, obtained negative results with 6 specimens. Specimens of $A$. tarsimaculatus sent from Trinidad to S. P. James, in England, failed to carry a Roumanian strain of $P$. falciparum. Earle (1936b) in Grenada dissected 17 A. tarsimaculatus which had fed on a human carrier of $P$. falciparum, and found 6 infected.

P. malariae. Darling (1910), in Panama, dissected one $A$. tarsimaculata which had fed on a patient with quartan malaria, with negative results. Davis and Shannon (1928) also reported negative results with one mosquito.

b. Infection in nature. Reports of infection in nature have been made by Boyd (1926), de Verteuil (1933) and Earle (1936b). Those prior to 1931 are listed by Covell $(1927 ; 1931 b)$.

c. Epidemiological.

A. tarsimaculatus was formerly suspected as an important vector in Panama (Simmons et al. 1939), and the reduction in incidence of malaria at certain forts subsequent to the canalizazation of nearby tidal swamps on the Atlantic coast suggests that the salt water variety (A. aquasalis) may have been con-

3 A. aquasalis (Rozeboom and Gabaldon). cerned. De Verteuil (1933) concluded that A. tarsimaculatus was the most important vector in Trinidad, and more recently de Verteuil and Spence (1937) stated that of the 13 species of anophelines in Trinidad the only two that are important vectors are A. tarsimaculatus Goeldi and A. bellator var. cruzii D \& K. Earle (1936b) stated that $A$. tarsimaculatus was taken in houses, preferred human blood, and was the main carrier in Grenada and St. Lucia. On the other hand, Giglioli (1938a, b), in British Guiana, observed that A. tarsimaculatus Goeldi is relatively infrequent in houses, prefers to feed on animals and is prevalent in localities usually free from endemic malaria. Tournier (1937) reported that $A$. tarsimaculatus Goeldi, which is apparently adapted to brackish water, is the only anopheline found in the coastal region of French Guiana and that it and A. albitarsis are the principal vectors.

In spite of the early confusion as to the identity of $A$. tarsimaculatus ( $A$. aquasalis), the available evidence indicates that it is probably an important vector of malaria in many localities. This species deserves further experimental study.

\section{Anopheles (Kerteszia) bellator Dyar and Knab 1906}

There are no data of experimental infection and the data for infection in nature are inadequate, but de Verteuil and Spence believe that $A$. bellator var. cruzi is a dangerous vector of malaria in Trinidad, and that in native villages it is probably responsible for about 20 per cent of the malarial mortality. De Bezerra (1936), in Brazil, remarked that $A$. bellator var. cruzi is thought to carry malaria, though it has not been proved to do so in the laboratory. This mosquito should be studied experimentally.

\section{Anopheles (Kerteszia) neivai Howard,} Dyar and Knab 1917

There are no data of experimental infection or of infection in nature and the epidemiological data are inadequate. The conclusion is that $A$. neivai should be studied to determine its susceptibility to infection and its relation to malaria. 
TABLE III

ANophelines Considered or SUSPected as EFrective Vectors of Malaria.

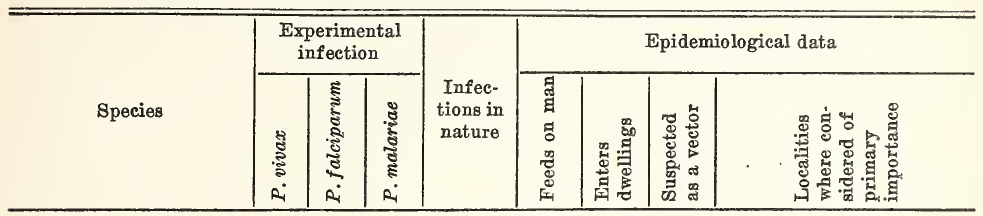

Nearctic region

\begin{tabular}{|c|c|c|c|c|c|c|c|c|}
\hline $\begin{array}{l}\text { A. quadrimaculatus } \\
\text { A. maculipennis (U. S.) .... }\end{array}$ & $\begin{array}{l}+ \\
+\end{array}$ & + & + & + & $\begin{array}{l}+ \\
+\end{array}$ & $\begin{array}{l}+ \\
+\end{array}$ & $\begin{array}{l}+ \\
+\end{array}$ & $\begin{array}{l}\text { Southern and Eastern U.S. } \\
\text { Pacific Coast and South- } \\
\text { west U. S. }\end{array}$ \\
\hline 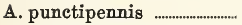 & + & + & + & (1) 8 & + & +- & + & \\
\hline A. crucians & + & + & - & + & + & +- & + & \\
\hline (1) Inland type & + & + & - & & 4 & +- & + & \\
\hline (2) Coastal type ............... & + & + & & & + & +- & + & \\
\hline
\end{tabular}

Neotropical region

\begin{tabular}{|c|c|c|c|c|c|c|c|}
\hline 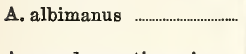 & + & + & + & + & + & + & $\begin{array}{l}\text { Central Am., W. Indies \& } \\
\text { Northern So. Am. }\end{array}$ \\
\hline A. pseudopunctipennis ....... & + & + & + & + & + & + & Mexico, Argentina \\
\hline A. tarsimaculatus ..................... & + & + & + & + & + & + & $\begin{array}{l}\text { Trinidad, Grenada, St. } \\
\text { Lucia, French Guiana }\end{array}$ \\
\hline $\begin{array}{l}\text { A. argyritarsis } \\
\text { A. darlingi }\end{array}$ & 1 & + & $\begin{array}{l}+ \\
+\end{array}$ & $\begin{array}{l}9 \\
+\end{array}$ & $\begin{array}{l}9 \\
+\end{array}$ & $\begin{array}{l}+ \\
+\end{array}$ & $\begin{array}{l}\text { Rio de Janeiro, Brazil } \\
\text { Brazil, British Guiana, and } \\
\text { Venezuela }\end{array}$ \\
\hline $\begin{array}{l}\text { A. albitarsis (Panama } \\
\text { type) }\end{array}$ & & + & & P & 8 & - & \\
\hline (Brazil type) & - & & + & + & + & + & Brazil and Venezuela \\
\hline A. punetimacula & + & + & + & + & + & + & Panama Jungle Areas \\
\hline A. hectoris & + & + & & + & & + & Guatemala Highlands \\
\hline A. bellator & & & & + & 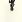 & + & Trinidad \\
\hline
\end{tabular}

\section{Conclusion}

In this chapter data have been presented which, though incomplete, serve to indicate the scope of our knowledge of the roles played by North American anophelines in the transmission of malarial fevers. Seven nearctic and 23 neotropical species have been included, and in each instance consideration has been given to the published information concerning the relation of the mosquito to malaria as indicated by laboratory infections, natural infections, and epidemiological data. Tables III and IV provide an index summarizing certain information about the more important species, but they do not indicate the adequacy of the studies made of these species.

Considered as a whole, the information now at hand fails to indicate the relative importance of all the anophelines named. Certain species believed to be the most important vectors, such as $A$. quadrimaculatus in the temperate zone and $A$. albimanus in the tropics, have been studied extensively, while others have been neglected on the assumption that they are unimportant. Even with the former group, the experimental information is somewhat restricted to investigations made in a few localities. Thus it fails to indicate the relative susceptibility of the different strains of the mosquito species to the different strains of malarial plasmodia which may be encountered in each of the various regions of its geographical distribution. Finally, much of the data collected is of questionable value because of the present confusion as to the identity of certain of the North Ameri- 
TABLE IV

ANophelines INFected With Plasmodia but not Adequately Studied to Determine their ReLation to Malaria

\begin{tabular}{|c|c|c|c|c|c|c|c|}
\hline \multirow[b]{2}{*}{ Species } & \multicolumn{3}{|c|}{ Experimental Infection } & \multirow[b]{2}{*}{$\begin{array}{l}\text { Infections } \\
\text { in nature }\end{array}$} & \multicolumn{3}{|c|}{ Epidemiological data } \\
\hline & P. vivax & $\begin{array}{l}P . \text { falci- } \\
\text { parum }\end{array}$ & $\begin{array}{l}\text { P. mala- } \\
\text { riae }\end{array}$ & & $\begin{array}{l}\text { Feeds } \\
\text { on man }\end{array}$ & $\begin{array}{c}\text { Enters } \\
\text { dwellings }\end{array}$ & $\begin{array}{l}\text { Suspected } \\
\text { as a } \\
\text { vector }\end{array}$ \\
\hline \multicolumn{8}{|c|}{ Nearctic region } \\
\hline $\begin{array}{l}\text { A. atropos } \\
\text { A. barberi } \\
\text { A. walkeri }\end{array}$ & $\begin{array}{l}+ \\
+ \\
+\end{array}$ & + & & + & $\begin{array}{l}+ \\
+ \\
+\end{array}$ & $\begin{array}{l}+ \\
+ \\
+\end{array}$ & $\begin{array}{l}+ \\
+ \\
+\end{array}$ \\
\hline
\end{tabular}

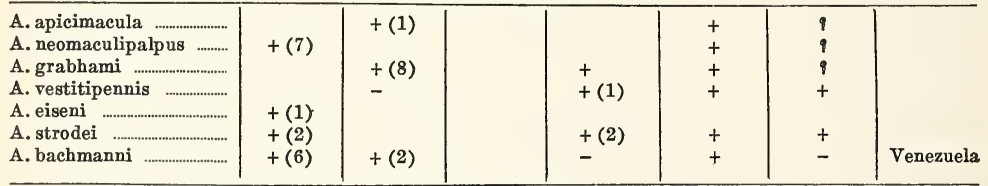

NoтE: The following anophelines have not been investigated to determine their relation to malaria: C. bathanus, A. kompi, A. parapunctipennis, A. xelajuensis, A. anomaphyllus, A. oswaldoi, A. neivae.

can anophelines and the possibility that various unrecognized species, subspecies or varieties were formerly included under such specific names as $\dot{A}$. maculipennis, $A$. crucians, A. tarsimaculatus, A. pseudopunctipennis, etc.

Obviously more exact methods are needed to determine the relative importance of different anophelines as vectors of malaria under all of the varied conditions to be encountered in the different regions where the disease is endemic. It appears that the development of such methods must depend primarily on the organization of increased facilities for fundamental research in medical entomology, and new techniques for appraising the effectiveness of anophelines as vectors.

Such a revised program must begin with the establishment of exact criteria for the differentiation and identification of the anopheline species, subspecies and varieties. Surveys should be made to determine the relative prevalence, breeding places and habits of each kind of mosquito at different seasons in the various regions. Their relation to malaria in each of these regions should be determined by carefully con- trolled epidemiological and experimental observations, which should, in so far as possible, be continued as a routine integral part of the local malarial control program.

In this connection it is believed that a continuous check on the progress of malarial control in a routine entomological laboratory might be just as helpful as are the bacteriological checks utilized routinely in determining the efficiency of a water purification plant. The information obtained might include the incidence of naturaily acquired infections in local anophelines at different seasons of the year and the probable sources of these infections; also changes in the susceptibility of local anophelines to infection with local and introduced strains of plasmodia from time to time. Such information collected over a period of years might throw light on some of the unexplained fluctuations in malarial morbidity and mortality.

In conclusion, it is desired to reemphasize the belief that the development of more adequate fundamental knowledge concerning the anophelines of North America should make it possible to more effectively control malaria in this continent. 


\title{
CYCLICAL VARIATION IN THE INCIDENCE OF MALARIA
}

\author{
By CHARLES F. CRAIG \\ SAN ANTONIO, TEXAS
}

Crclical variations in the incidence of the malarial fevers have been noted by practically all investigators since the etiology of these fevers has been understood, and long before we were acquainted with the malaria plasmodia writers upon the subject called attention to the seasonal variations and the occurrence of epidemies of these fevers in regions usually free or in endemic centers. These cyclical variations were noted to be of two types, i.e., of short amplitude, or seasonal, and of long amplitude.

\section{Cyclical Variations of Short AMPLITUDE}

Variations in incidence of short amplitude are usually called seasonal variations and occur to a greater or lesser extent in all malarial regions, whether endemic or hyperendemic. In the tropies these variations are connected with the rainy and dry seasons, but in temperate regions, as the United States, seasonal variations are confined to certain periods in the year and depend upon the presence of temperatures favorable or unfavorable to the breeding and development of the mosquitoes transmitting the malarial infections or to the development of the malaria plasmodia within the transmitting mosquitoes. Thus, infections with $P$. vivax, the benign tertian plasmodium, occur in the spring, summer and autumn months in temperate regions, while infections with $P$. falciparum, the malignant tertian or estivo-autumnal plasmodium, oceur almost entirely in the summer and autumn. One never observes an initial infection with $P$. falciparum in temperate regions much before the middle of June, while the vast majority of such infections occur in the late summer and early fall. The name "estivo-autumnal" is, therefore, truly descriptive of the occurrence of this type of malaria in the southern parts of the United States, where it is prevalent. Cyclical variations in the incidence of malaria of short amplitude occur yearly and in some localities are of short duration, due to seasonal conditions entirely.

\section{Cyclical Variations of Long AMPLITUDE}

Many malariologists in other countries have recorded cyclical variations in the incidence of malaria of long amplitude, in which increases in the amount of malaria present have occurred at intervals of years but with fair regularity. In the United States the only observer who has investigated this subject is Faust (1933, 1939b), who has been able to show that such variations occur in the mortality curve of malaria in the southern states, and, presumably, in the morbidity curve, and that these long-interval variations in the incidence have been more or less regular in type.

Since 1930, Faust has reported yearly upon the malaria mortality in the southern states and his observations have demonstrated that, beginning in 1927, the malarial death rate rose slowly to a peak in 1928 1929 , and then receded to a low point in 1931-1932. In 1933 the mortality rate again rose quite sharply and then slowly receded until a low point was reached in 1939

While it is admitted that mortality rates in malaria may not be an absolutely correct index of the incidence of these infections, owing to the possibility of many mistaken diagnoses, it is the only index that Faust could employ, as the morbidity statistics of the malarial fevers in the southern states 
were very unreliable until recently. The writer believes, with Faust, that the mortality statisties of malaria, as kept by the various Boards of Health of the southern states, are of such accuracy that the deductions based upon them are warranted, and that two cyclical variations of long amplitude have oceurred in the incidence of malaria in these states during the past 15 years.

\section{Causative Factors}

The factors causing cyclical variations of short and long amplitude are many and have to do both with the mosquitoes transmitting these infections and with the recipient of the infection, i.e., man. We are familiar with many of the factors causing cyclical variations of short amplitude but we know much less of those having to do with cyclical variations of long amplitude.

Factors operating on the vectors and parasites. Of the many factors operative in causing cyclical variations in the incidence of malaria of short amplitude, climate and season are probably the most important, not only in influencing the breeding and development of the transmitting mosquitoes but also in affecting the growth and development of the malaria plasmodia within the transmitting mosquitoes. It has already been stated that in temperate regions, as the United States, malaria is most prevalent during the summer and fall months and least prevalent in the northern portions of the United States, because of the colder climate which does not favor the breeding of anopheline mosquitoes; or, if it does not interfere with the breeding of mosquitoes, does prevent the development of the malaria plasmodia within the mosquitoes.

The influence of temperature upon the development of the various species of malaria plasmodia in mosquitoes has long been known and each species of plasmodium has an optimum temperature in which development occurs most rapidly and completely. Thus $P$. vivax develops best in the mosquito at a temperature of about $25^{\circ} \mathrm{C}$ and the mosquito becomes infective in about eleven days; $P$. malariae develops best in the mosquito at a temperature of $22^{\circ} \mathrm{C}$, and the mosquito becomes infective in from 18 to 21 days; while $P$. falciparum completes its development in the mosquito best at a temperature of $30^{\circ} \mathrm{C}$, and the mosquito becomes infective in from 10 to 12 days. This relationship of temperature to the development of the plasmodia in the transmitting mosquitoes readily explains why infections with the latter species of plasmodium occurs in temperate regions in the summer and early fall, when the highest temperatures are present, and are never observed, as initial infections, in the spring, when temperatures are too low for successful development in the mosquito.

Humidity is also of great importance in cyclical variations in malarial incidence. If there is not sufficient humidity present the malaria plasmodia will not develop in mosquitoes, and the nearer it is to saturation the more rapid is the development in these insects. Humidity also has a profound effect upon the breeding and development of the transmitting mosquitoes, and the amount and character of the rainfall very largely governs the number of anopheline mosquitoes in any locality. Thus, although malaria is present throughout the year in the tropics, it is always greatest in incidence during the latter portion of the rainy season and immediately following that season, and least prevalent during the dry season; while in temperate regions, as the United States, these infections are most common in the summer and early fall when conditions as regards rainfall and temperature are most favorable.

The species of anopheline mosquitoes present in any locality has much to do with cyclical variations. Many species of anophelines do not transmit malaria while others are either good or poor transmitters. If species which are good transmitters are present, i.e., those in which the malaria plasmodia develop best, and non-immunes are present in large numbers, malaria will occur in epidemic form, while if smaller numbers of infective mosquitoes are present and few non-immunes, malaria will be rarely observed. Cyclical variations in the 
prevalence of these infections are caused by cyclical variations in the number of efficient mosquito transmitters, depending upon conditions favorable to the breeding and development of these insects. In the United States, A. quadrimaculatus and $A$. maculipennis are the mosquitoes most frequently concerned in the transmission of malaria, especially the former, and wherever these species are present, provided human "carriers" are also present, malaria is more or less frequently encountered. It is probable that cyclical variations in the incidence of malaria of both short and long amplitude are due to variations in the species of anopheline mosquitoes present.

The influence of the species of anophelines upon the incidence of malaria is strikingly illustrated by the introduction of $A$. gambiae into Brazil from Africa, as recorded by Barber (1940). This species is especially active as a transmitter of malaria and its breeding habits are such as to favor its infection from man. Since its introduction into Brazil it has spread rapidly and malaria has greatly increased in amount and has become epidemic in many localities. Its spread into other countries of South America, and even into the United States, is possible and would undoubtedly be accompanied by a great rise in malarial incidence and Barber well says:

There is no doubt that this invasion of gambiae threatens the Americas with a catastrophe in comparison with which ordinary pestilence, conflagration, or even war are but small and temporary calamities. Gambiae literally enters into the very veins of a country and may remain to plague it for centuries.

The number of infective mosquitoes present in any locality, other things being equal, will determine the amount of malaria there present. It is not essential that a large percentage of anopheline mosquitoes be infective, for even in regions where malaria is common the percentage of infective mosquitoes, as determined by dissection, has been found to vary between 1.5 to 3 per cent, but in hyperendemic areas the percentage is higher, usually varying between 6 and 10 per cent. In the case of A. gambiae Barber (1940) found a sporo- zoite rate of 30.5 per cent in a town in West Africa, but the average for the surrounding districts was only 6.5 per cent. In South America, Barber found that the sporozoite rate of this species of mosquito varied from 2.7 per cent to 10 per cent, according to the length of time the species had been transmitting malaria in a particular locality.

The apparently low percentage of infected mosquitoes in regions where there are many human carriers of the plasmodia is largely due to the fact that only a small percentage of mosquitoes biting a carrier becomes infected and because repeated feedings are usually necessary before the insect becomes infected. Again, it has been shown that certain individuals are good infectors of mosquitoes while others seldom transmit the infection to mosquitoes, so that the number of efficient human carriers in any locality has much to do with the incidence of malaria.

Migration. The migration of nonimmune individuals into a malarial region is always followed by a rise in the incidence, and if such migrations occur at regular intervals, we may have cyclical variations in incidence of both short and long amplitude, depending upon the length of time between such migrations. If efficient human carriers of malaria are present, the mosquitoes of the region will be infected and it follows that the arrival of large numbers of non-immune individuals will be followed by the infection of many of them and a marked rise in the incidence of the malarial fevers in that locality. After infancy and childhood, the native population of such regions will have acquired a considerable amount of premunition $^{1}$ and immunity, so that symptoms are not frequently observed in the adult population, although infants and children suffer markedly from these infections. If a large number of individuals from regions free from malaria migrate into such a commu-

1 In the present instance "premunition" is employed to designate a state of resistance conferred by a substance or substances formed while a parasite is residing in the host and the resistance terminates with the disappearance of the germ. 
nity, there will be a great increase in symptomatic malaria in both pre-adult and adult new-comers, and severe epidemies of malaria may thus arise. The bearing of immunity to the prevalence of the malarial fevers and its relation to malaria in the community is fully considered in the contribution by Hackett (1937).

Importation of exotic strains. It has been conclusively demonstrated by numerous observers that the premunition and immunity acquired after repeated attacks of malaria are limited to the species of malaria plasmodia infecting the individual and to certain strains of these species. Thus, premunition or immunity acquired to $P$. vivax is largely inoperative against $P$. malariae, $P$. falciparum or $P$. ovale and vice versa. Not only is this true, but both are limited to certain strains of the particular species of the plasmodium, i.e., to the strain with which the individual has been infected. It follows, therefore, that if new species or strains of species of plasmodia be introduced into a malarial region the incidence of malaria will be raised because the inhabitants have no premunition or immunity to such species or strains of species. Such an occurrence might explain cyclical variations of both short and long amplitude.

As an illustration of the effect of the introduction of new species of malarial plasmodia into a locality, the author may mention a personal observation demonstrating its importance. In the state of Connecticut, in 1898, the only species of malarial plasmodium present in a certain region was $P$. vivax and benign tertian malaria was endemic in the locality for many years. After the end of the SpanishAmerican War, a company of the National Guard from this locality, the men of which had been on duty in a southern camp where infections with $P$. falciparum were numerous, returned to their homes. Within a few weeks cases of infection with this plasmodium began to appear, and before the end of autumn an epidemic of estivoautumnal malaria had occurred, with a few fatal infections. Thus, in this locality where this type of malaria had never before occurred, the importation of carriers of $P$. falciparum in the Guard company mentioned, during favorable climatic conditions, resulted in an epidemic of this foreign type of malaria among a non-immune population. 


\title{
TOPOGRAPHICAL AND RELATED FACTORS IN THE EPIDEMIOLOGY OF MALARIA IN NORTH AMERICA, CENTRAL AMERICA, AND THE WEST INDIES
}

\author{
By ROBERT BRIGGS WATSON and REDGINAL HEWITT
}

HEALTH AND SAFETY DEPARTMENT, TENNESSEE VALLEY AUTHORITY, WILSON DAM, ALABAMA

IN common with other diseases which require an insect vector, perhaps to the greatest extent, malaria is characteristically a disease of place. The constant close association of its regional prevalence with still water has been recorded by numerous writers during the period of 2000 years which preceded Ross' discovery of the definitive host of Plasmodium. The subsequent definition of the bionomics of the many species of Anopheles now permits rationalization of the occurrence of malaria, as regards both time and place, largely in terms of the natural history of the regional vector.

The density of the vector, probably more than any other factor, determines the rate of malaria transmission. Therefore, physiographical factors which tend to produce an environment favorable to the propagation of a regional vector are of considerable interest from an epidemiological standpoint. The following discussion seeks to correlate the occurrence of malaria in several regions with certain physical factors. Consideration is given chiefly to the influence of geological and topographical factors on the disposal of water, with special reference to this influence on the propagation of the regional vector; and to the influence of altitude and of temperature on the prevalence of anophelism and malaria.

Anopheles quadrimaculatus and A. maculipennis are certainly the principal vectors of malaria in the United States. The six other representatives of this genus which occur in this country are of relatively little consequence from an epidemiological standpoint.

East of the Rocky Mountains, A. quadrimaculatus is chiefly, if not wholly, re- sponsible for malaria transmission. This mosquito propagates in permanent or semipermanent collections of water under a wide variety of conditions. Its optimum requirements are clear, still water, partially shaded, with a pH of about 7.0 and containing emergent vegetation and floating debris. Perceptible current, gross pollution with sewage or industrial wastes, and salinity inhibit or prevent larval development. The diminished propagation of A. quadrimaculatus in polluted streams is probably due, either directly or indirectly, to the lack of dissolved oxygen in them. Very little malaria is found associated with the brackish water marshes along the coasts of the United States. This probably indicates that $A$. atropos, which propagates in such places, does not transmit malaria to a significant extent. While aquatic environments which approach the optimum for $A$. quadrimaculatus propagation occur principally in the Atlantic and Gulf coastal plains at an altitude less than 500 feet, neither this mosquito nor malaria due to its presence are confined to this area. $A$. quadrimaculatus probably occurs throughout the entire Southeastern United States, whether or not it can be detected by usual means (Watson and Spain 1937). That this statement may apply also to the Central and Northeastern states is attested by the recent occurrence of malaria in Ohio (Hoyt and Worden 1935) and New Jersey (Butts 1937).

Altitude does not of itself inhibit propagation of A. quadrimaculatus and malaria transmitted by it; it is the climatic and topographic circumstances which are usually associated with high places. In most 
instances, malaria in mountainous sections of the Eastern United States is associated with artificial lakes since the terrain prevents extensive natural collections of still water. A few cases of malaria have been found associated with Lake Norris since its impounding in 1936. The impounding of a small lake in Putnam County, Tennessee, caused an epidemic of malaria in 1925 . These situations are in the foothills of the Cumberland Mountains, at an altitude of about 1200 feet.

In general, the distribution of $A$. maculipennis is more northern than $A$. quadrimaculatus, although Hinman and Hurlbut (1941) found both species occurring together in Southern Ontario. It is found principally West of the Rocky Mountains, from Southern California to Alaska, and Eastward through Canada to Northern Maine. Its natural history, including its requirements for prolific propagation are quite similar to that for $A$. quadrimaculatus. The larvae are found in permanent or semi-permanent collections of water, such as roadside pools, along the margin of lakes and in irrigated fields. However, it accommodates itself better to the colder climates of high altitudes than does A. quadrimaculatus. It has been reported to occur in the Valley of Mexico at altitudes up to 8000 feet (Bustamente 1939). Boyd (1940f) has observed malaria due to $A$. maculipennis near Reno, Nevada, at an altitude of 4000 feet.

Anopheles albimanus is the principal vector of malaria in the Greater Antilles, Mexico, and Central America at elevations less than 3000 feet. This species exhibits a great adaptability to diverse environmental situations, perhaps more than any other mosquito in North America. While it prefers stagnant water which is exposed to direct sunlight, it may be found along the margins of flowing streams and in marshes containing brackish water. Perhaps the greatest deterrent to its propagation is dense shade. Its occurrence is not restricted entirely to lowlands, although, in general, it does not find favorable situations for propagation at altitudes considerably above sea level. It probably utilizes small, temporary collections of water to a greater extent than does $A$. quadrimaculatus, such as cattle tracks in seepage areas.

Anopheles tarsimaculatus occurs throughout the Lesser Antilles and on the mainland from Nicaragua southward. Its breeding situations are like those of $A$. albimanus and it exhibits much of the same adaptability of the latter mosquito to varying conditions. While its breeding places are usually found at sea level, it may occur at altitudes up to 3000 feet (Root and Andrews 1938).

A. pseudopunctipennis occurs in the semiarid and arid portions of tropical and subtropical America from the Southwestern United States to Northern Argentina. In the United States it is apparently not of much concern in the transmission of malaria but in the highlands of Mexico and in Central America it is probably the principal vector. The available evidence suggests that this species has at least two races or subspecies, the lowland form being relatively innocuous. Its larvae are found in pools in stream beds during the dry season, in roadside ditches and in irrigated fields, such as the rice fields of Mexico.

Until recently $A$. darling $i$ was thought to occur only in Brazil, but in the past two years has been found in various situations in Central America (Kumm 1940c). It is likely that it has a wider distribution than was believed formerly. This may be accounted for by the fact that the morphological characteristics of the adults are not unlike those of $A$. argyritarsis and may have been mistaken for this mosquito or for other white-footed species which occur in tropical America. Its detached occurrence in British Honduras suggests importation (Boyd 1940f). Because of its remarkable potentialities as a malaria vector, its occurrence anywhere is of considerable importance. At the present time, its distribution in Central America and the conditions under which it is found have not been defined accurately.

\section{Regional Geology and Topography in Relation to Endemic Malaria}

1. The United States. In the eastern 
United States the Atlantic Coastal Plain extends from New England to the southern tip of Florida, rising slowly to the Appalachian Highlands. Beyond these mountains lie the Central Plains and Great Plains of the Middle West, which extend to the Rocky Mountains, the Western Plateaus and the Pacific Ranges. The Gulf or Southern Coastal Plain borders the Gulf of Mexico, and leads to the Ozark Plateau, part of the Central Plains and the Piedmont Plateau in the north (Fig. 1).

The regions richest in swamps and marshes are, for the most part, located in the coastal plains. Extensive natural breeding places for A. quadrimaculatus are in general restricted to this lowland belt. The Gulf Coastal Plain is greatly widened in the vicinity of the Mississippi River, extending north in a pronounced embayment to southern Illinois. This area is a great alluvial plain covering 300,000 square miles, in which optimum conditions oceur frequently for the propagation of $A$. quadrimaculatus.

Two topographical features encountered in the coastal plains, namely, meandering, old-age streams and "solution topography" described by Boyd and Ponton (1933), are chiefly responsible for the prevalence of malaria in this region.

When a surface has been cut by erosion and solution to an elevation only slightly above that of the master streams of a region, the tributary streams flowing over the surface are unable to cut their channels deeper. Under these conditions the gradient of the tributary streams is lessened, they become sluggish, meandering across the flat surface in tortuous courses. Save for periods of flood, movement in these streams is slow and occasional pools may be almost stagnant. In periods of drought, movement of the water is often stopped and the stream may be converted into a series of pools.

During flood periods these old age streams frequently take more direct routes, cutting across the necks of loops in their normal courses. With the recession of the flood level, semi-circular ponds may be left where the former channel lay. The river, pursuing a new course through the "cut-off," leaves residual ponds in the old channel which are called "ox-bows" colloquially. These ponds may be refilled during flood

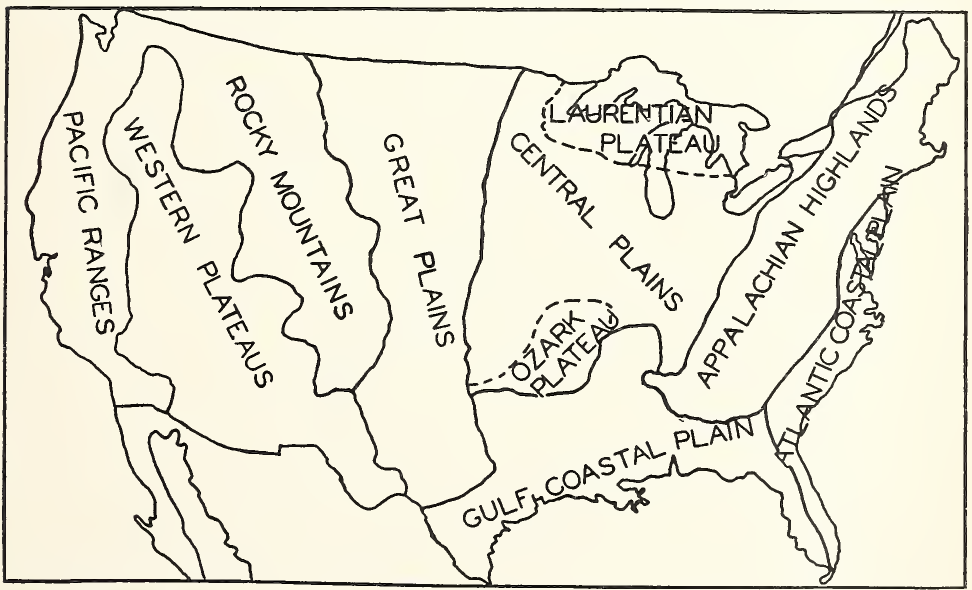

Fic. 1. Principle physiographic divisions of the United States (from Compton's Pictured Encyclopedia 1937). 
stages and may retain water for a long time. Such situations frequently form important breeding places for A. quadrimaculatus.

Large streams with low gradients usually build flood plains on either side of their channels. Flood plains are composed of materials which streams carry during periods of flood and deposit on the sides of their channels when their velocities return to normal. Most of the rivers in the southeastern United States have formed flood plains which, in the case of the larger streams such as the Mississippi and Tennessee Rivers, may be several miles in width. During periods of flood, often every spring, the rivers may overflow their flood plains and the rapidly moving water may cut shallow channels in them. These channels, or "chutes," may hold water for long periods after the flood-stage has subsided.

Even in years when no flood occurs these channels may be filled by rainfall to form temporary ponds; and often they may communicate with a spring or a small stream whose course has been blocked so that a swamp is formed. The blockage of tribu- tary streams by the formation of the flood plains is a common occurrence. Even though the stream may find a new course into the river, its channel below the point of blockage may still communicate with the river and may be filled with stagnant water from it. Situations of this sort are called "sloughs."

As the principal streams approach the coast, the land adjacent to them becomes flatter and more poorly drained. As a consequence, there is a tendency toward the formation of extensive swamps and marshes, the latter tending to become brackish near the sea coast.

A considerable portion of the southeastern United States is underlaid by limestone. This mineral is subject to solution by percolating ground water, especially when the latter contains carbonic and humic acids. As a consequence changes take place in the limestone substrata which result in the formation of a characteristic topography. In the early stages of the solution of the limestone caves are formed which become larger with the passage of time. If

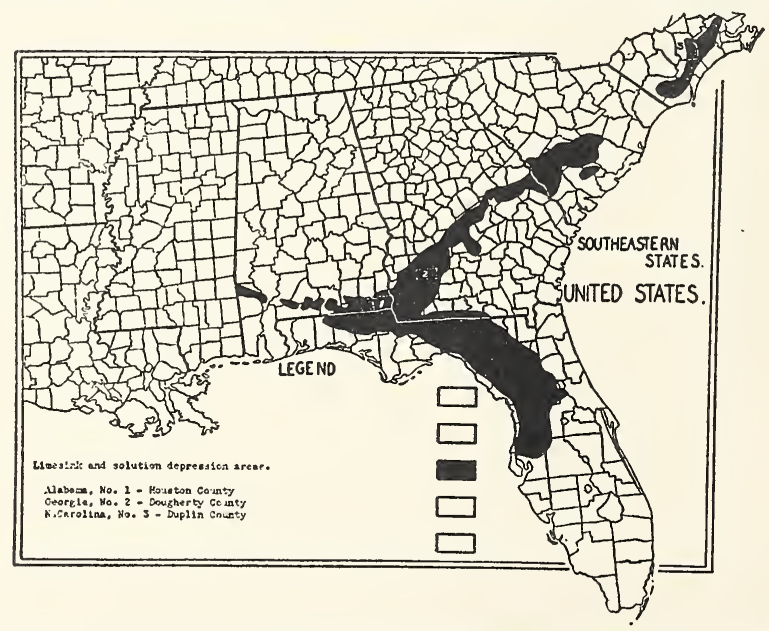

FIG. 2. Limesink and solution depression areas in the southeastern United States (from Boyd and Ponton 1933). 
the roof of a cave is near the ground surface and is not supported by other rock formations it finally becomes so thin that it collapses from the weight of its overburden. A depression results, the size of which is determined principally by that of the cavern. The depth may be little or as much as 100 feet; the diameter may be a few feet or several hundred yards. A region which is characterized by the presence of these depressions is referred to as having a "solution topography" (Fig. 2). Regions with little surface relief, which are underlain with limestone, offer the best opportunity for the development of solution topography. They are usually covered by deposits of clay which tend to hold rainwater under hydrostatic pressure, thus enabling it to take into solution more carbon dioxide.

The relationship between the bottom of a limesink depression and the ground water table and the nature of the region in which it occurred determine whether it will be flled with water of a permanent or semipermanent character. If the bottom of the depression lies well above the level of the ground water and vertical drainage is not obstructed, surface water flowing into it may pass quickly into underground channels. These conditions are seen frequently in eastern Kentucky, in the Tennessee Valley Area in Eastern Tennessee and in southwestern Virginia. In these places limesinks are common but, because of vertical drainage, they rarely hold water for more than a day or two, except during periods of heavy rainfall.

In situations where the surface run-off carries fine clay from the top soil into the depression, natural drainage may be impeded or prevented even though the bottom of the depression lies well above the water table. Water filters through this clay very slowly and a pond forms which is maintained by normal rainfall although its level may recede considerably during dry seasons.

When the level of the natural drainage of a depression is the same as, or below, that of the ground water table a permanent pond is formed, which is fed by the water table in addition to the surface run-off.
The size, age and permanency of collections of water in limesinks determine the nature of the vegetation which they contain. Permanent spring-fed ponds of considerable size and age often support dense growths of trees, such as tupelc gum and cypress. Nearly all of them contain at least marginal growth of dense emergent vegetation which consists of small trees and plants of various kinds. The $\mathrm{pH}$ of their waters depends in part upon the vegetation they contain. In the Tennessee Valley region the waters are usually neutral or alkaline although occasionally acid waters, due to dissolved carbon dioxide, may be encountered. In northern Florida, southern Georgia and Alabama small limesink ponds are sometimes acid in reaction, due to the decomposition of vegetable debris.

With the exception of propagation in collections of water which have been produced artificially, such as impounded water projects, irrigation, and water in excavations, the production of $A$. quadrimaculatus is associated principally with still water produced by the two topographic conditions described above. Moreover, it has been shown that the foci of endemic malaria in the southeastern Atlantic seaboard states and south Alabama are also associated intimately with these topographic conditions (Boyd and Ponton 1933). They call attention particularly to the occurrence of the most intense malaria in this region in the area lying between 100 and 500 feet above sea level.

These authors, as well as Andrews (1938), mention another type of topography associated with Anopheles production and malaria endemicity in the area which lies less than 100 feet above sea level. They call this the "sandy flat woods section" and Andrews says of this area in Georgia, "This land is notable for its flatness, and many of the water-holding basins are man-made borrow pits fed by flowing wells. The majority of these provide satisfactory conditions for the production of $A$. quadrimaculatus." Naturally, there is a low incidence of malaria in the sandy flat woods sections. Since the tree-grown ponds and swamps contain accumulations of vegetable matter 
which are not flushed away by floods, their water is acid and deters A. quadrimaculatus propagation. This applies also to similar areas in South Carolina and Florida. Solution topography is not encountered to any great extent in these coastal low lands.

Malaria in the southern states adjacent to the Mississippi River is likewise not associated to any great extent with limesink or solution topography. In this region the malarious districts center around one or more large river systems, and oceur especially along the flat alluvial valleys and fluvial swamps which follow the broad bed of the Mississippi. The whole western border of Tennessee is subject to overflow, and the valleys of tributaries adjacent to the master stream may be flooded several times during the year. Meleney, Bishop and Roberts (1929) point out that malaria in western Tennessee is confined essentially to these flood plains, and as such is a part of the malaria problem of the entire Mississippi delta. In eastern Arkansas, southeastern Missouri, Mississippi, and Louisiana highly endemic malarious regions are centered along the St. Francis, White, Arkansas, Ouachita, Red, Yazoo, and Mississippi Rivers. The broad alluvial plain between the Yazoo and Mississippi Rivers is responsible for a large part of the malaria in Mississippi (Barber and Komp 1929c). Numerous creeks and tributary streams of this region are sluggish and turbid during most of the year and "ox-bows" are commonly formed as described previously. Wooded swamps and spring-fed ponds also occur along the eastern border of the delta, and these provide breeding places for $A$. quadrimaculatus.

Fertile valleys in the eastern and southeastern parts of Oklahoma, drained chiefly by the Arkansas River, include most of the malarious counties of this state, and the endemic centers in Texas are situated chiefly around the Rio Grande, Red and Sabine Rivers.

In southwestern Kentucky relatively minor endemic foci are found associated with the Ohio, Mississippi, Tennessee, Cumberland, and Blood Rivers.

Along the upper Mississippi and Missouri
Rivers endemic foci or sporadic outbreaks of malaria have been reported recently, which are associated in part with impounded water projects to provide better navigation facilities and for flood control.

In the glaciated region north of the Ohio River, lakes, ponds, swamps and marshes occur that were formerly associated with malaria. The fact that this region is no longer malarious is due more to its social and economic development than to the absence of places for $A$. quadrimaculatus propagation.

Most of the situations referred to previously are confined to sea-level or near sea-level elevations, and the vector is $A$. quadrimaculatus. Quite a different topographical picture, however, from any so far described is found in the malarious districts of New Mexico and California. Barber and Forbrich (1933) define three widely separated regions of endemic malaria in New Mexico. The first occurs in the valley of the San Juan River near Farmington, at an elevation of 5300 feet. Here $A$. maculipennis breeds in large areas of swampy meadows and is considered to be the important vector, although $A$. pseudopunctipennis is also present. A second malarious district is located in Rio Grande Valley, in the vicinity of Espanola, at an elevation of 5600 feet. The third district is located in the southern part of the state near Las Cruces, at 3800 feet, in which drainage ditches and irrigation canals are the breeding places. Malaria in New Mexico, then, is associated with high elevations, irrigated river valleys, and the presence of $A$. maculipennis, a distinctly different epidemiological picture from that of the Gulf and Atlantic Coastal Plains.

In California malaria is transmitted by A. maculipennis and has always occurred principally in the San Joaquin and Sacramento River valleys which lie between the coastal mountain ranges and the Sierras. The San Joaquin flows northward, the Sacramento southward, and they join each other near the coast to empty into San Francisco Bay. In former times the vector found breeding places in fluvial marshes of the 
deltas of both rivers, particularly in their lower portions. Propagation also occurred in isolated pools in the upper portions of these rivers during the dry season.

Since 1915 there has been a conspicuous decline in malaria in California, due to control measures. However, most of the eases still occur in these river valleys (California Department of Public Health 1940), although much of it is attributed to A. maculipennis propagation in irrigated fields in these districts.

A. maculipennis finds suitable environments for propagation elsewhere in California and in the Pacific Northwest (Stage and Gjullin 1935) in spring and seepage water and along the grassy edges of permanent ponds and small lakes. Webber (1920) reports endemic malaria at an altitude of 5482 feet. However, it appears that malaria and anophelism elsewhere than in the river valleys named above are of relatively little consequence.

2. Mexico, Central America, and the West Indies. The elevated plateau of central Mexico is flanked on either side by a high chain of mountains, the Sierra Madres, which slope down to the Pacific Ocean on one side and to the Gulf of Mexico on the other. The peninsula of Southern California is a continuation of the American coast range, while the peninsula of Yucatan is composed entirely of low calcareous plains. The central Mexican plateau reaches an elevation of 8000 feet in the vicinity of Mexico City ; its southward slope is abrupt and its northward slope is very gradual. River systems are searce, and much of the northern part of the country is arid or semi-arid. Breeding sites for the several species of malaria vectors present in the country are spread widely, and occur in topography varying from the flat coastal plains to the high central plateau.

The basic topography of the Central American countries is not uniform throughout, but in general low-lying coastal plains rise to mountain ranges in the interior. Notable exceptions are the San Juan River and Lake Nicaragua regions in the southern part of Nicaragua, where a wide strip of lowland cuts the mainland from the Mosquito Bank to the Pacific Ocean. Large areas of swampland border the eastern

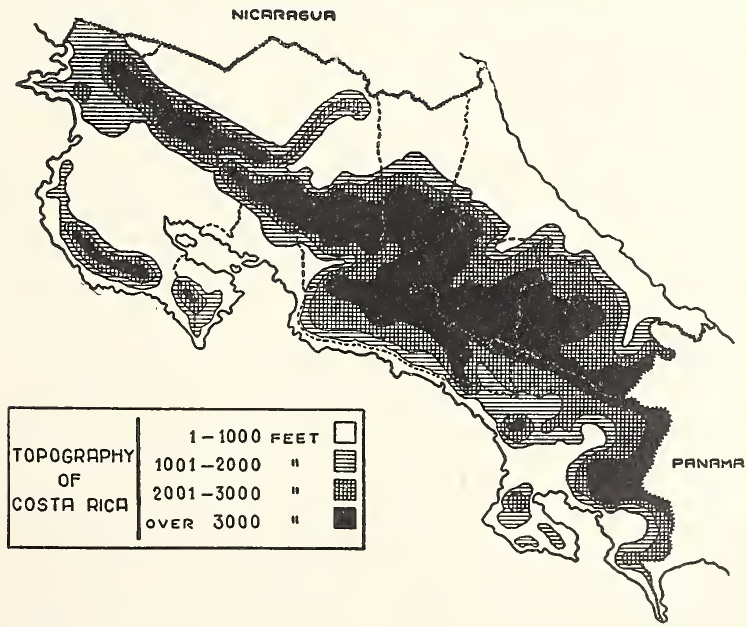

Fia. 3. Topographical map of Costa Rica (from Kumm and Ruiz 1939b). 


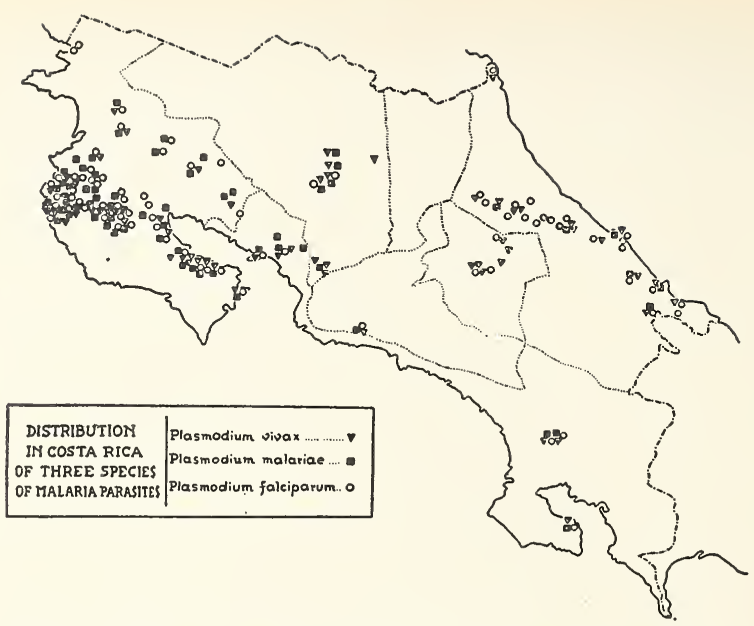

Fig. 4. Distribution of malaria in Costa Rica (from Kumm and Ruiz 1939b).

coasts of Nicaragua, Honduras and British Honduras.

The islands comprising the Greater and Lesser Antilles of the West Indies represent the summits of a submerged mountain chain. Hilly regions of various configurations occupy the interior of many of the islands, and coastal plains border the sea. Malaria throughout the West Indies is chiefly coastal in distribution, rarely reaching altitudes greater than 500 feet.

It is important to note that regional variations in topography do not always conform to the general conditions cited above. River valleys, seepage basins and sometimes, wide areas of flat country may be found in the interior of many of the Central American countries and the West Indes. These local deviations from the basic physiography may provide abundant anopheline breeding places (Kumm 1940c).

The lowlands adjacent to the Pacific Ocean and the Gulf of Mexico are well suited to the breeding of $A$. albimanus, particularly along the eastern coast where it is a dangerous vector in the state of Vera Cruz (Hoffmann 1929b). On the west coast
A. pseudopunctipennis predominates (Hoffmann 1928). However, A. albimanus oceurs abundantly at least as far north as Los Mochis (Boyd 1940f). The highlands in the central plateau are also spotted with endemic centers of malaria, particularly in the valley of Mexico at an altitude of approximately 8000 feet. Two species of anophelines occur in this region, $A$. pseudopunctipennis and A. maculipennis var. aztecas. It is claimed that the latter is the chief transmitter in Xochimilco, a short distance from Mexico City (Bustamente 1939). This is probably the highest altitude in North or Central America where endemic malaria has been known to exist for a long period of time. Clinical histories have been obtained from this region since 1875 (Bustamente). In the vicinity of Mexico City there are numerous lake beds and extensive swamp lands, combined with abundant rainfall at certain periods of the year and an average mean temperature of approximately $60^{\circ} \mathrm{F}$ in the spring and summer.

On the peninsula of Yucatan the lowlying coastal marshes provide abundant breeding places for A. albimanus (Hoff- 
mann 1934), and the malaria of this part of Mexico is restricted to sea-level conditions.

A wide variety of climate and topography are provided in the Central American countries of Guatemala, Salvador, Honduras, Nicaragua, Costa Rica, and British Honduras. For the most part, however, the flat coastal plains which flank the Pacific Ocean and the Caribbean Sea provide the most suitable breeding places for anopheline vectors, chiefly $A$. albimanus. Malaria occurs in the highlands of Guatemala at an altitude of 5000 feet (Raynal 1932) where $A$. pseudopunctipennis is the transmitter. $A$. albimanus, however, adapts itself to altitudes of 3280 feet or higher in Guatemala (Mira 1936). In a recent malaria survey of Costa Rica, Kumm and Ruiz (1939b) demonstrate that the most important areas of malarial endemicity lie in the coastal regions, at elevations less than 1000 feet. The accompanying maps (Figs. 3, 4 and 5) show the relationship of topography to the breeding areas of $A$. albimanus and likewise to the endemic centers of malaria. $A$. argyritarsis flourishes in the highlands but attains its maximum prevalence in places where malaria does not oceur; thus it is not considered to be an important transmitter.

The malaria problem in Panama centers chiefly around $A$. albimanus breeding sites provided by fluvial and coastal marshes, and by the low wet areas adjacent to the Chagres River. The lake systems provided for the Panama Canal serve as breeding places for A. albimanus, particularly Gatun Lake (Simmons et al. 1939).

The basic physiography of the islands included in the West Indies is essentially the same, and, for the most part, the endemic centers of malaria are coastal. The central portion of these islands is generally hilly or mountainous, and it is in the coastal marshes and river beds that anopheline breeding occurs.

The coastal plains are often almost flat and their water tables are near the surface. The rivers, except for short periods of flood, are sluggish and not infrequently their mouths are blocked by wave action. These conditions make for poor drainage of the coastal lands and for the formation of marshes and swamps. Seepage areas occur frequently and each pool of water, however small, becomes a potential breeding place

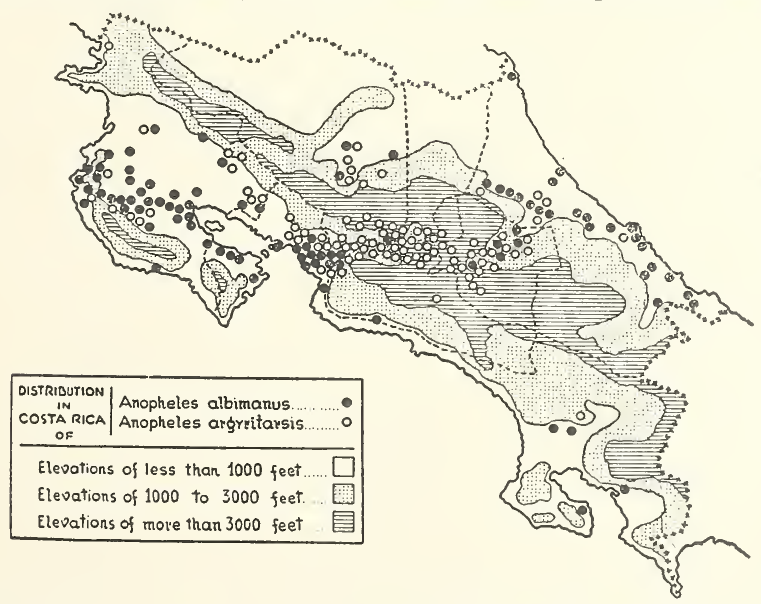

Fı. 5. Distribution of A. albimanus and A. argyritarsis in Costa Riea (from Kumm and Ruiz 1939b). 
for A. albimanus. Since the coastal lands are most suitable for agriculture, there is a natural tendency for the rural population to be concentrated near sea-level, a combination of circumstances which favors the transmission of malaria.

During the dry season some of the surface collections of water may disappear. The current in the rivers becomes slower and anopheline vectors may propagate in algal mats which form on the surface. In the upper portions of the rivers, particularly in the dry season, the running streams may give way to isolated pools and in them anophelines find suitable breeding places.

Malaria in Puerto Rico is most severe in the coastal plain (Earle 1930a), although it occasionally becomes epidemic in the foothills at the southern part of the island. $A$. albimanus, $A$. grabhami and $A$. vestitipennis are found on the island, and none of them occurs in abundance above an elevation of 600 feet. In all regions where epidemics have been investigated $A$. albimanus has been the predominant anopheline found. It has a wide range of habits and breeds well in both temporary and permanent waters in the lowlands (Earle 1930a).

A complete malaria survey of Cuba has not yet been made, but is now in progress under the direction of Henry P. Carr. In the first detailed report of the work to date (Carr, Melendez and Ros 1940) the prov-
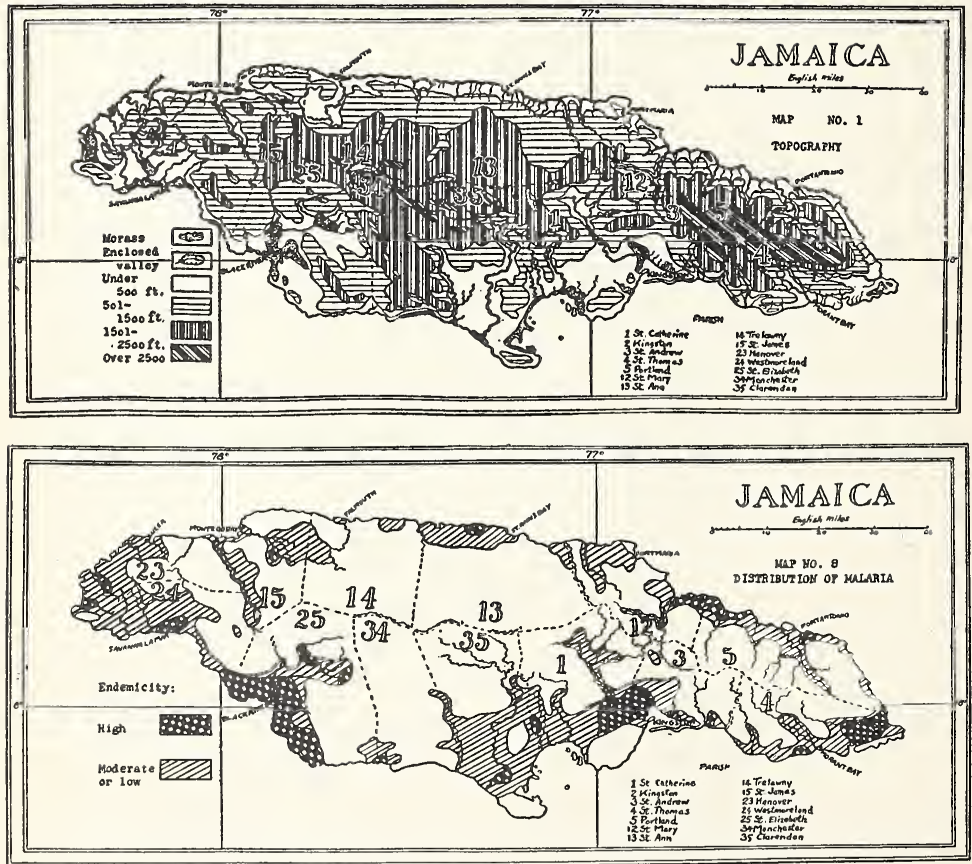

FIGs. 6, 7. Topographical map of Jamaica. Distribution of malaria in Jamaica (from Boyd and Aris, 1929). 
ince of Oriente on the most easterly part of the island is considered. Here the malaria problem oceurs in areas less than 1000 feet above sea-level where flat alluvial river valleys provide breeding places for $A$. albimanus. The greater part of Cuba, except Oriente, has a solution topography. However, the general physiography favors drainage rather than stagnation of water.

Similarly, in Jamaica (Boyd and Aris 1929) small areas of high endemicity occur in the lowlands, below 500 feet, as shown in Fig. 7, but the central plateau and mountainous areas are relatively free from malaria. In the lowlands $A$. albimanus is the dominant species and is regarded as the important malaria carrier. The accompanying figures (Figs. 6, 7) show the relation between the topography of the island and the endemic centers of malaria.

On the island of Haiti $A$. albimanus breeds in coastal swamps and springs near towns, at elevations "below 3000 feet" (Cook 1930) and, so far as present records indicate, malaria is restricted largely to the coastal regions.

In Trinidad (de Verteuil 1933) and

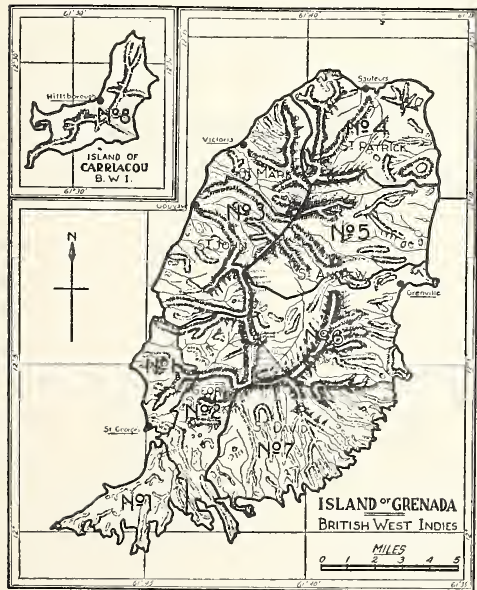

Grenada (Root and Andrews 1938) malaria occurs in the coastal regions, also, but the principal vector on these islands is $A$. tarsimaculatus. This species breeds in mangrove swamps and in connection with small streams and rivers along the coastal lowlands. The hilly interiors are not malarious.

The numerous other small islands located in the Lesser Antilles have not been surveyed for the distribution of malaria to any great extent, but from the observations which have been made in other parts of the island chain it is probable that coastal malaria predominates.

\section{Climatological Factors Which Influ- ENCE THE Distribution OF Malaria}

Aside from the more specific effects of regional topography on the distribution of malaria, it is important to remember also that the disease occurs only within certain climatological limits. In general, the northern and southern limits follow, although not precisely, the mean summer isothermal lines of $60 \mathrm{~F}$ ( $60 \mathrm{~N}$ to $40 \mathrm{~S}$ latitude). Different zones of malaria have been defined

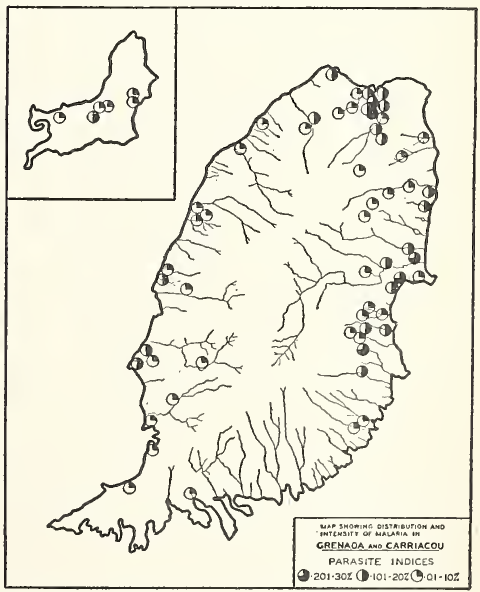

FIGS. 8, 9. Topographical map of the islands of Grenada and Carriacou. Parasite indices in Grenada and Carriacou (from Root and Andrews 1938). 
by James and Christophers (1922) and Gill (1938a). The former authors recognize temperate, sub-tropical and tropical zones, and the latter divides the distribution of malaria into 4 zones as follows :

\begin{tabular}{|c|c|c|}
\hline Zone & $\begin{array}{l}\text { Meteorological } \\
\text { characteristics }\end{array}$ & $\begin{array}{l}\text { Type- } \\
\text { area }\end{array}$ \\
\hline $\begin{array}{l}\text { Tem- } \\
\text { perate }\end{array}$ & $\begin{array}{l}\text { Temp. } 16-20^{\circ} \mathrm{C} \text { dur- } \\
\text { ing hot-test months. } \\
\mathrm{R} \text {. H. } 70 \text { per cent or } \\
\text { more. }\end{array}$ & Holland \\
\hline $\begin{array}{l}\text { Sub- } \\
\text { tropical }\end{array}$ & $\begin{array}{l}\text { Temp. } 20-25^{\circ} \text { C. } R . \\
\text { H. } 50 \text { per cent or } \\
\text { more. }\end{array}$ & S. Italy \\
\hline Tropical & $\begin{array}{l}\text { Temp. } 25^{\circ} \mathrm{C} \text { or more. } \\
\text { R. H. } 50 \text { per cent or } \\
\text { less. }\end{array}$ & N. India \\
\hline $\begin{array}{l}\text { Equa- } \\
\text { torial }\end{array}$ & $\begin{array}{l}\text { Temp. } 25^{\circ} \mathrm{C} . \mathrm{R} . \mathrm{H} . \\
70 \text { per cent or less. }\end{array}$ & $\begin{array}{l}\text { Ceylon } \\
\text { (WetZone) }\end{array}$ \\
\hline
\end{tabular}

Different epidemiological types of malaria exist in each of the roughly-defined endemic belts, and elsewhere in this monograph these types are described.

According to Gill's classification there are seven climatic zones of malaria in the two hemispheres. A single type of malaria is not universally distributed throughout each zone, however, since local topographical features (high altitudes in particular) may inhibit mosquito breeding. Furthermore, the presence of large bodies of water or deep mountain-rimmed valleys may affect the temperature and humidity of surrounding regions, and either favor or deter mosquito propagation.

Rainfall is another factor which is greatly affected by regional topography. Arid or semi-arid regions such as occur in the southwestern states and northern Mexico are the direct result of the Rocky Mountain and Pacific Coast ranges. In the western United States the atmospheric drift from the $\mathrm{Pa}$ cific Ocean gives part of California, Washington and Oregon a marine climate, with small annual or diurnal temperature changes. In California most of the rainfall occurs in the winter months. The western mountain ranges, however, precipitate most of the moisture brought from the $\mathrm{Pa}$ cific on their windward slopes, making the great region between the 120 th and 100 th

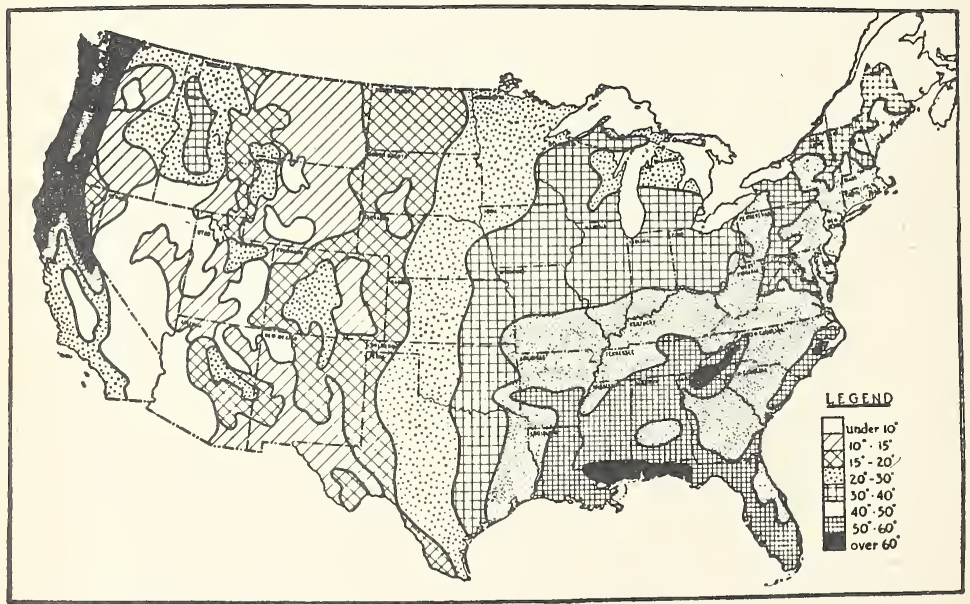

FIG. 10. Precipitation over the United States. The different shadings show the precipitation in inches as an average over a period of years (from Conservation in the United States by Gustafson et al., Comstock Publishing Co., 1939). 
meridians arid or semi-arid. Thus the desert regions of Utah, Arizona, Nevada and New Mexico do not provide favorable sites for the propagation of mosquitoes, unless, of course, man-made irrigation systems interfere with the natural land conditions. In the South, easterly winds from the Atlantic Ocean and Gulf of Mexico bring most of the southern states into the semi-marine or semi-tropical belts. Rainfall in this area generally exceeds 40 inches per year (Fig. 10).

Boyd and Ponton (1933) have shown that periods of excessive summer rainfall coincide with periods of increased malaria morbidity. In so far as generalization is permissible it may be said that when the season of highest temperature coincides with the season of greatest rainfall general conditions for the transmission of malaria are most favorable. Recent observations in the Tennessee Valley indicate that prolific propagation of $A$. quadrimaculatus occurs only during periods when minimum water temperatures of $70^{\circ} \mathrm{F}$ are maintained. This coincides roughly with the period when the mean daily temperature is $70^{\circ} \mathrm{F}$ or higher. High atmospheric humidity is conducive to longevity of Anopheles and probably influences favorably the development of the exogenous development of Plasmodium.

\section{Summary}

Malaria in the United States is confined largely to the Atlantic and Gulf Coastal
Plains. In this region it is transmitted by A. quadrimaculatus which propagates in collections of water associated principally with two types of topography, namely:

(1) Solution topography produced by erosion of limestone strata by percolating ground water. Subterranean caverns are formed and these tend to collapse, producing surface "sinkholes" which often contain water and constitute suitable breeding places for mosquitoes.

(2) Collections of water associated with meandering old-age streams of the region.

Relatively minor endemic foci of malaria transmitted by A. maculipennis occur in the Southwest and along the Pacific Coast, particulariy in the San Joaquin and Sacramento River valleys of California. For the most part, this malaria is due to $A$. maculipennis propagation in irrigation waters; to a minor extent it is correlated with natural breeding places of this mosquito.

Malaria in the lowlands of Mexico, Central America and the West Indies is associated with the propagation of $A$. albimanus and $A$. tarsimaculatus. These mosquitoes find breeding places in collections of water at altitudes less than 500 feet above sea level in the coastal plains of these places. In the high lands of Mexico A. pseudopunctipennis and perhaps $A$. maculipennis are the vectors. Their breeding places are not associated with a particular topography but rather with collections of water for irrigation. 


\section{MALARIA AND THE COMMUNITY}

\section{By L. W. HACKETT}

INTERNATIONAL HEALTH DIVISION, ROCKEFELLER FOUNDATION, BUENOS AIRES, ARGENTINA

THE most important aspect of malaria as it affects the human community is its endemicity, or degree of prevalence. This covers a wide range of manifestations, from sporadic cases to the hyperendemic situation in which few persons escape infection. But the more intensely malarious communities differ from the less malarious ones in more than the mere number of cases. With increasing intensity new factors intervene such as changes in the vitality and relative prevalence of the different species of malaria parasite, the accumulation of mixed infections and chronic cases in the population, and most important of all, a developing group tolerance to the effects of the disease which at a certain height begins to alter the picture of malaria in a radical way.

Since the nature and growth of this resistance is affected considerably by the racial composition of the population, the nature of the climate and such an inconstant factor as the relative prevalence of the plasmodial species, malaria assumes a particular expression in each community which is the function of a great many variables. Nevertheless, it takes its essential character from the rate of transmission, which is the frequency with which its inhabitants are bitten by infective anopheles -technically, the number of infective bites per 100 persons per unit of time. The frequency of inoculation will evidently depend on the numbers of the anopheline vectors, and of their sources of infection, the gametocyte carriers in the population.

\section{The ANophentNe Factor}

The efficiency of the local anopheles as vectors of malaria depends not only on their quantity, but on other factors of great importance, such as their susceptibility to infection, their longevity, their season of prevalence in relation to the periodicity of the local plasmodia, the accessibility of their hosts, the rapidity of passage and hence the vitality of the malarial organism, environmental conditions such as extremes of temperature and humidity, and many secondary circumstances which influence the host-parasite relationship but which fall outside the scope of this paper.

More important than any of these, to the human community, is the degree to which the local anopheles resort to man for food. Anopheles in general obtain their blood meals from a wide variety of sources, but it has been observed that any one species appears to have a range of preferred hosts, not necessarily the same from place to place, in the presence of which it ignores other accessible sources of blood. Man may or may not be included in this preferred group. If not, the anopheles are harmless, regardless of their numbers, as shown by the numerous malaria-free areas which produce anopheles in variety and abundance. If on the other hand, man is the host of predilection, malaria will be extraordinarily severe and spare no one in the community, as seen throughout the range of A. gambiae in Africa and in the recently invaded area of northeastern Brazil. Between these two extremes fall the other 200 odd species of anopheles; and the extent to which each acts as a vector is closely bound up with the degree of its host relationship to man.

Our knowledge of the degree of contact of the various species with man was based, before 1930 , on the place of capture and the percentage of females found infected in nature. Later the precipitin test as modified by Rice and Barber (1935) permitted the determination of the proportion of insects which give a positive reaction for human blood. This has been termed the 
anthropophilic index or better, human blood ratio, and is admittedly unreliable if based on selected groups of insects found resting in particular situations such as bedrooms or stables, which may not be representative of the anopheline population at large. Furthermore, engorged insects of certain important species are impossible to find in sufficient numbers for an index since they disappear after feeding. Recent developments in technique (Hackett and Bates 1938) have led to the cultivation of captive colonies of many anopheles including all the Palearctic vectors, and use of such material permits a more accurate determination of instinctive host preferences.

The extent to which this supposed natural order of host preference may be altered by the local predominance of certain kinds of animal is not known. The deviation of some anopheline species from man by domestic animals has been considered the cause of the "natural" recession of malaria from entire regions in Europe (Hackett 1937). It seems likely that with vectors only weakly attracted to man, the presence of livestock may considerably reduce the transmission of malaria. It is still a question what the effect of an animal barrier is in the case of a vector species with a strong attraction for human blood. (Hackett, Russell, Scharff and Senior-White 1938.)

The terms anthropophilic (or androphilous) and zoophilic have been loosely used to describe mosquitoes which prefer human and animal blood respectively. They are perhaps useful words to indicate tendencies, but they do not divide anopheles into groups, because the same mosquito may have both characteristics: it may prefer certain animals to man, and man to certain other animals. In fact this is usually the case even with dangerous vectors, while "zoophilic" species such as A. quadrimaculatus, A. philippinensis or A. pseudopunctipennis spread malaria by force of numbers even though man stands well down in the scale of preferred hosts.

The transmission rate, therefore, which is of such fundamental importance to the community, will depend as far as the anoph- eline agent is concerned not only on the quantity of mosquitoes produced, or the host-parasite relationship within the insect, but also in large measure on the degree of host relationship to man, all these factors combining to determine the local efficiency of the species as a vector of malaria.

\section{The Sources of Infection}

The number and distribution of gametocyte carriers in the population are the resultant of another complex of factors which have not been found easy to analyze. Malaria is characteristically a carrier disease, the true carrier being an individual with gametocytes in his circulating blood, hence potentially infective to anopheles. Every case, however treated, ordinarily becomes a carrier at one time or another. It might be expected that the number of carriers would bear some fairly constant ratio to the incidence of the disease, but there is a wide disparity in reports from different regions. For example, in Egypt, in untreated villages in 1939, we found that about 50 per cent of the positive thick-film blood specimens from apparently healthy children showed gametocytes, but Clark reports from Panama that thick-film blood examination of an infected community in an untreated area showed gametocytes present in from 8 to 15 per cent of those carrying malarial parasites in their peripheral blood. Such gross differences are due to the operation of a number of factors, many of which we can identify although we may not understand their mode of action.

1. The effect of clinical attacks. It is generally thought that gametocytes are not produced continually but in waves associated with acute attacks. Their production varies with the species of parasite: with $P$. vivax they closely parallel the attack; with $P$. falciparum they follow it and persist with fluctuating numbers for some time; and with $P$. malariae their appearance is delayed sometimes for months, though they continue to turn up at irregular intervals for a long period. However, Swellengrebel and his collaborators were impressed by the fact that in Holland cer- 
tain families continued to be foci of anopheline infection (vivax) for at least two successive autumns without the occurrence of fresh cases of malaria. Later they noted (1937) that children as often as not became carriers without a fresh attack, but adults usually were not good infectors without a recent history of acute symptoms.

2. The effect of season and environment. In the community gametocyte carriers are most numerous during and after an epidemic or at the height of the transmission season. A survey at these times will not give the same information as an interepidemic survey, or one which includes febrile cases as one based on apparently healthy carriers. The picture also changes with the season for other and more mysterious reasons, due to the long term relapses of $P$. vivax in spring, the dominance of falciparum in summer and fall, and the persistence of malariae into the winter. Seven patients infected simultaneously with $P$. falciparum and $P$. vivax by Boyd and Kitchen all had initial attacks characteristic of $P$. falciparum, but three relapsed in the following spring with $P$. vivax.

Gill (1938a) has proposed the theory that there may be environmental causes (possibly climatic or solar in origin) which operate periodically (in endemic regions) to produce waves of relapses which thus tend to occur simultaneously over great areas and stimulate gametocyte production on a large scale as the prelude, not the result, of epidemic outbreaks of the disease. We know nothing about this except from rather ambiguous epidemiological evidence.

3. The effect of immunity. A growing immunity represses gametocyte production; it does not stimulate it. Gametocytes are found earlier in relapses, but they are more constant and abundant in primary attacks, and diminish in number with each recurrence of symptoms and parasites. In the tropies where immunity is more rapidly and permanently established in the first decade of life than in temperate climates, it is usual to find the gametocytes under more stringent control than the trophozoites. Thomson found in native children of
Nyasaland that, while all forms of $P$. vivax and $P$. malariae had virtually disappeared from the circulation by the age of nine, all the children continued to show ring-forms of $P$. falciparum although no gametocytes of any kind were to be found.

In a temperate climate with a very high transmission rate and a long summer, the effect of immunity on $P$. vivax infections is almost as striking as in the tropics, since the tolerance is more rapidly acquired and survives the winter pause more easily than in the case of $P$. falciparum. Thus in a village in Sardinia, Missiroli found threequarters of the infants infected with $P$. vivax in the fall, but only one-third of the preschool group (2-5 years) and about a sixteenth of the older persons. $P$. vivax infections began to give way to $P$. falciparum during school age. There were twenty times as many vivax gametocytes in the school children as in the adults, and twice as many $P$. falciparum gametocytes (Hackett 1937).

4. The effect of diversity of species and strains of the parasite. Each species of plasmodium has its own characteristic output of gametocytes. In Egypt we found twice as many carriers in the $P$. falciparum cases as in the $P$. vivax cases, at all levels of intensity and in all seasons. A high inoculation rate will increase the $P$. falciparum carriers at the expense of the other two species, while a low transmission favors $P$. vivax and $P$. malariae. In towns in Algiers after a whole year in which extraordinary climatic conditions prevented anopheline breeding, Parrot and Catanei found the following situation:

Gametocyte earriers in $P$. falci-
parum positives:
Gametocyte carriers in $P$. vivax
positives:
Gametocyte carriers in $P$. malariae
positives:

Old cases of $P$. falciparum infection are the least likely and old cases of $P$. malariae infection the most likely to remain foci of anopheline infection.

An important point in connection with a diversity of strains and species is that 
there is relatively littlc cross immunity among them. An individual would soon become completely immune to any one organism with which he might be constantly reinfected, but each new strain, like a new species, finds the host defenceless and initiates a train of events culminating in an acute attack, and a period of gametocyte production.

5. The effect of age and exposure. Since immunity is developed by exposure except in very young infants, it has nothing really to do with age, but only with number and frequency of infections. A high inoculation rate tends to concentrate the persistent and dangerous carriers in the earlier agegroups. D. B. Wilson (1939), in a series of important papers on malaria in East Africa, describes such a situation among the Bantu in Tanganyika, who live in an area of high endemicity. All the babies are infected before they are 5 months old and the period of acute infestation lasts about 18 months. There is little danger to life after 6 months. Gametocytes reach their height at the end of the second year, and are seldom seen in adults. There are all gradations between such a situation and areas of sporadic malaria with no measurable immunity at all. In rural districts of Kenya 29 per cent of the natives under 10 years of age were gametocyte carriers, but less than 2 per cent of the rest, while Clark and Komp in Panama, with a less established immunity, found 41 per cent of the children carriers, and 29 per cent of the adults.

In very young babies there appears to be a resistance to infection derived possibly from the mother. Clark (1937) found in Panama that infants enjoyed almost complete protection in the first two months of life although even more exposed to mosquito bites than adults. Strickland, Sen Gupta and Mazumdar observed in India that the average age of infants at the first attack was from 3 to 4 months depending on the season, while Barber, Mandekos and Rice found in Macedonia that older infants were more susceptible to infection than younger ones.
6. The effect of treatment. None of the drugs now at our disposal is able to prevent malaria from relapsing, or the appearance of gametocytes. We can possibly cut down the number of relapses somewhat, but never sufficiently to protect a community from continual reinfection. Even small groups under discipline cannot be sterilized. Ciuca found that neither quinine nor atabrine prevented the appearance of gametocytes in as many as 149 out of 269 cases of induced $P$. falciparum infections in hospital. Simmons (1939a) reports that in Panama more than 20 per cent of soldiers receiving the standard treatment of quinine followed by 15 grains daily for 3 months, became carriers while still taking the drug. Bispham claims that with suitable atabrine treatment, over 90 per cent of $P$. vivax carriers can be cleared of parasites. His experience was confined to encampments of young men, while communities with children and babies are quite another problem. Even so, the clearing of only 90 per cent of parasites would hardly be adequate protection for a community. Barber, Rice and Mandekos present proof that both in the Balkans and in Liberia even one heavy carrier in a village could raise the sporozoite index of the anopheles and cause a notable increase in transmission.

Neither in Spain nor Holland, where the malaria is principally a mild tertian, nor in Sardinia or the Balkans where the malaria is severe, nor in Panama where the climate is tropical and infection goes on the year round, have the most intensive and persistent treatment campaigns been able to destroy the seedbed of the disease.

7. The effect of race. The subject of carriers is complicated in many regions, such as Panama, Africa and the Southern United States, by the presence of a mixture of races. Negroes have a notable tolerance for $P$. vivax infections and while they are susceptible to $P$. falciparum and $P$. malariae, the clinical course of the disease is milder than in the white race. In the southern states, the white children have more $P$. vivax parasites and the negro children more $P$. falciparum. Thus in the 
Wilson Dam area, T. V. A., the peak of malaria for whites is in June and for negroes in August. These differences are confirmed in the laboratory. Among G. P. I. patients, negroes exhibit a high degree of tolerance to $P$. vivax inoculations, which is ordinarily absolute for the strains now being employed in malaria therapy. This tolerance is found in children as well, which suggests that it is racial and not acquired.

In areas where $P$. falciparum infections predominate the matter is not so clear. D. B. Wilson (1939) believes that in the black population in East Africa, inherited tolerance plays little part in the ultimate acquisition of immunity, for the babies suffer from intense infections, and while they rapidly acquire resistance, this is not dependent upon race but upon frequency of infection. This is supported by van Nitzen who says that natives of Ruanda when they come to the highly malarious mining camps of Katanga, get attacks comparable to those of Europeans, with cerebral forms and blackwater fever. Acclimatization takes about 3 years. Nevertheless Giglioli (1938c) points out that in British Guiana, negroes and East Indians live under exactly the same conditions and are equally exposed to malaria. The negroes become infected with malaria like the Indians and the parasite rates are comparable (60 and 74 ), but the negroes tolerate the infection better and react less violently with a spleen rate of 13 as compared with 70 among the Indians. The fact that the parasite rates are so nearly equai shows that the tolerance is to the effects of the parasites and not to the parasites themselves, and negro communities, while not great sufferers from clinical malaria, may serve as continual foci of infection to other racial groups.

8. The influence of sex. Significant differences are occasionally reported between the sexes. Russell found the parasite rate for boys to be 32 and for girls 22 in some Indian villages, and I have noted the same thing in Sardinia. This is not a constant finding, and is usually attributed to differences in exposure to infection.
9. The effect of individual and family disposition. Clark found in Panama that the bulk of the malaria was confined to the same individuals or families year after year, and Barber and Komp, out of their long experience, came to the considered opinion that "it is the occasional heavy carrier who is most dangerous to a community."

10. The influence of social factors. Malaria has long been classed as a social disease, and the implication is that economic stress reflected in low standards of living, undernourishment, lack of medical care, overcrowding and so forth, creates a situation so favorable to the persistence of malaria in the individual and in the group that it cannot be eradicated without drastic changes in living conditions. Small, substandard aggregations of people living under such conditions at the edge of more prosperous communities, are seed beds of malaria for the general population. Thus the negroes in our South, like the gypsies in Hungary, are foci of infection which insure a constant supply of gametocytes, but whether this is to be attributed to social factors or to the fact that these unfortunate groups are almost always more accessible to mosquitoes than the towns people is not clear. The whole question is very controversial and a considerable literature has grown up about it (Hackett 1937).

Social factors may produce a lag in the rise or fall of malaria endemicity in a community, but this has never been measured or proved to exist.

11. The influence of habits and occupation. It is unnecessary to dwell on the fact that some people are more exposed than others to infection by reason of their manner of life. In the hill towns near Rome, malaria is an occupational disease of the farmer who descends daily to the plain early in the morning and does not return to his hilltop until after the anopheles have become active in the evening.

Summing up, we find that anopheles in proportion to their efficiency as vectors, build up a carrier group in a community which is fluctuating and indeterminate in 
size and distribution, and is favored or limited by a variety of factors. This, however, results in a certain transmission rate which determines the character of the malaria in the community. At every level, this transmission rate is attended by a corresponding tolerance made up of individual specific reactions to plasmodial infection, and at high intensities, the group immunity intervenes forcefully to protect the population. It pushes the acute struggle back to the earlier ages, and when completely established, restricts it to the first two years of life. Such intensely malarious communities do not usually increase in numbers, and the adults are able to live a relatively normal and active life.

\section{Chronic Cases, Mrxed Infections and Parasite Formulas}

It is known (through induced malaria in paretics) that the immunity conferred by infection is solid and lasting in the case of $P$. vivax; solid also, but less easily acquired and more evanescent in $P$. falciparum. $P$. vivax immunity may last as long as 7 years, and that to $P$. falciparum several months at least, sufficient to cover the ordinary transmission season. It is clear that no one could develop chronic malaria by repeated infection with any one parasite. Persistent splenic enlargement is not produced in the laboratory even with several strains. James repeatedly reinfected one of his cases over a period of 5 years, first with $P$. vivax ( 7 times), then with $P$. falciparum, then again with $P$. vivax and finally with $P$. ovale without producing anything but a high resistance to all three parasites.

But this immunity is very specific. A person acquires immunity to the particular strain employed and not to any other strain. There is a slight spread of tolerance in $P$. vivax infections; but hardly any in $P$. falciparum. As for different species, it has been shown that a current or recent infection with either $P$. falciparum or $P$. vivax parasites in no way interferes with successful inoculation with the other.

Chronic malaria, then, is due to overlapping infections of different species and heterologous strains of plasmodia. Mixed infections must be the rule and not the exception in localities with even a moderate transmission rate. Christophers has pointed out that there will be overlapping infections even with relatively low endemicity, for if one hundred infections were distributed at random among a hundred individuals, the chances are that:

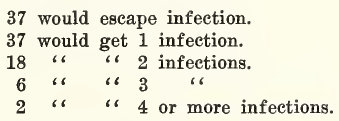

There is thus being formed a nucleus of individuals who have been infected twice or more, and in fact 26 per cent will already have mixed infections.

It is well known that these mixed infections are not found in the ordinary microscopic survey of blood specimens. Naturally we should not expect to recognize strains, but the species ought certainly to show up. Yet mixed infections are reported as present in only 2 or 3 per cent at most of the preparations. There is an antagonism between the three common species which ordinarily enables only one of them to remain in the circulating blood in sufficient number to be discovered by routine examination. It seems that $P$. falciparum usually commands during the acute stage, $P$. vivax tends to dominate the long term relapses, while $P$. malariae, a tenacious parasite, outlasts them both.

The only way to obtain an approximate idea of the percentage of mixed infections in a population is to follow a group through the year with persistent blood examinations. The proportion found will naturally correspond to the intensity of the malaria. Barber and Komp found 45 per cent of a group of children in the southern United States varying in type of plasmodium during the year, and Balfour reported the same percentage in a Greek village, although not more than 3 per cent of mixed infections were found at any one examination. We conclude that children can not grow up in a malarious locality of even moderate endemicity without acquiring a 
representative assortment of all the species and many of the strains of plasmodia with which the local anopheles are infected.

But if every chronic case of malaria is harboring many different strains of parasites and usually more than one species, the parasite formula, or relative prevalence of parasite species found in a blood survey of a community, is not an indication of the actual distribution of the parasites but only of the frequency with which each happens to be dominating the blood picture in infected individuals at the moment of taking the blood samples. We must look then for other factors besides the chance distribution of infective bites, which might account for the notable variations in the relative prevalence of the different species in the blood of populations at different times and under different conditions. They are internal factors which govern the host-parasite relationship and have to do with the peculiar biology of each plasmodial species as well as with the differential reaction of the human organism to the specific infections. P. falciparum causes an acute disease and builds up its numbers very rapidly. It is greatly favored by a high transmission rate and acquires a long lead over the other forms in epidemics. It loses ground as rapidly when transmission begins to fail, possibly because it requires more gametocytes than $P$. vivax to infect anopheles. The more persistent $P$. vivax takes its place, characterized by latent periods and long term relapses. $P$. malariae is masked by either of the other species in the acute stage: but it long outlasts them both and clings indefinitely to the human organism. Mühlens reports one case which relapsed, with parasites in the blood, 19 years after infection. Much of the so-called mystery of quartan fever is explained by the persistence of the parasite and its tendency to be recessive.

The waxing and waning of human immunity also plays a part in the appearance and disappearance of parasites from the circulation, and hence in the parasite formula. From all these considerations, it is clear that the parasite formula determined from groups containing febrile cases will differ from that based on apparently healthy carriers.

The mixed character of apparently simple infections is shown by the fact that with low transmission one parasite increases at the expense of the others. Thus in Sierra Leone, Gordon and Davey found the total malaria relatively constant, but the proportion of positives for $P$. malariae varied all the way from 0 to 22 per cent in 15 years. In tropical areas where the transmission rate is continuously high $P$. falciparum dominates the blood picture at all seasons and in all ages. Other species are present in due proportion during the first years of life, but are encountered more and more rarely later on.

There is finally the differential effect exerted on the relative prevalence of the various organisms by their specific incubation periods in the mosquito. That of $P$. vivax may be several days shorter than that of $P$. falciparum, and only half that of $P$. malariae. Since under some climatic conditions, these periods may approximate the life span of the insect, they may notably influence the parasite formula of the community.

Thus the multiform character of the plasmodium and the specificity of the immunity produce in the malarious community, as in the constantly exposed individual, a continuous immunological activity which becomes more and more complex as the rate of inoculation increases. The frequency of successive heterologous infections, the waxing and waning of specific tolerances, and the behavior peculiar to each species of the parasite, together determine the aspect of malaria, which is reflected in a way we are not able fully to analyze in the blood picture of the individual and the parasite formula of the community.

\section{Endemic and Epidemic Malaria}

The transmission rate gives malaria its volume and intensity, but the diversity of organisms creates that fluctuating and polyvalent immunity which characterizes 
chronic malaria in the individual and endemic malaria in the community. Endemic malaria is malaria which is constantly present in a degree to give a measurable amount of morbidity or splenomegaly. Any rapid increase of acute cases is called an epidemic and may take the form of an outbreak in a small community, or the expected seasonal wave of morbidity in temperate climates, or a widespread regional exacerbation of the disease on a large scale. Almost always, the epidemic manifestations have an endemic basis.

It is possible of course to have pure epidemic malaria, caused by the advent of a carrier or carriers into an uninfected area where there are anopheline vectors. This happens once in a while in the northern states. However, where periodic epidemics occur, the sources of infection are usually present, widely though often sparsely distributed throughout the population. If there is little evidence of malaria in the interepidemic season, this is called hypoendemic malaria. Such a situation comes about through a very low transmission rate or a very short transmission season. Still, the malaria may have a certain intensity in summer and carry on from year to year. In western Spain the summer epidemic may involve 30 per cent of the population, yet the winter parasite and spleen rates may be nearly zero. The inhabitants have almost but not quite time enough to recover between seasons.

At the other extreme, where transmission is constant at a higher or lower level, we may have pure endemic malaria, although this too is rare. It is approached in such tropical places at Laos in Indo-China where malaria prevails everywhere without intermission, and with little seasonal or annual variation. Epidemics are unknown, though hospital returns indicate an increased prevalence during the rains. Where the transmission rate is very high and constant, complete immunity may be established, as in certain Bantu tribes on the East African coast, described by D. B. Wilson (1939). Everyone is infected a dozen times a year and there is little illness after 2 or 3 years of age. No ill effects in older children or adults are seen which ean be attributed to malaria. The parasite counts in such a population are very interesting. They range from 8,000 per ec in infants to less than 200 in the age group 9-25. The tolerance is like immunity to sunburn, obtained only by frequent and prolonged exposure.

Between these extremes lies the broad field of endemic malaria with periodic epidemic waves. Transmission is seasonal, and if it is high, we have overlapping infections and chronic eases, while if it is low. the infections are widely spaced and the cases are mainly acute. The malaria is called hyperendemic if the spleen rate is constantly above 50, and sporadic or hypoendemic if the winter spleen rate is insignificant.

Within these limits two general situations occur, depending on the transmission rate, which are not sharply divided from each other, but grade from one into the other. One of these is characterized by poorly developed group immunity, and the type is chiefly epidemic, with a permanent and measurable endemic seed-bed. Such malaria can be identified by :

(a) Its high seasonal peaks.

(b) Little adult immunity to infection.

(c) No pronounced tendency to restrict splenomegaly, high parasite densities and carrier incidence to the early age groups.

The high seasonal and periodic increases in malaria may have several causes: a seasonal increase in anopheline production due to rain or lack of rain depending on the species; the introduction of a new anopheles, as in Calcutta and northeastern Brazil, which can build up serious epidemics on the foundation of a mild pre-existing endemic malaria; or possibly a wave of gametocyte production in the population provoked by some environmental cause. Gill (1938a) has recently called attention to this phenomenon in connection with the great Ceylon epidemic of 1934 . He believes this began with a simultaneous outbreak of relapses in latent cases due to unusual meteorological conditions, and cites in support of his theory the low incidence among babies and unimportant mortality in the population during the first weeks of 
the epidemic. The epidemic, according to Gill, broke out everywhere on virtually the same day all over the epidemic area, and could not therefore have been built up by an unusual prevalence of anopheles. This is characteristic, however, of malaria epidemies which are always explosive and do not spread centrifugally from a few foci of infection, as in many diseases. The seedbed is scattered far and wide and the epidemic is limited to the area in which conditions of spread are favorable. It can not spread beyond this area, and it does not actually spread within it, since the new cases arise in every part of it at once, giving a static character to the epidemic.

We have no good proof that the observed phenomena cannot be produced by the ordinary gametocyte prevalence, through the agency of an enormously increased anopheline population.

We have been discussing a malaria situation which is mainly epidemic in character with an endemic seedbed. Under more intense transmission, this is converted into a type of malaria which is chiefly endemic, but with periodic waves of increased incidence. Mixed infections prevail with a consequent preponderance of chronic cases. Such malaria is characterized by :

(a) Reduced epidemic manifestations.

(b) Adult immunity well developed.

(c) Splenomegaly, high parasite densities and high carrier incidence restricted to the earlier age groups.

This well-established group immunity is the result of a high transmission rate during a long season.

The commonest measure of endemicity is the spleen rate. However, Wilson and Wilson (1937) point out that frequency of infection in the non-immune portion of the population-that is in early life-is the most accurate index of endemicity. A reduction in the parasite count reveals developing immunity. Variation with age in spleen and parasite rates is the best basis for classification of communities according to the degree of developed immunity. It is clear that in localities with seasonal transmission, the spleen and parasitic rates taken during the interepidemic period will measure the importance of the endemic component of the local malaria, while those secured during or immediately after the transmission season will indicate the extent of the epidemic oscillation.

\section{SUMMARY}

Malaria in the community derives its character from the rate and constancy of transmission, and from the immunological diversity of the parasites. The frequency of infection depends on the one hand, on the density and efficiency of the anopheline vectors, and particularly their host-relationship to man, and on the other, on the incaleulable fluctuations in the source of infection, the gametocyte carriers. At every level, the transmission rate, thus determined, creates a corresponding tolerance which is made up of highly specific reactions to the numerous and immunologically independent strains and species of the parasite. The picture is one of growing multiplicity of mixed infections, resulting in chronic malaria and the attendant phenomenon of mutual parasite antagonisms. The mass effect, however, of group immunity at high levels of intensity is one of powerful protection of the older age groups and the shifting of the struggle to childhood or even infancy. The blood picture of the individual and the parasite formula of the group are resultants of complex factors arising from the biological diversity of the plasmodia and the differential immune reactions of the human host.

The discontinuity of transmission due to seasonal influence is responsible for the epidemic waves characteristic of endemic malaria. Only very rarely do we find pure epidemies without an endemic seedbed, or a hyperendemic situation without seasonal or annual exacerbations. The rate of transmission is best measured by the frequency of inoculation among infants, and the degree of endemicity is indicated by the developed immunity which determines the proportion of acute cases, the scope of the epidemic manifestations, and the age distribution of splenic enlargement, parasite densities and gametocyte carriers. 


\title{
THE ROLE OF ANOPHELINES IN THE EPIDEMIOLOGY OF MALARIA
}

\author{
By ROBERT MATHESON \\ DEPARTMENT OF ENTOMOLOGX, NEW YORK STATE COLLEGE OF AGRICULTURE AT \\ CORNELL UNIVERSITY, ITHACA, N. Y.
}

IN 1897 and 1898 Sir Ronald Ross discovered, for the first time, that mosquitoes are the transmitters and definitive hosts of the malarial organisms of birds and man. These results were confirmed by Grassi, Bignami and Bastianelli in a series of papers in 1898 and 1899. Since that time it seems well established that the etiological agents (Plasmodium vivax, $P$. malariae, $P$. falciparum and also probably $P$. ovale) of malaria of man are transmitted only by mosquitoes belonging to the genus Anopheles (in the broad sense). Since 1900 innumerable papers have been published on human malaria and on their transmitters, the anopheline mosquitoes. Summarizing the results of all this intensive research, it may be stated that it seems established that certain species of Anopheles are effective and dangerous transmitters of human malaria while others may be considered of minor or insignificant importance. Furthermore certain so-called "good" or "dangerous" anopheline transmitters are known from all the endemic malarial regions of the world. Again, it appears established that in some regions the "good" transmitters are especially effective in transmitting malaria, and the introduction of such species into other endemic malarial centers may, and frequently does, bring about epidemic conditions (as, for example, the introduction of $A$. gambiae from Africa to Brazil, and $A$. albimanus into Barbados, Jamaica, and St. Croix). It is also apparently well established that certain species of Anopheles may serve as "dangerous" transmitters in one region of the world and in another region be unimportant or of minor importance in the transmission of malaria (e.g., A. pseudopunctipennis is a "dangerous" species in
Argentina, Mexico, etc., while in the United States it is of minor or of no importance; A. subpictus (rossi) and A. hyrcanus are "dangerous" in the East Indies, etc., but not of importance in India; ete.). Again it is reported that in some regions (Dutch Guiana) the human population has become so attuned or adapted to the malarial organisms that the clinical symptoms of the disease have almost vanished and the people carry on quite effectively despite the parasites within them (the "efficient parasite" of Swellengrebel 1940).

Studies of the biology and taxonomy of anopheline mosquitoes have been carried on intensively in many parts of the world, particularly in Europe. In 1900 scarcely anything was known about the biology of mosquitoes and for many years thereafter it was generally considered that the biology of all species of mosquitoes was quite similar. In recent years $(1916-\ldots)$ this conclusion has been rudely shattered and we feel certain that the biology of each species is a very complicated and, in most cases, unsolved problem. Similarly with taxonomy. In 1900 very few species of anophelines were known, while today over 170 species are recorded from the world, and as we learn more about them the species becomes ever more complex (as, for example, the $A$. maculipennis complex in Europe, Asia and North America).

This brief paper is to deal with the role of anophelines of North America in the transmission of malaria. What are the species with which we have to concern ourselves? At present this is a somewhat difficult problem for we are not certain just what our species are and certainly we are far from knowing their biologies, distribution, habits, habitats, or their preference 
for man (androphilic) or animals (zoophilic). We can present only the data that are available. At the present time there have been recognized the following species of Anopheles: barberi Coq., boydi Vargas (?franciscanus MeCracken), crucians crucians King, crucians bradleyi King, crucians georgianus King, quadrimaculatus Say, punctipennis Say, pseudopunctipennis Theo., atropos D. \& K., walkeri Theo., occidentalis D. \& K., occidentalis freeborni Aitken., albimanus Wied.

All of these species have been infected experimentally with one, two or all three species of plasmodium of human malaria except $A$. boydi, $A$. crucians georgianus, $A$. occidentalis and $A$. occidentalis freeborni. ${ }^{1}$ At the present time it is generally agreed that A. quadrimaculatus and A. occidentalis and its varieties are the principal transmitters of human malaria in North America. That this assumption is correct seems doubtful to the writer. Only a few years ago we knew nothing about $A$. walkeri, yet today we know that it is widely distributed, that it readily and eagerly bites man and that it has been experimentally infected with tertian and aestivoautumnal malaria. Recently it was found naturally infected with malarial sporozoites. Only a few years ago $A$. barberi was not found north of Washington, D. C., yet today it is recorded from many localities despite its minute size and the difficulties of finding it. We know very little of its biology or its feeding habits; experimentally it has been shown to transmit tertian malaria from man to man. A. pseudopunctipennis is known to transmit malaria in Mexico and Argentina, but our form appears to play no part in its transmission. More information is required about this species in the United States.

The question before us is the role of anophelines in the epidemiology of malaria. From our present knowledge the following

1 Though Herms and Gray (1940) record this species as the transmitter of malaria in Calif. and N. Mex., I can find no data of either experimental infection or infected specimens caught in the wild, except the work of Barber and Forbrich (1933) (New Mexico, for A. maculipennis). factors would seem to have to be present in order that an epidemic might occur in a known malarious region: (a) a susceptible population exposed to mosquito attack; (b) a new strain of malaria (either a heterologous strain or an increase in the virulence of the prevalent strain); (c) a species or subspecies of anophelines present and readily susceptible to infection and androphilous; (d) favorable breeding grounds for a marked increase of the anopheline transmitters; and (e) human carriers with viable gametocytes of a new strain (herologous) or an increase in the virulence of the prevailing strain.

Early work, based on the mosquitotheory, was carried on by Doty (19011909) on Staten Island, and in some small towns near New York City by Berkeley (1901) where malaria occurred in epidemic form. Their work was very successful and malaria soon disappeared (in 1909 only 5 cases on Staten Island). Probably the first city-wide organized malarial control in the United States took place at Ithaca, N. Y., beginning after an epidemic of typhoid in 1903 and an epidemic of malaria in 1904, when there were over 2000 cases in a population of 13,000 . The health officer was given authority to require every physician to report each week every case of malaria and its location, to require a blood examination of every suspected case, to appoint an inspector and two assistants to find and eliminate anopheline breeding places, and to issue instructions as to how malaria is contracted, how to avoid infection and in regard to isolating patients. There were 1000 cases in 1905; none in 1908, and since that time Ithaca has been practically free of malaria.

In 1905, when Ithaca suffered from a severe flood, there were extensive cat-tail marshes in the northern, southern and western portions of the city in which Anopheles bred in immense numbers. Though A. punctipennis bred commonly about the city and still does, A. quadrimaculatus was recorded as the dominating and most prevalent form. Beginning in 1905 and continuing until 1912, all these 
marshes and all breeding places within private property in the city were treated each week or ten days during the mosquito breeding season with kerosene oil. Then the work became more or less haphazard until 1928, when it was again organized and is still carried on. Practically all the marshes have been drained or filled.

Only a few specimens of $A$. quadrimaculatus have been taken within the city since 1918. Now there is no malaria and the anti-mosquito work continues mainly to get rid of such mosquitoes as Aedes vexans, Culex pipiens and certain spring breeding forms.

A detailed study was made by Dr. Smillie (1927) of the results of a water impoundment at Gantt in Alabama. During 1922 (the year work was begun) there were recorded 16 cases of malaria in a population of about 250. The dam was completed in late 1923. The lower half of the dam site was partially cleared while the upper half remained uncleared with high water surrounding trees, shrubs, and all kinds of floating debris. The malaria cases in this area were 3 in 1921, 16 in 1922, 6 in 1923 (water impounded in late autumn), 238 in 1924, and 132 in 1925 (105 relapses and 27 new cases). Of the 238 cases in 1924 only 21 had a previous history of malaria and of these only 5 had the attack during the previous two years. The epidemic began slowly in June and July and reached its peak in October, 1924.

A detailed study was made of the anopheline population during 1923, 1924 and 1925. As there were practically no anopheline breeding areas surrounding the dam very few adults of $\boldsymbol{A}$. quadrimaculatus were present except in the late autumn. $A$. crucians and $A$. punctipennis were also present in small numbers. During 1924 A. quadrimaculatus was very abundant, reaching a peak in late September. The other two species showed no marked increase over the previous year. The eurve of malarial cases followed closely that of the abundance curve of $A$. quadrimaculatus. During late 1924 active measures were taken to improve the impounded water by clearing and in- creasing the use of oil or other larvicides. The water was again impounded (June 1) without the upper half of the dam being cleared, resulting in excessive breeding of A. quadrimaculatus in the upper portion of the dam. Control by larvicides was not successful and there developed 21 new cases of malaria about the uncleared area. The water was again withdrawn on July 10 , the control of anopheline breeding was established and malaria stopped.

The dam was now cleared of all timber, shrubs, and a clean shore-line was established well above the high-water line. The results as summarized by Smillie are: $\boldsymbol{A}$. quadrimaculatus is essentially a pond breeder, $A$. crucians was prevalent during spring and summer, A. punctipennis was present throughout the year but most abundant during the winter months. Epidemiological evidence clearly demonstrates that $A$. quadrimaculatus is the vector of malaria in this area.

It is very unfortunate that in this interesting and illuminating study of a malaria epidemic in a restricted area no dissections of anophelines were made.

In the late summer of 1935 a rather striking epidemic of malaria occurred in Camden, N. J. From 1912 to 1934 a total of 61 cases of malaria had been reported from the entire county of Camden. In the late summer of 1935 Butts (1937) reports a total of 120 cases in a comparatively small section of Camden City, in the northern area close to low, marshy ground near creeks. A case of malaria had returned from South Carolina shortly before the appearance of new cases in this vicinity. However the city has a central airport near the marshy area and heavy traffic from all parts of the country passes through this district. On September 11 and 12 collections of mosquitoes were made which showed the presence of small numbers of A. quadrimaculatus and A. punctipennis. From September 14 to September 28 daily trappings were made and a total of 2748 mosquitoes was secured, of which 53 were female Anopheles (species not indicated).

At the time of Butts' report (August 14, 
1936) there were 16 relapsing cases and several relapsing cases (number not stated) observed in 1936 that were not included in the 120 cases reported for 1935 . The origin and course of this malarial epidemic is rather obscure. Butts reports no new cases up to August 14, 1936, but only relapses. All infections recorded were due to $P$. vivax.

The state health officer (Dr. Mahaffy) reports only 1 case in 1937, none in 1938, 1 in 1939 and 1 in 1940.

A peculiar epidemic occurred, in 1934, in Aurora, Ohio, a small village of about 1,000 inhabitants where no malaria had been known, at least since 1920. On September 3, 1934, seven cases were reported, two of which had parasites $(P$. vivax) in their blood. The publicity of these cases soon resulted in the discovery of 22 cases that developed prior to September 3. Later there were 10 new cases in September and 5 in October, or a total of 37 cases between August 21 and October 23, or 3.7 per cent of the total population. The origin of this epidemic is obscure, though Hoyt (1935) reports a case of malaria in a house painter who had malaria in Florida and a relapse in May in Aurora (1934). Search for mosquitoes demonstrated the breeding of $A$. punctipennis but not of $A$. quadrimaculatus. However, A. quadrimaculatus was found in the houses but a dissection of six adults proved negative. As mosquito control measures were started at once no further information could be obtained.

Probably as interesting and as sharp an epidemic as recorded is one reported for a small section of the Elk River which empties into Wheeler Lake, Alabama. Smith (unpublished thesis 1939) investigated this outbreak and; his report may be briefly summarized. The area is part of the T.V.A. impoundment of Wheeler Reservoir. In this locality (Limestone County) not a single breeding place could be found before impoundment within two miles of its high-water line. After the reservoir was filled certain localities along the Elk River furnished favorable places for breeding of A. quadrimaculatus. A malarial survey in
1934 (two years before impoundage) showed 22 cases in a small peninsula in this area. This land was purchased by the Authority and depopulated before impoundage.

During the summer of 1937 certain collecting stations in this localized area showed high mosquito counts and in October of the same year came a report of a malarial epidemic. The area in question is populated by tenant farmers $(2 / 3)$, and land owners $(1 / 3)$. About one-third of the tenant farmers move each year, but in general the population is quite stable, the same people remaining year after year. Since the Authority had complete information of every house within one mile of the reservoir it was not difficult to make a detailed survey. The entire population numbered 842 in 176 houses. Blood smears were collected (in early October) from 743 of these people. Of these, 106 (14.2 per cent) had malaria parasites (68 with $P$. falciparum; 38 with $P$. vivax). Case histories showed a much higher incidence in August, at least 50 per cent of the population. The antimalarial drugs taken by most of the people tended to prevent the showing of the parasites in the blood. As malaria was not common in this locality the case history records are of great value. In 1936 only 94 gave positive case histories, while in 1937 there were 370 with positive case histories and 106 (in October) showed malaria parasites in their blood.

What was the source of this epidemic and how to account for its severity (50 per cent of the entire population) in an area about 12 miles along the Elk River and for a distance of fully one mile on each side cannot be answered with certainty. Some 92 persons gave positive case histories for $\mathbf{1 9 3 6}$ and a number of tenant farmers from other endemic areas moved into the area that spring. The anopheline breeding in this area during 1936 showed an average weekly catch of $\boldsymbol{A}$. quadrimaculatus per station (Nos. 15, 17, 18 and 19) of .25, 2.83, 2.58, and .06 ; in 1937 these same stations had an average weekly catch of $16.70,15.86,50.69$ and 10.04 of $A$. quadrimaculatus. The 
greatest intensity of the epidemic was about stations 17 and 18 and these were closest to the known breeding areas of the anophelines. For example, house 1125 had 8 positive blood smears (all the inhabitants) and none of them had positive case histories the previous year. Houses 1095 (8), 1096 (7), 1097 (7), also close to station No. 17, showed a hundred per cent malaria, except 1096, where 4 out of the 7 were infected. A similar condition existed close to collecting station No. 18 .

From this study it may be inferred that given a susceptible population, a "good" transmitter in reasonable numbers, and a limited source of gametocytes at first there is an almost assured epidemic of malaria. It is unfortunate that this epidemic was not observed through its entire course and that no dissections of $A$. quadrimaculatus are recorded.

It is not possible to give even a cursory survey of the West Indies, Mexico and Central America in regard to epidemies of malaria because we are not certain of the species of anophelines present or their distribution. Malaria is endemic in many parts of this area and epidemies occur from time to time, as that of St. Croix in 1931 and Barbados in 1927. In St. Croix only 15 cases of malaria were recorded from 1918 to 1930 . Yet in 1931 (the wettest year on record for the island, 69.81 inches of rainfall compared with the average of 45.54 in.) a severe epidemic occurred, over 900 cases in a population of about 15,000 . In Barbados there were reported over 1,000 cases in 1927, and up to that time no anopheline was known from the island. However, A. albimanus was found present and widely distributed.

In Jamaica, B. W. I., Washburn (1933) records a sudden outbreak of aestivoautumnal malaria in 1931 in Falmouth and surrounding area. The town of Falmouth and nearby areas have a population of about 8,000. During 1931 (July 1931 to February 1932) some 4,442 cases of malaria developed with 138 deaths. The island experienced heavy rainfalls in 1931 after two years of low rainfalls (92 inches in
1931 compared with the normal of 76 inches; in Falmouth the rainfall was 60 inches while during the preceding four years the average was only 33 inches). From 1923 to 1930 only a small number of malarial cases were treated at the Falmouth public hospital (less than an average of 20 cases per year; 34 in 1930). Falmouth has extensive saline marshes and mangrove swamps around it. A road passed through this area separating the marsh from the mangrove swamp but connected by a 12 foot channel. In 1928 this channel was closed. During the dry seasons of 1929 and 1930 parts of the mangrove swamp were cleared (it lies below sea level). The heavy rains of 1931 converted many of the saline pools to almost fresh water, especially in the mangrove swamp which was now closed to its sea connections. Mosquitoes appeared in immense numbers and $A$. albimanus, A. grabhami, and A. vestitipennis were the anophelines. From January to June, 1931, only 205 cases of malaria were treated at the Public hospital. In July 505, August 1,455, September 731, October 401, November 401, December 448, January 211 and February 54 cases of malaria were treated in clinies, or a total of 4,442 , in a population of less than 8,000. During this period anti-mosquito measures were carried on, the channel into the mangrove swamp was reopened and its waters gradually became saline. By February 1932 the catches per night of $A$. albimanus (from twelve stations) dropped from 1,209 in October to 233 in February. However, A. grabhami showed an increase from 98 in October to 130 in February. In March the epidemic had subsided.

Most epidemics of malaria seem to have an almost "explosive" character, developing with great rapidity in the whole area and then subsiding rather rapidly. Such results may be explained by the fact that there are many strains of each kind of malaria and that a population soon acquires a certain immunity to the strain present. Probably the anopheline carrier also becomes more or less refractory (as indicated by many workers demonstrating the fail- 
ure of oocysts to develop in experimental or wild caught anophelines). A new epidemic may occur if a heterologous strain is introduced or a more susceptible anopheline transmitter invades the region. However we have certain anophelines that seem almost perfect transmitters, as $A$. gambiae (costalis), and epidemics follow its introduction into an endemic region.

As pointed out in the beginning, of the nearly 200 anopheline species in the world, scarcely more than a score are known to be efficient or "dangerous" transmitters. Ex- perimental work indicates that all anophelines, so far as tested in any numbers, have a high degree of susceptibility to infection. If this is true, why should we not have more intensive malaria transmission? Recent work in many lands and by many workers has demonstrated that many of our anophelines are not androphilic. Furthermore, it has been demonstrated that in one of our best known anopheline transmitters ( $A$. maculipennis of Europe) there are several distinct races or sub-species, only two or three of which are androphilic. 


\title{
THE INFECTION IN THE INTERMEDIATE HOST: SYMPTOMATOLOGY, GENERAL CONSIDERATIONS
}

\author{
By MARK F. BOYD \\ INTERNATIONAL HEALTH DIVISION, ROCKEFELLER FOUNDATION, TALLAHASSEE, FLA.
}

Since the introduction of cinchona, comparatively few physicians have had the opportunity to observe the natural evolution of malarial infections to a spontaneous termination. This is in striking contrast to typhoid, for example, for which the lack to date of any specific bactericidal agent prevents a physician from brusquely interrupting the evolution of the disease. Hence in the period from the beginning of specific medication until the introduction of naturally induced malaria as a therapeutic agent in the treatment of neurosyphilis, the personal acquaintance of medical men with the natural history of these diseases greatly deteriorated.

Malarial infections are either naturally or artificially acquired. Naturally acquired infections, excluding instances of congenital malaria, result from the parenteral inoculation of sporozoites by an infected mosquito. Depending upon whether the inoculation was effected by the chance bites of wild mosquitoes in a free condition, or by the deliberate application of experimentally infected insects, we may distinguish autochthonous from induced infections. To the latter class belong the few reported instances where inoculation has been effected with suspensions of sporozoites prepared from the glands of infected anophelines (James, Nicoll and Shute 1927). Artificially acquired infections follow the parenteral inoculation of trophozoites in infected blood. Probably the only autochthonous infections of this class are the instances of congenital malaria. A further exception to the foregoing statement is afforded by the recent report by Shortt and Menon (1940) of their success in infecting 6 of 7 rhesus monkeys with $P$. knowlesi, and 7 of 17 chickens with $P$. gallinaceum by dropping defibrinated infected blood into their open mouths. Most are induced either deliberately in malaria therapy, or accidentally as a consequence of transfusion (Hutton and Shute 1939), or intravenous medication (Black 1940), or the communal apparatus for the preparation of "shots" by gregarious nareotic addicts (Biggam 1929; Himmelsbach 1933; Faget 1933; Helpern 1934; Most 1940b).

The minimal number of sporozoites which can initiate infection in a susceptible intermediate host is not known. The circumstance that the mere insertion of the proboscis of a single infected anopheline can introduce an infecting dose does not answer the question, as the density of infection in different insects varies widely, so that some specimens might only introduce a few, others many hundreds or even thousands. Sporozoites removed from infected glands may be suspended in Locke's solution and injected by a syringe (James, Nicoll and Shute 1927). Using such a method, Shute (1937) reports having effected inoculations with as few as fifty. De Sanctis Monaldi (1935) reports takes after incubation periods of from 15 to 17 days in 3 of 7 patients intravenously inoculated with from 2,500 to 100,000 suspended sporozoites. The length of these incubation periods he regarded as normal for the strain.

While it is probable that most of the sporozoites introduced by an anopheline are discharged into the blood stream, since immediate excision of the tissues about the site of the insertion of the proboscis will not forestall an infection, the slender fusiform shape of the sporozoite suggests an 
ability to penetrate the tissues in passage to a blood vessel, if not directly introduced therein. This is confirmed by the infections resulting from the application of infected mosquitoes to blisters (Boyd and StratmanThomas 1934e). It is not known whether the crushing of an infected anopheline on the skin might result in infection.

Artificial inoculations may be ranked in order of increasing reliability as subcutaneous, intramuscular and intravenous. The first two methods are not only highly unreliable, but if successful are followed by long incubation periods. It is difficult to understand how trophozoites introduced by either of the first two routes make their way to the blood stream. Entirely apart from the dosage of trophozoites introduced, the duration of the incubation period or even the success of the inoculation following intravenous inoculation may depend on whether the blood of the donor and recipient are compatible or incompatible (Polayes and Derby 1934). These observers report mean incubations, presumably of vivax, of 4.3 days when the bloods are compatible and of 8.2 days when they are incompatible. The injection of incompatible blood soon gives rise to an elevation of temperature, chills, nausea and dyspnoea. The necessity of typing in order to avoid this reaction can be avoided by effecting inoculation with not over 10 ee quantities of blood.

We are likewise ignorant of the minimal number of trophozoites of the parasites of man which can initiate infection, although Coggeshall (1938a) found that in Macaca mulatta a single trophozoite of the highly virulent $P$. knowlesi could induce infection. Stauber (1939) working in Huff's laboratory has successfully initiated an infection with $P$. cathemerium by the inoculation of a canary with a single merozoite. We have effected inoculations of $P$. vivax with as few as ten trophozoites on intravenous inoculation, and are of the opinion that the number might still be further reduced. Distinction of the age of the trophozoites must be made, as an inoculation effected with 10 presegmenters would have a different significance from one effected with 10 young amoeboid forms.

The possibility of a malarial infection occurring in the recipient of a transfusion from a donor with a latent infection is always a matter of concern to a surgeon, although if this possibility is borne in mind, and blood smears are taken from the recipient in the event a pyrexia develops, there is little actual risk. Infected donors usually have latent infections, with parasite densities so low that the recognition of the infection by microscopical examination is hopeless. Consequently surgeons should avoid, or at least regard with suspicion, donors for direct transfusions who have resided in endemic regions, or give a history of past malaria infection (Hutton and Shute 1939). An instance has even been reported where, on direct transfusion, the recipient infected the donor. While all species of parasites may be encountered in this connection, the extreme and unpredictable duration of latent quartan infections makes them an especial hazard.

Malarial infections also merit consideration in connection with the growing practice of storing blood under refrigerated conditions for emergency transfusions. Johns (1931) succeeded in infecting paretic patients with defibrinated blood containing 1 per cent dextrose which had been stored at $0^{\circ} \mathrm{C}$ for $16-18$ days. Since these conditions closely coincide with those used on blood destined for storage, malarial transfer might be a possibility, although the use of citrate as an anticoagulant would be detrimental to the parasites.

Assuming that the route of inoculation is adapted to the parasite, an infection or take will follow their introduction. If the patient presents some degree of immunity, the infection may be of short duration and even remain subclinical in degree. When an infection is artificially induced by intravenous inoculation with the donor at the bedside, and the volume regulated by the degree of the donor's parasitemia, some degree of take is almost inevitable unless the recipient is a refractory hyperimmune. On the other hand greater uncertainty fol- 
lows natural inoculations, even when the mosquitoes are proved to be infected. Some of our results are shown in Table I.

TABLE I

Proportion of Successful Infections SubseQUENT TO THE APPLICATION OF VARYing NUMBERS OF DEMONSTRABLY INFECTED MosQUITOES

\begin{tabular}{l|c|r|r|r}
\hline Parasite & $\begin{array}{c}\text { Number } \\
\text { of mos- } \\
\text { quitoes }\end{array}$ & $\begin{array}{c}\text { Persons } \\
\text { inocu- } \\
\text { lated }\end{array}$ & Takes & $\begin{array}{c}\text { Per } \\
\text { cent } \\
\text { takes }\end{array}$ \\
\hline P. falciparum & 1 & 11 & 7 & 63.6 \\
& $2-5$ & 42 & 22 & 52.3 \\
& $6-10$ & 39 & 24 & 61.4 \\
& $11-15$ & 11 & 7 & 63.6 \\
P. vivax & 1 & 54 & 43 & 79.6 \\
& $2-5$ & 150 & 123 & 81.8 \\
& $6-10$ & 155 & 130 & 84.2 \\
& $11+$ & 35 & 24 & 68.6 \\
Total & 103 & 60 & 58.3 \\
& Total & 394 & 320 & 81.3 \\
\hline
\end{tabular}

These data suggest that the presumptive dosage of sporozoites has had little bearing on a successful natural inoculation.

\section{The Intrinsic Incubation Period}

As in the case with inoculation by any infecting agent, a period of clinical quiescence or incubation ordinarily intervenes between inoculation with malarial parasites and the onset of symptoms. Practical criteria to mark its termination are either (a) the first detection of parasites in the peripheral blood, or (b) the first elevation of temperature to $100^{\circ} \mathrm{F}$. In most infections this period can be explained as the interval necessary for a scanty number of invading organisms to multiply until their numbers are sufficient to produce an effect on the host. In malarial infections artificially induced this interpretation appears adequate, but subsequent to natural inoculation the operation of another factor appears likely. The incubation period subsequent to natural inoculation of the intermediate host is called the intrinsic incubation period, to distinguish it from the period of non-infectiousness following infection of the definitive host, which is known as the extrinsic incubation period, q.v.

Although the duration of the incubation period following artificially induced infections may vary with the tissues into which the parasites are introduced, yet if the intravenous route of transfer is followed, the duration to a great degree will vary inversely with the dosage of trophozoites administered (Table II). Sufficient parasites may even be given to make them immediately microscopically detectable in the

TABLE II

Showing the Inverse Relation Between the Dosage of Trophozoites and the Length of the incubation Prriod in artificially Induced Malaria (Intravenous)

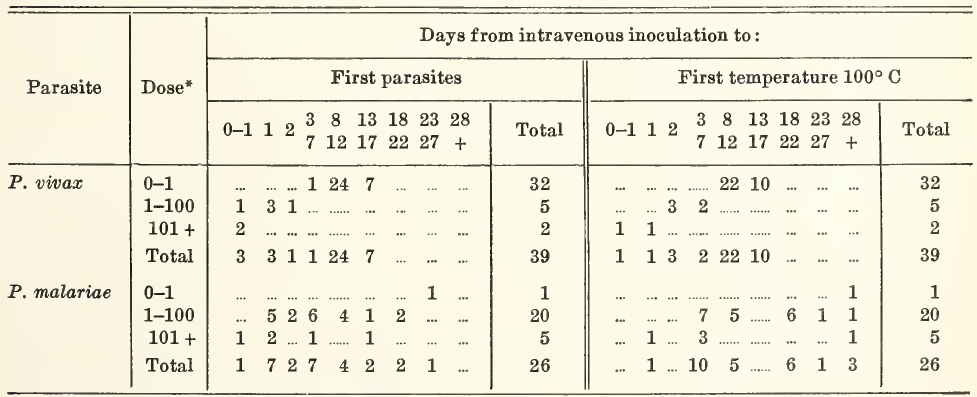

* Doses are in millions of trophozoites. 
circulating blood of the recipient, while furthermore with such large doses, the recipient may clinically experience passive paroxysms, attributable to the division of the original parasites received from the donor (Boyd and Kitchen 1936c).

Depending on the species of parasite, the intrinsic incubation may vary inversely with the dosage of sporozoite inoculum, as roughly measured by the number of infected mosquitoes applied (Boyd 1940e; Boyd and Kitchen 1937c; and Boyd and Kitchen 1937e). This is shown by the summary presented in Table III.

It will be noted from Table III that even by the crude method of appraising the relative sporozoite dosage, an inverse relation between the dosage and the time elapsing to the first detection of trophozoites is evident with the vivax inoculations but is not apparent with the falciparum inoculations. This suggests that the latter parasite may have a very low minimal infecting dose, which conversely is likely much higher for $P$. vivax. But more significant still, it will be noted that even with the maximum inoculum, the intrinsic incubation period not only has not been suppressed, but has not been reduced below a characteristic minimal interval for each species.

Furthermore, natural inoculation even by massive doses of sporozoites, is followed by a period in which the blood is free from trophozoites, in marked distinction to arti- ficial inoculation. This has been demonstrated for $P$. vivax by Boyd and StratmanThomas (1934c), for P. falciparum by Ciuca et al. (1937a) as well as by Boyd and Matthews (1939), while Warren and Coggeshall (1937) have shown that this characteristic is also exhibited by the $P$. cathemerium of birds. Using massive inoculations of 250 cc of blood, Raffaele (1937a) found the blood of a naturally inoculated vivax patient infectious as early as the fifth day after inoculation. These observations indicate that it is unlikely that any trophozoites of $P$. vivax or $P$. falciparum are in the blood stream earlier than the 5th day subsequent to natural inoculation. This is strong evidence against the view that sporozoites directly initiate schizogony, and perhaps explains the general failure of all efforts to confirm the claim of Schaudinn (1902-03) to have observed the penetration of erythrocytes by sporozoites in vitro.

The facts just related give support to James' (1931) hypothesis of the existence of a hitherto unrecognized fixed tissue cell stage intervening between the sporozoite and the trophozoite, which was suggested in an endeavor to explain why quinine is not effective as a causal prophylactic.

The criteria by which the termination of the incubation period is recognized do not usually coincide, although as seen from Table IV, they usually vary directly with each other. The extent to which they do

TABLE III

Showing the Relation Between the Length of the Incubation Period and the Number of Infected Mosquitoes Applied (Sporozoite Dosage) in Naturally Induced Malaria

\begin{tabular}{|c|c|c|c|c|c|c|c|c|c|c|}
\hline \multirow{2}{*}{ Parasite } & \multirow{2}{*}{$\begin{array}{l}\text { Number of } \\
\text { infected } \\
\text { mosquitoes }\end{array}$} & \multicolumn{9}{|c|}{ Days from inoculation to first detection of parasites } \\
\hline & & $0-1$ & 1 & 2 & $\begin{array}{l}3 \\
7\end{array}$ & $\begin{array}{c}8 \\
12\end{array}$ & $\begin{array}{l}13 \\
17\end{array}$ & $\begin{array}{l}18 \\
22\end{array}$ & $\begin{array}{l}23 \\
27\end{array}$ & Total \\
\hline P. vivax & $\begin{array}{l}1-5 \\
6-10 \\
11+ \\
\text { Total }\end{array}$ & $\begin{array}{l}\ldots \\
\ldots \\
\cdots \\
0\end{array}$ & $\begin{array}{l}\ldots \\
\ldots \\
0\end{array}$ & $\begin{array}{l}\ldots . . \\
\ldots \\
0\end{array}$ & $\begin{array}{l}\ldots \\
\ldots \\
\ldots \\
0\end{array}$ & $\begin{array}{r}38 \\
33 \\
3 \\
74\end{array}$ & $\begin{array}{r}74 \\
7 \\
1 \\
82\end{array}$ & $\begin{array}{l}9 \\
\ldots \\
\ldots \\
9\end{array}$ & $\begin{array}{l}\ldots . . \\
\ldots . . . \\
\ldots . . . \\
\ldots . . .\end{array}$ & $\begin{array}{r}121 \\
40 \\
4 \\
165\end{array}$ \\
\hline P. falciparum & $\begin{array}{l}1-5 \\
6-10 \\
11+ \\
\text { Totai }\end{array}$ & $\begin{array}{l}\ldots \\
\ldots \\
0\end{array}$ & $\begin{array}{l}\ldots \\
\ldots \\
\ldots \\
0\end{array}$ & $\begin{array}{l}\ldots \\
\cdots \\
\cdots \\
0\end{array}$ & $\begin{array}{l}2 \\
\ldots \\
\cdots \\
2\end{array}$ & $\begin{array}{l}18 \\
37 \\
15 \\
70\end{array}$ & $\begin{array}{r}11 \\
9 \\
4 \\
24\end{array}$ & $\begin{array}{l}\ldots \\
\ldots \\
\ldots \\
\ldots\end{array}$ & $\begin{array}{c}\ldots . . . \\
\ldots . . . \\
\ldots . . . \\
\ldots . . .\end{array}$ & $\begin{array}{l}31 \\
46 \\
19 \\
96\end{array}$ \\
\hline
\end{tabular}


TABLE IV

Correlation Between the Periods from Inoculation to Rise in Temperature and to Detection of PARASITES

(A) P. vivax-8 strains

\begin{tabular}{|c|c|c|c|c|c|c|c|c|c|c|c|c|c|c|c|c|c|}
\hline \multirow{2}{*}{$\begin{array}{c}\text { Days to } \\
\text { rise of } \\
\text { temperature }\end{array}$} & \multicolumn{16}{|c|}{ Days to detection of parasites (prepatent period) } & \multirow{2}{*}{ Total } \\
\hline & 8 & 9 & 10 & 11 & 12 & 13 & 14 & 15 & 16 & 17 & 18 & 19 & 20 & 21 & 23 & 30 & \\
\hline No fever & $\ldots$ & 1 & ....... & $\ldots . .$. & ....... & 3 & 1 & 1 & $\ldots . .$. & 2 & 1 & 1 & $\ldots$ & ... & $\ldots$ & 1 & 11 \\
\hline 8 & 1 & 1 & $\ldots . .$. & $\ldots . .$. & $\ldots . .$. & 1 & ....... & $\ldots$ & ....... & $\ldots$ & $\ldots$ & $\ldots$ & $\ldots$ & $\ldots$ & $\ldots$ & $\ldots$ & 3 \\
\hline 9 & 1 & 2 & 3 & 1 & ...... & 1 & ....... & ....... & $\ldots . .$. &.. & $\ldots$ & $\ldots$ & ... & $\ldots$ & $\ldots$ & $\ldots$ & 8 \\
\hline 10 & $\ldots$ & 3 & 5 & 3 & 2 & 3 & ....... & $\ldots . .$. & ....... & $\ldots$ & $\ldots$ & $\ldots$ & $\ldots$ & $\ldots$ & $\ldots$ & $\ldots$ & 16 \\
\hline 11 & 1 & 4 & 10 & 11 & 13 & 6 & 4 & 1 & ....... & $\ldots$ & $\ldots$ & $\ldots$ & $\ldots$ & $\ldots$ & $\ldots$ & $\ldots$ & 50 \\
\hline 12 & 1 & 2 & 3 & 15 & 7 & 9 & 3 & $\ldots . .$. & $\ldots . . .$. & $\ldots$ & $\ldots$ & $\ldots$ & $\ldots$ & $\ldots$ & $\ldots$ & $\ldots$ & 40 \\
\hline 13 & $\ldots$ & 2 & 4 & 11 & 12 & 14 & 6 & 2 & $\ldots . .$. & $\ldots$ & $\ldots$ & $\ldots$ & $\ldots$ & $\ldots$ & $\ldots$ & $\ldots$ & 51 \\
\hline 14 & 1 & $\ldots . . .$. & 1 & 9 & 10 & 9 & 1 & 2 & $\ldots . .$. & $\ldots$ & ... & $\ldots$ & $\ldots$ & $\ldots$ & $\ldots$ & $\ldots$ & 33 \\
\hline 15 & $\ldots$ & $\ldots . .$. & ....... & 3 & 7 & 6 & 7 & 2 & 4 & $\ldots$ & $\ldots$ & $\ldots$ & $\ldots$ & $\ldots$ & $\ldots$ & $\ldots$ & 29 \\
\hline 16 & $\ldots$ & $\ldots . .$. & 1 & 2 & 2 & 4 & 7 & 6 & 4 & 1 & $\ldots$ & $\ldots$ & $\ldots$ & $\ldots$ & $\ldots$ & $\ldots$ & 27 \\
\hline 17 & ... & $\ldots .$. & $\ldots . .$. & $\ldots .$. & $\ldots \ldots$ & 5 & 1 & 2 & 5 & 3 & 1 & $\ldots$ & $\ldots$ & $\ldots$ & $\ldots$ & $\ldots$ & 17 \\
\hline 18 & $\ldots$ & ....... & ...... & 1 & 1 & $\ldots . . .$. & 2 & 4 & 1 & 2 & 2 & ... & $\ldots$ & $\ldots$ & $\ldots$ & $\ldots$ & 13 \\
\hline 19 & $\ldots$ & ....... & ....... & $\ldots . .$. & $\ldots . .$. & $\ldots . .$. & 1 & 2 & $\ldots . .$. & 1 & $\ldots$ & $\ldots$ & 1 & $\ldots$ & $\ldots$ & $\ldots$ & 5 \\
\hline 20 & $\ldots$ & $\ldots .$. & $\ldots . .$. & $\ldots . .$. & ....... & $\ldots . .$. & ....... & 1 & $\ldots . .$. & $\ldots$ & $\ldots$ & 2 & $\ldots$ & 1 & $\ldots$ & $\ldots$ & 4 \\
\hline 21 & $\ldots$ & $\ldots . .$. & ....... & $\ldots . .$. & $\ldots . .$. & $\ldots . . .$. & ....... & $\ldots . .$. & $\ldots . .$. & $\ldots$ & $\ldots$ & 1 & $\ldots$ & $\ldots$ & 1 & $\ldots$ & 2 \\
\hline 22 & $\ldots$ & $\ldots . . .$. & ....... & $\ldots . .$. & ....... & ...... & ....... & ....... & $\ldots . .$. & $\ldots$ & $\ldots$ & ... & $\ldots$ & 1 & $\ldots$ & $\ldots$ & 1 \\
\hline 23 & ... & ....... & ....... & $\ldots . .$. & $\ldots . . .$. & $\ldots . . .$. & $\ldots . .$. & $\ldots . .$. & $\ldots . .$. & $\ldots$ & 1 & 1 & $\ldots$ & $\ldots$ & ... & $\ldots$ & 2 \\
\hline 24 & $\cdots$ & $\ldots .$. & ...... & $\ldots .$. & ...... & $\ldots . .$. & 1 & ...... & ....... & $\ldots$ & $\ldots$ & $\ldots$ & $\ldots$ & $\ldots$ & $\ldots$ & $\ldots$ & 1 \\
\hline Total & 5 & 15 & 27 & 56 & 54 & 61 & 34 & 23 & 14 & 9 & 5 & 5 & 1 & 2 & 1 & 1 & 313 \\
\hline
\end{tabular}

(B) P. falciparum -5 strains

\begin{tabular}{|c|c|c|c|c|c|c|c|c|c|c|c|c|c|c|c|}
\hline \multirow{2}{*}{$\begin{array}{l}\text { Days to rise of } \\
\text { temperature }\end{array}$} & \multicolumn{14}{|c|}{ Days to detection of parasites (prepatent period) } & \multirow{2}{*}{ Total } \\
\hline & 6 & 9 & 10 & 11 & 12 & 13 & 14 & 15 & 16 & 17 & 20 & 22 & $\cdot 24$ & 25 & \\
\hline No fever & $\ldots$ & 1 & ....... & $\ldots . .$. & $\ldots . .$. & $\ldots . .$. & $\ldots$ & $\ldots$ & 1 & $\ldots$ & $\ldots$ & $\ldots$ & $\ldots$ & $\ldots$ & 2 \\
\hline 7 & $\ldots$ & 1 & 1 & ....... & $\ldots \ldots$. & ....... & $\ldots$ & $\ldots$ & $\ldots$ & $\ldots$ & $\ldots$ & ... & $\ldots$ & $\ldots$ & 2 \\
\hline 8 & $\ldots$ & $\ldots . .$. & 1 & 1 & $\ldots . .$. & $\ldots . .$. & $\ldots$ & $\ldots$ & $\ldots$ & $\ldots$ & $\ldots$ & $\ldots$ & $\ldots$ & $\ldots$ & 2 \\
\hline 9 & 1 & $\ldots . .$. & $\ldots \ldots$. & $\ldots . .$. & $\ldots . .$. & $\ldots . .$. & $\ldots$ & $\ldots$ & $\ldots$ & $\ldots$ & $\ldots$ & $\ldots$ & $\ldots$ & $\ldots$ & 1 \\
\hline 10 & 1 & 8 & 3 & 3 & $\ldots . .$. & $\ldots . .$. & 1 & ... & $\ldots$ & $\ldots$ & $\ldots$ & $\ldots$ & $\ldots$ & $\ldots$ & 16 \\
\hline 11 & $\ldots$ & 1 & ....... & 3 & $\ldots . .$. & $\ldots . .$. & $\ldots$ & $\ldots$ & $\ldots$ & $\ldots$ & $\ldots$ & $\ldots$ & $\ldots$ & $\ldots$ & 4 \\
\hline 12 & $\ldots$ & 8 & 4 & 32 & 2 & 4 & $\ldots$ & 1 &.. & $\ldots$ & $\ldots$ & $\ldots$ & $\ldots$ & $\ldots$ & 51 \\
\hline 13 & $\ldots$ & 2 & 1 & 7 & 2 & 1 & 2 & $\ldots$ & $\ldots$ & $\ldots$ & $\ldots$ & $\ldots$ & $\ldots$ & $\ldots$ & 15 \\
\hline 14 & $\ldots$ & 2 & 3 & 19 & 2 & 5 & $\ldots$ & ... & $\ldots$ & ... & $\ldots$ & $\ldots$ & $\ldots$ & $\ldots$ & 31 \\
\hline 15 & $\ldots$ & $\ldots . .$. & $\ldots . .$. & 4 & 3 & 5 & $\ldots$ & $\ldots$ & 1 & $\ldots$ & $\ldots$ & ... & $\ldots$ & $\ldots$ & 13 \\
\hline 16 & $\ldots$ & ....... & $\ldots . .$. & 4 & 1 & 1 & $\ldots$ & 1 & $\ldots$ & $\ldots$ & $\ldots$ & $\ldots$ & $\ldots$ & $\ldots$ & 7 \\
\hline 17 & ... & 1 & $\ldots . .$. & $\ldots .$. & 1 & $\ldots . .$. & 1 & ... & 1 & $\ldots$ & $\ldots$ & $\ldots$ & $\ldots$ & $\ldots$ & 4 \\
\hline 18 & $\ldots$ & $\ldots . .$. & ...... & 2 & $\ldots . .$. & 1 & $\ldots$ & $\ldots$ & $\ldots$ & $\ldots$ & $\ldots$ & ... & $\ldots$ & $\ldots$ & 3 \\
\hline 19 & ... & $\ldots . .$. & ...... & $\ldots . .$. & ....... & $\ldots . .$. &.. & $\ldots$ & $\ldots$ & 1 & $\ldots$ & $\ldots$ & $\ldots$ &..$:$ & 1 \\
\hline 22 & $\ldots$ & ...... & ....... & $\ldots . .$. & $\ldots . .$. & $\ldots . .$. & ... & $\ldots$ & $\ldots$ & $\ldots$ & $\ldots$ & 1 & ... & $\ldots$ & 1 \\
\hline 25 & $\ldots$ & ....... & ...... & ....... & ....... & $\ldots . .$. & ... & 1 & $\ldots$ & $\ldots$ & $\ldots$ & ... & $\ldots$ & $\ldots$ & 1 \\
\hline 26 & $\ldots$ & $\ldots . .$. & ....... & ....... &..... & $\ldots . .$. & $\ldots$ & $\ldots$ & -. & $\ldots$ & 1 & $\ldots$ & $\ldots$ & 1 & 2 \\
\hline 27 & $\ldots$ & $\ldots .$. & ...... & $\ldots . .$. & $\ldots .$. & ...... & $\ldots$ & $\ldots$ & $\ldots$ & $\cdots$ & $\cdots$ & 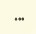 & 1 & $\cdots$ & 1 \\
\hline Total & 2 & 24 & 13 & 75 & 11 & 17 & 4 & 3 & 3 & 1 & 1 & 1 & 1 & 1 & 157 \\
\hline
\end{tabular}


(C) P. malariae-2 strains

\begin{tabular}{c|cccc|c}
\hline \multirow{2}{*}{$\begin{array}{c}\text { Days to } \\
\text { rise of } \\
\text { temperature }\end{array}$} & $\begin{array}{c}\text { Days to detection of parasites } \\
\text { (prepatent period) }\end{array}$ & \multirow{2}{*}{ Total } \\
\cline { 2 - 5 } & 23 & 32 & 34 & 37 & \\
\hline 30 & 1 & $\ldots$ & $\ldots$ & $\ldots$ & 1 \\
35 & $\ldots$ & 1 & $\ldots$ & $\ldots$ & 1 \\
36 & $\ldots$ & $\ldots$ & 1 & $\ldots$ & 1 \\
40 & $\ldots$ & $\ldots$ & $\ldots$ & 1 & 1 \\
49 & $\ldots$ & $\ldots$ & $\ldots$ & 1 & 1 \\
Total & 1 & 1 & 1 & 2 & 5 \\
\hline
\end{tabular}

or do not coincide in the data given is as follows :

TABLE V

Variations in Incubation Period

\begin{tabular}{|c|c|c|c|}
\hline & Falciparum & Vivax & Malariae \\
\hline $\begin{array}{l}\text { Parasites \& fever } \\
\text { same day }\end{array}$ & 10 & 52 & 0 \\
\hline Parasites precede & 129 & 180 & 5 \\
\hline Fever precedes ... & 16 & 70 & 0 \\
\hline No fever & 2 & 11 & 0 \\
\hline 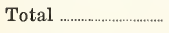 & 157 & 313 & 5 \\
\hline
\end{tabular}

From this table it is to be seen that the parasites are usually, but not always, detectable on or before the clinical onset. Most clinical onsets have occurred between the 11th and 14th days, while attention should be especially directed to the prolonged intrinsic incubation period of quartan. We have never observed the incubation periods of falciparum to exceed the limits shown in Table IV, but occasionally we have found longer periods for vivax.

The number of eases of this type which we have observed contemporary to the cases with normal limits are listed in Table VI.

These five cases constitute about 2 per cent of the McCoy strain inoculations. Other strains of $P$. vivax propagated abroad have shown a much higher proportion of inoculations followed by deferred onsets. Thus autochthonous Dutch strains employed in Holland have been followed by deferred onsets in 38 per cent of those inoculated, but when the use of the exotic Madagascar strain was begun, the propor-
TABLE VI

Protracted Incubation in Simple Vivax INOCULATIONS

\begin{tabular}{c|c|cc|cr}
\hline \multirow{2}{*}{$\begin{array}{c}\text { Case } \\
\text { num- } \\
\text { ber }\end{array}$} & $\begin{array}{c}\text { Date } \\
\text { inoe. }\end{array}$ & Mosquitoes & \multicolumn{2}{|c}{ Days to } \\
\cline { 3 - 6 } & & Lot No. pos. & $\begin{array}{c}\text { First } \\
\text { parasites }\end{array}$ & $\begin{array}{c}\text { First } \\
\text { fever }\end{array}$ \\
\hline 106 & $12 / 15 / 32$ & 122 & 3 & 97 & 104 \\
108 & $12 / 15 / 32$ & 122 & 3 & 85 & 86 \\
285 & $6 / 21 / 37$ & 570 & 4 & 304 & 302 \\
305 & $11 / 19 / 37$ & 690 & 4 & 280 & 302 \\
340 & $8 / 1 / 38$ & 747 & 7 & 30 & 56 \\
\hline
\end{tabular}

tion dropped to six per cent (Swellengrebel and de Buck 1938).

Autochthonous vivax malaria in Holland shows a high vernal incidence. Schüffner, Korteweg and Swellengrebel (1929) demonstrated that autumnal vivax inoculations by lightly infected mosquitoes gave rise to clinical attacks in the following summer, while heavier autumnal inoculations resulted in autumnal attacks after "normal" incubations, which were followed by numerous vernal relapses in the spring (Swellengrebel 1933). James, Nicol and Shute (1936) also express the opinion that light inoculations with sporozoites are commonly followed by protracted incubation periods, and state that they also occur in persons who are under the influence of atebrine at the time they are inoculated. Our own experience does not suggest that small dosage is a universal explanation for deferred onsets.

The duration of the intrinsic incubation period might conceivably show a variation with the strain of parasite. Thus our Coker and Long strains have given the results listed in Table VII.

Although the Long strain has shown greater uniformity than the Coker in the days elapsing to the first detection of parasites (Boyd and Kitchen 1937a), the mean duration of their elinical incubation periods is practically identical.

The elinical incubation periods of two vivax strains are shown in Table VIII.

The mean durations of incubation for these two strains of widely different prove- 
TABLE VII

Strain Variations in the Length of the Intrinsic Incubation Period of $P$, falciparum.

\begin{tabular}{|c|c|c|c|c|c|c|c|c|c|c|c|c|c|c|c|}
\hline \multirow{2}{*}{ Strain } & \multicolumn{14}{|c|}{ Days to first fever of $100^{\circ} \mathrm{F}$} & \multirow{2}{*}{ Mean } \\
\hline & 0 & 7 & 8 & 9 & 10 & 11 & 12 & 13 & 14 & 15 & 16 & 17 & $18+$ & Tot. & \\
\hline Coker ......... & 1 & $\ldots$ & $\ldots$ & 1 & 4 & 1 & 14 & 4 & 7 & 7 & 1 & $\ldots . .$. & 2 & 42 & 13.1 \\
\hline Long .......... & 1 & 1 & 1 & ... & 11 & 3 & 34 & 9 & 24 & 6 & 5 & 1 & 3 & 100 & 12.9 \\
\hline
\end{tabular}

TABLE VIII

Strain Variation IN THE Length of Intrinsic Incubation Period of $P$. vivax

\begin{tabular}{|c|c|c|c|c|c|c|c|c|c|c|c|c|c|c|c|c|c|c|}
\hline \multirow{2}{*}{ Strain } & \multicolumn{17}{|c|}{ Days to first fever of $100^{\circ} \mathrm{F}$} & \multirow{2}{*}{ Mean } \\
\hline & 6 & 7 & 8 & 9 & 10 & 11 & 12 & 13 & 14 & 15 & 16 & 17 & 18 & 19 & 20 & $21+$ & Tot. & \\
\hline Madagascar* & 3 & 8 & 16 & 34 & 60 & 59 & 96 & 99 & 109 & 78 & 62 & 50 & 29 & 16 & 13 & 14 & 746 & 13.6 \\
\hline 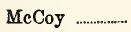 & $\ldots$ & ... & 3 & 7 & 12 & 40 & 33 & 43 & 32 & 23 & 23 & 15 & 10 & 5 & 4 & 4 & 254 & 13.6 \\
\hline
\end{tabular}

- After James (1931).

nance are equal. On the other hand, the indigenous Dutch strain previously mentioned is stated to have a "normal" incubation of from 15 to 30 days, with an average length of 21 days (de Buck 1936).

The number of merozoites formed at schizogony varies with these different strains of $P$. vivax as follows:

Madagascar Av. 17-18 (Swellengrebel and de Buck 1938)

Dutch Av. 12-13 (Swellengrebel and de Buck

MeCoy Av. 16.1

\section{Simultaneous Inoculation with Two SPecies of Parasites}

A period of intensified malaria transmission is not infrequently followed by two epidemic waves. The first wave, consisting largely of recognized infections with $\boldsymbol{P}$. falciparum, is closely connected chronologically with the transmission period; the second wave, occurring in the following year, is less obviously related, and consists of infections with $P$. vivax. This is exemplified by the data presented in Fig. 1.

It has been shown (Boyd and Kitchen 1937f) that anopheline mosquitoes may be simultaneously infected with both $P$. vivax and $P$. falciparum, and can simultaneously transmit both species. Neither species of parasite present in a simultaneous infection appears to exercise a significantly injurious or inhibitory effect upon the other (Boyd, Kitchen and Kupper 1937).

While both species of parasites are detectable in the blood of patients simultaneously infected at about the same time after the lapse of a "normal" incubation period, the center of the stage is soon assumed by falciparum, as it is the first to increase to densities in excess of the minimal microscopical level, while the first clinical reaction displays the characteristics of the disease which it initiates. The exhibition of quinine at this period, so necessary to restrain the exuberant multiplication of $\boldsymbol{P}$. falciparum, has a more destructive effect on the lagging $P$. vivax, which is driven down to submicroscopic levels. In some individuals who did not receive a great deal of quinine, a return of $P$. vivax is noted after several weeks, during which time $\boldsymbol{P}$. falciparum may markedly decline. Thereupon clinical activity displays all the characteristics of vivax malaria. $P$. falciparum may later return to clinical levels while $P$. vivax is still abundant, in which 


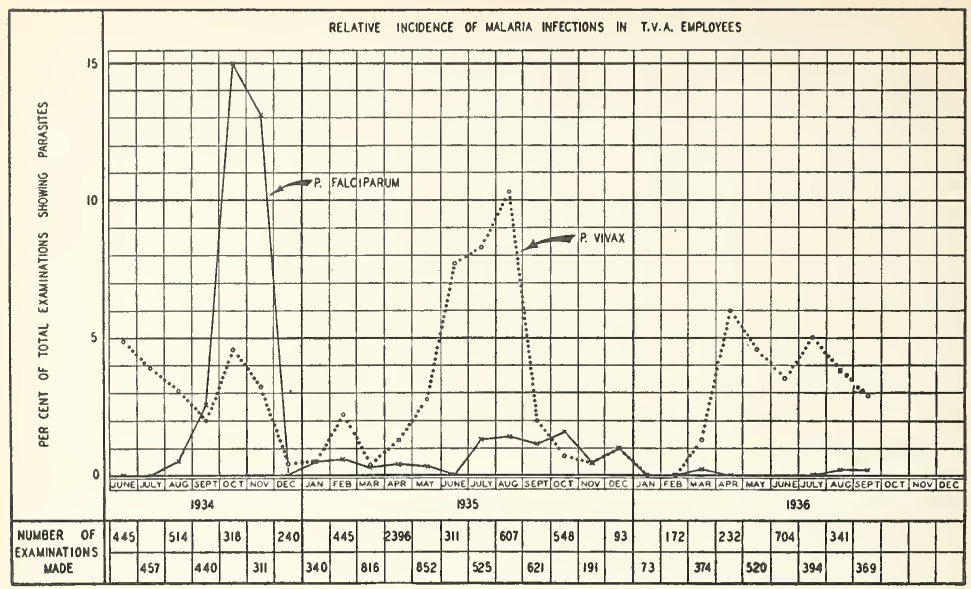

FIG. 1. Incidence of malaria infection during several consecutive years among employees of the Tennessee Valley Authority. Active transmission of infection is to be inferred during the first year (1934) when $P$. falciparum dominated the picture. The rise in $P$. vivax in 1935 and 1936 is ascribable to the activation of infections acquired in 1934. (Courtesy Health and Safety Division, Tennessee Valley Authority.)

event it is difficult to determine the responsibility of either parasite alone in the continuing illness (Fig. 2).
Our series of seven cases of simultaneous inoculation was notable for the clinical activity of vivax in three patients more

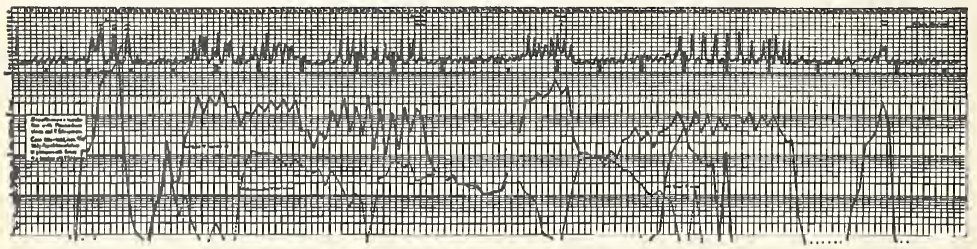

Fig. 2. Showing Clinical Attack Experienced by Patient 220-1085 Simultaneously Inoculated on May 29, 1936, by 16 Quadrimaculatus Infected with $P$. vivax and 17 Quadrimaculatus Infected with $P$. falciparum.

The chart covers a period of observation of 156 days. The upper portion represents the temperature curve, the black bars in the same portion represent the degree of splenomegaly observed. The lower portion with semilogarithmic ruling represents the daily parasite counts of each species of parasite per cubic millimeter, the lower line of which represents a count of 10 per cubic millimeter.

The solid line in the lower portion represents the total falciparum count, the line of àashes the total count of gametocytes of this parasite. The line of dots represents the total count of $P$. vivax. Sporadic $\boldsymbol{P}$. vivax are represented by dots below the bottom line of the chart.

The patient received two doses of 5 and 10 grains of quinine sulphate respectively on the 14th and 16 th days following inoculation and three doses of 10 grains on the 18th day. On the 66th and 67 th days, three doses of 5 and 10 grains each were given. On the 90 th day a single dose of 10 grains was given. No further quinine was given until the $143 \mathrm{rd}$ day when he received three doses, one of 7 and two of 10 grains respectively. On the 147 th day intensive quininization by Sinton's method was begun and continued until the 154 th day. (Am. Jour. Trop. Med., 17 (1937), 857.) 


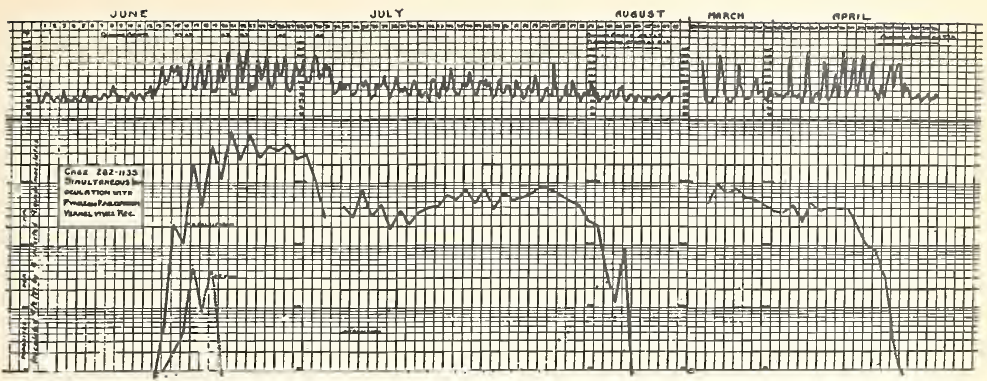

Fig. 3. Showing clinical attack experienced by Patient 282-1133 inoculated on June 2, 1937 , by 6 . quadrimaculatus simultaneously infected with both $P$. vivax and $P$. falciparum. The chart covers two periods of observation of 68 and 27 days. It will be noted that subsequent to inoculation the two species of parasites appeared practically simultaneously. Five-grain doses of quinine were given on the 15th, 16th, 20th, 22nd, 26th, and 30th days after inoculation. Coincident with the dose given on the 20th, $P$. vivax diminished to submicroscopical levels and was not thereafter noted during the first period of observation. After the 19th day the clinical attack exhibits the characteristics of falciparum malaria. From the 59th to the 66 th day intensive quininization by Sinton's method was effected. After an interval of 227 days, the patient experienced an attack from $P$. vivax in the following spring. (Am. J. trop. Med., 18: 512.)

than six months after the inoculation. This incidence of 42.9 per cent is in marked contrast to the 8.05 per cent of renewed activity after six months following simple vivax infection. None of the long term recurrences took place in patients who experienced vivax attacks immediately following the first period of falciparum activity, although all patients had received intensive treatment with quinine (Fig. 3) (Boyd and Kitchen 1938a). The renewed activity of vivax occurred 235, 236 and 282 days, respectively, after the onset of the original falciparum dominated attack, or at intervals of 226, 174 and 233 days, respectively, after its induced termination.

The experience of these patients appears similar to that observed in the epidemic situations cited.

\section{The Threshold or Prrogenic Level}

The minimal density which trophozoites must attain in the blood before a patient presents the first symptoms of illness is known as the threshold or pyrogenous level. The density prevailing at this time, we believe, should be distinguished from that which may prevail during the subsequent course of the illness and infection. Ross (1910), one of the earliest who considered this question, estimated that the parasites will not generally be numerous enough to cause illness unless there is at least 1 parasite to 100,000 erythrocytes; that is 50 parasites in $1 \mathrm{cmm}$ of blood, or 150,000,000 in a man of 142 pounds ( $64 \mathrm{~kg}$ ) in weight. It would require on the average a 15 minute search of a thin smear to detect a parasite at this density.

Assuming that the first elevation of temperature to $100^{\circ} \mathrm{F}$ or higher marks the clinical onset, we (Boyd 1938) have observed the parasite densities at the time of onset in induced vivax malaria shown in Table IX.

It is thus seen that $P$. vivax may induce a clinical reaction with densities of 10 or fewer trophozoites per $\mathrm{cmm}$ of blood regardless of the manner in which the attack was induced. It is to be further noted that the greater the disparity between the first detection of parasites and the first fever, the higher will be the pyrogenic level. The works of other observers eited by Sinton et al. (1931) lead them to suggest that there 
TABLE IX

Parasite Density at the Trme of Clinical Onset of Primary Induced Vivax Malaria

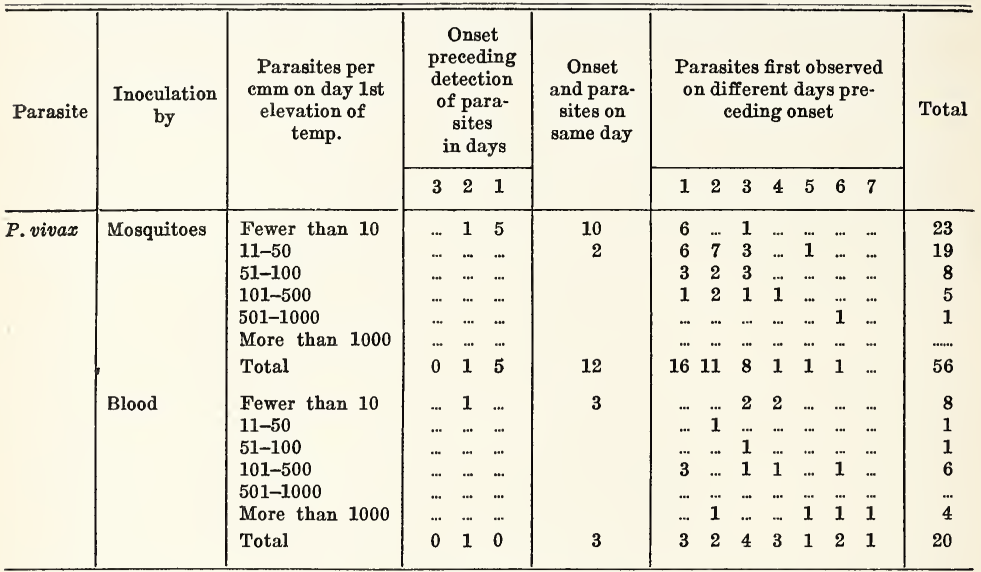

is not a constant pyrogenic threshold for all strains of this parasite.

A still higher threshold is observed at the onset of relapses in chronic infections. Thus studies by Sinton et al. (1931) indicate that under this circumstance a pyrexial level of about 5,000 per cmm prevails.

Our own experience leads to the view that varying susceptibility of patients rather than varying virulence of different strains of parasites is chiefly responsible for the variations in density noted at the onset.

\section{The Parasites in Relation to the Sxmptoms}

The intermittent and periodic febrile paroxysms experienced by patients with an active malaria infection are, as first shown by Golgi, directly ascribable to the maturation and asexual division of a brood or generation of parasites. The relationship is shown in the accompanying figure (Fig. 4). Smears made at the onset and during a few days shortly thereafter show a great diversity in the development of the parasites observed at any hour, although even at this period a majority may be in the same stage. In this circumstance the patient may exhibit a remittent, rather than an intermittent, fever. As the infection progresses the parasites, for reasons not clearly understood, tend to become more closely synchronized, so that all of a given generation complete their development within a few hours of each other. The generations in falciparum infections do not usually become as closely synchronized as in the case of the other species. Several may mature at different periods on the same day, thus greatly prolonging the duration of the fever, which may show several distinct peaks.

A schizogonous generation of $P$. vivax and $P$. falciparum requires 48 hours and one of $P$. malariae requires 72 hours for its completion. During the growth of the parasites the infected person will not present any markedly significant symptoms connected with the cycle. With the completion of schizogony and the liberation of the merozoites from the divided schizonts in the shells of the erythrocytes in which they were formed, the patient, provided the pyrogenic density of parasites is attained, 


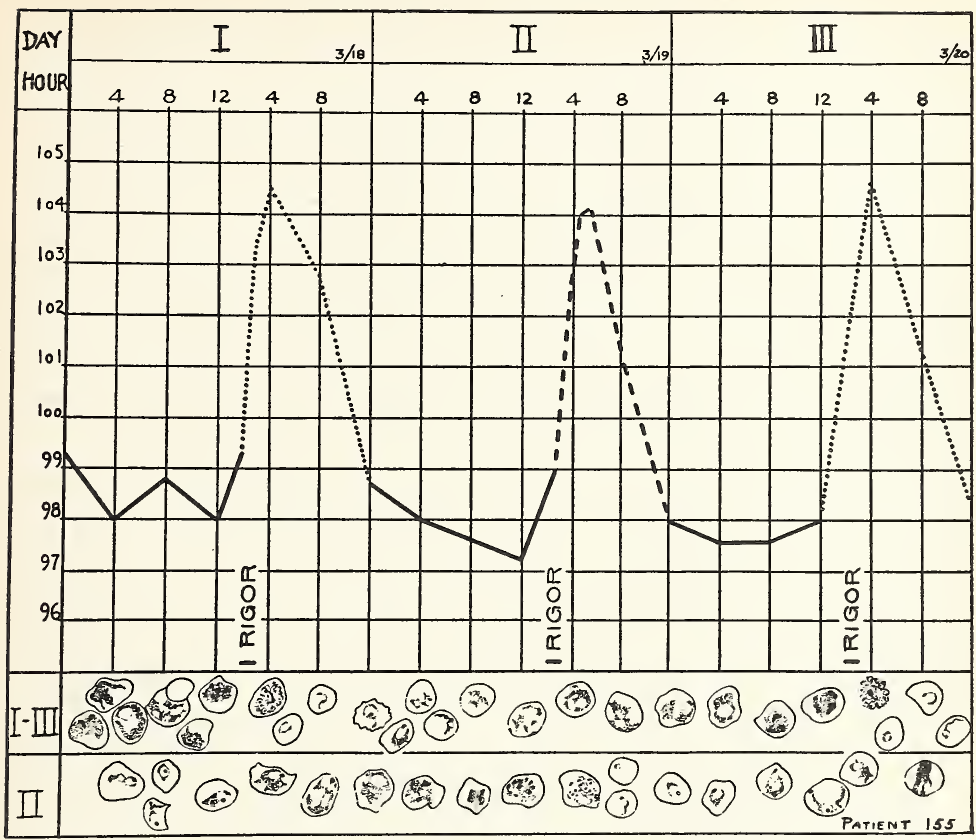

FIG. 4. Showing three days of the quotidian elinical attack experienced by Patient 155, infected with $P$. vivax. Periods on which the temperature ranged within normal limits are shown by solid lines, the elevations on the 1st and $3 \mathrm{~d}$ days (dotted lines) are due to the multiplication of the same cycle of parasites, that occurring on the $2 \mathrm{~d}$ day (dash line) is due to a second eycle maturing on alternating days. The short bar shows the relation of the period of the rigor to each febrile paroxysm. Beneath are shown a few representative parasites sketched from blood smears taken at each four hour interval when the temperature was observed, grouped according to the relation of their sporulation to the corresponding paroxysms.

experiences a febrile paroxysm, and will experience further paroxysms on subsequent days as each further generation matures. If the infection is dominated by but a single generation of $P$. vivax or $P$. malariae, the paroxysms will recur at tertian or quartan intervals. More commonly patients experiencing an initial infection with vivax present two generations maturing on alternate days, which will produce quotidian paroxysms. It is important to note that vivax infections are commonly initiated by intermittent quotidian parox- ysms, even when the patient was inoculated on only one occasion. The occurrence of two or more cycles or generations thus cannot be attributed to inoculation on successive days. In the case of quartan infection, a third cycle must arise in order to produce quotidian paroxysms. If the time for the maturation is either less or more than 24 hours, it will be noted that the successive paroxysms do not recur at the same hour, but either regularly anticipate or postpone.

A clinical attack of either disease characterized by quotidian paroxysms may ab- 
ruptly change to a tertian or double quartan, or the latter may similarly become a simple quartan. Such changes are attributable to the partial suppression of the corresponding parasite generation. The nature of the mechanism, particularly the manner by which its action is limited to one of the parasite generations, is not understood. Conversely, one may observe the introduction of new cycles in a tertian or quartan series, changing the attack to a quotidian. The effect of the new generation may be manifested either gradually or abruptly.

The paroxysm of intermittent fever presents a succession of well defined stages. It may or may not be initiated by a cold stage, otherwise known as a chill or rigor. This stage is not, however, observed at the onset of primary infections, and it may not be evident until the disease has continued for one or two weeks. The cold stage is initiated by a sensation of chilliness, rapidly progressing to shivering, often to an extreme degree. The skin is pale and bloodless, while the nails and lips are livid, the pulse is weak and rapid, and breathing is fast and shallow. Nausea is experienced and vomiting may be distressing. Convulsions are not infrequent in children. The patient will usually request all available cover. Before the end of this period, which may last from 1-3 hours, the temperature is already above normal, and the patient experiences a brief interval of comfort. The temperature continues to rise and may attain considerable elevation, persisting for 1-2 hours. The face is flushed, the skin hot and dry, the pulse is full and bounding, the head usually aches and some delirium may be evident. The bed clothes are discarded. The stage of fever is terminated by the appearance of a profuse perspiration, during which the temperature rapidly falls to normal or below. The patient may feel exhausted, but after a brief sleep awakes refreshed and tranquil. This stage may last from 2 to 4 hours.

The paroxysms of quartan last longer than those of vivax, and leave the patient with a sense of prostration not usually observed after the latter. In the intervals between paroxysms vivax patients will go about their usual pursuits. Some sporulation will have taken place before the onset of the rigor, but in general the rigor coincides with the time of sporulation. Most of the merozoites will have entered fresh erythrocytes before the end of the hot stage.

Although, as shown, the initiation of symptoms coincides with the maturation of certain minimal numbers of parasites, the manner in which this process initiates the paroxysms has not been satisfactorily elucidated. Brown (1912) observed that a solution of alkaline hematin injected intravenously into rabbits produced a paroxysm characterized by a short prodromal stage, a stage of chill and rising temperature, and a hot stage. He concluded that the human paroxysm is, at least in part, referable to the toxic action of this pigment. In this connection it may also be mentioned that the studies of Sinton and Ghosh (Ghosh and Nath 1934) indicate that the haemazoin of $P$. knowlesi, whose pigment has been studied most intensively, appears to be identical with hematin. The rise in the plasma potassium level which occurs at the time of sporulation of the parasite has been studied by Zwemer, Sims and Coggeshall (1940). It is not clear whether the rise is attributable to the release of potassium from parasitized erythrocytes or has been hiberated from body cells in general. That the high potassium values may be causably associated with the rigors is indicated by the observation of Beeson and Hoagland (1940) that the intravenous injection of 10 ec of a 10 per cent solution of calcium chloride will effect an almost instantaneous cessation of the rigor. In view of the relation of the adrenal cortex to the maintenance of a potassium equilibrium, continuation of these studies may have extremely significant results. Abrami and Senevet (1919) suggest that the paroxysm, in part at least, is an anaphylactic reaction, resulting from the sudden liberation into the plasma of the protein of burst parasites. They point out a parallelism 


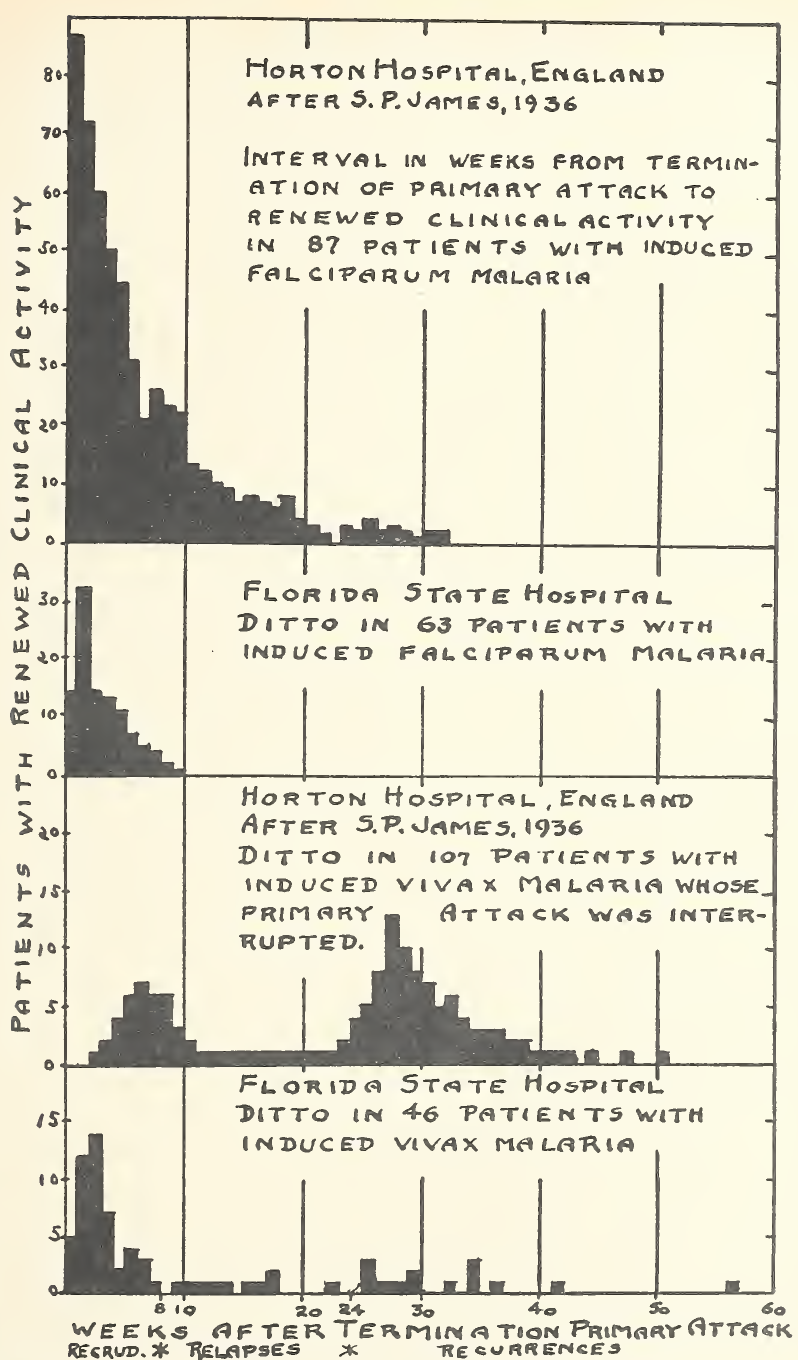

FiG. 5. Showing interval between termination of primary attack and periods of renewed clinical activity in falciparum and vivax malaria (See Table XI). 
between certain phenomena which precede the rigor and the reaction or "haemoclasis" resulting from the inoculation of certain foreign substances. These include a lowered arterial tension, leucopenia, diminution in the number of erythrocytes and changes in coagulability of the blood.

\section{Renewed Clinical Activity}

Malarial infections, even when untreated, frequently, almost characteristically, produce a series of clinical attacks separated by varying periods of quiescence. The first period of clinical activity following inoculation is known as the primary attack while the subsequent periods of secondary activity, depending upon the length of the interval between the termination of the primary attack and the reactivation, are, following James, variously designated as (a) recrudescence-renewed activity within 8 weeks, (b) relapse-renewed activity within from 8 to 24 weeks, and (c) recurrence-renewed activity after 24 weeks.

There is no certain criterion by which the end of the primary attack may be recognized. We have arbitrarily considered that the beginning of a quiescent interval at least equal to the suppression of two complete consecutive parasite cycles (5 days) marks its end. We have, however, reasons to believe that with vivax infections at least an interval of three weeks would be a surer criterion.

The duration of an uninterrupted primary attack in a highly susceptible person, as well as the duration of the quiescent intervals before secondary clinical activity, varies with the species of parasite, while the likelihood of secondary attacks may vary with the strain. Thus we have observed vivax patients to have an unbroken series of quotidian or tertian paroxysms for more than 60 days, quartan patients to have quotidian, double quartan or simple quartan paroxysms for more than 300 days, and falciparum patients a course lasting for more than 35 days. In vivax infections we have not observed secondary attacks when the primary attack exceeded 48 days in duration, while they may occur in falciparum infections in which the primary attack attains the maximum duration. These infections can maintain themselves in a chronic latent condition for indeterminate periods following the permanent cessation of clinical activity. It is impossible to assign even approximate limits to their persistence, but it appears that it is shortest for falciparum and longest for quartan, which latter may persist for several years. It is important to note that artificially induced infections in human subjects do not exhibit the chronicity which characterizes the naturally induced infections, although artificially induced avian infections appear to present a chronicity comparable to the naturally induced. The significance of this will be considered later.

The frequency with which secondary clinical activity has been observed subsequent to natural inoculation is shown in Table X.

From this table it is seen that secondary attacks oceur in a considerable proportion of patients whose primary attacks terminate spontaneously. They are very nearly

TABLE $\mathrm{X}$

Frequenct of Secondary Clinical Activity in RELATION TO MANNER OF TERMINATION OF the Primary AtTack

\begin{tabular}{|c|c|c|c|c|}
\hline \multirow{3}{*}{$\begin{array}{c}\text { Termination } \\
\text { of primary } \\
\text { attack }\end{array}$} & \multicolumn{4}{|c|}{ Per cent with secondary attacks } \\
\hline & \multicolumn{2}{|c|}{ P. vivax strains } & \multicolumn{2}{|c|}{$\begin{array}{c}P . \text { falciparum } \\
\text { strains }\end{array}$} \\
\hline & MeCoy & $\begin{array}{l}\text { Mada- } \\
\text { gascar }\end{array}$ & $\begin{array}{l}\text { Vari- } \\
\text { ous }\end{array}$ & $\begin{array}{l}\text { Vari- } \\
\text { ous }\end{array}$ \\
\hline $\begin{array}{l}\text { Spontaneous, } \\
\text { without sub- } \\
\text { sequent treat- } \\
\text { ment }\end{array}$ & 58.0 & $\ldots . . . . .$. & 42.8 & ........... \\
\hline $\begin{array}{l}\text { Induced, but } \\
\text { without sub- } \\
\text { sequent treat- } \\
\text { ment ........................ }\end{array}$ & 100.0 & $\ldots$ & 85.6 & .......... \\
\hline $\begin{array}{l}\text { Induced, with } \\
\text { subsequent } \\
\text { treatment }\end{array}$ & 25.0 & $47.4^{\mathrm{a}}$ & 8.3 & $80.6^{b}$ \\
\hline
\end{tabular}

s James, S. P. (1931). b James, Nicol and Shute (1932). Includes naturally and artificially inoculated patients. 
TABLE XI

Relative Frequency of Renewed Activity at DIFFERENT InTERVals Following Termination of Primary A tTack (Fig. 5)

\begin{tabular}{l|cc|cc}
\hline \multirow{2}{*}{$\begin{array}{c}\text { Interval } \\
\text { subsequent } \\
\text { to primary } \\
\text { attack }\end{array}$} & \multicolumn{2}{|c|}{ Per cent with secondary attacks } \\
\cline { 2 - 5 } & $\begin{array}{c}\text { McCoy } \\
(46)\end{array}$ & $\begin{array}{c}\text { Mada- } \\
\text { gascar } \\
(107)\end{array}$ & $\begin{array}{c}\text { Vari- } \\
\text { ous } \\
(63)\end{array}$ & $\begin{array}{c}\text { Vari- } \\
\text { ous } \\
(87)\end{array}$ \\
\hline $\begin{array}{c}\text { Under } 8 \\
\text { weeks } . . . . .\end{array}$ \\
$\begin{array}{c}\text { 8-24 } \\
\text { weeks ...... } \\
\text { Over 24 } \\
\text { weeks ...... }\end{array}$ & 13.7 & $17.8^{\mathrm{a}}$ & 2.9 & $24.2^{\mathrm{a}}$ \\
\hline
\end{tabular}

a James, Nicol and Shute (1936).

b Primary attacks interrupted.

inevitable when the attack is interrupted, but not followed by intensive treatment. It is interesting to note that the exotic strains in the hands of James have shown a much higher proportion of secondary attacks even when intensively treated.

The extent to which this characteristic may be related to the strain of parasite, is shown by the occurrence of secondary attacks in 68 (45.6 per cent) of 149 patients inoculated with the McCoy strain of $P$. vivax, while of 21 other patients inoculated with six other strains, only one (4.7 per cent) had a secondary attack (Boyd and Kitchen 1937d).

Some observations on the frequency of secondary attacks at varying intervals subsequent to the termination of the primary attack are shown in Table XI.

While the falciparum observations cited from James, Nicol and Shute (1936) are not strictly comparable with our own, probably due to the use of different criteria for distinguishing the termination of the primary attack, the data as a whole, nevertheless, present an agreement in certain essential characteristics. It will be noted that renewal of activity after the lapse of 24 weeks is more definitely a characteristic of vivax strains than of falciparum strains, while the New World strains have exhibited a decidedly less frequent tendency to become reactivated after long intervals of quiescence than have the Old World strains.

While it is likely that our criterion of the end of the primary attack is based on too short a period of quiescence, which might better be marked by a three-week interval, it appears unquestionable that the secondary attacks occurring within 8 , and perhaps even 12 , weeks of the termination of the primary attack are really a direct continuation of the latter.

TABLE XII

P. vivax: Interfal Elapsing from the End of the Primary Attack to Various Periods of Renewed Activity, in Relation to the Length of the Primary Attack

\begin{tabular}{|c|c|c|c|c|c|c|c|c|c|c|c|c|c|c|}
\hline \multirow{3}{*}{$\begin{array}{c}\text { Duration } \\
\text { primary } \\
\text { attack } \\
\text { in days }\end{array}$} & \multicolumn{8}{|c|}{ Natural inoculation } & \multicolumn{6}{|c|}{ Artificial inoculation } \\
\hline & \multirow[t]{2}{*}{$\begin{array}{l}\text { Total } \\
\text { cases }\end{array}$} & \multicolumn{3}{|c|}{$\begin{array}{c}\text { Secondary } \\
\text { attack } \\
\text { in weeks }\end{array}$} & \multicolumn{3}{|c|}{$\begin{array}{c}\text { Further } \\
\text { secondary } \\
\text { attacks } \\
\text { in weeks }\end{array}$} & \multirow[t]{2}{*}{$\begin{array}{l}\text { Total } \\
\text { cases }\end{array}$} & \multicolumn{3}{|c|}{$\begin{array}{c}\text { Secondary } \\
\text { attack } \\
\text { in weeks }\end{array}$} & \multicolumn{3}{|c|}{$\begin{array}{c}\text { Further } \\
\text { secondary } \\
\text { attacks } \\
\text { in weeks }\end{array}$} \\
\hline & & $0-7$ & $8-24$ & $24+$ & $0-7$ & $8-24$ & $24+$ & & $0-7$ & $8-24$ & $24+$ & $0-7$ & $8-24$ & $24+$ \\
\hline $0-6 \quad \ldots \ldots$ & 13 & 2 & $\ldots$ & $\ldots$ & 1 & $\ldots . .$. & $\ldots$ & 7 & 2 & $\ldots$ & $\ldots$ & $\ldots$ & $\ldots$ & $\ldots$ \\
\hline $7-13$ & 44 & 13 & $\ldots$ & 3 & 3 & 3 & 4 & 7 & 1 & $\ldots$ & $\ldots$ & $\ldots$ & $\ldots$ & $\ldots$ \\
\hline $14-20$ & 27 & 15 & 1 & 1 & 9 & 2 & $\ldots$ & 8 & 5 & ... & $\ldots$ & 2 & $\ldots$ & $\ldots$ \\
\hline $21-27 \ldots$ & 25 & 16 & $\ldots$ & 1 & 5 & 2 & 3 & 7 & 5 & $\ldots$ & $\ldots$ & 1 & $\ldots$ & $\ldots$ \\
\hline $28-34$ & 16 & 4 & $\ldots$ & 1 & 1 & 1 & 1 & 2 & ....... & $\ldots$ & $\ldots$ & $\ldots$ & $\ldots$ & $\ldots$ \\
\hline $35-41 \ldots \ldots \ldots \ldots$ & 12 & 4 & 2 & 1 & 1 & 2 & $\ldots$ & 4 & $\ldots . .$. & $\ldots$ & $\ldots$ & $\ldots$ & $\ldots$ & $\ldots$ \\
\hline $42-48$ & $\mathbf{5}$ & 3 & 1 & $\ldots$ & $\ldots . .$. & $\ldots . .$. & $\ldots$ & 3 & $\ldots . .$. & $\ldots$ & $\ldots$ & ... & $\ldots$ & $\ldots$ \\
\hline $49-69$ & 7 & $\ldots . .$. & $\ldots$ & ... & $\ldots \ldots$ & $\ldots \ldots$. & $\ldots$ & 3 & $\ldots . .$. & ... & $\ldots$ & $\ldots$ & $\ldots$ & $\ldots$ \\
\hline Total & 149 & 57 & 4 & 7 & 20 & 10 & 8 & 41 & 13 & 0 & 0 & 3 & 0 & 0 \\
\hline
\end{tabular}


TABLE XIII

P. vivax: Relation of Period of Renewed Acrivity to Period of Inoculation (BOYD AND KITCHEN 1937d)

\begin{tabular}{|c|c|c|c|c|c|c|c|c|c|c|}
\hline \multirow{3}{*}{$\begin{array}{l}\text { Period of } \\
\text { inoculation }\end{array}$} & \multicolumn{10}{|c|}{ Period of renewal } \\
\hline & \multicolumn{5}{|c|}{ Quiescent 8-24 weeks } & \multicolumn{5}{|c|}{ Quiescent more than 24 weeks } \\
\hline & Winter & Spring & Summer & Fall & Total & Winter & Spring & Summer & Fall & Total \\
\hline Winter ............. & ... & $\ldots$ & 1 & $\ldots$ & 1 & $\ldots$ & ... & ... & $\ldots$ & $\ldots$ \\
\hline 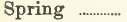 & ... & ... & 2 & 4 & 6 & 2 & 1 & ... & ... & 3 \\
\hline Summer ........... & .... & ... & ... & 1 & 1 & ... & 4 & $\ldots$ & $\ldots$ & 4 \\
\hline Fall ……................ & 2 & 1 & $\ldots$ & .. & 3 & 1 & 2 & 2 & $\ldots$ & 5 \\
\hline Total .......... & 2 & 1 & 3 & 5 & 11 & 3 & 7 & 2 & 0 & 12 \\
\hline
\end{tabular}

Another feature of interest in relation to these long term recurrences is shown in Table XII.

It is to be noted that we have not observed long term recurrences subsequent to artificial inoculation. It may be mentioned that neither Yorke and Macfie (1924a) nor James (1931) have observed renewed activity following the termination of attacks induced by artificial inoculation after periods of quiescence of more than 8 weeks duration.

The season in which renewed activity after a long quiescence has occurred is compared with the season of inoculation in Table XIII.

It is thus seen that renewed activity has, in our experience, less frequently followed inoculations made in the winter months than those made at other seasons. It is interesting to observe that most secondary attacks after the longest intervals of quiescence have occurred in the winter or spring. The longest interval we have observed between the termination of a primary vivax attack and a secondary attack has been 394 days.

These long term recurrences present another feature of interest as may be seen from Table XIV. If we assume that renewed activity within 8 weeks of the termination of the primary attack may be regarded as essentially a part of the latter, and group our cases accordingly, it will then be noted that either the primary attack or an early secondary attack had been interrupted in all patients who experienced clinical activity 24 weeks after the termination of the primary attack, and that interference had also been practiced in the

TABLE XIV

Showing the Manner in Which the Primary Attack and Early Secondary Attacks Were Terminated, in Certain Cases of Vivax Malaria who Subsequently Experienced LONG TERM RECURRENCES

\begin{tabular}{|c|c|c|c|c|c|c|c|c|c|}
\hline \multirow[t]{2}{*}{$\begin{array}{l}\text { Termination } \\
\text { of primary } \\
\text { attack }\end{array}$} & \multirow[t]{2}{*}{$\begin{array}{l}\text { Duration of } \\
\text { original at- } \\
\text { tack (days) }\end{array}$} & \multicolumn{2}{|c|}{$\begin{array}{c}\text { Recrudescence } \\
\text { 0-7 weeks: } \\
\text { termination }\end{array}$} & \multirow{2}{*}{$\begin{array}{c}\text { Persons } \\
\text { with re- } \\
\text { lapse in } \\
\text { 8-24 } \\
\text { weeks }\end{array}$} & \multicolumn{2}{|c|}{$\begin{array}{c}\text { Previous } \\
\text { recrudescence } \\
0-7 \text { weeks; } \\
\text { termination }\end{array}$} & \multicolumn{2}{|c|}{$\begin{array}{c}\text { Previous re- } \\
\text { lapse } 8-24 \\
\text { weeks ; termi- } \\
\text { nation }\end{array}$} & \multirow[t]{2}{*}{$\begin{array}{c}\text { Recur- } \\
\text { rences } \\
\text { after } 24 \\
\text { weeks }\end{array}$} \\
\hline & & Spont. & Induced & & Spont. & Induced & Spont. & Induced & \\
\hline Spontaneous & $\begin{array}{c}7-20 \\
21-41 \\
\text { Over } 42\end{array}$ & $\begin{array}{l}2 \\
1 \\
\ldots\end{array}$ & $\begin{array}{l}\cdots \\
\cdots \\
\cdots\end{array}$ & $\begin{array}{l}2 \\
2 \\
1\end{array}$ & $\begin{array}{l}\cdots \\
\cdots \\
\ldots\end{array}$ & $\begin{array}{l}\ldots \\
2 \\
\ldots\end{array}$ & $\begin{array}{l}\cdots \\
\ldots \\
\cdots\end{array}$ & $\begin{array}{l}\cdots \\
\cdots \\
\ldots\end{array}$ & $\begin{array}{l}\ldots \\
2 \\
\ldots\end{array}$ \\
\hline Induced & $\begin{array}{c}7-20 \\
21-41 \\
\text { Over } 42\end{array}$ & $\begin{array}{l}\cdots \\
1 \\
\cdots\end{array}$ & $\begin{array}{l}1 \\
2 \\
\cdots\end{array}$ & $\begin{array}{l}2 \\
3 \\
1\end{array}$ & $\begin{array}{l}\ldots \\
1 \\
\ldots\end{array}$ & $\begin{array}{l}2 \\
\cdots \\
\cdots\end{array}$ & $\begin{array}{l}\cdots \\
\cdots \\
\cdots\end{array}$ & $\begin{array}{l}1 \\
\cdots \\
\cdots\end{array}$ & $\begin{array}{l}6 \\
4 \\
\cdots\end{array}$ \\
\hline
\end{tabular}


case of 6 of the 11 patients who experienced renewed activity in from 8 to 24 weeks. The effect attributed to our use of quinine in the patients simultaneously inoculated with two species of parasites may be recalled. This suggests that recurrences after the longest intervals may be related to the prematurely early administration of a drug.

It is thus seen that in vivax malaria there is a striking parallelism, as pointed out by James (1931), in the length of the interval between inoculation and onset in those patients who experience protracted incubation periods and the length of the interval between the termination of the primary attack and the beginning of a recurrence. In this connection we may reiterate that: long-term recurrences are only observed in naturally infections; the clinical activity which marks the termination of the period of quiescence is most commonly observed in the spring; and they are frequently observed in patients who were receiving a plasmodicidal drug at the time of inoculation or shortly after the clinical onset.

In this connection we may also reiterate the following. significant facts previously brought out: the failure to observe the penetration of erythrocytes by sporozoites; the non-infectiousness of the blood for several days following natural inoculation; the inability to shorten the duration of the intrinsic incubation period by the employment of massive doses of sporozoites; and the complete elimination of an incubation period following massive doses of trophozoites.

These facts are not inconsistent with the hypothesis of the existence of a stage of the parasite living in fixed tissue cells which intervenes between the sporozoite and the trophozoite, the development of which may either be retarded, or inhibited or narcotized by a drug.

Added interest and significance is afforded this view by the recent discoveries of an exo-erythrocytic schizogony in several species of avian malaria parasites. In $P$. gallinaceum, in which this stage has been most extensively studied, the exo-erythrocytic phases are passed in endothelial cells. According to James (1939) they are observed subsequent to either natural or artificial inoculation. However, subsequent to the former inoculations they are observed at the onset; in the latter, only at a late stage of the disease. He consequently concludes that in this parasite the stages of schizogony can alternate between erythrocytes and fixed tissue cells. It seems very probable that something similar occurs with the human parasite subsequent to natural inoculation. Since chronic infections do not follow artificial inoculations with the human parasite, while they do with certain species at least of the avian parasites, it is suggested that in the human infection this stage only follows the sporozoite.

\section{Diagnosis}

Although many cases of malarial infection presenting the characteristic symptoms of intermittent febrile paroxysms regularly recurring at quotidian, tertian or quartan intervals can be diagnosed with reasonable certainty from the symptomatology, the diagnoses for those patients in whom the fever is remittent or the paroxysms irregular, in whom the onset is accompanied by alarming symptoms of a dangerous portent, or in whom the infection has become latent must be based on the demonstration of the parasites in blood smears. Additional clinical signs observable as the infection progresses are an enlargement of the spleen and a progressive anemia.

The practitioner or clinician is most often concerned with the diagnosis of clinically active infections in individual patients; the epidemiologist or malariologist, with the recognition of latent infections among a population group.

Objective criteria of varying value for the diagnosis of malarial infections are afforded by: (a) The detection of parasites in the blood; (b) the detection of splenomegaly; (c) changes in the normal hematological picture; (d) serological tests; and (e) biochemical tests. 
(a) The detection of parasites. The detection of the parasites in a blood smear is incontestable proof of the existence of a malarial infection. Excepting for a period of a few days to a week subsequent to the clinical onset of an attack in highly susceptible untreated patients, they are more or less readily detectable on microscopical examination of blood smears taken during an attack. As clinical activity diminishes and the infection becomes latent their numbers gradually diminish to submicroscopical levels. The importance of the subject justifies its separate consideration in an independent section (q.v.). It is deplored that the method is not more widely used by medical practitioners.

In order to facilitate the detection of parasites when their density is too low to be revealed in ordinary thick smears, various procedures have been devised which can only be briefly mentioned. Bass (1915) developed a technique by which the parasites in 10 ec of blood were concentrated by centrifugation at the top of the blood column, from which thick smears were made. The culture technique of Bass (1912), as modified by Thomson and Thomson (1913), is at times used by Indian clinicians to aid in the detection of parasites (Knowles and Senior-White 1927).

Reliable means to reactivate latent infections, either to facilitate diagnosis or to prolong therapeutic infections, are not available. The administration of different drugs for this purpose shows that the responses evoked in general fall into two classes. Some, such as epinephrine hydrochloride and amyl nitrite, may produce an increase in the number of parasites in the peripheral circulation within a few hours of their administration. Since the time elapsing until the increase in density is observed is too short to attribute this result to their multiplication, they must have been expelled from some viscus. The administration of others, such as tuberculin and typhoid vaccine, may bring about a gradual rise extending over a period of several days, indicative of multiplication. The circumstances suggest that in the latter case the immune mechanism has been depressed.
The most reliable means of excluding the existence of an otherwise undetectable latent infection is to subinoculate a susceptible patient with 10 or more cubic centimeters of the blood of the patient suspected of being infected. The opportunities to apply this procedure are limited to institutions where malarial therapy is practiced.

(b) Splenic enlargement or splenomegaly. Enlargement of the spleen may be detected by palpation within a few days after the clinical onset. The rate of increase in size is more rapid in persons who have some degree of immunity, while the degree of enlargement is roughly proportional to the duration of the clinical attack. On cessation of the clinical attack, particularly if interrupted by treatment, the enlargement rapidly diminishes and may altogether subside. The persistence of enlargement implies the continuance of a latent infection and the probability of relapse. Further enlargement will follow a renewal of clinical activity (Boyd 1930b).

(c) Hematological changes. The hematological changes are significant from the standpoint of the symptoms exhibited, but at the best only supply information of indirect or inferential diagnostic value.

As may be expected in an infection the parasites of which prey on the erythrocytes, malarial infections are characterized by marked destruction of red blood cells and of hemoglobin. Consequently anemia is a prominent symptom. Rate of destruction varies with the species of parasite, and is often more rapid than can be explained by the assumption that each parasite in the course of its growth exhausts only a single host cell. Some of this discrepancy may be explained by the phagocytosis of both infected and uninfected erythrocytes in the splenic pulp, while hemolysis also may occur.

The different parasites usually vary materially in the maximum density which they ordinarily attain and hence differently influence the rate of destruction. The densities in quartan are usually the lowest, rarely reaching 20,000 per $\mathrm{cmm}$; in vivax malaria, 50,000 per cmm is but rarely ex- 
ceeded, while in falciparum malaria no limit is apparent. In the latter infections counts of 500,000 per emm are a bad prognostic sign.

One possible factor, at least in determining the potential density, would appear to be the available numbers of erythrocytes of different ages for which different species of parasites appear to show a specific predilection. Thus the studies of Kitchen (1939b) indicate that $\boldsymbol{P}$. malariae prefers mature or perhaps even aged erythrocytes, $\boldsymbol{P}$. falciparum is indifferent to the age of the erythrocytes it attacks, while $P$. vivax prefers reticulocytes. As might be expected, therefore, the rate of blood destruction is most rapid in falciparum infections and slowest in quartan. In the former, rapid blood destruction may conceivably be a factor in producing a fatal outcome, as a consequence of an anoxemia. On the other hand, clinical activity in vivax infections frequently comes to a spontaneous cessation when the erythrocyte density is lowered to the neighborhood of $1,750,000$, with 5 grams of hemoglobin. In the attacks of longest duration restoration of erythroeyte density and hemoglobin is evident before the termination of the attack. Restoration of the former proceeds more rapidly than that of the latter.

The anemia does not present distinctive characteristics. A moderate degree of anisocytosis will be observed, while the color index is variable. Evidence of regeneration of erythrocytes, afforded by the presence of reticulocytes and normoblasts, is quite constant in acute infections. Since the iron from the destroyed cells is retained in the body, the anemia is not hypochromic. Individuals who experience a protracted chronic relapsing infection may develop an intense anemia, probably of an aplastic character.

Less striking but nevertheless noteworthy ehanges occur among the leucocytes. In general, particularly in the apyrexial intermissions, these infections are characterized by a leucopenia, although at the time of a paroxysm a slight leucocytosis is evident. Furthermore, in the intervals between paroxysms there is noted a marked increase in the proportion of cells of the mononuclear varieties, so that they fluctuate inversely with the temperature. During these intervals the polymorphonuclear leucocytes decline from 70 to 50 per cent of the total leucocytes, while the mononuclear leucocytes rise from 25 to 45 per cent or even higher. The increase is most evident in the monocytes. As a paroxysm declines much phagocytosis of pigment by polymorphonuclear leucocytes may be observed.

(d) Serological methods. Serological methods of diagnosis are still in the experimental stage. One of these, based on the fixation of complement, appears to be of promise and will be discussed in the section on humoral immunity (q.v.).

(e) Biochemical methods. In 1927 Henry, assuming that the "melanotic" and "yellow ferruginous" pigments formed in the tissues of malaria patients might either give rise to the formation of specific antibodies, or disturb the colloidal state of the serum so as to impart to it certain floceulating properties, reported on certain tests designed to reveal such changes. These were designated as the melano-reaction and the ferro-flocculation tests, respectively, in which, owing to the difficulty of securing true malaria pigments, choroidal melanine and certain organic iron compounds were employed as "antigens." The latter test soon fell into disuse but reports on the former indicate that it merited some consideration. It has, however, attracted but little attention in this country.

As originally devised, ehoroidal melanin secured from ox-eyes was used as antigen, but difficulties in securing a uniform suspension led Greig, van Rooyen and Hendry (1934) to modify the test so as to employ melanin pigment extracted from human hair.

The demonstration that melanin and organic iron compound were antigenically inert, with the observation that positive melano-reactions were secured from the serum of patients ill with other diseases in which neither of these pigments were produced, plus the further cireumstance that 
positive sera became negative after exposure to heating to $55^{\circ} \mathrm{C}$ for 30 minutes, indicate that the reaction does not have an antibody basis. On the other hand, several observers have noted a parallelism between the melano-reaction and a flocculation in mixtures of serum and distilled water. Observations by Chorine and Gillier (1934) indicated that the substance flocculating in distilled water is water-insoluble euglobulin, probably associated with other substances such as cholesterol, lecithin and uric acid of low water solubility. It thus finally appears that the reaction of Henry is due to a disequilibrium of serum proteins brought about by an increase in serum globulin, in which the melanin served as an indicator.

Based on these principles, Proske and Watson (1939) report a simple colometric test to detect an increase in euglobulin, which avoids the need for an expensive photometer required for the original Henry test. "The procedure is based on the fact that proteins possess a chromogenic property which can be measured quantitatively against the color produced by pure tyrosine in the presence of a phenol reagent. This chromogenic value is constant for a given protein and the intensity of the color produced can be used as a measure of the amount of the protein examined. Serum euglobulin is precipitated from the serum to be examined by the addition of $13.5 \mathrm{per}$ cent sodium sulphate solution, according to the method of Howe. The tyrosine chromogenic index (TI) is determined by comparison with standards prepared from pure tyrosine (Pfanstiehl).",

"The tyrosine index for euglobulin fluctuates between 50 and 80 , while that for serum from malaria patients ranges from 80 to 280 , or higher. The test was found to be indicative of the presence of malaria in 97.4 per cent of known cases of malaria examined, as compared with 81.9 per cent positive thick blood films examined at the same time." 


\title{
THE INFECTION IN THE INTERMEDIATE HOST SYMPTOMATOLOGY: VIVAX MALARIA
}

\author{
BY WARREN K. STRATMAN-THOMAS
}

DIVISION OF PREVENTIVE MEDICINE, UNIVERSITY OF TENNESSEE SCHOOL OF MEDICINE, MEMPHIS, TENN.

Vivax malaria, due to infection by Plasmodium vivax, is the predominant malarial fever in temperate endemic zones. The terms "tertian malaria" or "benign tertian malaria," by which it is commonly known, while descriptive in so far as the cycle of the parasite is concerned, are perhaps unfortunate in that they do not tend to give a representative impression of the clinical course.

\section{OвJECTIVE SYMptoms}

During the incubation period there are no objective symptoms unless one accepts the first detection of parasites in the peripheral blood smears as marking the end of the incubation period. In the experience of Boyd and Stratman-Thomas (1934c), this most commonly occurred on the 13th day following inoculation. In some instances this marking point occurs a few days following the clinical onset.

Fever. Fever of $100^{\circ} \mathrm{F}$ marking the clinical onset is usually not observed before the 10th day after natural inoculation. If, however, a large number of infected mosquitoes has been used to inoculate a patient (as has been done in the administration of therapeutic malaria), a temperature of $100^{\circ} \mathrm{F}$ or higher may appear as early as the eighth day.

The first objective symptom to appear is a fever of about $100^{\circ} \mathrm{F}$ or above, which occurs within 1 to 3 days preceding or following the first appearance of $P$. vivax in the blood. The fever may be continuous or remittent over a period of 1 to 3 days (initial stage of James 1926) or may be an intermitten quotidian or, rarely following the onset, tertian. No chills occur during the period of continued fever. In $P$. vivax infections, unlike $P$. falciparum infections, a period of continued fever is rarely seen excepting at the end of the incubation period. The intermittent febrile paroxysms may assume a variety of sequences, quotidian fever being followed by tertian; a series of tertian changing to quotidian, even isolated quartan intervals have been noted. In patients exhibiting the continued quotidian and tertian fever, these generally follow one another in the order named.

The fully developed paroxysm consists of the three classical stages, namely, the period of chill or rigor, the pyrexial stage, and the sweating stage, or period of defervescence. Only rarely is the first paroxysm preceded by a chill. In the majority of patients 5 or more days of intermittent fever occur before the first chill is experienced. A peak temperature of less than $102^{\circ} \mathrm{F}$ is rarely preceded by a chill. Onset of an attack by a paroxysm attended with a chill suggests either (a) that the attack represents a relapse or (b) that the patient has had previous experience with malaria.

The paroxysms may occur at any time of the day, and those due to the same parasite cycle may recur at approximately the same time. However, by anticipation or postponement those of one cycle in successive paroxysms may oceur a short time before or after the hour of their immediate predecessors. Thus a certain cycle may gain or lose 24 hours or more over a period of time. When the paroxysms are quotidian, sometimes those of one cycle may occur in the morning and those of the other in the afternoon. When the fever is quotidian, rigors may accompany only one cycle and thus may be experienced only every other day. As the clinical course continues, the duration of the rigor may increase from 5 minutes to an hour, although 25 to 40 minutes is their usual duration.

In nearly all instances in which the clin- 
ical attack terminates spontaneously the rigors stop before the fever paroxysms. A progressive decline in the height of the quotidian or tertian fever peaks is prognostic of the imminent cessation of the rigors; further decline in the height of the temperature after the chills have stopped forecasts an early spontaneous termination of the clinical attack.

Observations made by Kitchen (1940) on $P$. vivax paroxysms showed that (1) 90 per cent of the paroxysms in their patients occurred during the post meridian hours and 70 per cent from 3 to 9 P.M., (2) the majority of paroxysms initiated by rigors reached an observed maximum temperature of between $104^{\circ}$ and $105^{\circ} \mathrm{F}$, whereas the majority of rigorless elevations attained an observed maximum of between $100^{\circ}$ and $101^{\circ} \mathrm{F},(3)$ fewer paroxysms exhibited rigors in the lighter attacks, (4) the maximum fever experienced by an individual during his attack is not usually reached until about a week after clinical onset, (5) the majority of rigors fell within a duration period of 45 to 60 minutes, and (6) over 70 per cent of rigors commenced with a temperature of less than $100^{\circ} \mathrm{F}$ and the largest group (36 per cent) terminated with a temperature of between $103^{\circ}$ and $104^{\circ} \mathrm{F}$.

Excepting their shorter duration, the paroxysms of $P$. vivax infections have little to distinguish them from the paroxysms of the other two malarial fevers of humans. The most noteworthy characteristic of the $P$. vivax infection is the rapid improvement in the patients condition within a few hours after the temperature has returned to normal. The average duration of the $P$. vivax paroxysm is shorter than that due to $P$. malariae or $\boldsymbol{P}$. falciparum. The febrile paroxysm has the samejoutline as that of quartan and but seldom presents the double peak often seen in the fewer curve of falciparum malaria.

Nausea and vomiting. Vomiting may be an early sign of an attack of $P$. vivax malaria. The patient may experience a violent nausea and the attack of vomiting may be almost projectile in nature. This generally occurs a few minutes before the malaria paroxysm, and may recur just before each succeeding malaria chill.

Jaundice is much less common in $\boldsymbol{P}$. vivax than in $P$. falciparum infections. It is seldom seen during the first week or ten days of the clinical attack and is observed only in patients in whom a high degree of parasitization of erythrocytes has produced a rapid and extreme anemia. Jaundice is more apt to be observed in cases in which the red blood cell count has fallen below $2,000,000$ per cmm within a period of a week or ten days, than it is to be seen in cases where this degree of anemia has been attended by an acute clinical attack of three weeks or more. It would appear that the jaundice is due to rapid destruction of red blood cells and not to biliary obstruction.

Herpes labialis is extremely common. The herpes ordinarily does not occur until the clinical course is well advanced. Occasionally the lesions may be so severe as to involve all the oral region and local intercurrent infection may result. These lesions disappear in a few days or within a week after the clinical attack is terminated.

Urticaria is not an uncommon finding in $P$. vivax infections and appears 1 to 2 hours after the rigor begins. It is transient, disappearing within 8 or 10 hours after its first manifestation, and may recur at the time of the next paroxysm. Its origin is not clear.

Oedema. In a very few cases of $P$. vivax malaria a pronounced oedema of the legs and ankles is observed after the patients have experienced an acute clinical attack of 2 to 4 weeks duration and the red cell count is below two million. Ascites does not follow this oedema as may be the case in quartan malaria. There is no ready explanation for this phenomenon which disappears rather slowly, 3 to 4 weeks after cessation of the acute clinical attack.

Splenic enlargement is not evident until the clinical course of the infection is well established (Stratman-Thomas 1935). In white adults the spleen is usually not palpable until about 7 days after the first detection of parasites. It was noted in Cyprus 
that white infants, 6 weeks old, who were infected with $P$. vivax presented spleens reaching to the umbilicus. In infants and young children, $P$. vivax produces a greater degree of splenomegaly more rapidly than in adults. After the termination of a $P$. vivax infection (whether spontaneous or by use of drugs) the size of the spleen decreases rapidly and within 6 or 8 weeks it may not be palpable at the costal margin, even on deep inspiration. Persistently enlarged spleens in $P$. vivax malaria are occasionally noted and are indicative that the patient will have a relapse.

Malaria cachexia. If we define cachexia as a condition of wasting and general ill health occurring and persisting for months or years after an acute illness, then "malaria cachexia" in $P$. vivax malaria, as evidenced by observations on paretics undergoing malaria therapy, does not exist. Whenever the clinical course is interrupted with quinine or when the clinical course terminates spontaneously the red cell count and the hemoglobin return to normal within a month or 6 weeks after cessation of the clinical attack. The enlarged spleen, the enlargement of which has varied directly with the severity and length of the clinical attack, decreases in size, except in a few cases. Other than this transient splenic enlargement, there is nothing present after an attack of $P$. vivax malaria as described under malaria cachexia. The persistently enlarged spleens found in the field are attributable to successive infections with different species and different strains of plasmodia which bring about a long series of clinical attacks.

Instead of a cachetic condition following a $P$. vivax attack, the opposite condition obtains. One of the most outstanding effects noted following the use of $P$. vivax malaria for the therapy of paresis is the increase in weight after cessation of the clinical attack, even when the attack terminutes spontaneously and antimalarial drugs are not administered. A gain in weight of 10 pounds over the weight of the patient before malaria therapy was instituted is not unusual, and in a few in- stances a gain of as much as 20 or even 30 pounds has been noted.

From these observations it appears that it is extremely unlikely that a cachexia due to vivax malaria exists in nature unless the disease is complicated by other factors which may of themselves produce a cachexia, such as intercurrent infections and malnutrition. Emaciation, oedema, nephritis or secondary anemia are not present, and subjective symptoms such as loss of appetite, malaise and lassitude and "a general condition of nervous exhaustion" are not met with.

\section{SubJective Symptoms}

Subjective symptoms indicative of an impending clinical attack of $P$. vivax malaria are usually experienced before the end of the incubation period. The first symptoms are vague and may be represented by insomnia, lassitude, a degree of apathy, loss of appetite and a feeling of nausea. Some 2 to 12 hours later, headache, pains in the muscles and joints may occur as well as a feeling of cold. These symptoms last only a few hours and the patient feels well, but these same symptoms may reappear at approximately the same time the next day. This is especially noted in the case of the feeling of cold, the first chill often occurring at the hour of the day at which this sensation was previously experienced.

During the initial stage of continued fever the patient may be extremely miserable and experience more malaise than at any other time during the clinical attack. For several days the headaches may be very severe and the "rheumatic" pains in the muscles and joints be extremely painful. The patient is decidedly uncomfortable and he has no respite until the first intermission marks the end of the period of continued fever.

During the intervals between the paroxysms of the clinical attack subjective symptoms may be completely absent. The patient may do his work with a certain degree of efficiency and even forget that he is ill. Just before the next paroxysm 
occurs, however, the patient usually "knows he is going to have his chill," but a lucid description of the subjective symptoms which give him this information is difficult to obtain. A sense of giddiness, nausea and feeling of cold is about all that is present within 15 to 30 minutes before the paroxysm occurs, although some hours earlier the headache and the muscle and joint pains may be experienced.

During the rigor the outstanding sensation is that of cold. The patient's mind is not greatly disturbed and he can think clearly. When the chill is over there is a pleasant feeling of warmth. When the fever rises, however, there is present euphoria and a considerable amount of mental dissociation as evidenced by the patient's conversation and inability to put his thoughts on paper. The latter may be aggravated by a certain degree of muscular incoordination which exists at the height of the febrile attack.

The stage of defervescence commences as the temperature begins to drop, which it usually does rather rapidly. Perspiration occurs during this stage and may become so profuse as to saturate the bed clothes. Often the patient will fall into a refreshing sleep. As a rule subjective symptoms disappear a few hours after the temperature has returned to normal and the patient feels quite well again.

It is important to note that there are no subjective symptoms in a chronic or latent $P$. vivax infection. After the clinical attack of benign tertian malaria has terminated, either spontaneously or by the use of quinine, the patient "enjoys good health" unless he experiences a relapse of which there are few warning symptoms more than a few hours before the paroxysm occurs.

\section{Characteristics of the Infection}

The method of inoculation (natural or artificial) appears to have some influence on subsequent events. Boyd (1940b) pointed out that in artificially induced attacks : (1) the incubation period is usually shorter and may even be eliminated if the dosage of trophozoites be large enough, (2) the detection of parasites in the peripheral blood smears more frequently precedes the initial fever, (3) renewed clinical activity occurs only within eight weeks of the termination of the primary attack, and (4) when the donor of the trophozoites had been naturally inoculated, the subsequent attacks in the recipients were usually briefer than when the donor had been artificially inoculated.

Other factors may also influence the course of the attack. These are race, residential environment and season of the year. Boyd (1934) noted that negroes show a high degree of tolerance for $P$. vivax. He observed that but 5 of 14 colored patients developed infection following their inoculation with this parasite. Furthermore, only 3 of the 5 developed pyrexia. This was light in degree and of less than a week's duration. These results were in marked contrast to those observed in white patients inoculated with the same mosquitoes.

In areas where $P$. vivax infections are endemic the likelihood of a resident contracting the disease varies directly with the constancy of his exposure. It is quite common for persons who have spent their lives in such areas to exhibit some degree of immunity toward this parasite, and when reinfected with other strains of the same species the resulting illness may be quite mild.

It was observed by Boyd, Kitchen and Muench (1936) that inoculations made during the winter quarter of the year were followed by a lower proportion of takes; the latter had longer incubation periors, shorter courses, and renewal of clinical activity was confined to an eight-week period following termination of the primary attack. On the other hand, inoculations made in the other quarters of the year, and particularly those in the summer quarter, were characterized by the largest proportion of takes, the shortest incubation periods, the longest clinical attacks and the greatest likelihood of renewed clinical activity as late as 24 weeks after termination of the primary attack. 
Malaria in infancy. Young infants are early infected with malaria in endemic localities. In a highly endemic region, as Leopoldville in the Belgian Congo, one-half of the infant mortality in 1928 was due to malaria. In Cyprus was observed that nearly 100 per cent of infants over a month old had malaria and exhibited enlarged spleens of malarial origin, occasionally reaching to the symphasis pubis when they were two months old. The splenic enlargement is proportionately greater in infants under 6 months of age.

The clinical symptoms of malaria and the classic paroxysm is seldom observed in children under 6 years. In infants and young children, the clinical attack is ushered in suddenly. Fretfulness, refusal to nurse, and vomiting may be the first sign. The malarial chill, which is uncommon in children under 6 years of age, is represented by a paleness of nails and lips; the hands and feet show the same pallor and there is a shrinking of the skin of the pads of the toes and fingers as is seen in adults during a malarial chill. The skin of the hands and feet is cold to touch and a slight degree of cyanosis may be present.

In infants and young children the fever due to $P$. vivax infection is continuous or remittent in type, rather than of a well defined intermittent form.

A clinical diagnosis of malaria in infants and young children is far more difficult to make than in the adult, but examination of a blood smear on 3 consecutive days will show whether the fever is due to malaria.

Obscure symptoms and convulsions can be attributed to $P$. falciparum rather than to $P$. vivax and will not be discussed here. Likewise, such sequelae as cachexia, "pasty appearance," secondary anemia and mental dullness, if they ever do follow an acute attack of malaria, are more probably attributable to concurrent hookworm infection or malnutrition.

Malaria in pregnancy. In countries where the endemicity of malaria is high this disease is credited with causing a large number of maternal, fetal, and neonatal deaths, and there is no doubt that an acute attack of $P$. vivax malaria during pregnancy is prejudicial to women already in a poor physical condition because of hookworm, malnutrition, etc.

Spontaneous interruption of a pregnancy before term is stated to be common, repeated clinical attacks are credited with bringing about miscarriages or premature labor, and death of the fetus in utero is described.

In cases where there is a marked degree of anemia due to $P$. vivax malaria, the resistance to intercurrent infection may be so lowered that the patient develops puerperal sepsis. It has been stated that women with grave anemia seldom reach term, and if so, fetal deaths due to anemia may be ascribed to $P$. vivax. It must be borne in mind, however, that it is only the acute clinical attack and not a chronic $P$. vivax infection that can be incriminated in having a harmful effect on either pregnancy or the puerperium. It is very doubtful that a $P$. vivax infection is a predisposing factor in the toxemia of pregnancy. Likewise, there are no data to show that such infections prolong the time of labor or predispose to hemorrhage after delivery.

There is good reason to believe that childbirth may precipitate an attack of acute clinical malaria in a woman with a latent $P$. vivax infection. However, the difficulty of finding adequate data on this point is almost insuperable. In the South it is not uncommon to find a well informed practitioner who routinely administers quinine to the mother shortly before labor and during the puerperium in order to prevent the development of an acute clinical relapse. As in the case of $P$. falciparum infections, $P$. vivax can be demonstrated in placental smears when no parasites are to be found in the peripheral blood of the mother. It has been stated that malarial parasites persist in the placenta and so generate a relapse. This reasoning is not quite clear, except that pregnancy itself may conceivably protect the mother from an acute clinical attack of malaria, just as we have observed in experimental trypanosomiasis that pregnant female rats survive 
longer than non-pregnant females. While it has never occurred to anyone to interrupt a pregnancy because of an acute attack of benign tertian malaria, some physicians hesitate to use quinine during pregnancy lest an abortion or premature labor occur. It is the consensus of opinion that therapeutic doses of quinine have no oxytocic effect except, perhaps, at term.

Congenital malaria. While there is no reason to believe that the incubation period of benign tertian malaria in infants is different from that of adults, we have no exact information on this point. Taking eight days as the shortest time after mosquito inoculation that $P$. vivax can be demonstrated in blood smears of adults, we cannot with scientific accuracy classify under "congenital" malaria $P$. vivax infections observed in children more than 9 or 10 days old. It is of the utmost importance to inquire if the infant received a blood transfusion, as in our own cases of "congenital malaria" this was their origin. Seemingly authentic instances of $P$. vivax infection in infants under 10 days old have been observed. In these cases the child was likely infected with maternal blood through an abrasion at birth and not infected in utero.

Duration of infection and renewed clinical activity. The duration of an infection with $P$. vivax is not accurately known. Data available from blood transfusion records are inconclusive, but it appears from the best authenticated cases wherein $P$. vivax malaria was transmitted by blood transfusion, that the parasite may still be present as late as 2 years after the primary clinical attack. In this circumstance the infection persists in a clinically inactive or latent status. It is difficult to appraise the effect produced on the host by the scanty parasites which persist. The period of latency or quiescence is frequently broken or interrupted by one or more intervals of renewed or secondary clinical activity. These secondary attacks manifest all of the symptoms, both subjective and objective, which characterised the primary attack.
The likelihood of renewed clinical activity, appears, according to Boyd and Kitchen (1937d) to be more likely with certain strains of $P$. vivax than with others.

As the primary attack approaches termination, not infrequently the hours when one or more paroxysms are due will be passed without clinical activity. Not until one such period of quiescence has attained a duration of from 2 to 3 weeks, is it likely that the termination of the primary attack has actually been observed. This practically includes any activity occurring within 60 days of the onset.

Renewed clinical activity was observed by Boyd and Kitchen in slightly more than 50 per cent of the patients whose primary attack terminated spontaneously and who did not subsequently receive intensive therapy. When termination of the attack was followed by intensive therapy, cases of this character have not shown further activity. Patients whose primary attacks were interrupted by small doses of quinine have invariably had a return of clinical activity unless they subsequently received intensive therapy.

According to Boyd and Kitchen, patients having primary attacks lasting for more than 48 days have not experienced renewed clinical activity, which has also but rarely been observed in persons whose primary attack lasted only from 7 to 34 days. The period of renewed activity is usually of shorter duration than the primary attack, but it may sometimes be longer. The first such period is usually longer than the second or later periods. Some may consist solely of a single isolated paroxysm.

Variations with strains. Variations in the virulence of different strains of $P$. vivax, as judged by the severity of clinical symptoms and length of the acute clinical attack, have been reported. There is only a small amount of tangible evidence for these conclusions. It must be remembered that too few first-hand observations have been made by competent observers in an adequate number of patients with the same status of immunity under controlled con- 
ditions and in the same geographical locality to ascertain with any certainty what real difference actually exists. If an accurate study is to be made, many other complicating factors, such as dosage of sporozoites and age of sporozoites, must also be considered. We know, however, from practical experience that certain strains of $P$. vivax will produce a longer clinical course in paretics than other strains, which because of the shortness of the acute clinical attack are unsuitable for malarial therapy.

Alleged manifestations and sequelae. The innumerable clinical manifestations, symptoms and sequelae which have been ascribed to malaria are unflattering demonstrations of the imagination and credulity of the human mind. Fortunately for the reviewer, most of these conditions have been ascribed to infections by $P$. falciparum and not to $P$. vivax and were previously noted under malaria eachexia.

Cause of death, case fatality. When death occurs from $P$. vivax malaria the anemia due to the destruction of erythrocytes by the parasites must be regarded as responsible in nearly all cases. Rupture of the spleen must occur very seldom, indeed, as we have never observed this in over 300 patients with neurosyphilis treated with $P$. vivax malaria. The many serious complications attending $P$. falciparum infection, due to the selective places for sporulation of this parasite, are not met with in $P$. vivax malaria.

The fact that $P$. vivax malaria has a low case fatality has given it the name of "benign tertian." Some information from hospitals where malarial therapy has been used indicate that the number of deaths range from 0 to 8 or 10 per cent. It must be remembered that many of the patients inoculated were already in poor physical condition and often given malaria as the last therapeutic resort. It is difficult to ascertain from hospital records if case fatality varies with different strains, but judging from the severity of the clinical courses produced such must be the case. 


\title{
THE INFECTION IN THE INTERMEDIATE HOST: SYMPTOMATOLOGY, QUARTAN MALARIA
}

\author{
By S. F. KITCHEN \\ INTERNATIONAL HEALTH DIVISION, ROCKEFELLER FOUNDATION, TALLAHASSEE, FLA.
}

QUARTAN malaria, least common of the malarias and uncommon in North America, shows a number of differences from falciparum and vivax malaria in symptomatology. It is more like the latter, however, and in this connection, certain characteristics of $P$. malariae, the causative organism, are of interest.

Firstly, as in the case of $P$. vivax, sporulation of the mature schizont occurs, at least in part, in the peripheral circulation and therefore there is present much less evidence of localized damage to the viscera, such as is frequently characteristic of falciparum infections. Secondly, this parasite requires 24 hours longer than $P$. vivax and $P$. falciparum to complete the schizogonous cycle. Thus the evolution of the attack is slower, the density of parasites in the peripheral circulation rarely exceeds 20,000 per cmm, and the marked invasiveness of $P$. falciparum is not, as a rule, evident. Thirdly, the schizogonous stages of the parasite are better synchronized than those of $P$. falciparum, resulting in great regularity of the individual paroxysms, such as is seen in vivax infections. Fourthly, in our experience $P$. malariae appears to be more toxic in relation to parasite densities attained than does either $P$. vivax or $P$. falciparum.

In a recent article, Boyd (1940b) has reviewed our experience (5 naturally and 38 artificially induced infections) with quartan malaria and the data therein presented have been drawn upon freely in the preparation of the following account.

Prodromal period. In common with the other malarias, the quartan infection will usually, though not invariably, provoke prodromal symptoms not particularly distinguishing in nature. In the case of quartan malaria, however, such prodromes will usually occur during a period when the parasites are demonstrable in the blood smears, inasmuch as the appearance of parasites in almost every instance precedes the clinical onset by at least a few days. The usual premonitory symptoms of lassitude, anorexia, possibly nausea, headache and vague general aching or chilly sensations may be present for one or more days prior to the onset of paroxysms.

Fever. From the clinical aspect, quartan malaria is usually classified according to the arrangement of the paroxysms. The fact that the life cycle of the schizont is about 72 hours in duration and that there may be 1, 2 or 3 cycles of parasites suffciently active to produce fever provides 3 possible classes. The infection may be (a) simple in type, exhibiting a paroxysm at intervals of 72 hours; or (b) double quartan, in which case there is a paroxysm on two successive days followed by a day without fever, then 2 more successive paroxysms and an afebrile day and so forth; or (c) triple quartan, in which there occur daily paroxysms.

The febrile reaction in these infections is usually one of regular periodicity from the start and the paroxysms as a rule are distinct, although one may occasionally observe that two adjacent paroxysms are so close together that the first temperature curve has not quite returned to normal before the second rise commences (Fig. 1, No. B-2814). James (1910) considered the temperature curves of his quartan patients to be irregular although from the appearance of his charts it is assumed that he referred to the appearance and disappearance of eycles. There is rarely observed a preliminary exhibition of remittent fever such as one frequently sees at the onset in $P$. vivax malaria. The onset in quartan 
infections may be with a single, double, or triple arrangement of paroxysms but most frequently with single or double.

The complete quartan paroxysm presents the three classical stages, namely, (a) the cold, or stage of rigor, (b) the hot, or febrile stage, and (c) the sweating, or stage of defervescence.

The cold stage, in our experience, has most frequently begun with a temperature below $100^{\circ} \mathrm{F}$. Infrequently the temperature has been subnormal at this time, which appears to be to a large extent a characteristic dependent upon the individual. Occasionally the cold stage has been ushered in with a fever as high as $103^{\circ}$ or $104^{\circ} \mathrm{F}$ but this is not common. This stage is usually initiated by chilly sensations, generally beginning in the extremities. They may then extend in area and increase in degree until an actual rigor occurs with its accompanying cyanosis, particularly of the lips and finger nails, cold extremities, rapid and small pulse and quick but brief respirations. Headache may be severe.

On the other hand, the patients may have rigorless paroxysms. The severity of the latter may be confined to chilly sensations and a subsequently elevated temperature or there may occur merely a slightly elevated temperature. Our experience regarding the incidence of rigors has been almost the opposite of Marchiafava and Bignami (1901) who state that they are "not apt to be absent." As Boyd (1940b) pointed out, (a) rigors most frequently initiate simple quartan paroxysms, (b) in the case of double quartan fevers the rigor more frequently precedes only one of the two paroxysms and (c) when the paroxysms are quotidian, the incidence of rigors is likewise most often quotidian.

While, as mentioned before, the individual paroxysms of quartan infections tend to be regular, one cannot as a rule say the same for their incidence throughout an attack. As illustrated in Fig. 3 (No. B-2813), by an additive process, a double quartan course may become triple (quotidian) in type, and conversely, by a subtractive process, a quotidian course may revert to a simple form. Also through the

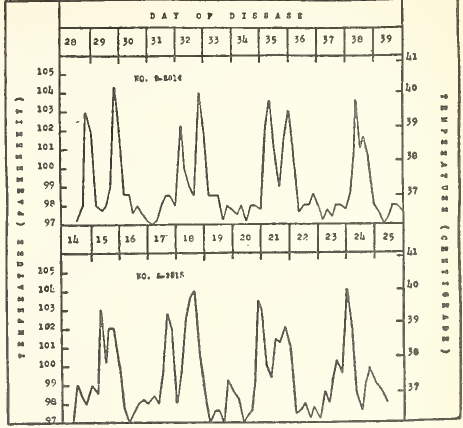

Fig. 1. No. B-2814 (P. malariae): Postponement of one cycle eventually results in its fusion with a second cycle. No. B-2815 (P. malariae): New cycle appears (14th, 17 th, etc., days) as old cycle (15th, 18th, etc., days) disappears.

substitution of a new cycle for one that has dropped out, the clinical type (single, double, triple) will remain unchanged (Fig. 4, No. B-2802). The appearance of new cycles or the reappearance of dropped cycles and the disappearance either of original or added cycles has been charac-

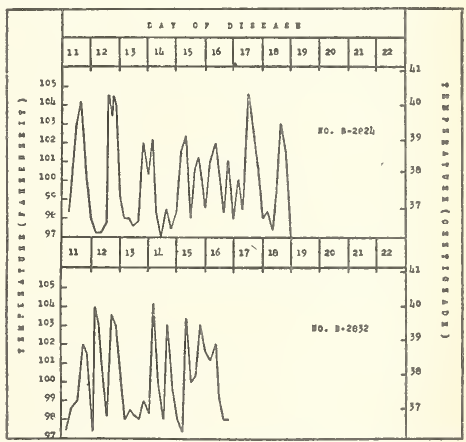

Fig. 2. No. B-2824 (P. malariae): Note paroxysms on 12th and 13th days with double peaks and later, further breaking up of the chief paroxysms into several minor ones (two a day on the 15th and 16th days). No. B-2832 ( $P$. malariae): Parasites showed extreme invasiveness in this instance. Unusually high parasite densities. Note two paroxysms on 12 th and 14 th days with terminal remittency. Fatal termination. 


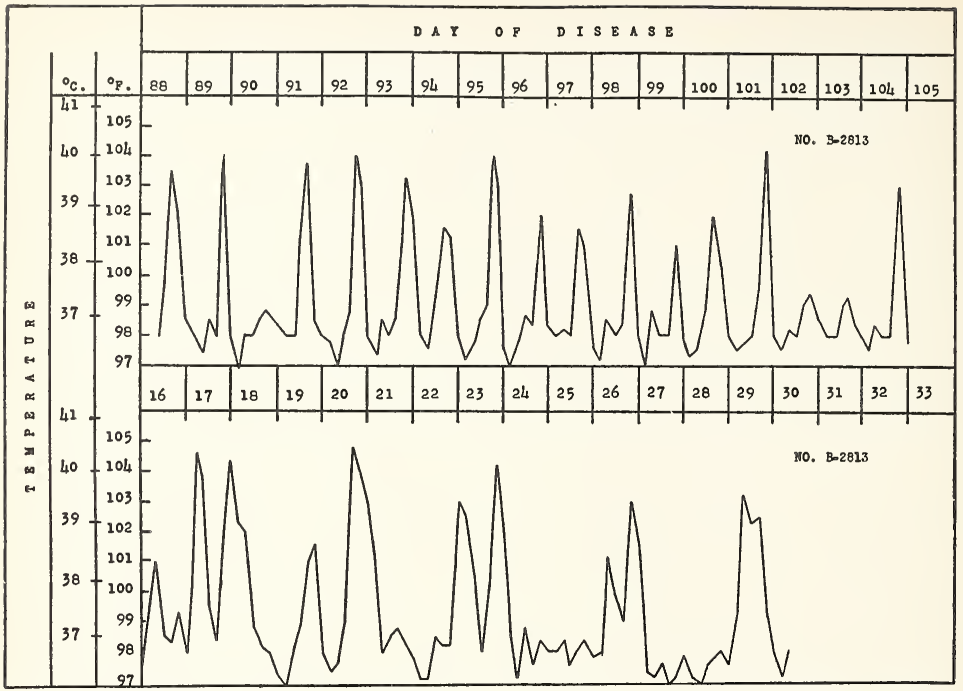

FIG. 3. No. B-2813 (P. malariae): Upper panel shows a double quartan course becoming quotidian. Two cycles disappear resulting in a simple quartan course. Lower panel shows postponement of one cycle and its final merger with another cycle.

teristic of our artificially induced infections. Thayer and Hewetson (1895) and Craig (1909) appeared not to have seen as many instances of multiple cycles as has been our experience. It ought to be pointed out that these authors were dealing wholly with naturally inoculated patients. As we shall mention later, our naturally induced infections were also of a simple pattern. Boyd (1940b) further observed that in the case of paroxysms prior to remissions of over 8 days in artificially inoculated patients, most of the quotidian paroxysms occurred in the patients who experienced primary attacks of less than 73 days' duration; and where the attacks lasted over 144 days they chiefly consisted of simple quartan paroxysms and no quotidian experience was evident. On the other hand, in the case of naturally inoculated patients, with pre-remission attacks of less than 73 days duration, none exhibited quotidian paroxysms though these were noted in a few attacks of longest duration.
In comparing the parasite densities at the onset of paroxysms in 23 artificially induced quartan infections, with the lapse of time between first appearance of parasites and first paroxysms, there was noted a significant positive correlation (Table I). As Boyd (1940b) observed, the tolerance for these parasites is surprising in view of the scarcity of quartan malaria in this country.

TABLE I

Correlation Between Tolerance for $P$. malatiae AND INTERVAL FROM FIRST DETECTED

Parasites to First ParoxysM

\begin{tabular}{|c|c|c|}
\hline \multirow[t]{2}{*}{$\begin{array}{l}\text { Parasite } \\
\text { density per } \\
\text { cmm at } \\
\text { clinical onset }\end{array}$} & \multicolumn{2}{|c|}{$\begin{array}{l}\text { Days elapsing between first } \\
\text { detection of parasites and } \\
\text { clinical onset. Number } \\
\text { of patients }\end{array}$} \\
\hline & $0-7$ & $8-14$ \\
\hline $\begin{array}{c}10-500 \\
500+\end{array}$ & $\begin{array}{r}15 \\
1\end{array}$ & $\begin{array}{l}1 \\
6\end{array}$ \\
\hline & \multicolumn{2}{|c|}{$\mathrm{P}<0.01$} \\
\hline
\end{tabular}




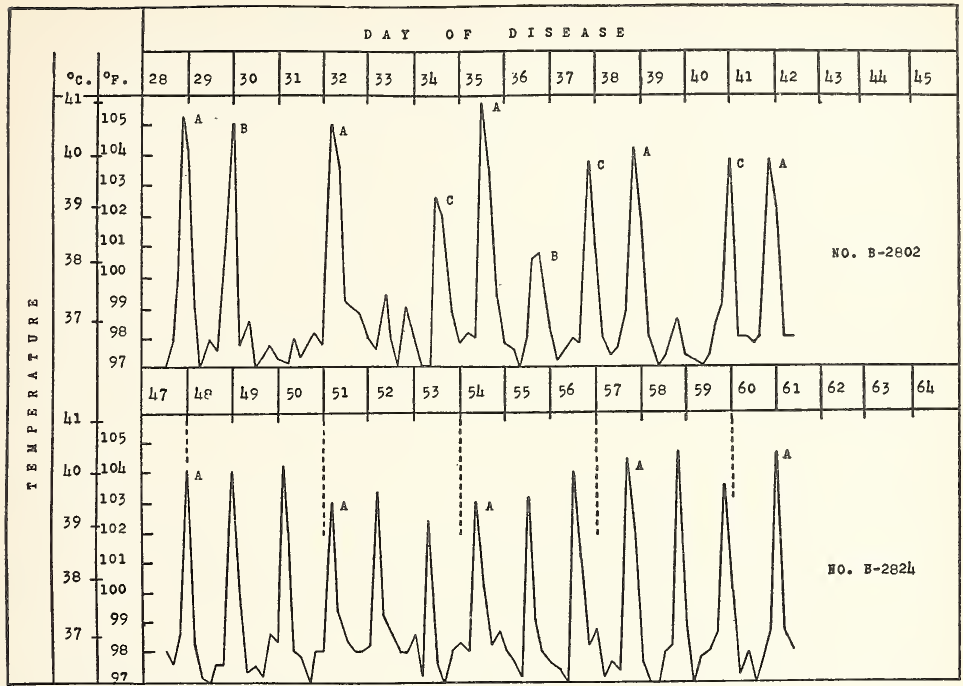

FIG. 4. No. B-2802 ( $P$. malariae): Illustrates the maintenance of a double quartan course by the replacement of one cycle (B), which disappears, by a new cycle (C). No. B-2824 (P. malariae): Constant postponement of one cycle (A) results, it occurring 24 hours late at the end of a thirteen-day period.

On the other hand, we know little regarding immunity in $P$. malariae infections.

If one classifies the three cycles as Boyd (1940b) did (cycle " $A$ " whose paroxysms occur 1st, 4th, 7th, etc., days following inoculation, cycle " $B$ " whose paroxysms fall on the $2 \mathrm{~d}, 5$ th, 8 th, etc., days, and cycle " $C$ " with paroxysms on the $3 \mathrm{~d}, 6 \mathrm{th}$, etc., days), it is evident that a patient could exhibit one or more of seven possible single and multiple arrangements of cycles (A, $\mathrm{B}, \mathrm{C}, \mathrm{AB}, \mathrm{AC}, \mathrm{BC}$, or $\mathrm{ABC}$ ) during the course of his attack. Examining the paroxysms from this standpoint, it was noted that (a) cycle "A" was never seen where an artificially inoculated patient experienced a simple quartan course throughout, (b) twenty-seven patients, artificially inoculated, exhibited 5 or more of the possible 7 combinations and five patients experienced all seven, (c) naturally inoculated patients tend to a simpler pattern of cycle combinations, (d) one-half of the arti- ficially induced attacks were initiated by " $\mathrm{C}$ " cycle and 37 per cent terminated on the "A" cycle, (e) naturally induced attacks were most frequently initiated by "A" cycle, (f) the paroxysms, following either the natural or artificial method of inoculation tend to show progressive ( $\mathrm{C}$ to $\mathrm{BC}$ to $\mathrm{B}$ to $\mathrm{AB}$ to $\mathrm{A}$ etc.) rather than regressive ( $C$ to $\mathrm{CA}$ to $\mathrm{A}$ to $\mathrm{AB}$ to $\mathrm{B}$ to $\mathrm{BC}$ etc.) changes in the cycle alterations. In naturally inoculated patients the cycle intervals are relatively more stable and the "A" cycle is most prominent. This may be significant inasmuch as in artificially induced infections there is a marked tendency to work around to the "A" cycle before the termination of clinical activity.

In addition to the above alternations, there also occurs a true anticipation or postponement (Fig. 4, No. B-2824) of paroxysms. Successive paroxysms may consistently anticipate the preceding one of the same cycle by several hours until 
finally the paroxysm may occur on a different cycle day. Such anticipation or postponement of paroxysms, when occurring consistently, may have part basis in postor pre-maturation of the cycle and part in some immunological factor, of which we are not yet cognizant, which results in the ultimate simplification of the pattern of the paroxysms.

Some authors have stated that most malarial paroxysms occur in the morning and that this point is of value in differential diagnosis. Craig (1909) noted that the paroxysms of quartan malaria usually occur during the afternoon or late morning. In our series of quartan infections, almost three-quarters of the paroxysms of artificially inoculated patients occurred post meridian. The largest single group, on the basis of eight-hourly divisions comprised over 33 per cent which took place between noon and 8 P.M. Of the naturally induced paroxysms, 94 per cent occurred post meridian and 52 per cent between noon and 8 P.M. Of considerable interest in this connection are the observations of Young, Coatney and Stubbs (1940). They found that the sporulation time of $P$. malariae could be altered by changing the hours of the patients' activities and rest.

Anemia. The development of anemia is much slower in quartan infections than in those due to $P$. falciparum or $P$. vivax. This may be due partly to the lower parasite densities usually characteristic of quartan malaria and partly to the tendency, as shown by the writer (1939a), of $P$. malariae to invade chiefly the mature cells, thus not interfering with the replacement.

In our series it was noticeable that the rate of decline of the erythrocytes and hemoglobin was most rapid during the first and second months of clinical activity, and slowest in the case of the shortest clinical attacks. Of interest is the fact that in the case of patients with the infections of longest duration, the erythrocyte count commenced a sustained increase several months prior to the cessation of clinical activity. A marked increase in the mean erythrocyte level was noted during remissions. Those with the longest recurrences, who had had the shortest initial attack, experienced a greater diminution of erythrocytes during the recurrence than during the initial period of clinical activity. In general the trend of the hemoglobin fluctuations paralleled that of the erythrocytes and the color index was less than unity.

Splenomegaly. We have noted that the spleen does not attain the size in quartan infections that it may in $P$. vivax attacks. The period of detectable enlargement is proportional to the duration of the clinical attack and splenomegaly takes place more rapidly in the case of short clinical attacks and more slowly in the long incursions. No spleens have been noted to extend below the umbilicus. Enlargement of the spleen during the course of an infection is not necessarily consistently progressive to the maximum but may be interrupted by contractions. Splenomegaly developed very slowly in two naturally inoculated patients and was not detected in a small group of negro patients.

Albuminuria and oedema. At some time during the course of the attack all of our patients developed some degree of albuminuria and in the case of 14 it amounted to more than a trace; the clinical attack had to be interfered with in 12 of these. The tendency toward an appreciable degree of albuminuria was less marked in the naturally induced infections (due to frequency of quartan courses?). Occasionally when the quantity of albumin has been recorded as 4 plus we have noted erythrocytes, even in large numbers; hyaline and granular casts have not been uncommon with lesser degrees of albuminuria. We have had no evidence of hemoglobinuria in our cases.

Of 6 patients who showed varying degrees of oedema, 4 showed albuminuria to the extent of 4 plus. In the other 2 the albuminuria did not exceed a trace. James (1910) cited the case of 2 patients in whom there was a marked oedema and clinical signs of acute diffuse nephritis. They gave a history of mild attacks of fever over a long period of time and had taken no quinine. Giglioli (1929), working in British Guiana, stated that untreated quartan malaria was 
largely responsible for the considerable amount of chronic nephritis he found. $\mathrm{He}$ believed long-continued, unchecked fever to be the cause. Manson-Bahr (1931) remarked that there was "some evidence that quartan malaria is more prone to be associated with subacute nephritis than are the other forms of malaria." In a study of plasma proteins, Boyd and Proske (1941) found that in 2 patients with quartan malaria, albuminuria', to the extent of a trace, was associated with a depression in the plasma albumin, and that oedema occurred at the time of such depression; when the plasma albumin was depressed to 2 grams per cent, oedema occurred unless a compensatory increase in globulin was developed. In both of these instances a nephrosis had developed.

Icterus. In one of 4 of our artificially inoculated patients who developed jaundice, this symptom did not appear until after termination of the primary attack.

Duration, remissions and chronicity. This disease is notorious for its persistence and is the most chronic of the three classical malarial fevers. It comprises a primary attack which is usually followed by one or more remissions and secondary attacks and has been reported to last one or more years in the known absence of opportunity for reinfection. In our artificially induced, spontaneously terminating infections the duration varied between 19 and 169 days with a mean duration in the case of white patients of 81.2 days, and in the case of negroes, 53 days (in patients receiving specific medication which might have been expected to interfere with the natural course of the infection, there was observed a variation of from 16 to 303 days with a mean of 98.6 days. This suggests that these patients might otherwise have had longer and more severe attacks). In naturally inoculated white patients the mean duration of the course has been 170 days. The comparatively long duration of attacks in quartan malaria is of interest in view of the slow development of these infections (this inverse relationship is notably the opposite of that which obtains in $P$. falciparum malaria; here the organism grows rapidly and the infections are less given to chronicity than even those due to $P$. vivax).

While remissions, in our experience, have shown much variability both as to number and length, we have not observed renewed activity following a quiescent period which lasted as long as 53 days. Boyd's (1940b) study of remissions showed that: (a) their mean duration varied directly with the length of the attack, (b) most of them of 14 days or less duration were spontaneous in origin but the majority of the longer ones followed interference, and (c) about 40 per cent of the spontaneous, and about 56 per cent of induced remissions occurred within 50 days of the onset of the illness.

Prognosis. Quartan malaria is not prone to develop pernicious symptoms and the parasite density has rarely exceeded 20,000 per $\mathrm{cmm}$ in our patients. Twenty nine per cent of our artificially, and 60 per cent of the naturally, induced infections came to a spontaneous stop. James (1910) recorded spontaneous recovery in 25 per cent of his patients and described the disease as milder in tropical than in temperate zones. Craig (1909), however, felt that ". . . in tropical regions the prognosis in the quartan infections, especially, should be guarded. . . ." None of our 5 naturally induced infections terminated fatally but 3 of the 38 artificially inoculated patients died. Of these 3 , in only one case did it seem likely that the quartan infection was a major factor in the fatal outcome. This species has ordinarily been quite readily controlled with quinine when necessary. Interference (usually employing small doses of quinine) was practised in 23 of our patients, largely to control the severity of the infection. On the other hand, interruption was indicated when the patient was not supporting the infection well or when some intercurrent condition developed. Frequently after brief interference it was possible for a patient to carry on to a spontaneous termination and no recurrence was encountered following intensive quinine treatment, given either to terminate the attack or subsequent to spontaneous cessation. No sequelae have been observed in any of our patients. 


\title{
THE INFECTION IN THE INTERMEDIATE HOST: SYMPTOMATOLOGY, FALCIPARUM MALARIA
}

\author{
By S. F. KITCHEN \\ INTERNATIONAL HEALTH DIVISION, ROCKEFELLER FOUNDATION, TALLAHASSEE, FLA.
}

Falciparum malaria is essentially, from a clinical standpoint, a very different disease from $P$. vivax and quartan malaria. Among the reasons for this difference are certain fundamental characteristics probably peculiar to the causative organism.

These characteristics, by reason of which $P$. falciparum malaria in particular of the three classical infections may be attended by great hazard and well deserves the term "malignant," are: (a) Marked invasive. ness. Extraordinarily rapid and great increases in the numbers of parasites occur and the amount of debris and foreign protein released into the circulation at sporulation time is thereby greatly increased. The toxicity of this species of plasmodium, per $s e$, is probably not remarkable; in the writer's opinion it is less than that of $P$. vivax or $P$. malariae at equivalent parasite densities. (b) Internal sporulation. As Marchiafava and Bignami (1894) noted almost half a century ago, "the fission of the amoebae is effected for the most part in the eapillary system of certain of the viscera." Very large numbers of parasites are conducive to the blocking of capillaries, especially in view of the agglutinative trait of the parasitized erythrocytes that has been described, together with a tendency for them to adhere to the vascular walls. Furthermore, one or more organs may bear the brunt of this process. This explains the multiplicity of symptoms that will be noted later and justifies the term "malarial mimicry," used by Castellani and Chalmers (1919). (c) Asynchronization. This parasite does not lend itself readily to regimentation of sporulation. As will be described subsequently, this is reflected in the irregularities of the febrile reaction exhibited by the host.

Prodromal symptoms. Usually in typical primary attacks in wholly susceptible persons the patient will complain for 2 or 3 days prior to the onset of paroxysms of general malaise, loss of appetite, perhaps a vague feeling of ill-being or exceptional fatigue and localized aching. The temperature during this time may or may not show slight daily elevations, and chilly sensations may be experienced. Prodromal symptoms, however, may be almost negligible or entirely wanting, and the onset may be abrupt, possibly with coma as the first indication. A sudden onset is frequently the case in individuals possessing some degree of resistance to the infection. Resistance may even be of such an order that symptoms are not evident until a relatively high density of parasites in the peripheral circulation has been reached. Following onset the type of attack experienced by a person will naturally depend upon many factors concerning the individual, such as inherent resistance to infection, previous malarial infection and general physical condition.

Fever. The paroxysm, which may be regarded as the unit of the patient's febrile reaction to the infection, is, in $P$. falciparum as in the other malarias, usually ushered in by chilly sensations, if not a frank rigor, and usually terminates with a period of diaphoresis. Presumably it is due to the release of the merozoites at the time of sporulation, which act as a foreign protein; certainly the symptom complex is comparable to that seen following the intravenous introduction of a foreign protein. Whether the intact merozoites can initiate the paroxysm or whether a preliminary breaking down of some of them is necessary, is not known.

In the majority of instances the temperature is below $100^{\circ} \mathrm{F}$ and not infrequently 
it is less than $99^{\circ} \mathrm{F}$ at the onset of the paroxysm; a normal temperature is not unusual. Aching is usually more severe in $P$. falciparum infections and if a rigor is present it is ordinarily less marked than in vivax or quartan malaria. Vomiting occurs most frequently at the end of the cold stage or early in the febrile phase. Pain over the epigastric or splenic areas is commonly severe. The long duration of fever is an outstanding feature of $P$. falciparum paroxysms.

Periodic elevation of the temperature is, by definition, the only constant characteristic of the malarial paroxysm. Rigors are not as frequent constituents of the falciparum paroxysm as of those due to $P$. vivax. Marchiafava and Bignami (1901) expressed the opinion that the fact that sporulation in estivo-autumnal malaria occurred "in the vessels of the internal organs under relatively stable conditions and the parasitic forms which finally produce the fever are not circulating" had a bearing on the frequent absence of the chill. In our experience with several strains of $P$. falciparum which we have utilized (ranging in point of origin from the southern United States, Cuba, Mexico and Central America), the rigor.has been an unusual predecessor of the febrile stage in the American negro, in whom we recognize some degree of racial immunity. In an unselected group of 20 such patients, 136 paroxysms were observed and only one of these was initiated by a rigor. These patients, however, commonly complain of "dumb chills" (chilly or cold sensations). In white patients, on the other hand, the picture has been somewhat different. Here a much larger proportion of the paroxyms are accompanied by rigors; for example, of 231 paroxysms experienced by 10 persons, 69 , or practically 30 per cent, commenced with a rigor (even this incidence of rigors is well below the usual finding in susceptible white patients with $P$. vivax infections).

Some of the early Italian writers evolved elaborate classifications of the pyrexial reactions of individuals infected with $P$. falciparum. This would seem neither necessary

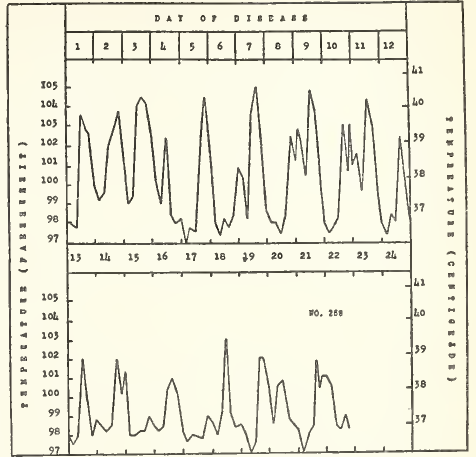

Fig. 1. No. 258 (P. falciparum) : Onset with remittent-quotidian type of fever which becomes intermittent. Breaking up of lesser cycle into several peaks on 8 th and 10th days, producing remittency again.

nor desirable in considering our observations in this country. Mannaberg (1905), aiming toward simplification of nomenclature, rightly stated that such differentiation was lacking in practical value. The same author noted that "the form of the fever and the severity of the infection do not correspond."

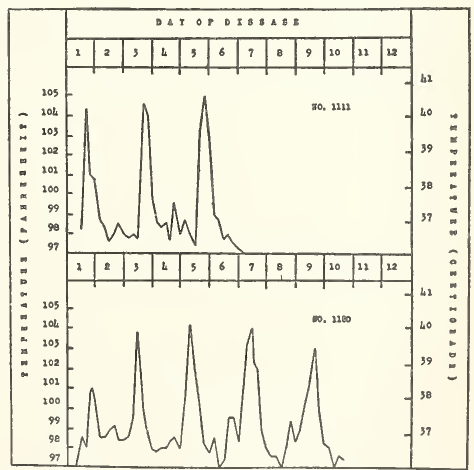

FIG. 2. No, 1111 and 1150 (P. falciparum): Both experienced brief tertian courses. In both instances a second cycle showed a tendency to develop but did not reach pyretogenic levels. 


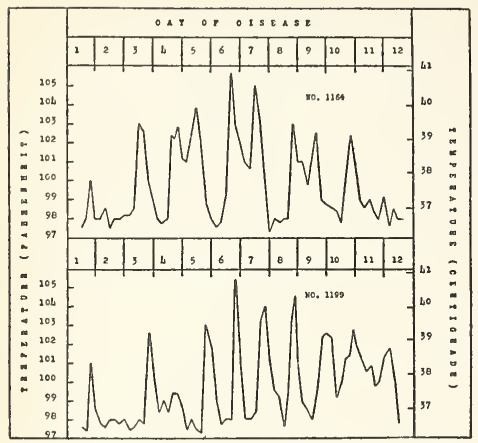

FIG. 3. No. 1164 (P. falciparum) : Fever of intermittent-remittent type. Two adjacent paroxysms pairing off to produce remittency. No. 1199 ( $P$. falciparum): Course commenced as tertian, became quotidian, then remittent (temperature remained over $37.2^{\circ} \mathrm{C}$ for 64 hours). Fatal termination.

Occasionally at the onset of a falciparum infection, the temperature presents a remittent type of curve (Fig. 1, No. 258). It is probable that in this case sporulation was almost continuous. Later, a variable degree of regimentation of sporulation usually appears but it is very seldom as orderly as one usually sees in $P$. vivax infections.

We have occasionaly seen pure tertian

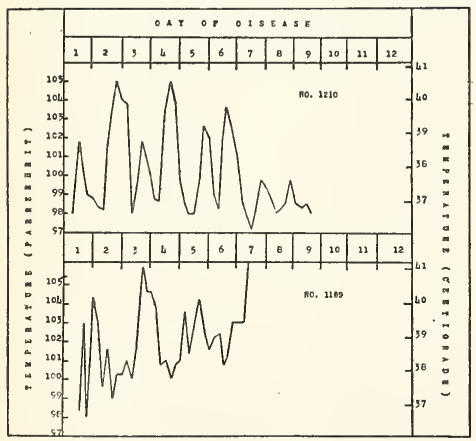

FIg. 4. No. 1210 (P. falciparum): One cycle shows higher temperature peaks and broader bases than other. No. 1189 (P. falciparum): An example of continuous remittent fever. Uncontrollable by quinine. Fatal termination. courses (Fig. 2, Nos. 1150 and 1111) in $P$. falciparum infections and recognize that the schizogonous cycle of the parasite is primarily of 48 hours duration. We have observed also the presence of two cycles, resulting in quotidian paroxysms. Pure tertian or quotidian courses, in our experience, have been uncommon (the hypothesis that daily paroxysms were induced by a subspecies, $P$. falciparum quotidiannum (Craig 1909), is not generally accepted). Their occurrence is probably dependent, at least in part, on immunological factors which we do not as yet understand. Courses that are tertian throughout do suggest some degree

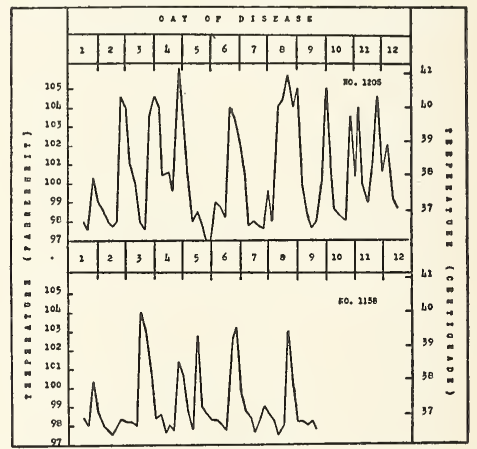

Fig. 5. No. 1205 (P. falciparum): Course commenced as quotidian, became tertian and, regaining lost cycle, terminated as quotidian. No. 1158 ( $P$. falciparum): Brief course commencing as tertian. Original cycle disappears ( $3 \mathrm{rd}, 5$ th and 7 th days) as new one appears (4th, 6th and 8th days).

of immunity on the part of the patient. The paroxysms in falciparum malaria, even when in simple quotidian arrangement, commonly have a broader base than do those due to $P$. vivax. Frequently, therefore, two closely adjacent paroxysms may partly merge and show an intermission in which the temperature does not fall to normal, creating a remittent curve (Fig. 3, No. 1164). This may occur over a period of days, the paroxyms pairing off to produce a series of remittent curves, between each of which pairs the temperature drops to normal. In such cases the interval between 
the two peaks of the remittent paroxysm will commonly be less than 24 hours, and that between the two peaks on either side of the intermission, greater than 24 hours. Marchiafava and Bignami (1901) described the typical estivo-autumnal tertian fever curve as comprising the invasion of fever, the period of pyrexia, the pseudo erisis, the precritical elevation, and the true crisis. Their graphic illustration resembles essentially what we deseribed as a partial merger of two paroxysms to form a remittent curve. Perhaps this should be called an intermittent-remittent arrangement when continued throughout. As Mannaberg notes, however, this type of curve is not by any means constant for this infection. Occasionally one observes that in quotidian courses one cycle provides paroxysms of short duration and the other, elevations of temperature with broader bases (Fig. 4, No. 1210). Patient 1199 (Fig. 3) exhibited remittency involving three paroxysms. Here the temperature remained above $99^{\circ} \mathrm{F}$ for at least 64 hours.

In courses that start as tertian in type one frequently notes the sudden or gradual appearance of a second cycle (Fig. 3, No. 1199) resulting in quotidian paroxysms; or one may see one cycle drop out of a quotidian course (Fig. 5, No. 1205), leaving tertian paroxysms. Occasionally, as in the case of Patient 1205 (Fig. 5), one cycle may disappear temporarily, perhaps missing one or two paroxysms, to return again. Also a second paroxysm may appear in a tertian course following which the original cycle may drop out. Many variations may thus be provided by the appearance and disappearance of cycles. In addition to irregularities of the intermittent, remittent, or continuous types of fever curve, one may note that the arrangement of the paroxysms shows irregularities, These features frequently combine to form febrile courses which, on superficial examination, would appear to be entirely without order. On closer study, however, despite the irregularities most of them will be seen to possess some design. Basically they comprise one or more tertian cycles with modifications such as (a) the appearance and disappearance of extra cycles, (b) the transitory and close approximation of two quotidian cycles so as to produce a remittent curve, or (c) the breaking up of a single fever peak to produce two or three secondary elevations (Fig. 1, No. 258) during the course of the paroxysm and a very broad base thereto. The tendency of falciparum paroxysms to anticipate or postpone in relation to the time of the previous one is much more irregular than in the case of $P$. vivax infections.

As previously indicated, the lack of regimentation, or synchronization, in sporulation of $P$. falciparum is apparently responsible for the irregularities noted both in the individual paroxysm and in the arrangement of paroxysms in the course of the infection. It is realized that variation in individual sensitiveness may, of course, play some part, but the irregularities are so nearly constant that this factor would seem to be of relatively minor importance. Thompson and Woodeock (1922) and James (1922), however, prefer to ascribe the disorderly paroxysms to the variable time required by individual parasites within a group to complete schizogony. It would seem more reasonable to assume that such irregularities are due to various broods of parasites sporulating asynchronously rather than to presuppose a confused alternation in the length of the schizogonous cycle of groups of parasites. Unfortunately smears from the peripheral blood do not usually reveal the mature forms or schizogonous cycle of $P$. falciparum.

Relationship between parasite density and the paroxysms. Following are several points of interest in the relationship between parasite density and the incidence of paroxysms. Between individuals of the white and black races there is quite a marked difference in tolerance for $P$. falciparum. We have commonly observed in infections among negroes that the parasites may attain densities of several thousand per cmm before a febrile reaction is provoked. A count of over 71,000 was noted on the first day of fever in one instance. In this case the parasites had first appeared in the 
peripheral circulation three days prior to the onset of paroxysms. It has been very rare in our experience to observe a febrile reaction prior to the first appearance of parasites in colored patients. In white persons the attainment of high densities by the parasites before the onset of fever is, on the other hand, uncommon, and frequently the initial fever precedes the first detected parasites by one or more days.

It is noteworthy that the parasite density at the onset of fever is usually much lower

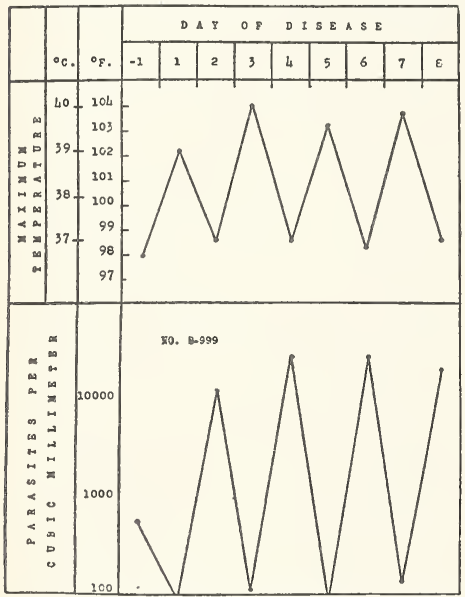

Fig. 6. No. B-999 (P. falciparum): Shows the association of high parasite density and normal temperature on alternate days, and of low parasite density and elevated temperature on the intervening days.

than that which obtains subsequently at the onset of recurrences. In other words, some tolerance for the parasite has developed and a greater number of them is necessary to provoke a febrile response. As a corollary, one usually observes that the parasite count is greater at the termination of the primary attack than it was at the onset.

In the case of infections with paroxysms occurring at tertian intervals an interesting phenomenon may be present (Fig. 6).
When one examines smears made at intervals of 24 hours and enumerates the parasites per cmm it may sometimes be noted that the parasite density shows a daily alternation between low and high levels and that there is an inverse relationship between the level of the parasite density and the incidence of paroxysms; the latter occur on the day of the lower parasite count and are absent on the following day when the parasite density is greater. Such alternation may be present over a period of several days.

Clinical types of falciparum malaria. The clinical classification of $P$. falciparum infections has been made very cumbersome. Systems of classification based on the febrile reaction alone are not satisfactory chiefly because the degree or type of fever is not consistent. Elaborate divisions and subdivisions on the basis of clinical types and syndromes serve only to indicate that this disease is very protean in its manifestations and therefore must be considered in making a diagnosis of an illness in any person who has been in a position to contract it.

It is convenient to consider, in the first place, that $P$. falciparum malaria may (a) be of a simple type, involving the body as a whole but attacking no system of organs in particular, or (b) show localizing symptoms denoting involvement of one or more organs predominantly; these infections usually arise from those of the first group.

The former group, though they also occur in tropical areas, comprise the majority of estivo-autumnal attacks that occur in subtropical zones. Although many of them are relatively benign in character, it is wise to consider that all $P$. falciparum infections are endowed with fatal potentialities (the type of illness which follows inoculation is dependent upon individual factors and the administration of specific medication). The parasites, though giving no signs of localization, may show an extreme degree of invasiveness and if unchecked, multiply rapidly until the patient is overwhelmed and death ensues. Of course in many such instances intercurrent disease, poor physical condition or lack of resistance for other reasons 
are secondary contributors; nevertheless this inherent dangerous tendency of $P$. falciparum must not be minimized. Other patients may show quite a degree of tolerance for relatively high densities of parasites and after a comparatively mild attack symptoms will disappear. The attacks, as Boyd and Kitchen (1937a) have pointed out, tend to be of shorter duration than those due to $P$. vivax. The mean in our series was 10.8 days, although the maximum duration noted was 36 days (it must be remembered that our data were gathered from persons undergoing therapeutic malaria and as little quinine as was compatible with their safe management was administered during these infections).

of the group of estivo-autumnal infections which tends to attack predominantly some system of the body, the majority are characterised by a severity which has earned them the name pernicious. These forms, distinguished by symptoms which indicate a danger to life, are found chiefly in the tropics and particularly under epidemic conditions or in areas of high endemicity. Sternberg (1884) notes that in temperate zones pernicious symptoms are usually preceded by one or more simple paroxysms, and Craig (1909) states that the "great majority of pernicious attacks occur in persons who have suffered repeatedly from malarial paroxysms which have not been properly treated ... and ... often develop ... during an apparently mild paroxysm." Certain predisposing causes undoubtedly aid the development of malignant symptrms. Among these may be debility (as in convalescence), malnutrition, excessive fatigue, chronic alcoholism, heat prostration, drug addiction (Most 1940a) and pre-existing anatomic lesions. In this type of infection there is usually a high density of parasites, though it is not necessarily evident in the peripheral circulation. The degree and type of fever does not correlate with the pernicious features of these attacks. Mannaberg (1905) concluded that "perniciousness is associated with no definite elevation of temperature and no particular type of fever" although he recog- nized that "the majority of pernicious cases manifest a subcontinued fever."

Manson-Bahr (1931) divided the pernicious types into two groups, (a) the cerebral forms and (b) the algid forms. In the cerebral group he included those patients whose symptoms pointed predominantly to damage of some part of the nervous system. The chief feature of the algid group, as the name suggests, is coldness of the body surface. Collapse and a tendency to syncope are prominent features. Among this group are infections involving conspicuously the gastro-intestinal tract, hemorrhagic forms, syncopal types and acute hemolytic ancmia.

Inasmuch as these pernicious forms are not disease entities but are caused by the same parasite that produces mild infections, it is proposed not to describe an almost endless series of syndromes that have been met with, but rather to list systems of organs that have been particularly involved, together with syndromes and symptoms that have been observed:

(a) Nervous system: Hemiplegia, paraplegia, localized paralysis, epileptiform seizures, neuritis, headache, irritability, restlessness, delirium, convulsions, coma, tremors, ataxy, speech changes, aphasia, amnesia, psychoses and milder mental changes.

(b) Gastro-intestinal system: Syndromes of acute appendicitis, hemorrhagic pancreatitis, acute gastritis, cholera, dysontery and acute peritonitis. Vomiting (bilious or hematemesis), icterus, diarrhoea and melena.

(c) Cardiovascular and hemopoietic systems: Rupture of spleen, symptoms of thrombosis and hemorrhage, syncope, heart failure, dyspnoea, hypotension, angina-like pain, anemia and leucopenia.

(d) Respiratory system: Pneumonia, bronchitis.

(e) Genito-urinary system: Syndromes of nephritis and nephrosis (albuminuria, casts), hemoglobinuria, hematuria, orchitis, and oophoritis.

$(f)$ Other systems: Involvement of special senses as hearing, sight (optic neuritis, retinal hemorrhage) and mastitis.

Splenomegaly. Most writers agree that 
enlargement of the spleen is a constant occurrence during the paroxysm. It tends to become smaller during the interval, however, and may not be palpable during the first few paroxysms in a primary infection. As the course of the infection progresses the swelling increases and a relatively large volume may be attained. The usual tendency is for the tip of the enlarging spleen to swing diagonally across the abdomen toward the lower right quadrant. Pain is sometimes complained of and may be due to perisplenitis or stretching of the capsule. Tenderness on palpation, particularly during the paroxysm, is commonly experienced. Rupture of the engorged spleen has been met with but it is usually consequent upon trauma.

It has been our experience that the splenomegaly in primary $P$. falciparum attacks does not reach the proportions that may be noted in vivax malaria in highly susceptible individuals. Probably this is largely due to the fact that primary $P$. falciparum attacks are of shorter duration than those caused by $P$. vivax. In negroes the attacks have been relatively brief and splenomegaly has seldom been evident. Our white patients have experienced longer attacks, but here the degree of enlargement has not exceeded the Number 2 size of Boyd's classification (1930a).

Anemia. The great densities which $P$. falciparum may quickly attain undoubtedly accelerate the development of anemia. Too, as the writer pointed out, their tendency to invade both mature and immature erythrocytes may be a factor of importance. We do not definitely know of destruction of the erythrocytes other than by direct action of the parasite, although some authors feel that circumstances necessitate the hypothesis of some additional factor, such as a hemolytic toxin. It must be remembered, however, that we only see the parasitized cells that appear in the peripheral circulation. It is not uncommon to note that in the course of a week of infection a patient may lose from one to two million erythrocytes per $\mathrm{cmm}$ and from two to five grams of hemoglobin per $100 \mathrm{cc}$ of blood. There is not necessarily a correlation between the blood destruction and the apparent clinical severity of the infection. It is noteworthy, furthermore, that as the degree of anemia increases the rate of its development slackens. It is remarkable how quickly the blood restoration becomes evident following the termination of an attack. In some instances we have noted that the destruction is more than compensated for as the number of parasites is decreasing and perhaps just before the paroxysms have ceased.

Leucocytic reaction. The total leucocyte count is altered both in relation to the individual paroxysm and to the course of the infection. It is generally agreed that a leucopenia exists during the paroxysm; it probably becomes evident prior to the onset of the latter. This reaction, however, may be of the same general type as the usual body response to the introduction of a foreign protein and therefore relatively non-specific, in so far as the malarial infection is concerned. James (1922) states that "a true leucocytosis is by no means infrequent during the very early stage of an ordinary acute attack (especially in malignant tertian infections) and for a longer period in pernicious cases while the fever is rising." Marchiafava and Bignami (1901) quote Kelsch as observing a leucocytosis in the pernicious infections. Craig (1909) agrees on this point. We have noted a leucopenia in our falciparum infections, thougi not a very marked one as Fig. 7 shows. In this chart the means of the leucocyte counts for ten patients have been plotted over a period of from 3 days before until 12 days after the first appearance of parasites. It will be noted that the leucopenia begins to appear before we are able to detect parasites in thick smears and that the lowest point in the curve occurs 6 days after parasites were first detected. Frequently a low count persists for some time after the onset of the infection. We have occasionally observed a greater degree of leucopenia to occur, as a result of a relapse with its accompanying increase in parasite density, than there was evident during the primary infection. 


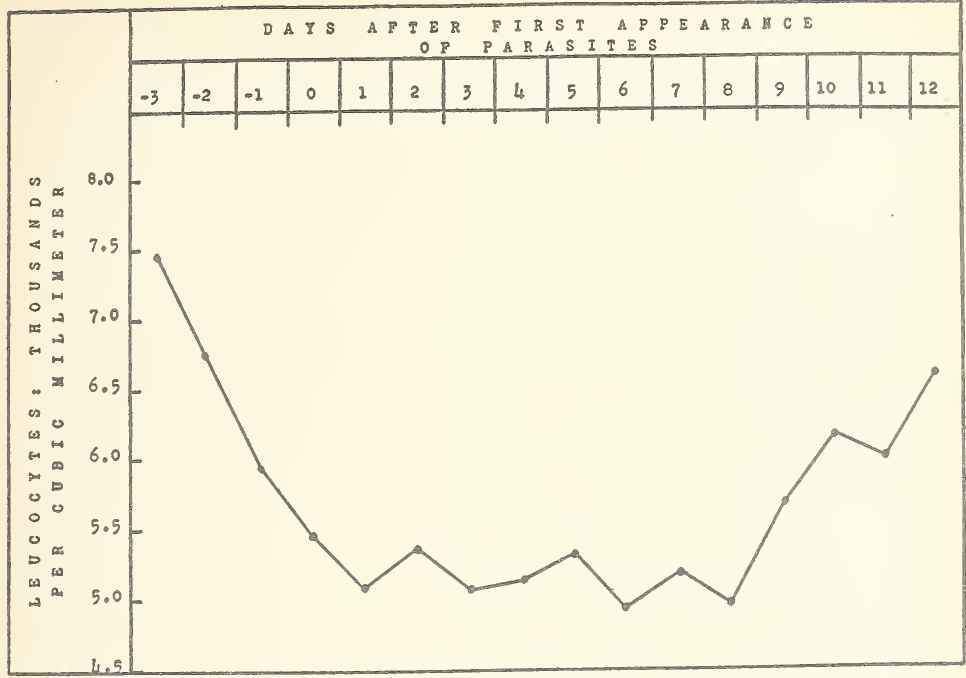

FIc. 7. Mean leucocyte counts for ten patients with falciparum malaria. Note that the count commences a steady drop prior to the detection of parasites in the blood. Lowest point occurred on the sixth day after the first appearance of parasites.

Regarding the differential white cell count, James (1922) quoted Thomson as observing that the proportion of nononuclear elements varies inversely with the temperature. During the apyrexial period they might rise as high as 80 per cent. James (1922) noted that during the afebrile stage the polymorphonuclear forms fall from about 70 to 50 per cent and that the mononuclears rose from about 25 to 45 per cent. The proportion of these latter forms is greater than normal in active malaria, and monocytes containing variable amounts of pigments are commonly seen in blood smears from falciparum-infected patients.

Prognosis. If the infections are seen early, and if proper treatment is instituted promptly and pernicious symptoms are not evident, the prognosis should be good. The appearance of intercurrent infections calls for a guarded prognosis. We have noted that in the event that very high densities have been attained by the parasites or that an intercurrent infection has set in, quinine is not as effective as under ordinary circumstances. Naturally the prognosis is grave, though not always hopeless, when pernicious symptoms have developed. Over a ten-year period (1922-1931) reports of the United Fruit Company showed that 43,535 patients were treated for $P$. falciparum malaria in their hospitals. There were 525 deaths, or a mortality rate of 1.2 per cent. The case fatality ratio was $829: 1$. This is undoubtedly a great deal lower than obtains under general conditions in endemic areas. Of all fatalities due to malaria during that same period, $P$. falciparum infections were responsible for 86.3 per cent.

Malaria during pregnancy. The gravid female generally experiences a more severe infection than the non-pregnant, other things being equal. This is understandable, inasmuch as she already has an added call on her resources. Perhaps the gravest problem she faces is that of anemia and even in 
lighter infections this may be of considerable moment. Reports from various localities assign to malaria varying degrees of responsibility as the etiological agent of abortions. Mannaberg states that: "the abortion does not require a fever paroxysm for its production. The foetus is usually dead before the abortion occurs." Hehir (1927) notes that: "the later the infection occurs during pregnancy, the greater the liability to miscarriage." Although the child of a malarious mother may survive birth, it frequently dies within a week.

Congenital malaria. Marchiafava and Bignami (1901) were of the opinion that at the time of their writing, no incontrovertible proof had been presented in favor of the existence of congenital malaria and cited instances in which evidence had been searched for and not found in foetuses and new-born of heavily infected mothers. On examining peripheral (maternal) placenta and cord blood smears in 400 patients, Clark (1916) found 19 estivo-autumnal infections. Of these 19, all showed parasites (commonly segmenting and presegmenting forms) in the placental smears, some in dense numbers, whereas only 8 peripheral blood smears were parasite-positive. One cord smear revealed parasites and in this instance there was a history of accident of pregnancy in relation to the placenta. Blacklock and Gordon (1925) likewise found a much higher incidence of $P$. falciparum in placenta smears than those from the peripheral blood. They found a positive correlation between maternal infection and death of the child in utero, or immediately after birth (without infection of the foetus or child). It is reasonable to assume that there must be absorption of toxic substances by the; foetus and interference with the foetal nutrition in the case of a heavily infected placenta. The concensus of opinion seems to be that infection of the foetus occurs only as a result of damage to the placenta. Diagnosis of congenital malaria on the basis of an enlarged spleen and fever in a child born of an infected mother is not justifiable in the absence of parasites from the child's circulation.
Malaria in children. McGown (1849) has the following to say on this subject: "Children under 5 years of age are less liable to remittent fever than those who have passed this age. Those between 2 and 5 years of age appear to be more liable to it than those under 2 years, but it does not appear to occur very frequently in the former. Those under 2 years of age, so far as I am aware, are seldom the subjects of remittent fever, though they are not entirely exempt from it. . . . It appears that a very large majority of the cases of congestive fever occur among adults, or those who have arrived at the age of puberty; though it is not exclusively confined to them; as children of 3 years, or 5 years and upwards, are sometimes the subjects of it." Cleghorn, Parry and Wharton (quoted by Sternberg 1884) supported the view of McGown. Sternberg (1884) felt that if the very young enjoyed an apparent immunity, it was probably due to lesser exposure. Craig (1909), speaking of the malarial fevers in general, stated that susceptibilty varied inversely with age.

The paroxysm is generally atypical in children particularly with reference to the cold and diaphoretic stages. These are less marked than in adults. Splenomegaly and anemia tend to develop more rapidly than in adults. Convulsions are not unusual and are mostly associated with the febrile stage. Pernicious symptoms are not uncommon in endemic areas and death is frequently due to the intensity of the parasitic invasion, often with cerebral symptoms. In those over 5 years the paroxysms more nearly resemble the adult type. One frequently sees in endemic areas, children who have experienced several infections due to $P$. falciparum, with protuberant abdomens as a result of chronically enlarged, "caked" spleens.

Chronic malaria. Reinfections. Cachexia. There is a tendeney on the part of some writers to include reinfections when speaking of chronic malaria. This does not seem to us to be permissible in the strict sense of the word. James (1922) in speaking of indigenous inhabitants of endemic 
areas who are repeatedly infected and who receive little or no treatment between attacks says "the result is that they pass into a condition which is correctly named 'chronic malaria.', Marchiafava and Bignami, on the other hand, believe that distinction should be made between chronicity following a first attack and that resulting from a succession of infections. In a recent paper on chronic malaria, Fondé and Fondé (1939) expressed the opinion that statistics regarding the incidence of malaria, based on the demonstration of parasites rather than on clinical evidence, are not a true indication of the extent of malaria. We deplore the promulgation of this doctrine and feel that there is already far too great a tendency to blame malaria for many diverse debilitating conditions and elevations of temperature in the absence of demonstrable parasites, and chiefly because a diagnosis of malaria, rightly or wrongly, had once been made. Mannaberg defined chronic malarial infection as one continuing for months and specified that the continuance was not dependent on reinfection "but on obstinate persistence of the virus." It is perhaps timely to express the opinion that $P$. falciparum malaria is an infection which is not as prone to chronicity as is generally thought. Our own experience leads us to believe that it is less given to chronicity than is the disease induced by $P$. vivax. Manson-Bahr (1931) commented on the greater persistence of benign tertian infections.

The tendency to a few relapses is a recognised characteristic of $P$. falciparum malaria and we have noted as many as four or more of these before immunity has developed to a point where they cease. In our own experience renewal of clinical activity beyond an eight-week period from the cessation of the primary attack has been rare and no recurrence has been noted after a six-month period. In a previously mentioned paper by Boyd and Kitchen (1937a) it was observed that patients inoculated in the last quarter of the year have shorter incubation periods, longer attacks and fewer instances of renewed activity after cessation of the primary attack, whereas infections induced during the winter tend to have longer incubation periods, shorter attacks, and a greater tendency toward renewal of clinical activity. The incidence of relapses and recurrences may thus be taken as an indication of the effectiveness of the immunity built up during the primary attack. Both chronic malaria and malarial cachexia are stated to be the result of untreated or inadequately treated attacks of malaria. In our experience, lack of treatment during the primary attack in a person in good physical condition is conducive to more rapid development of specific immunity, and interference with the primary attack will directly influence the relapse rate. Thus two-thirds of our patients (Boyd and Kitchen 1937a) receiving small doses of quinine during the primary attack (to control the therapeutic infections) exhibited relapses, whereas slightly more than a quarter of those receiving no quinine did likewise. Over 90 per cent of our patients who received a week's course of quinine (after the method of Sinton 1930) subsequent to the primary attack experienced no relapses. It is recognized that the development and maintenance of immunity in malaria are adversely affected by poor hygienic conditions and concurrent or intercurrent infections, and it seems likely that these factors have been responsible for much of the chronicity for which $P$. falciparum has been primarily blamed. Inadequate, irregular, "hit or miss" treatment may lead to chronicity by reason of delaying or interfering with the immunogenic process. We believe, however, that much of the so-called chronicity and cachexia in $P$. falciparum malaria is due to causes other than lack of or inadequate treatment.

There undoubtedly exist in endemic areas several immunologically distinct strains of $P$. falciparum and it would seem to be rather difficult to differentiate between relapses and reinfections in all instances during the season of transmission, although it is likely that a reinfection would involve a greater parasite density and a more severe clinical reaction. Milder superinfections 
with heterologous strains might be indistinguishable. We (Boyd, Stratman-Thomas and Kitchen 1936a) have observed that a patient convalescent from an infection with one strain of $P$. falciparum may, when reinoculated with a second (heterologous) strain, develop an equally severe attack. Many of the pernicious infections which are said to develop suddenly during a relapse, may well be the result of a reinfection with an heterologous strain of the parasite.

Mannaberg states that: "In order to diagnose chronic malaria the following clinical symptoms must be present: (a) occasionally recurring paroxysms of malaria for months (relapses) which may be normal or latent, (b) a certain degree of anemia, (c) enlargement of the spleen and eventually enlargement of the liver, (d) the same species of parasites in the blood throughout the whole period, (e) general characteristic appearance." (These conditions could easily be met by reinfections.) Under appearance he cites the tawny color of the skin with its pallor and not infrequent icteric tint. He refers also to the anemia and its accompanying dyspnoea and palpitation on exertion. The patients frequently complain of malaise, headache, vertigo and insomnia.

It is difficult, and sometimes impossible, to differentiate between what some authors describe as chronic malaria and others as malarial cachexia. Some consider them as synonymous. Again, some authors (James 1922; Hehir 1927) divide malarial cachexia into two types, acute and chronic. The acute type, as described, would not seem to differ greatly from the condition frequently seen following some of the severe types of malarial infection. James (1922) feels that under endemic conditions " cachexia' is only exceptionally an accurate term to apply to the condition of patients who have suffered for a long time from repeated malarial infections and reinfections." Severe anemia and marked splenomegaly are the out-standing symptoms. Dyspnoea, weakness, emaciation and diarrhoea are usual and there may be oedema of the lower extremities, possibly ascites. A description of this syndrome, credited to Dr. R. E. Little of Quincy, Florida, appeared in MeGown's (1894) text. The latter author noted that ". . . this cachectic condition ... (in certain areas) ... is most frequently the sequelae to malarious diseases."

Complications and sequelae. Complications are not infrequent in estivo-autumnal malaria, particularly in highly endemic areas and in individuals debilitated by repeated infections with $P$. falciparum. Indeed, in so far as the latter group of individuals is concerned, malaria may be said to be a disease of complications. Often the form of the intercurrent disease is modified by the malarial infection. Respiratory infections frequently complicate the picture; of these, bronchitis, pneumonia, and influenza may be mentioned. When the latter, under epidemic conditions, attacks patients with malaria, the mortality rate is high. Manson-Bahr (1931) stated that "pulmonary tuberculosis is very prone to supervene in cachectic cases," but Marchiafava and Bignami (1901) do not agree on this point. It is likely, however, that malarial infections tend to cause a tuberculous focus to extend. The last-named authors are of the opinion that it induces a miliary form. Staphylococcic and streptococcic infection may occur. These, though local at first, may take the septicaemic form. Dysentery and cholera occasionally become associated with malarial infections. Rupture of the spleen when due to trauma, as it usually is, may be regarded as a complication. The usual outcome is fatal and death, due to the hemorrhage, is rapid. Kala-azar and ankylostomiasis are other diseases which not infrequently complicate estivo-autumnal malaria. There are many other, though less frequent, infections which may supervene.

Among the sequelae that have been noted following malaria are certain psychic disturbances. These may amount to frank psychoses, more or less prolonged. James (1922) quoted Porot and Gutmann as ob- 
serving that malarial psychoses are always primarily of the confusional type but may be transformed into more protracted forms. James also stated that malarial neurasthenia, sometimes with accompanying signs of physical debility, and psychopolyneuritis may be observed. Various neuropathies resulting from focal damage to the brain are cited by Mannaberg (1905). He stated that motor irritative symptoms were less frequent than degenerative symptoms. The duration of these symptoms varied greatly.
Paraplegias due to spinal cord injury have been observed, as have also parasthesias, anasthesias, and neuralgias. Most writers mention an hepatomegaly that may persist for some time after the malarial attack. Thayer (1897) was convinced of a malarial origin of chronic nephritis and Marchiafava and Bignami observed that acute and subacute nephritis developed shortly after cessation of a grave malarial infection. The latter authors feel that these changes are toxic in character. 


\title{
THE INFECTION IN THE INTERMEDIATE HOST: BLACKWATER FEVER
}

\author{
By S. F. KITCHEN \\ INTERNATIONAL HEALTH DIVISION, ROCKEFELLER FOUNDATION, TALLAHASSEE, FLA.
}

THus syndrome is now generally accepted to be intimately related to malaria although the exact nature of the relationship is still the cause of much controversy.

Etiology. Forty years ago Marchiafava and Bignami (1901) speaking of instances of this disease, in some of which attacks malaria parasites could not be found, and some of which were cured and others provoked by quinine, stated: "But in all these cases, although occurring under the most varied conditions, the one fact which can be affirmed with certainty is the etiological importance of the malarial infection." Stephens (1913), working in the Panama Canal Zone, offered statistical evidence supporting this relationship as regards incidence. Deeks and James (1911), studying blackwater fever in the same region, considered malaria as "a predisposing factor of great importance" and showed that "hemoglobinuric fever prevails among all races in direct proportion to the amount of malaria among them" ; they went further and concluded that the amount of blackwater fever is in direct proportion to the intensity of estivo-autumnal malaria. Foy and Kondi (1937), working in Macedonia, also established a definite correlation between malaria and blackwater fever in a malaria-sensitized population. Nevertheless there are yet many points concerning hemoglobinuric fever that are obscure.

On the other hand, however, we recognize several established facts regarding its etiology: (a) It occurs chiefly in nonimmune persons who are relatively recent arrivals (usually a few months to a few years resident) in endemically malarious areas. Ross (1932) has shown that it is particularly prevalent in persons with occupation in which the opportunity for constant exposure to infected mosquitoes is present. Residential environment is likewise important in this connection. (b) In the case of those who develop blackwater fever there is usually, though not invariably, a history of repeated attacks of malaria. Ross (1932) concluded that frequency of previous malarial infection in patients with blackwater fever was a factor of undoubted significance. Leishman (1912) stated that "there is certainly no direct relationship to the number or severity of the antecedent attacks of malaria." We have not observed any evidence of blackwater fever in over 200 patients with naturally induced $P$. falciparum infections; most of these have been in colored persens and few have been reinfected James, Nicol and Shute (1932) reported two instances of hemoglobinuria in their induced $P$. falciparum infections. According to Stephens (1913), it is usually during the second year of residence in the tropics that the greatest incidence of blackwater fever is noted and he considered this as evidence of the part played by repeated malarial infection. The disease has not been known to occur where malaria does not exist. (c) Negroes and other native races of endemic zones show a high degree of immunity to blackwater fever and this immunity is noticeably linked up with that to malaria. Furthermore, measures which decrease the incidence of malaria will do likewise for blackwater fever.

The common association of blackwater fever and $P$. falciparum malaria has undoubtedly led some workers, such as Robertson and Moore (1923) and Thomson (1924), to regard the former disease either as a pernicious form of the latter or as the same disease. Marchiafava and Bignami (1901), however, had stated "that a special place should be reserved for hemoglobi- 
nuria among the clinical forms of the malarial infection... (and it) is a phenomenon which may be manifested during the course of an active infection as well as in one which has spent its course; it is intimately related to malaria, but there is no direct causal relation between this phenomenon and the malarial parasites, such as there is, for example, between the coma of a pernicious attack and the parasitic invasion of the capillary vessels of the brain. Nor do we believe that we can class together, as some have done, hemorrhagic malarial infections in general and hemoglobinuria.",

The finding of $P$. vivax and $P$. malariae in blood smears from blackwater fever patients has been considered by some workers as evidence against $P$. falciparum as the constant etiological factor. This must be regarded as circumstantial evidence. Ross (1932) stated that". . . it would seem that too much significance is placed upon the species then present as indicating the variety of malaria which has led to the development of susceptibility." In this connection Boyd and Kitchen (1937f) have noted that in induced mixed infections with $P$. falciparum and $P$. vivax, only $P$. vivax, for example, may be observed in the blood smears at a given time even though there exists a concurrent $P$. falciparum infection. In addition to this, the hemoglobinuric paroxysm may have occurred after clinical termination of a falciparum attack and yet coincidentally with the appearance of a new $P$. vivax or quartan infection. Furthermore, in the majority of blackwater fever attacks, though parasites may be demonstrated prior to the paroxysm, their incidence in the peripheral blood afterwards diminishes rapidly. This also could account for the frequent absence of $P$. falciparum.

Among additional predisposing factors in the etiology of hemoglobinuric fever there may be considered: (1) Familial disposition. Many authors have cited instances in which several members of certain families showed a marked tendency to develop hemoglobinuria, particularly in some cases following the use of small doses of quinine. Deaderick (1914), in a study of 34 patients, recorded evidence of familial tendency. Stephens (1937) concluded, “' 'Disposition,' whether personal-and even hereditary-or familial may involve some at present unknown factor, but the known factor malarial infection appears always to be present." (2) Age and (3) Sex. The reports regarding these two factors are somewhat conflicting and it would seem that they both are subordinate to a dominating factor, viz., opportunity for exposure to malaria. (4) Deeks and James (1911) considered that syphilis was an important predisposing cause by reason of its tendency to provoke relapses of malaria.

Among the exciting causes of the hemoglobinuric attack, parturition, over-exertion and fatigue, exposure to cold and quinine have been chiefly mentioned. Foy and Kondi (1936) could find no evidence favoring the existence of hemolytic strains of plasmodia or such other specific parasites as have also been suggested as etiological factors. Deeks and James (1911) felt that malaria should be considered not only as a predisposing cause but also as a precipitating cause.

The rôle of quinine in provoking an attack of hemoglobinuria is a very puzzling one. It is a well established fact that very small doses of this drug have repeatedly been followed by hemoglobinuria in some persons. Quinine can be shown to produce hemolysis in vitro, but the corresponding amount required to do so in vivo could not be tolerated by the human organism under any circumstances. On the other hand, it is known that quinine has been given in blackwater fever with impunity. Many patients appear to be tolerant of a certain dose but exhibit hemoglobinuria when that amount is exceeded. The maximum incidence of hemoglobinuria as a reaction to quinine appears to occur within six hours of administration.

An excess of lactic acid due to deficient oxygenation was advanced by Blacklock and Macdonald (1928) as the cause of hemolysis in blackwater fever, but Ross 
(1932) was unable to demonstrate a hyperlactacidemia in the blood of blackwater fever patients. Cort (1929) referring to the hemoglobinuria, suggested that a specific factor was indicated by the evidence, "supposedly a malaria parasite, but a variety capable of elaborating a potent hemolysin." Numerous writers have felt that the hemoglobinuric attack was an anaphylactic manifestation. Many other hypotheses, largely speculative in nature, have been offered to account for the phenomenon. For a synopsis of these the reader is referred to Stephens' (1937) very useful summary.

Pathogenesis. The crux in the pathogenesis of blackwater fever is the intravascular hemolysis, whatever its cause. Whether it occurs in the peripheral circulation or, as advocated by Yorke, Murgatroyd and Owen (1930), in the sinuses of the spleen and liver, is still an open question. Lytic substances have not been demonstrated in the serum or plasma of blackwater fever patients. The last named authors consider that several hemolytic crises rather than an isolated hemolytic episode are involved in the erythrocytic destruction. They also note that but a small proportion of the released hemoglobin is found in the plasma. Fairley and Bromfield (1934) observed that in any intravenous hemolysis only a very small amount of the liberated oxyhemoglobin finds its way into the urine. They felt that the factor which provokes the hemolysis acts on the oxyhemoglobin to convert it largely into methemoglobin since the latter could not be demonstrated in washed corpuscles of these patients and yet was the predominant pigment in the plasma. The latter workers concluded that much of the hemoglobin was taken care of by the reticulo-endothelial system and subsequently the liver. In their cases there was evident a hyperbilirubinemia. Also in two patients studied for this purpose, a five to sevenfold concentration of bilirubin was found in the gall-bladder. As Ross (1932) said, it is possible that the relationship between the amount of hemoglobin con- verted into bilirubin and the amount excreted by the kidneys may vary in different individuals. He also noted that the liberation of hemoglobin in malaria is not usually followed by hemoglobinuria. If a renal threshold for hemoglobin exists and it "is not reached, the hemoglobin will be disposed of solely by the reticulo-endothelial system, whereas if it is exceeded both this and the kidney undertake the duty of disposing of the excess." The rapidity and degree of hemolysis would obviously influence this mechanism and the resulting symptoms.

Onset and symptoms. Although Thomson (1924) considered that there were premonitory symptoms evident in blackwater fever and Manson-Bahr (1931) described a "pre-blackwater" state, Yorke, Murgatroyd and Owen (1930), as well as Ross (1932), could not support this opinion. It is quite possible that in some instances symptoms due to a concurrent malarial infection have been mistaken for premonitory symptoms of blackwater fever.

In the majority of instances, the hemoglobinuric paroxysm is ushered in by a rigor which varies widely in its severity. Similarly its duration may vary from a fraction of an hour to as much as four hours. In a study of 162 patients with blackwater fever, Ross (1932) observed rigor to be present in 114, or 70 per cent. In about 11 per cent of these the rigor did not occur until after the appearance of hemoglobinuria. Multiple rigors are not uncommon. Stephens and Stott (1915) noted that the mode in the time incidence of rigors in their patients occurred between 9 A.M. and 12 M. Ross' (1932) experience was the same.

The constant accompanying symptom of the hemoglobinuric attack is fever, the duration of which varies with the course of the illness. The intimate association of pyrexia and the attack is of interest inasmuch as Barratt and Yorke (1914) showed that the injection of red cell stromata (but seldom hemoglobin) intravenously into rabbits usually caused convulsions and death. Fever may not commence to rise 
for half an hour after the rigor and as a general rule the peak of the fever curve occurs near the beginning of the attack and the temperature tends to fall as the hemolysis decreases. Exceptions to this are, (a) the drop to normal or subnormal level in the case of anuria and (b) the continuance of fever beyond the hemoglobinuric phase ("post-hemoglobinuric pyrexia"). Instances of hyperpyrexia have been described but appear to be uncommon.

Vomiting is not an uncommon symptom in hemoglobinuric fever but is more prone to occur in the severe toxic and anuric types. Commonly it is bilious in appearance although some writers have described a black color distinguishable from the "coffee-grounds" vomitus of yellow fever. This distressing symptom may be limited to the onset or may continue for a variable period during the attack in an intermittent manner. Much bile may be brought up. Hiccoughing is not infrequently associated with vomiting and like it seems to be most common in the same (severe) types of blackwater fever.

Splenomegaly and Hepatomegaly. Deeks and James (1911) found the spleen always to be enlarged and tender as long as the fever persisted. This is of interest because in primary attacks of $P$. falciparum malaria the spleen is rarely palpable. Where "post-hemoglobinuric fever" exists, the liver is more enlarged and tender than in malaria alone. Blacklock and Macdonald (1928) considered the splenomegaly as a necessary preliminary to hemolysis and Manson-Bahr (1931) stated that the spleen shrank as the hemolysis cleared up and considered this as evidence that hemolysis occurred primarily in the spleen.

Urine. Hemoglobinuria is, of course, the cardinal symptom of blackwater fever and it usually appears within an hour or so after the rigor occurs. Occasionally it is delayed by as much as 12 hours or more and in many of such instances the delay is probably due to some degree of suppression. Spectroscopic examination may show the presence of both oxyhemoglobin and methemoglobin in the urine. The former is more frequently found. In 102 specimens which showed absorption bands, Ross (1932) noted that 55 per cent contained oxyhemoglobin, 39 per cent both, and 6 per cent methemoglobin. The urine, however, may be negative spectroscopically and yet give a positive benzidine reaction. Pseudomethemoglobin, the new pigment described by Fairley and Bromfield (1934), has not been found in the urihe although it does occur in the plasma. These authors (1937) concluded that this pigment in the plasma had been "erroneously regarded as methemoglobin by all observers." Foy and Kondi (1938) found no methemoglobin in the sera of blackwater fever patients in Greece. More recently Fairley (1939) stated that the "haem" of the hemoglobin molecule is oxidized to hematin which unites with the serum albumin to form methemalbumin (pseudo-methemoglobin). The concentration of hemoglobin in the urine frequently shows considerable variation when samples are examined over a period of time, and, of course, is influenced by the volume excreted. Occasionally the first specimen voided after the rigor may be clearer than later ones. The variation may also be affected by other possible factors such as additional hemolytic events and irregular release of hemoglobin into the main blood channels from certain areas (spleen?, liver?). The duration of hemoglobinuria is likewise variable. It is commonly less than 24 hours but may vary from 12 hours to a week or more. It naturally depends upon the degree of hemolysis and its rate as well as the amount of urine excreted. Possibly there are other factors. Yorke and Nauss (1911) advanced the hypothesis that hemoglobin is excreted by the epithelial cells of the convoluted tubules which are damaged in the process.

Deeks and James (1911) noted that "in all types at the onset of the attack there is some suppression of urine, followed as a rule by hypersecretion." They had but one recovery among their patients experiencing complete suppression. Barratt and Yorke (1909) advanced the hypothesis that anuria was due to precipitation of the 
hemoglobin in the renal tubules and was facilitated by any factor which interfered with secretion of water by the malpighian bodies. Occasionally a small amount of urine may be voided after anuria has set in; such a specimen may contain much albumin (solidifying on boiling) and may be free of hemoglobin. Ross (1932) suggested that this might indicate that the fluid is largely inflammatory lymph from denuded surfaces of the tubules. There may be considerable variation in the incidence of oliguria and polyuria from patient to patient; while many authors consider the latter a favorable sign, Ross (1932) noted it in two toxic fulminating attacks.

The urine is usually light red to black in color and contains urobilin in irregularly increased amounts. Albuminuria has been recorded in degrees as high as 50 per cent and frequently persists for a few days after the hemoglobinuria. Bile pigment and bile salts are recognized as uncommon in blackwater fever urine. Ross stated that bilirubinuria was not observed in his patients unless the plasma yielded a direct Van den Bergh reaction to the extent of four units. The urinary sediment consists largely of blood pigment. This is present as (a) a majority of amorphous material and (b) easts which tend to increase in number as the disease progresses. Frequently they persist for some time and epithelial and hyaline casts may be seen later. The observations as to the presence of erythrocytes are conflicting. Some careful observers, however, have indicated that they undoubtedly have been seen in some instances.

Icterus. The degree of icterus occurring in blackwater fever is, as one might reason, dependent upon (a) the extent of the hemolysis, (b) the amount of hemoglobin excreted by the kidneys and (c) the efficiency of the biliary system in excreting the extra production. Obviously, then, there is going to be a certain amount of variation. Early, the Van den Bergh test gives the indirect reaction of hemolytic jaundice; later in anuria cases one may get the direct reaction characteristic of the obstructive type of jaundice. Icterus is infrequently absent, usually appears early and may be evident prior to the rigor or the detection of hemoglobinuria. It is marked in the fulminating toxic type of infection if the patient survives over 24 hours. Likewise icterus is usually quite definite when the hemoglobinuria is prolonged, either in the continuous or intermittent type of blackwater fever. The greatest degree of jaundice, however, is noted in those with anuria. Ross (1932) makes an interesting point that gall-stones were frequent in those who had had either blackwater fever or severe malaria; that stones removed from such individuals invariably were composed entirely of calcium-bilirubinate.

Blood. The destruction of erythrocytes is, of course, the outstanding feature in blackwater fever. The rate and amount of loss varies considerably and may be as great as one million red cells in $\mathbf{2 4}$ hours. Actual counts of less than a million erythrocytes per $\mathrm{cmm}$ and hemoglobin values as low as ten per cent have been observed. As the bone marrow responds, the immature forms in the erythrocytic series may be found in the smears. Anisocytosis, poikilocytosis, macrocytes and microcytes have been reported. The sedimentation rate is usually increased. There is much inconsistency in the different observations regarding the total leucocyte count and it is difficult therefore to determine possible correlation with clinical reaction. It is generally agreed upon, however, that an increase in mononuclear leucocytes is commonly, though not invariably, found and that there is frequently a lymphopenia.

Nitrogenous retention may reach high values in patients with anuria, yet these persons usually do not show signs of uremia. Bilirubin in the blood plasma reaches its greatest concentration in the toxic or the anuric patients and may range from normal to 80 or more units. The color of the plasma is dependent upon the relative degree of hemoglobinemia and bilirubinemia. Methemalbumin (Fairley 1939) is another pigment which occurs in the 
plasma. Usually, though not always, a hypocholesterinemia is present. The alkali reserve may be normal or show a decrease.

Prognosis. The death rate in blackwater fever is about 20 to 30 per cent and is about 10 per cent higher in the case of multiple attacks than in initial episodes. In approximately 65 per cent it occurs during the first week. Cardiac failure is usually assigned as the cause of death. Prognostic signs of bad omen are: (a) uncontrollable vomiting, (b) singultus, (c) anuria, (d) a marked degree of icterus, (e) a sudden drop in the temperature with prostration, (f) grave anemia and (g) coma.

Pathology. The lesions found in blackwater fever depend to some extent upon the stage of the disease at which death occurred. The liver is enlarged, congested and contains much bile. The bile capillaries stand out distinctly. Hemosiderin and hemoglobin may be noted in the hepatic cells and malarial pigment is seen in the swollen endothelial cells. Necrosis of the parenchymal cells may occur. The spleen is swollen and shows much evidence of phagocytosis. Much pigment and red cell debris may be present, the venous sinuses are usually dilated and hyperplasia of the malpighian follicles is commonly seen. The kidneys may show some enlargement. The epithelium of the convoluted tubules undergoes degenerative changes and the lumina contain casts made up largely of hemoglobin, erythrocytic and other debris and epithelium. The collecting tubules likewise contain casts, chiefly erythrocytic in origin.

Treatment. The literature, unfortunately, is cluttered up with suggestions for the treatment of this disease but many of these have not been given adequate trial and will not be mentioned here. As yet no specific to control the hemoglobinuria is known. Absolute rest and good nursing are, of course, of primary importance. There would seem in most instances no good reason for, and some against, the use of quinine. In this circumstance atabrine may serve as an alternative although hemoglobinuria has been observed following its administration. The parenteral injection of saline if done judiciously appears to be of some value. Transfusions, perhaps several, are apparently desirable in cases of severe anemia. In the main, however, the treatment is essentially symptomatic. Recently Krishnan (1939) has reported the successful use of an adrenal cortex preparation concurrently with ascorbic acid and glucose in seventeen patients. 


\title{
SOME PATHOLOGIC ASPECTS OF HUMAN MALARIA
}

\author{
BY PAUL R. CANNON \\ DEPARTMENT OF PATHOLOGY, UNIVERSITY OF CHICAGO, CHICAGO, ILL.
}

HUMAN malaria is a type of infectious disease in which the duration, severity and outcome are affected by a number of variables, such as the species of parasite, the variations in its biologic properties, the length of infection, the age and race of the host, the differing grades and kinds of resistance, the effects of relapse, the kinds and amounts of therapy used and the concomitant influences of intercurrent and terminal infections. It is apparent from a consideration of these facts that malaria can no more develop according to a simple pathologic pattern than can tuberculosis, syphilis or any other infectious disease with a variable clinical course. Rather than attempting to describe pathologic details, therefore, this discussion will be directed toward those general pathologic aspects of malarial infection which have a direct relationship to infectious disease as a whole.

A satisfactory understanding of any infectious process requires the study of properly fixed and stained necropsy material from all stages of the disease. It is because of this requirement that the story of the pathologic effects in human malaria is as yet incomplete. There are several reasons for this. Very few patients die from tertian or quartan infections ; furthermore, in estivo-autumnal infections death does not usually occur for days or weeks, and the pathologic pieture is that of the end stage, with this frequently obscured by a terminal infection. Too frequently, also, necropsies are performed hours after death, thus interfering with satisfactory fixation and staining of the tissues. Correct interpretation as to the site and extent of distribution of malarial pigment in cells and organs has also been hampered by the widespread use of formalin-fixation, with the attendant uncertainty caused by the presence in cells of the confusing "formalin pigment." It is because of these difficulties that, although a great deal is known about the pathology of human malaria, many of the current ideas about its earlier phases are inferential or derived from experimental sources.

The essential facts about the pathology of human malaria may be summarized briefly as follows (Dock 1894; Barker 1895; Craig 1938; Councilman and Abbott 1885; Seyfarth 1926) :

1. Malarial parasites, after their initial entrance into red blood cells and their consequent dissemination throughout the body, go through the characteristic cycles of asexual development which produce, in large measure, the symptoms of the disease. The ultimate course of events depends upon the ability of the host to prevent the continued accumulation of the parasites and the ensuing parasitization of other red blood corpuscles. Under ordinary circumstances, as with tertian or quartan parasites, the defensive mechanisms of the infected individual soon come into action and, in time, the parasites disappear from the blood stream, tending to become localized predominantly in three organs, viz., the liver, the spleen (Clark 1928) and the bone marrow. In those instances where the parasites continue to multiply in the tissues, as in infection with Plasmodium falciparum, localization in these organs, although occurring to some extent, fails to counterbalance the unrestricted reproduction of the parasites and the infection may ultimately become overwhelming and lethal.

2. The selective localization of parasites in the liver, spleen and bone marrow occurs because of anatomic and physiologic characteristics peculiar to these organs 
(Cannon, Sullivan and Neckermann 1932). They differ particularly from other organs in possessing blood sinuses in addition to ordinary capillaries; this changes the character of the blood flow. These sinuses, and the loose tissues around them, are lined with or contain potentially phagocytic cells along or between which the parasitized erythrocytes must pass. While doing so the parasitized cells are very likely to come into contact with phagocytes and be engulfed. Proof of this is seen in benign malaria, where these macrophages become engorged with parasites and pigment but eventually cleanse the blood of infected red blood cells. In malignant malaria, on the other hand, such phagocytosis, for various reasons, is presumably less effective, and the infection progresses at times to the lethal stage. Phagocytosis, therefore, stands out as the determining element in the progress of malaria infection and in the resulting pathologic picture of the disease.

3. Because of this selective localization of the parasites, these three organs undergo hypertrophy, hyperplasia and pigmentation, all characteristic features of the pathologic response; and in these processes additional pathologic complications may ensue, as, for example, fatty and toxic changes in the liver, hemorrhages or infarctions of the spleen, and focal necrosis of the spleen and bone marrow. The main reaction between tissues and parasites in all organs, however, is essentially mesenchymal and the parenchymal lesions which may occur are presumably secondary and for the most part incidental.

4. Participation of lymphoid tissues is active in all three organs, although most evident in the spleen, as seen by the proliferative reactions of the splenic corpuscles and by the toxic necrosis, pigmentation, and "depletion" of the lymphoid structures. It is probable, therefore, that, as emphasized in the studies of simian malaria by Taliaferro and associates $(1936 ; 1937$; this volume p. 239), the lymphoid tissues constitute an important part of the reaction to malarial infection and act as a sort of "backlog" or mesenchymal reserve from which mononuclear phagocytes may be derived.
5. The parasitization of the red blood cells and their dissolution during the course of schizogony leads to the anemia, so important a feature of malaria. This varies in severity from the comparatively mild forms in chronic benign malaria to the fulminating types seen in pernicious malaria or in blackwater fever (Whipple 1927). The influence of the infection upon the bone marrow is further reflected in the failure of the polymorphonuclear leukocytes to enter the blood stream, so that the usual blood picture is that of a leukopenia with a relative lymphocytosis. The large mononuclear elements, however, are frequently increased in the blood and at times may comprise twenty-five per cent or more of the colorless corpuscles.

6. Parasitic localizations in organs other than the liver, spleen and bone marrow are exceptional, and when they occur, as in pernicious malaria, are supposed to be due to embolic occlusions of capillaries and arterioles. These embolic occlusions apparently follow no general rule, although they are seen more frequently in the brain, heart and gastrointestinal tract. They may occur, however, in almost any organ of the body, as for example in the kidneys, pancreas, abdominal fat, thyroid glands, lungs or suprarenals.

7. Because of these vascular disturbances, widespread toxic and necrotizing lesions may develop. These include the so-called ring hemorrhages of the brain, the perivascular areas of necrosis, the so-called malarial granulomas, the degenerative changes in the cells of the nervous tissues, the toxic changes in the tubules of the kidneys, particularly as seen in blackwater fever, and the hemorrhagic and necrotizing lesions in the myocardium, suprarenals and elsewhere (Dudgeon and Clarke 1917; Gaskell and Millar 1919-20; Allen 1926).

Consideration of these pathologic facts suggests the similarity of malaria to bacterial infections and engenders the hope that common pathologic principles may govern both types of infection. Looked at from the standpoint of an infectious disease, malaria, on the basis of an evaluation of its pathologic effects, may be considered. 
as a particular type of infectious process which follows the characteristic pattern of any disseminative type of infection. It may thus be compared to septicemia, pyemia, tuberculosis, syphilis, typhoid fever, plague, undulant fever and the like.

In the natural history of all of these diseases, certain general principles may be deduced as follows:

1. They may all, initially, be generalized infections, with the infectious agents widely disseminated through the blood stream. An essential difference is that the bacteria are carried in the blood plasma whereas the malaria parasites are carried within the red blood corpuscles (Ratcliffe 1928).

2. The clinical severity depends upon the parasitizing potentialities of the infectious agents and the ability of the invaded host to restrict their development, their toxic effects and their tissue localization. Such terms as virulence, invasiveness and toxicogenicity describe the bacterial proclivities, whereas, in malaria, the varying effects are commonly supposed to be due to the differing potencies of the "toxins" liberated with the merozoites at each segmentation. In both types of infection humoral and cellular mechanisms of defense determine the varying grades of natural and acquired resistance. In tertian and quartan malaria, for example, the parasitizing abilities of the microorganisms are usually counterbalanced by the resistance of the host; in pernicious malaria, on the other hand, the invasive potentialities of the parasites not infrequently overcome the resistive capacity of the host. But whether the infection is bacterial or malarial, the question whether it will be non-lethal or lethal depends upon the ability of the infected person to convert a generalizing, progressive type of infection into a localized, regressive one. The most important problem which confronts the host in any type of infectious disease, therefore, is either that of confining the initial infection to a localized area, or, if this is not immediately possible, of mobilizing defensive mechanisms which may ultimately localize the infectious agents. An adequate comprehension of this process in malaria, as revealed by pathologic facts, should aid materially in the better understanding of the genesis of the disease and the significance of its clinical symptoms.

The similarity in the pathologic course of events in benign malaria and in typhoid fever is striking. In both infections there is the initial distribution of the infective agents throughout the body, followed by their predominant localization in the liver, spleen and bone marrow. This localization, in malaria, is evidenced by the greater deposition of malarial pigment, whereas, in typhoid fever, it is characterized by the presence of "focal necroses." In both infections there is hypertrophy and hyperplasia of the reticuloendothelial elements; in both, the usual blood response is a leukopenia. In both, injury to erythrocytes and increased erythrophagocytosis lead to secondary anemia; in each there is a characteristic increase in the number of mononuclear leukocytes in the blood. It is not surprising, therefore, that in the past the two diseases were so frequently confused, and that the term "typhomalaria" was used so commonly to describe symptoms which might pertain to either disease alone or to their concomitant occurrence.

Pernicious malaria, on the other hand, may more appropriately be compared with those fulminant and overwhelming bacterial or viral infections in which death occurs speedily because of the unusual virulence of the invading agents, as, for example, in hemorrhagic smallpox, measles, scarlet fever, meningococcemia, streptococcic bacteriemia, etc. Under these conditions an unusually invasive type of microorganism presumably overcomes the resistance of the host because the defensive mechanisms of the latter are unable to localize it. So, in infection with $\boldsymbol{P}$. falciparum, the effects are particularly serious because (1) of its greater ability to multiply and (2) of its greater toxicogenicity. This species attains a higher concentration in the blood, has a shorter incubation period and thereby causes an earlier manifestation of symptoms. This may be due to the fact that it produces more merozoites per schizont 
than do either $P$. vivax or $P$. malariae. It is also more toxic, in the sense that it causes graver symptoms, a severer anemia, greater damage to the central nervous system, more regressive changes in the infected erythrocytes (the so-called "brassy" cells) and more severe degenerative changes in such parenchymatous organs as the heart, liver, kidneys and suprarenals. It may also lead to widespread fatty degeneration of blood vessel walls, leukopenia, vascular hemorrhages, and purpuric lesions.

In both types of fulminating infection, the adverse effects may be explained on the basis of unrestricted parasitic growth in "virgin soil." This conclusion is supported by the statement of Marchiafava (1931) that "only recent infections or early relapses develop into pernicious malaria." According to him "it is especially in organisms virgin to the malarial infection that the estivo-autumnal parasites multiply more acutely, reaching at times enormous numbers; and, developing greater virulence, give rise to pernicious symptoms. And, on the other hand, with the persistence of infection in the organism, this acquires a relative and partial immunity, an immunity against the pernicious feature of the infection, such as we see exemplified every day among the inhabitants of malarious communities." He states, furthermore, that the hyperplasia of the spleen in pernicious malaria is always acute, with the pulp soft and almost fluid and that the spleen never shows evidence of a chronic hyperplasia.

In all of these infectious processes, therefore, the primary pathologic effects are centered in the activities of the system of macrophages or, in other words, in the reticuloendothelial system. The contribution of myeloid elements to the circulating blood may or may not be a significant part of the bone marrow response, but the fixed tissue reticular cells of the marrow act to engulf the foreign elements circulating in the blood. In the liver, spleen and bone marrow, which have been aptly called the "primary blood filters," the first clash between invader and invaded occurs, and here the immediate outcome of the infection is determined. If the disease is fulminating or malignant, death may occur because these organs are unable to localize the developing infection. In more favorable cases, on the other hand, localization is accomplished and, in the hypertrophied macrophages of the splenic cords, the Kupffer cells of the liver and the reticular cells of the bone marrow, are seen the malarial and blood pigments as the characteristic effects of the successful struggle. All other pathologic effects of malarial infection, therefore, may be regarded as departures from the primary purpose of blood cleansing and organ localization, and depend for the most part on local peculiarities, massiveness of infection, embolic accident or pure chance.

One may ask the question: Why does malaria show such a definite predilection for the reticuloendothelial system? It is equally proper to ask, however, why in bacterial septicemia, typhoid fever, undulant fever, miliary tuberculosis and in acute syphilis, the same predilection is manifested? Why, for example, in tertian and quartan malaria and in bacterial septicemia, are such organs as the myocardium, thyroid gland, pancreas, suprarenals, lungs, brain, kidneys and gastrointestinal tract so rarely affected? The answer requires a true understanding of the conditions which influence the removal of infective agents from the circulating blood; this, in turn, requires a more detailed consideration of two fundamental problems, viz., the essential nature of endothelium and the dynamies of capillary blood flow.

There has long been confusion regarding the essential nature of endothelium and its part in the localization of infectious disease. This is due to the fact that many persons have believed that all cells lining blood vessels are, by definition, endothelial cells, and that because some are actively phagocytic, all are likewise so. The popularity of the term "reticuloendothelial system" has also encouraged many to believe that all endothelium is actively or potentially phagocytic and that from it arise the circulating mononuclear leukocytes of the blood. 
The facts of infectious disease, however, prove beyond a doubt that under the ordinary conditions of blood flow phagocytosis by endothelium is extremely uncommon. And even when there is some evidence that it may occur, the possibility cannot be excluded that the infectious agents are merely passing through the endothelial cell rather than having been phagocytosed. In many infectious diseases, including malaria, numerous attempts have been made to demonstrate the rôle of endothelial phagocytosis in common endothelium (Bruetsch 1932a), but the evidence of the commonness or the importance of such activity is scanty and unconvincing.

It is not surprising that this is so. Common endothelium, as it lines capillaries, arterioles and larger blood vessels, consists of stretched-out cells with but little cytoplasm available for phagocytic action. Maximow (1927b) described them as "fixed, highly differentiated cells" which belong to the group of fibrocytes. According to this eminent histologist "the rôle of the endothelium in the adsorption and neutralization of toxins and in other general reactions combating infections or intoxications may be important, but it is not manifest in appreciable morphologic changes." Endothelium, therefore, may be thought of as a blood-tissue barrier which keeps formed elements constantly confined to the blood stream but which allows diffusion of oxygen, carbon dioxide and metabolites of the blood and tissues. So long as it remains uninjured, infectious agents in the blood stream may pass into and out of most organs until, in their passage through the liver, spleen or bone marrow, they encounter altered conditions of blood flow and come into contact with the phagocytic cells. It is because of contact of the parasites with these specialized cells, sometimes called "special endothelium," and not because of contact with common endothelium, that they are engulfed and removed from the blood stream. This entire selective mechanism may well be regarded as a wise adaptation of nature, a specialization of organic function, whereby vital organs are shielded from many of the more serious vicissitudes of life.

It is well to think, conversely, of what might happen if common endothelium were functionally active and participated energetically as a phagocytic tissue. In every bacteriemia, phagocytosis by common endothelium would interfere with the normal circulation of blood through many important organs; the ensuing circulatory disturbances and the resulting localized inflammations would surely make common such conditions as thyroiditis, myocarditis, pancreatitis, myositis, adrenalitis, orchitis, oophoritis and the like. The fact that this does not happen constitutes excellent presumptive evidence, therefore, that under ordinary conditions of blood flow, but little phagocytosis by endothelium occurs.

Furthermore, under the usual conditions of blood circulation through capillaries and larger vessels, the rapidity of blood flow makes it unlikely that many opportunities may be afforded endothelial cells for effective phagocytosis. And even under conditions of blood stasis, the extent of phagocytosis by endothelium is slight. In instances of extreme sluggishness of blood flow, as for example, in association with the hemoconcentration of shock (Moon 1939), some agonal phagocytosis by endothelium might contribute to the process of capillary occlusion, so prominent a feature of pernicious malaria.

This problem of capillary occlusion in pernicious malaria has been a perplexing one for many years. Although vascular occlusion by parasitized erythrocytes is particularly common in the white matter of the brain, the fact that it may also occur in other organs, as for example, in the myocardium, gastrointestinal tract or elsewhere, suggests that local causes do not explain the condition adequately. These embolic occlusions cannot be attributed to the effects of swelling of the erythrocytes, because these cells tend to enlarge in infection with $P$. vivax, where such embolization does not occur, and tend often to shrink in infections with $P$. falciparum.

One feature of pernicious malaria which 
is noteworthy is the vascular injury, as revealed by generalized fatty degeneration, hemorrhages into the brain, purpura, etc. Such a condition should certainly predispose to loss of fluid elements of the blood similar to that in shock, and as it does particularly in the algid forms of pernicious malaria. There is also evidence of hemoconcentration in pernicious malaria at times, which might also lead as aforementioned, to an increased tendency to blockage of capillaries.

A factor not adequately considered in the genesis of capillary occlusion in pernicious malaria is that of agglutination of parasitized red blood cells, although Cropper, many years ago (1908), in smears taken from the blood of a patient two hours before death from pernicious malaria described the clumping of red cells in masses, a phenomenon which he said "so far as I know has not been previously observed in peripheral blood, though it is commonly seen in the brain and other organs affected in pernicious attacks after death. ... I would conjecture that such masses must occur in the internal organs in intense infections, but that as a rule they are not seen, being eliminated and filtered out from the blood in the spleen or liver, thus giving rise not only to enlarged spleen but also to bilious, remittent attacks and malarial hepatitis. Where, however, other organs such as the brain, the kidney, the pancreas or intestinal walls are affected, cerebral symptoms, etc., develop."

Dudgeon and Clarke, more recently (1917), refer repeatedly to the presence of "agglutinated" red blood cells in thrombi seen in the brain, kidneys and suprarenals of patients dying of pernicious malaria.

These observations merit more investigation in the light of Knisely's recent demonstration by cinematophotography that, in severe infections of rhesus monkeys with $P$. knowlesi, erythrocytes actually agglutinate in the circulating blood stream following which the agglutinated masses, while passing through the liver, are quickly engulfed by macrophages.

Evidence further substantiating this viewpoint is presented in the following case of pernicious malaria (Case \#3883, Department of Pathology, The University of Chicago).

The pathologic findings in this patient are of particular value because she had probably had no previous malarial infection; the present infection was unsuspected and no anti-malarial treatment was given; furthermore, necropsy was performed two hours after death. The patient, a woman 50 years of age, developed symptoms uf epigastric pain, nausea and vomiting, followed by irregular fever, rapid pulse and moderate leukopenia. She had recently been on a boat trip to Central America and these symptoms first appeared about 8 days after her return. The total duration of illness from the first chill until death was 9 days. Figs. 1-6 inclusive furnish evidence of the unequal distribution and concentration of the malarial parasites in various organs. Fig. 5 is especially interesting in that it shows intravascular agglutination of parasitized erythrocytes. Fig. 3 also shows the accumulation of parasitized erythrocytes in a mucosal capillary of the ileum with an adjacent venule containing no parasitized red blood cells. Such an appearance can be explained by the gradual "screening out" of parasitized erythrocytes as they pass through capillaries, with non-parasitized ones thus comprising the bulk of the venous outflow. Such an agglomeration cannot be readily explained by chance, but could be due, in the lungs, to a similar adherence of parasitized erythrocytes on their passage through intra-alveolar capillaries and their occasional outflow into pulmonary veins.

These evidences of intravascular agglomeration are to be expected, if malaria is a type of infectious disease which follows the general pattern of infectious processes. Agglutination of bacteria in the tissues is a recognized phenomenon (Sullivan, Neckermann and Cannon 1934). It is due, presumably, to the deposition of antibodyprotein on the surface of the antigenic particles, thus changing their surface proper- 
ties and making them more cohesive, buth to themselves and to surrounding tissues. The demonstrations of agglutination in pernicious malaria, therefore, suggest the presence of an antibody whose action becomes manifest after a proper interval of time and when the parasitic population in the blood is large enough to afford opportunities for contact between numerous parasitized red blood corpuscles. If this explanation is correct, the prevention of intravascular agglutination would seem to require early, intensive and adequate chemotherapy in order to prevent the increase of the parasitic population to the critical level.

In summary, then, there are many striking similarities, as revealed by pathologic studies, between malarial and bacterial infection. If the conclusion is correct that the two types of infection are essentially similar, a better understanding of malaria should develop from the further application to its study of ideas and methods which are now well established for bacterial infections.

FIGS. 1-6 incl. Photomicrographs from sections of patient $(\$ 3883)$ who died from untreated pernicious malaria. Fixation in Zenkers solution (without acetic acid). Stained with hematoxylin-eosin azure II.

FIG. 1. $\times 1575$. Note the accumulation of parasitized erythrocytes in a capillary of the left myocardium. FIG. 2. $\times 1250$. Note the scarcity of parasitized erythrocytes in a small renal vein.

FIc. 3. $\times 1300$. A capillary in the mucosa of the ileum is obstructed by parasitized erythrocytes whereas a contiguous venule contains no parasitized red cells in the focal plane examined.

FIG. 4. $\times 1150$. Many parasitized erythrocytes are concentrated in the red pulp of the spleen. Note the absence of conspicuous phagocytosis.

Fig. 5. $\times 1425$. A cross-section of a pulmonary vein showing an agglutinated clump of parasitized erythrocytes surrounded by non-parasitized red cells.

Fit. 6. $\times 575$. A "depleted" splenic corpuscle surrounded by many parasitized erythrocytes in the red pulp. 


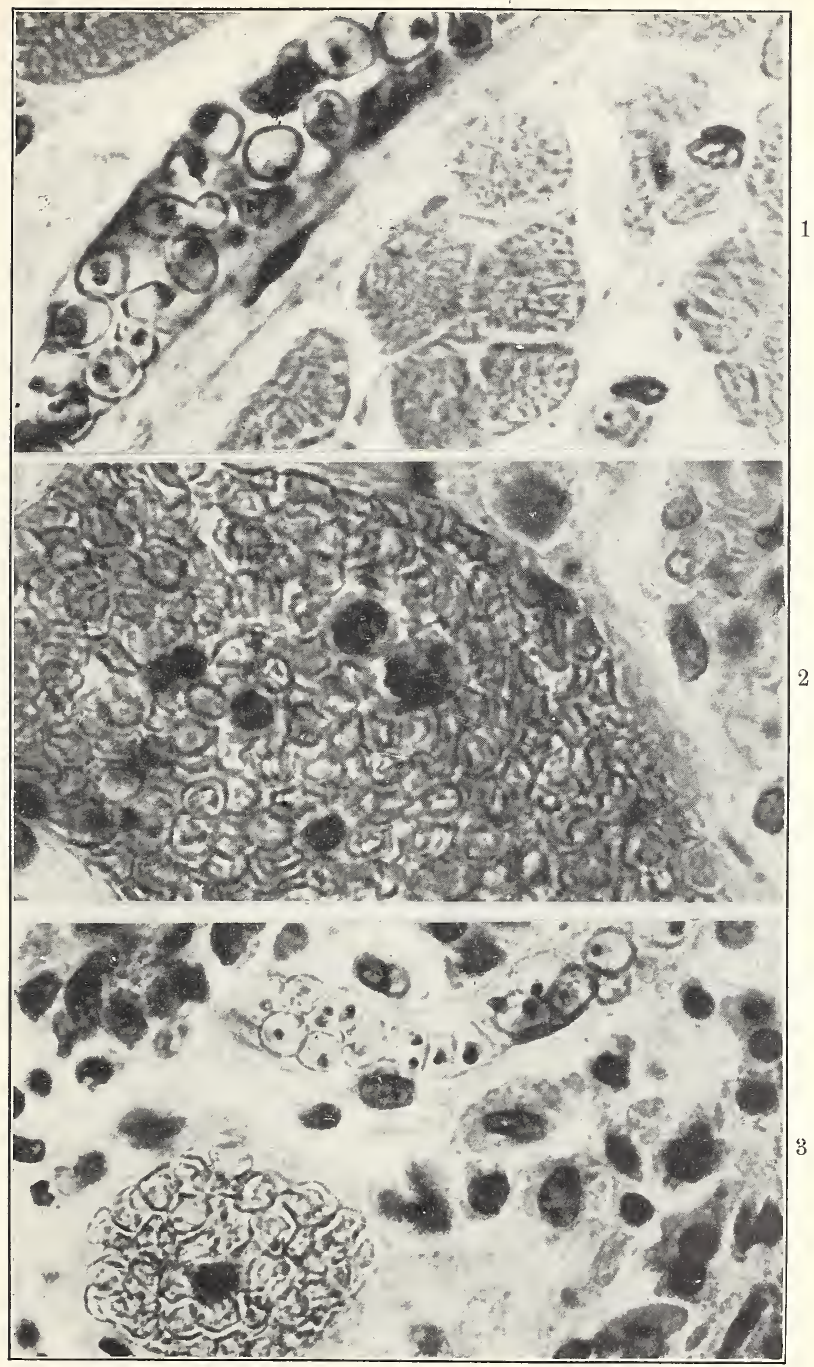




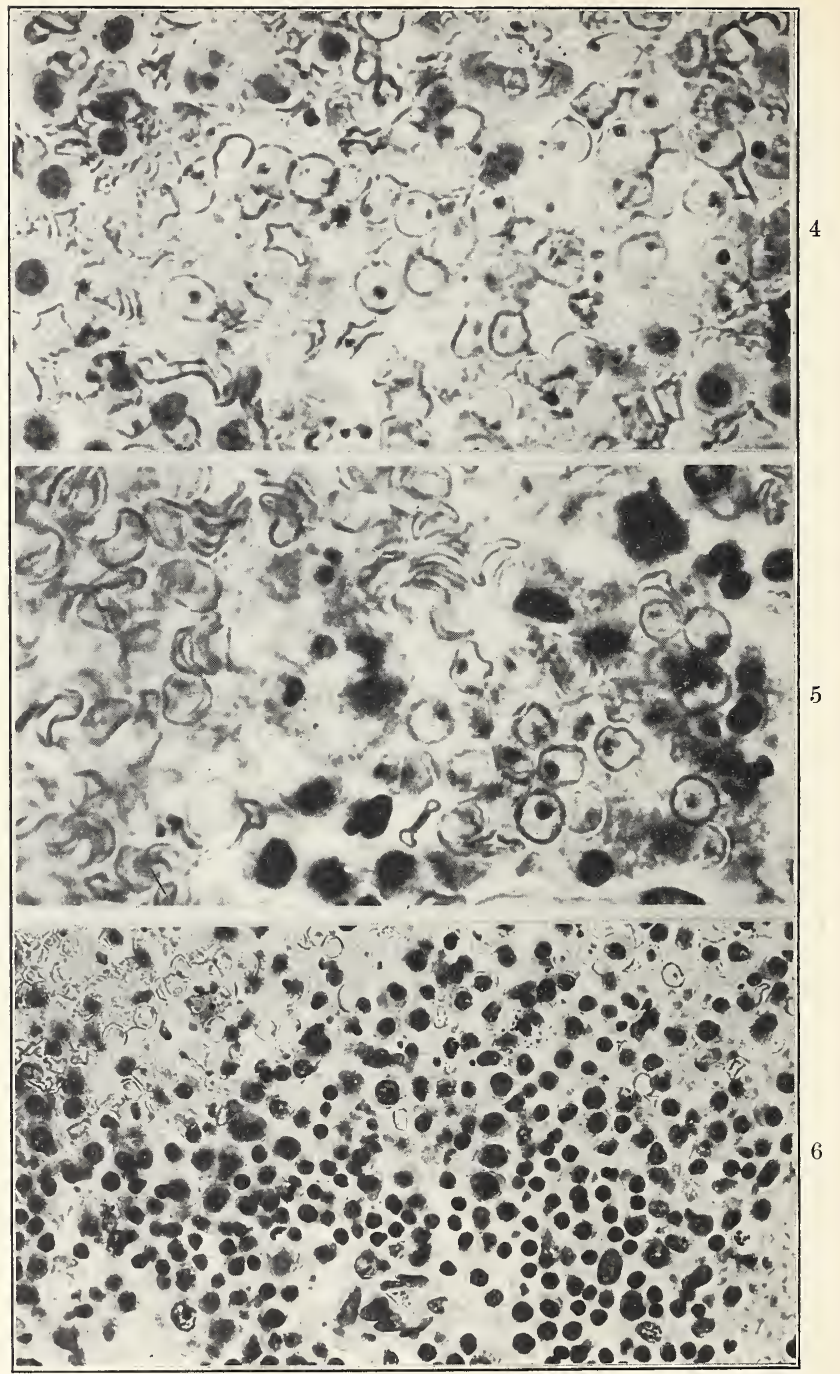




\title{
THE PHYSIOLOGICAL PATHOLOGY OF MALARIA
}

\author{
By HENRY E. MELENEY \\ DEPARTMENT OF PREVENTIVE MEDICINE AND PUBLIC HEALTH, VANDERBILT UNIVERSITY \\ SCHOOL OF MEDICINE, NASHVILLE, TENN.
}

THERE is no phase of the study of malaria which offers more promise of interest and accomplishment to the worker in the clinical and biochemical fields than the physiological changes produced in human subjects and experimental animals by the various species of malarial parasites. These changes may best be appreciated by reviewing briefly the effects produced by the asexual cycle of the parasite in the human host. When schizogony has been completed, the schizont ruptures the erythrocyte, liberating hematin, salts, cell constituents and possibly toxins into the blood plasma. The chill and fever are clinical manifestations of this event when large numbers of parasites complete schizogony at one time. When large numbers are constantly completing schizogony, a continuous or irregular high fever with other severe clinical manifestations occurs.

The continuous repetition of the asexual cycle has further effects on the blood and other organs of the body. The number of erythrocytes is reduced and an increased proportion of young erythrocytes appears in the circulation. The proportion of erythrocytes to plasma is decreased. The plasma proteins, lipoids, carbohydrates and salts are thrown out of normal balance. The erythropoietic function of the bone marrow is stimulated. The secretory and excretory activity of the liver and kidneys is increased, and the functions of other glands, such as the suprarenals, may be stimulated or disturbed. In addition, the occurrence of chills and fever makes greater demands on the carbohydrate and protein metabolism, thus depleting the storage of these substances in the liver and muscles. The phagocytic mechanism of the body is stimulated, causing multiplication and in- creased activity of the reticulo-endothelial cells.

Two other phenomena which produce serious physiological disturbances in malaria are the plugging of capillaries by erythrocytes parasitized by $P$. falciparum, and the sudden intravascular hemolysis known as blackwater fever which severely alters the constituents of the blood plasma and often places an insuperable strain on the excretory functions of the kidneys.

It is now appropriate to discuss in detail the changes which malarial infection produces in the constituents of the blood and in the functions of various organs of the body. Although the subject of this symposium is human malaria, certain additions to our knowledge have been contributed by recent research on monkey malaria, and these will be drawn upon to supplement observations on human subjects. Space permits reference to only a limited number of papers in the enormous literature in this field.

\section{BLood Proteins}

Ghosh and Sinton (1935) reviewed the observations made previously on the blood proteins in human cases of malaria. Most of the work had been done on blood serum. There was general agreement that the total of serum proteins was reduced, both in the acute and in the latent stage of the disease. This reduction involved principally the serum albumin. The serum globulin was usually not reduced and was often increased and the proportion of euglobulin was always increased. The globulin-albumin ratio was always increased though usually not above unity. These changes were more marked in acute than in chronic infections. Radosavljevice and Ristic (1926) found that in the blood 
plasma there was an increase in fibrinogen at the height of each malarial paroxysm with an absolute decrease of albumin and that these conditions were reversed during the apyrexial period.

Chopra, Mukherjee and Sen (1935) studied the serum proteins in human cases during and after the paroxysm and found that during the rigor there was a considerable decrease in the albumin and a slight increase in the euglobulin while the pseudoglobulin remained practically normal. After the rigor had subsided the changes in proteins were found to be similar to those found during the rigor but the changes were less marked.

The increase of euglobulin in malaria is associated with the development of Henry's melano-floceulation test for the diagnosis of the disease. Chorine and his co-workers $(1934,1938)$ and a number of others have shown that this reaction is not dependent upon antigenic properties of melanin specific to malaria, but is due to the floceulation of euglobulin with melanin as an indicator. Chorine and Gillier (1934) showed that the flocculation depended upon the molecular concentration of the serum, flocculation being prevented by the solubility of euglobulin if the molecular concentration was increased. On the basis of this work, Proske and Watson (1939) developed a protein tyrosin color test for the diagnosis of malaria in which they completely precipitated the euglobulin by the use of 13.5 per cent sodium sulfate.

The blood urea apparently undergoes no significant change in malaria unless there is severe damage to the kidneys either in malignant malaria or associated with chronic malaria or blackwater fever.

One of the interesting phenomena associated with malaria, and probably related to the blood proteins, is the development of positive reactions to serological tests for syphilis. Although a number of conflicting reports have appeared from time to time, Kitchen, Webb and Kupper (1939) have shown that positive tests are obtained regularly during some period of the clinical disease. The duration of the seropositive period varied considerably but was as much as four weeks in nearly half of the cases. The percentage of positive reactions was highest during the third week following the last previous paroxysm. These findings emphasize anew the non-specificity of the present serological tests for syphilis.

Physico-chemical changes which are probably related to the blood proteins have been found in both human and monkey malaria. Sinton, Orr and Ahmad (1928), studying human cases, found a slight decrease in the surface tension of the serum during the rigor with a return to normal afterward. In three out of four cases they observed a rise in the refractive index of the serum during the rigor. Kehar (1936) studied these and other physico-chemical changes in monkeys infected with $P$. knowlesi. He found that the surface tension of the serum decreased considerably as the disease progressed, and that there was a slight but persistent rise in the refractive index during the acute period of the infection, but that no variations were observed in the specific gravity of the serum.

The sedimentation rate of the erythrocytes in malaria has been studied by a few investigators. Radosavljevié and Ristic (1926) found that during the rise of temperature there was a decrease of the sedimentation rate associated with a decrease of total plasma "albumen" and an increase of plasma fibrinogen. During the fall of temperature and in the following feverfree period the sedimentation rate increased to a level above normal while the "total albumen" and fibrinogen showed only a partial return toward normal. During the active stage of the disease these changes became progressively more marked, but returned to normal gradually during spontaneous remissions or more quickly after quinine treatment. They found these changes not only in the clinical stages of the disease but also in the incubation period and just before relapses, and believed that they were related to the sporulation of parasites rather than to the mere 
presence of parasites in the peripheral blood. They suggested that the clinical phenomena were evidences of hypersensitivity to the parasites and that spontaneous remission was an evidence of desensitization.

Kehar and Harbhagwan (1937), reporting observations made by Mulligan, found a considerable increase in the sedimentation ratc during the acute phases of human infections with both $P$. vivax and $P$. falciparum.

\section{BLOod LIPOIDS}

Cholesterol and lecithin have been measured in a few instances in both human and monkey malaria. Kehar (1937) found an increase in cholesterol in human malaria during the rigor stage and a decrease to below normal during the afebrile periods before and after the paroxysm as well as in chronic infections. There was a high correlation between the cholesterol content and the temperature but the fall in cholesterol preceded the fall in temperature. In monkeys infected with $P$. knowlesi he found a slight fall in cholesterol during the primary acute attack. Since this infection is not characterized by paroxysms of fever, as in human malaria, the relation of the cholesterol to such episodes could not be determined. In monkeys dying of overwhelming infection without hemoglobinuria the cholesterol continued to fall until death. In monkeys in which hemoglobinuria occurred as a terminal event there was a sharp terminal rise in cholesterol. Lecithin determinations demonstrated a close correlation between the amount of this substance and the amount of cholesterol in the serum both before and during malaria infections.

Krishnan, Ghosh and Bose (1936) also studied the cholesterol changes in monkeys infected with $\boldsymbol{P}$. knowlesi. They found wide fluctuations during the early period of parasitemia and a distinct tendency toward a decrease when the infection became intense. If the cholesterol remained normal or rose to normal or above after the occurrence of hemoglobinuria the hemoglobinuria invariably disappeared. These authors concluded that cholesterol was capable of exerting an inhibiting influence on hemolysis and hemoglobinuria resulting from intense infection in monkeys. Kehar (1937), however, was unable to protect monkeys from the onset of hemoglobinuria by the administration of cholesterol.

\section{Blood Sugar}

Sinton and Kehar (1931) reviewed the literature on the blood sugar in malaria and made observations on human cases of $P$. vivax and $P$. falciparum infections. There was always a rise during fever, sometimes to a level slightly above normal limits (maximum $149 \mathrm{mg}$ per cent) and a fall after the fever to a level usually slightly higher than before the fever. In three patients followed at short intervals during the febrile period it was found that the peak of the blood sugar level was reached slightly before the peak of temperature, and began to decline slightly before the temperature. In one case of $P$. falciparum similarly studied the rise in blood sugar was much greater than in the $P$. vivax cases, reaching $206 \mathrm{mg}$ per cent. The rise in temperature was not so marked as in the vivax cases but was of longer duration, and the blood sugar began to fall before the maximum rise of temperature was attained. The authors discussed the possible causes of the rise in blood sugar and concluded that it was chiefly due to an increased glycogenolysis following an increased activity of the adrenal glands with an increased secretion of adrenalin. They called attention to the similarity of this mechanism to that occurring in protein shock.

An interference with the storage of glycogen in the liver has been emphasized by a number of workers who studied the functional capacity of the liver in malaria. Sinton and Hughes (1924) found a decrease in levulose tolerance and Ruge $(1935,1939)$ a decrease in galactose tolerance in human subjects, indicating a disturbance of the glycogenetic function of the liver. 


\section{Blood Salts}

Changes in the concentration of various salts in the blood plasma due to malarial infection appear to be limited to the period immediately after the rupture of large numbers of erythrocytes. The only salts which have a higher concentration within the erythrocytes than in the plasma are the potassium salts. The amounts of other salts are so much greater in the serum than in the erythrocytes that the addition of the amounts contained in ruptured erythrocytes does not materially affect their percentages in the plasma.

Zwemer, Sims and Coggeshall (1940) studied the plasma potassium level in human and monkey malaria. In eleven human cases of $P$. vivax infection the plasma potassium was found to rise suddenly from an average of $22 \mathrm{mg}$ per cent to an average of $34 \mathrm{mg}$ per cent at the time of the chill. It fell rapidly before the peak of fever was reached and attained its previous level in an average of 14 hours. This rapid fall was probably due to excretion through the kidneys. In monkeys infected with $P$. knowlesi, which undergoes schizogony every 24 hours, it was more difficult to observe these sudden rises in plasma potassium, but they were observed when specimens were obtained exactly at the time of sporulation. When the infection became intense and the erythrocyte count became low the wholeblood potassium fell off to approximately 33 per cent of the initial values, and the plasma potassium level showed a gradual decrease in the base line between periods of sporulation. The increase in potassium level at the time of sporulation was sometimes too great to be accounted for by the potassium content of parasitized erythrocytes, and the authors presumed that potassium might also have been released from body cells in general, due either to the toxic action of the released potassium or to cellular damage produced by the elevated temperature. These authors called attention to the toxic effect of high plasma potassium on the adrenal cortex.

Junior and Brandão (1937) described a series of cases of malaria which presented a syndrome of adrenal insufficiency, including the picture of Addison's disease. Chessa (1938) studied cases which clinically suggested adrenal hypofunction and showed that 35 per cent of them had a hypersensitivity to insulin. Paisseau and Lemaire (1916) and Natali (1934) described profound changes in the adrenal cortex in autopsies on patients who had died of $P$. falciparum malaria. Natali also found similar changes in monkeys dying of infection with $P$. knowlesi.

\section{Blood Pigments}

Hematin (haemozoin). The composition of the pigment found in malaria parasites was the subject of discussion from the time the parasite was discovered. Its resemblance to melanin and the fact that it did not give the ferrocyanide reaction for iron led many to believe that it was actually melanin. This was the basis of the use of melanin in the Henry reaction for diagnosis. Brown (1911a) was the first to make an extensive chemical examination of malarial pigment and came to the conclusion that it was identical with hematin. Sinton and Ghosh (1934a, 1934b) and Ghosh and Sinton (1934) firmly established the identity of the two substances, showing that haemozoin gave all the chemical and spectroscopic reactions of hematin, that when purified it had the same rate of solution, and that its chloride and iodide salts were identical with similar salts of hematin.

The hematin formed in the malaria parasites during their development in the erythrocytes apparently retains its chemical structure for some time after parasitized erythrocytes which have been phagocytosed by the reticulo-endothelial cells are digested. Brown (1911b) showed that powdered hematin remained for weeks in tissue cells with but slight and slowly progressing alteration. It is also probable that much of the hematin liberated into the blood plasma with the fully developed schizonts remains in particulate form and is phagocytosed by the reticulo-endothelial cells, since these cells contain more hematin than can easily be explained by their phag- 
ocytosis of parasitized erythrocytes. The hematin which is liberated into the blood plasma may be a factor in causing the malarial paroxysm since Brown (1912) showed that alkaline hematin injected intravenously into experimental animals caused a similar paroxysm. He also reported (Brown 1913a, 1913b; Brown and Loevenhart 1913) that hematin produced pathological changes in the blood, circulation and kidneys somewhat comparable to those seen in malignant malaria.

Hematin probably acts as one of the stimulants to the reticulo-endothelial system in malaria. It may also produce a blocking effect on the reticulo-endothelial system, such as is produced by the intravenous injection of inert particulate matter, and may thereby produce a disfunction as well as a hyperfunction of these cells.

Hemoglobin. Probably not all of the hemoglobin in parasitized erythrocytes is changed into hematin by the time the mature schizonts rupture the erythrocytes, and consequently some of it is probably liberated into the blood plasma. It is not ordinarily detectable in the blood plasma in malaria because of its rapid transformation by the reticulo-endothelial cells into bilirubin with the liberation of iron. The bilirubin is quickly discharged into the blood plasma and in heavy malarial infections is detectable there in considerable quantities, producing an indirect Van den Bergh reaction. It is absorbed by the parenchymatous cells of the liver and secreted in the bile. In excessive amounts it probably contributes to disfunction of the liver and constitutes an important part of the thickened bile which may be responsible for the so-called bilious symptoms of some severe cases of malaria.

If the iron from the hemoglobin is not immediately utilized in the reformation of new hemoglobin, it may be found in the form of hemosiderin in the parenchymatous cells of the liver, kidneys and other organs.

In blackwater fever, where a sudden massive hemolysis of the erythrocytes occurs, hemoglobin is found in the blood plasma in large quantities. Since it has a fairly low threshold of excretion in the kidneys some of it escapes by this route. Most of it, however, is quickly changed to bilirubin by the reticulo-endothelial cells. It is not the province of this paper to deal with blackwater fever, but since there are many cases of malaria in which a small degree of hemolysis occurs without the clinical picture of blackwater fever it seems desirable to call attention to another change which may take place in the hemoglobin liberated into the blood plasma. Fairley and Bromfield (1934) discovered a pigment very similar to methemoglobin in the blood plasma of a case of blackwater fever. They first called this pigment pseudomethemoglobin; later they found that it did not contain globin but was a combination of hematin with crystalbumin, an albumin found in the sera of only man and monkeys, and renamed it methemalbumin (Fairley 1938). Further investigations by these authors (Fairley and Bromfield 1937) and by Foy and Kondi (1938) showed that this pigment occurred in the plasma of most of the cases of blackwater fever which they studied and that methemoglobin, which was previously supposed to be the brown pigment present in the serum of these cases, did not occur in the plasma but was formed in the urine as a product of hemoglobin. Methemalbumin is never excreted in the urine, apparently because of the large size of its molecule.

\section{Red Blood Cells}

The progressive anemia produced by malarial infection is usually of the secondary type with evidence of normal or stimulated functioning of the bone marrow, although in some cases of chronic infection both with $P$. falciparum and $P$. vivax a primary type of anemia has been described. The two conditions usually can be differentiated in the blood itself by the Price Jones curve for measuring the diameters of the cells (Fairley and Bromfield 1933). With the progressive reduction in erythrocytes, the oxygen carrying capacity of the blood is reduced and this, of course, interferes with 
tissue respiration and stimulates compensating mechanisms such as respiration and heart rate. The dilution of the blood plasma may also affect tissue metabolism and encourage edema.

Reticulocytes are usually found in the blood in proportions slightly higher than normal during the course of malarial infection and usually are increased greatly after the acute stage of the disease has passed. The maturation of reticulocytes after discharge from the bone marrow into the circulation has been questioned by Mermod and Dock (1935). These authors found that reticulocytes were less resistant than mature erythrocytes to certain hemolysins such as saponin, and stated that reticulocytes did not participate in carrying oxygen to the tissues because they used oxygen themselves. The question as to whether, as they state, reticulocytosis is a necessary evil preceding erythrocyte regeneration or is a direct evidence of regeneration must await further investigation. The attention which reticulocytes have received recently in relation to their infection by malaria parasites deserves special comment. It has been definite established that $P$. vivax has greater affinity for reticulocytes than for mature erythrocytes. A number of workers have also shown that $P$. falciparum is apparently indifferent to the stage of erythrocyte which it invades, and Kitchen (1939b) has produced evidence that $P$. malariae prefers mature erythrocytes to reticulocytes.

\section{White Brood Cetls}

Attention has already been called to the stimulation of the monocytes of the blood as part of the reticulo-endothelial system. Mulligan (1929), using supravital stains demonstrated that the reticulo-endothelial cells in the peripheral blood were increased in both acute and chronic malaria. In acute malaria both the younger monocytes and the older clasmatocytes were markedly increased. In chronic malaria the increase in monocytes was similar to that in acute cases but the increase in clasmatocytes was less. It is probable that these increases in circulating mononuclear cells reflect the increased activity of the reticulo-endothelial system rather than increased leucopoietic activity of the bone marrow.

\section{Reticulo-Endothelial System}

The stimulation of the reticulo-endothelial system throughout the body in malaria has received great attention and is manifested histologically by both a hyperplasia of the system, chiefly of the spleen, bone marrow and liver, and an increased phagocytic activity of the individual macrophages. Increase in phagocytic activity of the individual macrophages is at first gradual, but Cannon and Taliaferro (1931), Taliaferro and Cannon (1936) and Taliaferro and Mulligan (1937) have demonstrated that in bird and monkey malaria there is a marked increase in phagocytic activity at the time of the sudden decrease of the parasites. They interpreted this as an immune reaction of the opsonic type and regarded it as the principal immune phenomenon in malaria (Taliaferro, this volume, p. 239). The recent demonstration of positive complement fixation reactions (Coggeshall and Eaton 1938a), agglutination reactions (Eaton 1938), and protective substances (Coggeshall and Kumm 1937) indicates the presence of humoral antibodies which may also have their source in the reticuloendothelial cells (Taliaferro, this volume, p. 239).

It is apparent that in malaria all of the functions of the reticulo-endothelial system are probably brought into play. These functions include phagocytosis, the formation of bilirubin from hemoglobin, and the formation of various immune substances. In addition, the apparent absence of parasites from the blood for the first few days following infection with sporozoites (Boyd and Stratman-Thomas 1934c) indicates that the reticuloendothelial system may serve as an incubator for the development of merozoites from sporozoites and may hold the secret to true chemoprophylaxis in malaria.

\section{Circulation}

The effect of malaria on the circulation 
is often profound, but most of the observations on man have been purely clinical. Brown and Loevenhart (1913) found that the injection of alkaline hematin into dogs and cats caused dilatation of the splanchnic vessels, constriction of the cutaneous vessels and a marked fall in blood pressure. The heart rate was slowed and the cardiac output diminished. These effects were apparently due mainly to toxic action on the vasomotor and cardio-inhibitory centers. Dudgeon and Clarke (1917) reported fatty degeneration of the heart muscle in fatal cases of malaria, similar to that seen in diphtheria. They also reported thrombosis of vessels in the brain, spleen, kidneys and adrenal glands.

Serious circulatory disturbances in human malaria occur almost exclusively in $P$. falciparum infections. The two factors primarily responsible for these disturbances seem to be the plugging of capillaries by parasitized erythrocytes and the liberation of toxic substances from these erythrocytes on the completion of schizogony. The massive infections often seen in $P$. falciparum infections would appear to be sufficient to produce severe toxic symptoms, particularly since $P$. knowlesi infections in monkeys produce fatal toxic symptoms when the infection becomes massive, even though there is no plugging of capillaries. These symptoms are partly circulatory and are not unlike the toxic effects of hematin described above.

The plugging of capillaries in $P$. falciparum infections is an additional, and probably often the principal, cause of the alarming symptoms which develop. The severe headaches, drowsiness and coma, often periodic in occurrence, can be explained on the basis of obstruction to cerebral capillaries. The choleraic and dysenteric types of so-called "algid" malaria may also be due to obstruction to eapillaries in the intestinal mucosa. Furthermore, it is probable that the mechanical embarrassment of the circulation tends to exaggerate the symptoms produced by the toxic substances liberated from the parasitized erythrocytes.

\section{LIVER}

What has been said above concerning blood destruction and pigment metabolism in malaria indicates the load which is placed upon the liver in this disease. It is called upon to dispose of bilirubin, hemosiderin and possibly hematin. Its storage of glycogen is called upon and its relation to lipoid metabolism may be affected. The secretion of large amounts of thick bile rich in bile pigments and the possible effect of this secretion on the gastro-intestinal tract have given the name of "bilious remittent fever" to one clinical form of $P$. falciparum malaria. The lowering of levulose and galactose tolerance gives evidence of injury to its glycogenetic function, and the increase of cholestrin and lecithin in the blood at the time of the malarial paroxysm suggests its increased activity in connection with these lipoids. Histological evidence of damage to the parenchymatous cells of the liver in fatal cases of malaria is not uniform, but both degenerative changes and fat infiltration are sometimes found.

\section{KIDNEYS}

Kidney function is not usually disturbed in clinical cases of malaria. Small amounts of albumin may be found in the urine, usually associated with febrile periods, but damage to the water, salt or nitrogen secretory functions of the kidneys are not usually demonstrable in acute malaria. There is, however, an interesting picture which is not infrequently seen in chronic malaria, least often in $P$. vivax, more often in $P$. falciparum and most often in $P$. malariae infections. This is a picture of water retention with edema, and with albumin in the urine, often in large amounts. Hyaline and granular casts are usually present. There is usually little or no retention of nitrogen. This is the picture which is usually given the name lipoid nephrosis. Its occurrence in chronic quartan malaria has been studied particularly by Goldie (1930) in Palestine, and it has also received attention in many other parts of the world. Usually in $P$. vivax and $P$. falciparum in- 
fections the condition rapidly disappears on treatment with antimalarial drugs. This often occurs also in chronic quartan infections, but some cases have gone on to fatal terminations despite antimalarial treatment (Fabry, Guillerm and Ragiot 1937). In some of the quartan cases, particularly in older adults but also in some children, a chronic glomerular nephritis manifested by nitrogen retention has been seen, and in the young cases at least this condition seems to be a late result of the malarial infection (Giglioli 1932). In some overwhelming cases of $P$. falciparum malaria the general kidney function is profoundly affected and the patient may die with a picture of uremia. Acidosis is not a prominent symptom in malaria. The carbon dioxide combining power of the blood may be temporarily lowered during the febrile stage but is usually normal at other times.

In blackwater fever, kidney function is, of course, profoundly disturbed by the products of hemolysis which the kidneys are called upon to excrete. The formation of methemoglobin and its precipitation in the kidney tubules is apparently the most important element in the development of anuria and uremia which characterizes the clinical picture.

\section{SUMMARY}

The physiological pathology of malaria involves primarily the blood and circulation, with secondary effects on many organs of the body. The blood euglobulin is increased, cholesterol, lecithin and glucose rise during the chill but are usually slightly decreased at other times, potassium is greatly increased by the rupture of erythrocytes, erythropoiesis is stimulated and the reticuloendothelial system is activated and possibly partly blocked. A non-specific positive serological test for syphilis is obtained. The circulation is disturbed by the blockage of capillaries and probably by toxic products of the infection. Tissue respiration and nutrition are affected. The liver and kidneys suffer from the heavy demand upon their excretory functions. The adrenal glands show cortical depletion possibly due to postassium poisoning. The heart shows toxic changes and is further embarrassed by the blood changes and by the effects on other organs. The brain is also involved in toxic and circulatory changes. In short, there are few infectious diseases of man which, in their severe manifestations, produce functional changes in more organs and systems of the body than malaria. 


\title{
IMMUNITY TO HUMAN MALARIA: CHAR- ACTERISTICS OF IMMUNITY
}

\author{
By W. B. REDMOND \\ DEPARTMENT OF BIOLOGY, EMORY UNIVERSITY, GA.
}

IMMUNITY to human malaria should be considered from two standpoints. (1) A study of the literature reveals the importance of the protection resulting from years of infection in areas of hyperendemic malaria. This protection is apparent especially in the adult population, in contrast to the high infection in all below the age of 15 years. A single infection under these conditions affords no protection probably because of the numerous heterologous strains present and the constant rate of reinfection by mosquitoes. (2) A different picture is presented in experimental malarial infections. Complete protection against reinfection with the same strain is afforded in about one case out of every three, but when the reinoculated parasites are of a different strain the protection is not so marked. The resulting infection may be lower in number of parasites, and the febrile attacks are generally fewer and less severe. There seems to be no protection, however, to heterologous strains of $P$. falciparum.

The use of malarial therapy in the treatment of paresis first employed by WagnerJauregg initiated the laboratory study of human malaria. Previous to any experimental work on human malaria it was realized by some malarialogists and physicians in highly malarious districts that some persons could live among infected peoples in conditions optimum for the transfer of malaria and still not be infected. Laveran (1908), however, stated that a first attack of malaria did not produce immunity, and that some times following the primary attack an individual was more susceptible than previously. Bass (1919) came to the conclusion that no effective and lasting immunity to malaria was developed. He found that 50 per cent of the population in certain parts of Mississippi had malaria each year; about half of these were relapses and half were reinfections. The adult population had a very high rate of infection, but not as high as the children. His criterion of immunity was based on a failure to find parasites in the blood.

Persons who appeared to be resistant to attacks may or may not have had histories of previous infection. Bass reported that blood examinations of individuals without any previous history of malaria showed 16 per cent to be harboring parasites in the blood. They were without symptoms and were unaware of the fact that they were infected. Daniels (1901) and Gill (1914) noted the enlargement of the spleen and were able to correlate the spleen rate with the immunity. Children under two or three years of age had the highest infections and most severe attacks. During this period the spleen increased in size, but with the decrease in malarial attacks after 5 years of infection the spleen rate began to diminish. In adults attacks were infrequent and mild, but the high spleen rate was indicative of continued infection. According to Daniels this freedom from febrile attacks is associated with continued inoculation of sporozoites without interruption over a period of years. Gill concluded that a constant spleen rate over an extended period was significant of a definite immunity. Where the inoculation of parasites is interrupted by conditions not suitable for the natural transfer by mosquitoes, such as seasonal changes, the fluctuating infections do not result in a very effective immunity. A low spleen rate is considered to be indicative of a high susceptibility, and an infection rate of 5 per cent in such an area could result in an epidemic should 
the conditions for transfer become optimum. By correlating these facts it has been possible to predict fairly accurately when and where an epidemic of malaria may be expected. Following an epidemic Christophers (1924) found a high spleen rate which began to fall off rapidly after about 2 years, if the infection rate was low. The average length of time before another epidemic may occur in the area is 6 years. Generally at the end of that time the spleen rate has fallen to about 10 per cent. In hyperendemic centers where a constant infection rate is maintained the resistance appears to be kept at a high degree and no epidemics occur.

Not until Wagner-Jauregg introduced the malaria therapy of paresis were there any controlled experiments in immunity to human malaria. This technique has been used in various countries and has added much to our knowledge of malaria.

Yorke and Macfie (1924a) first pointed out the fact that on recovery from an infection of $P$. vivax the patient showed a considerable degree of resistance to reinoculation with the same strain of parasites. That recovery from normal attacks of induced malaria produces in the patient a resistance to further attacks of the same strain of parasite has been amply demonstrated by Antic (1925), James (1926), Plehn (1926), Ciuca, Ballif, and Chelarescu-Vieru (1928), Boyd and StratmanThomas (1933c) and others. Most of these workers also observed that there was some resistance to infections with other strains of the same species of parasite, but none to other species. This fact is made use of in the malarial therapy of paretics where it is necessary to treat a patient more than once.

Until mosquitoes were available for controlled transfers the inoculations were made by injecting a small amount of blood from an infected individual into a susceptible person. Following recovery from the subsequent attacks, provided the attacks were allowed to run their normal course, it was impossible in most cases to produce further attacks by inoculations with the same strain of parasite. In chronic cases showing a few parasites in the blood following recovery from the primary attack a second inoculation generally produced an increase in the number of parasites in the peripheral blood, but no further febrile attacks in the majority of cases. This led Sinton, Harbhagwan, and Singh (1931) to suggest that the resistance was manifested in two ways, an effect on the toxic products producing the symptoms, and a reduction in the number of parasites in the blood. The primary attack of $P$. vivax malaria is followed by one or more recrudescences. These attacks are light and generally end in spontaneous recovery. Then follows a period in which no parasites can be found. This period, however, lasts for only about 8 to 10 months and a recurrence of fever and appearance of parasites follows. The recurrence generally ends spontaneously after one or more recrudescences within a period of 2 or 3 weeks. Rarely is the recurrence as severe as the primary attack even though the parasite count may be almost as high. Febrile attacks are fewer in number and occur for a period of only a few days. Previous to the recurrence a reinoculation may result in a slight attack, but afterwards it is impossible in most cases to get any response from reinoculations with the same strain of parasites.

Gill (1933) thinks that the immunity following induced malaria may not be analogous to the resistance produced in normal individuals since the subjects used in such experiments are syphilitic, and the treatment with arsenical drugs, and reactions produced both by these drugs and the disease may materially alter the reaction of the body to the malarial parasites.

\section{DeVelopment of ImMunity}

As indicated above very little immunity follows a single infection unless that infection is continued over a long period of time by relapse and recrudescences. Termination of the infection at an early stage by drug treatment has been found to interfere with the normal development of the immunity. In controlled cases of therapeutic 
malaria in the hospitals it is possible to obtain some indication of the rate of development of the immunity following various types of infections.

It has been indicated in the previous section that an immunity can be established to a certain strain of malarial parasite in a period of approximately one year by allowing the infection to relapse and recrudesce with no treatment except when the febrile attacks reach a condition endangering the life of the individuals.

Ciuca, Ballif, and Chelarescu-Vieru (1934) found that only 34 per cent of their patients were resistent to $P$. vivax after the first infection. Following the second infection the number was increased to 72 per cent, and to 87 per cent after the third. The remaining 13 per cent of susceptibles developed a solid immunity only after the fourth infection. All were resistant to the fifth inoculation. Using $P$. malariae on 322 patients they found that 35 per cent resisted a second inoculation, but this had increased to only 81 per cent following the fifth inoculation. In order to produce immunity in all cases it was necessary to inoculate some for the sixth time. After the tenth inoculation with $P$. falciparum 3 per cent of 544 persons still were susceptible.

Sinton (1940a) found that 48 per cent of $P$. ovale infections produced immunity to a second inoculation. A third inoculation showed that 41 per cent were still susceptible, but the intensity and the duration of the attacks had considerably decreased. During the third infection only 40 per cent of those susceptible developed fever and the attacks lasted on an average less than one day.

An apparent contradiction to these experimental findings occurs in hyperendemic areas where a constant high infection is found up to the tenth year. The febrile attacks fall off but may continue intermittently for about 5 years more after which the individual usually remains free from attacks though parasites may be found in the blood. The two different pictures presented can now be readily explained on the basis of heterologous strains of each species of plasmodium. Even during the period following the cessation of febrile attacks while there are still numerous parasites to be found in the peripheral blood a heterologous strain will provoke a new attack. The implication here is that one is at least partially susceptible until he has experienced attacks and recovered from all the strains within the area of infection. Garnham (1935) found the population of certain endemic centers immune to subtertian and quartan malaria, but very susceptible to $P$. vivax which was not endemic to the area. In areas where little protection against infection can be provided children under two years of age have the highest parasite count. Christophers reports that in some areas in India the average is 10,000 parasites per cubic millimeter of blood. The spleen is palpable in 88 per cent and attacks are almost continuous. Garnham (1935) finds that the children of Kenya are highly infected, but suffer little from febrile attacks. There are very few deaths among the natives, but cutside children are much more severely affected and deaths occur frequently. Gill (1914) has found that a constant spleen rate over a period of years is correlated with the immunity, but likewise is dependent on almost unbroken infections of parasites. Even a seasonal fluctuation of transmission interferes with the development of the immunity.

\section{SPECIFICITY}

As shown by Boyd and Stratman-Thomas (1933e) and Ciuca, Ballif and ChelareseuVieru (1933) the immunity produced to one strain of parasites is much less effective against all other strains of the same species. Some resistance is shown to a heterologous strain, the resulting infection producing fewer febrile attacks and ending spontaneously. There seems to be no limit to the number of strains, however, as individual infections found within a radius of a few miles differ enough to provide only partial protection to each other. This fact may explain the length of time 
necessary for protection in hyperendemic areas. Each strain must run its course of infection and result in a homologous resistance, thus multiplying the interval by the number of strains. It has been estimated that a period of 15 years of almost constant malarial attacks is necessary before an individual can be considered immune.

The only indication of a resistance to one species being effective against another species in man is that reported by James and Ciuca (1938). They found that patients who showed considerable immunity to $P$. vivax were likewise resistant to infections with $P$. knowlesi, a species of monkey malaria. When this species is inoculated into persons immune to $P$. vivax a resistance is noted similar to that found when a heterologous strain of this parasite is inoculated. Primary attacks of $P$. knowlesi are similar to the primary attacks of $P$. vivax, but are less severe in white people.

In all four species of human malaria with the exception of $P$. falciparum there has been demonstrated a considerable degree of resistance to heterologous strains. Following the inoculation of a heterologous strain the parasites increase in number and may reach the normal level for the strain, but in most cases are far below this level. Even when the parasite count is at the peak the febrile attacks are less intense and of short duration. The infection generally terminates spontaneously with very few or no recurrences.

\section{IMmUNITy IN THE Different SPECIES}

Only in $P$. ovale has any critical study of the variations in infections with different strains been made. Although Sinton, Hutton and Shute (1939b) were able to reinoculate some of their patients with strains originally obtained from areas separated by great distances, some from other continents, immunity to the heterologous strains was high. Only 18 febrile cases followed reinoculation, the average duration in these being only 4.5 days. The average length of the period of the primary attacks was 18 days. Only one strain (strain D) showed any marked degree of difference from the other three. Strain D appeared to be more virulent than other strains, the primary attack being considerably prolonged in a number of cases. When a person who had recovered from an infection with another strain was reinoculated with this strain of parasite, a febrile attack with numerous parasites followed. Reciprocal crosses were not made as it was not possible to maintain all the strains at any one time in the hospital. Other strains showed very little heterologous properties. A parallel case is found in avian malarial strains that produce heterologous immune reactions. A virulent strain of $P$. relictum produces a low degree of infection in a bird that is carrying a latent infection of a very avirulent strain of the same species. The reciprocal cross, however, differs in that the more virulent strain confers a complete immunity to inoculation with the avirulent strain. Redmond (1939) has indicated that the difference in the degree of immunity may be largely dependent on the relative number of parasites occurring during the primary infections of these two strains.

Although $P$. ovale was at one time considered to be a strain of $P$. vivax, the immune reactions indicate for it a greater degree of individuality. Fewer febrile relapses are found following the primary attack of $P$. ovale, but a chronic infection may persist for as long as 3 months. In both malarious and non-malarious countries of Europe very little resistance to $P$. ovale has been found by Sinton.

It has been shown by Ciuca that more reinoculations are necessary to produce homologous immunity with $P$. falciparum and $P$. malariae than with $P$. vivax. In subtertian malaria 22 per cent were resistant to reinoculations following the first infection but 10 inoculations were required to produce immunity in 35 persons of 544 used in the experiment. Missiroli (1932) considers that the immunity developed against repeated inoculations of $P$. vivax is more solid than that developed against P. falciparum. Boyd, Stratman-Thomas and Kitchen (1936a) found good homolo- 
gous but little heterologous immunity to the latter species.

Thomson (1934) reports that in the district around Kisumu "Immunity to quartan malaria develops early and rapidly becomes well-nigh perfect." Ciuca states that quartan malaria is less likely to recover spontaneously than benign tertian and although 36 per cent of cases become immune after the first infection, seven reinoculations are necessary to increase the number to 100 per cent. Young and Coatney (1940) report almost complete protection against a second inoculation of quartan malaria. In one or two cases a few parasites were observed, but no clinical attack appeared.

Reinoculations of $P$. knowlesi following recovery from primary attacks in man indicate that a good homologous immunity is developed. Ciuca, Tomescu, and Badenski (1937) had only one febrile attack in 20 reinoculations; Milam and Coggeshall (1938) reported no febrile attack following a second inoculation. In five of Ciuca's patients a slight rise in the parasite count was noted. Although James and Ciuca reported that infections of $P$. knowlesi produced a heterologous immunity to $P$. vivax, the reciprocal cross is evidently not protective. Milam and Kusch (1938) inoculated 7 paretic patients with $P$. vivax that had been previously treated with infections of $P$. knowlesi. They did not state the nature of the resulting infections but indicated that they were normal.

COMPARISON OF IMMUNITY IN INFECTIONS INITIATED WITH SPOROZOITES AND WITH TROPHOZOITES

Sinton (1939b) inoculated 22 patients with sporozoites and compared the primary attacks with those following blood inoculations. There appeared to be very little natural resistance to the sporozoite infections. On the other hand the primary attacks of the patients inoculated with infected blood were much shorter and less intense. Later he (1940b) compared the immunity resulting from the two methods of infection and found that the patients with sporozoite inoculations acquired a greater degree of resistance than those with blood inoculations. Likewise, when the reinoculating dose was sporozoites the resulting infection was more pronounced than when infected blood was used. James, Nicol and Shute (1932) could not differentiate between infections of $P$. falciparum one group of which was inoculated with sporozoites and the other with trophozoites. Resistance to reinfection appears to be against the trophozoite stage of the parasitic cycle of $P$. vivax and has very little effect on the sporozoites. According to Boyd and Kitchen (1936b) a reinoculation with sporozoites results in the usual incubation period before the resistance comes into play, but the resulting trophozoites in the erythrocytes are as susceptible as those following blood inoculations. Jerace(1934) says that $P$. vivax infections induced by sporozoites are more likely to produce quotidian febrile attacks than when induced by trophozoite inoculations. These observations have led some workers to adopt the sporozoite inoculation method of giving infections to their patients, either by allowing infected mosquitoes to transfer the sporozoites by feeding on the patients to be infected or by injecting the sporozoites obtained from the mosquitoes.

\section{DURATION OF IMMUNITY}

A very important question regarding malarial immunity is its duration. The immunity to avian malaria has been established as lasting while the infection remains latent. If the bird loses the infection the immunity very soon disappears. Thomson (1933) states that immunity to human malaria, likewise, is associated with a latent infection. Others have failed to find a latent infection in cases that proved to be immune. In numerous cases subinoculations have been made with the idea of determining the presence or absence of a latent infection. If the subinoculated individual becomes infected this establishes the infection in the case, but on the other hand no infection does not prove the absence of a latent infection. Splenectomy 
will often cause a latent infection to become active in monkeys after all other attempts have failed.

If a latent infection is necessary for a lasting immunity, the question arises as to which strain or species is latent, or whether there is a latent infection of each strain to which the individual is immune. Since one strain produces only a partial immunity to other strains and none to other species the latter alternative must be considered further. In highly endemic areas where the infection rate in the adult population is high, but the febrile attacks mild and few, the individual is constantly exposed to reinoculation. This cannot be considered to be a condition of latency, but one of subnormal infection without clinical manifestation. In areas where the transmission of malaria is seasonal the immunity is much less effective in preventing infection. These latter conditions would be optimum for the establishment of a latent infection, but the immunity is found to fluctuate, the spleen rate to decrease and the degree of susceptibility to increase. Following epidemics of malaria the spleen rate remains high for two or three years, then gradually, then more rapidly begins to decrease. During this time individuals are more or less susceptible.

If the immunity is dependent on a latent infection it would appear that a single infection would be sufficient to establish the latent condition and the subsequent immunity. In only about 35 per cent of cases is there a resistance to reinfection following a single infection. This could be explained by the supposition that more than one infection is required to establish the latent infection in man, or that only 35 per cent carried a latent infection following the first infection. Although a few isolated cases of long standing immunity have been reported in the apparent absence of infection the indication is that the immunity gradually loses its power of preventing reinfection when the tissues are free of all parasites. Boyd, StratmanThomas and Kitchen (1936c) were able to demonstrate an effective immunity in one case three and one half years after recovery from a previous infection. Subinoculations of massive doses of blood failed to show the presence of a latent infection prior to the reinoculations. James and Ciuca (1938) found that one of their patients had remained immune apparently for 5 years.

The effectiveness of the immunity in preventing further attacks, however, does not depend on a complete sterilization of the blood. In cases of constant reinoculation where the individual has a low grade infection the immunity is maintained at a high level by the parasites present in the tissues. Under hyperendemic conditions in India Christophers found that the rate of infection in adults was about 50 per cent, but the parasite count was low and attacks occurred on an average of once in six months. These attacks were relatively mild and of short duration.

\section{Factors Influencing Development OF IMMUNITY}

Children are generally thought to be more susceptible to most diseases than are adults. However, where there have been specific studies made on this point the adult resistance appears to have been acquired by either an acute or latent infection. Kligler (1930) and Kligler and Mer (1933) measured the difference between children and adults in their powers to resist malarial infection following treatment. Up to 4 years of age they found the reduction of the infection to be less than 4 per cent, while for a group between the ages of 4 and 9 years the reduction in infections was 23 per cent after two weeks of treatment. Nothing is said, however, of the great degree of immunity developed by the older group in comparison with that of the younger group as a result of twice as many years of malaria infection. The hyperendemic conditions make it practically impossible for the older group to have escaped infection. Since children of this age are seldom found in the hospitals for the insane, little controlled experimentation has been possible. It is apparent that further facts are necessary before a 
decision can be reached. Christophers (1924) was able to compare the susceptibility to infection in the same age groups but of different origin and of children who presumably had not been exposed to malaria. He found that in groups up to 6 years of age the first 2 years of residence in malarial districts showed the highest rate of infection and the highest parasite count. There was only a slight difference in this age group in children born in the hyperendemic community and those born elsewhere and moving into the area. However, in age groups over 6 years of age there appeared to be a marked resistance in children moving into the area. $\mathrm{He}$ was not able to preclude the possibility that the resistance shown by these children had not been acquired by exposure to malarial infections previous to entering the hyperendemic center.

Boyd and Stratman-Thomas (1933d) have very good evidence that the resistance to $P$. vivax found in negroes is a natural racial condition and not influenced by any previous exposure to this species of malaria. They have found it necessary to use $P$. falciparum in treating negro paretics. The few cases that show parasites have too few febrile attacks to be of therapeutic value. Two test cases each bitten by 16 mosquitoes infected with $P$. vivax failed to become infected. Twelve white patients each bitten by from 1 to 3 of the same mosquitoes all became infected. In another experiment a negro child 5 years of age was inoculated with $P$. vivax by allowing infected mosquitoes to feed at two different times. No infection developed. Examinations over a long period of time previous to the test did not show any indication of an infection. Proof that the refractoriness of negroes to $P$. vivax is a natural resistance and not acquired as the result of infection is lacking, but this one case studied by Boyd is indicative that there is a natural racial tolerance in the negro to $P$. vivax infections.

The aboriginal natives of one of the hyperendemic areas in India were no less susceptible than other tribes who were more recent immigrants to the commun- ity, according to observations made by Christophers (1924).

In the absence of any marked resistance among the negroes to other species of malaria it appears that a racial tolerance must have been developed somewhere in the past and has become inherent in the race. It is possible that $P$. vivax has been endemic in the negro race for centuries and through long association the natural resistance shown by man to plasmodial infections has become enhanced to the degree of almost complete resistance. The fact that there is a preponderance of $P$. falciparum in Africa at the present time lends credence to the hypothesis of the close association of $P$. vivax and the negro race.

Malarial infections have also been observed to reappear following accidents, operations and child-birth. Garnham (1938) believes the loss of large numbers of reticulo-endothelial cells with the placenta is responsible for the reappearance of malaria infections following child-birth. Likewise an accident or a major operation that requires the full component of the body's resistance may leave the body without sufficient protection from the malaria, whereupon latent infections may become active. It is thought that diseases and similar factors may act in the same way as blockade of the reticulo-endothelial tissues.

\section{Effect of Drug Treatment on Immunity}

There is considerable disagreement on the question of the effect of drugs either directly or indirectly on the immunity to malaria: The most important factors appear to be the period during the infection at which the drug is administered and the extent of the treatment. When the treatment is begun early in the development of the infection before the parasites have multiplied to any considerable extent and is continued so long that the infection does not show up after the treatment is stopped, it has been found that no immunity develops. On the other hand, if the infection is allowed to run its course the resultant immunity is highly effective in preventing another infection. Yorke and Macfie (1924a) 
put forth the theory that quinine destroys numerous parasites if given during a period of heavy infection thus liberating large quantities of antigen that in turn stimulates the immune mechanism to greater activity resulting in a greater degree of immunity. Sinton (1938) has admirably reviewed the question and both his experimental work and references to observations and experiments of others bear out the results obtained in other types of immune response, namely, that within certain limits the degree of the immune response is proportional to the amount of antigen absorbed. A large quantity of antigen made available in a short period of time will be less effective than the same amount over a longer period, however. On this basis a high infection of malaria terminated by drugs after a few paroxysms will produce only a slight degree of immunity, while if allowed to continue to spontaneous recovery followed by relapses and recrudescences the immunity will be of such a degree that no new infection with the homologous strain will be possible. Drug treatment interferes with the immunity only to the extent that it interferes with the normal development of the parasitic infection. Since the clinical symptoms are affected only indirectly, that is, by the effect of the drug on the parasite, any control of febrile attacks will in a corresponding measure limit the number of parasites available for stimulation of the immune mechanism.

From these facts it would appear that in isolated cases of malaria where the possibility of reinfection following cure was very slight interference with the development of immunity would be of little importance and intensive drug treatment could be used effectively. However, in hyperendemic areas where reinfection is certain the development of immunity is important. A cure under these conditions would be followed by reinfection and would probably result in the lowering of the resistance of the individual. Kligler (1930) found that two weeks after treatment and cure of malaria in children under 10 years of age the infections were more severe than before. Such individuals cannot be protected from reinfection under these conditions and it is doubtful that alternate eure and reinfection would be of material benefit in the development of immunity.

The results obtained by Sinton and others with monkey malaria coincide with the observations on most human experiments. When infections of $P$. knowlesi in $S$. rhesus are held to a low parasite count the infection will relapse as soon as the treatment is withheld and the monkey will die unless the same treatment is again used. If the infection is allowed to develop, with treatment sufficient to save the life of the animal, the resultant immunity will prevent further infection with the same strain. In view of this fact therapeutic measures employed to reduce the incidence of malaria will greatly reduce the effectiveness of the immunity resulting from natural infections. 


\title{
THE CELLULAR BASIS FOR IMMUNITY IN MALARIA
}

\author{
By WILLIAM H. TALIAFERRO
}

DEPARTMENT OF BACTERIOLOGY AND PARASITOLOGY, THE UNIVERSITY OF CHICAGO, CHICAGO, ILL.

THERE are four main aspects of the functional role of cells in malarial immunity: (1) the phagocytosis by various leucocytes and, in particular, by macrophages of parasites and other materials incident upon the infection; (2) the production of new phagocytes; (3) various reparative proliferations other than the production of phagocytes; and (4) the elaboration of specific antiparasitic antibodies. Of these the first three are of importance in both natural and acquired immunity and can be studied directly in histological preparations. The fourth is the specific characteristic of acquired immunity and has not been studied in malaria. In fact, it has only been investigated under the most favorable conditions by indirect methods which have given plausible but not conclusive results. The literature on these and related subjects is so voluminous that it can be only treated summarily with comparatively few references to specific investigations. It has been critically reviewed in detail by Taliaferro and Mulligan (1937).

In considering the physical basis for malarial immunity, it should be kept in mind that the defense reactions against any infectious agent are fundamentally the same. The distinguishing characteristics of malarial immunity arise largely from the localization of the parasite in the blood stream, since such a localization determines where the parasites will come in contact with phagocytic cells (see Taliaferro 1934).

Detailed studies on the cellular basis for immunity in the malarias of man are handicapped in the following ways (Cannon, this volume p. 214). Most human necropsy material represents terminal stages of the infection in which the defense mechanisms of the host have been broken down. Most of the work has been done on infections of
$P$. falciparum in which various degenerative manifestations have overshadowed the defense aspects. Studies on monkeys indicate that certain cardinal aspects of the immune process last for only a short time and are generally missed unless the experimental hosts are sacrificed at closely spaced intervals. Finally, much of the human material has not been fixed immediately after death and handled with the specialized techniques necessary for the finer cytological details which are important in studying the histogenesis of macrophages. Much of the underlying theoretical part of the following account is, therefore, based on simian malaria with as many facts as possible fitted in from the literature on human infections.

\section{Cellus Involved in Immunity}

Ordinarily, malarial parasites are introduced parenterally through the epidermis so that there is no opportunity for the manifold nonspecific factors, such as epithelial barriers, the outward flow of mucous or urine, or the action of digestive fluids, to come into play as they do in natural immunity to some potential invaders.

The cells chiefly involved in inflammation and the so-called defense reactions against parenterally introduced materials belong to the connective tissue which is derived embryologically from the mesenchyme. The connective tissue includes the blood and lymph, the reticular or bloodforming tissues, loose connective and adipose tissues, cartilage and bone and performs a wide variety of physiological functions including respiration, intermediate metabolism, storage and various types of mechanical support. Of these various components, the cells most active in immunity belong to the blood and lymph and the 
reticular and loose connective tissues and owe their functional importance to their wide distribution over the body, their capacity for phagocytosis, their ability to secrete enzymes and antibodies, and their retention of various mesenchymal potencies to develop into other blood and connective tissue cells. Their functions in immunity are, to a large extent, and may be entirely an accentuation of their activities in normal metabolism.

Many factors have contributed to the present complex and, in part, almost chaotic classification of the cells of the blood and connective tissue. Chief among these are: (1) cells now believed to have identical functions have long-standing different names which date from early histological descriptions ; (2) different groups of workers, such as anatomists, pathologists and hematologists, often use different terminologies; and (3) most important of all, there is no consensus of opinion as to the identification, classification, developmental potencies or even existence of some of the important cells.

The following simplified classification is taken largely from the author (1941) with special reference to the cells functional in malarial immunity. The views regarding the developmental potencies and relationships of the cells follow Maximow (1927a, 1927b) and Bloom (1938a). A more detailed consideration of the cells in relation to malaria is given in Taliaferro and Mulligan (1937).

\section{Predominantly Fixed Connective Tissue Cerus}

Of the many connective tissue cells which are predominantly fixed, the most important in defense reactions are macrophages, as defined in this paper, fibroblasts and endothelial cells.

(1) Macrophages. The term macrophage is used in this chapter in much the original sense intended by Metchnikoff to denote any large mononuclear cell that is phagocytic or can immediately become phagocytic without any pronounced change in morphology. It includes the following four categories of cells : the reticular cells, littoral cells and pericytes, which retain the embryonic or mesenchymal potency to develop into all other types of cells of the blood and connective tissue, and the macrophages of the loose and dense connective tissue, which are believed to have fewer potencies for heteroplastic development. In the older pathological literature, all these cells were frequently termed endothelial cells (see below). The concept that cells in the connective tissue of the adult body retain mesenchymal potencies is due largely to the work of Marchand (1924) and Maximow (1927a, 1927b). It is not known to what extent they lose their potencies for heteroplastic development when they become engorged with foreign material. From the viewpoint of immunity it is important that all these cells can be phagocytic in their fixed position (fixed macrophages) or after rounding up and becoming free (free macrophages). They are, especially as free macrophages, indistinguishable from cells developed from the lymphoid free mesenchymal cells (lymphocytes or hemocytoblasts). They can all proliferate by mitosis and under suitable stimuli can become transformed into fibroblasts (see below). Both mitosis and transformation into fibroblasts are seen to a limited extent after they contain phagocytosed material. In the following brief descriptions, the four categories of macrophages are arranged in order of their functional importance in malaria.

1. The reticular cells, together with fibers, form the stroma of all reticular (myeloid and lymphatic) tissues. It is probable that the stroma cells of various lymphoid tissues, such as the lamina propria of the gut, may have identical potencies and functions.

The reticular cells of the spleen and, to a lesser extent, of the bone marrow are of paramount importance in immunity to malaria. Except under unusual circumstances, as in the occlusion of blood vessels, local hemorrhage and the like, the reticular cells of the lymph nodes or stroma cells of the gut are not important. 
2. The littoral cells of Siegmund line the sinuses of the reticular tissues (e.g., the spleen, lymph nodes and bone marrow) and the sinusoids of the liver, hypophysis and adrenal. In the liver, they are known as Kupffer cells. Those of the reticular tissues are true reticular cells, but the exact nature of the littoral cells of the liver, hypophysis and adrenal is not sufficiently known although they are admittedly similar to reticular cells. In any case, the separation of the littoral cells from the previous category is convenient as they behave somewhat differently because they are located along the blood stream. It is unfortunate that they are still frequently termed endothelial cells and are grouped with true endothelial cells because the latter are more limited in their potencies for phagocytosis and development. An accurate, but confusing, custom is to group the littoral cells under the designation special endothelium as contrasted with true endothelium.

The littoral cells of the liver and to a lesser extent of the other organs are functional in immunity to malaria.

3. The fixed, outstretched, undifferentiated cells of Maximow in the adventitia of the small blood vessels of loose connective tissue throughout the body have broad mesenchymal potencies. They are widely distributed over the body, but are only incidentally functional in malarial immunity. Thus, after intense malarial stimulation, pericytes in the dense periportal connective tissue (Glisson's capsule) of the liver are phagocytic for malarial material. Whenever the blood vessels are occluded with resulting hemorrhage, they are phagocytic and because of their location are frequently erroneously considered to be true endothelial cells.

4. The macrophages, variously known as histiocytes (Aschoff and Kiyono), clasmatocytes (Ranvier), resting wandering cells (Maximow) and rhagiocrine cells (Renaut), comprise about half of the cells in the loose and dense connective tissue throughout the body. As in the case of the perivascular cells they phagocytose malarial material only incidentally. Thus, the macrophages of the skin are active when infected blood is introduced intradermally and they join with the perivascular cells of the loose connective tissue in phagocytosing malarial material during vascular occlusion in various organs. The nature of the phagocytes or glial cells, which are similarly active in the brain, is not entirely clear.

(2) Fibroblasts and endothelial cells. Fibroblasts which, together with the macrophages, are the chief cellular constituents of loose and dense connective tissue generally assume a functional role in the later stages of defense. They repair connective tissue, fill in cavities and wall off foreign material that cannot be removed by phagocytosis. They can proliferate by mitosis. A few investigators, especially v. Möllendorff (for a discussion and criticism of this work, see Maximow 1927a, 1927b; Bloom 1938b), believe that they, like some of the macrophages, are endowed with full mesenchymal potencies, but most workers consider them highly differentiated in man and believe that they are rarely phagocytic and do not generally develop into other cells. In lower animals, however, as for example during repair and regeneration of the spleen after malarial infaretion and necrosis in the canary, they can transform into reticular cells with full mesenchymal potencies for development. It is possible that under exceptional conditions such ordinarily latent potencies may be invoked in man.

The role of the fibroblasts in malarial immunity is probably limited to connective tissue repair, the filling in of cavities and scarring, all of which are associated with infarction and similar injuries seen most frequently in infections with $P$. falciparum.

The endothelial cells, as designated in this chapter, are sharply differentiated from the littoral cells and are restricted to the cells which line the larger blood vessels and capillaries. They, like the fibroblasts, have only limited capacities for defense under ordinary conditions. Thus, they can proliferate by mitosis, are only rarely phagocytic and do not generally develop into other cells.

In malaria the endothelial cells assume 
a reparative role under the same conditions as do the fibroblasts and are largely limited to the production of new blood vessels of developing connective tissue. The frequent reports of phagocytic endothelial cells are due chiefly to (1) the reversion to the widespread older conception that the macrophages in categories 1,2 and 3 originate embryologically and, frequently, in the adult organism from true endothelial cells and that all endothelium can be phagocytic either in situ or after desquamation; (2) confusion of adventitial cells (both undifferentiated perivascular cells and macrophages of the perivascular loose connective tissue) with endothelial cells; and (3) confusion of circulating macrophages which have become closely applied to the vessel wall with endothelial cells. Although not well substantiated, true endothelial cells may be phagocytic under unusual conditions, such as in stasis associated with occlusion of the brain capillaries in P. falciparum.

\section{Free Connective Tissue and Blood Cells: Cellis of the Blood AND LYMPH}

The cells of the blood and lymph are generally divided into those of myeloid and those of lymphoid origin, although during ectopic myelopoiesis the myeloid cells may arise in organs other than the bone marrow, and, under certain conditions, lymphatic nodules may occur in the bone marrow.

(1) Myeloid cells. The myeloid cells include (1) the red blood cell series, of which the youngest free cell is the erythroblast and the mature cell is the erythrocyte; (2) the blood platelets, which probably develop from the megakaryocytes; and (3) the granulocyte series, of which the youngest free cells are the three types of myelocyte and the mature cells are the heterophil or special (=in man the neutrophil polymorphonuclear), eosinophil and basophil leucocytes. In malaria erythropoiesis is important as a "reparative proliferation" because red cells are destroyed either directly by the parasite or indirectly by toxic action and erythrophagocytosis (Taliaferro and Cannon 1936). Among the remaining cells only the heterophils show evidence of a functional role (see below under hematology). They are rarely phagocytic under ordinary conditions, but may be phagocytic in heavy infections of $P$. falciparum.

(2) Free mesenchymal cells: lymphoid cells. There is fair agreement among investigators regarding the nature of the predominantly fixed cells with mesenchymal potencies, but there is a wide diversity of opinion regarding the nature of the free cells. It is generally agreed that in addition to the lymphocytes of lymphatic tissue, free lymphoid cells, more or less similar to lymphocytes, occur under physiologieal and pathological conditions in the myeloid and loose connective tissues and that they are the stem cells of the various myeloid cells of the preceding paragraph, but there is no unanimity of opinion as to whether they are all lymphocytes or differ in structure and in developmental potencies.

The work of the present author (Taliaferro and Mulligan 1937) has convinced him of the essential soundness of the unitarian view of Maximow (1927a, 1927b) and Bloom (1938a) to the effect that all of the stem cells of the myeloid tissue (hemocytoblasts) are identical in morphology and developmental potencies with the lymphocytes of lymphatic tissue. This does not mean that the lymphocytes of lymphatic tissue and the hemocytoblasts of myeloid tissues develop indiscriminately into myeloid and lymphoid cells under physiological conditions. This view holds rather that the lymphocytes of lymphatic tissue give rise only to lymphoid cells and the hemocytoblasts of myeloid tissue only to myeloid cells under ordinary conditions, but that both types of cells are able to develop into any cells of the blood and connective tissue under abnormal stimuli. Thus, lymphocytes develop into macrophages in malaria, as is described later. As a corollary to this concept of the free mesenchymal cells, the author agrees with 
Bloom (1938a) that the free stem cells of the proposed polyphyletic theories, such as lymphoblasts, monoblasts, ete., do not exist as cell entities separate from lymphocytes. The free mesenchymal cells or lymphocytes are self-perpetuating by mitosis and unquestionably may arise from the fixed mesenchymal cells previously described.

In malaria the free mesenchymal cells are of obvious importance as stem cells for the lymphoid and myeloid cells of the blood and connective tissue. In addition (see below) the lymphocyte is probably the single most important source of new macrophages in malarial immunity.

\section{Systems of Cells in Immunity}

Modern concepts of systems of connective tissue cells distributed over the body, which play a major part in immunity, have arisen fundamentally from studies on inflammation. Among the early workers, great credit should be given Metchnikoff (1892) for stressing the essential role of the connective tissue cells in inflammation and to Cohnheim (1867), Ziegler (1891), Marchand (1924), and Maximow (1927a, $1927 \mathrm{~b}$ ) for studying the histogenesis of the local inflammatory reactions. Metchnikoff laid the basis for the modern concept of the immunological significance of fixed and mobile cells of the connective tissue by phagocytosis. His concept was physiological. $\mathrm{He}$ distinguished (1) microphages which are identical with heterophils and (2) macrophages as used in this chapter except that he included phagocytic glial cells of the brain. Furthermore, studies on vital staining and the storage of colloidal dyes, chiefly by Renaut, Maximow, Goldman, Tschaschin, Kiyono and Aschoff have laid the basis for the modern concept of macrophages. Aschoff's (1924) reticulo-endothelial system, as broadly defined and as generally used, consists of the macrophages as outlined in this discussion and is synonymous with the macrophage system of many recent authors.

Particular mention should be made of the Gefässwandzellen of the MarchandHerzog school (Marchand 1924). This sys- tem of cells includes not only endothelial cells, but also pericytes and adventitial macrophages which are supposed to arise from the endothelium of developing vessels. Unfortunately, many authors have interpreted the Gefässwand to signify only endothelium and to consider, therefore, that true endothelium is phagocytic either in situ or after desquamation or migration.

One of the frequent accompaniments of immunity in malaria and other infections is the local increase of cells of the macrophage or reticulo-endothelial system. This hyperplasia is frequently assumed to be caused exclusively by a proliferation of the macrophages or elements of the reticuloendothelial system. As will be shown later, such a proliferation is an admitted source of the new macrophages, but detailed studies on simian malaria by Taliaferro and Mulligan (1937) indicate that most of the new macrophages arise from lymphocytes, with or without the intervention of a monocyte stage. In order to group together under one term both macrophages and all macrophage precursors which develop homoplasticly from preëxisting macrophages or heteroplasticly from agranulocytes (lymphocytes and monocytes), Taliaferro and Mulligan (1937) proposed the term, lymphoid-macrophage system. This term would include not only macrophages and lymphocytes, but other mononuclear exudate cells (i.e., Maximow's polyblasts) which form the cellular exudate in inflammation and which are so prominent in the spleen in malaria (see below).

(1) Phagocytic phases of malarial immunity. Malarial immunity is chiefly concerned with parasiticidal effects. There is no complete or lasting inhibition of reproduction of the parasites. As originally noted by Golgi (1888) in infections of $P$. vivax and $P$. malariae and as has been found subsequently in infections in both man and animals, the parasites formed by asexual reproduction show a high death rate from the beginning of the infection. At first this death of the parasites is a natural immunity and represents the un- 
suitability of the normal host for the parasite, but as the initial infection is suppressed, it greatly increases and then represents an acquired immunity. Various degrees of acquired immunity are generally manifested from the suppression of the initial attack onward throughout the infection and during superinfection with the same strain.

All evidence indicates that the death of parasites during both natural and acquired immunity is associated with phagocytosis. Several early observers, notably Golgi (1886e, 1888), Marchiafava and Celli (1887), Metchnikoff (1887) and Laveran (1889), studied the phagocytosis of malarial material and postulated that the process is functional in defense. Many subsequent workers, working on human necropsy material, established beyond question that the macrophages, chiefly of the spleen, liver and bone marrow, and to a lesser extent of other sites, such as the adrenal and around areas injured by the localizations of $P$. falciparum, as in the brain, phagocytose free and intracorpuseular parasites and pigment. As evidence accunulated, some authors questioned the primary functional role of the phagocytes in suppressing the infection. Various theses were maintained, such as that the activity of the macrophages is limited to removing dead or effete material rather than vigorous viable parasites; that although macrophages ean ingest vigorous parasites, they cannot digest them; or that macrophages are ineffectual in immunity because they are more active in acute pernicious malaria than in eases of spontaneous cure. Many of the ideas, however, were incident upon the use of human material which precluded closely spaced observations and rarely came to the necropsy table unless the defense mechanisms of the body were overwhelmed.

Doubt of the functional significance of phagocytosis has been largely dispelled by the closely spaced serial observations of tissues during initial infections and superinfections first made by Cannon and Taliaferro (1931) in birds and Taliaferro and Cannon (1936) in monkeys. The latter observations on Central American monkeys infected with $P$. brasilianum seem directly

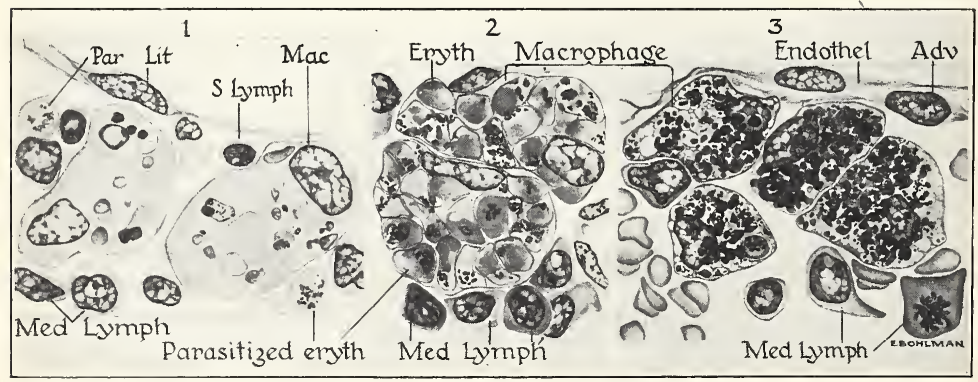

Plate I

Comparative phagocytic activity of the splenic macrophages in the Billroth cords at different stages in malaria. Central American monkeys infected with $P$. brasilianum. $\times 1200$. Modified from Taliaferro and Cannon.

Fig. 1. Sluggish phagocytosis during the natural immunity of the initial acute rise of the infection. The macrophages contain a small amount of parasitic and red cell debris.

Fig. 2. Intense phagocytosis during the acquired immunity at the erisis which is associated with the termination of the initial acute rise. The macrophages are engorged with parasitized erythrocytes.

Fig. 3. Pigment and comparatively indigestible debris remaining in the macrophages about two days after the crisis. These materials disappear within a few months. 
applicable to $P$. vivax and $P$. malariae infections in man because they agree with the necropsy material as far as the latter go. They are also probably applicable to infections with $P$. falciparum except for the fact that infections with $P$. falciparum are frequently overlaid with general and particularly with local degenerative changes.

The main conclusions from the investigations on monkeys are as follows: During the initial acute rise of the infection when death of the parasites represents a natural immunity, the free merozoites and intracorpuseular parasites in all stages of development are phagocytosed sluggishly by macrophages of the spleen, liver and bone marrow (Pl. I, Fig. 1). At the erisis when the initial acute rise is terminated, the parasitized erythrocytes are regionally concentrated in the splenic cords and are probably agglutinated or adhere to the macrophages. After a day or so, the parasitized erythrocytes are avidly eaten by the macrophages of the spleen, liver and bone marrow (Pl. I, Fig. 2). This phagocytic activity is many times greater than that observed before the erisis and represents the beginning of the heightened activity of the immune reaction. Once this immune phagocytosis is initiated, the infection is generally subdued and held at a comparatively low level. The red cells and parasites within the phagocytes disappear rapidly, but the pigment is not metabolized for several months (Pl. I, Fig. 3). When animals which have recovered from an infection are superinfected with the homologous strain to which they are immune, the sequence of splenic filtration, phagocytosis, etc., is initiated within an hour instead of several weeks or months as in the initial attack. In other words, aequired immunity takes time to develop, but once developed responds immediately.

The limitation of phagocytosis to the spleen, liver and bone marrow probably follows from the fact that the blood in these organs, at least periodically, flows slowly and comes into direct contact with active macrophages, whereas other organs, such as the lymph nodes, lung, ete., which are also rich in macrophages, do not have this intimate contact with the blood except after a hemorrhage due, for example, to infaretion. The fact that the adrenal is similar to the liver and yet is only sporadically involved may be due to the richness and swiftness of the blood flow. In any ease there are differences in the activity of the macrophages in these various organs. Thus, Taliaferro and Mulligan (1937) have shown that even in the overwhelming infection of $P$. knowlesi in the rhesus monkey in which almost every available cell of the lymphoid-macrophage system is phagocytic, the same quantitative relation is maintained, i.e., the individual macrophages of the spleen are most active, those of the liver are less active and those of the bone marrow are least active ${ }^{1}$ while those in other organs are only rarely active. The generalized character of the malarial infection and the limitation of the immune reaction to a few organs in which the macrophages are oriented to phagocytose material from the blood led Taliaferro (1934) to suggest that many of the so-called general immunities are actually loeal immunities in strategically placed organs.

The objective histological differences between aequired immunity and natural immunity are: (1) a greatly increased rate of phagocytosis by individual macrophages and (2) a local increase of macrophages in some of the strategically placed organs, in particular in the spleen and to a lesser extent in the bone marrow. The increased rate of phagoeytosis by the individual macrophages is specific (i.e., is largely operative against homologous strains of parasites) and is the chief characteristic of aequired immunity. It is probably assoeiated with the opsonic activity of specific antibodies and is considered in detail by Coggeshall (this volume p. 250). It suggests a fundamental difference between the phagocytosis of natural and aequired immunity. Such a conclusion is further

1 Although the individual macrophages of the liver are less active than those of the spleen, the total activity of the liver may be greater due to its greater size. 
'strengthened by the work of Gingrich (1934, 1941). He found that blockade with foreign red cells markedly affects acquired and not natural immunity of birds to $P$. cathemerium. The local increase of macrophages in strategic sites has been noted repeatedly in man, chiefly in the spleen and bone marrow. Experimental work with animals indicates that it varies in extent and is nonspecific, i.e., it is not necessarily associated with acquired immunity. If the initial attack is long enough, it may begin before the immune reaction is initiated.

Before considering specifically the origin of new macrophages, certain proliferations should be mentioned. In the bone marrow, a myeloid hyperplasia may oceur which involves an increase in the cells of the erythrocyte and heterophil elements (see reparative proliferations). In the spleen, which is the lymphatic organ most affected by malaria, lymphoid hyperplasia characteristically occurs and involves the mitotic proliferation of, chiefly, the medium lymphocyte and, to a lesser extent, the large tissue lymphoeyte (the lymphoblast of some hematologists) and is a characteristic part of the development of the enlarged "chronic" (as contrasted with the early hyperemic) spleen of malaria. Various proliferations and accumulations of lymphocytes may take place in the liver, bone marrow and other organs, but are not as characteristic of man as of the monkey. Whenever lymphoid hyperplasia occurs in lymphatic tissue or myeloid hyperplasia occurs in the bone marrow, the reticular cells of the stroma proliferate. These cells are macrophages and their mitotic proliferation supplies new macrophages. In the fresh tissues of monkeys, however, which have been particularly studied, the number of mitoses in lymphocytes is many times that found in reticular cells and, as will be pointed out below, the lymphocytes are the source of the majority of the new macrophages. Botl the reticular cell and lymphoid hyperplasias form a prominent part of Siegmund's "mesenchymal activation."

(2) Mobilization and formation of macrophages. As pointed out by Taliaferro and Mulligan (1937), many macrophages are available for malarial defense from the beginining of the infection. Chief among these are (1) the fixed and free reticular cells in the spleen and bone marrow and (2) the littoral cells of the sinuses or sinusoids of the spleen, liver, bone marrow and adrenal. In addition, new macrophages replacing those which go to pieces as a result of the malarial infection and accounting for the "hyperplasia of the reticuloendothelial system" so characteristic of malaria, particularly in the spleen, arise both homoplastically and heteroplastically.

Homoplastically, macrophages arise by the mitotic division of (1) a few engorged macrophages in the spleen, liver and bone marrow, (2) a few littoral cells, particularly of the liver, and (3) large numbers of outstretched unengorged reticular cells. Bruetsch (1927, 1932a, 1932b) has studied

Plate II

Comparative histology of a venous sinus of the spleen (1) of a normal rhesus monkey and (2) of a rhesus monkey infected with $P$. cynomolgi in which intense heteroplastic cytogenesis of macrophages from lymphocytes is seen. $\times 1400$. From Taliaferro and Mulligan.

Fig. 1. A portion of a venous sinus and of a Billroth cord in the red pulp of the spleen from an uninfected monkey. Note within the sinus the typical nongranular leveocytes and within the cord the rounded macrophages and the typical reticular cells with indeterminate cytoplasm.

Fig. 2. A comparable region from the spleen of a monkey killed 10 days after infection and 7 days after parasites appeared in its peripheral blood. The animal underwent a typical infection and was killed at about the time of the crisis. The sinus contains many lymphocytes with an inereased amount of cytoplasm and many large phagocytic cells which do not oceur in the normal spleen. In addition, it contains a progressive series of mononuclear cells from lymphocyte to macrophage as is indicated by Polyblasts 1-5. Polybl. 1 has a lymphocyte type of nucleus, but contains one piece of malarial pigment, whereas at the other end of the series Polybl. 5 is a typical macrophage. Within this series of cells, the extent of phagocytosis varies approximately with the size of the cell. The same intermediate stages can be found in the cords. Med. Lym. 1 possesses a typical lymphocyte nucleus and contains two small granules of malarial pigment in its cytoplasm. 
Monocytoid lymphocyte Sinus Plasma cell Large lymphocyte Small lymphocyte

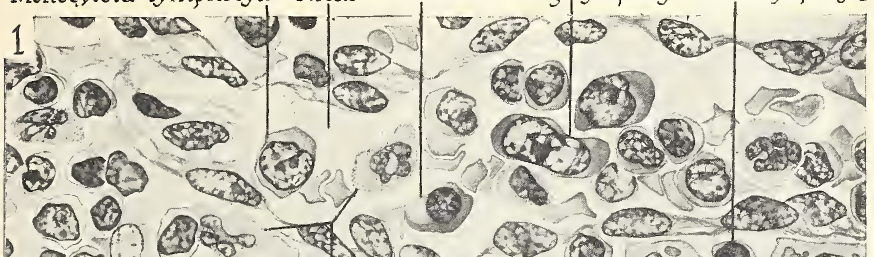

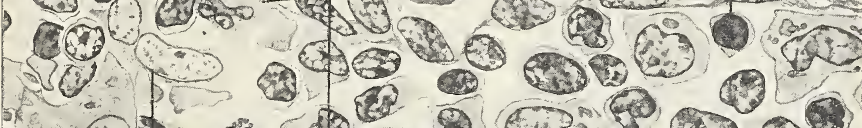

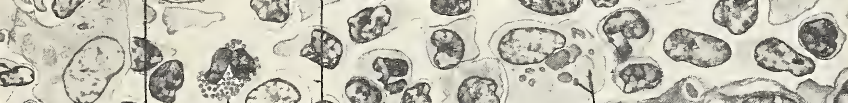

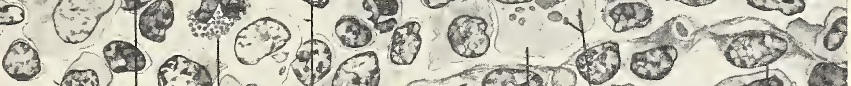
(5)

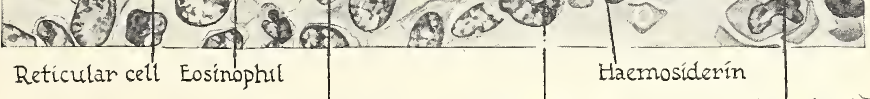
Heterophil Macrophage Liftoral cell Littoral cell Sinus Malarial piogment Med. lym.1

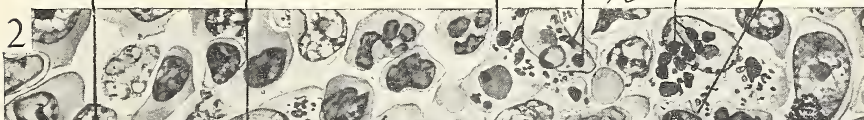
(3)

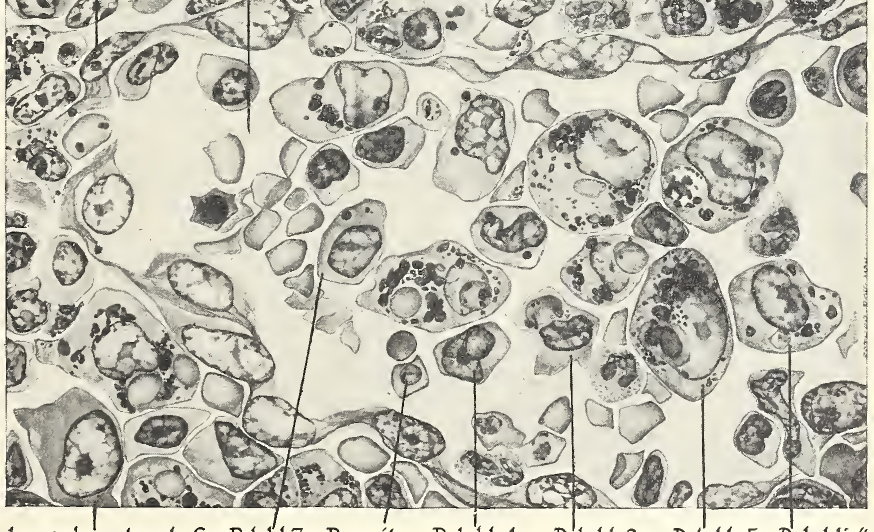
Largelymphocyte G Polybl.3 Parasite Dolybl.1 Dolybl.2 Dolybl.5 Polybli.4 
in detail this origin of macrophages in pareties inoculated with tertian malaria. $\mathrm{He}$ concludes that the specific endothelium (littoral cells) of the liver, spleen, bone marrow and lymph nodes and other macrophages of the connective tissues are stimulated to form what he terms "macrophagic tissue" in various organs. He believes that the blood macrophages arise primarily from littoral cells and to a lesser extent from ordinary endothelium, especially of the brain. The last conclusion regarding the capillary endothelium has been questioned by Taliaferro and Mulligan (1937).

As has been demonstrated in malaria of monkeys and as probably occurs in malaria of man, although it has not been adequately studied in the latter, by far the majority of new macrophages arise from lymphocytes with or without passing through an intermediate monocyte stage. This heteroplastic cytogenesis of macrophages takes place largely in the spleen and results in the occurrence, after sufficient malarial stimulation, of every conceivable intermediate stage between lymphocyte and macrophage or from lymphocyte through monocyte to macrophage (PI. II; $C f$. Fig. 1 with Fig. 2). These intermediate stages oceur only rarely under physiological conditions. During malaria they have been indiseriminately elassified by many investigators as large mononuclears or macrophages. In general, the degree of phagocytic activity is proportional to the amount of cytoplasm of the cell. Under the extreme conditions of exceptionally heavy or pernicious infections, small cells, even including a few undoubted lymphocytes. may be phagocytic (Pl. II, Fig. 2). The transformation of lymphoeytes into macrophages in the spleen involves lymphocytes chiefly formed in situ, but some may be brought to the spleen from other sites by the blood. The cytogenesis of macrophages also takes place in other parts of the body, although to a lesser extent. Thus, hemocytoblasts, the equivalent of lymphocytes, in the bone marrow undoubtedly produce some macrophages.
The occurrence of intermediate stages between agranulocytes and macrophages does not indicate the direction of the process, but the fact that lymphocytes actively proliferate by mitosis whereas the various macrophages do so to a much lesser extent indicates that the lymphocyte is actually the "stem cell." Finally, the investigations of Maximow and others on the developmental potencies of the lymphocyte and the recent investigations on the origin of macrophages in malaria and in other infections ( $c f$. Conway 1939) supply a functional explanation for lymphoid hyperplasia and indicate that the lymphocyte is not an end cell with completely unknown functions, but that it, together with the partially differentiated monocyte, constitutes an easily mobilized source of macrophages.

(3) Hematology. Reviews of the hematology of human malaria may be found in Pöch (1903), Seyfarth (1926), Schilling (1924), Schilling et al. (1924) and Taliaferro and Klüver (1940). In general, changes in the peripheral blood show a high correlation with those in the organs in regard to erythropoiesis, heterophil myelopoiesis and monocytopoiesis, but, although they indicate the changes, they do not quantitatively reflect those in the organs in regard to phagocytosis or the heteroplastic development of macrophages. Special mention should be made of the following points which are discussed in detail by Taliaferro and Klüver (1940): Rubitschung (1925), Bunker (1926) and others for man and Taliaferro and Klüver for monkeys have found a pronounced transient heterophil leucocytosis associated with the segmentation of the parasites. As heterophils are only rarely phagocytic in either the peripheral blood or organs, and then generally only to a minor extent (Taliaferro and Mulligan 1937), the funetional significance of this finding is not clear unless they remove the less easily identifiable or more easily digested products of segmentation. Furthermore, many authors for man and Taliaferro and Klüver 
for monkeys have found that the large mononuclears, which have been variously defined to include the larger blood lymphocytes, monocytes and small macrophages but which consist for the most part of monocytes, generally increase in relation to segmentation of the parasites and to the infection as a whole. This increase reflects to a minor extent the cytogenesis of macrophages in the organs.

(4) Reparative proliferations. Many of the cellular proliferations in malaria are not related to the suppression of the infec- tion, but are fundamentaiìy reparative. Thus, cells are frequently destroyed during malaria which may, thereafter, be regenerated. This is notably true of erythropoiesis and may occur in the regeneration of other tissues, as in the liver. It is also true to a greater or lesser extent of lymphoid hyperplasia, macrophage proliferation and the heterogenic cytogenesis of macrophages because these cells, particularly in the pernicious infections with $P$. falciparum, are often destroyed by the toxic action of the parasites. 


\title{
HUMORAL IMMUNITY IN MALARIA
}

\author{
By L. T. COGGESHALL \\ LABORATORIES OF THE INTERNATIONAL HEALTH DIVISION, ROCKEFELLER FOUNDATION, NEW YORK
}

THE humoral aspects of immunity in malaria, like those of any other infectious disease, are concerned with the production and behavior of specific antibodies, the study of which has been handicapped chiefly by the lack of a method of cultivation or a suitable means of concentrating the malarial plasmodia. This is especially true with those species pathogenic for man, which, incidentally, present an additional difficulty as they are not known to be infectious for a lower animal. In spite of these difficulties, it has been conclusively demonstrated that the development of immunity in malaria is accompanied by the development of specific antibodies in the circulating blood. The identity, nature and mode of action of the various antibodies thus far shown to be directly concerned with recovery from an acute attack or relapse in malaria have been obtained almost entirely from the study of the disease in lower animals. However, since the behavior of most of the experimental infections, especially those occurring in monkeys, closely resembles that of the disease observed in man, important inferential evidence may be obtained.

\section{Passive Immunity}

The early attempts to transfer passively protective antibodies in malaria were based upon the use of convalescent serum obtained from patients with long standing chronic infections. Soteriades (1917) obtained serum from patients who presumably had a high degree of immunity, as judged by the presence of circulating parasites, and no clinical symptoms. He administered this serum to another patient during an acute attack and reported improvement in the recipient. More recently Lorando and Soteriades (1936, 1937), repeating the experiments with whole blood, have reported that a beneficial effect is obtained when this procedure is used as a therapeutic measure. Kauders (1927) showed that serum obtained after recovery from attacks of induced malaria would ameliorate an active infection in paretics and that acute or normal human serum was entirely ineffective. These experiments suggested the presence of circulating protective substances but were not conclusive since the effects could have been nonspecific or entirely coincidental.

Protection tests in animals were attempted by numerous investigators with consistently negative or inconclusive results until it was shown by Coggeshall and Kumm (1937) that the serum of rhesus monkeys with chronic $P$. knowlesi malaria protected normal monkeys against death due to infection with this very virulent parasite. In the same study it was discovered that homologous immune serum would attenuate the less severe $P$. inui infection and that normal serum was entirely without effect against either infection. There is a quantitative relationship between the amount of immune serum and the number of parasites used in the inoculum for a protection test; smaller dosages of parasites require proportionally lesser anounts of immune serum for effective protection (Coggeshall and Eaton 1938b). This is a probable explanation for earlier failures with protection tests in that dosages were excessive and overwhelmed the action of the antibodies. More recently Hegner and Eskridge (1938), Manwell and Goldstein (1940), Mosna (1938), and Taliaferro and Taliaferro (1940) have also been able to demonstrate that the serum of animals with chronic malarial infections contained protective antibodies. The blood serum obtained from pareties after induced $P$. knowlesi malaria exerts a protective 
action when administered to rhesus monkeys against infections by the same parasite (Coggeshall 1940b). However, the serum of these same individuals before the infection is without effect.

These studies seem to furnish ample evidence that neutralizing antibodies are found during an attack of malaria and, although not experimentally proved, it seems probable that they are concerned with the specificity of the disease. This assumption was given strong support by the studies of Boyd et al. (1938), who showed that paretics with induced $P$. vivax malaria had a complete immunity against the Floridian strain of the parasite, with which they were initially inoculated, yet were readily susceptible to Panamanian and Cuban strains of $P$. vivax. The fact that an immune host can resist reinfection following massive doses of the homologous organism, yet react as a normal when injected with a closely related strain, indicates that some factor other than a highly active cellular defense mechanism is responsible for the marked degree of specificity encountered with malarial infections.

The behavior of the protective antibody during the long latent period of malarial infection has not been thoroughly studied, although there is evidence to support the belief that the concentration fluctuates with the changes in the rate of multiplication of the parasite. For example, preliminary studies in this laboratory have shown that serum obtained immediately before a parasitic relapse in $P$. knowlesi infections contains practically no protective antibodies, while serum obtained after the spontaneous termination of the relapse in the same animal often has an extremely potent effect when used in a protection test. It seems probable that recovery from malaria is accompanied by a gradually increasing titer of protective antibodies resulting from a series of relapses (during which parasites may or may not be found in the circulating blood stream) until eventually a complete cure is attained. Experimental evidence supporting this conclusion comes from the finding that the titer of serum from monkeys with chronic $P$. knowlesi infections can be enhanced considerably by repeated injections of massive doses of living parasites (Coggeshall and Kumm 1938). Undoubtedly the occurrence of spontaneous relapses is a mechanism of autohyperimmunization which results in a more efficient humoral immune mechanism.

The exact role of the protective antibody in malarial immunity has not been clearly demonstrated, although a likely possibility is that it sensitizes the parasite and renders it more susceptible to phagocytosis. A greater protective effect in protection tests in monkeys and chicks is obtained with serum that has been incubated at $37^{\circ} \mathrm{C}$ with the inoculating dose of parasites instead of merely allowing the immune serum and parasites to come in contact before inoculation of the mixture or the separate injections of the two components (Coggeshall and Eaton 1938b; Taliaferro and Taliaferro 1940). There is a union between the protective antibody and the parasite because the protective effect of an immune serum ean be removed by absorption with an excess of living parasites. These and other related experiments where immune serum renders viable parasites noninfectious indicate that an immune malaria serum ean act upon the asexual plasmodia, although the removal of the circulating parasites from an infected host is undoubtedly the function of the macrophages (Taliaferro, this volume, p. 239). Although the above mentioned findings are suggestive, further studies are necessary to elucidate clearly the exact mechanisms of action of the protective antibody.

\section{Complement-Fixing Antibodies}

A specific complement fixation reaction for malarial infections has been the object of many studies, and a summary of the results up to 1927, although contradictory, indicates the presence of complement-fixing antibodies in the serum of patients with chronic malaria (Taliaferro 1929). The most significant work was that of Kingsbury in 1927 who used infected $P$. falciparum blood from internal organs in an 
attempt to obtain an antigen of low anticomplementary property but of high specificity and sensitivity. Saline emulsions of heart blood gave specific results, yet sera from 25 known cases of malaria fixed complement in only 48 per cent of the instances. The chief difficulty encountered in all of the investigations was the lack of sufficient quantities of parasitic material to serve as an antigen. This obstacle was overcome by the utilization of $P$. knowlesi parasites from rhesus monkeys which, unless treated, usually die from an overwhelming parasitemia (Coggeshall and Eaton 1938a). In these animals it was found that specific cornplement-fixing antibodies appeared shortly after the acute attack was terminated either by quinine or by immune serum and that the antibody titer dropped to a more or less stationary level. During the chronic stage of the infection when parasite relapses occur at irregular intervals, the titer is low preceding each relapse and is elevated considerably following each relapse. There is no evidence to show that specific complementfixing antibodies are identical with the protective antibodies, although frequently animals with a high protective serum titer show a marked concentration of complement-fixing antibodies. Complement-fixing antibodies can be produced with ease in rabbits and monkeys by the injection of killed $P$. knowlesi parasites, although this procedure does not confer an active immunity in the monkey or produce protective antibodies in the rabbit. Possible explanations are that separate immunity mechanisms are involved or that perhaps it is only a quantitative effect.

The complement-fixing antigen is present in some unidentified constituent of the parasite, and it also can be readily detected as a soluble antigen. The greatest concentration of the soluble antigen oceurs in the blood serum at the height of the acute attack of $P$. knowlesi malaria, and, as would be expected, the amount is related directly to the parasite count. The antigen has the ability to fix complement in immune animals and probably is concerned with the production of the complement-fixing antibody; at least these antibodies are readily produced in normal animals by injection of the soluble antigen. When normal monkeys are immunized, apparently only complement-fixing antibodies are developed as no agglutinins or protective antibodies appear in the serum, and there is no resistance to infection. Clinically, the antigen is unstable in acids and alkalies, is heat-stable up to $56^{\circ} \mathrm{C}$ and appears to be a constituent of the albumin fraction of the serum.

\section{Complement Fixation Reaction in Human Malaria}

In the studies on the $P$. knowlesi complement-fixing antigen one of the most interesting developments was the discovery of the broad antigenic power of the antigen. It would bind complement in human malaria serum with the same degree of sensitivity as was found with homologous monkey immune serum (Eaton and Coggeshall 1939). This finding immediately suggested the investigation of the practicability of the reaction as a diagnostic aid in malaria, which is a real necessity, especially in those low-grade infections or treated cases where parasites are too scanty for microscopical detection. As pointed out above, all previous tests depended upon infected human material as a source of the antigen, which naturally limited their usefulness. In one average-size rhesus monkey it is possible to obtain approximately $10 \mathrm{ec}$ of packed red cells with over 60 per cent containing malaria parasites. The antigenic property of this material can be shown to exist in dilutions up to $1: 320$, and as the anticomplementary range is absent or extremely low, it would seem to be a widely adaptable test even for large-scale use. It was found that the test was highly specific for malaria in that practically no false positive reactions were obtained although large numbers of sera from normal individuals, from convalescents from a variety of infectious diseases, and from patients with strong positive Wassermann reactions were used as controls. Patients either with 
or without luetic infections and negative malaria complement fixation tests before induced therapeutic malarial infections develop the maximum titer of complementfixing antibodies about 3 weeks after the onset of the central acute attack. In paretics the antibodies persist from 2 to 5 months after the disappearance of parasites from the blood smears, an important consideration, as these studies were conducted with a strain of malaria characterized by infrequent relapses. It is possible that serum from patients in endemic areas of malaria would be positive for even longer periods. The value of this test as a diagnostic procedure depends upon its being positive in chronic infections without detectable parasites in the peripheral blood stream. Siratman-Thomas and Dulaney (1940) have employed the above described antigen and technique for diagnostic purposes with promising results. Although the test has yet to be completely evaluated, preliminary observations indicate that it may be an important aid not only for diagnostic purposes but more important as a means of studying parasitic activity and host response.

\section{AggLUTinins}

The specific agglutination of malarial parasites by an immune serum was originally demonstrated in 1938 by Eaton with $P$. knowlesi. This observation has been confirmed by Somogyi (1939) and Singh and Singh (1940). Recently in a preliminary publication Mulligan and Russell (1940) reported the specific agglutination of $P$. gallinaceum sporozoites in dilutions of homologous immune serum as high as $1: 8000$. Thus the discovery of agglutinins in malarial infections adds more evidence to the belief that the immune response within a host differs little whether the pathogen is a virus, bacterium, or a protozoan.

It is possible to detect agglutinins in $P$. knowlesi infections about the time that an acute infection is being transformed into a chronic one. Although only weakly positive at this stage, the reaction becomes pro- gressively stronger so that finally a demonstrable effect may be obtained in serum diluted 1:1000. Only the mature parasites located within red cells that presumably have more permeable cell membranes, or parasites artificially released from the red cells, have the capacity of being agglutinated by an immune serum. Other related findings indicate that the agglutination phenomenon in malaria is a true antigenantibody reaction. Unlike the complement fixation reaction where the antigen is unable to distinguish between antibodies produced by closely related parasites, the agglutination reaction is very specific. This test might possess important practical applications if it were possible to obtain sufficient organisms. Unfortunately, the reaction with schizonts is very unstable, and only viable organisms can be used. The specific agglutination of $P$. knowlesi parasites by the homologous immune serum is strong presumptive evidence that the parasites are sensitized in vivo and thus rendered highly susceptible to phagocytosis by the macrophagic cells of the host. As mentioned above, the cellular defense mechanism undoubtedly plays the final role in the concentration and destruction of the malaria parasite. In the initial stages of the fight, however, the host's attempt to recover from an infection may be dependent upon specific antibodies. At least a highly active macrophage system is effective only against an infection originally responsible for its activation (Taliaferro, this volume p. 239).

\section{Precipitins}

Several investigators (Pewny 1918; Ziemann 1924; Taliaferro and Taliaferro 1928; and Row 1931) have attempted to demonstrate specific precipitins in malaria immune serum, and although suggestive, none of the reported results appear to be entirely conclusive. The work of the Taliaferros was the most extensive, as they tried 75 separate parasite antigens. The best antigen was prepared from infected placentas by removing and drying the parasites, then digesting with $0.05 \mathrm{~N} \mathrm{HCl}$, and 
neutralizing with $\mathrm{NaOH}$. A positive test was obtained in 29 instances using the sera from 34 patients showing circulating parasites. However, the use of antigens prepared in the same manner in different areas, did not furnish such promising results. Repeated, unsuccessful efforts have been made in this laboratory to demonstrate a specific precipitin reaction in avian and monkey malaria. Antigens prepared from practically pure concentrations of $P$. knowlesi parasites and known to have a high antigenic potency for the malarial complement-fixing antibodies failed to precipitate antibodies in the serum of animals obtained during the various stages of the acute and chronic infection or even with hyperimmunized serum. From the studies on humoral immunity in malaria it seems highly probable that there should be specific precipitins at some stage of the infections and that the failure of consistent demonstration is due to low concentrations of the antibodies and a weakly reactive antigen.

\section{Summary}

Humoral immune substances in the form of specific antibodies have been conclusively demonstrated in malarial infections of birds, monkeys and man. Those identified thus far are protective antibodies, agglutinins, and complement-fixing antibodies. There is also suggestive evidence for precipitins, although their specificity is in doubt and they cannot be demonstrated consistently. The development of immunity to malaria can be shown to accompany the formation of the specific antibodies. Although not definitely proved, there is much inferential evidence pointing to the specificity of the immune response in malarial infections as being the concern of humoral immune bodies. 


\title{
CINCHONA AND ITS ALKALOIDS IN THE TREAT- MENT OF MALARIA
}

\author{
By ERWIN E. NELSON \\ DEPARTMENT OF PHARMACOLOGY, TULANE UNIVERSITX OF LOUISIANA SCHOOL OF \\ MEDICINE, NEW ORLEANS
}

THE cinchona official in the United States Pharmacopoeia XI is the dried bark of the stem or root of three species of cinchona, viz., Cinchona succirubra, or red cinchona, C. Ledgeriana and C. Calisaya or yellow cinchona, or of hybrids of these species with other species. This bark must yield not less than 5 per cent of total alkaloids. The chief source of cinchona bark at present is the East Indian island of Java, where the Dutch have developed its cultivation to a high degree. The consumption of cinchona derivatives in the United States is not exactly known, but the Department of Commerce estimates it at about 5,000,000 ounces per year (Concannon 1940). Since the war broke out in Europe there has been a definite increase in the importation into the United States. Considering importations of bark on the basis of their alkaloidal content, there was imported between September 1, 1939, and March 1, 1940, the equivalent of $5,000,000$ ounces of cinchona alkaloids, as compared with $2,270,000$ ounces for the corresponding period of 1938-39. As a result of these increases in importation the Department of Commerce believes that there are in the United States approximately $10,000,000$ ounces of quinine sulfate, or enough to last for a period of more than two years. In the latter part of 1939, the War Department purchased 800,000 ounces to be held for emergency (Concannon 1940).

The small fraction of imports that has in the past come from Latin American countries has also increased extensively. According to the figures of the Department of Commerce, the importation of cinchona from Guatemala, Ecuador and Colombia for the first 5 months of 1940 amounted to nearly 81,000 pounds, as compared with less than 19,000 for the corresponding months of 1939 (Chapman 1940). The only justifiable uses of the cinchona alkaloids in the opinion of this reviewer are for their effect on the heart in certain types of disordered rhythm and for their action against the organisms responsible for malaria. Actually much of the material imported goes into hair tonics, headache remedies, and the so-called chill tonics. Restriction of the use of the cinchona alkaloids to the treatment of malaria would be a very proper emergency measure.

From the bark of various species of cinchona there have been isolated more than 20 alkaloids. Although the constitution of not all of these is known, those which are of importance to the present discussion are members of a closely related series whose general formula is given in Fig. 1.

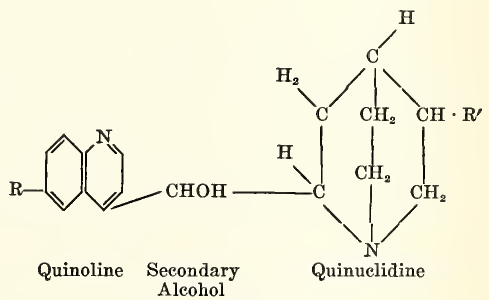

Fig. 1. General formula for the quinine series of alkaloids (modified from Findlay 1939).

In quinine and quinidine $\mathrm{R}=$ methoxy $\left(-\mathrm{OCH}_{3}\right)$, and $\mathrm{R}^{\prime}=\operatorname{vinyl}\left(-\mathrm{CH}=\mathrm{CH}_{2}\right)$. In cinchonine and cinchonidine $\mathrm{R}=\mathrm{H}$, and $\mathrm{R}^{\prime}$ remains $\left(-\mathrm{CH}=\mathrm{CH}_{2}\right)$. When $\mathrm{R}$ becomes $-\mathrm{OH}$, there is formed the base cupreine. Formation of the hydro-bases of cupreine and of the above alkaloids 
occurs when the unsaturated vinyl grouping is replaced by the saturated chain $-\mathrm{CH}_{2} \cdot \mathrm{CH}_{3}$. Alkyl substitution for the $\mathrm{R}$ of dihydrocupreine gives rise to a series of substances much studied for their chemotherapeutic action (optochin, eukupin, vuzin) but of no present interest because of their lack of any important degree of action against the malarial plasmodium. These chemical relationships are shown in Table I. to alkaloids of the opposite sign (Dawson et al. 1933).

The historical aspects of the use of cinchona have recently been reviewed by others (Dawson 1930, Fischl and Schlossberger 1932) and will not be discussed here. Up until the introduction of plasmochin (1926) and atabrine (1933) preparations of cinchona or its alkaloids were the only established substances for the specific treatment of malaria. Although these newer

TABLE I

The Principal Cinchona Alkaloids

(Fischl and Sehlossberger 1932, p. 153)

\begin{tabular}{l|l|l|l|l}
\hline \multirow{2}{*}{ Formula } & \multicolumn{2}{|c|}{ Sign of rotation } & \multicolumn{2}{c}{ Substitutions } \\
\cline { 2 - 5 } & \multicolumn{1}{|c|}{ Laevo } & Dextro & $\mathrm{R}$ & $\mathrm{R}^{\prime}$ \\
\hline $\mathrm{C}_{10} \mathrm{H}_{22} \mathrm{ON}_{2}$ & Cinchonidine & Cinchonine & $\mathrm{H}$ & $\mathrm{CH}=\mathrm{CH}_{2}$ \\
$\mathrm{C}_{10} \mathrm{H}_{22} \mathrm{O}_{2} \mathrm{~N}_{2}$ & Cupreine & Cupreidine & $\mathrm{OH}$ & $\mathrm{CH}=\mathrm{CH}_{2}$ \\
$\mathrm{C}_{20} \mathrm{H}_{24} \mathrm{O}_{2} \mathrm{~N}_{2}$ & Quinine & Quinidine & $\mathrm{OCH}$ & $\mathrm{CH}_{3} \mathrm{CH}_{2}$ \\
$\mathrm{C}_{20} \mathrm{H}_{24} \mathrm{ON}_{2}$ & Hydrocinchonidine & Hydrocinchonine & $\mathrm{H}$ & $\mathrm{CH}_{2} \mathrm{CH}_{3}$ \\
$\mathrm{C}_{18} \mathrm{H}_{22} \mathrm{O}_{2} \mathrm{~N}_{2}$ & Hydrocupreine & Hydrocupreidine & $\mathrm{OH}$ & $\mathrm{CH}_{2} \mathrm{CH}_{3}$ \\
$\mathrm{C}_{20} \mathrm{H}_{24} \mathrm{O}_{2} \mathrm{~N}_{2}$ & Hydroquinine & Hydroquinidine & OCH & $\mathrm{CH}_{2} \mathrm{CH}_{3}$ \\
$\mathrm{C}_{21} \mathrm{H}_{23} \mathrm{O}_{2} \mathrm{~N}_{2}$ & Optochin & & $\mathrm{OCH}_{2} \mathrm{CH}_{3}$ & $\mathrm{CH}_{2} \mathrm{CH}_{3}$ \\
\end{tabular}

There are four asymmetric carbons in the formula given in Fig. 1. This of course gives rise to the possibility of isomerism among the members of the group. Thus, quinine and quinidine form a pair, differing only by the sign of rotation of polarized light. Similarly, cinchonidine and cinchonine form a pair. However, it has not been possible to relate therapeutic effectiveness of the alkaloids against the malarial organisms to the sign of rotation. Often it is the case that the laevo-rotatory member of a pair of isomers has the greater biological activity. This certainly does not hold for the quinine-quinidine pair. Such information as is available indicates that in the treatment of malaria they are essentially equivalent (vide infra), while in toxicity and depressing effect on the various parts of the circulatory mechanisms the dextro-isomer quinidine is clearly the stronger member of the pair (Nelson 1927a). The chief significance of the isomerism lies in the fact that idiosyncrasy to alkaloids of one sign does not necessarily carry over substances are apparently coming to be of greater and greater importance, quinine still holds a major place. A recent report of the Malaria Commission of the Health Organization of the League of Nations (1937) carries the following statement in its conclusion: "Among those drugs [i.e., those used for curative or prophylactic mass treatment] quinine still ranks first in current practice by reason of its clinical effectiveness and almost complete absence of toxicity, coupled with widespread knowledge of its use and dosage" (page 1015).

While historically the use of bark or of galenicals made from it in the treatment of malaria is of considerable interest, such preparations do not play any important role in the treatment of malaria by organized medical and public health agencies in this country. In other parts of the world the necessity for supplying drugs for mass treatment at the lowest possible cost has led to the introduction of the use of extracts of bark without complete separation and isolation of the alkaloids. The Malaria 
Commission of the Health Organization of the League of Nations has set up standards for a preparation of the "total alkaloids" under the name "Totaquina" which has been widely used throughout the world (Pampana and Fletcher 1934). This preparation was adopted into the British Pharmacopoeia in 1933, and its adoption for the U. S. P. XII has been urged on the ground that this would establish standards for the preparation used in the Philippines. It can be produced in the Philippines and quite possibly in Central America. Any dislocation of commerce with the East Indies that cut off supplies of alkaloids would in all probability at the same time disturb traffic with the Philippines. The flow of supplies from Central America, however, should be free from interruption.

The most important alkaloid in einchona is quinine. The most extensive experience in this hemisphere in the treatment of malaria is that making use of quinine or of its salts. The emphasis in the rest of this paper will therefore be placed on a discussion of this substance. The pharmacology of quinine and its relatives will be discussed only in so far as this may relate to their use in malaria.

Fundamentally, it may be said that the action of quinine, in adequate dosage, is to destroy all forms of $P$. vivax and $P$. malariae in the peripheral blood of the human host, and the trophozoites but not the gametocytes of $P$. falciparum (Craig 1939). Treatment with quinine in the usual dosage does not affect the patient's general condition adversely, and generally has no depressive or toxic effect, if the period of administration is limited to the strictly necessary number of days. This summary statement should of course be amplified. It is well recognized that there are differences in the action of quinine on the different species of plasmodium eausing malaria, and possibly also on different strains within the same species. Further, as already indicated, different stages in the life cycle of the plasmodium react differently. It is generally held that quinine is parasiticidal, in the sense of causing their disappearance, to both trophozoites and to the fully developed gametocytes of $P$. vivax and $P$. malariae in the circulating blood. On the fully developed gametocytes of $P$. falciparum, quinine has only a slight action, if any. It is believed by some (Parrot, Catanei and Ambialet 1937) that quinine, if not toxic to fully formed gametocytes, does in fact retard their formation. These workers insist that any "drug with schizonticidal properties ipso facto possesses gametocidal properties in regard to sexual forms in progress of development." The correctness of this view has not been established and further evidence is needed. The point is of considerable importance in relation to the control of malaria. So far as treatment of the individual is concerned, it makes no difference whether he carries sexual forms in his blood or not.

The relief of clinical symptoms and the disappearance of the parasites from the blood stream are more or less parallel. In the benign tertian infection the trophozoites disappear about the third day, and the clinical symptoms are relieved at the same time. For quartan fevers clinical improvement comes on in about the same time. With malignant tertian the trophozoites persist a day or more longer, and similarly there may be three or four paroxysms after treatment is started. In no ease, however, can the relief of symptoms be taken to indicate that the body has been freed of the invading organisms. Cessation of treatment is followed by relapse in a considerable number of cases. In primary infections treated by the ordinary doses of quinine as high as 50 per cent may relapse.

This tendency to relapse has resulted in the development of two quite different practices in the treatment of malaria. The National Malaria Committee recommended as a plan of treatment quinine sulfate, 0.6 gm 3 times per day for 8 days, followed by $0.6 \mathrm{gm}$ daily for 8 weeks. Craig (1939) using this treatment finds not over 5 per cent of relapses. The League of Nations Malaria Commission on the other hand recommends 1.0 to 1.2 grams daily for 5 to 7 days, with treatment of relapses only 
when they occur. The arguments for and against these respective plans lie outside the scope of this paper.

With respect to the mode of action of quinine on the malarial plasmodium, it cannot be said that any real explanation exists. The earlier workers believed that the drug exerted a simple direct antiplasmodial action, and current texts on pharmacology to some extent still reflect this view. But the matter is not so simple. In the first place it has long been known that incubation of malarial blood with relatively high concentrations of quinine does not destroy the infectiveness of this blood in the production of therapeutic malaria (Kirschbaum 1923). The concentrations reached in the blood after either oral or intravenous administration are in the first place not high, and secondly they are not maintained (Chopra, Roy and Das Gupta 1934) for more than a very brief period. It is difficult, therefore, to think that the action of the quinine in the blood stream or on the blood cells is a direct one on the parasite. The suggestion has been made that the drug retards the reproduction rate of the parasites. Acton and Chopra (1927), for example, hold that "it probably hinders reproduction by the formation of a smaller number of merozoites." Krishnan (1933) added that quinine might increase the length of time necessary to complete an asexual cycle. There have been a number of experimental studies on bird malaria which indicate that for these forms at least such disturbances of reproduction occur, both by retardation of the cycle and reduction of the number of merozoites formed at schizogony (Manwell 1934; Boyd and Allen 1934; Lourie 1934; Boyd and Dunn 1939). Observations such as these do seem to offer an explanation of the puzzling observation that a drug like quinine, so effective in reducing the number of parasites, fails to destroy all of them. They are also consistent with the observation that quinine is ineffective in reducing the number of gametocytes. Boyd and Dunn (1939) emphasize the fact that these actions on reproduction in experimental malaria in birds are observed when quinine is given during the growth period of the trophozoites. In human beings, James (1933) found that pretreatment of the patient with quinine did not prevent or retard the development of naturally induced malaria.

The observations just listed do not offer a complete explanation of the action of quinine. At various times it has been held that some of the breakdown products of quinine might be responsible for the antimalarial action, since quinine itself is not very toxic for the malarial organism. Another way of getting around this difficulty has been to suggest that quinine favored the production or activation of some natural antibodies (Yorke and Macfie 1924b). Other observers have assigned to the reticuloendothelial system a role in the activity of quinine and other antimalarial drugs. This view is based on the finding that pretreatment with trypan blue, which blocks the reticulo-endothelial system, reduces the effectiveness of antimalarial drugs in the treatment of experimental infections (Kritschewski and Demidowa 1934).

While quinine is the alkaloid present in cinchona bark in greatest amount, other alkaloids are present to an extent justifying investigation of their effectiveness against malaria. Investigations of the effectiveness of these alkaloids have been complicated by the difficulty of obtaining completely pure specimens of the individual alkaloids. The complete separation of these alkaloids is apparently not feasible except as a research project, and the ordinary medicinal preparations are far from pure. In Table II are given the probable impurities, according to Dawson.

The first and still the most extensive comparative study is that of the Madras Cinchona Commission (1867-68). This work was done before the discovery of the malarial parasite, and the eriterion of cure was "cessation of febrile paroxysms." Although the alkaloids used certainly were not pure, the results obtained serve as convincing evidence that there is no great difference in clinical effectiveness of the four major alkaloids. In Table III is given a 
TABLE II

Approximate IMPURities in Medicinal Preparations

\begin{tabular}{|c|c|}
\hline Alkaloid & $\begin{array}{c}\text { Approximate percentage of } \\
\text { impurities }\end{array}$ \\
\hline Quinine & $\begin{array}{l}\text { Cinchonidine } 2 \% ; \text { Hydroqui- } \\
\text { nine } 1.5 \%\end{array}$ \\
\hline Quinidine & Hydroquinidine $6-30 \%$ \\
\hline Cinchonine & Hydrocinchonine $10 \%$ \\
\hline Cinchonidine & $\begin{array}{l}\text { Quinine } 10 \% \text {; Hydrocinchoni- } \\
\text { dine } 8 \%\end{array}$ \\
\hline
\end{tabular}

brief summary modified from Dawson's extensive discussion of this important report in the report of the Madras Cinchona Commission. These early observations have been essentially confirmed by the more recent workers in this field. The exact relationship varies, but it can be assumed that any of the four alkaloids listed is effective. In India, in the treatment of chronic benign tertian malaria, Sinton and Bird (1929) concluded that the percentage of cases showing relapse was about the same for the four alkaloids. According to Fletcher (1928) this is true not only for benign tertian, but also in estivo-autumnal infections. In the latter, however, cinchonine and cinchonidine are perhaps somewhat inferior. Quartan fevers yield to any of the four alkaloids. Since by far the largest amount of alkaloid in the currently available Dutch barks is quinine, the use of the other alkaloids is of importance only in those cases showing an idiosyncrasy to quinine. As already mentioned, in some cases at least, idiosyncrasy to quinine does not extend to its isomer of the opposite sign so that it is possible to treat malaria

TABLE III

Effectiveness of Four Chief Cinchona Alkaloids

\begin{tabular}{l|r|r|c}
\hline & $\begin{array}{r}\text { Total } \\
\text { cases }\end{array}$ & Cured & $\begin{array}{c}\text { Per cent } \\
\text { cured }\end{array}$ \\
\hline Quinine ....... & 846 & 840 & 99.2 \\
Quinidine ..... & 1040 & 1025 & 98.5 \\
Cinchonidine & 762 & 745 & 97.7 \\
Cinchonine ... & 969 & 946 & 97.6 \\
\hline
\end{tabular}

in an individual showing abnormal reactions to quinine with quinidine (Dawson et al. 1933).

No general discussion of the pharmacology of quinine or the related alkaloids seems desirable here. It should always be kept in mind that there are two groups of untoward reactions that may follow these alkaloids, namely, the symptoms of overdosage, or cinchonism, which may be expected in any individual if enough alkaloid is given, and the symptoms of idiosyncrasy, occurring in a small number of individuals, even from small doses. By cinchonism is meant the well known complex, characterized chiefly by headache, ringing in the ears, sensation of fullness in the head, disturbances of vision. While this complex is undoubtedly more easily brought on in certain individuals than in others, there is reason to believe that all individuals would show it after adequate dosage. Idiosyncrasy to quinine, however, is shown by a different group of symptoms. The most common of these are skin eruptions which may take a variety of forms. Other effects may be pseudo-asthmatic attacks, or gastrointestinal disturbances, with vomiting or diarrhea. Some patients according to Bastedo (1937) may show "typical anaphylactic phenomena with swelling of face, hands, and feet, a sense of suffocation, colic and prostration." The mechanism of idiosyncrasy is unknown. In many such cases, quinidine has been substituted successfully (Dawson et al. 1933).

Absorption of quinine or of its salts from the gastro-intestinal tract is reasonably rapid and probably complete. Quinine can be detected in the urine within 15 minutes after oral administration and it continues to be excreted by the kidney for some time. Excretion is most rapid in the first 24 hours, but traces occur in the urine for as long as three days. Only about one-third of that ingested can be recovered from the urine. The fate of the balance is unknown. After intravenous administration quinine disappears from the blood stream very rapidly, 95 per cent of it within 5 minutes. A part of this can be recovered from some 
of the organs-lungs, liver, kidneys, heart, and brain (Hatcher and Weiss 1926). A considerable amount, however, cannot be accounted for. Weiss and Hatcher (1927) believe that the liver destroys the greater part of an intravenous dose. Determinations of the quinine content of human blood after intravenous quinine therapy give results consistent with the above mentioned experimental findings. Hatcher and Gold (1927), for example, found only traces of quinine in 60 ce of blood after the intravenous administration of $650 \mathrm{mg}$ of quinine hydrochloride.

A detail of the pharmacology has to do with the effect of quinine or quinidine when given intravenously. Such a procedure is rarely necessary. According to Maxey (1928) such a route of administration has per se no superiority over the oral one, and should be reserved for occasions when oral administration is impossible. When quinine or quinidine is given intravenously there always results a fall in blood pressure, due in part to a depression and weakening of the heart muscle, and in part to a dilatation of blood vessels. The degree of fall is in part dependent on the concentration of the solution and in part on the rapidity of injection. Such an injection should therefore be carried out very slowly, and should be of a well diluted solution. The statement is commonly made that injections of epinephrine may be used to counteract the circulatory depression. It does not seem to be widely known that one of the characteristic actions of quinine and quinidine is to antagonize the pressor effects of epinephrine, so that it is very much less effective under these circumstances than is normally the case (Nelson 1927b).

Another point of practical concern in the pharmacology of quinine is its action upon the pregnant uterus. It is commonly believed to be hazardous to administer quinine to a pregnant patient. Further, quinine is credited with being an effective oxytocic substance and is often used by obstetricians for its stimulating action upon the uterus. Whether such an action actually exists may be questioned. Recently Reed (1940) has reiterated the view, previously expressed by others, that the danger from miscarriage in untreated malaria is greater than the likelihood of stimulation of the uterus to evacuation by the use of quinine. This reviewer, speaking as a pharmacologist and not as a clinician, has long felt that the oxytocic action of quinine was very much overestimated. There still remains, however, the possibility of injury to the fetus in utero by the administration of quinine.

There are a number of preparations of quinine available differing chiefly in their solubility. This matter of solubility probably modifies the taste more than it does the rate of absorption. The preparation that has had the most extensive use in the United States is the sulfate, which is a relatively insoluble form ( $1 \mathrm{gm}$ in $810 \mathrm{cc}$ water at $25^{\circ} \mathrm{C}$ ). Clinical experience certainly indicates that it is absorbed fairly readily and promptly. Quinine, the base, is only half as soluble as the sulfate, and is considerably less bitter. The tannate and ethyl-carbonate are still less soluble and practically tasteless. They make a satisfactory dosage form for administration to children. For intravenous administration there are three soluble preparations available, quinine dihydrochloride, quinine and urea hydrochloride, and the N.F. ampule of quinine hydrochloride and urethane. Since the size and frequency of dosage and the duration of treatment are taken up elsewhere in this volume, they will not be considered here.

Cinchona and its alkaloids have long played a most important role in the attack upon malaria. While the drug does not meet every requirement of modern medicine, it still remains one of the most powerful weapons in the physician's armamentarium, and certainly is not to be discarded until more effective substances are available. 


\title{
ANTIMALARIALS OTHER THAN QUININE
}

\author{
By HANS MOLITOR
}

MERCK INSTTTUTE FOR THERAPEUTIC RESEARCH, RAHWAY, NEW JERSEY

Medicinal agents other than quinine used in the treatment of malaria may be divided in three groups: (a) synthetic compounds with a specific chemotherapeutic action against the malaria parasites and structurally related to quinine; (b) synthetic compounds with a specific chemotherapeutic effect against malaria parasites, but structurally not related to quinine; (c) drugs of synthetic or natural origin with general chemotherapeutic activity, but not specifically directed against the malarial parasites.

The introduction of synthetic compounds with antimalarial properties, equal or superior to those of quinine, is one of the greatest triumphs of systematic chemotherapeutic research and the first real advancement in the field of antimalarial therapy since the introduction of quinine. The credit for this achievement belongs to a group of workers at the Research Laboratory of the I.G., the great German chemical trust. The work of these men, among whom Hörlein, Kikuth, Mietzch, Roehl, Schoenhofer, Schulemann and Wingler are prominent, opened new approaches to malarial therapy and led to the synthesis of thousands of compounds by chemists in all parts of the world. However, none of the compounds made since the introduction of Plasmochin in 1928 and Atabrine in 1933, has proved superior to these two agents; and for brevity's sake we may therefore confine our discussion to them.

\section{Plasmochin}

The structure of plasmochin is chemically somewhat related to that of quinine, being an amino quinoline derivative ( $\mathrm{N}$-diethylaminoisopentyl-8 - amino - 6 - methoxyquinoline $\left(\mathrm{C}_{2} \mathrm{H}_{5}\right)_{2} \mathrm{~N}\left(\mathrm{CH}_{2}\right)_{3} \mathrm{CH}\left(\mathrm{CH}_{3}\right) \mathrm{NH}$ $\left.\mathrm{CH}_{3} \mathrm{O} \mathrm{C}_{9} \mathrm{H}_{5} \mathrm{~N}\right)$. However, Schulemann and his co-workers (1927) who synthesized the compound, arrived at it not through an attempted modification of the original quinine molecule, but rather through an alteration of the structure of methylene blue, the antimalarial properties of which had already been recognized by Ehrlich in 1891.

Plasmochin is one of the most specific and potent antimalarials. Its effective dose in Proteosoma praecox infected canaries, when administered daily by mouth for 6 days, is $0.02 \mathrm{mg}$, and its lethol dose is $0.6 \mathrm{mg}$ per bird (Roehl 1926). The corresponding figures for quinine hydrochloride are $1.25 \mathrm{mg}$ and $5.0 \mathrm{mg}$, respectively. Thus, its chemotherapeutic index is $1: 30$, as compared to that of quinine 1:4. In experiments in vitro, a concentration of plasmochin of $0.2 \mathrm{mg}$ per ec will kill $P$. praecox after incubation for 1 hour, as compared to $0.5 \mathrm{mg}$ per ec of quinine (Borchardt 1930). In a comparison of the effect of quinine and plasmochin on proteosoma infected canaries, a peroral dose of 0.01-0.1 gm of plasmochin greatly reduced the number of merozoites as well as their rate of formation, while a peroral does of 0.25 to $0.5 \mathrm{gm}$ of quinine only reduced their number, but did not materially alter their rate of formation (Boyd and Dunn 1939). Although exerting but slight effect on schizonts of the parasites of tertian and quartan, it possesses a powerful action against the gametocytes of $P$. falciparum (Roehl 1926; Kikuth 1932) and even in minimal doses renders them non-infectious for mosquitoes. (Barber, Komp and Newman 1929b). This gametocidal effect is not manifested by quinine, which possesses only antischizontic properties. The systematic administration of plasmochin to carriers of tropical malaria should greatly aid in the eradication of this disease through the destruction of the sexual forms of the 
parasites, which are necessary for the propagation of the disease in the carrier mosquito. However, the fact that the administration of plasmochin is safe only under constant medical supervision presents a serious obstacle to this form of malarial control. Unlike as in quinine prophylaxis, it seems generally agreed that the administration of plasmochin must be restricted to patients who can be seen regularly by a physician.

Toxicity. The toxicity of plasmochin has been investigated in most laboratory animals (LeHeux and van Wijndgaarden 1929; Tskimanauri 1931). It varies considerably with the species, not only quantitatively but also qualitatively. Two forms of symptoms are generally observed, one with an acute onset of the toxic signs shortly after administration of the drug and a preponderance of nervous, circulatory and respiratory disturbances; the other, a more delayed type, with cyanosis, methemoglobinemia and eventual respiratory failure. The acute type prevails in rabbits and canaries, while in mice, cats and dogs more often the delayed type is found; animals, which have recovered from the shock immediately following administration of a lethal dose of plasmochin may still succumb several hours or even days later from methemoglobinemia. This is particularly the case after peroral administration of the drug.

The difference between the peroral, subcutaneous and intravenous toxicity of plasmochin is not very great except in the rabbit, for which the peroral toxic dose is 60 to 70 times, the subcutaneous 5 to 6 times, larger than the intravenous. Prolonged intravenous or subcutaneous administration of plasmochin in therapeutic doses causes no pronounced toxic effects (Tskimanauri 1931). After the first few doses, the animals show a temporary loss of weight which, however, later becomes stabilized. Combination of plasmochin with quinine (known as Quinoplasmin or plasmochinum compositum) which has often been advocated in order to improve the therapeutic effectiveness, does not change the toxicity of plasmochin (Le Heux and de Lind van Wijndgaarden 1928).

The pathological findings in animals dying from excessive doses of plasmochin are unspecific and due mainly to the methemoglobinemia. They consist of a brownish discoloration of the lungs, muscle, omentum, and mucous membranes. In dogs, cats and canaries the intestines are hyperemic and the blood vessels of the adbominal region are markedly dilated.

The formation of methemoglobin is one of the most pronounced toxic effects of plasmochin. It occurs in man after daily administration of $0.08-0.1 \mathrm{mg}$ (Fischer and Rheindorf 1928) and is readily observed in most animal species (horses, cows, sheep, pigs, cats, dogs), with the exception of rabbits and mice, in which it occurs only after the blood has been hemolyzed (Le Heux and de Lind van Wijndgaarden 1928). The minimal blood concentration of plasmochin causing methemoglobin formation is $1: 12,500$ in cats and dogs, $1: 1500$ in rabbits. In the latter, peroral doses of $6 \mathrm{mg}$ per $\mathrm{kg}$ convert up to 7 per cent; $22 \mathrm{mg}$ per $\mathrm{kg}$ up to 40 per cent of the total hemoglobin into methemoglobin (Messini 1928). However, the methemoglobin formation is reversible in vivo as well as in vitro (Le Heux and de Lind van Wijndgaarden 1928) ; all signs disappear after discontinuation of the drug (Messini 1928) and the process is not associated with an increased destruction of erythrocytes or damage of the liver, as evidenced by negative bilirubin and urobilinogen tests even at the height of the methemoglobinemia. Plasmochin in concentrations greater than 1:5000 causes hemolysis in vitro (Le Heux and de Lind van Wijndgaarden 1928).

The effect of plasmochin on the blood pigments is not its only toxic property. Far more serious is its adverse effect upon the circulatory system, which is found in all species, including man, and which may be observed in the isolated organs as well as in situ. In the isolated frog and rabbit's heart concentrations of $1: 250,000 \mathrm{up}$ to $1: 1$ million induce irregular beat; 1: 100,000 causes an irreversible diastolic 
standstill (Le Heux and de Lind van Wijndgaarden 1928). In the heart-lung preparation, concentrations as small as 1: 500,000 greatly decrease the ability of the heart to overcome increased peripheral resistance and a concentration of $1: 50,000$ induces diastolic standstill. The minimal concentrations causing such an effect are, for plasmochin, 1:2000; for atabrine, 1:500; and for quinine, 1:300. Plasmochin, in small concentrations, causes vasoconstriction; in large concentrations, vasodilatation in the frog's leg, rabbit's ear, coronary and pulmonary vessels. It lowers the blood pressure, primarily due to a direct effect on the heart. In a comparison of plasmochin with atabrine and quinine in the electrocardiogram of man and dog, all three drugs were found to be negative chronotropic and negative dromotropic. All three cause in the isolated monkey-heart irregular beat and diastolic standstill (de Langen and Storm 1935). In contrast to quinine, which in submaximal doses increases the minute volume and does not affect the vasomotor center, both plasmochin and atabrine in comparable doses decrease the minute volume and impair the function of the vasomotor center.

The pharmacological effects of plasmochin on other organs or organ systems are not pronounced. It does not alter the diuresis (Le Heux and de Lind van Wijndgaarden 1928) and, up to sublethal doses, does not influence experimental fever, such an induced by the injection of Bacillus coli or puncture of the temperature regulating center in the midbrain (Girndt 1929). Even if injected into the brain or directly applied to the temperature center, it lowers the body temperature only for the duration of the severe toxic effects.

Plasmochin is probably rapidly absorbed from, and not to any great extent destroyed in, the gastro-intestinal tract, since the toxic signs follow very rapidly its peroral administration. It appears in the urine within 20 minutes after administration, but the amount excreted is only small, e.g., of a total of $127.5 \mathrm{mg}$ administered only 2 $\mathrm{mg}$ were excreted during the first 2 days; probably it is quickly destroyed in the tissues.

\section{ATAB'RINE}

Atabrine was synthesized in 1933 by Mietzch and Mauss. It is an acridin dye of the formula 2-methoxy-6-chloro-9- $\alpha$-diethylamino- $\delta$-pentylaminoacridin and is structurally not related to quinine. It is a powder of light yellowish color, with a M.P. of $245-255^{\circ} \mathrm{C}$. In neutral solution it is readily water soluble, at room temperature up to 2.5 per cent; at $40^{\circ} \mathrm{C}$ up to 7 per cent. In ultraviolet light it shows a strong fluorescence which is easily detectable in aqueous solutions up to 1 part in a million. In its antimalarial action it resembles quinine far more than plasmochin, having the pronounced antischizontic effect of the former and lacking the gametocidal effect of the latter (Kikuth and Giovannola 1933). Its activity in proteosoma infected canaries is about 4 times greater than that of quinine but 15 times less than that of plasmochin. Its chemotherapeutic index is $1: 30$, the same as that of plasmochin.

The toxicity of atabrine varies considerably with different species and with the mode of administration, ranging from 200 $\mathrm{mg}$ per $\mathrm{kg}$ in cats and mice to $500 \mathrm{mg}$ per $\mathrm{kg}$ in rabbits (Koyu 1937; Hecht 1933). Particularly noteworthy is the great variation in toxicity with different methods of administration. Thus the intravenous lethal dose is 20 to 40 times smaller than the peroral, depending greatly upon the rate of injection (Dawson, Gingrich and Hollar 1935); e.g., intravenous injection of $5 \mathrm{mg}$ per $\mathrm{kg}$ to dogs over 30 to 60 seconds resulted in the death of 21 per cent of the animals while a similar injection of an even greater dose (7.5 mg per $\mathrm{kg}$ ), but over a period of 60 to 90 seconds, caused only 4.8 per cent deaths. Because of the marked increase in toxicity upon intravenous injection it has been suggested that for therapeutic purposes this mode of administration should be avoided except in emergencies. The cumulative effect of atabrine and its very slow and prolonged excretion are additional factors which suggest caution 
in its administration. Atabrine has been found in the urine of patients as late as 9 weeks after the termination of the course of treatments (Roehl 1926), and it has therefore been suggested that treatment should not be repeated within an interval of less than 8 weeks and that the total amount given in any one period of treatment should not exceed $2 \mathrm{gm}$ ( $0.3 \mathrm{gm}$ per day).

Cumulative effects have been observed in animals (Martin, Cominole and Clark 1939; Dawson et al. 1935). Thus, with a single peroral administration the lethal dose of atabrine in dogs was $300 \mathrm{mg}$ per $\mathrm{kg}$, but a dose of $100 \mathrm{mg}$ per $\mathrm{kg}$ was fatal when administered daily over 15 to 27 days, and even a dose as small as $10 \mathrm{mg}$ per $\mathrm{kg}$ resulted in a loss of weight in all dogs.

The signs of atabrine poisoning are anorexia, salivation, vomiting, epigastric pain of colicky type, occasional headache and yellowing of the skin (the latter due to the staining properties of the drug and not to liver damage (Kingsbury 1934)). Occasionally psychic disturbances have been reported after administration of relatively small therapeutic doses (6 to 15 tablets) starting within $1 \frac{1}{2}$ to 9 days after initiation of the treatment and lasting up to several days. However, while in these cases the mental disturbances were sufficiently severe to necessitate hospitalization in a mental asylum, the frequency as well as the danger of this complication is rather small (Craig 1939). The cause of this side-effect, which is not found with other antimalarials, is not yet definitely established; it may be due to a direct effect of the drug on the brain or to an increased liberation of parasitic toxins during the first few days of treatment.

The pathological findings in atabrine poisoning consist of a marked hyperemia and toxic central injury to the liver and a fatty degeneration of the renal tubules.

In liver and kidney function tests on dogs it was found (Clark, Cominole and Martin 1939) that the degree of liver impairment depends upon the dose and the length of administration. The kidney function, tested by urea clearance, non-protein nitrogen in blood and phenolsulphonphthalein, showed no evidence of damage in 11 out of 12 dogs after daily administration of 17 to 66 per cent of the minimum lethal dose for a period up to 40 days.

In experiments with the Warburg method on isolated brain and testicular tissue atabrine did not depress the oxygen consumption, indicating that it is not a general protoplasma poison. Unlike plasmochin it does not cause methemoglobin formation or hemolysis in vitro or in vivo (Hecht 1933).

Atabrine is rapidly absorbed from the gastrointestinal tract and within a few minutes after administration is found evenly distributed over the body tissues (Hecht 1936). After $2 \frac{1}{2}$ hours a greater concentration is found in the liver, gall bladder and intestines, and it accumulates subsequently in the duodenum and upper ileum, while only little is found in the lower portion of the small intestine and in the feces. After 5 days it is still detectable in the gall bladder and upper portion of the gastrointestinal tract and it requires 7 to 8 days before the last traces disappear from all organs. The accumulation of atabrine in the liver offers an explanation for the great difference between the peroral and intravenous toxicity (Blaze and Simeons 1935). Atabrine is probably selectively adsorbed by the erythrocytes and parasites, since it has been shown (Fischl and Singer 1934) that even washing with alcohol fails to extract it completely from blood.

Atabrine is only occasionally found in the cerebrospinal fluid and appears in the brain only after injection of lethal doses. It is excreted in part in chemically unchanged form; the amounts present in the urine and feces are about of the same order of magnitude. After discontinuation of the administration, the excretion drops to a low level which is maintained for several days (Tropp and Weiss 1933).

The distribution of atabrine in various body fluids and tissues has been studied in cats, dogs and rabbits with an extremely sensitive method, capable of detecting 0.5 micrograms in 1 gram organ (Hecht 1936). 
After 10 successive daily peroral administrations of $50 \mathrm{mg}$ per $\mathrm{kg}$ to dogs, the maximal concentrations were found in the spleen (2.75 $\mathrm{mg}$ per gram), the kidney (2.5 $\mathrm{mg}$ per gram) and the liver $(2 \mathrm{mg}$ per gram); the minimal in the skin $(0.11$ $\mathrm{mg}$ per gram) the muscle $(0.07 \mathrm{mg}$ per gram) and the brain (0.035 $\mathrm{mg}$ per gram). Three weeks after a course of 5 daily intravenous administrations, the largest amounts were in the lungs $(0.08 \mathrm{mg}$ per gram), the liver $(0.01 \mathrm{mg}$ per $\mathrm{kg})$, the kidney (0.004 $\mathrm{mg}$ per gram) and the spleen $(0.0025 \mathrm{mg}$ per gram). The organs in which the greatest amount is deposited vary with the mode of administration. Thus, the proportion of the atabrine content in lungs, kidneys, and liver is after intravenous injection $3: 2: 1$, whereas after peroral administration this proportion changes to $1: 1: 10$.

The pharmacological effects of therapeutic doses of atabrine on various organs and organ systems are not characteristic. Toxic doses affect the central nervous system, causing clonic convulsions, and depress the respiratory and circulatory systems. The blood pressure is lowered (Koyu 1937), general vasodilatation takes place and the electrocardiogram shows after administration of small doses slight changes in rhythm and a disturbance in the atrioventricular conductivity ; large doses induce paroxysmal tachycardia, 2:1 heart bloc, ventricular flutter and fibrillation (Motta 1937). In the isolated monkey heart atabrine dilates the coronary vessels, and causes extrasystoles and rhythmic disturbances (Storm 1935), which respond favorably to intravenous injection of adrenalin. Since a similar beneficial effect of adrenalin is observed in the heart in situ (Storm 1935), it has been recommended that whenever atabrine is injected intravenously, adrenalin should be added to the injection fluid in a ratio of $0.5 \mathrm{cc}$ of the standard 1:1000 solution of adrenalin to each ce of a 3 per cent solution of atabrine. An additional advantage of such a combination is the vasoconstrictor effect of ad- renalin on the spleen which aids in the mobilization of hidden parasites.

Atabrine exerts a moderately strong antipyretic effect on experimental fever produced by the injection of $B$. coli; in the eat $0.1 \mathrm{gm}$ per $\mathrm{kg}$ lowers the temperature by 0.6 to $0.9^{\circ} \mathrm{C}$, the lowest point being reached 2 hours after the peroral administration. Smaller doses cause occasionally transitory hyperthermia.

The effect of atabrine on the uterus has been investigated in the isolated organ as well as in situ in pregnant and non-pregnant guinea pigs, rabbits, and cats (Sapeika 1934). In the isolated organ, concentrations of $1: 200,000$ increase the muscular tone and frequency of contraction, while 1: 20,000 decreases both and renders the uterus irresponsive to adrenalin, pilocarpine and barium chloride. Intravenous injection of 1 to $4 \mathrm{mg}$ per $\mathrm{kg}$ in a pregnant eat is followed by a gradual increase of uterine contractions, which however return to the norm after a few minutes. Since these doses are proportionately many times larger than those administered for therapeutic purposes in man and since furthermore the latter are given by mouth and not injected intravenously, it is generally felt that in contrast to quinine the use of atabrine in pregnancy is not contraindicated.

It would seem justified to limit a discussion of effective antimalarials to the quinine, plasmochin and atabrine group. Optimal therapeutic results may be obtained with these and the great number of other agents still used in the treatment of malaria consist either of once popular drugs which are prescribed through force of habit or of new chemicals in the state of clinical investigation. Although quinine and atabrine control effectively the schizonts of the malarial parasites and plasmochin is equally effective as a gametocide in $P$. falciparum infection, none of them can be regarded as a true chemoprophylactic agent. The ideal antimalarial still has to be found and it is therefore obvious that every new chemotherapeutic agent is being 
tried in malarial infections. Methylene blue, inorganic and organic arsenicals, acridin dyes, bismuth preparations, antimony compounds have all been used with some degree of success. However, none of them approaches in efficacy and safety the performance of the specific antimalarials previously discussed; and interest in them today is only historic.

The situation is different with chemotherapeutic agents belonging to the socalled organic. sulfur compounds. This group, of which prontosil was the first representative, consist now of thousands of synthetic compounds, among which sulfapyridine, sulfanilamide, and sulfathiazole are the best known. Some of its members have been tried in the therapy and prophylaxis of malaria, but no definite conclusions as to their usefulness in this field have as yet been reached. While none of the compounds so far investigated compared favorably with quinine, plasmochin or atabrine, the number of those which have undergone sufficient trials is still very limited. Certain experimental and clinical findings seem to justify a systematic investigation of this group, particularly for its value in chemoprophylaxis. Not only have favorable clinical results been reported following the use of sulfonamide compounds in tertian, quar$\tan$ and $P$. falciparum malaria (de Leon 1937; Hill and Goodwin 1937; van der Wielen 1937, Chopra, Hayter and Sen 1939; Chopra, Das Gupta, Sen and Hayter 1939; Farinaud and Ragiot 1939), but it has been shown that sulfapyridine destroys the sexual as well as the asexual forms of $P$. vivax and that no relapses of the patients occur after treatment with large doses of this drug. In simian malaria sulfanilamide (Coggeshall 1938c), soluseptazine (Chopra and Das Gupta 1938) and sulfapyridine (Chopra and Das Gupta 1939) were found to possess not only curative but also prophylactic value. For soluseptazine the latter property has also been reported in man (Sinton, Hutton and Shute 193a). However, since in these experiments the prophylactic dose was rather large and the duration of the protection did not exceed 32 hours, these findings have no practical significance and are important only from a theoretical viewpoint because they represent the first instance of a chemoprophylactic effect in man with a non-toxic dose of an antimalarial. Although successful experiments along similar lines have been reported with plasmochin (James 1937), the doses required were distinctly in the toxic range.

In view of the fact that effective prophylaxis is the goal of all malarial control and that chemoprophylaxis is bound to play one of the most important parts in this work, the future efforts of research workers will probably be directed towards the finding of new compounds with prophylactic properties, rather than towards further modifications of quinine, plasmochin or atabrine which, although therapeutically highly effective, possess no chemoprophylactic properties. This work will necessitate not only the most careful investigation of possible damages produced by very prolonged administration, but also the development of a dependable and practical method for the testing of prophylactic properties. Whether organic synthesis will provide us with the new agents or whether they will be isolated from one of the many plants used in the native tropical medicine, such as Gelipea jasminiflora (Peckolt 1899a), Tachia guanensis (Peckolt 1899b), Helianthus (Danzel 1929), etc., only future research will show. Up to this time only the synthetic compounds belonging to the sulfanilamide group have offered some promise, but it must be remembered that the great majority of tropical plants has not yet been investigated except in a superficial and incomplete manner. Existing reports are usually many years old and based on questionable statements collected from natives by explorers who themselves were laymen in the fields of pharmacology and medicine. Pharmacological analyses have been carried out either with inadequate methods or under conditions which do not exclude the possibility of missing 
chemotherapeutic properties because of of the bird malaria test has been subjected decomposition of the active principle or lack of proper pharmacological technique. Almost none of the hundreds of alkaloids and glucosides isolated from tropical plants during the period prior to the introduction to a pharmacological analysis which today could be regarded as adequate. It may therefore be justified to expect unforeseen and valuable results from a thorough reinvestigation of this field. 


\title{
EXPERIMENTAL CHEMOTHERAPY IN MALARIA By JOHN MAIER
}

\author{
LABORATORIES OF THE INTERNATIONAL HEALTH DIVISION, ROCKEFELLER FOUNDATION,
} NEW YORK

Present methods of malarial control are inapplicable in many regions of the world because of the high cost of antimosquito or antilarval measures. In these areas the use of antimalarial drugs probably constitutes the only practical control measure. Clark and his associates in Panama (1940) have shown that the intensive and widespread use of quinine and atabrine over a period of 9 years has not significantly altered the incidence of malaria; indeed, an epidemic occurred during the 9 th year of the study. There is no question of the value of quinine or atabrine from the point of view of the individual with acute malaria. However, from the broader aspect of the control of malaria as a world problem, antimalarial drugs have been deficient.

The failure of drug control of malaria is attributable principally to two well recognized limitations: (1) The present antimalarial drugs do not have any specific prophylactic action against the sporozoite. (2) They do not always sterilize the acute or chronic infection. This permits the occurrence of clinical and parasitic relapses, and the accumulation of large reservoirs of carriers who are periodically infective for mosquitoes.

It is possible that a drug superior in either respect to quinine, plasmochin or atabrine would be more efficient in malarial control. Further research in malarial chemotherapy is desirable, in the hope of discovering such a drug. Several considerations as to the best procedure are apparent. First, many thousand quinoline and acridine derivatives have already been synthesized and tested in the research which led to the discovery of plasmochin and atabrine. These latter drugs, together with quinine, may be regarded as the best of their type. Further testing of related chemicals does not offer much hope of suc- cess. Accordingly, the best method of approach is the routine testing of unrelated chemicals until one is discovered which has an effect on either the sporozoite, trophozoite, or gametocyte. The only requirement known for a potential antimalarial drug is that it must be able to penetrate the red cell as suggested by Hegner et al. (1928) and Shaw (1928). When some degree of activity has been found in a compound, all its derivatives can then be investigated. Up to this point, however, the work must be more or less empirical. No completely rational selection of chemicals is possible because the mode of action is not known for any chemotherapeutic agent.

Methods available for testing antimalarial drugs are as follows:

1. In vitro testing

2. Experimental malaria

a. Monkey malaria

b. Canary malaria

c. Chick malaria

3. Human malaria

a. Autochthonous infections

b. Induced infections in pareties

\section{In Vitro Testing}

Because of the expense and difficulty of using large numbers of animals, a reliable in vitro test would greatly facilitate the testing of drugs. Fulton and Christophers (1938) found that quinine and atabrine produced inhibition of respiration of $P$. knowlesi as measured in a Warburg manometer. This method was studied as a means of evaluating potential antimalarial drugs (Coggeshall and Maier 1941). One ce of blood from a heavily infected monkey is placed in a conical Warburg flask with 2 ec of $\mathrm{M} / 15$ phosphate buffer, $\mathrm{pH}$ 7.35. An accurately weighed sample (10 to $20 \mathrm{mg}$ ) of the drug to be tested, dissolved or suspended in 0.5 ec of Ringer's solution, is 
placed in the side-arm of the flask, which is then connected with the manometer and shaken in a water bath at $37^{\circ} \mathrm{C}$. The oxygen uptake is measured for an hour in order to be sure that all flasks have the same rate of respiration. The drug is then tipped in from the sidearm and the respiration measured for an hour, with two flasks without drug as control. The oxygen uptakes are calculated per hundred million parasites so that all experiments are comparable. The difference between the oxygen uptake in the control flask and in the drug flask, divided by the uptake of the control flask, gives a percentage coefficient of inhibition for the drug used.

Results of representative experiments are shown in Table $I$. Here it is seen that and sulfadiazine, which also produce sterilization of the infection in the host, produce no inhibition of respiration in vitro. This is to be expected from the nature of the two compounds. Sulfadiazine is insoluble, only $12 \mathrm{mg}$ per $100 \mathrm{cc}$ dissolving at $37^{\circ} \mathrm{C}$, and therefore would not be expected to show activity in vitro. In the monkey it is apparently converted into a soluble derivative since the effect is just as great as that of sodium sulfathiazole or sulfanilamide. Prontosil is a complex azo dye of which the sulfanilamide molecule is a part. In vitro it has no activity; in vivo it is converted into sulfanilamide (Trefouël 1935 et al.; Fuller 1937) and is fully as effective as this drug.

Further experiments summarized in

TABLE I

Chemotherapeutic Activity of Various Drugs Against $P$. knowlesi

\begin{tabular}{|c|c|c|c|c|c|c|}
\hline \multirow{2}{*}{ Drug } & \multirow{2}{*}{\multicolumn{2}{|c|}{$\begin{array}{l}\text { Concentration } \\
\text { in flask }\end{array}$}} & \multicolumn{2}{|c|}{$\begin{array}{c}\mathrm{O}_{2} \text { uptake } \\
\left(\mathrm{mm}^{3} / \mathrm{hr} / 10^{8} \text { parasites }\right)\end{array}$} & \multirow{2}{*}{$\begin{array}{c}\text { Coefficient } \\
\text { of } \\
\text { inhibition } \\
\text { I-II/I }\end{array}$} & \multirow{2}{*}{$\begin{array}{l}\text { Therapeutic } \\
\text { effect }\end{array}$} \\
\hline & & & $\begin{array}{l}\text { Control } \\
\text { flask } \\
\text { I }\end{array}$ & $\begin{array}{c}\text { Flask } \\
\text { with drug } \\
\text { II }\end{array}$ & & \\
\hline \multirow{3}{*}{ Quinine dihydrochloride ...... } & $m g$ & molar & $m m^{2}$ & $m m^{3}$ & & \\
\hline & 1.6 & 0.001 & 15.1 & 9.7 & 36 & H \\
\hline & & & 10.3 & 6.7 & 35 & \\
\hline \multirow[t]{2}{*}{ Atabrine } & ....... & 0.001 & 27.2 & 9.0 & 67 & $H$ \\
\hline & & & 15.1 & 4.4 & 71 & \\
\hline \multirow[t]{5}{*}{ Sodium sulfathiazole ............. } & 10 & 0.01 & 19.3 & 9.8 & 49 & $H+$ \\
\hline & & & 19.3 & 10.9 & 44 & \\
\hline & & & 11.2 & 6.8 & 40 & \\
\hline & & & 32.0 & 18.0 & 44 & \\
\hline & & & 25.7 & 15.3 & 41 & \\
\hline Sodium sulfapyridine ............. & 10 & 0.012 & 14.2 & 7.9 & 44 & $H$ \\
\hline \multirow[t]{2}{*}{ 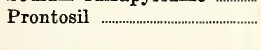 } & 10 & 0.004 & 27.2 & 28.0 & 0 & $H$ \\
\hline & 20 & 0.008 & 27.2 & 26.6 & 0 & \\
\hline Sulfadiazine & 10 & 0.010 & 23.0 & 23.0 & 0 & $H+$ \\
\hline
\end{tabular}

+ Arrest of acute infection without sterilization.

+ Sterilization of acute or chronic infections.

0.001 M quinine dihydrochloride inhibits the respiration of $P$. knowlesi from 35 to 36 per cent; $0.001 \mathrm{M}$ atabrine produces 67 to 71 per cent inhibition. The inhibition produced by sodium sulfathiazole (40 to 49 per cent) is approximately the same as that produced by sodium sulfapyridine (44 per cent); both these drugs produce complete sterilization of the infection in the rhesus monkey. However, prontosil
Table II show the effect of $0.01 \mathrm{M}$ sodium sulfathiazole against various plasmodia. It is seen that the inhibition of respiration produced for $P$. inui and $P$. cynomolg $i$ is 58 and 50 to 51 per cent, respectively, which is greater than the inhibition produced for $P$. knowlesi. This is in contrast to the activity of the drug against the three infections in vivo. It sterilizes $P$. knowlesi infections completely, but when given in 
TABLE II

The Chemotherapeutic ACtivity of $0.01 \mathrm{M}$ Sodium Sulfathiazole Against VARIOUS Plasmodia

\begin{tabular}{|c|c|c|c|c|}
\hline \multirow{2}{*}{$\begin{array}{l}\text { Plasmo- } \\
\text { dium }\end{array}$} & \multicolumn{2}{|c|}{$\begin{array}{c}\mathrm{O}_{2} \text { uptake } \\
\left(\mathrm{mm}^{3} / \mathrm{hr} / 10^{8}\right. \\
\text { parasites })\end{array}$} & \multirow{2}{*}{$\begin{array}{c}\text { Coeffi- } \\
\text { cient } \\
\text { of } \\
\text { inhibi- } \\
\text { tion } \\
\text { I-II/I }\end{array}$} & \multirow{2}{*}{$\begin{array}{l}\text { Thera- } \\
\text { peutic } \\
\text { effect }\end{array}$} \\
\hline & $\begin{array}{c}\text { Con- } \\
\text { trol } \\
\text { flask } \\
\text { I }\end{array}$ & $\begin{array}{c}\text { Flask } \\
\text { with } \\
\text { drug } \\
\text { II }\end{array}$ & & \\
\hline P. cathe- & $m m^{3}$ & $m m^{3}$ & & \\
\hline merium ... & $\begin{array}{r}9.5 \\
21.4\end{array}$ & $\begin{array}{r}6.1 \\
13.7\end{array}$ & $\begin{array}{l}36 \\
36\end{array}$ & 0 \\
\hline $\begin{array}{l}\text { P. cyno- } \\
\quad \text { molgi }\end{array}$ & $\begin{array}{l}5.9 \\
2.2\end{array}$ & $\begin{array}{l}2.9 \\
1.1\end{array}$ & $\begin{array}{l}51 \\
50\end{array}$ & + \\
\hline $\begin{array}{l}P . \text { inui } \\
P \text {. lophura... }\end{array}$ & $\begin{array}{r}6.4 \\
12.2\end{array}$ & $\begin{array}{l}2.7 \\
9.5\end{array}$ & $\begin{array}{l}58 \\
22\end{array}$ & $\begin{array}{l}+ \\
0\end{array}$ \\
\hline
\end{tabular}

0 No effect.

+ Temporary diminution in eirculating parasites.

the same dosage and the same manner to monkeys with $P$. cynomolgi or $P$. inui infections, it produces only a temporary diminution in number of circulating parasites, without sterilization.

Sodium sulfathiazole also produces inhibition of respiration of $P$. cathemerium ( 36 per cent) and $P$. lophurae (22 per cent). It has no demonstrable effect on the infections produced by these parasites in canaries and chickens, respectively.

It can be stated, therefore, that inhibition of in vitro respiration cannot be used as a sole guide to the chemotherapeutic efficiency of a drug, but should be used only in conjunction with other information. The in vitro method is valuable for comparing the effectiveness of closely related compounds in a series. However, its usefulness is limited to relatively soluble chemicals of simple structure, active in their original form, and not converted from an inactive to an active form in the body. Since the mode of action of sulfanilamide and its derivatives is not known, there is no explanation of the lack of correlation between the marked in vitro effect on the respiration of $P$. inui and $P$. cynomolg $i$ and the slight in vivo effect. Similarly, there is no explanation for the complete lack of effect of sodium sulfathiazole upon $P$. cathemerium and $P$. lophurae infections, although the drug inhibits the respiration of both parasites in vitro. It can only be said that some factor other than a direct effect of drug on parasite as evidenced by inhibition of respiration, is responsible for the sterilization of $P$. knowlesi infections.

\section{Experimental Malaria}

It is apparent from these facts that in vivo testing is necessary to exclude inactive drugs and to give some indication of the activity of effective drugs to be tested further. Of the common laboratory animals, rhesus monkeys, canaries, and chicks are available for testing purposes.

a. Monkey malaria. Three plasmodia which are infectious for monkeys are available. Of these, $P$. knowlesi is the best for drug testing because of the highly standardized type of infection which it produces. It causes death in 5 to 6 days from the time of appearance of circulating parasites in over 99 per cent of normal animals. Hence, any animal which lives after being given a drug can be said to have been protected by that drug, within a very small margin of error. Sulfanilamide was shown by Coggeshall (1938b) to sterilize $P$. knowlesi infections in rhesus monkeys. Since then sulfathiazole, sulfapyridine, prontosil, neoprontosil, and sulfadiazine have been shown to be equally effective. The only sulfauilamide derivative which has not produced complete sterilization of this infection has been quinine sulfanilamide bisulfate. Its failure is probably due to the fact that only 28 per cent of the molecule is sulfanilamide, and doses large enough to include the necessary amount of sulfanilamide are fatal.

There is no indication that any of these drugs have any significant effect on human malaria. There is apparently some characteristic peculiar to $P$. knowlesi which renders it susceptible to sulfanilamide and allied compounds. Perhaps the fact that it is one of the most virulent of the malarias makes it more readily affected by a drug, 
just as only virulent strains of hemolytic streptococei are susceptible to sulfanilamide.

$P$. cynomolgi produces a milder infection in the rhesus monkey, never fatal in our experience, and characterized by a chronic course of long duration, with a few parasites (2 to 10 per 10,000 red cells) almost constantly in the circulating blood over a period of months. $P$. inui produces the same type of infection but of shorter duration. $P$. cynomolgi can be transmitted by A. quadrimaculatus, which makes it possible to test drugs for effect on the sporozoites of this parasite. These parasites are much less susceptible to sulfanilamide and its derivatives than is $P$. knowlesi. By giving $0.5 \mathrm{gm}$ intraperitoneally and $1.0 \mathrm{gm}$ by mouth on 2 successive days, it is usually possible to free the blood temporarily of circulating parasites, which, however, reappear in about 2 weeks, so that there is no sterilization comparable to that achieved with $P$. knowlesi. With $P$. cynomolgi and $P$. inui infections sterilization is the only reliable criterion on which to evaluate a drug inasmuch as the course of the infection is so variable. For this reason, they are less satisfactory for chemotherapeutic testing than $P$. knowlesi infections where protection from death can be regarded as demonstrating the effect of a drug.

b. Canary malaria. Canaries are more satisfactory for the routine testing of large numbers of new drugs of unknown toxicity. The Hartman strain of $P$. cathemerium produces a standardized type of infection to which normal canaries are uniformly susceptible. The acute infection results in parasite counts of varying height and may progress to death or to a crisis after which parasites disappear from the blood stream within 1 or 2 days. Because the behavior of a given acute infection cannot be predicted or controlled, we do not use its response to treatment in the evaluation of the effectiveness of a drug. Increase in the incubation period of the infection, with retarded appearance of the parasites in the blood stream, is a better index, uncompli- cated by factors of immunity, as suggested by Roehl (1926). Birds are inoculated in the pectoral muscle with 0.02 ee of blood from a heavily infected canary (parasite count 1000 per 10,000 red cells or more). Parasites first appear in the circulating blood in 3 to 5 days. Solutions or suspensions of drugs to be tested are fed by stomach tube (No. 9 soft rubber eatheter attached to a tubereulin syringe) for 6 days, beginning on the day of inoculation. Delay in the time of appearance of the parasites is taken as a measure of effectiveness against the parasite. Quinine dihydrochloride ( $2 \mathrm{mg}$ per day per bird) when given in this manner will delay the incubation period to 15 days or more. Birds treated with atabrine (1 $\mathrm{mg}$ per day per bird) or plasmochin (2 mg per day per bird) usually fail to show circulating parasites for periods up to a month, which is as far as experiments have been carried. Such birds in several cases were successfully reinoculated, proving that they had been protected against the infection completely.

It is also readily possible to obtain sporozoites of $P$. cathemerium from Culex pipiens in order to test drugs for prophylactic action.

Sodium sulfathiazole given in very large doses (62.5 mg per day per bird) failed to prolong the incubation period over that of the controls. In addition, the parasite count rose to levels as high as those found in the controls. $P$. cathemerium seems to resemble human plasmodia closely in its behavior toward drugs and should be a more reliable guide in chemotherapeutic testing than $P$. knowlesi seems to be. This is another reason why canary testing is superior to monkey testing.

c. Chick malaria. $P$. lophurae is the only parasite available which is infectious for chicks. It has not been extensively used in chemotherapeutic tests because of the variability and short duration of the infection and the necessity for intracardiac or intravenous inoculation. Wolfson (1940) has stated that ducks are very sus- 
ceptible to this parasite, which would overcome the difficulties encountered with chicks.

\section{Human Malaria}

In testing a drug which has been found to be effective against one or more of the experimental malarial infections, field trials in endemic or epidemic areas, while difficult to control, are nevertheless probably more satisfactory than tests against induced infections in paretics. Induced infections are frequently more sensitive to the effect of drugs than are naturally occurring infections. In addition, it seems unjustifiable to test a drug early in an induced infection in a paretic because if the malaria is interrupted the patient may be deprived of the beneficial effect of the malaria upon an otherwise fatal disease. If one waits until the paretic has completed the therapeutic course of the infection, it is difficult to evaluate the effect of a drug because of the possibility of spontaneous termination of the malaria.

On the other hand, pareties are suitable for the testing of the prophylactic effect of drugs which are believed to have a direct effect on the sporozoite. If the drug is ineffective, the patient develops malaria without delay; if it proves effective and he is protected against the disease, he can be reinoculated with trophozoites. In addition, when testing prophylactic effect on therapeutic malaria, all necessary controls such as reinoculation with sporozoites or trophozoites are possible. Field experiments could not, of course, be controlled in this manner, and such factors as immunity due to pre-existing infection could not always be ruled out. 


\title{
A SUMMARY OF TEN YEARS OF OBSERVATIONS ON MALARIA IN PANAMA WITH REFERENCE TO CONTROL WITH QUININE, ATABRINE, AND PLASMOCHIN, WITHOUT ANTI- MOSQUITO MEASURES
}

\author{
By HERBERT C. CLARK \\ GORGAS MEMORIAL LABORATORY, APARTADO 1252, PANAMA CITY, R. P. \\ WILLIAM H. W. KOMP \\ UNITED STATES PUBLIC HEALTH SERVICE, GORGAS MEMORIAL LABORATORY, PANAMA CITY, R. P.
}

IT is probably true that anti-mosquito measures are the most efficient methods of malaria control, but in many instances their cost has been prohibitive, particularly if it is necessary to apply them in rural areas in the tropics, where economic resources are low, and the cost per capita of the measures would be high. Considerable work in attempting to control malaria in rural native populations has been done by British workers in India, Malaya, and other parts of the East, and by the Italians in Italy, using anti-malarial drugs alone, or in combination with anti-mosquito measures. We have been interested in the possibilities of drug control of malaria, without the use of antimosquito measures for two reasons : it seems logical to assume that a great reduction in the number of infected mosquitoes might follow a reduction in the malarial parasite rate by means of drugs administered to a given population; and we wished to investigate the possibilities of the newer synthetic anti-malarial drugs, under conditions which made outlays for mosquito control measures impossible.

In 1929 the Republic of Panama granted us the privilege for an indefinite period of using some villages located near the middle of the Chagres River basin for an experiment in drug control of malaria. At the beginning of our investigations the towns selected had no means of communication with the outside world except by small dugout canoes propelled by paddle and pole.
The houses of the villagers were of the usual type common in the tropies, with thatched roof, side-walls of cane, and dirt floor. The villages were located along the river banks, just above flood level. There was no sanitation; water for drinking and cooking was obtained from the river, which was also used for bathing and laundering. There was no local medical attention and no drugs were available in any of the towns. The river with its small branches and many shallow lagoons, filled with aquatic vegetation, provided large breeding areas for the larvae of anopheline mosquitoes. These villages could be reached from our laboratory in Panama City in about one and one-half hours, by automobile and outboard motorboat.

At the beginning of our experiment, we knew that two large-scale construction projects would soon be under way near the villages, one of which was the Madden Dam across the Chagres River, some few miles above the villages, and the other of which was the Madden Highway from Panama City to Madden Dam, a distance of about 24 miles. The imminence of these projects and the probability that other changes would take place in the towns themselves, such as the installation of latrines, better schools, and the introduction of our own medical service, made them especially suitable for long-continued observation. We have limited our experiment to 10 successive years of observations. The towns se- 
lected all lie between the Madden Dam and the eastern boundary of the Panama Canal Zone, and are situated on the banks or tributaries of the Chagres River. The towns of Santa Rosa, Guayabalito and Gatuncillo are on the right (north) bank of the river; Las Guacas lies opposite Santa Rosa on the left bank, at the junction of the Chagres and its Moja Pollo tributary. This latter town is almost surrounded by water full of aquatic vegetation, making ideal conditions for anopheles mosquito breeding. In February, 1937, the town of Agua Clara was added to the number of towns under observation. This town dates from 1936, when a group of people who formerly lived on Gatun Lake cleared the jungle from several hilltops just across the eastern boundary of the Canal Zone at boundary marker No. 50. The elevation of this town is about 100 feet, and the distance from Santa Rosa is about one and one-half miles.

During these investigations, we used chiefly the population located along the Madden Highway, in the towns of Chilibre, Aguas Buenas, and Buenos Aires as controls. These towns are located about 5 miles to the south of the left bank of the Chagres River, and about $\mathbf{1 5 0}$ feet higher than the river towns.

The populations of just such rural areas as these in which our observations were made are the sources from which large business organizations must draw their labor forces.

\section{Survey Methods}

Blood parasite surveys made monthly in all villages during the ten-year period have been used to measure our results. The thick-film technique of Barber and Komp (1929b) has been used throughout the period, and the staining and examination of the films has been done by experienced technicians, most of whom have been with us throughout the experiment. It was found that not all the inhabitants of the villages could be surveyed at any one survey during the month, but during the days for the period of treatment and during inspections, we were able to collect blood-films from all the permanent inhabitants. The migratory habits of some of our people have been a source of much inconvenience to us. It was impossible to examine or treat individuals adequately who did not reside permanently in the towns, and such persons might be undiscovered carriers of sexual malarial parasites, capable of infecting mosquitoes.

\section{Treatment Methods}

During the first year of our observations (Sept. 1930-Aug. 1931) we used quinine sulphate in all villages. A native Panamanian graduate nurse (assisted by school teachers and the corregidors (mayors) of the villages) was supplied with the list of names of those found positive in each monthly survey. Twenty grains (1.29 gm) of quinine sulphate to each positive per day for a period of 10 days constituted a course of treatment during the first year.

During the second year (Sept. 1931-Aug. 1932) quinine was used in the same manner, but in addition, plasmochin simplex in doses of $0.01 \mathrm{gm}$ twice a week was given to all positives in all the villages under treatment except New San Juan.

During the third year (Sept. 1932-Aug. 1933), quinine sulphate was used in the same dosage throughout the year in New San Juan, but in the four other villages under treatment we used quinine sulphate during the first 4 months, and atabrine 0.1 gm three times a day for 5 days from January 1933 to August 1933.

During this latter period, one of us (W. H. W. K.) stayed a week during each month in the villages, and personally administered the atabrine to all those found positive in the preceding monthly surveys. It is only during this period that we are absolutely sure that the drug was administered according to schedule. It is well to mention that during this period blood-films were taken at the completion of treatment from all persons who received the drug; in every case but one these films were negative for asexual parasites at the end of the 5-day course of atabrine. Crescents, which are 
little affected by atabrine, were of course present in many cases.

During the fourth year (Sept. 1933-Aug. 1934) tablets of quinine sulphate 15 grains $(0.972 \mathrm{gm})$ a day for 5 days were given to positives in New San Juan, followed 2 days later by plasmochin simplex $0.01 \mathrm{gm}$ twice a day over a succeeding period of 5 days. The populations of Santa Rosa, Guayabalito, Gatuncillo and Las Guacas were treated with atabrine $0.1 \mathrm{gm} 3$ times a day for 5 days, followed 2 days later by 5 days' course of plasmochin simplex $0.01 \mathrm{gm}$ twice a day.

These treatment methods were continued in the towns named up to and including August 1940. The town of Agua Clara was added to the atabrine-plasmochin treated villages in February 1937.

During the fourth year (Sept. 1933Aug. 1934) a definite policy of using nonmedical personnel for the administration of the drugs was established. Six native girls who were residents of their respective villages were selected by the river supervisor (an intelligent native man), after consultation with the people of each village.
These "nurses" were under the direction and inspection of a medical member of our staff, and thus soon succeeded in obtaining the necessary cooperation and administered the drugs in a fairly satisfactory manner. Weekly inspection trips to the villages by the medical staff made it possible to examine and treat cases of clinical malaria that developed between the monthly bloodfilm surveys.

\section{Presentation of Data}

For the period from Sept. 1930-Sept. 1940 the monthly and annual malarial parasite rates, the annual rainfall, incidence of malaria by age-groups, incidence of species of parasite, numbers of erescentcarriers and heavy infections, the incidence of malaria in infants, records of anopheline collections and dissections, and the relation of all these to the results of treatment can be more easily and briefly presented in tables.

Table I shows that the malarial parasite rate, as determined by the monthly bloodfilm examinations, bears no direct relation to the seasonal rainfall, because anopheles

TABLE I

Consolidated Report on Villages by Months. Adults and Childiren Combined

\begin{tabular}{|c|c|c|c|c|c|c|c|c|c|c|c|c|c|c|c|c|c|c|c|c|c|c|}
\hline \multirow[b]{2}{*}{ NokTBS } & \multicolumn{2}{|c|}{$1932-31$} & \multicolumn{2}{|c|}{$1931-32$} & \multicolumn{2}{|c|}{$1932-33$} & \multicolumn{2}{|c|}{$1933-31$} & \multicolumn{2}{|c|}{$1934-35$} & \multicolumn{2}{|c|}{$1935-36$} & \multicolumn{2}{|c|}{$1936-37$} & \multicolumn{2}{|c|}{$1937-38$} & \multicolumn{2}{|c|}{$1938-39$} & \multicolumn{2}{|c|}{$1939-40$} & \multicolumn{2}{|c|}{ TEN YEARS } \\
\hline & Matwitu & $\begin{array}{l}\text { Resintal } \\
\text { tonthos }\end{array}$ & 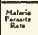 & $\begin{array}{l}\text { Rosetes } \\
\text { inches }\end{array}$ & Males: & 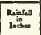 & 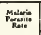 & Remplal & Matwi: & $\begin{array}{l}\text { Dosimlall } \\
\text { tachithes }\end{array}$ & Materit: & $\begin{array}{l}\text { Rumblit } \\
\text { tominger }\end{array}$ & Motur: & $\begin{array}{l}\text { Hoingas } \\
\text { tocido }\end{array}$ & 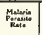 & 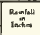 & Molatio & $\begin{array}{l}\text { Reiscoll } \\
\text { tached }\end{array}$ & Mathos & 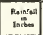 & 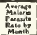 & 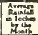 \\
\hline Septembet & 16.2 & 12.65 & 23.1 & 17.52 & 21.2 & 10.16 & 22.8 & 4. 20 & 6.2 & $10.5 s$ & 6.9 & 13.94 & 16.0 & 15.62 & 2.4 & 20.87 & 5.5 & 7.5 & 20.5 & 11.4 & 16.5 & 12.16 \\
\hline October & 15.6 & 10.15 & 215 & 8.05 & 23.4 & 17.09 & 20.2 & 7.54 & B.1 & 18.51 & 9.6 & 10.4 & 10.9 & 16.03 & 6.6 & 13.51 & 5.9 & 15.52 & 13.5 & 14.62 & 13.7 & 13.4 \\
\hline Nonember & 20.0 & 3.08 & 25.1 & 29.23 & Wo Hecord & 22.41 & 18.3 & 14.59 & 10.9 & 20.55 & 13.4 & 33.52 & 10.5 & 9.99 & 6.0 & $17.5 s$ & 98 & $12.7 \mathrm{~s}$ & 11.2 & $18 .-17$ & 23.6 & 12.19 \\
\hline Derember & 27.0 & O.8s & 15.7 & 1.65 & 26.5 & 6.97 & 11.0 & $7 \pi \pi$ & 13.2 & 5.90 & 16.4 & 6.07 & 13.1 & 0.65 & 4.8 & 18.51 & 78 & 11.25 & 17.9 & iss & 16.1 & 7.12 \\
\hline Jattuary & 21.5 & 0.24 & 23.2 & 1.06 & 21.2 & 1.01 & 19.1 & 1.41 & 25.6 & 0.84 & 16.0 & 0.08 & 11.8 & 1.52 & 6.9 & 0.20 & 10.8 & 0.02 & 14.2 & 2.16 & 17.0 & O.8S \\
\hline Fobreary & 20.6 & 0.13 & 12.2 & 0.22 & 13.5 & 0.0 & 11.1 & 0.17 & 21.9 & 0.46 & 9.7 & 0.07 & 14.7 & 0.25 & 8.1 & 006 & 12.1 & 0.01 & 16.7 & $0.5 s$ & 13.8 & 0.19 \\
\hline March & 15.6 & $2.5 s$ & 16.5 & 0.38 & 15.9 & 2.47 & 10.1 & $0.12^{\circ}$ & 28.0 & 0.12 & 10.7 & 0.09 & 9.5 & 0.13 & 6.1 & 0.65 & 6.7 & 006 & 8.7 & $0.0 s$ & 12.7 & 0.64 \\
\hline April & 21.1 & 2.19 & 16.2 & 5.74 & 13.6 & 0.23 & 10.4 & 4.47 & 22.4 & 1.61 & 9.6 & 1.02 & 8.5 & 0.90 & 3.9 & 3.67 & 5.7 & 0.11 & 11.2 & 0.15 & 12.0 & 2.08 \\
\hline May & 26.5 & 11.08 & 16.7 & 17.35 & 10.8 & 12.11 & 8.9 & 18.77 & 116.6 & 16.35 & 10.0 & 12.00 & 75 & 10.98 & 6.3 & 13.26 & 7.5 & 3.29 & 9.9 & 5.59 & 12.1 & 11.99 \\
\hline June & 26.6 & 13.72 & 25.8 & 11.49 & 16.4 & 10.75 & 6.4 & 11.66 & 16.9 & 13.30 & 10.6 & 6.88 & 11.1 & 10.59 & 6.0 & 15.92 & 6.3 & 11.16 & 73 & 6.99 & 12.5 & 11.22 \\
\hline fuly & 20.2 & 12.81 & 17.9 & 6.79 & 16.5 & 10.34 & 7.6 & 9.60 & 10.2 & 22.52 & .13 .6 & 13.00 & 7.1 & 9.48 & 6.7 & 2.97 & 6.9 & 7.05 & 9.1 & 7.73 & n.s & 10.72 \\
\hline \multirow[t]{2}{*}{ Auguet } & 27.8 & 9.06 & 121 & 11 ss & 11.1 & 12.68 & 6.0 & 11.21 & 11.5 & 16.56 & 11.5 & 8.82 & 11.8 & 20.43 & 8.4 & 9.80 & 11.2 & 11.95 & 80 & 12.24 & 22.1 & 11.62 \\
\hline & $\begin{array}{c}21.6 \\
\text { Axerse. }\end{array}$ & 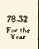 & $\begin{array}{r}16.8 \\
\text { Antrise }\end{array}$ & 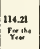 & $\begin{array}{c}16.8 \\
\text { Anesen }\end{array}$ & 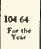 & $\begin{array}{c}12.8 \\
\text { Arwese }\end{array}$ & $\begin{array}{l}98.51 \\
\text { se.5. } \\
\text { Futed }\end{array}$ & An:man & 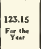 & Aness & 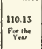 & $\begin{array}{r}10.8 \\
\text { Anerare }\end{array}$ & 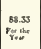 & $\begin{array}{r}6.6 \\
\text { Anement }\end{array}$ & 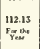 & $\begin{array}{r}8.0 \\
\text { Amerane }\end{array}$ & $\begin{array}{l}\text { 83.58 } \\
\text { Prothe } \\
\text { Prete }\end{array}$ & $\begin{array}{r}12.2 \\
\text { Aners.n }\end{array}$ & 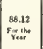 & 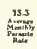 & 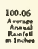 \\
\hline
\end{tabular}


mosquito production is not dependent upon rainfall in our village areas, as most of the larval breeding occurs in lagoons and backwaters of the Chagres River, which are always filled with aquatic vegetation, except during infrequent periods when this vegetation is washed out by flood-waters. Water impoundment behind Madden Dam, which was begun Sept. 1934, has exerted more influence on mosquito production than any other single factor. The gates of this dam are sometimes closed for months, and during such periods the only water released is that which passes through the hydroelectric plant at the dam. This impoundment causes a low water level, and sluggish current. Conditions of stagnation are most favorable for the growth and spread of aquatic vegetation, which at such times covers the river near all its small tributaries and in all its many shallow lagoons. The production of Anopheles mosquitoes is tremendously increased while such conditions exist.

TABLE II

average Monthly Parasite Rates. Treated Groups COMPARED WITH UNTREATED Control Groups

\begin{tabular}{c|c|c}
\hline \multirow{2}{*}{ Year } & \multicolumn{2}{|c}{ Parasite rate } \\
\cline { 2 - 3 } & $\begin{array}{c}\text { Treated } \\
\text { groups }\end{array}$ & \multicolumn{1}{|c}{$\begin{array}{c}\text { Control } \\
\text { groups }\end{array}$} \\
\cline { 2 - 3 } $1930-31$ & 21.6 & 17.5 (12 months) \\
$1931-32$ & 16.8 & 27.5 (12 months) \\
$1932-33$ & 16.8 & 20.5 (8 months) \\
$1933-34$ & 12.8 & 22.5 (12 months) \\
$1934-35$ & 15.8 & 18.5 (12 months) \\
$1935-36$ & 11.5 & 16.2 (12 months) \\
$1936-37$ & 10.8 & 16.4 (1 survey) \\
$1937-38$ & 6.6 & 20.8 (1 survey) \\
$1938-39$ & 8.0 & 32.7 (4 surveys) \\
$1939-40$ & 12.2 & \\
\hline
\end{tabular}

These control records are from various areas on the Madden Dam Highway where the migratory population made a very poor control group. Many of those who had malarial attacks went to Panama City for treatment and were not caught in the bloodfilm surveys.

The cumulative rate is obtained by divid-
TABLE III

Malarial Parasite Rates. adult Rates ComPARED WITH CHILDREN'S (15 YEARS AND UNDER) Rates, These Rates are Cumulative FOR THE YEAR. YEARS 1935-1940

\begin{tabular}{c|c|c|c}
\hline & Adults & Children & Total \\
\cline { 2 - 4 } & $\%$ & $\%$ & $\%$ \\
$\begin{array}{c}\text { Initial survey 1929. No } \\
\text { treatment or control }\end{array}$ & 37.2 & 56.5 & 45.6 \\
$\begin{array}{c}\text { measures* ................ } \\
\text { Cum. records Sept. 1935- }\end{array}$ & 29.1 & 41.5 & 35.3 \\
$\begin{array}{c}\text { Aug. 1936. All villages } \\
\text { Cum. records Sept. 1936- } \\
\text { Aug. 1937. All villages }\end{array}$ & 30.3 & 42.7 & 36.5 \\
$\begin{array}{c}\text { Cum. records Sept. 1937- } \\
\text { Aug. 1938. All villages }\end{array}$ & 21.2 & 28.8 & 25.0 \\
$\begin{array}{c}\text { Cum. records Sept. 1938- } \\
\text { Aug. 1939. All villages }\end{array}$ & 25.0 & 30.8 & 27.9 \\
Cum. records Sept. 1939- & 37.9 & 42.0 & 39.9 \\
Aug. 1940. All villages & 37.9 \\
\hline
\end{tabular}

* The rates found in the initial survey represent but one examination, while the cumulative rates include 12 monthly surveys, and all positives discovered. This shows very clearly that a single survey uncovers only a fraction of the annual malaria rate, even when drug control is in use. One favorable effect of treatment is shown in the decreasing children's rate relative to the adult rate, as treatment progressed. Usually children show a parasite rate almost double that of adults. After continued treatment, the two rates become more nearly equal. This is partially explained by the fact that children are more likely to be present at surveys, and can be more thoroughly examined and treated.

ing the total number of individuals positive during the year by the total number of persons examined.

It is seen from the last line in Table IV that from 1 to 5 examinations of the same individual during the year give average annual parasite rates of 19.3 per cent, while 12 consecutive monthly examinations of the same individual increase these rates to 47.6 per cent. These data mean that approximately half the population of our area had malarial parasites in the blood at least once during the year.

The table also indicates one of the diffculties encountered in surveying and treating native population groups in the field, either in villages or in labor camps. Our river supervisor lives in one of the towns, 
TABLE IV

parasite Index. Three Survey Groups. All Villages

\begin{tabular}{|c|c|c|c|c|c|c|c|c|c|}
\hline \multirow[b]{2}{*}{ Years" } & \multicolumn{3}{|c|}{1 to 5 surveys } & \multicolumn{3}{|c|}{6 to 11 surveys } & \multicolumn{3}{|c|}{12 surveys } \\
\hline & $\begin{array}{l}\text { No. ex- } \\
\text { amined }\end{array}$ & $\begin{array}{l}\text { No. } \\
\text { posi- } \\
\text { tive }\end{array}$ & $\begin{array}{l}\text { Per } \\
\text { cent } \\
\text { posi- } \\
\text { tive }\end{array}$ & $\begin{array}{l}\text { No. ex- } \\
\text { amined }\end{array}$ & $\begin{array}{l}\text { No. } \\
\text { posi- } \\
\text { tive }\end{array}$ & $\begin{array}{l}\text { Per } \\
\text { cent } \\
\text { posi- } \\
\text { tive }\end{array}$ & $\begin{array}{l}\text { No. ex- } \\
\text { amined }\end{array}$ & $\begin{array}{l}\text { No. } \\
\text { posi- } \\
\text { tive }\end{array}$ & $\begin{array}{c}\text { Per } \\
\text { cent } \\
\text { posi- } \\
\text { tive }\end{array}$ \\
\hline $1935-36$ & 789 & 152 & 19.2 & 379 & 197 & 52.0 & 230 & 134 & 58.2 \\
\hline $1936-37$ & 623 & 133 & 21.3 & 426 & 230 & 54.0 & 190 & 82 & 43.1 \\
\hline $1937-38$ & 689 & 100 & 14.5 & 410 & 156 & 38.0 & 171 & 63 & 36.8 \\
\hline $1938-39$ & 694 & 123 & 17.7 & 383 & 155 & 40.5 & 142 & 61 & 43.0 \\
\hline $1939-40$ & 583 & 155 & 26.6 & 407 & 226 & 55.5 & 71 & 43 & 60.6 \\
\hline Totals & 3378 & 663 & 19.3 & 1805 & 964 & 53.4 & 804 & 383 & 47.6 \\
\hline
\end{tabular}

* The intervals are from Sept. 1 to Aug. 31 of the following year.

has motor-boat transportation, and often visits all towns even outside of the regular treatment periods following blood-surveys. Our medical staff visits the towns on an average of 4 times a month. Yet with all these opportunities to collect blood-films from those not present on regular survey days, we found it impossible to secure blood-films from every individual for 12 successive months. Some of this difficulty may be explained by the habits of the population. Some of the inhabitants move from the towns, and others take their places; others have residences in the towns, but divide their time between these homes and their farms, which may be several miles from the villages; other persons examined are transient visitors over a period of weeks or months. The persons examined in 6 to 11 surveys and in 12 surveys per year, a total of 2,609 individuals, formed the permanent population of the area during the period of the survey. The remainder, 3,378 individuals, were transients who made satisfactory treatment of the persons examined very difficult because their presence in the villages increased the chances that mosquitoes would become infected, for many of them were untreated carriers of sexual forms of the malarial parasite.

For the past 3 years a steady diminution in the population of our towns has been taking place. However, enough inhabitants remain to show the benefits of drug control of malaria. In August, 1940, the atabrine-treated towns had a parasite rate of 11.5 per cent; the quinine-treated town a rate of 12.7 per cent; the Madden Highway untreated control group, mostly school children, a rate of 32.7 per cent; and Rio Pescado, another untreated group, a rate of 65.1 per cent. The number of heavy infections found was also much higher in the control areas than in our treated towns.

The age group from 5 to 10 years and from 10 to 20 years always have the highest rates, proving the importance of malaria in young adult labor force.

Both Tables V and VI show that inci-

\section{TABLE V}

Cumulative Incidence of Malaria BY AgE Groups FOR TEN YEARS IN THE TREATED Villages. Migratory aNd PermaNENT INHABITANTS

\begin{tabular}{|c|c|c|c|c|c|c|}
\hline \multirow[b]{2}{*}{$\begin{array}{l}\text { Age in } \\
\text { years }\end{array}$} & \multicolumn{3}{|c|}{$\begin{array}{l}\text { Atabrine- } \\
\text { Plasmochin }\end{array}$} & \multicolumn{3}{|c|}{$\begin{array}{l}\text { Quinine- } \\
\text { Plasmochin }\end{array}$} \\
\hline & 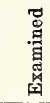 & $\begin{array}{l}\stackrel{8}{ \pm} \\
.7 \\
07 \\
0 \\
01\end{array}$ & 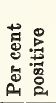 & 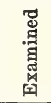 & $\begin{array}{l}\text { 量 } \\
\text { 䒼 } \\
0 \\
0\end{array}$ & 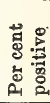 \\
\hline $0-5$ & 665 & 162 & 24.3 & 635 & 190 & 29.9 \\
\hline $5-10$ & 397 & 177 & 44.6 & 388 & 173 & 44.6 \\
\hline $10-20$ & 712 & 293 & 41.1 & 618 & 274 & 44.3 \\
\hline $20-40$ & 855 & 237 & 27.7 & 811 & 215 & 26.5 \\
\hline $40-60$ & 452 & 146 & 32.3 & 336 & 60 & 17.8 \\
\hline Over 60 & 122 & 40 & 32.7 & 140 & 21 & 15.0 \\
\hline Totals & 3203 & 1055 & 32.9 & 2928 & 933 & 31.8 \\
\hline
\end{tabular}


TABLE VI

Individuals Surveyed 12 Consecutive Months*

\begin{tabular}{|c|c|c|c|c|c|c|c|c|c|c|c|c|}
\hline \multirow{2}{*}{ Ages } & \multicolumn{3}{|c|}{ Chagres } & \multicolumn{3}{|c|}{ New San Juan } & \multicolumn{3}{|c|}{ Totals } & \multicolumn{3}{|c|}{ Controlst } \\
\hline & $\begin{array}{c}\text { Ex- } \\
\text { amined }\end{array}$ & $\begin{array}{l}\text { Posi- } \\
\text { tive }\end{array}$ & $\begin{array}{l}\text { Posi- } \\
\text { tive }\end{array}$ & $\begin{array}{c}\text { Ex- } \\
\text { amined }\end{array}$ & $\begin{array}{l}\text { Posi- } \\
\text { tive }\end{array}$ & $\begin{array}{l}\text { Posi- } \\
\text { tive }\end{array}$ & $\begin{array}{c}\text { Ex- } \\
\text { amined }\end{array}$ & $\begin{array}{l}\text { Posi- } \\
\text { tive }\end{array}$ & $\begin{array}{l}\text { Posi- } \\
\text { tive }\end{array}$ & $\begin{array}{c}\text { Ex- } \\
\text { amined }\end{array}$ & $\begin{array}{l}\text { Posi- } \\
\text { tive }\end{array}$ & $\begin{array}{l}\text { Posi- } \\
\text { tive }\end{array}$ \\
\hline years & No. & No. & $\%$ & No. & No. & $\%$ & No. & No. & $\%$ & No. & No. & $\%$ \\
\hline $0-5$ & 165 & 65 & 39.1 & 114 & 49 & 43.0 & 279 & 114 & 40.8 & 228 & 70 & 26.2 \\
\hline $5-10$ & 135 & 71 & 52.6 & 69 & 41 & 59.4 & 204 & 112 & 54.9 & 1020 & 385 & 37.7 \\
\hline $10-20$ & 69 & 45 & 65.2 & 38 & 28 & 73.7 & 107 & 73 & 68.2 & 1028 & 415 & 40.3 \\
\hline $20-40$ & 63 & 23 & 36.5 & 50 & 24 & 48.0 & 113 & 47 & 41.6 & 293 & 67 & 22.8 \\
\hline $40-60$ & 50 & 19 & 38.0 & 15 & 6 & 40.0 & 65 & 25 & 38.4 & 119 & 35 & 29.4 \\
\hline Over 60 & 15 & 5 & 33.3 & 17 & 5 & 29.4 & 32 & 10 & 31.2 & 25 & 4 & 16.0 \\
\hline Totals & 497 & 228 & 45.9 & 303 & 153 & 50.5 & 800 & 381 & 47.6 & 2713 & 976 & 36.0 \\
\hline
\end{tabular}

* Period covered September 1935 to and including August 1940.

f Controls: Madden Highway schools, 4 surveys; Chilibre (town on Madden Highway), 1 survey; Rio Pescado, Gatun Lake shore, 1 survey.

dence is highest in ages 5 to 20 and that those above 60 years of age, who have spent their lives in these highly endemic centers, are not immune to malaria.

In addition to the figures given above, the Buenos Aires schools (on the Madden Highway) were examined 4 times (Oct. 1939, Jan., May and Aug. 1940). The number of positives found was 203 . No treatment was given in this area, except quinine sulphate, which was provided for the positives, to be used if they so desired. There was no supervision of treatment. The results of the survey show that of the 203

\section{TABLE VII}

Individuals (405) Positive for Malaria who Were SURveyed Regularly for 12 CoNsecutive Months. Period of Sept. 1, 1935-AUg. 31, 1940

\begin{tabular}{c|r|r|r|r}
\hline $\begin{array}{c}\text { Times } \\
\text { positive }\end{array}$ & Chagres & $\begin{array}{c}\text { New San } \\
\text { Juan }\end{array}$ & Chilibre & Total \\
\hline 1 & 124 & 75 & 2 & 201 \\
2 & 54 & 35 & 5 & 94 \\
3 & 27 & 18 & 5 & 50 \\
4 & 12 & 13 & 2 & 27 \\
5 & 8 & 7 & 2 & 17 \\
6 & 1 & 4 & 4 & 9 \\
7 & 2 & 3 & 0 & 5 \\
8 & 0 & 0 & 1 & 1 \\
9 & 0 & 0 & 1 & 1 \\
Totals & 228 & 155 & 22 & 405 \\
\hline
\end{tabular}

positives, 115 were positive once; 55 twice; 23 three times; and 10 four times. Thus 88 , or 43.3 per cent, were positive from 2 to 4 times.

We believe that the larger proportion of positive findings is due to relapse, not to new infections. This is well shown in those who were repeatedly positive over long periods. These repeaters were certainly less tolerant to malaria than others living in the same area, for they kept a high parasite rate even though they were subjected to treatment at frequent intervals. Certain families, regardless of the location of their homes, show very low tolerance to the disease, and these people defeat our efforts to attain a further reduction in malarial parasite rates.

Mixed infections are no doubt more common than is indicated in Table VIII, as it is often necessary to examine bloodfilms made on successive days to discover such infections. Table VIII shows that $P$. falciparum infections constitute the majority of our positives and that $P$. vivax and $P$. malariae infections are more common in the Madden Highway groups than in the Chagres River villages.

The crescent rates among $P$. falciparum infections seem very high, yet during 9 years of our observations only about 43 heavy crescent-carriers were found in the 
TABLE VIII

Species of Malaria Parasites Found in 2,939 Positives

\begin{tabular}{|c|c|c|c|c|c|}
\hline $\begin{array}{l}\text { Species of } \\
\text { parasites }\end{array}$ & 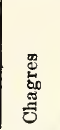 & 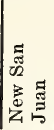 & 总 & 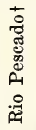 & 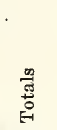 \\
\hline P. falciparum & 782 & 744 & 528 & 54 & 2,108 \\
\hline P. vivax & 163 & 86 & 135 & 21 & 405 \\
\hline P. malariae & 21 & 10 & 52 & 4 & 87 \\
\hline P. vivax & 90 & 80 & 99 & 4 & 273 \\
\hline $\begin{array}{l}\text { P. falciparum \& } \\
\text { P. malariae ..... }\end{array}$ & 15 & 10 & 16 & 1 & 42 \\
\hline $\begin{array}{l}\text { P. vivax and } \mathrm{P} \text {. } \\
\text { malariae }\end{array}$ & 2 & 1 & 11 & 0 & 14 \\
\hline $\begin{array}{l}\text { P. falciparum, vi- } \\
\text { vax \& malariae }\end{array}$ & 3 & 2 & 5 & 0 & 10 \\
\hline 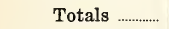 & 1,076 & 933 & 846 & 84 & 2,939 \\
\hline
\end{tabular}

* Five years records of Chilibre and Madden Highway schools. Infrequent surveys.

t One survey, August 1940, in a town on Gatun Lake. A period of ten years for New San Juan and the Chagres villages. Monthly surveys.

5 Chagres River villages. Of course this figure was obtained from the results of single examinations; if a series of examinations had been made in the same individuals, probably more would have been found. However, it is not an easy task to find in any one survey a suitable crescentcarrier for mosquito infection experiments. This is true of the untreated control areas, as well as of the treated groups. The

TABLE IX

Incidence of P. Falciparum Crescents in Five Chagres River Villages and in Madden HighWAY CONTROL GROUPS

\begin{tabular}{c|c|c|c}
\hline \hline Years & $\begin{array}{c}\text { Chagres } \\
\text { Villages }\end{array}$ & $\begin{array}{c}\text { Madden } \\
\text { Highway }\end{array}$ & $\begin{array}{c}\text { Rio } \\
\text { Pescado }\end{array}$ \\
\hline $1931-32$ & 13.2 & 28.7 & \\
$1932-33$ & 29.2 & 34.6 & \\
$1933-34$ & 22.8 & 17.9 & \\
$1934-35$ & 28.2 & 25.3 & \\
$1935-36$ & 26.5 & 18.3 & \\
$1936-37$ & 39.8 & 31.7 & \\
$1937-38$ & 44.0 & 27.2 & \\
$1938-39$ & 37.9 & 25.6 & \\
$1939-40$ & 45.1 & 54.8 & 38.9 \\
\hline
\end{tabular}

crescent rate is apparently higher in these treated groups, but this may be only the result of more frequent examinations. The gametocyte carrier is still a great problem in areas where drug control is attempted.

Table $\mathrm{X}$ shows the interesting and im-

TABLE $\mathrm{X}$

annual Percentage of Heavy Malarial inFeCtions DURING the Past Ten Years

\begin{tabular}{c|c|c}
\hline Years & $\begin{array}{c}\text { Five Chagres } \\
\text { River villages } \\
\text { (treated) }\end{array}$ & $\begin{array}{c}\text { Madden High- } \\
\text { way and Dam } \\
\text { (controls) }\end{array}$ \\
\hline $1930-31$ & 16.7 & 6.5 \\
$1931-32$ & 11.6 & 8.8 \\
$1932-33$ & 21.8 & 19.6 \\
$1933-34$ & 15.0 & 12.2 \\
$1934-35$ & 20.0 & 17.4 \\
$1935-36$ & 15.0 & 11.3 \\
$1936-37$ & 19.3 & 10.5 \\
$1937-38$ & 17.3 & 10.0 \\
$1938-39$ & 20.4 & 12.9 \\
$1939-40$ & 23.1 & 10.3 \\
\hline
\end{tabular}

portant fact that a greater percentage of all malarial infections were "heavy" among the cases found in the treated Chagres River groups than in the untreated control groups. Heavy infections were more numerous in the treated groups after 10 years of treatment than they were in the early years of our work. Yet the average annual malarial parasite rate in the treated groups in 1939-40 was only 12.1 per cent, while in the initial survey made in 1929 the rate was 46.5 per cent.

We believe that a partial explanation of this otherwise discouraging fact lies in the possibility that treatment of all parasitepositive cases rids so many of them of parasites that immunity is lost. When such individuals with lowered immunity become newly infected, they are more likely to be clinically ill, with many parasites in the blood, than are others who retain a few parasites at all times but rarely become actively ill.

Of the 20 infants found positive in the control areas, the first infection of at least one was found in each month of age from 
TABLE XI

SURters of Infants 12 Months or Less of Age. SEPT. 1935-AUg. 1940

\begin{tabular}{|c|c|c|c|c|c|c|}
\hline \multirow{2}{*}{$\begin{array}{l}\text { Period } \\
\text { covered }\end{array}$} & \multicolumn{2}{|c|}{$\begin{array}{c}\text { Chagres } \\
\text { River } \\
\text { towns }\end{array}$} & \multicolumn{2}{|c|}{$\begin{array}{l}\text { Control } \\
\text { areas }\end{array}$} & \multirow{2}{*}{ 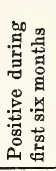 } & \multirow{2}{*}{ 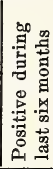 } \\
\hline & 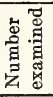 & 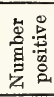 & 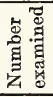 & $\begin{array}{l}\text { 岁. } \\
\text { 营 } \\
\text { 总 } \\
\end{array}$ & & \\
\hline $1935-36$ & 53 & 5 & 13 & 1 & 0 & 6 \\
\hline & 59 & 8 & & & 4 & 4 \\
\hline $1937-38$ & 53 & 1 & & & 1 & 0 \\
\hline 1938 & 65 & 1 & & & 0 & 1 \\
\hline $1939-40$ & 42 & 1 & 7 & 3 & 1 & 3 \\
\hline Totals & 272 & 16 & 20 & 4 & 6 & 14 \\
\hline Per cent & & 5.9 & & 20.0 & 30.0 & 70.0 \\
\hline
\end{tabular}

the second to the twelfth. The highest incidence was 4 in the seventh month, but too few infections were recorded to give the result significance. Of the 20 infants found positive, five had $P$. vivax infections and 15 had $P$. falciparum infections, a ratio which is quite comparable to that existing for these types in the general population.

The infant rate of infection is a yardstick with which to measure transmission. Table XI shows an infant rate of 5.9 per cent among the babies in the treated villages, surveyed 12 times a year, as compared with a rate of 20 per cent among infants living in the control areas, which are surveyed only at infrequent intervals.

\section{Crclical Variations in Malaria Inci- DENCE in Populations Under OBSERtation}

During the 10 years of our observations, great fluctuations in the malarial parasite rate have been noted, in both our treated towns and in the control groups. The chart which will be found on page 112 of our fifth annual report (1936) indicates these fluctuations for the first five-year period, and Table $I$ of the present paper shows the variations in the rates observed in the treated population since 1935 . The existence of such fluctuations is undeniable, as shown in the chart and the table, but the authors are not in agreement as to their causes. The first author (H. C. C.) believes that they are caused by unusual increases in the numbers of anopheles mosquitoes in the villages, owing to favorable conditions for larval production caused by periodic lowering and stagnation of the waters of the Chagres River. Such favorable conditions recur annually at the beginning of the dry season (January), and have been particularly marked since the beginning of impoundment of water behind Madden Dam (Sept. 1934). Unfortunately no data as to mosquito prevalence at various seasons of the year are available, except for the last two years of our study (Sept. 1938-Sept. 1940). The figures for these years show that mosquito production is heaviest during the dry season (JanuaryMay), when low water, sluggish current and bright sunlight combine to make larval breeding conditions very favorable. In support of the idea that increased mosquito production is responsible for high malarial rates, it is pointed out that two notable peaks of incidence occurred in the villages along the Chagres River, in the midst of the breeding areas, at the beginning of the dry season in 1933, and again in 1935. Since 1935, only one such peak has occurred, and this increase began in August, 1939, and continued for several months thereafter. In 1936 and 1937, malarial rates were higher in the first 5 months of the year, corresponding with the dry season, but in 1938 and 1939, higher rates were obtained in the second half of the year, particularly in 1939, when a parasite rate of 17.5 per cent was found in August in the 4 Chagres River villages. This was higher than any rate noted in these villages since the great epidemic of the first 4 months of 1935, when parasite rates as high as 28.8 per cent were obtained in the 4 Chagres River villages. This relatively high rate of 17.5 per cent occurred in the early part of the rainy season, and was more than three times as high as the rates found during the dry season months of March, April and May of the same year, when they averaged about 5.4 per cent. 
The evidence that cyclical variations in malarial parasite incidence is due to some other factor than mosquito abundance is contained in the tables showing parasite rates obtained during the past 10 years. In Table III the high parasite rate of 45.6 per cent for the initial surveys (including New San Juan) is mentioned. These surveys were made in September and December, 1929, during the heavy rainy season, 5 years before Madden Dam was completed. The next surveys were made a year later (Sept. 1930), revealing a parasite rate in the same population of only 16.2 per cent. Meanwhile no treatment had been given. This diminution in parasite rate, which was not in any way influenced by treatment, was apparently the normal decline from a peak of high incidence, in which we had unwittingly made our initial surveys. Increases in parasite rate were noted during the first year of our treatment, until in August 1931 they reached another peak with a rate of 27.8 per cent, in spite of treatment. They declined thereafter almost steadily, even through the dry season, until 1933, when high rates were again found, which continued throughout the year in spite of intensive treatment of 4 towns with atabrine, personally administered by one of us (W. H. W. K.). In 1934, rates dropped precipitately, even through the dry season, and remained at low figures throughout the year. Then in the first 4 months of 1935 we experienced an epidemic, when rates rose to high levels (28.8 per cent in the treated towns) during the dry season (January through March), and declined rapidly thereafter (see chart on p. 112 of our fifth year's observation). No significant increases were noted in 1936 , 1937, or 1938, and none occurred in 1939 until August, when the rate increased to 17.5 per cent from 6.9 per cent in the preceding month. Thus 4 dry seasons passed without any significant rises in parasite rates; the first such increase occurred in August, 3 months after the rains had begun. Apparently, judging from this experience, an epidemic may occur at any season of the year.
If river conditions favoring increased production of anopheles mosquitoes are the cause of increased parasite rates, such increased rates should reoceur each dry season, after the completion of the Madden Dam (September 1934), which impounded water during the dry season. However, with the exception of the epidemic in the early months of 1935, the next 5 years passed with no significant rise in parasite rates in any dry season. The first notable increase came in the month of August 1939, several months after the rains had begun.

If mosquito density were the answer to the problem of cyclical increases, towns along the river bank should have the highest parasite rates, as they are nearest the source of mosquitoes. The town of Las Guacas, which is nearly surrounded by water and in which the highest density of mosquitoes was found in 1932, has had consistently the lowest rate of any of our villages. On the other hand, New San Juan, which lies some 2 or 3 miles to the north of the Chagres River, has had consistently the lowest density of mosquitoes of any of our towns. Yet at times the parasite rate in New San Juan has been higher than in any of the other villages.

Malarial parasite rates in our area exhibit large variations over periods of varying lengths. One of us (W. H. W. K.) believes that these variations are caused by great increases in new infections, following a loss of tolerance, which may be due to natural causes or to treatment. Relapses certainly play a part in the increases noted, but they are believed to be significant as compared to the numbers of new infections found during epidemic periods.

The same author believes that the increased numbers of "heavy infections" noted as occurring since our drug treatment was begun is also due to a decrease in tolerance brought about by ridding the population of its parasites. When malaria attacks such a non-immune population, the number of clinical cases is higher than in a control group, which has had little or no treatment. This point is brought out strongly in our fifth annual report (2), and 
is confirmed by the figures in Table $\mathrm{X}$, which show a steady increase of "heavy infections" among our treated population.

Whatever may be the cause of the cyclical variations which occur in the malarial parasite rate, their existence must be considered in evaluating the results of control measures. By an unfortunate chance, our initial surveys made in 1929 showed high rates, and much of the success of our efforts has been referred back to these high rates as a base-line. If, however, we had made our initial surveys in September 1930, we would have been much less encouraged by our results, as the 1930 figures, 16.2 per cent, were low. In fact, they were a fraction lower than the average monthly rate for the $12^{\circ}$ months ending August 1932, after two years of treatment with quinine and plasmochin (Table 1 of our second year's observations, 1932).

"In order to reach satisfactory conclusions in regard to the value of anti-malaria measures, the natural trend of the disease must be observed over a period sufficiently long to pass through several cycles of the disease. Otherwise, what may appear to be a success may be only a swimming with the tide. In order to correctly evaluate a drug, it should be administered over a period long enough to include one of the cyclical upswings of the malaria rate. Only if it is successful under these conditions can it be considered of any value in community malaria control or prevention." The value of our observations is enhaneed because they have been made over a period of time long enough to encounter a number of normal eyclical variations. There has been a steady diminution in average annual parasite rate, as determined by monthly surveys in the treated groups. As shown in Table $I$ the average annual rate for these groups was 21.6 per cent, in the first year of treatment. Nine years later, in 19391940 this rate was reduced to 12.1 per cent. A comparable decrease was not noted in the control groups, so far as figures for these groups are available. We believe that the reduction in the annual parasite rate in our treated groups is a fair measure of the success of our work.

\section{SUMMARY}

1. This report deals with an experiment in the control of malaria with drugs alone, conducted in a rural region of high endemicity in Panama, where no anti-mosquito measures were used. The experiment has extended over a period of 10 years. The population concerned were native Panamanians, with a large negroid strain, living in six villages located on the banks of the Chagres River between Madden Dam and the eastern boundary of the Canal Zone; and a control group living about five miles away on Madden Highway. Breeding of A. albimanus, the principal malaria vector, was abundant in the aquatic vegetation along the river-banks and in the many lagoons and backwaters near the towns. Such breeding became extremely abundant at periods of low water, caused by the impounding of water behind Madden Dam, which resulted in slow current and semi-stagnant conditions in the river.

2. During the entire period of our studies, no correlation between the monthly malarial parasite rate and monthly rainfall was noted. The annual rainfall varied from 73.32 inches to 123.15 inches, with an annual average of about 100 inches.

3. The population involved in our studies was divided into three groups, two of which were treated, and the other used as a control. In all our work, only those individuals who were found parasite-positive, as indicated by examination of blood-films, were treated. Mass treatment of the population was not attempted at any time. The control group was provided with quinine sulphate, which was taken voluntarily, with no attempt at supervision of treatment. This method was used in the control groups throughout the course of the experiment.

From September 1930 to September 1932, the two treated groups were given quinine sulphate alone, or with plasmochin. In September 1932, one group was treated with atabrine 0.1 gram 3 times a day for 5 days, followed 2 days later by plasmochin simplex 0.01 gram twice a day for 5 days. The other group was treated with quinine sulphate (tablets) 0.972 grams a day for 5 
days, followed 2 days later by plasmochin simplex 0.01 gram twice a day for 5 days. These methods of treatment were continued from September 1932 to September 1940.

During the course of these experiments, one case of quinine idiosyncrasy was noted. Plasmochin simplex, given either following quinine or atabrine, gave trouble in many instances when administered twice a day for 5 days: We found one family the members of which cannot take plasmochin in any quantity without experiencing abdominal distress.

4. Blood-film surveys for malarial parasites were made monthly throughout the entire ten-year period, to measure our results. The thick-film technique of Barber and Komp (1) has been used, and the staining and examination of the films have been done by experienced technicians.

5. Administration of treatment during the first years was in the hands of a native graduate nurse, assisted by the local school teachers and civil authorities. One of the staff also had charge of the administration of the drugs in the Chagres River towns.

A definite policy was later established of employing native girls to give the treatments under the supervision of the river supervisor. Thus the administration of the drugs was by non-medical personnel; visits of inspection were made by a member of our staff 4 days a month.

6. During the later years, large shifts of population in our towns interfered seriously with the continuity of our work. The population has declined in number steadily since about 1938. Many families have moved to Madden Highway, where better transportation and school facilities are available. Greatly increased labor demands in the Panama Canal Zone have drawn many of our villagers from the river towns. A large number of these people had been permanent inhabitants of the towns from the early years of our work.

7. The part played by new infections, as compared with relapses from previous infections, is believed to be relatively small. In the quinine-plasmochin treated group,
51.6 per cent of all positive for malarial parasites were positive from 2 to 7 times during a year's observations. In the atabrine-plasmochin treated group, this percentage was 45.6 per cent. A certain portion of the percentage of repeated positives is doubtless due to insufficient or incomplete treatment, but in the main it is the result of low tolerance to the disease. Persons repeatedly positive keep up the parasite rate, even though given frequent complete courses of treatment. These individuals defeat our efforts to effect a further reduction in the parasite rate. We believe that they represent cases of relapse, in most instances, rather than new infections. Some confirmation of this view is found in the low monthly parasite rates, and the low percentages of infections in infants, which point to low transmission rates.

8. During the past 10 years, the incidence of the several species of malaria parasites among all positives has been as follows: $P$. falciparum, 77.8 per cent; $P$. vivax, 19.3 per cent; $P$. malariae, 2.9 per cent.

The percentage of crescent-carriers in $P$. falciparum infections has increased rather than diminished from year to year; however, exceedingly few carriers are found in any year which are good subjects for mosquito infection experiments. The percentage of heavy infections found among positive individuals has not decreased in the treated groups; however, the majority of positives in these groups show very few parasites in the films, while positives in control areas show many parasites, requiring less time to discover them.

9. Surveys of infants under one year, from 1935 to 1940 , show positive rates of 5.9 per cent in the 272 examined in the treated areas, as against 20 per cent of 20 examined in the control areas.

10. The highest parasite rates were found in the age-groups from 5 to 10 years, and from 10 to 20 years. The latter group contains the young adults who form a large 
percentage of any tropical labor force. Adequate treatment of this latter group is necessary, therefore, to maintain its effciency under conditions of hard labor and exposure. Such treatment can be administered in the field with small personnel and little expense.

11. Non-medical personnel selected from camp or village residents can be instructed to administer anti-malarial drugs in a reasonably satisfactory manner. However, a physician qualified and interested in tropical and industrial medicine should supervise such non-medical personnel, making visits at least once a week to the areas under treatment.

12. We consider quinine sulphate and atabrine to be of equal therapeutic value in the treatment of malaria. In our work, atabrine has the advantage that it is preferred to quinine by the native population. It is, of course, considerably more expensive than an equivalent course of quinine treatment. We do not believe that plasmochin has played a very important role in our work. 


\title{
GENERAL CONSIDERATIONS IN PLANNING MALARIA CONTROL ${ }^{2}$
}

\author{
By JUSTIN ANDREWS \\ DIVISION OF MALARIA AND HOOKWORM SERVICE, GEORGIA DEPARTMENT OF PUBLIC HEALTH, \\ ATLANTA, GA.
}

WHAт constitutes a malaria problem is more than a rhetorical question. In theory, any place in which man, infectible mosquito and malaria parasite are accessible to one another under favorable natural conditions may constitute a malaria problem. In practice, however, these situations vary in malaria intensity with respect to the quantitative and qualitative aspects of these factors. Since the malariologist can rarely serve all of them at once, it becomes imperative for him to develop some selective policy and procedure by means of which his services and resources shall become available first in places where they will do the most good. This aphorism, then, states both the necessity for, and the objective of, malaria-control planning.

As a preliminary step, therefore, the public health malariologist might define a malaria problem as a situation in which proved malaria morbidity, or morbidity and mortality, prevail at such heights as to stimulate concerted group activity aimed at suppressive efforts. This stimulation may be spontaneous or induced. It will be the former if morbidity and mortality are sufficiently severe, i.e., under epidemic or hyperendemic conditions; it may be induced if these effects are not impressive enough to alarm the public, though they result in demonstrable economic loss which may not be realized until the malariologist points it out.

\section{First Consideration : Developing the General Picture of Malariousness}

The first essential in planning malaria control is to visualize the usual intensitydistribution of the disease over the entire area of responsibility. For this purpose, 1 Contribution No. 6. information may be obtained from the offcial vital statisties pertaining to malaria, or from malaria reconnaissance. Neither of these methods is completely satisfactory. Theoretically the former is preferable, for the best measure of malaria should be the amount of sickness and death which it causes. Unfortunately these attributes are not reported with numerical accuracy under routine conditions and are, therefore, of limited dependability.

Deaths from malaria are generally overreported. Many medical practitioners in malarious areas are prone to accept any antemortem sign or symptom suggestive of malaria as evidence of infection and, in the absence of some more obvious factor, to certify it as the cause of death. Blood smears, and even more rarely thick blood films, are made on only a fraction of cases. Dauer and Faust (1937) state that of the reported malaria deaths in Mississippi for the year 1935, the causes of only 34 per cent of the deaths of colored persons were confirmed by laboratory diagnosis. Meleney (1937) showed that of 115 malaria deaths reported in Tennessee in 1935, blood smears had been made on only 40 per cent, all of which were positive. Brown (1940) analyzed 101 malaria deaths reported in Georgia in 1937 and found that blood smears had been made on 51 per cent of the cases.

Industrial life insurance policies costing from 5 to 25 cents a week and requiring no medical examination are very popular in the South, especially among negroes. They pay death benefits of from $\$ 70$ to $\$ 350$. Many of them contain the following limited liability clause or its equivalent ". . . this Policy shall be void if the insured ... has ever had before said date 
(of application), paralysis, cerebral hemorrhage, tuberculosis or other pulmonary diseases or chronic bronchitis, asthma, cancer, pellagra, or any disease of the heart, liver or kidneys or any venereal disease...." These exclusions, to which homicide is commonly added, leave a comparatively limited series of causes, including malaria, from which a negro may die without forfeiting his life insurance. Of late years the effect of this stipulation is offset to a considerable extent by another which provides that, after a stated period of one or two years or more, the policy shall be incontestable except for non-payment of premiums; nevertheless, in some contracts payment is not made under any circumstances if venereal disease is involved. The net effect of such policies is to influence the physician to write in malaria rather than tuberculosis or syphilis as the cause of death to insure payment to the beneficiary.

On the other hand, some true deaths from malaria are not reported as such. Mistakes in diagnosis, as indicated by Meleney (1937), may be responsible for missed malaria deaths. Very poor families, remotely situated, who cannot afford doctors, funerals, or caskets, frequently bury their own dead without any official record of the event.

As a rule, cases of malaria are not as well nor as adequately reported as deaths. Whereas death reporting suffers mainly from errors of commission, case reporting suffers from errors both of commission and of omission. In endemic areas, malaria is commonly regarded as a trivial, insignificant complaint which rarely justifies the expense of calling a physician. In those instances where the doctor is consulted, the patient or his family observes that the diagnosis is usually based on a meager combination of case-history and physical examination. Some physicians make a conscientious and thorough investigation-including a blood examination-before they arrive at a diagnosis of malaria, but all too frequently the doctor merely inquires how long the patient has been ill and if he has had a fever or a chill. He then feels the patient's pulse, looks at his tongue, suggests that he may have a "touch" of malaria and prescribes quinine or atabrine-perhaps with calomel and a purgative. There is nothing in this pattern of diagnostic procedure that the patient or his family cannot do themselves, and so the next time the same set of symptoms appear, the doctor is by-passed and the quinine or atabrine-or a substituted chill tonic-is obtained without prescription. Thus the great majority of what the laity believes to be cases of malaria are self-diagnosed and self-treated; they never come to medical attention and so cannot be reported. Boyd (1930c) states that not over 35 or 40 per cent of the cases studied in southeastern Missouri had consulted a physician. Unfortunately, many of these which have sought medical advice are not reported, especially if the diagnosis was made on a purely clinical basis.

Case-reporting is subject to local influences which may affect statistics without having true morbidity significance. The arrival of a new doctor accustomed to reporting malaria or of making blood smears as a diagnostic aid may suddenly increase the apparent malaria morbidity in a county, whereas the "increase" is only in the reporting of morbidity. This improvement in reporting malaria-as well as other communicable diseases-invariably follows the establishment of an active local health agency. In some states, it is customary to include in routine morbidity statistics all blood positives found in malaria surveys. This adds appreciably to the apparent morbidity in surveyed counties in contrast to other counties not surveyed but in which there is every reason for believing that malaria is as serious a problem. Thus the malariologist reviewing statistical evidence of malaria morbidity must carefully scrutinize and satisfy himself as to the circumstances behind each reported increase.

It must not be supposed, however, that, because of their defects, mortality or morbidity statistics are entirely useless. Fig. 1 shows the reported annual case and deathrates for a single malarious state, Georgia, 


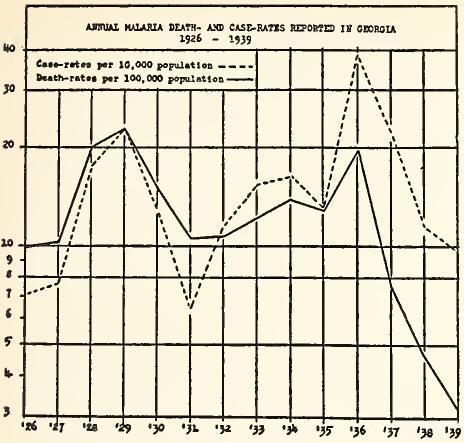

FIG. 1

for the years 1926-1939, inclusive. It is apparent that either graph would serve to show the relative annual intensity of malaria. Incidentally the graphs show the effect of sustained efforts at improving the reporting of malaria cases and deaths. Prior to 1932, the reported case-fatality ratio was ten or less, whereas, after 1931 it exceeded ten. During the last three years shown, it has been from 25 to 30 . Similarly, Fig. 2 is an array of 10 -year, monthly malaria case- and death-rates for the same state. Considering the known defects in-

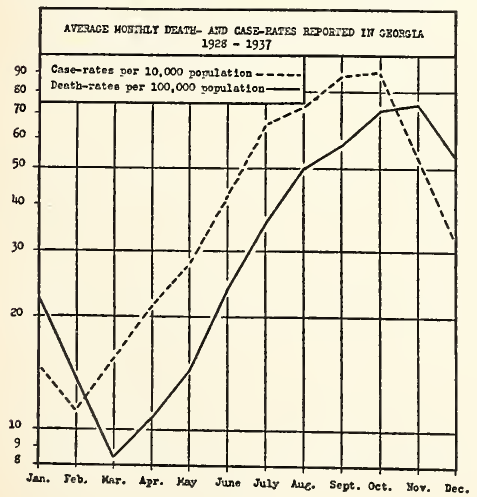

FIG. 2 herent in the data, the two graphs exhibit a remarkable degree of parallelism in trend. This gives a useful picture of the seasonal occurrence of each attribute exemplifying the well-known lag in the rise and decline of deaths as compared to cases. It also shows what is either a reportorial or actual difference in the case-fatality ratios in the winter versus the other months.

Thus, employed conservatively and with a proper understanding of their limitations, either morbidity or mortality statisties may serve to point out certain useful time-malaria relationships, provided the area or population involved is sufficiently large. They may also be used to advantage in defining certain space-malaria relationships, again provided that the time span is great enough. Some extra caution, however, is required in interpreting the distributional maps thus developed, of which Fig. 3 is an example.

While geographic distributions of mortality are probably more dependable than those of morbidity for the reasons pointed out above, it must be remembered that they show little more than the intensity-distribution of estivo-autumnal fever. This may or may not be associated with tertian malaria. Where tertian predominates to the exclusion of other types of malaria throughout the season, morbidity should be plotted instead of-or as well as-mortality, assuming that cases have been reported with some regularity for several years.

Rate maps and unit maps have different distributional significance. The first show variations of attributes expressed as proportions of the population. Thus in areas including large cities many of whose inhabitants are at minimal risk of malaria, rate maps may be misleading because they indicate low rates but do not reveal the effect of their application to large populations. A good example of this is shown in Fig. 3. Chatham County, in the most easterly projection of the state, has a low malaria deathrate, but this is due mainly to the fact that 95,271 of 116,803 persons in Chatham County live in Savannah where exposure to malaria is virtually negligible. Never- 


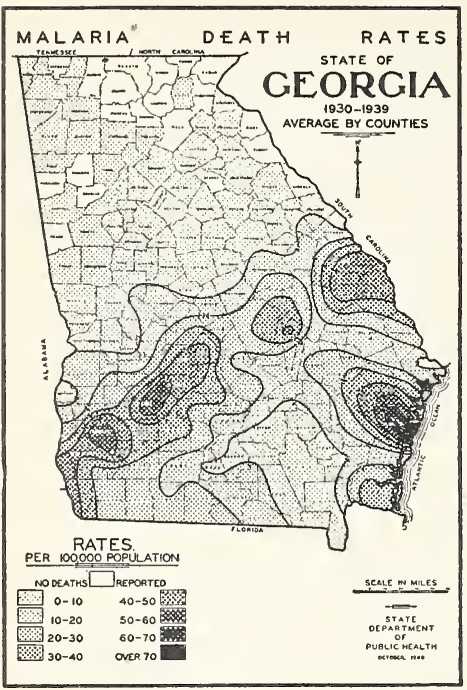

Fig. 3

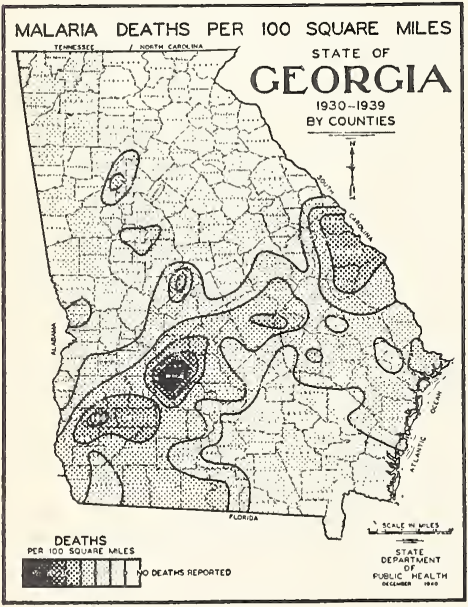

Fig. 4 theless, the number of persons dying from malaria in the County is frequently much larger than in other counties where malaria death-rates are higher.

In their simplest form, unit maps-the familiar "spot map"- -show the actual number of cases or deaths either accurately located or expressed numerically in terms of political or other arbitrary subdivision, such as counties, parishes, or military districts. The difficulty with these maps is that certain subdivisions may be several times the size of others and so are not comparable. This defect may be overcome by adjusting the attribute per subdivision to a constant unit area, as 100 square miles. This has been done in Fig. 4 with the data used in Fig. 3. It will be observed that the same general distribution of incidence and intensity is expressed in both maps, but that in the unit map, Fig. 4, the urban counties-Fulton (Atlanta), Muscogee (Columbus), Bibb (Macon), Richmond (Augusta), and Chatham (Savannah)-have greater prominence in the malaria picture. Thus it shows the aggregate effect of low exposure potentials in urban areas as well as the consequences of high endemicity in rural areas. The rate map emphasizes intrinsic malariousness whereas the adjusted unit map depicts aggregate population experience with malaria. Both types of maps are of value and should be made and studied carefully to acquire a good understanding of the intensity-distribution of the disease.

In the event that morbidity or mortality records over a suitable period of time are not available or are patently unreliable, the general picture of relative malaria intensity may be sketched in by making an areawide malaria reconnaissance. This procedure is aimed at determining the current or recent prevalence of malaria in man. It is usually achieved by making a census of splenic enlargement or parasitemia, or both, among public school children throughout the area at any time during the year, though more useful data are obtained toward the end of the malaria season. These are plotted on a map to show their 
geographic distribution and areas of concentration.

In competent hands, splenometry is a rapid and inexpensive method of estimating malariousness. Since details of the technique are given in Dr. Elmendorf's paper, they will not be included here. Certain practical difficulties in applying it on a large scale may be mentioned, however. To obtain reliable and comparable information, spleen palpation should be done by well-trained and experienced medical personnel, desirably by the same individual. Thus either the area of reconnaissance is restricted to what one person can handle in a given season or several individuals must be involved. This last is not easily arranged because experienced splenometrists are rare and it is difficult to enlist the interest of the health officer in the procedure. His acquaintanceship with malaria splenomegaly is usually restricted to observations made on patients acutely ill with malaria. Their spleens are enlarged, but the increase in bulk is due mainly to congestion. They are soft, doughy, and mobile and it is not easy to define their lower margins by palpation. Thus the physician who later becomes a health officer is frankly skeptical about the value of splenometry as a measure of malariousness. If he can be induced to try it on any considerable scale, he soon experiences the thrill of finding for himself the firm, unmistakable, easily-defined spleens of chronic malaria and may become an enthusiastic advocate of the method. Perhaps the most serious impediment to its use is that even after considerable experience with spleen palpation, different examiners may express diverse opinions about the presence and extent of small degrees of splenic enlargement. As these constitute the major portion of the enlarged spleens encountered in this country, the main difficulty in depending upon splenometry in malaria reconnaissance is on obtaining reliable and comparable measurements on a large scale.

A further consideration is the fact that the spleens of white children are more responsive to malaria infection than are those of negroes. This is particularly true of tertian malaria which, in the negro, is comparatively rare and almost always asymptomatic. Thus it is common experience to find more enlarged spleens than positive bloods among white children, while the reverse is true among negro youngsters. It follows that geographic distributions of malariousness based on spleen size alone may be misleading in areas where the racial composition is highly variable.

Blood-smear reconnaissance technique likewise has its pros and cons. The advantages are: (1) that smears can be collected by nurses, engineers, or other non-medical personnel, (2) that positive findings are objective and can be preserved for future verification, and (3) that species and gametocyte determinations can be made. Their great disadvantages are: (1) the time and expense of processing and examination, and (2) their easy deviation by antimalarials which gives spuriously low infection rates.

In the author's opinion, the most productive and economical reconnaissance practice in this country would be to have the spleens of white children palpated by physicians and to have non-medical assistants make blood smears from negroes. These examinations may be restricted to rural populations, as little, if anything, is gained by including children living under strictly urban conditions.

\section{Second Consideration: Selection of Malaria-Control Areas}

Having developed the general picture of malaria intensity-distribution on a regionwide basis, the next step in planning malaria control is to decide just where it shall be done. Theoretically, this should be in the areas where it has been shown that the hazard is greatest or affects most people. Wherever possible, this public health principle should be allowed to dominate all others. Actually, malaria-control operations cannot be carried out without cash or labor; therefore, except in situations where support is not locally derived, selection will probably be conditioned as much by con- 
sideration of available resources as by known degrees of endemicity. Even where means are at hand, a vigorous promotional and educational campaign may be necessary to secure their utilization.

The size of area to be selected deserves mention. Every effort should be made to avoid units so small that nullification of all malaria hazards within them will not appreciably affect malaria rates. On the other hand, it is difficult to maintain malariacontrol interest in an area so large that the effectiveness of control can be appreciated by only a minor fraction of its inhabitants. Generally speaking, it is advisable to adhere to existing political subdivisions, because vital statisties, public expenditure, local health agencies and other participating organizations are usually related to them. Occasionally a major situation, such as the head-watershed or impoundment of a large river system, may make it necessary to involve several subdivisions. Again, malaria prevalence may be intense but so limited in extent within a county that the county population is unwilling to endorse public expenditures for its control. Under such circumstances, the project must be developed and studied on less than a county basis.

\section{Third Consideration : Malaria Surveys}

Assuming that these choices have been made, the third step in malaria-control planning is to assemble detailed malariological information about each area. Such a study is called a malaria survey. It differs from a reconnaissance in that its scope is intensive rather than extensive. Whereas the reconnaissance is only a preliminary investigation aimed at defining areas of malaria importance within a region, the object of the survey is to show how and at what levels malaria is being transmitted and what ean be done to reduce that level in areas where malaria control is proposed. Thus it involves not only measurements of malaria in man, but studies of local anophelines and of the specific environmental conditions which influence the development and transfer of parasites from man to in- sect and vice-versa. Essentially, it will try to distinguish between primary focalizations and secondary extensions of endemicity and to determine the specific location and nature of the breeding places from which the anophelines responsible for transmission in each primary focus are derived.

Inasmuch as Dr. Elmendorf's contribution treats of the techniques, analysis, and interpretations of malaria surveys, no mention is made of these phases of the subject in this presentation, noting only that the comprehensive survey report will contain, in addition to particularized malariologic information, some account of the social and economic conditions prevailing locally. Is the population composed of family units, including children, or of adult, male laborers employed on some remote construction project? Is it their custom to sit outof-doors or to travel about in the evening? Are they housed adequately in buildings that, at a reasonable cost, might be made mosquito-proof? What is the per capita or per family income and how is it spent? Do property-owners, renters, wage-hands, or share-croppers predominate in number? How many of them pay taxes? How much of the land is owned by absentee landlords? Are the intellectual capacity and experience of the people such that they can accept responsibility for the proper use of sereens, bed-nets, or antimalarial drugs provided with prophylactic intent? Are they subject to any sort of discipline, direct or indirect, or must all cooperation be voluntary?

In obtaining public funds, health departments must compete with other public services. An expanded program of roadbuilding, of schoolhouse construction, of providing old-age pensions, ete., may seriously jeoparidize public interest in and maintenance of major malaria-control undertakings. Can the present health allotment be expanded to defray the costs of malaria control? If not, what special fiscal provisions can be made for this purpose? What is the total assessed value of private property and public utilities? Would a special malaria tax be constitutional or would it require enabling legislation? 
What is the limit of local bond issuance and the present outstanding indebtedness?

Likewise, malaria control must vie with other health improvements for its support. What is the importance of malaria relative to other health problems in terms of mortality, morbidity and dollars and cents? How much proprietary and ethical antimalarial medication is consumed and what does it cost? Is there reason for believing that malaria affects or is affected by other local, chronic disabilities such as malnourishment, undernourishment, hookworm disease, etc.? Is the health officer keenly interested in malaria or is he preoccupied with other health problems? These considerations may be important in determining the immediate scope, the continuity rate and the final success or failure of the malaria control project.

Malaria is usually a rural disease and is, therefore, frequently associated with populations dependent upon agricultural pursuits for their livelihood. Is the local economy based upon products whose market is fluctuating, whose prices are speculative? Are agricultural practices up-to-date? Is any attempt being made to apply new methods of planting, cultivation and harvesting, to introduce new crops or farming activities, to develop new markets? These considerations may seem remote from the general subject of malaria prevalence and control, yet in planning the initial scale and ultimate extent of the program, they are determinants of primary importance.

\section{Fourth Consideration : Selection of Malaria Control Measures}

The survey report will undoubtedly include recommendations for control and a supporting budget. These should be prepared on a somewhat idealized basis to reflect clearly the relative malaria importance of each area and the preferred methods-and anticipated costs-of dealing with each of them.

Desirable malaria-control practice calls for permanent, preventive measures wherever possible. Essentially, those involve the complete removal of man or the trans- mitting species of Anopheles. Depopulation is rarely feasible on a large seale because of the expense involved and the reluctance of people to leave their homesteads. It does have minor application, however, in sub-marginal lands and in the relocation of workers' quarters on plantations. In the vicinity of wildlife refuges and reservations where drainage and larvicidal measures are not sanetioned, population removal is the ideal method of protection to be recommended.

The filling of anopheline breeding-places has the unique advantage of being immediately and permanently effective without requiring further expenditure. Its capital cost is so great, however, that its use is virtually restricted to small ponds, pools and pits in or near cities.

All things considered, drainage is probably the most satisfactory control method now used. It should be recommended wherever the dewatering of breeding places can be accomplished without violating vested, recreational or wildlife interests whose values are believed to transcend those of health improvement in the area. The construction costs of drainage are high, and are largely dependent upon the type of soil moved and the method used. Handlabor excavation costs from 40 to 50 cents per cubic yard. Machine (dragline) digging costs run from 15 to 20 cents per cubic yard but require a huge yardage to justify the purchase or rental of the equipment. In swampy places where a heavy dragline cannot be readily moved or where large stumps must be dislodged from the ditch line, dynamite excavation may be employed to advantage though its cost is a little above that of dragline operation.

If drainage is the method of election, an index of ponds should be included in the report, showing precisely the malariogenic status of each and the type of drainage construction proposed. It will then be possible to devise a desirable sequence of drainage operations by hand labor, or, if justified, by machine.

The greatest defect in planning for drainage control is failure to provide for 
the annual maintenance necessary to keep the channels functional. The costs are exceedingly variable but can usually be anticipated in different terrains with a reasonable degree of accuracy. To a considerable extent, these maintenance costs may be reduced by paving ditch-bottoms with concrete, clay, brick, or masonry and by stabilizing the slopes of open ditches. The construction costs of such procedures limits their application to urban and suburban situations where, generally speaking, it is better practice to invest a little more money and install a closed, subsoil drainage system if possible. While these refinements in design and construction do reduce maintenance costs, they rarely, if ever, succeed in eliminating it.

Certain breeding-places, however, represent interests vested in power, wild-life, recreational or ornamental values and cannot be destroyed. In such situations the only alternative to depopulation is temporary or semipermanent control measures such as larvicidal applications, mosquito-proofing, or medication. Good anopheline larvicidal effect may be obtained with either oil or Paris green. Oil has the advantage of being lethal to all mosquito larvae whereas Paris green is mainly effective against anopheline larvae. The material costs of oil are about twice those of Paris green and the radius of application is somewhat less. Thus oil is more expensive but provides nullification of a pest problem as well as a health hazard. Larviciding should be employed either as a supplement to or during the development of a drainage program. It is rarely economical to substitute it for drainage. If, however, it can be demonstrated that the annual costs of area-wide larviciding are less than the annual maintenance and amortization costs of drainage for the same area, the proposition should be seriously considered.

Most malarious states have regulations promulgated for the control of artificial impoundments. Such bodies of water present very special malaria problems due to the fact that natural fluctuation of pool level in response to rainfall and run-off does not occur as in natural ponds. Fluctuation is an effective anti-larval method and, where feasible, means for achieving it in artificial impoundments should be specified in the malaria survey report. Clearing, bank-sloping, herbicidal treatment, marginal drainage, and larviciding may be necessary as supplementary measures to suppress anopheline production in impounded ponds and lakes. The cost of malaria control operations on revenue-producing impoundments should be borne by the owners.

The use of larvicides presupposes that under local conditions sufficiently effective control of breeding can be obtained to reduce malaria rates and enough people live within flight-range of the treated ponds to justify the costs. This is not always true. Using ground or boat equipment, oiling costs about $\$ 20$ per season-acre, Paris-green dusting, \$15; airplane dusting, if the volume justifies it, is less expensive, about $\$ 7.50$ per season-acre according to Kiker, Fairer and Flanary (1938). Thus it may be more economical to provide mosquitoproofing than larviciding in the vicinity of ponds where population is scanty. The Tennessee Valley Authority has encountered situations where standard Paris-green airplane dusting does not kill enough larvae to control local malaria morbidity, and has, therefore,. substituted screening and mosquito-proofing for anti-larval measures.

An insect-proof house is part of a minimum standard of living and should not have to be promoted as a specific antimalaria measure. Nevertheless, thousands of unprotected houses exist within flightrange of anopheline breeding-places. Where filling, drainage, or anti-larval measures are not practicable, some of these houses can be screened at a reasonable fraction of their present value. It is in such situations that mosquito-proofing should play a part in the malaria control program. The effectiveness of this measure is dependent upon the residents' understanding its purpose and that its value will be reduced 
by abuse or by not remaining behind screens after dark.

There always remain houses in the areas where drainage and larviciding cannot be done which cannot be protected against insect invasion except by major reconstruction at a cost which neither private owners nor local government will assume. In such places, medication is about the only thing left to recommend. The treatment of gametocyte carriers and of indigents is justified by ample precedents in public health practice and, irrespective of its lack of effect upon the intrinsic malariousness of the area, should be recommended in the report.

As an example of comparative malaria control costs, the following is abstracted from a survey report entitled "A Study and Analysis of Malaria in Morgan County, Alabama" (1939) by E. A. Philen. ${ }^{2}$ The drainage of 158 ponds and the larvicidal treatment of 46 others is recommended as being the cheapest and most effective means of control. This would require, if machine drainage were employed, annual expenditures of $\$ 16,150$ for 9 years and $\$ 8,075$ yearly thereafter. Hand-labor drainage would cost at least $\$ 100,000$ more and would require a longer construction period. The annual cost of county-wide, larvicidal control is estimated to be $\$ 20,670$; of screening and mosquito-proofing, $\$ 36,388$, plus an initial outlay of $\$ 181,940$; of medication, $\$ 64,625$.

If the project is to be supported on nonlocal funds, there will probably be little occasion for departing from the desirable sequence of operations suggested by the surveyor. If, on the other hand, it is to be maintained on local resources, it may be necessary to evolve a feasible works program based upon joint consideration of the control recommendations, the means available for their fulfillment and perhaps certain circumstances of local psychology and politics.

Assuming the project to be of the latter type, an inventory of all resources should be prepared. Local governments may sup-

2 Made available by the Alabama Department of Public Health whose courtesy is hereby acknowledged. ply funds derived from special taxes, appropriations or bond issues. In addition, they may be able to provide trucks, excavation machinery, cement-mixers, etc. In some counties, convict labor is available and may be used for physical control operations. In addition to local facilities, certain federal agencies may contribute materially to malaria prevention while working towards their own social objectives. The names and functions of these will doubtless change and perhaps disappear with the years, but at present the Works Progress Administration, the Farm Security Administration, the Soil Conservation Service, the National Youth Administration, the Civilian Conservation Corps, and the U. S. Housing Authority are able to participate directly or indirectly in anti-malaria activities. Not infrequently, private property and public utility owners can be prevailed upon to supply labor, equipment, dynamite or mosquito-proofing materials for use on their own premises under the technical supervision of local health officials. The final malaria-control plan should utilize each of these assets to the utmost while adhering as closely as possible to the sequence and procedure recommended in the survey report.

Certain pressures will be brought to bear against the proposed program. These must be squarely faced and nullified if effective and economical malaria reduction is to be accomplished. Wild-life enthusiasts and sportsmen will argue against the dewatering of aquatic areas and will urge the substitution of impermanent control methods. Drainage is necessary for certain types of land improvement. While such a consequence of malaria-control drainage is desirable, it must not dominate the selection of drainage sites. Self-interested landowners will urge drainage of their property at public expense with little or no actual malaria justification. The acceptance of such projects is fatal to the future of a malaria-control program. Similarly, ditch-lining may provide enhanced facilities for dealing with storm-water runoff or pest-mosquito breeding incidental to anoph- 
eline reduction. These are desirable improvements, but they should not be done under the aegis of malaria control if no malaria problem is involved. Many public officials are entirely willing to spend public funds in dusting ponds on private property, but are not willing to participate in the costs of mosquito-proofing private homes. In spite of the obvious economy of complete drainage development of one section of a county at a time, the people at risk of malaria in other sections may demand a more equitable distribution of these operations or perhaps treatment at public expense while the dragline remains on the other side of the county. Finally, certain procedures, such as sereening and medication, that are dependent upon individual cooperation and responsibility may not succeed in particular localities without either disciplinary control of the population or a special program of education and inspection. These factors must all be considered in the integration of desirable malaria-control practice with local resources and cireumstances.

\section{Fifth Consideration : Evaluation of Malaria Control}

Measurable malaria attributes fluctuate not only with the season of the year but from one year to the next (Fig. 1). In many instances there is manifested a certain regularity in their trends which suggests some inherent cyclicity or periodicity, the reasons for which are obscure. Malariacontrol operations started during the descent of these attributes are naturally predestined to success. It is, accordingly, very easy to deceive oneself about the success of malaria-control efforts. Probably the most objective method of avoiding such pitfalls is to maintain under careful observation one or more malarious areas where no preventive measures are being carried on. Reconnaissance methods, as well as vital statisties analysis, may be applied year after year to note the natural movements of malaria prevalence in these comparsion areas and to gauge departures from them in places where control programs are in effect.

In the final analysis the best indications of success in malaria control are favorable expressions on the part of low-income families living in previously malarious sections and of tax-paying land-owners who can compare their individual expenditures for malaria treatment before control with their tax bills and land values after control. 


\title{
MALARIA SURVEY: METHODS AND PROCEDURES
}

\author{
By JOHN E. ELMENDORF, Jr. \\ INTERNATIONAL HEALTH DIVISION, ROCKEFELLER FOUNDATION, PENSACOLA, FLORIDA
}

A MALARIA survey is a quantitative as well as a qualitative procedure directed toward securing information which wili determine the status of malaria in a community. The survey should serve as a blueprint for the guidance of the malaria department and it should provide an original base-line from which all subsequent measurements of the course of the disease are determined.

Lists of the equipment necessary for a survey are given in detail by such writers as Boyd (1930a) and Christophers et al. (1936).

The staff will depend upon the scope of the program, but generally speaking the essential personnel should be: a medical malariologist, who should also be the director of the group ; an entomologist with special training in culicidology and the use of larvicides; and a sanitary engineer with special training in hydraulies.

A malaria survey should include the collection of data on demography and incidence. Demographical data, describing for minor civil divisions the composition of the population by race, age and sex, may be secured from official censuses; but if data from such sources are not available, the investigator may be obliged to make a census or at least a very careful estimate.

The incidence of malaria in a population cannot be exactly determined. Morbidity reporting, so useful in many lines of practical epidemiology, has undeveloped possibilities as applied to malaria. At present, however, the utility of available reports is slight, even where competent medical service is conveniently available, because the diagnosis of malarial infection is often not made with precision. Moreover, many infected persons do not consult a physician during their attacks. Furthermore, many states accept totals of cases purportedly seen without requiring the particularized identifying data so essential in epidemiological inquiries. Consequently, the malariologist is usually obliged to gather his own data.

A malariologist with proper appreciation of his problem of diagnosis will limit himself to the collection of objective data, significant in relation to the existence of a malarial infection. Unfortunately, there is not available at present any single infallible criterion by which malarial infection may be recognized. Comparatively few persons encountered in the course of a survey may be acutely ill at the time. The detection of parasites in a blood smear is incontrovertible evidence of the existence of infection, but in many persons with chronic and latent infections the parasites may, at the time when a blood smear is collected, be at submicroscopical levels, and hence undetectable. Malarial infections invariably produce some degree of splenomegaly, which maý persist when the infection becomes chronic, particularly in inadequately treated persons. While other infections may also produce acute enlargement of the spleen, these subside quickly. Therefore in most communities, where other acute infections have not recently been epidemic, the presence of any number of persons with splenic enlargement may be significant from a malarial standpoint. If enlargement is due to malaria, these persons will usually show a significantly higher proportion with parasites in their blood than will be found in those with normal spleens. Furthermore, considerable variation in the size of different enlarged spleens will be observed. For a consideration of other aspects, including the effect of race, etc., the reader is referred to Boyd (1930a).

Since it is usually impracticable to 
examine all persons in a community, the malariologist will generally limit his observations to a sample, the size of which will depend on the total population, the time available, the trend of the current results and the character of the objective data sought (Boyd 1930a). Some malariologists will find it most practicable, for some particular reason or personal preference, to limit themselves to observations on the incidence of parasites or splenomegaly alone or to consider both criteria together. If both are considered the observations should be made concurrently on the same individuals. It should be remembered that the detection of parasites is more particularly indicative of infections recently active; the detection of splenomegaly, of a developing immunity. Hence the two criteria have a different significance.

From the standpoint of practical employment a spleen survey has wider usefulness, especially if performed in the confirming manner described, than a parasite survey, because it permits the examination of a larger sample with the further advantage that the results are immediately available. The collection of blood smears from all persons examined, because of the greater time required per person, reduces the size of the sample, and its examination may delay the availability of the results for many months.

Since schools include the younger age groups of both sexes, various races, and representatives of differing social status, they, are convenient centers in which to conduct the major investigations of the clinical survey. It is frequently unnecessary to examine children over the age of 12 , and many limit themselves to those under 10. However, sometimes other age groups, including infants and adults, should be included; to reach them domiciliary visits will be required.

The clinical survey should be made at, or immediately following, the period of greatest intensity of the disease. Once this period has been ascertained on the basis of history, mortality or morbidity data, or previous surveys, the examinations should be repeated if required, at the same time each year in order that the results may be comparable.

The examination for splenomegaly is preferably made with patients in the recumbent position with knees flexed and abdomen bared. The examiner, standing on the patient's right side, palpates lightly with the right hand which is held more or less at right angles to the costal margin. Two requisites are essential for successful examination: light pressure of the examiner with the hand flat upon the abdomen, and relaxation of the patient, associated on his part with full, deep, abdominal breathing. Thoracic breathing with tense muscle can prevent the detection of a spleen which otherwise would be readily palpated below the costal margin.

The record should state the relative position of the lower border of the enlarged spleen. For this purpose Boyd's classification of splenic enlargement has been found practical. His classification divides enlarged spleens into five groups, namely those palpable on deep inspiration (P.D.I.), and numbers $1,2,3$, and 4 . The ascending numbers indicate progressive degrees of enlargement, varying from No. 1 at the costal margin on normal inspiration, to No. 4, which extends below the umbilicus. No. 2 enlargement extends to any point lying from the costal margin to half-way to the umbilicus, and No. 3 from the former limit to the umbilicus.

In order to demonstrate the extent to which the observed splenomegaly is attributable to malarial infection, it is desirable to take blood smears from each person with an enlarged spleen. Smears furthermore should be taken on a certain proportion (every second, third, fourth, etc.) of those persons presenting negative spleens. An analysis of the incidence of parasites among the persons with different spleen classes reveals the degree to which the observed splenomegaly is indicative of malarial infection. The number of those positive expressed as a percentage, however, does not constitute a parasite index, although it may be estimated therefrom. 
If blood specimens are collected from all persons in the sample the parasitic index may be computed. This is, of course, most time-consuming. The presence of parasites indicates recent spread of the disease, and hence recent infection.

The persons in the sample subjected to splenic examination will necessarily include, if malaria be present, a varying, probably small, proportion of acute clinical cases, as well as many latent or chronic infections. Those with detectable enlargement include a few persons in whom blood examinations are parasite negative, possibly representing recovered infections. On the other hand, a few whose blood examinations are positive will not exhibit detectable splenomegaly. The latter, in some instances, represent very recent infections.

It is most convenient to record the data pertaining to each person on a special record card with keyed perforations along the margins, such as are now commonly used in recording statistical data. The punching out of these holes for positive data simplifies subsequent analyses. The minimal information gathered should include the following: date, locality of examination, name, age, sex, race, residence and length of residence in the house and the locality, spleen classification, identifying number of blood smears, species of parasite, gametocytes, occupation, malarial medication taken, condition of dwelling and protection against mosquitoes (diffcult to secure without a domiciliary visit), attacks of fever over a period of the last year, hemoglobin percentage if desired.

Four points of great importance are to be elucidated by the entomological survey: the anophelines of the local fauna, their role in the transmission of malaria, the water collections wherein they breed and the habits of the adults.

Entomological investigations are usually limited to the area to be controlled and a bordering area about a mile in depth. These investigations require field observations of the larval and adult insects. The ova are also studied at times, but observa- tions of them are not usually essential to the practical ends of a survey. If information on seasonal and yearly variations in incidence is required, studies are continued for a year or more. Exigencies of practical control work will rarely permit this.

The location of the residences of infected persons on a spot map affords a lead to the production areas, at least those of major significance, although all water areas within average flight range of the district in which control is contemplated should be studied. If early in the season and breeding is just beginning, search for larvae alone is most practicable, whereas if the season is more advanced, it is better to carry on both lines of investigation simultaneously.

The entomologist must become acquainted with the local terrain. Topographical maps are of assistance in the discovery of breeding places. If not available, these maps should be prepared concurrently by the engineer.

As minimal equipment in the search for larvae there are required a dipper and pipettes, rubber boots and labelled specimen bottles. If time permits recurring observations, the interval should not be in excess of ten days.

All observations should be entered on permanent record cards. The records pertaining to larvae should afford the following minimal data: exact location and date, type of water collection and actual level; degree of exposure to sun, last significant precipitation, aquatic vegetation, number of larvae collected by instars, pupae and pupal shells collected, local distribution of breeding and its density, identifications, and distance to possible shelters and source of blood meal for adults. All breeding places discovered should be represented on maps by conventional signs and the presumptive permanency of each area should be noted.

Observations on adults are secured from nocturnal catches, the use of traps, and captures in diurnal shelters, called capture stations. Capture stations may be any place where anophelines congregate during 
the day, in or under a house, a latrine, a shed, stable, in a culvert under a bridge, a tree hole, or any locality affording shade and humidity. They may or may not be related to a source of a blood meal. All collections from each location should be kept as separate units for identification.

As minimal equipment in the search for adults there are required : a flashlight; collection tubes, either suction or chloroform; labelled pill boxes for individual collections; and record cards, including labeled spaces for recording places of capture, date and hour; type of station, diurnal or nocturnal eatch; weather conditions during previous night; time spent in capture; species identified and numbers of males and females.

As control work progresses, routine entomological investigations afford a check on the work, and routine visits at weekly or ten-day intervals should be made to areas selected after preliminary investigations have shown that the sites are frequented by adults. They should be chosen with definite relation to known breeding areas so that they cover the total area to be studied in a comprehensive manner.

If it be necessary to demonstrate the importance of certain breeding areas, particularly if located at about the maximum flight range of a species, the recovery of stained adults in nature as well as in special catching stations may be attempted.

Some expression of the density of the anophelines should be adopted. When density is not great, comparison may be made between total catches, but when more abundant, results may be expressed as the number caught per man-hour (Boyd 1930a).

Analysis of the entomological data should yield the following information: density and distribution of local fauna, actual and potential breeding areas, relation of production areas to clinical cases, flight range and direction of ingress or infiltration, and seasonal variations. The data should be entered on entomological spot maps.

It is not expected that each survey would require a specific appraisal of the actual or potential role of the local anophelines as vectors. Information already available will generally indicate the vectors, since the results of intensive studies performed in a few typical and sample localities will likely afford significant information applicable to wide areas.

If required, pertinent information bearing upon this question may be received from the results of precipitin tests performed on the blood removed from a series of stomachs of freshly blooded anophelines to ascertain the host from which the blood meal was taken. If the presumptive evidence suggests a species to be anthropophilic, its susceptibility to infection with malarial parasites should be determined, and demonstration of the infection of this species under natural conditions may be attempted. The technique of the precipitin test is described by Rice and Barber (1935).

If the terrain is unfamiliar and maps are not available, these deficiencies must be supplied by the engineer. Maps for the spotting of clinical and entomological data must be available immediately. Hulse's (1922) sketch mapping for public health work is a very good introduction to the practical preparation of work maps. Attention must be given to all residences and their distinguishing identifications and to water courses and collections, of water and depressions which may hold water temporarily. Information relating to the geological formation of the region and types of soil, as well as the past and current records of precipitation, may also be required.

Furthermore, for convenience in the analysis of the data, the area covered by the maps may be subdivided according to civil or arbitrary subdivisions in order to accentuate local differences in data.

\section{Analysis of the Data Secured in the SURVEY}

The different portions of the surveys are complementary to each other, and in an appraisal of the situation their results 
should be considered together. An analysis of the survey data gives information on various subjects: (1) the scope of the malaria problem in the community, (2) the intensity of the malaria problem, (3) the vectors involved and their areas of production, (4) consideration of control measures which are applicable, (5) preparation of a control project.

The perforated eards pertaining to each geographical unit should be analyzed separately. From these cards information is quickly tabulated showing: the age, sex, and race groups of those examined; the numbers with splenomegaly in each of the above groups; as well as the total $;^{1}$ the incidence of parasitemia in each spleen group; and the incidence of splenomegaly in each geographical subdivision.

An index of malarial infection may be determined by choosing some criterion for infection and determining the number of persons satisfying the criterion per 100 or 1000 of the sample examined. Since no one criterion will infallibly reveal all cases of infection, the computed index of infection will almost invariably be too small.

In case all persons constituting the sample are examined for splenomegaly, and parasites are sought in blood smears taken from each of them, it is possible to calculate readily the crude spleen and parasite indices for the entire group as just described. Of course, a parasite index cannot be directly computed from the parasite incidence observed only in those with splenomegaly. Each index should be computed from its own data. In addition to the crude index for the entire population it is desirable to compute indices for different age groups. The percentage of children under 10 years of age in whom parasites are found has been called the

1 Whether the P.D.I. spleens should be regarded as significant or grouped with the negatives depends on the circumstances. If the incidence of parasites among persons with such spleens is significantly higher than among those with normal spleens. The P.D.I. spleens may in the case of white children be regarded as significant. In the case of negroes, they are definitely enlarged. endemic index by Stephens and Christophers.

The smaller the proportion of the individuals in the group or sample examined to all persons with the same characteristics of age, sex or race in the general population of the area surveyed, the greater the likelihood that chance variation might reveal considerable difference in the results if further samples were examined. On the other hand, when all children between certain age limits are examined at school on a certain day, sampling errors are practically eliminated, as the group examined includes nearly the whole population within those limits of age, sex and race. A more extended discussion of these questions will be found in Boyd (1930a).

The scope of the malarial problem is the first subject of interest. The local geographic distribution of residences of infected persons should be shown by a spot map. A map indicating the extent of malaria-whether the incidence observed was of epidemic or endemic prevalenceshould be supplemented with data covering, among other considerations : the infection measured by splenomegaly and blood positives, by age groups; numbers of the splenic group also positive to parasites; size and character of spleen; parasites in the general population or in the splenic negative group; numbers found with different species of the parasite, and gametocytes present in blood smears.

The expression endemic prevalence acknowledges the perennial prevalence of malaria with some degree of possible fluctuation, depending upon whether the endemic level is normally low, medium, high or even greater. The limits of annual fluctuation are greatest under conditions of low endemicity and limited season and diminish as the level of endemicity increases. Malaria may attain epidemic prevalence after its introduction into areas from which it has been previously absent or from which it has spontaneously disappeared, and after such an introduction remain endemically established for an indefinite period. Furthermore, the wider 
upward fluctuations under conditions of low endemicity are essentially epidemics.

If opportunities for malarial transmission are so widespread that all occupants of an area, inhabitants and visitors alike, may be bitten not once but several times during the transmission season by infected mosquitoes, it is unlikely that the chance visitor or neweomer will pass the transmission season and escape infection. This condition may occur temporarily, as during a severe epidemic, or it may prevail year after year, in which event the situation is characterized as a hyperendemic.

In the latter conditions, all newcomers, whether immigrants or newborn, will soon be inoculated, and presuming they possess the maximum susceptibility will soon suffer from the active disease. This experience will be repeated probably until they acquire an immunity to all the prevalent local strains of parasites. The newborn who survive this initial infection will experience a great deal of clinical malaria during infancy and childhood, but with adolescence clinical attacks will become rarer, and as adults they will seldom or never experience a clinical attack and may not even exhibit any objective signs of infection. Under these conditions we find a high incidence of infection in children, a low incidence in adults.

As the grades of endemic intensity diminish the chance of a susceptible person's

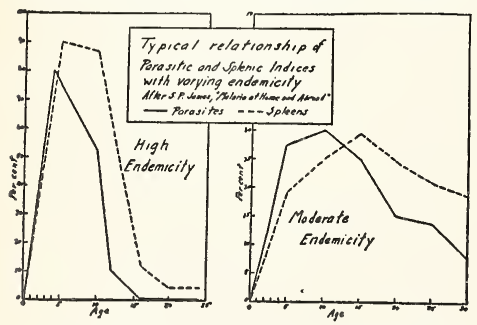

FIG. 1. Typical relationship of parasitic and splenic indices with varying endemicity. (After s. P. James, "Malaria at Home and Abroad." By permission of John Bale, Sons and Danielson, Ltd.) (From Boyd, "Introduction to Malariology," 1930, Harvard Univ. Press.) passing through the transmission season without being bitten by an infected anopheline increase until conditions are reached where such an occurrence will be rare or unusual. Consequently many, or even most, persons may reach adolescence or adult life before they experience their first infection, if they do at all. Thus, as the grades of endemicity diminish, the disparity between the incidence in children and adults will also diminish, until when of moderate or low degrees, the incidence of infection at different age periods may be very nearly uniform and but a small proportion of the population will possess an immunity (Fig. 1).

Populations living in regions of low endemicity and with low levels of herd immunity will respond to an intensification of transmission by the exhibition of an epidemic. Under this circumstance the level of incidence at all age periods will rise, without presenting a disparity between children and adults. This appraisal may be useful in forecasting the difficulties which will require surmounting in order to establish effective control. Satisfactory results are much easier and more rapidly attained where the incidence is a low endemic or an epidemic, than where it is high or hyperendemic.

Differentiation of the endemic picture requires the computation of specific indices for different age groups, including representatives of the adult population. In general the curve of the specific parasite indices will more closely conform to the pictures sketched, regardless of race, than will the specific splenic indices, because negroes are less subject to splenic enlargement than whites.

To discover whether there are seasonal variations, histories, diagnostic laboratory examinations, outpatient records and morbidity reports must be secured through continued surveys. It is important to have knowledge of seasonal variation, since it has a definite value in planning for control because seasonal malaria is easier to control.

Attention must be given to the propor- 
tion in which the different species of plasmodium are present ( $P$. vivax, $P$. falciparum, $P$. malariae). In regions where prevalent, a high relative incidence of $P$. falciparum suggests recent transmission at fairly high levels of intensity. A relatively high incidence of $P$. malariae, especially if $P$. falciparum is low, suggests a low level of transmission during recent years. Recent transmission is revealed by a high level of infection in non-immunes, such as infants and immigrants.
If the entomological survey has incriminated the vector species the problem of species sanitation may be taken up. Searches for larvae and adult captures may have indicated the production areas of the vector. The correlation of the distribution of clinical cases with the known production areas will indicate whether all likely production areas have been discovered. Knowledge of the bionomics of the vector may suggest possibilities for methods of biological or naturalistic control. 


\title{
METHODS DIRECTED AGAINST ADULT MOS- QUITOES IN THE CONTROL AND ERADICATION OF MALARIA
}

\author{
By DANIEL M. JOBBINS \\ GORGAS MEMORIAL LABORATORY, PANAMA CITY, R. P.
}

\section{Destruction by Hand}

THE systematic collection of mosquitoes in dwellings as a prophylactic measure against malaria originated in the Canal Zone according to LePrince and Orenstein (1916). There it was adopted as a standard procedure following its successful use in a temporary camp at Cocoli in 1908 . Universal application has been found for modifications in the technique of collecting mosquitoes with a cylindrical glass tube, about 4.5 inches long and one inch in diameter, in the closed end of which were located cut up rubber bands saturated with chloroform. The capture of live specimens has been facilitated by the use of glass tubes having an inward opening funnel fused in one end and a suction tube affixed in a cork at the other end.

LePrince (1926) stressed the value of destroying anopheles in dwellings, particularly when permanent measures temporarily fail, and noted that their resting habits frequently make the fly-swatter and collecting tube effective in the reduction of malarial transmission even in unsereened buildings.

Reitler estimated that malaria was reduced to one-fifth on a construction project in Palestine largely through the collection of female $A$. superpictus Grassi and $\boldsymbol{A}$. sergenti Theo. from resting places among dark woolen garments. Collections were made with test tubes wetted on the inside with kerosene.

In Europe, Hackett (1937) remarked that programs for collection of adults were unsuccessful in groups of individuals not under rigid discipline because there was no way to compel villagers to capture mosquitoes and a few recalcitrants might render the whole campaign ineffective.
Mosquito collections in houses enabled Davey and Gordon (1933) to reduce to a mathematical formula the relationship between infected anopheles densities and the possibility of inoculation with malaria from different species or in different localities.

\section{NETS}

Since ancient times protection has been obtained from the bites of mosquitoes by the use of nets. As an emergency measure they have been used as bed nets, head nets and as accessory parts of other devices.

Russell and Nono (1934) considered it likely that nets are the most important defense against malaria in the Philippines where permanent screening is out of the question because of the loose construction of rural habitations. These authors found that the locally made sinamay netting of about 16 by 20 mesh per inch with fibers averaging 0.13 inch diameter would exclude all anopheles and most other mosquitoes. Bed nets illustrated in this report were made completely of netting for maximum circulation of air. Marzinovsky recommended bed nets with cloth tops to keep dust from falling on the sleeper and cloth bottom edges to exclude scorpions and snakes.

The use of nets for protection from $A$. gambiae was urged by Thomson in Southern Rhodesia and by Thornton in Natal. Gater concluded that in Malaya, 22/23 mesh cotton net was adequate to exclude anopheles. In Africa, netting of 25/26 mesh cotton afforded protection from $A$. gambiae and $A$. funestus, according to J. Y. Brown (1934) and to Hargreaves (1936). Covell (1931a) pointed out that the mosquito netting supplied to the British Army in India is $25 / 26$ mesh woven cotton thread 
of 25,200 yards to the pound. Copper wire screening in the $16 / 16$ mesh, 28 S.W.G., recommended by LePrince and Orenstein (1916) for the Canal Zone, was found to possess the maximum safe aperture for use in Africa by Davey and Gordon (1938). Mulligan and Majid (1932) stated that apertures somewhat larger were satisfactory for India. Earle (1932) judged that 12-mesh copper of 0.015 inch diameter wire was probably the best screening material for Puerto Rico.

\section{Traps}

Traps providing an attractive resting place for adult mosquitoes have been extensively used in India. Those reviewed by Covell (1931a) have been supplemented by the feather duster and earthen pot traps described by Gore (1937a, 1937b). Russell and Santiago (1934) devised a box-like trap for anopheles which had a layer of soil one inch thick held in place on the inside walls and roof by 16-mesh screening. A dripcan on the roof kept the soil moist. The trap bottom was open and the one end not boarded up was partially closed at night by a black cloth which hung to within 6 inches of the ground. The box was placed directly on the ground and collections were made with glass vials in the morning. The smallest effective trap of this type used in the Philippines was 2 feet wide, 2 feet high and 3 feet long.

In the Orient human-baited traps have been effectively used. Manalang (1931) captured females of $A$. funestus Giles (minimus) in a wire screen cage baited with a man sleeping under a mosquito net. The collector did not risk contracting malaria and mosquito collection was made relatively easy. Urbino (1936, 1938) and Ejercito (1938) also reported the use of man-baited traps to determine the density of A. minimus var. flavirostris. Gater (1933) found that a double bed net, of which the outer had openings that were closed with flaps at intervals during the night, was many times more effective than hand catches in the collection of Malayan anopheles.
In Panama a $\mathrm{V}$-shaped ridge of screening with a slit cut in at the apex enclosed in a screen wire housing was used with success by Bath (1931) to entrap mosquitoes attracted to human scent. The unit was mounted at the top of a window or in the wall of a building. Mosquitoes entering the slit were confined between the ridge and housing screens. In the West Indies, South and Central America, animal baited traps have reached their highest state of development. Magoon (1935) illustrated a stable trap for the capture of anopheles mosquitoes in Jamaica. Earle and Howard described the portable stable trap employed in Puerto Rico and stressed the value of studies of mosquito densities in the direction of control work. Farle (1934, 1937) used animal baited traps for a number of years to determine the feeding preferences of anopheles and to trace the reduction in density of $A$. albimanus Wied. resulting from mosquito control measures. Carr, Melendez and Ros, by means of calf-baited traps, found $A$. albimanus most abundant in the areas of greatest malarial incidence in the Province of Havana. Pereira Barreto captured $A$. darlingi var. paulistensis and other Brazilian anopheles attracted to a horse, and Gabaldon illustrated the burrobaited stable trap used in Venezuela.

In a comparison of animal-baited and mechanical mosquito traps in Panama, Curry stated that the mechanical suction fan light trap was notably inefficient in obtaining information as to density of flights. A portable muslin and netting cage which could be suspended over an animal by cords was devised by Shannon (1939), principally for the capture of nonanopheline mosquitoes. In the capture of A. maculipennis emerging from hibernation quarters in Central Russia, Khelevin (1938) employed glass plates covered with a mixture of one part castor oil and two parts colophony.

In the eastern United States electric light traps have been widely used for sampling mosquito populations. The evolution of the New Jersey light trap from an illuminated sugar can to electric fan suction 
models equipped with time clocks can be traced in the reports of Headlee (1932). In these traps a vertical sheet metal cylinder is covered by a conical roof spaced some distance above its upper end. The attractive light source is located directly under the roof which acts as a reflector. An electric fan is mounted in the upper portion of the cylinder and a screen funnel emptying into a cyanide jar is placed below the fan. Insects attracted to the light are sucked through the fan and are killed in the cyanide jar. The value of these instruments in evaluating anti-mosquito work has been demonstrated by Mulhern (1940). Bishopp, Cory and Stone (1933), in a mosquito survey of the Chesapeake Bay section, observed that $A$. quadrimaculatus and $A$. punctipennis did not enter these light traps in proportion to their relative abundance. In Florida, Bradley and McNeel (1935) traced seasonal variations in the abundance of $A$. crucians and other mosquitoes by means of New Jersey traps. Carnahan (1939) found about 6 per cent of all specimens collected in these light traps near Miami, an airport of possible entry of exotic species into the United States, were A. crucians Wied., A. walkeri Theo., $A$. quadrimaculatus Say and $A$. atropos D. and $K$. represented in that order of abundance.

Johnson (1937) captured A. walkeri in abundance in Tennessee with similar traps in an area where hand catches were negative to that species. As various colors were tested the attractiveness of the light increased through the visible spectrum from red to violet.

Infra-red and photographic red light did not attract anopheles but ultra-violet did so, slightly.

Reports of mosquito collections by means of New Jersey type light traps include Botsford and Turner (1933), Stearns, McCreary and Newhouse (1933), McCreary (1939) and Shields (1938). Storage battery operated devices patterned on these traps have been used by Butts (1937) in New Jersey and in a Works Progress Administration Project in Delaware (1939).

\section{Sprays}

Previous to the widespread adoption of pyrethrum in kerosene oil sprays for the destruction of adult mosquitoes, Mansell (1930) recommended a spray composed of 1 per cent pure carbon tetrachloride, 2 per cent methyl salicylate in second quality kerosene with the addition of one-fourth pound naphthalene to each gallon, applied at the rate of 5 fluid ounces to 1000 cubic feet, and Barber (1936a) found a saturated solution of naphthalene in kerosene cheap and moderately effective.

In Africa, Thornton (1934, 1936), Booker (1935, 1936), and Ross (1936) reported campaigns against $A$. gambiae and $A$. funestus in which the systematic and efficient spraying of native dwellings with a pyrethrum spray prevented epidemic malaria in every case in which they were instituted. Huts representing a population of more than 50,000 natives were included in certain spray programs.

In Europe, Swellengrebel (1934) conducted extensive experiments on the destruction of adult mosquitoes in buildings with pyrethrum sprays. Nijkamp and Swellengrebel (1934) recommended a spray for stables which was composed of kerosene 550 cubic centimeters, vaseline oil 450 cubic centimeters, methyl salicylate 20 cubic centimeters, oil of sassafras 10 cubic centimeters and pyrethrum extract 10 grams. Spraying was considered a routine and not an exro nental procedure by Schüffiner and Swellengrebel (1938). These authors concluded that spraying against anopheles in a state of sexual inactivity in houses in late summer prevented from 50 to 92 per cent of the malaria that would otherwise occur in the following year.

Nabokov and Tiburskaya (1936) obtained excellent results against anopheles in daytime shelters in peat bogs near Moscow with a 10 per cent benzine extract of pyrethrum with the addition of phenyl and methyl salicylates when applied at the rate of 10 fluid ounces per 1000 cubic feet. Viktorov estimated that at least 90 per cent of mosquitoes in houses and tents were destroyed by spraying with a water solution 
of soft natron, soap with the addition of methyl alcohol.

In India, Sinton and Wats (1935) tested the efficiency of various insecticidal sprays in the destruction of adult mosquitoes and found that a solution of one part of a standardized concentrated extract of pyrethrum flowers (Pyrocide 20) in 19 parts of refined kerosene gave results approximately equal to those of the best proprietary brands at about half the cost. These authors stated that the spray was improved by the addition of 5 per cent oil of citronella, $\frac{1}{2}$ to 1 per cent oil of sassafras or 4 to 5 per cent oil of pine. Manifold (1939) remarked that a popular formula for the spraying of huts, barracks and tents was made up of paraffin oil, second grade, $124 \mathrm{oz}$., extract of pyrethrum $2 \mathrm{oz}$., carbon tetrachloride $4 \mathrm{oz}$., oil of citronella $8 \mathrm{oz}$. and gasoline (Petrol) $22 \mathrm{oz}$.

Sprays of pyrethrum extract (Pyrocide 20) in 19 parts of kerosene yielded satisfactory results in many anti-mosquito campaigns. Chopra (1938) added one part of pine oil to the mixture; Covell, Mulligan and Afridi (1938) obtained favorable cost comparisons with temporary anti-larval measures; de Burca (1939) believed reduced incidence of malaria among troops in a cantonment followed a spray program and Russell and Knipe (1939) concluded that the weekly spraying of houses and sheds prevented malaria transmission to a marked extent. Barreto (1929) added pyrethrum to the kerosene and carbon tetrachloride spray used originally in the Rio de Janeiro yellow fever epidemic in order to destroy adult anopheles in Brazil.

In the United States outdoor gatherings have been provided temporary protection from mosquito attack by using sprays. Ginsburg (1935) reported a series of satisfactory trials of a pyrethrum larvicide spray for this purpose. In one formula an emulsion of 100 gallons of kerosene, containing extract of pyrethrum in the amount equal to 100 pounds of flowers, 6 pounds of "Gardinol" concentrated wetting agent and 50 gallons of water was diluted with 10 or 12 parts of water. Power sprayers applied the diluted mate- rial to the vegetation and as a mist in the area to be protected. In Florida, King, Bradley and MeNeel (1937) obtained a high degree of mosquito repulsion in an uncleared area with pyrethrum extract, pine tar oil, oil of citronella and kerosene sprays. In a cleared area, however, none of these sprays was effective during the first mosquito flight period. The behavior of the insects suggested that they were affected chiefly upon coming in close contact with the sprayed vegetation rather than by odors carried through the air.

Among the methods used for destroying disease transmitting mosquitoes in aircraft, Symes (1937) recommended spraying the compartments immediately after departure from an airport with a mixture of 1 part concentrated extract of pyrethrum, 16 part of white kerosene and 68 parts of carbon tetrachloride. Ross (1938) described the use of aqueous base and carbon tetrachloride extracts of pyrethrum in aircraft of the Imperial Airways. Application was made with dry mist vaporizers and sprayers operated by a soda water siphon bulb containing carbon dioxide under 400 pounds pressure.

Griffitts (1933) demonstrated the ability of mosquitoes to enter the United States from Central America in aircraft and endorsed the procedure of fumigating airplanes under emergency conditions and preceding overhaul with as much as 8 ounces of hydrocyanic acid per 1000 cubic feet in the form of Zyklon discoids.

Williams (1940) outlined the procedure of the Public Health Service to prevent the introduction into the United States of mosquitoes infected with yellow fever from South American ports; to prevent the introduction of $A$. gambiae from eastern South America; and to prevent the introduction of any anopheles from the west coast of the United States into the Hawaiian Islands. The methods of spraying the fuselage of all planes from South America with a concentrated pyrethrum spray (2 grams pyrethrins in 100 cubic centimeters of light oil) as described by Williams and Dreesen (1935) and by Welch (1939) was changed to spraying the interior of the 
planes at a port removed from both the infected territory and the nearest United States port. A power sprayer was especially designed with an accurate metering device and an adjustable nozzle with a pressure release to prevent dripping of insecticide.

\section{Fumigation}

Sulfur burned alone or in combination with other materials has long been a moderately effective method of fumigation against adult mosquitoes in spite of its disadvantages. Ananyan (1929) burned a mixture of dung and sulfur to drive $A$. maculipennis into box traps on the top of cow sheds in Armenia, and Clyde found that burning a mixture of equal parts of sulfur and powdered waste tobacco was an effective fumigant. To reduce fire hazard, Nabokov (1929) experimented with the generation of sulfur dioxide from sodium sulfite and sulfuric acid. $\mathrm{He}$ also constructed an apparatus in which the fumes from burning sulfur or tobacco on three dishes placed one above another in a metal cylinder were carried off by a pipe affixed to a conical lid.

James (1935) cleared barracks of anopheles by burning pyrethrum to drive them into a net bag mounted on a dark cloth stretched over a window. Rooms were darkened so that the trap bag was the only lighted area visible to the anopheles. The less effective fumigants, cresol and sulfur, were also used. Treillard (1934) recommended the dripping of cresol on a hot plate in fumigating against anopheles in Indo-China.

A finely ground slaked limedust impregnated with 5 to 10 per cent anabasine sulfate and applied at the rate of 5 ounces per 1000 cubic feet was effective against hibernating mosquitoes in Russia according to Kremer and Kuvichinskii (1937) and Pivovarov and Guterman (1937). Benyaminson and Nabokov destroyed hibernating mosquitoes in freezing temperatures with 2 ounces of pyrethrum dust per 1000 eubic feet, and May found vapors of hexachlorethane toxic but not very practical in the control of mosquitoes in closed spaces.

\section{PoIsons}

In experiments with poisoned baits for destroying adult anopheles, Lischetti (1927) mixed honey with mercury bichloride, boric acid, arsenious anhydride, potassium arsenite or potassium cyanide. Potassium arsenite proved most satisfactory; potassium cyanide, though most active, soon lost its toxicity.

The supposed toxic effect of coumarin on malarial parasites when ingested from clover by female anopheles was not supported by experimental evidence (Mayne 1930a). Stratman-Thomas (1931) considered that any correlation between the planting of leguminous plants and reduction in malarial incidence was due to drainage necessary in growing the crop.

\section{REPELLENTS}

Coogle (1925) repelled mosquitoes from unscreened and dilapidated houses by pressure spraying creosote at the rate of one gallon per 450 square feet of interior wall surface.

Gutzevitch and Podolyan (1935) repelled mosquitoes with smoke candles made from a mixture of $100 \mathrm{gm}$ of pyrethrum powder (pyrethrin content .28 per cent), $50 \mathrm{oz}$ semi-liquid wheat flour paste, $25 \mathrm{oz}$ potassium nitrate, $25 \mathrm{oz}$ sawdust and 50 to 70 fluid oz water. Candles about one centimeter square cut from the viscous mixture were dried and burned at $\frac{1}{2}-2 \frac{1}{2}$ oz per 1000 cubic feet as a repellent or $1-1 \frac{1}{2}$ oz per 100 cubic feet as a fumigant.

Repellents for the protection of individuals from mosquito attack have been concocted on a rather empirical basis until recently. Bunker and Hirschfelder (1925) set forth the desirable characteristics of such substances for personal use and tested the effect on mosquitoes of most of the materials recommended in earlier literature. Twenty substances listed in their general order of effectiveness were: (1) citronellol, (2) caprylic alcohol, (3) benzyl alcohol, (4) geranyl acetate, (5) linalyl acetate, (6) amyl salicylate, (7) acetophenone, (8) oleum picis liquidae rectifactum, (9) phenyl-propyl alcohol, (10) oliver oil, (11) citronellal, (12) eamphor, (13) vanillin, 
(14) methyl cinnamate, (15) menthol, (16) cedarwood oil, (17) citrol, (18) coumarin, (19) beta-naphthol-ethyl ether, (20) geraniol. These authors suggested that the influence of certain atomic groups on insects might act as a guide in the search for more effective repellents. Alcohols, ketones, and aldehydes were in general more efficient than other groups. Rudolfs (1926, 1930) studied the response of mosquitoes to many of the organic compounds associated with animal and human odors and tested a large number of oils, solvents and organic compounds as repellents for non-anopheles. Gibson (1935) reported tests on several well-known repellent formulae.

Oil of citronella, one of the most effective and popular repellents, was mixed with an equal part of olive oil by Cooley and others. Freeborn found a mixture of $3 \mathrm{oz}$ oil of citronella, $1 \mathrm{oz}$ spirits of camphor, $1 \mathrm{oz}$ tar oil, $\frac{1}{4}$ oz oil of pennyroyal and $4 \mathrm{oz}$ castor oil effective against Aedes mosquitoes, and Manifold (1939) stated that the British army in India, Egypt and Palestine used a modification of Dover's repellent consisting of oil of citronella 18.25 per cent, camphor 1.00 per cent, cedarwood oil 9.00 per cent, paraffin duram 26.75 per cent and paraffin molle-white 45.00 per cent. MacNay (1939) recommended $\frac{1}{2}$ fluid oz oil of thyme, 1.0 fluid oz extract of pyrethrum ( 0.2 pound of flowers) and 2 to 3 fluid oz castor oil as being effective for 3 to 5 hours against Aedes species.

Granett (1940) gave results of comparative tests of established repellent substances and a recently developed proprietary synthetic organic chemical mixture containing diethylene glycol monobutyl ether acetate, diethylene glycol monoethyl ether, ethyl alcohol, corn oil and perfume. Developed from tests of nearly 1000 compounds and mixtures, this repellent was found to be definitely superior to citronella and 42 representative proprietary products in lasting power and desirable accessory properties.

\section{Deviation}

In Macedonia the investigations of Bar- ber and Rice (1935), comparing the attraction to humans of $A$. elutus and A. maculipennis varieties typiens and messeae, illustrated the necessity of knowing the degree to which local anopheline species are attracted to animals before judging the effectiveness of animal barriers.

In China, although $A$. hyrcanus var. sinensis Wied. was found to be zoophilous, Toumanoff and $\mathrm{Hu}$ (1935) judged deviation by animals was not complete. In the Philippines, Russell (1934) was unsuccessful in protecting a native house from $A$. minimus var. flavirostris by the use of four water buffalos. In Kenya, Symes (1930) showed that cattle stabled within native dwellings afforded the inhabitants little protection from $A$. gambiae and $A$. funestus.

In Argentina, precipitin tests by Davis and Shannon (1928)' showed 50 per cent of A. pseudopunctipennis taken in houses to have fed on man. In Venezuela, Hill found $A$. albimanus fed on man in large numbers and in Puerto Rico precipitin tests confirmed observations that $A$. grabhami preferred animal blood to that of man. In Mexico, the majority of $A$. pseudopunctipennis captured in houses in Temixco by Vargas (1938) contained human blood.

In Canada, Hearle observed that $A$. maculipennis attacked animals in preference to man on the prairies; and in the United States, Boyd (1930d) suggested (from precipitin tests on blood from anopheline stomachs in North Carolina) that the nocturnal presence of cattle in the vicinity of dwellings was associated with reduced malaria and that this was probably accomplished by a diversion of $A$. quadrimaculatus from man to cattle. Christophers and Missiroli (1933) judged that in general this use of cattle will be confined as a practical measure to conditions where the cattle form an integral part of an agrarian scheme. Hackett, Russell, Scharff and Senior-White (1938) considered deviation the only naturalistic measure showing promise of success or worthy of the expenditure of public funds. 


\title{
HOUSING WITH SPECIAL REFERENCE TO MOSQUITO-PROOFING FOR MALARIA CONTROL
}

\author{
By CALVIN C. KIKER \\ SANITARY ENGINEER HEALTH AND SAFETY DEPARTMENT, TENNESSEE VALLEY AUTHORITY, \\ WILSON DAM, ALABAMA
}

Housing may influence regional health in many ways. Certain diseases are increased by the crowded condition found in the slums of great cities, while others are favored by the inadequacy of houses in certain of the rural sections. The discussion here will be limited to housing with particular reference to its relation to malaria transmission in the Southeastern States.

It must be stated in the beginning that health organizations in the Southern States, perhaps without exception, consider the most effective and satisfactory approach to malaria prevention to be through the control of $A$. quadrimaculatus, the only important vector of the disease in this region. The soundness of this approach is not questioned since there can be no malaria transmission in the absence of the vector. $A$. quadrimaculatus is highly selective as to its place of propagation, and for the most part the aquatic development takes place only in the still parts of shallow natural or artificial ponds where the surface is overgrown with vegetation or is covered with flotage. There are many ways of preventing or limiting emergence of the vector which are discussed in detail elsewhere in this volume; hence, only brief mention will be made of them here.

The only completely effective mosquito control measures are those which eliminate the breeding areas permanently, usually by drainage. The principle of this method is applied also to bodies of water which cannot be eradicated, such as impounded lakes. In them environment unfavorable to anopheline propagation is produced temporarily at appropriate intervals by changing the water level, combined with other measures which tend to prevent the growth of marginal vegetation. All other measures directed toward the prevention of mosquito propagation are of secondary efficiency.

Likewise, measures directed toward the prevention of malaria transmission must be considered to be secondary control measures. This does not imply that they lack importance. The general nature of the malaria problems of the United States requires the use of secondary control measures in many instances. In situations where malaria is most prevalent it is often associated with collections of water which, from an economical standpoint; cannot be eliminated by drainage or filling; nor can mosquito propagation in them be accomplished by other biological control measures or by larvicides. Under these conditions, the improvement of homes by the application of mosquito-proofing, to lower the rate of biting frequency, is probably the most practical, economical, and effective malarial control measure available.

Shortly after the discovery of the rôle played by anopheles, mosquitoes in malaria transmission, the principal bionomic characters of $A$. quadrimaculatus were defined. The fact that this mosquito confines its search for blood to evening and night hours, when rural families are usually in their homes, was soon discovered. This observation led naturally to a search for methods which would prevent the entrance of mosquitoes into homes, and mosquito-proofing was advocated by many health workers.

In spite of the fact that improved housing, including mosquito proofing, has been advanced for many years as a practical 
means for securing malarial control, remarkably little progress has been made in the past 20 years in the application of this measure on a group or regional basis. This may be due, in part, to the discovery in 1922 that Paris green was a useful anopheline larvicide. The years which have followed its first use have shown, however, that it possesses the limitations inherent in all larvicides.

\section{Rural Housing in the Southeastern United States}

Rural homes of the tenant class in the Southeastern States are usually very poor. The average dwelling of this class usually has many cracks, knot holes and other openings in the floors, walls and ceilings. These openings must be closed, in addition to applying screens to doors and windows, before the house offers a reasonable protection against mosquitoes.

Fullerton and Bishop (1933) presented a detailed program and plans for improved rural housing in the malarious areas of Tennessee. A bulletin of instructions, structural plans, and specifications was published and made available to builders through county health departments. It was shown that, by proper planning and construction, houses could be built at very little increased cost which would offer protection against mosquitoes. Moreover, the planned houses were arranged more conveniently and offered greater protection against the cold of winter and the heat of summer. Attention was directed to the possibilities of effecting economies, conveniences and sanitary improvements by planned grouping of houses. It was emphasized that by making a study of available sites the house or group of houses might be located beyond flight range of $A$. quadrimaculatus breeding areas.

This possibility of lessening malaria transmission on large plantations or tracts of land by systematically locating houses beyond flight range of mosquito breeding areas is deserving of more attention. It would in no way interfere with the normal use of land as pasturage, and agriculture would continue as usual, but it would require restriction of the use of land, within flight range of breeding places, to daytime occupancy.

The Tennessee Bulletin on rural housing set out plans for improved construction of houses from materials now in common use. In this connection there is a construction method deserving of mention, which is as yet little used in the south. It is the type of construction where walls of the house are made of "pisé du terre," or rammed earth. It has been fully explored and tried in the United States and elsewhere and has proved economical, serviceable and durable. The usual construction is to make the walls 14 to 16 inches in thickness of rammed earth, with floors, ceiling, roofs and door and window frames of the usual wood construction. A foundation of concrete or masonry is required to extend a few inches above the ground line. The best material for rammed earth construction is a sub-soil clay or loam containing 30 to 50 per cent sand. Pure clay is not suitable because it tends to crack and flake upon drying. The walls are usually constructed in short sectional courses about 3 feet in height. Three or 4 forms are all that are necessary as their use may be alternated. The forms must be constructed of heavy material and tied with steel rods, as in forms for concrete. An alternate to the monolithic construction is the pre-cast rammed earth blocks which, upon being cured, are used in building the walls as brick, tile or concrete blocks would be used.

It is important that the earth contain just the proper amount of moisture. Ramming must be thorough, with tools designed for the purpose. Further details may be found in the literature listed in the references under Betts and Miller (1937), Ellington (1938), Patty (1936), Patty and Minimum (1933) and Williams-Ellis-Clough (1920). This type of structure tends to facilitate complete mosquito-proofing since cracks and crevices, so common to the walls of frame structures, are minimized.

The Resettlement Administration built 7 houses of rammed earth construction at 
Mt. Olive, a suburb of Birmingham, Alabama, in 1937. These houses were examined in 1940 after 3 years' use. They were all occupied and in good condition and were reported to be unusually cool in summer and warm in winter.

There is a real need for study of rural housing by health agencies, looking toward specifications which would permit construction at a cost of $\$ 100$ per room. Rammed earth construction of homes of simple design, the forms for which might be made available through a county agency, may be a partial solution of the problem. Construction with conventional materials does not appear to offer hope that the goal is attainable.

\section{Mosquito-Proofing}

While improved housing is necessary for satisfactory and durable mosquito-proofing, health workers are reluctant to wait until such a profound change can be brought about in areas where secondary control measures are indicated. Mosquito-proofing is not an easy task in the average rural house in areas of endemic malaria, for malaria apparently begets poor housing through lowering the economy, and the mild climate of the deep South is certainly not conducive to special efforts to making the houses tight as protection against extremes of temperature.

Some of the principal difficulties encountered in mosquito proofing these houses, most of which are constructed of poorgrade lumber are: (1) Cracks in the floors between the planks which also frequently sag due to inadequate support; (2) cracks and knot holes in the walls and ceiling (in some cases there are no ceilings); and (3) deteriorated door and window frames having such uneven faces that replacement is necessary for adequate fitting of screens and doors.

In spite of the poor condition of most rural houses in the South, health organizations have developed reasonably effective methods of rendering them mosquito-proof, and there have been notable cases of mass mosquito-proofing, some of which deserve mention.

In about 1927 the Lake County, Tennessee, Health Department undertook the promotion of a county-wide screening program which in the next 3 years resulted in some improvement to practically all rural dwellings in the county (Meleney and Crabtree 1934).

In 1926-1928, C. P. Coogle, epidemiologist with the U. S. Public Health Service, directed the screening and mosquito proofing of 500 rural houses in LeFlore County, Mississippi. The experiences on this and other work in Mississippi were set out in bulletins for use of others engaging in the work. A control of nonmosquito-proofed houses was established for observation in the LeFlore County work, and the differences in malarial rates in the two areas is given in the following table:

EfFects of ScReening on Malarial Rate

\begin{tabular}{|c|c|c|c|c|c|c|c|}
\hline \multirow{2}{*}{ 范 } & \multicolumn{4}{|c|}{ Screened houses } & \multicolumn{3}{|c|}{ Unscreened houses } \\
\hline & 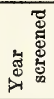 & 总 & 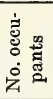 & 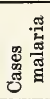 & 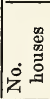 & 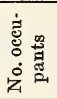 & 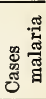 \\
\hline 1926 & 1926 & 104 & 416 & 24 & 104 & 467 & 84 \\
\hline 1928 & 1927 & 500 & 2057 & 206 & 500 & 2140 & 814 \\
\hline
\end{tabular}

This screened area was visited by the writer in October, 1940. Most of the door frames were found to be in serviceable condition, although the original screen wire was gone in many cases. Some of the original window screens located in protected places were in serviceable condition. Some limited maintenance had been carried out by the owners or occupants of the houses.

In 1930 representatives of the Alabama State Department of Public Health visited the mosquito-proofing work in Tennessee and Mississippi for the purpose of obtaining information on methods and procedures. Subsequently, for a period of about 2 years, organized effort at mosquito-proofing was carried out in a number of Alabama counties. The volume of the mosquito-proofing resulting was not large, but 
the work was considered to have value as an educational measure.

The completion of mosquito-proofing on a group of 700 rural houses located about the Tennessee Valley Authority's Wheeler Reservoir on the Tennessee River in North Alabama provides more recent information and data on this subject (Watson and Maher 1940; Kiker and Breedlove 1941; Watson and Rice 1941).

The marginal areas of the central part of the Wheeler Reservoir are characterized by extensive shallows where a combination of rich alluvial soil, moisture and watertolerant plants has made it very difficult, if not impracticable, to provide completely effective control of $A$. quadrimaculatus through biological means. In attempting to control or limit mosquito production from these areas Paris green dust was applied as a larvicide by use of airplanes. Routinely, however, it was found difficult to obtain a kill of larvae approaching 90 per cent with Paris green dust. This failure to obtain a complete kill of larvae permitted a significant emergence of $A$. quadrimaculatus, since the size of the breeding areas in question is very large. A further difficulty was that mosquito production occurred from natural breeding areas bordering the reservoir which were in no way affected by the impoundage. It was seen, therefore, that regardless of the degree of mosquito control obtained on the lake, sufficient A. quadrimaculatus would emerge from the natural ponds for serious malaria transmission.

The question of alternative methods of control was placed before the Tennessee Valley Authority's Board of Malaria Consultants $^{1}$ which recommended that mosquito-proofing be tried and studied over a period of some 5 years to obtain information on effectiveness and costs, from which

1 Dr. Mark F. Boyd, Rockefeller Foundation; Col. George C. Dunham, U. S. Army; Comm. C. S. Stephenson, U. S. Navy; Dr. T. H. D. Griffitts, Dr. L. L. Williams, Jr., Mr. J. A. LePrince, U. S. Public Health Service; Mr. G. H. Hazlehurst, Alabama State Department of Public Health; Dr. W. V. King, U. S. Department of Agriculture; Dr. Robert Matheson, Cornell University. a decision might be made as to the place of mosquito-proofing in malaria control associated with impounded waters. The Alabama State Department of Public Health agreed to employment of mosquito-proofing as a temporary substitute for larvicide in certain areas. The Department administered the construction work, which was financed by the Tennessee Valley Authority from an allotment for malaria control on Lake Wheeler. Controls of nonmosquito-proofed houses were established. The study was begun in 1938 and has been earried out as a cooperative endeavor between the State Department of Public Health and the Health and Safety Department of the Tennessee Valley Authority.

The initial mosquito-proofing work, completed in the spring of 1938 , embraced 100 houses in the Harris Station Area. The results obtained during the first season of transmission were inconclusive, but were so encouraging that additional groups totaling 243 houses were mosquito-proofed during 1939. The second season's observations tended to confirm the first and a further expansion was made during 1940 , bringing the total to 702 houses mosquitoproofed to date. Data bearing on effectiveness are shown in the following two graphs.

Total Morbidity Rates in THRee Test Areas, WHEELER RESERVOIR, 1939

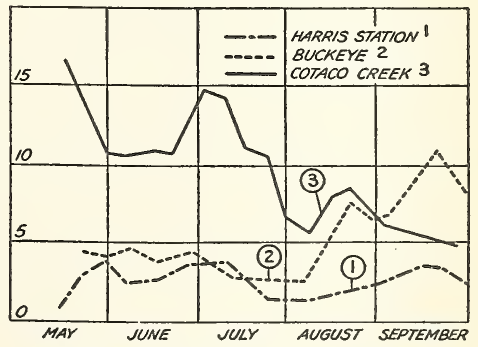

1. All houses mosquito-proofed. 2. Control area; no houses mosquito proofed. 3. Mosquito-proofing 50 per cent complete July 1; 75 per cent July 15; 100 per cent August 1. Scale on the left is the number of cases per 1000 persons. 
Malaria Parasitemia as Determined by Surveys in Three Test areas, Wheeler Reservorr, 1939

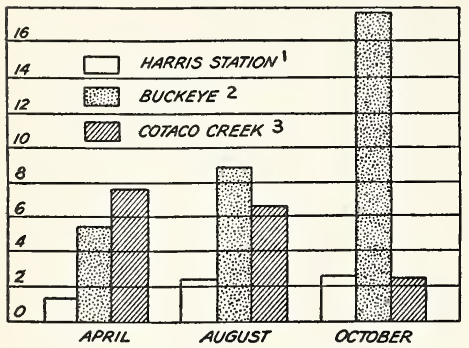

1. All households mosquito-proofed. 2. Control area; no houses mosquito proofed. 3. Mosquitoproofing 50 per cent complete July 1; 75 per cent July $15 ; 100$ per cent August 1 . Scale on left is number of cases per 100 persons.

The findings thus far tend to confirm some earlier data indicating that mosquitoproofing is a reasonably effective measure against malaria. No final conclusions have been drawn, however, as it is believed advisable to draw conclusions only from experiences over a period of years which embrace, if possible, a complete cycle of high and low peaks of regional malarial transmission. Previous data have prompted the belief that this cycle in the Tennessee Valley has a period of about 5 years.

An unusual feature of the construction program was that the work was done by contract on a unit price basis which has proved highly satisfactory. The usual form of construction contract was employed, including specifications, proposal and contract. Bidders were required to submit a bidder's bond or certified check, with the successful bidders subsequently posting a security bond not only to guarantee the completion of the work on schedule, but also to guarantee the excellence of both workmanship and material.

Since the specifications may be of interest, those used on the last contract are quoted as follows:

Lumber. (a) Screen door frames shall be thoroughly dried clear cypress-full one inch thick and three inches wide-S-4-S. (b) Screen door hang- ing strip shall be thoroughly dried clear eypressfull one and one-sixteenth inches thick by three inches wide-dressed one side, two edges. (c) Door and window facings, flooring, ceiling, etc., shall be No. 1 pine-S-4-S.

Screen wires shall be galvanized wire screen of 16 meshes per inch.

Construction of screen doors. The attached drawing supplementing the above mentioned bulletin shall be followed in constructing sereen doors.

Material for screen doors. (a) Reinforcing plate for screen doors shall be cut from square of 24 gauge galvanized sheet metal. (b) Hardware cloth. The screen wire in the bottom panel of the screen doors shall be reinforced with galvanized hardware cloth, $4 \times 4$ meshes per inch standard gauge wire.

Nails. The wire clout nails used in fastening the metal reinforcing plates on the outside of the screen door shall be of sufficient length to clinch $\frac{1}{8}$ inch. The inside reinforcing plates shall be put on over the screen wire and fastened with wire clout nails of length equal to the thickness of the wood frame.

Hinges. The hinges shall be $3^{\prime \prime} \times 3^{\prime \prime}$ loose pin butt type and fastened with No. 8, $1^{\prime \prime}$ screws.

Tacks. The screen wire shall be fastened to the doors with No. 6 bill poster tacks spaced 2" apart.

Other items. The door pulls, hook and eyes, and coil springs as specified in the bulletin shall be included.

Application of window screens. Window screens shall overlap the window frames full 1 " on top and sides. The bottom edge shall be folded under, overlapping sill two inches and securely fastened with a double row of tacks. Wire shall be fastened to the window frames with No. 6 bill poster tacks spaced 1" apart.

Mosquito proofing material. (a) Paper for covering walls and ceilings shall be 90 pound basic kraft weighing not less than 1 pound per 34 square feet. (b) Tacks. The paper shall be securely fastened to the walls with No. 6 bill poster tacks. (c) Roofing paper. The paper specified for covering floors shall be two-ply or medium weight roofing paper weighing 45 pounds per 100 -square feet. (d) Sheet metal for patching small holes in the floors and walls shall be twenty-eight gauge galvanized sheet metal and securely fastened.

Chimney protection. Omit the Napthalene cylinder and wire basket of moth balls for hanging in chimney. Fireplace openings shall be sealed with a removable ply board screen. This screen shall be constructed so as to seal effectively the fireplace opening. The unit price bid shall include the necessary repairs to the mantel facings and hearths to secure effective closure against the entrance of mosquitoes. Specifications are shown on the attached drawing.

These specifications were developed from earlier experience on such work in Alabama. Moreover, houses which had been 
mosquito-proofed several years previously on other projects were inspected very thoroughly to determine where improvement might be made to make the work more serviceable and durable. Some of the experiences with respect to construction are as follows :

(1) It is not advisable to use woods other than cypress in the construction of door frames.

(2) It is not advisable, generally, to attempt to utilize old existing screen door frames.

(3) It is very important to protect the screen wire of the bottom panel of the door with the heavy 1 inch mesh hardware eloth and to place a diagonal brace in the lower corner of the upper panel where a person might otherwise push against the sereen wire in opening the door.

(4) It is advisable to close fireplace openings with a removable fireplace screen of plyboard construction. Hanging a basket of moth balls in the chimney, sealing the top of the chimney with screen wire or covering with eanvas, elosing the opening with sacks stuffed with hay, paper, or other material have all been tried with indifferent success.

(5) The large-headed No. 6 bill poster tacks have proved satisfactory for fixing screen wire and paper.

(6) The 16-mesh galvanized screen woven of not less than .01 inch diameter wire has proved satisfactory. However, near the seacoast or about industrial plants where a corrosive atmosphere may exist, using a wire of larger diameter and more resistant composition, as bronze, should be considered.

(7) The 90 pound basic kraft paper is reasonably tough and to date has proved a very satisfactory material for covering walls and celings. There are other heavier and tougher papers, some even water-resistant, which are available at reasonable cost. The principal cost of papering walls and ceilings is the labor of application.

(8) It is advisable to replace deteriorated door and window frames.

(9) It is very important when applying roofing paper for closing eracks in floors to repair the planking by necessary nailing or bracing.

An analysis of the last contract completed shows that there was an average of 3 doors and 1 fireplace for closure per house. There was further, 112 square feet of screen wire, 1000 square feet of wall paper, 180 square feet of roofing paper for floors, and 180 board feet of lumber required in mosquito-proofing the average house, in addition to some miscellaneous items, as tacks, nails, springs and hooks for doors. The above materials cost $\$ 16$ for the average house. The contractor on the work indicated that an average of 37 manhours of labor per house were required to apply the materials. Assuming that 40e per hour was the average labor cost, then this item would amount to $\$ 15$, making the average total cost per house approximately \$31. In applying these data elsewhere it would be advisable to make adjustments for local costs of material and labor, including any necessary items for transportation, supervision and overhead.

The annual maintenance of the mosquitoproofing was also handled by contract in a similar manner. Items were added for refitting and repairing sereen doors and retacking paper and screen wires. In order to interest bidders in this type of work it was necessary to include an item for truck mileage since there was considerable travel in proportion to the volume of work specified.

During the spring of 1940 the 344 houses mosquito-proofed during 1938 and 1939 were gone over with a maintenance operation. Some new mosquito-proofing was required on houses built recently, rebuilt or oceupied since the original mosquito-proofing was done. All such work was lumped together with the regular rehabilitation and classified as an item of the maintenance of mosquito-proofing in the whole area.

The regular rehabilitation consisted of refitting and repairing screen doors and fireplace screens, patching screen wire, retacking sereens, paper and strips of tin and replacing door and window facings as required. At current wholesale prices the materials used have been estimated to average $80 \mathrm{c}$ per house. The contractor estimates that 4.4 man hours of labor were required which, if an average of $40 \mathrm{c}$ per hour is applied, would result in a total labor cost of $\$ 1.75$. The total cost for labor and materials would, therefore, average $\$ 2.56$ per house exclusive of transportation, supervision, overhead and profit charges.

The first year's maintenance operations indicate that the people really appreciate the work and are taking reasonable care of the mosquito-proofing. In no case has wanton or careless abuse been observed. 
The initial maintenance cost seems reasonable and it is believed that an annual rehabilitation job such as was done in 1940 will more or less perpetuate the mosquitoproofing, although 4 or 5 years will be required to reach the maximum annual maintenance cost.

\section{Financing}

The question arises as to how improved housing and mosquito-proofing may be obtained when they are indicated as a malarial control measure. Certain basic facts present themselves and must be taken into account in considering the problem.

Most public funds from which appropriations for public health work are made cannot be applied to improving housing because this may be construed as constituting improvement of private property. Malaria control drainage might also be considered in this light, for which considerable public funds are being expended but the legal aspects are somewhat different. The public also understands that the benefit to private property in the case of malaria control drainage is secondary and incidental to the public health benefit.

Thus, it is seen that at least for the present funds for improved housing and mosquito-proofing must be obtained from private sources directly or indirectly. Group taxation for a specific purpose is given as an example of how funds might be raised indirectly. For purpose of illustration reference is made to a recent malaria control bill which was introduced into the legislature of Alabama, adopted and later ratified by a state-wide referendum as an amendment to the constitution, which gives the people of Colbert County the privilege of levying additional taxes for the purpose of controlling malaria on a district basis. The bill is quoted as follows:

\footnotetext{
"The Court of County Commissioners of Colbert County, Alabama, is authorized to divide said County into drainage districts for the control of malaria, and said County is authorized and empowered to levy and collect in the several districts so formed, for use in the control of malaria, in addition to all other taxes now authorized by law, a special tax of three mills on all taxable property
}

situated in the several drainage districts so formed, based upon the valuation of such property as assessed for State taxation, and to be used exclusively for the control of malaria in the drainage district in which the said tax is levied and collected, provided such tax is authorized by a majority of the qualified electors residing in such drainage district voting upon such proposition at an election called and held for the purpose of authorizing such tax, and provided that said tax shall be levied and collected for a period of ten years from the time that it is authorized at the election held in such district. Such an election may be called at any time by the Court of County Commissioners of said County and shall be held and conducted and the results canvassed as now provided by law for holding and conducting and canvassing the returns of a regular election. The proceeds of the tax hereby authorized shall be used exclusively for the control of malaria in the drainage district in which it is levied and collected and shall be expended through the proper fiscal agencies of the County Government under the direction of the Governing Body of Colbert County, and the Colbert County Department of Public Health."

Material advancement is dependent on a strong individual and collective desire for improvement. Continuing public health education, both in and out of schools, should be an important approach to promotional effort. Health organizations should present a practical, logical and feasible control program. Long-term planning is important, but consideration should always be given to control measures of a temporary nature pending realization of the final objective. It is, of course, important that the program be predicated on a most thorough analysis of the problem from epidemiological, biological and engineering standpoints. The economic resources of the individual or the region must be taken into account. Malaria is a disease which exacts a tremendous toll in direct costs in addition to lost time and efficiency. Control program financing should show an economic saving if possible. General mass improvement of housing in rural areas of the south where malaria is endemic, and where biological control of its vector is not feasible, is difficult to foresee as an accomplishment of the immediate future. However, mosquitoproofing of existing houses is a practical possibility. Except for eradication of the vector, it is probably the most effective malaria control method. 


\title{
DRAINAGE AND FILLING METHODS FOR MOSQUITO AND MALARIA CONTROL
}

\author{
By NELSON H. RECTOR \\ MISSISSIPPI STATE BOARD OF HEALTH, JACKSON, MISS.
}

\section{Historical}

Sir Ronald Ross (1910) states: "Probably the ancients, and certainly the Italians, since the time of Lancisi (1717), were so well acquainted with the good effect of drainage that this principle was accepted as a dogma in all the medical sehools."

In 1902 Sir Malcolm Watson began using drainage in his successful malarial control work in Malaya. In 1904 General Gorgas, LePrince and others undertook the control of malaria (and yellow fever) in the Panama Canal Zone, utilizing all known methods for reducing the mosquito population, but placing major dependence on drainage. Between 1870 and 1920 an enormous amount of drainage was executed in every section of the United States. This period may properly be called the era of drainage.

Malaria control drainage is a very specialized type of work and should not be undertaken unless well qualified, experienced engineers are available to select, plan, and supervise the work. As a general rule, agricultural and malaria control drainage are not one and the same. The agricultural engineer desires to remove water quickly to prevent damage to crops, while the sanitary engineer aims to remove residual water before a brood of mosquitoes can develop.

\section{Man-made Malaria}

Much of the early drainage work carried on with relief labor was designed to eliminate mosquito-breeding areas that had been created by engineers along highways and railroads. Today highway and railroad engineers, realizing that good drainage along their rights-of-way makes the structures more stable and reduces maintenance costs, are not ereating any more undrained borrow pits.

\section{Preliminary Investigation}

After the malariologist and entomologist have determined that malaria is a problem in a locality, and that an anopheline vector breeds in the area, the engineer. begins his studies. He determines the feasibility of the project from the standpoint of: (1) whether a positive or suitable outlet is available, (2) its probable cost, and (3) whether the whole project seems to be practical. He will determine the area where drainage appears to be most feasible and will also decide on the projects to be undertaken first and will place the others in their proper positions, based on their relative importance. During this study, he will also keep in mind the types of drainage applicable and will determine if the ditch should be constructed by hand, with dynamite, with a road machine, with mules and slips, or by a dragline. He must also decide whether the drainage will be by means of open earth ditch, tile, vertical drainage, underground drain, or invert. The selection of the proper method is of paramount importance.

It would be foolish to spend the time necessary to make a scientific investigation if a preliminary study shows that the per capita cost would be prohibitive, that a positive outlet is not available, or that the drainage system would have to be very deep and traverse unstable soils. In a few places failure to follow these principles has cost the tax payers large sums. More than one drainage system has been rendered almost useless because the outlet canal was not carried into a positive outlet. Maintenance costs are always high on poorly designed drainage projects and in some instances the whole investment has been lost.

After it has been determined that the proposed work is feasible and necessary, the 
engineer in charge will secure topographical maps from which a base map may be prepared. A scale of one inch to 2000 feet will permit all the details required. When the base map has been completed, all water courses, ponds, sloughs, swamps, cisterns, wells and any other source of mosquitoes should be platted in their relation to the surrounding population.

\section{Methods}

On small projects, especially where relief labor is available, it will be economical for the community to construct the ditches by hand. On larger ditches through stable soil and where the property owner will permit a wide ditch, it is possible to effect a saving by the use of a road grader pulled by a Caterpillar tractor, or by mules and slips.

If the ditch follows a natural wooded slough or bayou and if the bottom of the ditch is soft and mucky, dynamite will often be the cheapest method for the installation of the drainage outlet. The work can be done easily and quickly and at a reasonable cost, as only one pound of dynamite is required to move a cubic yard of dirt. Large outlet ditches through deep ridges should be constructed with draglines.

Under most conditions vertical drainage is not considered a practical method. A notable exception to this rule is the vertical drainage project that was installed in Sheffield, Alabama, many years ago and is operating satisfactorily today. This method must not be used unless a permit has been secured from the state board of health, as the underground water might be contaminated by such procedure.

\section{SPECIFICATIONS}

Lines. The line of the main outlet ditch should be as straight as possible. However, if two routes are available, one traversing high ground where drainage is not needed, and the other being somewhat longer but following a natural depression, the second route should usually be selected. The construction and maintenance costs on

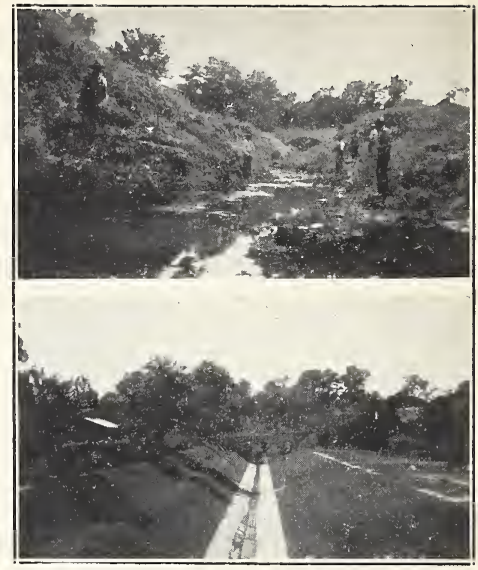

FIG. 1. Vicksburg, Mississippi. Upper, before malarial control drainage; lower, after construction of reinforced concrete invert with sodded banks.

the shallow ditch will probably be much less and the ditch on low ground will afford better drainage of mosquito-breeding areas. Rapid changes in alignment should be avoided and gentle curves substituted for abrupt ones.

Grade. The grade of a drainage ditch should be just enough to give a cleansing velocity but not enough to erode the banks. In general a grade of 0.05 foot per 100 feet is the minimum for open ditches. (Flatter ditches are satisfactory if under-drained with tile.) Ditches with this low grade will have a relatively high maintenance cost. The maximum permissible grade will vary with the soil. A slope exceeding 0.6 to 0.8 foot per 100 feet is not desirable in an open earth ditch. When steeper grades are encouniered, spillways constructed of concrete, masonry, or brick, or brush dams made of poles and tree tops laced together with wire, have been found invaluable for reducing the velocity and thereby preventing undue erosion.

Width. The width of the ditch depends on the watershed area, on the rainfall and 
on the type of terrain. Data are usually available in each of the state board of health offices which will enable an engineer to correctly design a drainage system or a part thereof for any fiven locality. In soils that do not erode, a small "U" shaped subditch should be constructed in the center of the large one. This small ditch will concentrate the low water flow, prevent the formation of bars, and discourage mosquito breeding (Diagram No. 1).

\section{DIAGRAM NO-I SUB DITCH IN BOTTOM OF DITCH}

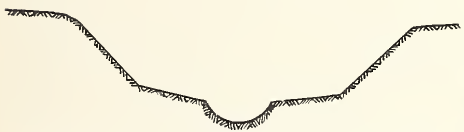

Side slope. The slope of the ditch banks will vary from a vertical or a $\frac{1}{2}: 1$ in stiff clay to as much as a $3: 1$ in sand. In eertain sections of Alabama ditches having a depth of at least 10 feet were constructed with vertical sides, and the soil was so dense that neither caving nor much erosion took place. In other sections where the soil is very unstable, it is difficult if not impossible to maintain a diteh in proper condition, even with very flat slopes, unless special measures are taken. Under these conditions, a line of poles accurately and securely pegged into the bank at the toe of slope will be found indispensable. These poles prevent the soil from eaving and reduce the width of the diteh at the bottom, thereby increasing the velocity and tending to prevent the formation of bars. The poles that remain under water will last indefinitely.

Berm. A berm of at least 6 or 8 feet should be left on large ditches. A threefoot berm is sufficient on small ditches. A better practice is to use this waste dirt for filling low depressions on either side of the ditch. If it is not practical to spread the spoil banks, short lateral ditches must be eut through to drain any low areas.

Protecting ditch banks. Under certain conditions it will be necessary to bring surface water over the banks in small paved inlets. If the flow of water in a lateral ditch is permanent but not of great volume, it can be carried through the ditch banks by means of underground drains.

Stabilizing banks. Willow poles have been used with much success in areas where unstable soils were encountered. Willow stakes were driven into the bank for at least 6 or 8 feet and long willow poles extending longitudinally were nailed to these stakes. There was enough moisture in the diteh bank to make these willows grow. Under such conditions this is a very economical and successful way to stabilize the banks.

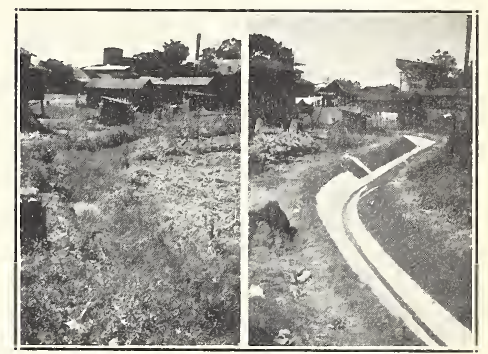

Fig. 2. Grenada, Mississippi. Left, before malarial control drainage; right, after construetion of brick and mortar invert with sodded banks.

Depth. The allowable depth of an openearth ditch is dependent on the method used for its construction. For example, it is never practical to construet a hand dug ditch which is over 10 feet in depth. A practical depth for a ditch made with a road grader is 5 feet. Dragline excavators are the most economical means of constructing large, deep ditches. The bottom of the ditch should be at least 6 inches lower than the bottom of the slough to be drained.

Cutoffs. Where a new ditch is constructed, making cutoffs across existing meanders of old streams, the excavated dirt must be utilized to fill the old ehannels (Diagram No. 2). Where the spoil dirt is 
DIAGRAM NO- 2 RE-CHANNELING CROOKED STREAMS

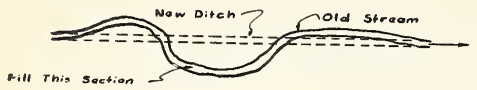

insufficient, these fills have been made with garbage, tin cans and other municipal wastes topped off with dirt. The tops of these fills should be sodded to prevent erosion, and a brick or riprap dam constructed at the upper end of the cutoff to prevent the stream from following its old course.

Junctions. Lateral ditches should always enter the main ditch at an acute angle in order that the flow shall take place with that of the main ditch and not across it. If possible, the lateral should enter the main ditch at an elevation above the grade of the main channel, and the grade of the lateral increased just before it enters the main ditch.

Seepage. The drainage of seepage or marshy areas fed by springs presents a very serious problem to the novice, and may offer difficulties for the experienced and trained engineer. Deep, narrow contour ditches, located along the toe of slope of the hill cut at right angles to the direction of ground-water flow, are utilized to intercept and collect the seepage flow. The elevation of these ditches can be determined accurately by locating the level of the ground water causing the seepage outerop with a post hole digger (Diagram No. 3).

DIAGRAM NO-3

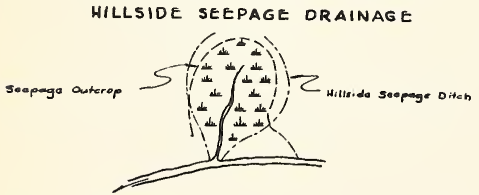

If the seepage is extensive, it may be necessary to construct several rows of contour ditches more or less parallel to each other. If the area is fed by a series of separate springs rather than a broad outerop, narrow individual laterals must be dug to each spring (Diagram No. 4).

$$
\text { DIAGRAM NO-4 }
$$

DRAINING SPRING-FED MARSHES

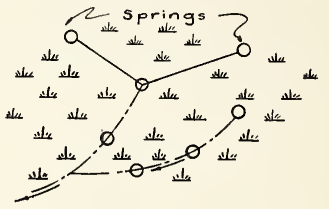

\section{Construction Operations}

The general practice is to begin operations on a new outlet ditch at the lower end in order that the erew may work in a dry ditch as long as possible. In cleaning out old channels, it is better to start work at the upper end in order that the trash and debris may be kept out of the ditch by floating it downstream as work progresses.

Experience has demonstrated the wisdom of dividing large erews into separate units. Each unit is assigned to a particular type of work, such as, clearing, grubbing, roughing out, finishing and sodding. By using the same erew on the same type of work continuously, the labor becomes more proficient and the work can be performed more economically.

\section{Cost Data}

The following is a table giving the actual cost of the different types of work:

\begin{tabular}{|c|c|}
\hline Type & Cost per cubic yard \\
\hline Hand & $\$ 0.25-0.50$ \\
\hline Grader & 0.19 \\
\hline Slips & 0.20 \\
\hline Dynamite & 0.13 \\
\hline Dragline & $0.10-0.18$ \\
\hline
\end{tabular}

These figures were derived from actual field operations in Mississippi from 1928 through 1931. The labor received approximately $\$ 2.00$ per day. 


\section{Special Problems}

Rice field workers in foreign countries have been able to control mosquito breeding in rice field by intermittent irrigation. If the rice field is irrigated for 3 days out of every 6 , it will be dry for at least 24 hours in every interval of 6 days, and breeding is thereby prevented. Any small undrained pools containing larvae should be treated with Paris green. In the Southern States rice fields are prolific sources of anopheles mosquitoes, but they have not been thought to be of much importance from a malaria standpoint.

Irrigation. There should not be much mosquito breeding on irrigation projects if they are well planned and well operated. In other words, the system should be planned to waste no water and to provide drainage ditches to take care of any seepage or waste. Unfortunately, but few are so prepared.

Pumping. In a few countries where there is insufficient fall, ditches discharge into a sump from which the water is pumped over the bank or levee into the outlet.

Modification of drainage structures. If the flow line of existing bridges or culverts is at a higher elevation than the territory directly above it, it is necessary to cut a trench through the bridge floor slab with air hammers. Care must be taken to construct adequate footings under the slab at the same time that the invert is made. This procedure must not be undertaken without first obtaining the written permission of the engineer in charge of the structure. Another means for securing additional fall is to utilize hydraulic jacks for pushing a pipe through the embankment at the correct elevation.

\section{Permanent Drainage}

Three types of drainage will be discussed under this heading; namely, invert construction, the laying of tile or underground drains, and the filling of swamps and ponds.

\section{Inverts}

A few years after the discovery of the mode of transmission of malaria, our Government initiated the construction of the Panama Canal. Officials of the United States Government, profiting by the mistakes of the French, incorporated malaria control and sanitation as a part of the construction work. LePrince and Orenstein (1916), in their book entitled Mosquito Control in Panama, deseribed in detail the permanent malaria eontrol work executed in Panama (1904-1910) and stated that the cost of the permanent construction was less than the maintenance cost on open earth ditches. The British studied this epochmaking work of Gorgas, Carter, and LePrince and began to copy it almost immediately in the far corners of the British

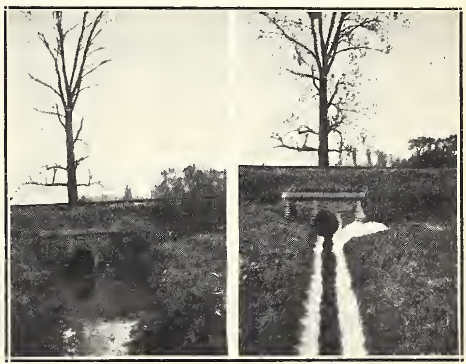

FıG. 3. Artesia, Mississippi. Left, before malarial control drainage; right, after construction of reinforced conerete invert with sodded banks.

Empire. But Americans waited approximately 30 years to imitate their own work. Some of the first invert construction was done in connection with field experiments of the U. S. Public Health Service in and near Memphis, Tennessee. It has been reported recently that the City of Memphis has lined more than 75 per cent of its water courses (anon. 1940) and several towns in other states are approaching this enviable situation.

Definition of inverts. The term "invert" means the bottom lining of an open ditch or water course with durable material. Inverts are of three types : masonry, monolithic, and precast. Masonry inverts are 
built of brick or stone set in mortar. In monolithic construction the concrete is poured continuously between forms, as in highway construction. The precast type is built of short units, usually not over three feet in length. Bermuda, earpet grass, or other suitable sod or concrete slabs are placed on the bank above the invert to prevent erosion.

Shape. A curved bottom is preferable to a flat one because, first, it concentrates the low water flow, thereby increasing the velocity in the center, eliminating mosquito breeding, and preventing the deposition of silt, and, second, the concrete extends well above the low water line and prevents erosion of the banks.

At least two states have adopted a design in which the depth of the invert equals onefourth of the width (Diagram No. 5). In

\section{DIAGRAM NO-5 \\ TYPICAL INVERT SMALL DITEH}

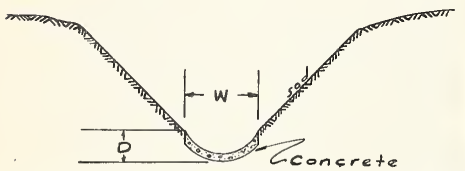

large eanals where the storm flow is large and the velocity high, shoulders are placed on each side of the invert (Diagram No. 6).

DIAGRAM NO-6

TYPICAL INVERT LARGE DITCH

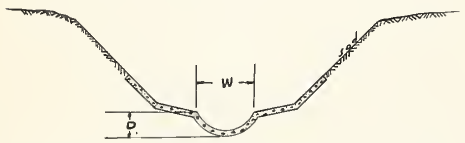

Table I gives the details of the invert being used in Mississippi and also the approximate amounts of material required for brick and eoncrete inverts.

Types. Several states seem to favor the monolithic conerete slab invert due to the fact that it can be poured rapidly and accurately with unskilled labor. The slab is 3 inches thick on most inverts, but is increased where the head of water is more than 8 or 10 feet or where the foundation is poor. When unstable soil is encountered, the inverts are reinforced with ehicken or hog wire, steel mesh or steel rods.

The Panama precast inverts are used with much success, and have been employed in several cities in Florida and Georgia. The sections of invert are cast in molds in a central factory, eured in a tank, pool, or with moist earth, and are rapidly laid on a carefully prepared subgrade. These inverts facilitate the collection of ground water by seepage through their joints; alignment and grade are readily restored

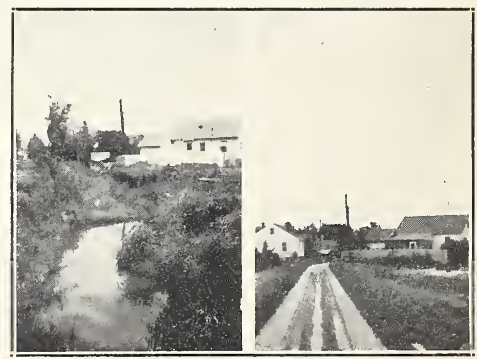

FIG. 4. Grenada, Mississippi. Left, before malarial control drainage; right, after construction of brick and mortar invert with sodded banks.

if settling occurs, while in the event the ditch is replaced by a storm sewer, they are all recoverable for use elsewhere (Magoon and Molloy 1936).

Jefferies (1936) has constructed a "U" shaped concrete lining by the use of silo staves laced together longitudinally and crosswise by cables. Experimental asphalt inverts were constructed in June, 1936, and are functioning satisfactorily today (1940).

Grades and velocities. In designing any drainage structure, the cross-section depends on the rainfall, on the size of the watershed and on the character of the terrain. After making a careful study of these factors, a size of the invert should be selected in which the resulting velocity will 
TABLE I

Mississippi State Board of Health INVERT DATA

\begin{tabular}{|c|c|c|c|c|c|c|c|c|c|}
\hline \multicolumn{4}{|c|}{ Conerete $\operatorname{mix} 1: 3: 5$} & \multicolumn{3}{|c|}{ For all slopes } & \multicolumn{3}{|c|}{ Mortar $1: 3$} \\
\hline & & & & \multicolumn{3}{|c|}{$100^{\prime}$ section con. bottom } & \multicolumn{3}{|c|}{$100^{\prime}$ section brick bottom } \\
\hline $\begin{array}{l}\text { Chord } \\
\text { length }\end{array}$ & $\begin{array}{l}\text { Radius } \\
\text { feet }\end{array}$ & $\begin{array}{l}\text { Depth } \\
\text { inches }\end{array}$ & $\begin{array}{l}\text { Cu. yds. } \\
\text { per } 100^{\prime}\end{array}$ & $\begin{array}{c}\text { Cement } \\
\text { sacks }\end{array}$ & $\begin{array}{l}\text { Sand } \\
\text { cu. yds. }\end{array}$ & $\begin{array}{l}\text { Gravel } \\
\text { eu. yds. }\end{array}$ & $\begin{array}{c}\text { Cement } \\
\text { sacks }\end{array}$ & $\begin{array}{l}\text { Sand } \\
\text { eu. yds. }\end{array}$ & $\begin{array}{l}\text { No. of } \\
\text { bricks }\end{array}$ \\
\hline $1^{\prime}$ & 0.63 & 3 & 1.3 & 5.6 & 0.6 & 1.1 & 4.8 & 0.5 & 545 \\
\hline $2^{\prime}$ & 1.25 & 6 & 2.4 & 10.5 & 1.2 & 2.0 & 9.1 & 1.0 & 1026 \\
\hline $3^{\prime}$ & 1.87 & 9 & 3.4 & 15.3 & 1.8 & 2.9 & 13.1 & 1.4 & 1490 \\
\hline $4^{\prime}$ & 2.50 & 12 & 4.5 & 19.9 & 2.3 & 3.8 & 17.1 & 1.9 & 1940 \\
\hline $5^{\prime}$ & 3.12 & 15 & 5.6 & 24.8 & 2.9 & 4.8 & 21.3 & 2.4 & 2420 \\
\hline $6^{\prime}$ & 3.75 & 18 & 6.6 & 29.4 & 3.4 & 5.6 & 25.3 & 2.8 & 2870 \\
\hline
\end{tabular}

Riprap or masonry construction requires approximately 25 sacks of eement and 3 cu. yds. of sand per 100 sq. yds. of invert. This table is for inverts whose thickness is three inches. Ten per cent should be added to the amounts for aprons, spillways, curtain walls, ete.

be more than 2 feet per second. In sections where it is absolutely impossible to secure velocities above 2 feet per second, small inverts should be used with shoulders on each side to take care of the storm water flow. If the velocity is found to be more than 4 feet per second, it will be necessary to construet spillways to drop sharply from one elevation to another. Increasing the grade of the invert on curves tends to prevent the deposition of silt.

Subgrade. On many projects it has been necessary to remove the muck, sometimes to a depth of 3 feet, and to replace this unsatisfactory material with clay, brickbats, broken stone, oyster shells, sand and gravel, or any other material that ean be satisfactorily compacted to form a solid subgrade. If the invert traverses a seepage area or one fed by springs, open jointed tile set in broken rock, brickbats, or gravel, is laid under, or to one side of, the invert to collect the underground water and stabilize the foundation. Hasell and Weathersbee have laid a relatively large amount of small bore butt joint tile for dewatering drainage ditches. They state (1938) that if an ample amount of porous material is placed around the tile to prevent the entrance of solid matter, these tile will function satisfactorily on a grade as flat as 0.1 per cent.

Protection of banks. Thick Bermuda or carpet grass sod has been found to be the best available material for the protection of ditch banks. Blanket sodding is much more satisfactory than strip or eheckerboard. The blocks of sod should be eut approximately 2 to 3 inches thick and about the same average shape. In laying the squares on the ditch bank, eare should be taken to lay them in the same manner as brick are laid, so as to eliminate erosion between the rows of sod and to conserve the water in it and rain water to promote its growth.

In urban areas, it is not always possible to secure the necessary width for the construction of inverts with sodded banks. Under these conditions, retaining walls constructed of rock, brick, or conerete offer the best solution. If a solid masonry or eonerete wall is used, the bottom width should be approximately 0.4 of the height, the height being the total distance from the bottom of the footing to the top of the wall.

Riprap made of broken concrete, native stone, brick set in mortar or precast concrete slabs are necessary to stop erosion on ditch banks on the outside of curves, under bridges, at junctions of ditehes above and below spillways, and in unstable soils. If the system is properly designed, it will not be necessary to use a large amount of riprap.

Low areas on each side of the invert system are drained into the paved ditches by 
lateral inverts, French drains, or ditches with sodded bottoms. Where the flow is constant, French drains or paved laterals are utilized, and where the flow is intermittent, a ditch with sodded sides and bottom has been found satisfactory to prevent caving of the sides and the formation of bars.

General specifications. The sand and gravel should be clean, well graded, and free from clay. The three-inch monolithic slab is poured of $1: 3: 5$ concrete. In the construction of brick inverts, the brick are laid flat with half-inch voids in the same manner as in constructing a brick wall. The brick are set in and covered with onefourth inch of 1:3 mortar. If soft brick are used, it is imperative that they be completely covered with mortar to prevent freezing and cracking during extremely cold weather. All brick must be soaked in water at least 12 hours before being used in the invert.

All inverts, either monolithic or precast, should be cured for at least 72 hours, by flooding, covering with a five-inch layer of moist dirt, by burlap mats, or by painting with an impervious material designed for this particular purpose.

Curtain walls should be constructed across the ditch under the invert to prevent any damage to the permanent lining by underflow. The depth of these walls in a 100 -foot section of ditch must be greater than the total fall. They should not be more than 3 inches in width. Weep holes are placed in the bottoms and sides immediately above the curtain walls at intervals not to exceed 50 feet; where much ground water is encountered the spacing may be reduced to 8 , or 10 feet. In sandy soil it may be necessary to construct a filter of graded gravel behind the weep hole to prevent the loss of sand.

Expansion joints should be left in the invert at intervals not to exceed 75 feet. These joints are made of burlap ropes soaked in liquid asphalt or of a soft material such as masonite. Construction joints are made in the riprap by pouring a thin countersunk slab about one foot wide up the bank at points where expansion joints fall. The bond between this slab and the riprap is broken by covering the slab with a sheet of newspaper.

Where round-bottomed inverts must join wide, flat-bottomed culverts or bridges, steps must be taken to reduce the width through the structure in order to increase the velocity sufficiently to prevent mosquito breeding and the deposition of silt. Many engineers are now building culverts with bottoms having the same curvature as the inverts.

Prior to construction work all water and gas pipes should be lowered below the invert in order to prevent the pipes from freezing and to remove an obstruction

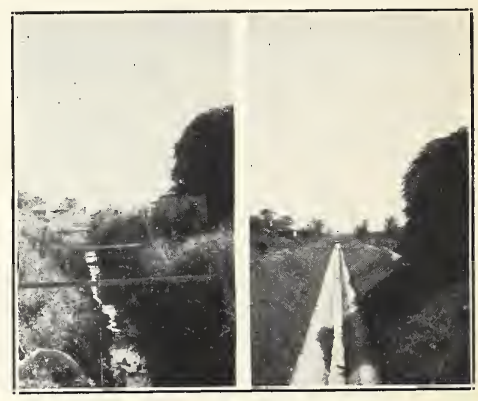

FIG. 5. Tunica, Mississippi. Left, before malarial control drainage; right, after construction of reinforced concrete invert with sodded banks.

which will block the flow, causing eddies and resulting in erosion to the banks. Where fences cross inverts, light swinging water gates made the same shape as the ditch should be swung across the invert.

Construction methods. The use of two templates on invert projects is well worth while. The subgrade template having a radius 3 inches greater than the standard enables the foreman to construct the subgrade accurately. The finishing template is used as a screed. By using it constantly in finishing the invert, it is possible to obtain an accurate, circular shape and its use also tends to result in a denser concrete. 
In order to obtain an accurate finished grade, batten boards are set across the ditch at intervals not to exceed 50 feet. Small blue top stakes are set on the center line of the ditch at six-foot intervals. The tops of these stakes are set on finished grade. It is relatively easy to obtain a true flow line by checking the flow line of the invert with a straight edge resting on the blue top stakes just before the concrete takes its initial set.

Benefits. The principal benefits resulting from inverts are: first, the elimination of mosquito-breeding areas with the corresponding reduction in the cost of mosquito control by oiling; second, the reduction in ditch maintenance costs; third, the protection of culvert and bridge structures; fourth, the reduction in the amount of household insecticides used; and fifth, the conservation and beautification of both public and private property.

\section{Underground Drains}

Underground drains were used by Gorgas and LePrince in Panama with much success. They are constructed of small tile set in broken rock, brickbats, or gravel and covered with at least 6 inches of straw, leaves, pine straw, or any other material which will act as a filter and prevent the dirt from filtering into the drain and filling the joints. In sandy soil it will be necessary to cover not only the top, but also the sides of the porous material to prevent sand from entering the drain (Diagram No. 7).

\section{DIAGRAM NO-7 \\ UNDERGROUND DRAIN USING TILE}

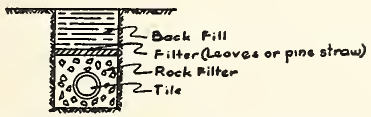

Satisfactory drains may be constructed of poles set in broken rock, brickbats, or similar material (Diagram No. 8). It is necessary to use more straw and filter material on pole drains in order to prevent filling of the voids by sand. In heavy

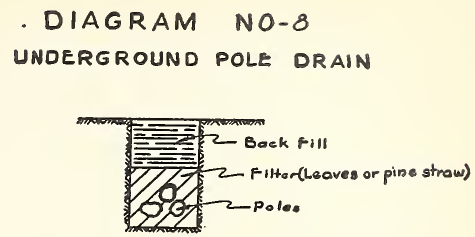

soils the pole drains may be covered with strips of sod cut the exact width of the drain and placed upside down to act as a filter.

These underground drains are frequently utilized in deep contour ditches to intercept the seepage flow, to carry the overflow from artesian wells, and as outlets for drinking fountains. Wherever possible this type of drainage should be used, as it eliminates mosquito breeding completely, reduces maintenance costs to a minimum, will be effective for a long period of time if properly executed, and constitutes an improvement to the property. Farmers favor this type of drainage as they can plow over the ditch, and city officials like it because the maintenance is very small.

\section{Filling}

Filling can be used in almost any town or city, without the expense of skilled supervision. Under certain conditions it is an ideal malarial control method, as mosquito-breeding areas are eliminated completely and permanently. In many towns and cities unsightly areas which formerly bred mosquitoes and were very difficult or impossible to drain have been transformed into parks by filling them with garbage, trash, or other debris. During fly-breeding season, dependable labor must be kept on the dump to sort the material, working the putrescible portion to the bottom. The fill is then topped with not less than six inches of dirt.

The popularity of this type of work has been due largely to the fact that local officials have reduced their hauling costs, have eliminated the need for oiling, and have created valuable building lots and beautified the city. 


\title{
THE MANAGEMENT OF WATER FOR MALARIA CONTROL
}

\author{
By E. HAROLD HINMAN \\ HEALTH AND SAFETY DEPARTMENT, TENNESSEE VALLEY AUTHORITY, WILSON DAM, ALABAMA
}

SINCE the malaria-transmitting mosquito spends the immature stages of its life cycle in an aquatic environment, the proper management of unremovable water affords an excellent prophylactic measure in the prevention of malaria. With the rapid increase of impounded water projects for flood-control, navigation, hydroelectric power development, water supply reservoirs, irrigation, and storage, the possibility for increasing "man-made malaria" is considerable. Despite the increase of the potential breeding surface it is possible in many cases to manage water levels so that the environment will not be suitable for production of the vector; or so that the vector may be destroyed during the larval stages at intervals frequent enough to prevent serious production of adult mosquitoes. To accomplish this, the future potentialities for mosquito propagation must be considered during the planning and construction of impounded water projects. Failure to do so has resulted repeatedly in unnecessary outbreaks of malaria.

\section{IMPOUNDED WATER}

A quarter of a century ago the importance of impounded water projects as a potential cause of increased malaria transmission was recognized in the Southern States. This resulted in the undertaking of joint studies by the U. S. Public Health Service and personnel of State Health Departments. Eventually regulations were adopted by various State Health Departments governing the impounding of water. In Alabama those first adopted in 1922 were subsequently held illegal on a technicality.

The regulations later adopted by the Alabama State Board of Health (1927) have the force and effect of law making it imperative that any person, firm, corporation, county or municipality desiring to impound water or who propose to raise the level of a previously existing pond must make formal application to the State Board of Health. Ponds of less than $1 / 10$ acre for watering stock or other domestic purposes or impoundages so located that no portion of them lies within one mile of any permanent human habitation are excepted. These regulations have served as a model for other states. The main objective has been to provide a naturalistic or biological control of the malaria transmitting mosquito through the development and maintenance of a clean water surface. This has been sought through: (1) proper reservoir preparation, (2) water level fluctuation, and (3) shoreline maintenance. The management of water level alone does not prevent the propagation of mosquitoes, for in the final analysis the control of mosquito breeding depends upon the control of vegetation, hence it is necessary to maintain a biological imbalance.

\section{Reservoir Preparation}

Smillie (1927) reported studies of an epidemic of malaria at the Gantt impounded area in Southern Alabama in an area of mild malaria endemicity. Impoundage in violation of regulations after only partial clearing of the reservoir resulted in a severe epidemic of malaria, beginning July 1 and extending to the middle of November, 1924. (This epidemic was only one of a series resulting from inadequate clearing of reservoirs prior to impounding:)

The principal requirements in reservoir preparation have been summarized by Kiker and Stromquist (1939) as follows: "That the reservoir be cleared so as to present a clean water's surface after impoundage between maximum and minimum water 
levels; and (2) that all depressions between maximum and minimum water levels be drained so as to provide water level fluctuation with the lake."

In preparing for most reservoirs it is necessary that the entire area to be flooded should be cleared of all trees and that all timber be removed. This is not only a necessary anti-larval measure, but it may be a requisite for removal of hazards to navigation and sources of drift which may be objectionable at the dam. In addition to the general clearing which extends up to the normal maximum summertime water elevation, there are certain special types of clearing, including (1) erosion clearingcarried back from 6 to 20 feet horizontally along steep shoreline to prevent trees falling into the lake; (2) clearing at heads of bights and indentations to provide space for stranding and removal of drift and flotage; (3) clearing of islands where they would be exposed to wave action and subsequently erode; (4) clearing for airplane dusting on points and at heads of indentations to facilitate larvicidal application; (5) clearing in the flood surcharge zone. Experience has shown that certain species of trees are intolerant to inundation and that temporary flooding during the growing season results in their death. Since these dead trees may fall into the reservoir and create a mosquito breeding hazard it is advisable to remove them prior to impoundage. Botanical studies earried on in the Health and Safety Department of the Tennessee Valley Authority have resulted in a classification of all woody species with reference to their ability to live under extended periods of inundation as: tolerant; semi-tolerant; and intolerant. In clearing practices only the latter group are remored from the flood surcharge zone. (6) Adjustment of clearing line to anticipated back water curves. In long narrow reservoirs having a considerable volume of inflow there may be a difference of several feet in water level between the lower and upper parts of the lake. In such instances it may be desirable to clear to higher elevations in the upstream portion of the reservoir.
Ten-foot zone conditioning above basic clearing line to remove dead and diseased trees, leaning trees, brush, logs, limbs, and debris lying on the ground to preclude the entrance of such material into the reservoir following impoundage.

The disposal of timber may be through sale, by piling and burning or, if below the minimum lake elevation, it may be fastened to the ground. The piling of timber along the margin should be discouraged since it creates a fire hazard. In marginal areas it is necessary that precautions be taken during the burning operation not to damage the standing timber by fire, since such trees may become diseased, die and eventually fall into the reservoir.

In large reservoirs the clearing operations may extend over one or several growing seasons, during which time most of the stumps will produce new shoots. Such coppice will constitute a favorable environment for Anopheles breeding following the filling of a reservoir. However, it is only in the marginal areas, where it reaches a height sufficient to break through the water surface, that it becomes a problem. In these areas also a rank growth of weeds is often produced. To combat this the Tennessee Valley Authority, since 1937, has pursued the policy of reconditioning the marginal zone during the autumn prior to filling, which should be done during the winter or early spring. This includes the cutting of all sprouts and larger herbaceous vegetation. Through the practice of low cutting of stumps it has been possible to use agricultural implements for this work. Either horse or tractor-drawn mowers and rakes make the operation a very economical one. After the material is dried it burns readily in windrows. By such treatment a clean shoreline results with marked savings in larricides during the initial season.

One species of tree has shown amazing ability to sprout from stumps, namely the black willow (Salix nigra). Even though the stumps be submerged for the major portion of the breeding season, as soon as the water level is drawn down sufficiently to expose them young shoots appear. If 
these reach sufficient height to break the water surface, the following spring they will continue to grow and will provide the protection necessary to anopheline larvae. Only submergence for a period exceeding a year will kill the majority of willow stumps. As a result, special investigation of the control of willow stumpage has been undertaken. It appears that hack-girdling (frilling) of the cut stump, just above the surface of the ground and the application of an arboricide, is the most efficient method of killing them. It is important that the hack-girdling shall completely and continuously encircle the stump, cutting through the bark and cambium layers. The hacked portion provides a trough into which the arboricide may be applied by means of a knapsack sprayer. Thorough application of the poison is necessary. The original treatment utilized by the Tennessee Valley Authority consisted of an aqueous solution of sodium arsenite (approximately 12.5 per cent). Owing to the dangers to laborers and also to livestock, a $30^{\circ}$ Baume diesel oil has been substituted and has been found to be fairly satisfactory.

Marginal drainage is a most important adjunct to reservoir preparation. All pools located between maximum and minimum lake levels must be connected to the main body of the lake to prevent isolated breeding pools upon fluctuation of the water level. Such work must be completed prior to filling the reservoir.

An important consideration with impounded water projects is the initial wintertime filling of the reservoir. It has been demonstrated repeatedly that if the impoundage is created during the growing season in the Southeastern States, a situation conducive to the propagation of $A$. quadrimaculatus will follow. When water rises slowly into terrestrial vegetation, it picks up fine debris (flotage) which makes a rich medium for larvae. Therefore, a new reservoir should be filled sometime after the close of one growing season and before the initiation of the following one. Preferably each project should have provision for a flood surcharge. This provides an oppor- tunity for filling above the normal summertime operating level and drawing down to this point just at the beginning of the breeding season. This results in the stranding of drift and flotage which would otherwise offer protection to larvae.

\section{Water Level Fluctuation}

Following adequate reservoir preparation, the next most important step in malaria control on impounded waters is the proper management of the water level. Carter (1914) was apparently the first to record the beneficial effect of the variation of pool level as an anti-larval measure. His first observations were made on power plants in which a weekly variation of elevation resulted from the elosing down of mills over the week-end. Carter, LePrince and Griffitts (1916) and Griffitts (1926) further studied the employment of this measure for anopheline control. Since these basic studies by the workers of the U. S. Public Health Service, certain power lakes in central Alabama, after clearing, have successfully utilized fluctuation for several years as the sole measure of control of anopheline larvae. In such cases a rather wide cyclical (weekly) fluctuation has been possible. Van Dine (1922) observed that the growth of vegetation along the margin of an impounded bayou was discouraged by a variable water level. The Alabama State Board of Health regulations governing the impounding of waters (1927) point to the advantageous use of a surcharge to strand flotage and drift and state that "during the mosquito breeding season, pronounced fluctuations of the water level may be, or may be made to be, a controlling factor in breeding. ... Changes in water level tend to prevent rank growth of aquatic plants and aid fish enemies (Gambusia) in reaching the mosquito larvae or flush the larvae within reach of the fish."

Hinman (1938) reported upon the biological effects of fluctuation of water level on anopheline breeding. The continuation of these studies forms the basis for the following discussion. Pool level fluctuation for malaria control may be employed along 
two distinct lines. A slow, continuous drawdown throughout the entire breeding season may be efficacious if of sufficient scope. Such a procedure is known as seasonal recession. On storage reservoirs where a wide drawdown fits into the requirements for power production, navigation or flood control, this simple measure is effective. However, there are many projects on which only a limited scope of water level variation is permissible. If stream flow or upstream reservoirs permit a regular pool level variation between constant limits, a cyclical fluctuation may be provided. This consists of a drawdown of a definite scope and refilling to previous elevation within a regular interval. Thus the elevation of the lake might be lowered one foot over a period of 3.5 days and refilled a foot over the same period. This would constitute a weekly cyclical fluctuation in level of one foot. The interval might be of any length but probably should not exceed 10 days (it should be less than the length of life of the larval stage of the mosquito). Such a type of fluctuation is particularly applicable where reservoirs are located in tandem and the drawing down process in one lake may coincide with the filling of the one immediately below it. However, any project utilized as a hydroelectric development will inevitably undergo a certain variation in water level. If this can be scheduled to be periodic it will be beneficial in preventing the development of anopheline larvae.

On the Tennessee Valley Authority lakes a combination of seasonal recession and eyclical fluctuation has been found to be most applicable under the conditions of operation. The graph at the end of this paper illustrates this type of schedule. The reservoir is lowered a definite amount during the first half of the cycle but the refilling process does not take it back to the original level, e.g., lowered 1.0 but refilled only 0.9. This results in a "staircase" type of schedule which has been found quite effective with a limited periodic fluctuation.

The mechanies by which fluctuation of pool level exerts its beneficial influence is not known exactly, but certain desirable effects are obvious. Each individual weekly drawdown, whether it be part of the cyclical fluctuation or of the seasonal recession, accomplishes several things, including strandage of debris which may have entered the reservoir recently, and strandage of larvae, pupae or eggs at the margin. In addition, certain larvae may be drawn out into open water where minnows and other predators have a greater opportunity to devour them. Finally, the drawdown diminishes the total area of potential breeding surface.

Cyclical fluctuation has a profound effect upon vegetation within the fluctuation zone. The drawdown, by its temporary dewatering, makes this zone uninhabitable by eertain species of submerged vegetation. Repeated refillings tend to exert a retarding influence on those species of terrestrial vegetation which would tend to advance constantly with the drawdown. Cyclical fluctuation also exerts an influence upon various species of erect emergent plants such as Saururus cernuus (lizard's tail) and Dianthera americana (water willow weed). It appears to defoliate the lower portions of stems in the water, thus producing less protection for the larvae and making the condition less attractive to them. Conversely, when reservoirs are filled above the scheduled elevations, the leafy portions of such vegetation are inundated and a higher density of larvae may be observed.

Unfortunately fluctuation of water level is not effective against all species of plants. Those types which are equally well adapted to either the terrestrial or the aquatic environment thrive when there are variations of water level. Similarly, those species which are suited to growth in deep water, e.g., Nelumbo lutea (lotus) and Nympheae advena (cowlily), are not inhibited.

Regardless of the exact mechanism by which fluctuation of water level exerts its beneficial effect, it should be considered as prophylactic rather than palliative. Through the control of water level the objective becomes one of making the environ- 
ment unsuitable to the development of anopheline mosquitoes rather than attempting merely to destroy developing larvae.

Reference is made to the graph showing the influence of various anti-mosquito measures on Lake Wilson. In 1935, owing to construction requirements at Wheeler Dam, it was essential that the lake be held constantly at maximum elevation throughout the mosquito breeding season. Despite the fact that about 50,000 gallons of oil and 41,000 pounds of Paris green dust mixture were utilized at a cost of approximately $\$ 25,000$, the mosquito control was unsatisfactory, as is evidenced by an average count of 11 female $A$. quadrimaculatus per station per week and a maximum weekly count of 46 mosquitoes. The counts were not decreased greatly until larvicidal measures were supplemented by marginal growth removal costing approximately as much as the larvicides. In 1936 irregular fluctuation was possible, resulting in some diminution in the expenditure for larvicides and a marked improvement in the station count (4 mosquitoes per station per week). In
1937 the combination of cyclical fluctuation and seasonal recession was employed for the first time. The decrease in larvicidal application, as well as in station count (less than 2 mosquitoes per station per week), was dramatic. The story for 1938, 1939 and 1940 likewise confirms the importance of water level fluctuation as an anopheline mosquito control measure on impounded water.

In the design of the dam and planning of an impounded water project, it is important to give consideration to the type of possible schedule. Each project will have to be considered in the light of its primary objectives. When storage is the important purpose, a slow, continuous drawdown throughout the mosquito breeding season may provide satisfactory control. If navigation is a major objective, a lower limit will exist beyond which the reservoir cannot be lowered. If reservoirs are constructed in tandem on a river, then provision for fluctuation can be designed and built into the projects. In any impoundages in an area where malaria is endemic,

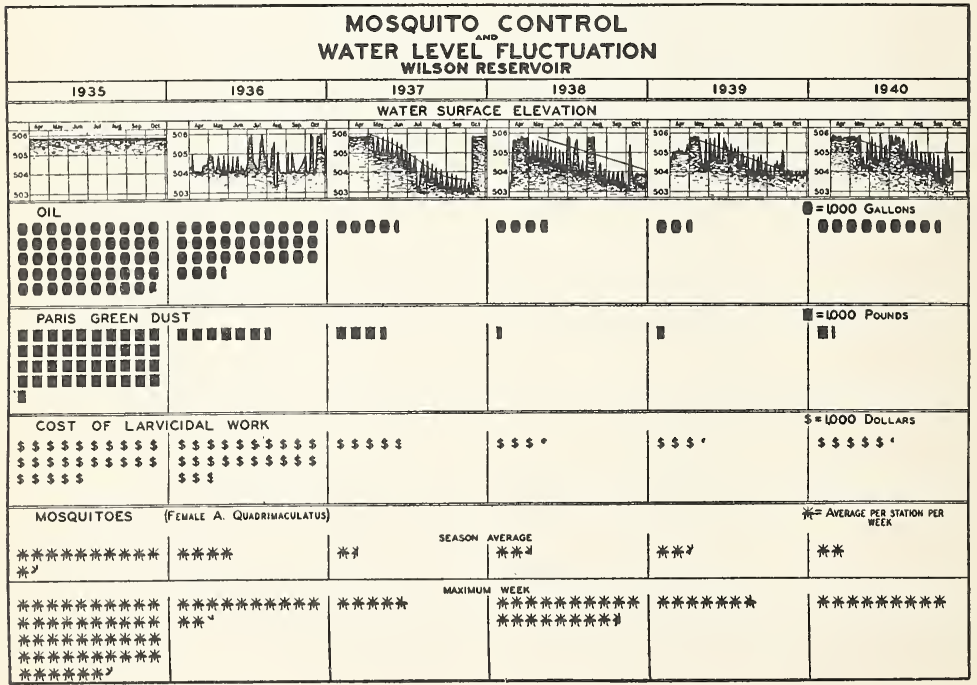


or may become endemic, it is desirable that a surcharge be provided so that drift and flotage may be stranded at the initiation of anopheline breeding. In addition to this, it is important that a seasonal recession be followed, combined with whatever weekly (cyclical) fluctuation is permissible in the operation of the project. The alternatives of either a wide seasonal recession or weekly fluctuation may be used if of sufficient scope.

\section{Shoreline Maintenance}

Although it appears to be established that water level fluctuation is an absolutely essential factor in the prevention of anopheline breeding in reservoirs, it must be repeated that the efficiency of this measure is dependent first on adequate reservoir preparation and, second, that it might be supplemented by shoreline maintenance. It is unfortunate that the latter is a continuing operation which may become expensive under certain conditions. The cost may be justified directly on the basis of anticipated savings from larvicidal operations. There are three types of shoreline improvement: (1) drainage maintenance; (2) drift removal; and (3) vegetation control.

As pointed out above, it is important that where depressions exist in the fluctuation zone, adequate drainage should be provided, otherwise standing pools would be left at the time of the drawdown. Obviously these ditches, whether constructed by dragline or by hand, must be maintained in a serviceable condition. The experience to date in the Tennessee Valley Authority has been that the cost of such maintenance is very low. Naturally this will vary with the type of soil encountered, the grade and section of the ditch, amount of silt being carried, etc. However, these factors can be taken into consideration in designing the ditches. Attention is called to the fact that this type of drainage differs from the usual surface drains in that it is subjected to extended periods of flooding.

Despite the greatest care in reservoir preparation, a considerable quantity of drift and flotage (smaller debris) will be found on any large reservoir following im- poundage. Furthermore, lesser amounts will continue to find entrance into the impoundage throughout the life of the project. Tributary streams are responsible for the entrance of a large amount of this material, especially during flood periods. The death and decay of marginal timber is the origin of much of it. In reservoirs in which a flood surcharge is not possible the removal of a certain amount of the larger drift will have to be considered. The operation should be undertaken at the time of minimum lake elevation. The drift may be handled either by hand or by mechanical equipment, mounted on a barge or operating from land. Any of these procedures are costly since the material usually must be piled, dried and burned. The latter operations may be facilitated by certain preimpoundage clearing at the heads of hollows and bays where drift may be expected to accumulate.

The problem of control of vegetation is of paramount importance and is not confined to malaria control on impounded waters. A. quadrimaculatus breeds amongst vegetation, in flotage or in a combination of the two. Since flotage is largely of vegetable origin, the control of anopheline larvae is intimately associated with control of vegetation.

The stumps of water-tolerant trees may survive extended periods of inundation and retain ability to send out young shoots following a drawdown. The control of such second growth is difficult and usually will necessitate hand removal at a season when the lake is at minimum elevation. The most effective method of control is through limitation of the rate of recession of water level during the first season of impoundage and the low cutting of stumps during reservoir preparation.

The problem of survival of woody species may be reduced to a minimum by proper planning. Unfortunately, such is not the case for herbaceous species. From a malaria control point of view, the herbs may be grouped into three classes: (1) terrestrial species, (2) wetland species and (3) aquatic species. In the present discussion 
the second and third groups will be discussed together.

Normally terrestrial species of vegetation are of slight consequence in anopheline propagation. With the employment of a seasonal recession there is a marked tendency for these species to invade the zone of drawdown, and if a summer flood refills the reservoir to top elevation they provide excellent breeding grounds for $\boldsymbol{A}$. quadrimaculatus. Furthermore, the tough vestiges of certain of these plants may remain throughout the winter and constitute a suitable environment for mosquito breeding the following year. In recent years the malaria control program of the Tennessee Valley Authority has included removal of such terrestrial vegetation and small coppice in certain critical areas. Mowing machines and rakes have been used to remove the growth very economically. Unfortunately, this operation is an annual process. Where growth removal can be carried out at a cost represented in larvicidal savings, it is to be urged since it has the added advantage of increasing efficiency. This operation is directed toward coppice and terrestrial herbaceous vegetation because most aquatic species are not impeded by this type of cutting and in certain instances may even be spread.

Wetland and aquatic species of vegetation are highly important in mosquito control. ${ }^{1}$ Fundamental to all plant control operations is a thorough knowledge of the morphology, and especially the ecology, of each species concerned. This includes the distribution, habitat requirements, competitive ability, rate of growth, phenology, and means of migration of each species. Although the methods of control of herbaceous plants are still in the process of evolution, they include (1) removal, (2) recurrent cutting and (3) herbicides.

Removal by digging, raking, pulling, or by a dragline is successful only when all the vegetative parts are eliminated and dis-

1 The following discussion is drawn exclusively from the experience of Dr. W. T. Penfound and Mr. T. F. Hall, who have been engaged in botanical studies for the Tennessee Valley Authority for the past several summers. posed of properly. Recurrent eutting has given very encouraging results during the past year on Tennessee Valley Authority reservoirs. It appears particularly applicable to such species as American lotus (Nelumbo lutea) and cowlily (Nympheae advena). Cutting either by underwater saw or a mechanized underwater weed cutter $^{2}$ has been efficient in deeper water. The treatment must be repeated at intervals of about three weeks, cutting the leaf blades just below the surface of the water. The number of cuttings required varies with the depth, turbidity, etc., of the water. The cut portions of the plants have not been found to promote anopheline breeding.

Considerable attention has been given to the utilization of herbicides. Sodium arsenite in weak solution (3 per cent) applied assiduously destroys those portions of the plant to which it is applied. Its use is limited, however, since it is highly poisonous to livestock and to man. In addition, the reservoir must be lowered sufficiently to expose the entire plant. During the summer of 1940 experimental application of powdered sodium arsenite ( $8 \mathrm{lb}$ per acre) was made by airplane. This was attempted in the hope of reducing the vegetative cover to a point where the necessity for larvicidal application would be greatly diminished. Actually about a 75 per cent reduction in cover followed each application which was made at monthly intervals. Where lotus (Nelumbo lutea) was present it was necessary to apply considerably in excess of $8 \mathrm{lb}$ per acre.

Liquid herbicides, noñ-poisonous to man or domestic animals, have been tested. Certain fuel oils and phenolic compounds have exhibited some promise. There is undoubtedly need for a powdered herbicide which may be applied by airplane and which is relatively non-toxic to man and his livestock.

\section{Flushing (Sluicing)}

Early experimental studies in the control of anopheline larvae by stranding and flushing were carried on by Russell (1932) in

2 Hockney type. 
the Philippine Islands. Periodic closing and opening of a dam, situated about half way along the length of the stream, twice on one day a week, brought about a marked reduction in larvae. This author has called attention to the fact that Bentley and others have advocated flushing as a method of mosquito control in Bengal. Blacklock (1939) refers to siphons which have been employed in anti-mosquito work, particularly in Malaya where Scharff, de Villiers, Williamson and others have developed their use. If sufficient fall can be obtained, it is possible, by means of a siphon, to produce a rapid transient rise of the water level in a stream in which anophelines are breeding. This author gives consideration to the various requirements of siphons, stressing particularly that for efficiency of flushing their action must be automatic. Various technical aspects of the utilization of siphons are considered and his paper should be read by all who have the opportunity of utilizing this unique method of malaria control. MacDonald (1939) has described a design of flushing siphon which has proven satisfactory in the control of anophelines in Malaya, Ismailia and Ceylon. The principle of control in this design is that a continuous flow, normally sufficiently slow to allow anopheline breeding in the stream or in the neighboring pools, is converted into an intermittent strong flow sufficient to destroy larvae. It is agreed by MacDonald and others that a very strong current is not necessary for this purpose and that any notable variation in the flow of water will destroy them by stranding or by washing them into the main current. Nicholas (1939) has described a four-day sluice.

It is not possible to summarize here the entire literature dealing with the control of anopheline larvae in running water by means of mechanical devices and flushing and flooding. However, reference must be made to the work of Ram (1935) Ejercito (1935) and Williamson and Scharff (1936). The latter authors have emphasized that sluicing can be effective only when used with other methods. The danger of anopheline breeding in impounded headwaters may be eliminated by erecting the sluices as high upstream as possible and by the use of Paris green.

Hackett, Russell, .Scharff and SeniorWhite (1938) summarized the main prineiples for making an efficient sluice for anti-larval fluctuation as follows: "That a sufficiently large volume of water should be discharged suddenly at a minimum of once a week, oftener in some cases, with the object of causing a wave to pass down the channel of the stream. This wave, as it flows onward, causes the stranding of mosquito larvae, pupae and eggs. Larvae which are not stranded are drowned by the turbulence of the flowing water. There is no doubt, too, that in sluiced channels, particularly those in fairly flat country, the soil disturbance and the liberation of silt and organic debris has a deterring effect upon further larval breeding." They have pointed out that the soft friable banks require special protection against erosion for a few yards below the outfall of sluices; also it may be necessary to eliminate side pools and seepages not reached by the flush. If a channel is too wide it is sometimes advisable to obstruct it with rocks in order to promote stranding or turbulence. They admit that the method's usefulness is restricted at present to fairly small streams and channels where there is plenty of water available.

\section{IRRIGATION}

The close association of malaria with the cultivation of rice and other crops requiring irrigation has long been recognized. Geiger and Purdy (1920) have considered the malaria problem of the rice fields of the United States. Earle (1925) has reported on the relation of irrigation on cane fields to the malaria problem of Puerto Rico and pointed out how the proper management of smaller irrigation ditches, which is in the hands of the cultivators, is of the greatest importance in malaria control. Sweet (1938) discussed the three types of irrigations systems in Mysore State and points out that lack of drainage remains as a common difficulty to all types. Herms and Gray (1940) state "it is a strange com- 
mentary on human intelligence that most irrigation districts have been organized, financed and constructed without any consideration of the problem of removing the inevitable seepage and surplus irrigation water." Russell (1938) has discussed malaria due to defective and untidy irrigation. From his recent personal observations he is of the opinion that the situation as regards irrigation and malaria is probably worse than ever before. He has summarized the specific ways in which irrigation produces malaria as follows: (a) Malaria-producing defects in construction, operations and maintenance on an irrigation system, and (b) general results of irrigation.

Russell's paper is amply illustrated with photographs of examples of most of these difficulties. He emphasizes the outstanding need for organization and cooperation between malariologists and irrigation engineers before and after an irrigation project is undertaken. It is not irrigation per se but defective and untidy irrigation which is the important source of malaria.

One hopeful approach to the control of malaria under systems of irrigation is that of intermittent irrigation. Smalt (1937) tried periodic draining in rice fields of the island of Bali and a 67 to 73 per cent reduction of anopheline larvae followed. In the laboratory, larvae of $A$. annularis and $A$. minimus survived for 145 and 175 minutes respectively in trays after they had been drained and for 40 and 60 minutes in dishes after the water had been removed.

Hill (1937) pointed out the possibility of utilizing intermittent irrigation of rice fields for malaria control in Portugal. Hill and Cambouruac (in press) presented the results of their studies since 1935 at the Louisville meeting of the American Society of Tropical Medicine, November, 1940. They found that periodic drying greatly decreases the number of mosquito larvae or completely suppresses them. Furthermore, the amount of water used is less than with the usual method of continuous irrigation and there is an increase in yield of certain species of rice per acre without detriment to its quality. As a result of these studies, the government of Portugal has passed a law making intermittent irrigation obligatory when recommended by the malarial service. A 17-day cycle was utilized by these workers.

\section{Summary}

The most important factor in the management of water for malaria control is the incorporation in the design of the project a provision for effective variation of water level. This requires the collaboration of malariologists and the engineers charged with the design of the project. In the case of artificial impoundages, proper reservoir preparation, followed by pool level fluctuation and supplemented by shoreline maintenance, are the cardinal features. Necessary maintenance of irrigation projects and sluices are likewise important. In all of these procedures it must be realized that the efficacy of variation of pool level for malaria control depends to a large extent on creating an environment unsuitable for breeding; actual destruction of the larvae may also be important. 


\title{
PETROLEUM PRODUCTS FOR MOSQUITO CONTROL ${ }^{1}$
}

\author{
By JOSEPH M. GINSBURG \\ DEPARTMENT OF ENTOMOLOGY, RUTGERS UNIVERSITY, NEW JERSEY AGRICULTURAL \\ EXPERIMENT STATION, NEW BRUNSWICK, N. J. \\ WILLEM RUDOLFS \\ DEPARTMENT OF WATER SUPPLIES AND SEWAGE DISPOSAL, RUTGERS UNIVERSITY, NEW JERSEY \\ AGRICULTURAL EXPERIMENT STATION, NEW BRUNSWICK, N. J.
}

WHERE permanent mosquito control, such as drainage and filling-in low areas, is either not possible or uneconomical, larvicides may be employed. Of the various chemicals tested for this purpose, petroleum oil, on account of its low cost and spreading properties on water surfaces is largely utilized for killing the aquatic forms of most species of mosquitoes, including Anopheles. That oils kill mosquito larvae and pupae has been known long before malarial control encouraged mosquito operations. It was not, however, until the experiments of Howard became known (1892, 1901) that their practical application was developed throughout the world. Since then many scientific workers have made researches in various mosquito oil problems, such as: (a) toxicology of petroleum oils; (b) amount of oil required to produce a killing film; (c) lasting properties of oil films; (d) efficiency of various oil larvicides; (e) injurious effects of petroleum oil to fish, water fowl, vegetation and man; (f) increasing toxicity, spreading and penetration of oils by incorporating various chemicals; (g) methods of application, and many others.

Field workers engaged in malarial control have used many kinds of petroleum products, including crude oil; various fuel oils, ranging from light distillates to heavy black oils; various waste oil products diluted with other oils; larvicides prepared from mixtures of various petroleum products with other toxic chemicals, etc. Con-

1 Paper of the Journal Series, New Jersey Agricultural Experiment Station, Department of Entomology. tributions from scientific investigators and practical field workers have accumulated sufficient information to enable those in charge of anti-malarial operations to select an oil which is most efficient for their purpose.

We have learned that the following properties are desired in an ideal mosquito oil: (a) it should exhibit a high toxicity to larvae and pupae; (b) it should spread quickly and uniformly on all kinds of waters; (c) it should penetrate rapidly through debris and thick vegetation; (d) it should form a stable and long lasting film; (e) it should be non-offensive to man and non-injurious to fish, water fowl, and plant life; and (f) it should be low in cost. Of course, not all of the enumerated characteristics are essential for all mosquito breeding waters. In certain areas, subject to intermittent flooding, long lasting properties are of no consequence. In other places, plant injury may offer no objectionable feature or may even prove beneficial. Nevertheless, certain specifications are absolutely indispensable for a satisfactory mosquito oil regardless of either the kind of breeding place or the mosquito species involved. These are: (1) toxicity; (2) spreading; (3) penetration into larval tracheae and (4) stability of film.

\section{ToXICITY}

Petroleum is a very complex mixture of hydrocarbons and other groups of compounds from which numerous products are manufactured, ranging from thick, black oil to colorless, harmless medicinal oil. Each product has distinct properties and 
performs definite functions. In order to either prepare or select a toxic mosquito oil it is important first to ascertain the properties responsible for toxicity. Various theories have been suggested to explain how petroleum oil kills larvae, among which the following deserve reviewing.

1. Suffocation. (A) The layer of oil on the surface of the water prevents the larvae and pupae from obtaining air (Celli 1900; Hardenburg 1922). (B) Oil impairs respiration of larvae by entering the tracheae effectually blocking them (Green 1924).

2. Toxicity of Oil Vapors. Toxic vapors from the inspired oil penetrate the tracheal tissue and produce a lethal effect upon larvae (Shaffer 1911; Takatsuki 1917; McFie 1917; Freeborn and Atstat 1918; Green 1924 ; Ramsay and Carpenter 1932).

3. Narcotic Effect. The toxic vapors from the inspired oil do not kill larvae directly but act as a narcotic, paralyzing

\section{TABLE I}

Summary of Laboratory Tests With Different Petroleum Oil Fractions on Larvae of Culex Pipiens AND Aedes Vexans (Copied from Ginsburg 1929)

\begin{tabular}{|c|c|c|c|c|c|}
\hline $\begin{array}{l}\text { Petroleum } \\
\text { oil tested }\end{array}$ & $\begin{array}{l}\text { Boiling } \\
\text { range } \\
\text { degrees } \mathrm{F}\end{array}$ & 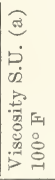 & 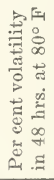 & 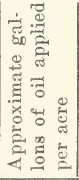 & 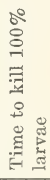 \\
\hline Fraction 1 & $200-300$ & 25 & 100 & 24 & $30 \mathrm{~m}$ \\
\hline 642 & $300-400$ & 28 & 94 & 24 & $15^{\prime \prime}$ \\
\hline “ & $400-500$ & 31 & 83 & 24 & $15^{\prime \prime}$ \\
\hline 66 & $500-600$ & 40 & 24 & 24 & $1 \mathrm{~h}$ \\
\hline "6 & $600-650$ & & 12 & 24 & $3^{6}$ \\
\hline i6 6 & $650-750$ & 182 & 5 & 24 & $14 ،$ \\
\hline $\begin{array}{l}\text { Mosquito } \\
\text { oil }\end{array}$ & $320-755$ & 50 & 50 & 24 & $30 \mathrm{~m}$ \\
\hline Fuel oil M & $360-725$ & 36 & 35 & 24 & $1 \mathrm{~h}$ \\
\hline $\begin{array}{l}\text { Fuel oil S } \\
\text { Light ma- } \\
\text { chine oils }\end{array}$ & $430-630$ & 40 & 22 & 24 & 26 \\
\hline $\begin{array}{l}\text { Straw } \\
\text { paraffin }\end{array}$ & $552-765$ & 65 & 10 & 24 & $18 " 6$ \\
\hline$\therefore \quad 6$ & $552-765$ & 65 & 10 & 100 & $9^{\prime \prime}$ \\
\hline 646 & $552-765$ & 65 & 10 & 200 & $9 ،$ \\
\hline $\begin{array}{l}\text { Diamond } \\
\text { paraffin }\end{array}$ & $638-770$ & 110 & 0 & 24 & $22 ،$ \\
\hline $66 \quad 66$ & $638-770$ & 110 & 0 & 100 & $12^{\prime \prime}$ \\
\hline
\end{tabular}

the nervous system. They are thus rendered helpless and subsequently drown (Barnes 1925).

Laboratory and field experiments conducted in New Jersey (Ginsburg 1928, 1929), with different commercial mosquito oils and with various fractional petroleum distillates indicate that the toxic properties are closely related to volatility and boiling range. Volatile, low-boiling distillates penetrate and kill larvae and pupae quickly, whereas oil fractions of high boiling range and of low volatility possess little or no direct toxicity, causing death primarily by suffocation but only after long periods of exposure.

From the results of these investigations it appears that petroleum oil films kill mosquito larvae by the following method. While in the process of breathing on the water surface, the larva also draws in oil from the surface film into its tracheal system by the flaps, in case of Anopheles, and by the breathing tube in case of other species. After penetration the toxicological effect will vary with the type of petroleum product used. Oils of low boiling range and of high volatility will exert a direct toxic action within a very short time. On the other hand, high boiling, non-volatile, viscous oil will slowly cause death by suffocation, after the tracheal stems have become flled with oil. The rate of penetration seems to be proportional directly to the thickness of the film and inversely to the viscosity of the oil.

The information secured from these experiments have simplified the problem of developing specifications for efficient mosquito oils. In general, the oil should contain enough of a low boiling petroleum fraction to insure quick penetration into the tracheal system and rapid kill of larvae and pupae; and a sufficient quantity of a high-boiling fraction to leave a lasting film. An example of such an oil is given in Table II. This oil has been effectively used in New Jersey during the last few years and can be readily obtained from most of the oil refineries at a cost varying from 5.5 to 8 cents per gallon depending on quantity pur- 
chased and transportation expense. In the field it spreads well, produces practically 100 per cent kill of larvae and pupae within one hour, leaves a stable film, and is not disagreeable to the oiling crew. These specifications are not rigid and can be readily modified to meet different requirements. The low boiling fraction may be decreased in areas where high temperatures prevail and where spreading and penetration are comparatively rapid, and increased where waters are cool or during seasons of low temperatures.

\section{TABLE II}

Type, distillate fuel; gravity (A.P.I.), 27-33; flash $130^{\circ} \mathrm{F}$ or higher; viscosity S.U. at $100^{\circ} \mathrm{F}$, $35-40$; odor, none offensive; distillation, $10 \%$ at $430^{\circ}-450^{\circ} \mathrm{F}$ and $50 \%$ at $510^{\circ}-550^{\circ} \mathrm{F}$ (the killing fraction), and $90 \%$ at $630^{\circ} \mathrm{F}$ and higher (the lasting fraction).

4. Spreading and Penetration. Ordinarily a non-viscous, toxic mosquito oil as above described spreads well on clear water surfaces, producing uniform film. On waters covered with flotage, debris, scum, thick vegetation and similar impeding objects, spreading speed is considerably slower, often resulting in unprotected areas where larvae may continue to develop undisturbed. This is especially apt to occur in early spring or fall, when the water temperature is comparatively low. In such instances application under high pressure may force the film through the-covered areas (Griffitts 1925 ; Coggeshall 1930; Watson and Bishop 1940). When power sprayers are not available the use of spreading agents in the oil may be advisable. The work of Langmuir (1917), Harkins (1917) and the more recent studies of Ginsburg (1927) and Murray (1939) have shown that oil-soluble organic compounds possessing either unsaturated bonds or polar groups in their molecule, such as $-\mathrm{OH}$, $-\mathrm{COOH}$, with marked affinity for water may greatly improve the spreading of petroleum oil. In practice, small quantities $(0.5-2 \%)$ of the following compounds were found helpful: cresylic acid, xylenol, oleic acid, turpentine, monohydric alcohols and sulphonated derivatives of petroleum.
5. Quantity of Oil per Acre. The amount of oil necessary to produce a uniform killing film in the field may vary from 25 to 60 gallons per acre, depending on the nature of the breeding area, toxic properties of the oil, species of mosquitoes, method of application and atmospheric factors such as prevailing wind and temperature. Usually more oil is required per acre of water surface covered with debris or vegetation than on clear water. It will also take more oil to cover a given area at low temperatures, such as prevail in early spring, than during the summer months. Where power sprayers capable of developing 100 pounds or more pressure are available, a killing film can be laid down with less oil than where hand sprayers are used. Anopheline larvae, because they stay near the surface most of the time, are effected by thinner films of oil and die during shorter periods of exposure (LePrince et al. 1916; Green 1924), than culex larvae or other bottom feeding species.

\section{OIL-Pyrethrum LaRVicide}

Mosquito control workers frequently encounter objections against the application of oil. There is a large number of private and municipal ponds, streams, and areas of impounded waters where fish and water fowl prevail and where aquatic plants are grown. Many of these places are favorable for mosquito breeding. Owners and authorities in charge of these places object, however, to the use of oil. Objections are raised also by preserve owners, game breeders, and those interested in propagation and conservation of wild life, waterfowl and game fish. In order to furnish mosquito control workers with an efficient substitute for oil, the New Jersey Pyrethrum Mosquito Larvicide was developed (Ginsburg $1930 ; 1934 ; 1939)$. Since its introduction it has been widely used in New Jersey and in other States (Vannote 1931; Smith 1932; Clarke 1936; and Rice 1936). It is primarily an oil emulsion of approximately the following composition: 66 per cent kerosene or similar light petroleum distillate; 0.07 per cent pyrethrins; 33.5 per cent 
water and 0.5 per cent sodium lauryl sulfate. This concentrated stock emulsion is mixed with about 10 parts of water before spraying. While the diluted spray contains only about 6 per cent oil the small quantity of pyrethrins greatly increases its toxic properties (Ginsburg 1937a), so that it is equally as efficient as straight mosquito oil in destroying larvae and pupae, but is at the same time non-injurious to fish, waterfowl and plants. This preparation was found useful wherever oil is objectionable and where a long lasting film is of no importance. It was also found valuable in protecting outdoor gatherings from mosquito annoyance (Ginsburg 1936; 1937b), and has been extensively used for this purpose in New Jersey, New York and other States.

\section{INDOOR SPRAYING}

Petroleum oil is also a valuable product to exterminate adult mosquitoes in human dwellings, barns, stables, trains and airplanes. An odorless, light petroleum distillate, containing small quantities of pyrethrins (about 0.1 per cent) proved useful not only in killing mosquitoes already indoors, but also in repelling those outside from entering living quarters through open doors, imperfect screens, etc.

\section{SUMMARY}

Petroleum oils, due to their surface spreading and toxic properties, may be universally employed in mosquito extermination operations where permanent methods of control are uneconomical. In the process of breathing on the water surface, the larva will inevitably draw in oil from the surface film into its tracheal system. Oils of low boiling range and high volatility exert a direct toxic action and cause death within a very short time; high boiling, non-volatile, viscous oils will slowly fill the tracheal stems and cause death by suffocation. The rate of penetration appears to be proportional directly to the thickness of film and inversely to the viscosity of the oil.

The following are the most essential characteristics of an efficient mosquito oil: (1) High toxicity to larvae and pupae; (2) quick, uniform spreading on water surfaces; (3) rapid penetration into the tracheal system; (4) stable, long lasting film on the surface.

On waters where application of concentrated petroleum oil is objectionable, the New Jersey pyrethrum mosquito larvicide offers an efficient substitute, being non-injurious to man and aquatic life. It has also proved efficient in protecting outdoor meetings from mosquito annoyance. 


\title{
PARIS GREEN (ACETO ARSENITE OF COPPER) AND OTHER STOMACH POISONS AS LARVICIDES AGAINST MOSQUITO LARVAE
}

\author{
By M. A. BARBER \\ J. S. PUBLIC HEALTH SERVICE, MALARIA INVESTIGATIONS, MEMPHIS, TENN.
}

LARVICIDES in powder form were first employed by Thibault (1918), who began with powdered pyrethrum which he found gave excellent results. Later he obtained "very good results" against both anopheles and culicines with various finely ground grasses and weeds, and he believed that the action of his powders was purely mechanical. We repeated his experiments without much success with any vegetable powder except pyrethrum.

Roubaud (1920) experimented successfully with trioxymethylene (paraformaldehyde) against anopheles. He was the first, I believe, to recognize that such powders are effective through ingestion by the larvae. Barber and Hayne (1921) introduced arsenicals, among them Paris green, into this sort of work.

The efficacy of larvicides in powder form against anopheles depends on two things: the toxicity of the poisons in the intestinal tract of the larvae, and the position of the particles of poison on the surface tension layer of the water of the breeding place within reach of the surface feeding larvae. The term "stomach poisons" is a good one with the understanding that the poisons may be effective in other parts of the gut. It is possible that the same larvicide may act both as a stomach and a respiratory poison.

\section{Chemical and Other Properties of PARIS GREen}

Paris green, a copper aceto arsenite, is a double salt of copper metaarsenite and copper acetate. In the pure state it contains the equivalent of 58.62 per cent of arsenious oxide. Under the United States Federal Insecticide Act of 1910, the name Paris green is restricted to products containing a minimum of 50 per cent of arsenic ealculated as oxide. One should be sure that the Paris green to be used as a larvicide contains at least that percentage.

Paris green is soluble in acids and ammonia, but in the pure state is practically insoluble in water. In the digestive tract of the larva it is presumably more soluble than in pure water. The solubility in water of ordinary commercial products of Paris green depends on the amount of free arsenious oxide in them. Such products contain about 1 to 3 per cent of free arsenious oxide and average about 1.4 per cent. U. S. Federal law prohibits interstate commerce in Paris green containing more than 3.5 per cent.

Paris green, then, is especially suitable as a larvicide because of its high toxicity within the gut of the larva and its low solubility in the water of the breeding place. Again, it is easily spread and penetrates reeds or grass covering the surface of the water. It is easily transported and is relatively cheap.

It has the disadvantage of failing to affect mosquito eggs or pupae, so that it must be applied at shorter intervals than oils. Small larvae, however, are poisoned as soon as they begin to eat, provided the Paris green used contains some fine dust, as is usually the ease. It is comparatively less efficient against culicines or other larvae not surface-feeding (see below under the topic "Paris green and culicines").

\section{Dilution with Dust}

When spread in powder form, Paris green is usually diluted with some inert 
dust, the diluent being used to prevent overdosage and to insure better distribution over the breeding place. Many sorts of dust have been used: road dust, fine gravel, sand, charcoal dust, talcum, soapstone, silica earth, ashes and slaked lime are examples. A dilution in common use is 1 part Paris green to 100 parts of inert dust by volume.

Dust mixtures should be deposited as nearly as possible only on the surface of the water and not made to penetrate the surface-tension layer. Therefore dusts must be dry, for particles of Paris green will stick to a moist vehicle and may be carried under the water. Some dusts, as slaked lime, sometimes cohere in small masses and submerge the poison. Dry sand or gravel is especially good for the treatment of certain less accessible breeding places, for one may project them considerable distances even through trees and brush. The greenish cloud which separates from the sand or gravel is particularly larvicidal. Larger particles of the vehicle are bound to sink, but we have shown by experiment that an effective percentage of Paris green will adhere to a large particle of dry gravel, even when projected several yards against the wind, and will be left on the surface when the gravel strikes the water.

The choice of a dust often depends on what is most available during the breeding season of the mosquitoes. Usually nearness to breeding places is an important factor, although dusts may be collected, sifted if necessary, and stored at any convenient time or place. Airplane dusting or the use of dust blowers may demand special-requirements.

Anopheline larvae ingest almost anything on the surface of the water if fine enough to enter the gut unless it consists of some substance distinctly repulsive to them.

Dusts are commonly thrown by hand or spread by dust blowers (also called "dust guns" or "dusters"). Spreading by hand has the advantage of requiring no machinery which must be carried by the workmen and which is likely to get out of order. Where dry dust, sand or fine gravel is present at the breeding place, spreading by hand is especially advantageous, for no transportation or sifting of the dust is required.

There is perhaps greater danger of over treatment of a breeding place where dust is spread by hand, although I have seen great abuse in this respect where blowers were used. "Dumping" of the larvicide burden instead of proper spreading may happen in any case-perhaps more often when the workman is burdened by the dustblower as well as by the dust.

Workmen accustomed to the use of dustblowers in agriculture make better distributors of larvicides because of their greater experience with the machinery and its operation. Such workmen have proved to be valuable in application of larvicides in Italy (Hackett 1925).

Dusts should be mixed well with the Paris green before spreading. Various sorts of mechanical mixers have been described, most of them consisting of a revolving box or barrel provided with baffles inside. Where no blower is carried and the dusts at the breeding place are employed, we have done successful mixing by hand in the pail in which the larvicides are carried.

Application of dusts by airplane will be described in another part of this paper.

\section{Dilution with Water of a Kerosene- TREATEd PARIS GREeN}

Barber, Rice and Mandekos (1936) have described a method by which Paris green is made more floatable by mixing with kerosene and is subsequently diluted with water at the breeding place. The ingredients are mixed in the proportion of $10 \mathrm{gm}$ of Paris green, 20 ce of kerosene and one ec of a solution of egg albumen. This mixture may be stored in bulk and eventually carried to the field in containers each with capacity of $15 \mathrm{cc}$ or $20 \mathrm{cc}$, quantities suited to the capacity of the sprayer. These containers are well closed and may be carried in a belt, cartridge-fashion.

At the breeding place (where water is always present) the mixture is thoroughly mixed with about 250 volumes of water. For breeding places little encumbered with debris of any kind, this proportion may be 
1 to 500 of water. This diluted larvicide is spread by means of a liquid sprayer. The mixture in water must be kept agitated somewhat, a purpose which is usually accomplished by the act of carrying and using the sprayer. One knows that he is keeping the contents of the sprayer well mixed if the spray-cloud continuously has a greenish tint.

The egg albumen makes an evener mixture in the sprayer and helps to prevent clogging of the nozzle. It is best applied by making a solution of about $5 \mathrm{gm}$ of dried egg albumen in $150 \mathrm{cc}$ of water and adding about 4 per cent of this solution to the Paris green-kerosene mixture. Or one may add the albumen solution to the water-diluted mixture in the sprayer, using, of course, an appropriately smaller percentage.

In the proportions employed in the Paris green-kerosene method, the kerosene does not act as a larvicide per se but functions as a vehicle for the Paris green and as a means of keeping it afloat. Eventually the kerosene evaporates and leaves the particles of Paris green free on the water surface, in which condition they are presumably more liable to be eaten by larvae than when mixed with a dust. The kerosene dilution method can be employed in any kind of weather, just so it is not raining at the time of application.

In one field experiment, about 100 square meters of breeding area, very grassy but relatively free from surface debris, was treated with 2,400 cc of a Paris green-kerosene-water mixture. The dilution was made in the proportion of one part of Paris green-kerosene to 500 parts of pond water, and the amount used represented $5 \mathrm{gm}$ of pure Paris green. Before treatment this area contained 1 to 20 larvae (A. maculipennis and $A$. elutus) per dip; while on the following day no living larvae could be found.

Barber, Rice and Mandekos (1936) devised a method for spreading the Paris green-kerosene mixture without the use of the sprayer or egg albumen-a method especially useful where an abundance of sand or fine gravel is present. The Paris green-kerosene mixture is well mixed in moist sand or gravel in the proportion of about 30 ec to 3 liters of sand or gravel. The degree of moisture of the diluent is important. If too wet, it cannot be easily spread. If too dry, the Paris green-kerosene will "paint" itself on the dry particles and may sink to the bottom with them; while particles suitably moist will give up their larvicides in the form of a blotch or film when they touch the water. A simple rule is to make the mixture of sand or gravel with the larvicide moist but not too wet to be easily strewn. One can often get the right moisture by blending wet and dry sand at the stream-side.

The sand or gravel can be easily strewn by hand and may be directed to any part of the breeding place, even against a strong wind. The larvicide thus prepared is less liable to get into the clothing or nostrils of the operator, for it does not separate from the moist vehicle until it reaches the water surface. One may add a spreader to the Paris green-kerosene mixture in the form of oleic acid (one per cent or less) or of some vegetable oil or resin. But such spreaders do not seem to be necessary for good filming except in cooler waters. Wind, of course, aids in the spread of films. One may use this form of larvicide immediately after a rain, if desired.

We made an experiment with Paris greenkerosene-gravel in a pool containing many anopheline larvae-over 3 per dip. The Paris green-kerosene mixture was spread over wet pebbles each of which was about $1.5 \mathrm{~cm}$ in diameter. Certain pebbles were distributed in water surfaces of known area and more or less confined by the bank or other natural barrier. From a single pebble the larvicide spread about one-half yard in one direction and from one and one-half to three yards in another, practically "sterilizing" this area. The next day we found many dead larvae (some of them with Paris green still demonstrable in the intestine) and only one living one. The water of this pool contained little debris and its surface had been cleared by a recent rain.

In a laboratory experiment, a single grain 
of Paris green-kerosene treated sand about $1 \mathrm{~mm}$ in diameter practically cleared of living larvae an area $10 \mathrm{~cm}$ in diameterabout the size of that in a large track-pool.

We have found the Paris green-kerosenemoist sand method very useful against larvae of $A$. gambiae in the partially dried river beds of Brazil (Barber 1940).

I have experimented in the laboratory with a Paris green made more or less floatable by treating it with a small amount of paraffin dissolved in kerosene. The kerosene was drained off by filtration and the powder subsequently well dried. This powder was mixed with moist sand and spread in some experimental bowls with very promising results.

Aziz (1939) used a "light Continental paris green" mixed with water from the breeding place with no addition of kerosene or egg albumen. In his earlier experiments he used a stock solution of about 1,000 cc of Paris green in 2,000 ec of water, which at the breeding place was diluted with stream water in the proportion of 20 to 25 ce of stock solution to 6 or 8 liters of water. This final dilution was applied by means of a sprayer. Later he discontinued the use of the stock solution and carried the Paris green to the breeding places in packages of a quantity sufficient to make one charge or a half-charge of the sprayer. It was then mixed just before use, either by pouring it into the sprayer and adding water or by mixing first in some container and then pouring it into the sprayer. Each liter of the mixture in the strength described should suffice to treat an area of about 60 square yards, the amount varying with the nature of the breeding place. Aziz thinks that in Cyprus conditions the Paris green-water method is less expensive and more effective than either the Paris green-dust or the Paris green-kerosene methods.

It seems to me that the modification of Aziz is promising, especially where a more or less floatable Paris green is available; and the method should be suitable for conditions other than those of Cyprus. It is to be remembered that the purpose of the kerosene is not only to make the Paris green more floatable, but also to aid in spreading it, for kerosene tends to spread widely before it evaporates. This filming, which is advantageous for most still waters, might be less useful in Cyprus, where anopheles larvae (mostly $A$. superpictus) are often found in flowing waters.

\section{Dosage, or Quantity of Paris Green to Be Used Per Unit of Breeding Area}

The toxicity of the larvicide is a prime factor; the minimum lethal dose for a single larva is, however, hard to determine. It might be done by picking out with a capillary pipette under microscopical control fragments of a crystal of a floatable sample of Paris green and later testing this dose on a single larva. One can get an approximation of a lethal dose by a simple experiment: A larva is put into a Petri dish containing water and kept under observation under a hand lens. When it is feeding well, a very small amount of Paris green is carefully put on the water surface near it. When it has ingested a small particle, it is removed by a pipette to a second dish, washed, and put into a third where the time of its survival is noted.

The size of particles efficiently toxic can be determined by another procedure: The sample of Paris green to be tested is mixed in ether and filtered through a paper filter of known permeability. The filtrate is placed on water in a suitable container and beside it a control with ether but no Paris green on its surface. When the ether has evaporated, which may require a day or two according to the temperature and amount, anopheline larvae are introduced into both control and treated containers, and the degree and time of mortality observed. The purpose of the ether is to afford a volatile substance which will leave the particles of larvicide on the surface of the water.

Laboratory experiments show clearly that amounts of Paris green so small as to escape visibility under the hand lens may poison all larvae in a container. In one laboratory we had a good deal of mortality among anopheline larvae in our routine breeding containers, an occurrence puzzling until we 
learned that Paris green was being mixed in another part of the building and that enough of it came through the open doors to kill our larvae.

Dosages of larvicide determined in the laboratory are applicable in open field work only to waters practically free from surface obstruction of any kind. In these waters the larvicides tend to disperse widely after they reach the water. When the spread of the larvicide is obstructed by scums, floating particles of wood, algae, or debris of any kind, the dosage of larvicide has to be increased. The dry larvicide may penetrate vertical vegetation, but some of it will be lost in passing through. Rain tends to break up scums and beat down algae and a treatment soon after a heavy shower may require comparatively little larvicide. Flowing streams require more than still water and often more careful spreading of the larvicide.

It is evident that to prescribe a definite dosage of larvicide may more often mislead than assist. The best procedure is first to determine roughly by dipping the numbers of larvae in the water to be treated. Try at first a light treatment of larvicide, then determine preferably on the following day, the results of this dosage. The presence of dead larvae on the surface of the water will often help in ascertaining results if the examination be not too long delayed. Such preliminary tests are almost necessary in beginning work in a new terrain.

The commonest fault is over-treatment. One sometimes sees treated pools fairly green with larvicide. A preliminary test would have shown that for such pools a dosage one-tenth as great would have sufficed. Generally the appearance of a green color on the water or the banks indicates that a part of the larvicide has been wasted.

Some approximation of proper dosages may be useful, at all events as a guide to preliminary tests. We have indicated the results of certain dosage in the Paris greenkerosene experiments. In dusting, Hackett (1925) showed that in waters with algae but with banks clean, the use of $0.02 \mathrm{cc}$ of Paris green per square meter gave 96.0 per cent killing of anopheles larvae; among reeds, a dosage of 0.04 ce per square meter gave a killing of 99.4 per cent; in heavy brush and cane, 0.05 ec per square meter, a killing of 98.0 per cent.

\section{Danger of Poisoning Man or Domestic animals by Paris Green}

In considering the danger to man or domestic animals by drinking waters treated by Paris green or other little soluble arsenicals, one should keep in mind the fact that such larvicides rest on the surface of the water and are effective against larvae not by arsenic in solution in water but by that in solution in the gut of the larva. Only a tiny amount scarcely detectible by chemical means is in solution in water, and this amount is negligible where a brand of Paris green little soluble in water is used and is applied in proper dosage. Even if the surface of the water is thickly covered by Paris green, it is unlikely that an animal drinking in the ordinary manner would get a toxic dose.

Although Paris green has been used for nearly 20 years as a larvicide for anopheline larvae, there is scarcely an authenticated case of serious poisoning of man or domestic animals from its use, at all events when applied in a proper manner. Hackett (1925) states that its use in wells, one gram to 20 square feet, is quite safe. Sweet and Rao (1934) treated wells in Bangalore City, India. Their routine was to treat a well weekly with 6 puffs with a hand-blower of a 2 per cent mixture of Paris green in road dust. Except immediately after the application of Paris green, the most delicate tests available failed to show its presence either in the water or in deposits from the bottom of wells which had been treated weekly for considerably over a year.

Missiroli (1917) states that accumulation. of arsenic through long application in the same water does not take place because any dissolved arsenic is volatized by the microflora of the water. He used as a test of arsenic in water the fungus Penicillium brevicaule, the growth of which produces a garlic odor in the presence of arsenic. 
Sometimes the fear of harm by the use of larvicides has been more troublesome than the larvicides themselves, especially in countries unaccustomed to the use of insecticides in agriculture. I have sometimes combatted this fear by a very simple demonstration: Water was set out in a container, larvae introduced, and treated with a dose of Paris green sufficient to kill all of them. A second container was placed beside the first and received the same dose of larvicide. After treatment I have myself drunk the contents of the second container (the first if one previously fishes out the larvae). Of course, no harm followed.

As in the case of arsenicals used in agriculture, the person spreading the poison should take some simple precautions against getting too much dust next to his skin or into the nostrils. Usually attention to the direction of the wind, proper clothing and a bath after work will suffice to prevent skin lesions. Where kerosene is used as a diluent, especially when on moist sand or gravel, such danger should be small.

\section{EFfect on Fish}

There is no published evidence that Paris green has any deleterious effect on fish or other water fauna predaceous on larvae.

\section{EfFect on Rice Plants}

Rao and Sweet (1937) measured carefully the effect of dusting certain rice fields in India. Their conclusion was that, with a one per cent dilution of Paris green in wood ashes used in quantities commonly employed for larval control, no effects could be demonstrated which indicated that Paris green decreased the rice and straw yield or increased the amount of chaff. There was no indication that stopping Paris green treatment was necessary during the period in which the rice plants were in flower.

\section{Frequenct of Application of Paris Green Treatments}

The interval between applications of Paris green to a breeding place must vary with conditions, especially with the water temperature, and that of about a week is suited to most conditions. The proper interval can usually be determined by a few preliminary observations. Barber and Olinger (1931) had to dust every 5 days in treating $A$. gambiae (costalis) in Africa. This species in the warm coastal region of West Africa will develop from egg to adult in about 7 days.

\section{Paris Green and Culicines}

Culicine larvae are poisoned by Paris green when the poison is ingested, but their manner of feeding below the surface makes the poison less accessible to them. Barber (1925) did many experiments to ascertain the effect of Paris green on culicines and determined that a fairly heavy mortality can be attained in shallow water, where the larvae feed on the particles of green which have sunk to the bottom. Also one may get a good killing where thick vegetation occurs in the breeding water just below the surface.

Griffitts (1927) mixed Paris green with wet sand in various proportions, in one experiment with one part of Paris green to 100 parts of sand. He attained in various experiments a killing of 95 to 100 per cent of the larvae of different species of culicines. The breeding water varied in depth from 2 inches to 1 foot. The wet sand served as a "sinker" to carry the larvicide to the bottom within reach of the bottomfeeding larvae.

King and McNeel (1938) attained under field conditions a high percentage of killing of Aedes taeniorynchus by means of Paris green mixed with water and applied with a small pressure sprayer. In another natural breeding place containing much vegetation and infested by Psorophora they attained a high mortality with Paris green diluted in water and spread by means of a sprinkling can. The depth of this water varied from a few inches to one foot. Their important experiment in treating Culex quinquefasciatus by means of Paris green spread by autogiro will be described in this paper under "Distribution by autogiro."

They found that calcium arsenite was about as effective as Paris green on culi- 
cines. King, Bradley and MeNeel (1939) warn against substituting calcium arsenate as a larvicide for the more toxic calcium arsenite. They (1939) state that "Paris green will destroy Culex quinquefasciatus in ditches, shallow ground pools, and catch basins, although oil is usually preferred, as it deters oviposition."

\section{Distribution of Paris Green by Airplane}

King and Bradley (1926) have done the pioneer work in the use of airplanes in the distribution of Paris green for larvicidal purposes. They made preliminary tests in which dry glass plates and dishes containing anopheline larvae were placed in an open field and treated by airplane. It was found that a dosage represented by 10 or more granules of Paris green per square inch gave a mortality of 100 per cent of larvae, that one trip of the airplane at an altitude of 15 to 30 feet above the ground effectively treated a strip 300 to 400 feet wide, and that the quantity of Paris green required was at the rate of about one pound per 20 acres. These figures would apply in natural breeding places only to waters free from surface debris, trees and aquatic vegetation.

In their subsequent tests over natural breeding places, the authors used a mixture of about 10 per cent of Paris green in Tripoli earth as a diluent. The amount of dust used was regulated by a hopper valve within the airplane and varied with the amount of brush and trees over the breeding place. In two final tests of 1924, made in lakes overgrown with aquatic vegetation, the percentage of anopheles killed varied from 88 per cent in the first test to 99 per cent in the second. Dew caused the dust to adhere to vegetation, but when the wind was not too strong work could be done after the vegetation had dried. It is essential to employ skillful pilots who can fly relatively low and make proper use of the wind in directing the dust cloud.

The cost of dusting ordinary breeding places such as were present in the Louisiana area was estimated at fifty cents to one dollar per acre, including the cost of the larvicide.

Williams and Cook (1927 and 1928) have continued the work of application of Paris green by airplane with the cooperation of the United States Marine Corps at Quantico, Virginia. There the production of anopheles occurred in flotage consisting of mats of eel grass and of water lilies. They found that the effective quantity of Paris green was one pound per acre, and they used principally powdered soapstone as a diluent. With wind velocities of less than 4 miles per hour and flying heights of 100 feet or less, a 25 per cent content of Paris green was effective; while with winds of greater velocity and flying heights of over 100 feet a dilution of 50 per cent was effective. When breeding was continuous and heavy it was necessary to dust at weekly intervals. The cost of the material was $\$ 0.724$ per acre.

Watson, Kiker and Johnson (1938) and Kiker, Fairer and Flanary (1938) report on the use of the airplane in certain impounded waters. Their experiments were done under the auspices of the Tennessee Valley Authority. It was found that onehalf pound of Paris green per acre would not afford uniformly effective results, but that the distribution of one pound per acre gave satisfactory control of anopheline breeding except in the presence of dense vegetation. Experiments are being continued by these workers and their results will appear in subsequent publications.

\section{Distribution by AUTOGIRo}

King and MeNeel (1938) used an autogiro equipped for spraying liquids and experimented with Paris green in water on the larvae of Culex quinquefasciatus. The larvae were contained in dishes arranged in two lines, these lines placed at right angles to the direction of flight and 500 feet apart. The plane was flown at a speed of about 45 miles per hour and at an altitude of 8 or 10 feet above the ground. In one experiment, in which 8 pounds of Paris green was mixed in 25 gallons of water, the mortality of the larvae varied from 61 to 96 per cent 
depending on their distance from the line of flight. The effective swath was at least 50 feet in width.

In these experiments the spray remained moist enough to sink and act on the culicine larvae. One would expect success on anopheles with a floatable larvicide or with a dry larvicide also spread by autogiro. The experiments of King and McNeel are of great importance in indicating the use of autogiros or other modifications of the airplane for various sorts of breeding places.

\section{Distribution by a Power Dusting Machine Carried in a Boat}

LePrince and Johnson (1929) have devised an apparatus designed for the treatment of lakes or other larger bodies of water. The blower can be operated by one man and the whole unit placed in a small boat. Hydrated lime containing 15 per cent of Paris green gave the most satisfactory results. In moderate breezes such a mixture gave a lethal path at least 525 feet wide. Material costs were as low as \$0.15 per acre.

It is well worth trying to devise a similar apparatus for spreading a Paris green made floatable by kerosene or by some other means and diluted with the water of the breeding place.

In connection with the spread of larvicide by airplane or other mechanical means, it is encouraging to remember that a partial destruction of anopheles in large breeding places may be very useful, because generally malarial transmission depends on the density of the mosquito vector. I have studied for a period of some years a pond in Greek Macedonia which supplies $A$. elutus and intense malaria to 5 or 6 different villages all situated within a radius of 5 kilometers. A partial control of this pond might at least protect the more distant villages. Certain anopheles, as A. pseudopunctipennis in Old Mexico and $A$. pharoensis in Egypt, apparently are serious vectors only where their density is great.

Howard and Andrews (1940) mixed Paris green with hydrated lime and applied it over a large breeding place. They deter- mined the character of the dust deposits at varying distances from the point of distribution and found that the lime went a greater distance than the Paris green. They warn against judging the area effectively treated by the visible clouds of dust.

\section{IMPROVEMENT OF LARVICIDES AND OF THE Methods of ApPLICATION}

In testing new stomach poisons for larvae the following points should be kept in mind: The larvicide must be very toxic in the gut of the larvae and practically insoluble in water. The larvicide should be inexpensive. Whatever success one has with a larvicide in the laboratory, it must be retested in the natural breeding places. These should include both shallow and deep water and scattered track-pools; also water with various surface covering.

These requirements hold for a larvicide which is designed for all sorts of breeding places. For special places, such as wells, cheapness may not be so essential.

My colleagues and I have made many attempts to find new larvicides and to improve the method of application of those we have; and a short account of some of our failures or partial successes may be useful.

Carbonate of copper filled the requirements of cheapness, insolubility and ease of spread, and in the laboratory killed the anopheline larvae. But in the field we found that its low toxicity required too heavy a dosage to make it practicable.

In certain field tests we found that iodoform diluted in road dust was practically as effective as Paris green. Iodoform has two disadvantages: It is relatively expensive and must be fresh or carefully preserved in order to be effective against larvae. But it might prove useful in wells where only small quantities are needed; and in the crude form at least it is easily manufactured, so that long storage would not be necessary. It is doubtful if persons with no hospital experience would object to the odor, at all events where very small quantities are employed.

For breeding places such as smaller ponds, pools and the like we have used Paris 
green incorporated in small masses or cakes These have the property of floating on the surface of the breeding place, slowly disintegrating and giving up a floatable Paris green to the surface of the water. The essential ingredients are: Paris green made floatable by treating it with paraffin dissolved in carbon tetrachloride, a plaster of Paris matrix to attain slow disintegration, and sufficient cork dust mixed in to make the whole mass floatable. It was found in field experiments that if a cake of larvicide sank it got lost in the mud of the bottom. This sort of larvicide should be easily distributed. In treating a series of pools one could throw a cake or two of larvicide into each, and theoretically one treatment should suffice for several weeks.

In the laboratory these cakes worked perfectly; but in the field, where pond levels varied considerably, the released Paris green did not spread well, sometimes because the cakes got lodged on the shore. I still think the idea a fertile one and that the technique of manufacture and distribution of this larvicide can be improved. In their present form, the cakes might be useful in places where rains are almost continuous. The rains would aid in the spread of a floatable larvicide but might render difficult the use of ordinary powders.

For a time we hoped that the mixture of stomach poisons in some non-evaporating oil or resin might aid in their application. Such substances spread widely, in broken patches if not in a film, and wind or rain should aid in their dispersal; so that application of the larvicide in one place might be sufficient for a whole pond. It is possible to get larvicides in solution in oils or to incorporate them in the form of fine granules. Larvae readily ingest particles of oil, as the dissection of anopheline larvae from oil-treated waters shows. Quantities of oil much smaller than those necessary for respiratory poisons should suffice. But we found that larvicides in oil pass into the gut and cause little mortality among the larvae. It seems that the oils or resins prevent the solution of the poison or otherwise prevent its entrance into the larval cells.
These experiments have served one useto warn against the use of too much paraffin or other insoluble substance in making Paris green more floatable.

Several brands of Paris green are on the market, some of them claiming greater fineness and floatability, both good qualities when there is no accompanying loss of toxicity for larvae. The toxicity of very fine particles can be determined by filtration through filters of known permeability (see under the topic "The dosage or quantity of Paris green, etc.)." Floatability can be estimated roughly by shaking a sample with water in a test tube and noting the percentage of green which comes to the surface.

\section{The Choice of a Stomach Larvicide and of Method of Application}

The choice of a stomach larvicide and method of application must depend on the conditions. For the present, it appears that Paris green is the most suitable larvicide. The method of application will depend on circumstances. In partially dry river beds with much sand and gravel I should try a moist vehicle mixed with Paris green made floatable by kerosene or by some other method. For continuously rainy weather some of the floatable Paris green methods may be especially suitable. In semi-desert countries with stony streams and a fair amount of dry dust available, I should prefer the distribution in dust by hand or the modification of Aziz. For very large bodies of water out of the reach of small dust blowers or of hand treatment, airplanes or other mechanical methods are most suitable.

\section{The Necessity of a Trained Inspector in Larvicidal WORK}

In some larvicidal enterprises which I have observed, the workman was given a bag of Paris green or a can of oil and left to look after the work with little or no supervision. The results were about as one would expect if brick layers or carpenters were given a blue print and told to build 
a house. The work must be begun properly by determining the dosage and method of application of the larvicides. Then during the season the work must be kept under observation and results must be measured by the density of mosquitoes in certain resting places or in suitable traps. The final test, that of the diminution of malaria, may have to be deferred to the end of the season or to some later time.

All of this is the essential bookkeeping of the business. Good inspectorship is the more essential since the results of the work may not stand out as would a finished bridge or wall, but appear in the far less conspicuous form of larvae in a pool or adult mosquitoes in a barn. Sometimes a conscientious workman will do wonders. after but little training, but such cases are unfortunately rare.

Controls are of course essential. Long observation has taught me that a single control village or pond is unsatisfactory because of the liability to wide natural variability. A group of such controls is far better.

\section{Discussion}

It is hardly necessary to state that in anti-mosquito work one prefers such permanent measures as filling or drainage. But there are many cases such as that of impounded water where such measures are impossible. Again, larvicides must often supplement engineering measures by preventing mosquito production in ditches and canals.

So that larvicides will be always useful, the development of new reagents and new methods of application should be encouraged.

\section{Summary}

1. Paris green (aceto arsenite of copper) is effective because of its high toxicity to mosquito larvae, low solubility in water, ease of application and relative cheapness.

2. Its disadvantages are its failure to affect pupae or eggs of mosquitoes and its inefficacy against culicine mosquitoes in certain types of breeding place.

3. It may be diluted with dry inert powders, with water after treatment with kerosene, or if made more or less floatable by any means it may be diluted without kerosene in water, in dry dust or in moist sand or gravel.

4. The quantity suitable per unit of breeding area varies with the nature of the breeding place and must be determined by preliminary tests. Frequency of application must also be predetermined by some tests, for this factor varies with temperature and species of mosquito. Some approximations are given here of both quantity and intervals.

5. If properly used, Paris green should be harmless to man, domestic animals, fish and rice plants.

6. Culicine larvae are killed by Paris green when it is within their feeding radius, as in shallow water or in water provided with certain types of vegetation.

7. Paris green has been spread by airplanes, autogiro, and boat-carried machines. Large-scale dusting should be useful even if it only diminishes the density of malaria vectors.

8. Experiments with larvicides other than Paris green and with various methods of distribution of larvicides are described.

9. The necessity of proper inspectorship for any kind of anti-malaria work is stressed. 


\title{
NATURALISTIC METHODS OF MALARIA CONTROL
}

\author{
By PAUL F. RUSSELL \\ INTERNATIONAL HEALTH DIVISION, ROCKEFELLER FOUNDATION, COONOOR, NILGIRIS, INDIA
}

A Naturalistic method of mosquito control may be defined as one that deliberately extends or intensifies natural control. Biologists speak of contending natural forces of potential creation and potential destruction (Chapman 1925). A pool that offers suitable conditions for larvae is a natural creative force; a growth of plant that eliminates larvae from the pool is a natural destructive force. Most mosquito control measures aim either to destroy mosquitoes or to eliminate forces that are potentially suitable for their creation. But naturalistic control differs from these measures in that it aims primarily to utilize specific natural forces inimical to mosquito density or dangerous activity. To poison larvae with Paris green is to cause death in an unnatural way; to put water underground in subsoil pipes is to destroy a natural mosquito-creating force. But to shade a breeding place is to intensify a specific natural limiting force. Sometimes it is cheaper to go with such natural forces than against them, hence the special significance of naturalistic methods.

Since the paper by Williamson (1935) and a League of Nations' bulletin (Hackett et al. 1938) naturalistic control has had increasing attention from malariologists, but there has not been agreement as to what measures should be included under this classification group. Probably too many measures have been put in this category, for there are secondary naturalistic aspects in many control procedures, such as sluicing and draining. A classification should be based on primary character, which in sluicing is management of water and in drainage is elimination of water, each largely by mechanical effort. The following discussion, with one or two exceptions, will be limited to measures that appear to be definable as primarily naturalistic.
It must be emphasized that (a) knowledge of anopheline biology is inadequate to permit fullest use of naturalistic measures; (b) all methods must be chosen to fit specific requirements; (c) all have inherent defects and dangers; and (d) there is no good reason (except experimental) why the application of any type of malaria control need be kept unmixed with the use of others.

\section{Naturalistic Measures Against Adult Mosquitoes}

Erecting plant barriers, either to repel the insects or to block their flight, administering drugs to modify perspiration so that mosquitoes would be repelled, treating bedrooms, houses, or compounds, to render them unattractive to mosquitoes, using natural predators (including bats), intensifying winter killing, or attempting to deviate vector species from man to animals for their food-none of these measures has yet been proved effective. All but the last-named can be dismissed with the hope that in time adequate experiments will make it possible to evaluate them.

Deviation of vector species from man to animals, zooprophylaxis, has had considerable attention, for undoubtedly the presence or absence of malaria in some areas depends somewhat on whether or not, and to what degree, local anophelines are naturally anthropophilic or zoophilic. "Anophelism without malaria," while sometimes mainly due to inadequate density of vector species, is probably frequently dependent on the fact that local anophelines prefer animal blood to that of man. Among the 180 or more species in the genus Anopheles, only about 24 have been proved to attack man as their preferred hosts and only about 15 have been incriminated as serious vectors. But the situation is complicated not only by 
marked differences in food preferences among species but sometimes by geographical variations in the habits of a given species. Moreover, some vector species, like A. minimus var. flavirostris in the Philippines, feed on man or animal as occasion permits without a well-marked preference.

Therefore, an experiment on zooprophylaxis requires as much information as possible about feeding habits of the local anopheline suspected of carrying malaria. Precipitin tests to determine the usual sources of blood meals of the vector are essential. For these the method of Rice and Barber (1935) is most suitable. It is also useful to make census studies of animal and human populations in relation to malarial prevalence, to study the flight, resting habits and time of feeding of the local vector.

In such experiments some attention should be paid to spacing between the animal barrier and the humans it is designed to protect for, when dealing with anophelines that have no strong preferences, it is possible to increase rather than decrease the danger by stabling animals too near a house (Russell 1934). In spite of a great deal of speculation and preliminary study, it is not possible to cite a definitely successful attempt to control malaria by zooprophylaxis.

\section{Naturalistic Measures Against Mosquito LARVAE}

Drying by planting. Not much can be said for attempts to control malaria by planting trees to dry water-logged land, although occasional reports have appeared regarding the use of such trees as Eucalyptus, Cassia, and Casuarina. Probably orthodox drainage would in most cases be preferable. However, Hopkins (1940) reports some success in using afforestation with Eucalyptus robusta to dry Uganda swamps where $A$. gambiae and $A$. funestus breed.

Altering flora. One sometimes observes the disappearance of anopheline larvae following some natural alteration in the flora of a breeding place. For example, a growth of Wolffia arrhiza over the surface of a pool will inhibit all mosquito breeding. This effect has also been reported in the presence of Lemna minor and of Salvinia cucullata and Azolla. It might be possible to use this natural force in mosquito control, but at present too little is known about transplanting such plants and encouraging the rate and luxuriance of their growth.

Some success has 'followed procedures for killing top-algae and stimulating bottom blue-green algae in salt-water fish ponds in Java. So, too, it is reported that by introducing a species of herbivorous fish, Puntus javanicus, into fresh-water fish ponds in Java the edges were kept clean-weeded and larva control by Panchax fishes was obtained (Hackett et al. 1938). However, one must avoid altering flora in such a manner that vector species are attracted rather than controlled by the changed conditions.

Agitating water surface. Although intermittent agitation in agricultural and domestic wells and gentle swells in a tidal basin are usually not sufficient to deter anopheline breeding, mosquito larvae and pupae are not found in water continuously ruffled by waves. Occasionally this method may be used in mosquito control.

Scharff (1935) used agitation successfully in some 200 agricultural pools on Penang Hill. He kept them free from $A$. maculatus breeding by changing the water supply line from small ditches to bamboo pipes with outlets four to six feet above the pools.

Kibbey (1923, 1925) reported using launches to create wave action for the control of A. quadrimaculatus breeding on some Alabama lakes. This device stranded floatage and, in conjunction with occasional hand removal of vegetation and debris, was effective.

Experiments in Madras with wind-activated "diddlers" were not successful in a small pool, but it appeared that further research might evolve practical control of this sort (Russell and Jacob 1939a).

Pollution. Anophelines in general will 
not breed abundantly in water polluted by industrial wastes, sewage, or sullage water. Tolerance to environment varies with species, and both in the laboratory (Russell and Mohan 1939, 1940, 1941) and in nature there are wide limits for a given species. The effect of pollution is probably due not only to toxic action but to some extent to microbic and physical effects on larvae and their food supply.

One seldom finds larvae of malarial vectors in buffalo wallows or in piggery waters. Streams polluted with bagasse from a Philippine sugarcane mill, pools catching refuse from a Ceylon sisal factory, and pits used for macerating canepa hemp in Italy were not patronized by malarial vectors (Hackett et al. 1938).

Williamson (1935) was perhaps the first to experiment with pollution. $\mathrm{He}$ tried herbage cover, a measure which covers shallow water in pools or ditches with green-cut grass, herbage, or leaves. The herbage is trampled into a compact mass a foot or more high. It should extend a foot or so beyond the edge of a pool to allow for rainy weather extension. In a ditch or small stream the packing may be held down by a double row of stakes. In the Philippines, good results have followed use of cocoanut husks and fronds in narrow streams. In Madras macerated cactus has been used in agricultural wells and tobacco stubble in borrow pits with success against anophelines.

Malariologists should be alert for opportunities to cheapen control by using industrial, agricultural, or home waste to pollute breeding places. Pig-raising may improve the economic status of a community while, with a little direction, it tends to free pools or ponds of malarial vectors.

Changing salt content of water. Entomologists have noted many instances of natural control of anopheline larvae following freshening or salting of a breeding place. Successful attempts at control have been based on this natural and potentially destructive force. Strictly speaking, the method probably should be classed under management of water rather than as a naturalistic method.
At Durazzo, in Albania, by using Calco tide-gates in reverse position, a brackish marsh was transformed into a sea-water lagoon, thus inhibiting $A$. elutus. "Within two years, breeding over an area of fifteen square kilometers was reduced to zero, while the profits to the Government of an existing fish concession, which has been greatly benefited, more than equalled the cost of the operations" (Hackett et al. 1938).

Excluding salt water has been useful in controlling both $A$. sundaicus and $A$. bar. birostris in Malaya (Hodgkin 1938). In North Holland it has been found that abundant irrigation in the new polders gradually washes salt out of the soil, causing the vector anopheline to disappear.

Before altering natural lagoons, a careful study should be made of the probable effect on anophelines. Washburn (1933) reported that in a malaria-free Jamaica town some seaside mangrove swamps became land-locked and partially dry. Then came heavy rains which filled them with water. A. albimanus soon appeared, with disastrous results. Spleen rates rose from 5 to 90 per cent. and there were 4400 cases of malaria with 138 deaths in a population of 8000 . When the fresh water was drained off and sea water readmitted, A. grabhami replaced $A$. albimanus and malaria subsided.

Shading. It has long been known in Malaya, Assam and elsewhere that clearing a jungle ravine of trees and shrubs, thus exposing a stream to sunlight, is frequently the prelude to epidemic malaria. So, too, for many years entomologists have noted that some anophelines, like $A$. umbrosus, prefer shaded breeding places, whereas others, like $A$. albimanus, prefer open, sunny places. This natural phenomenon has been developed into naturalistic control by shading, as for example, by Ramsay and Macdonald (1936) in Assam, Overbeek and Stoker (1938) in Java, and Carr (1938) in Cuba. The reverse, exposing to sunlight, has more often resulted disastrously than helpfully.

Shading may not control breeding directly; in fact, Thompson (1940) found 
that shade itself played no part in the control of breeding places of $A$. minimus by dense shading. The densest possible shade that could be produced in a field experiment was still attractive to gravid females. In fact, gravid $A$. minimus was strongly attracted by shade and would not oviposit in an unshaded position. Breeding continued until death of vegetation left the edge of the flowing drain completely denuded. In other words, the controlling effect against $A$. minimus was due entirely to secondary effects of shade, e.g., increased water movement. Effective control could be obtained by complete removal of all vegetation at the edges with full exposure of bare edges to light. Thompson (1940) notes that indiscriminate use of shading plants might do more harm than good.

In Assam, such plants as duranta, lantana, hibiscus, and tarapat have been planted along streams to control breeding of A. minimus. In Java, Tithonia diversifolia, a Mexican marigold, was used against A. maculatus. In Cuba, Carr (1938) used Ficus benjamina with excellent effect against $A$. albimanus. Good results have followed shading Malayan wells by erecting small thatched huts over them. In Assam woven fiber mats were used effectively to shade the edges of pools. But in South India we were not able to control $A$. culicifacies by shading casuarina pools (Russell and Jacob 1939a) nor have preliminary experiments with well-shading been successful. Breeding of this species in field channels can be controlled by planting rice. When these plants are a foot or more high culicifacies disappears. We are not able to state yet whether this is an effect of shade or of a barrier although the latter appears more probable.

Carr (1938) noted a double effect of shade. It not only of itself tended to drive out $A$. albimanus but it also prevented algae and other vegetation from flourishing, thus depriving larvae of protection against fishes.

This naturalistic method of shade cultivation is not so simple as may appear. The degree of shade required will vary and should be determined by experiment for a given species of mosquito. The shade plant must be carefully chosen. It should be easy to secure, should grow rapidly and be hardy enough to endure local climate and storms. Preferably, it should be evergreen and its foliage, fruit, and wood not attractive to man or animal. After planting, it may require cultivation and fencing and it must be inspected regularly and maintained.

Certain Assam ravines have been controlled suceessfully by planting and eultivating shade while allowing natural jungle growth to return, so that the end result has been dense, permanent shade which has given perfect control. It is, of course, much easier to preserve natural shade than to reproduce it. Undoubtedly, the method has possibilities in American tropies against $A$. albimanus, but it should always have preliminary experiments, such as Carr (1938) carried out in Cuba.

Use of natural enemies. Excepting fishes, not much can be said for use of natural enemies of mosquito larvae in malaria control. Such plants as Utricularia probably destroy some larvae but have had little experimental study in this regard. Undoubtedly, parasitic bacteria, fungi, viruses, and protozoa kill mosquitoes. Some species have been actively used with success against certain agricultural insect pests, but none has had a suitable experimental test against mosquitoes. So, too, certain nematodes, annelida, crustacea, and numerous hexapoda are natural enemies of mosquito larvae but have not yet been proved to be useful in malarial control. Even Notonectidae and Dytiscidae, perhaps the most active invertebrate predators, have not yet had suitable experimental trials. Various amphibians, reptiles, and birds have been observed to include mosquito larvae in their usual diets, but no convincing attempts have ever been made to stimulate them to give more than their natural help in controlling larvae. Only in the case of certain fishes has that potential, destructive force against mosquitoes 
which many invertebrates and vertebrates certainly possess, been intensified into successful naturalistic control.

This subject has been reviewed by Hinman (1934b). As he noted, one should remember that a predator that devours large numbers of larvae in an aquarium may not do so under natural conditions where perhaps its normal food takes preference. However, there have been successes in dealing with certain insect pests of agriculture by utilizing predators (Sweetman 1936).

Fishes that have been most useful in mosquito control belong to the order Cyprinodontes, of which the two chief families are the oviparous Cyprinodontidae, including Panchax, and the viviparous Poeciliidae, including Gambusia and Lebistes. The males of the Poeciliidae family are differentiated from those of the Cyprinodontidae by having an elongate anal fin which is developed into an organ of copulation. Lebistes reticulatus ("guppies" or "Barbadoes millions") is very prolific and has had considerable value in the West Indies and northern South America, especially in yellow fever prophylaxis. But the most important fish as regards malaria control is Gambusia, to some extent $G$. holbrookii, indigenous from Florida to Virginia, but chiefly G. affinis Baird and Gerard, of the Mississippi Valley, Texas, and North-Eastern Mexico (Rockefeller Foundation 1924; Covell 1931a).

This discussion will be limited to Gambusia affinis, which Hildebrand (1921) has called "by far the most important natural enemy of the mosquito known to date." The usefulness of these fishes depends on the following characters which they possess: (a) habitat corresponding closely with that of larvae; (b) alertness and tendency to push into emergent and floating vegetation; (c) a high food-preference index for larvae in natural surroundings; (d) a remarkable fecundity and a rapid rate of reproduction in nurseries and natural bodies of water; (e) hardiness and adaptability to waters deep and shallow, open and confined, clean and organically polluted, sweet and brackish, in tropical and temperate climates; (f) ability to withstand handling and transport; ( $\mathrm{g}$ ) worthlessness as food for man; (h) infrequency with which they have been known to be destructive to other fishes.

In the southern United States Gambusia usually can be obtained locally without difficulty, but to insure a ready supply it is advisable to choose a small shallow pond, having an area of 500 to 1000 square yards, for breeding of fishes. This stock pond, or hatchery, should be cleared of large fishes by seining with a fine fish net, or by draining and cleaning if it can be easily refilled. It is also possible to kill all fishes in a pond by exploding sticks of dynamite. The hatchery can then be stocked with from 100 to 1000 or more Gambusia. For propoganda purposes and to safeguard the fishes from use as live bait by fishermen, a sign post may be erected calling attention to the fact that the pond is a Gambusia nursery of the Health Department. At suitable places in the pond screened shelters of $3 / 16$ inch wire netting may be built to shelter young Gambusia from cannibalistic parents. If the stock pond has an outlet this should be screened with 14-16 mesh wire to prevent escape of the minnows. Inlets should be screened to prevent entrance of other fishes.

Gambusia fishes can be collected by using a small net, some three feet deep on a 12-foot handle or by use of a long bobbinet seine. For transportation, ordinary 5 to 10 gallon milk cans may be used, filling with water up to the point where the can narrows and using no stopper or else one with large perforations. Not more than 30 to 40 Gambusia should be transported in a 10-gallon can.

Gambusia in the latitude of the southern United States will have broods, depending on size of female, of up to 100 or more, averaging about 30 , every 4 to 6 weeks from May or June to September or October. The fishes arrive at sexual maturity in 4 or 5 months. Gambusia will not survive northern winters out-of-doors, but if pools and ponds are restocked in the 
spring from indoor aquaria the fishes multiply rapidly.

In the tropics it is usually best to build a concrete tank for use as a Gambusia nursery (Mulligan and Majid 1936). This may be from 4 to 10 feet or more square. It is a good plan to plant rushes in the corners and to add algae. The nursery may have to be screened against birds or animals preying on the fishes. In Madras we have had excellent results with three such nurseries $(30 \times 30 \mathrm{ft}, 14 \times 7 \mathrm{ft}$ and $10 \times$ $16 \mathrm{ft}$ ), feeding finely chopped raw meat to the Gambusia daily. Fishes have flourished in these concrete tanks with maximum temperatures of air and water up to $105^{\circ} \mathrm{F}$.

We have transported Gambusia in the tropics successfully in various types of native clay pots, adding some algae to each pot to prevent splashing. It is necessary to allow free access of air and to avoid overcrowding and splashing during transportation.

The numbers of Gambusia to be used in various types of breeding places to control mosquito larvae will depend on local conditions. More are required where the surface area is large or vegetation abundant. In a well, ten Gambusia may be enough to keep down all breeding. Ten to 50 may be needed in a cistern. Low-lying culverts holding water for a considerable time often may be kept free from larvae by stocking liberally with Gambusia, at least 5000 per acre.
Before stocking a well with Gambusia it is wise to treat the water with chloride of lime or "'bleaching powder," using 12 to 16 ounces to 800 gallons of water. This kills smaller fishes and brings the larger ones up for removal by netting. Sometimes a second treatment is required. The water becomes potable in a few days but it is best to wait 10 days before introducing Gambusia (Russell and Jacob 1939b).

Too few fishes or too much natural cover for larvae are primary causes of failure in mosquito control with Gambusia. The former defect is in turn usually due to (a) natural enemies, such as larger fishes or live-bait fishermen; and (b) neglect. The latter defect may consist of floatage or vegetation, especially when matted. In many situations, the cost of removing larger fishes or of keeping down larvaecover would be excessive and consequently fish should not be used. In other places, a little help by removing matted algae is all that the fishes require. Sometimes it is advisable to combine fluctuation in water level with the use of Gambusia to provide cleaner edges and so less shelter for larvae.

Some years ago Gambusia fishes were overrated in mosquito control. Now the tendency is to underrate them. Extensive trials throughout the world during the past two decades have proved that Gambusia can give effective results in certain types of breeding places and considerable help in others. 


\title{
ADAPTABILITY OF CONTROL MEASURES TO THE NIARCTIC FAUNA OF ANOPHELES MOSQUITOES
}

\author{
By HENRY A. JOHNSON \\ U. S. PUBLIC HEALTH SERVICE, WASHINGTON, D. C.
}

Distinct faunal regions of anopheles mosquitoes have been observed and Boyd (1930a) has delineated these divisions geographically. He classes in the Nearetic fauna those species of anophelines occurring in temperate and aretic North America, including the United States, Canada, and Greenland. This paper will be limited to a discussion of malaria and of the control of the anopheline vectors within these areas.

\section{Basic Problems}

"Although nine species of anophelines are represented in the Nearetic fauna only two are known to be important vectors of malaria, namely, A. quadrimaculatus and A. maculipennis. This is due to the particular breeding habits of the insects and their associations with man. A. quadrimaculatus is the chief vector of malaria in the greater portion of the malaripus sections. It breeds almost wholly in still water that is relatively clean. It requires some sunshine, never being found in dense shade. It is not found in waters wholly unshaded. Although A. quadrimaculatus will feed on animals as do other anophelines it will also feed voraciously on human beings and frequents human habitations. An ideal breeding place for $A$. quadrimaculatus is in freshly impounded water which floods a basin containing some underbrush and which is sparsely covered with trees. Such water quickly gathers floatage, dying land vegetation, twigs, and leaves among which algae soon appear. Such floatage offers the requisite amount of shade and an abundant food supply. The supply of minnows and aquatic insects will seldom be sufficient to prevent mosquito production.
"The normal detritus passing down a narrow stream will clog the interstices of a fallen tree or branch and ereate a dam. These natural impounded waters are excellent breeding places for A. quadrimaculatus. Swamps covered by a growth of virgin timber on the other hand are not good breeding places. They have a small seeding of $\boldsymbol{A}$. quadrimaculatus but not enough to perpetuate malaria. When the lumberman enters, cutting out the large trees and leaving the small ones, the branches and tree tops, he changes a safe water surface into one almost ideal for A. quadrimaculatus production" (Williams 1937a).

Agriculture on the other hand usually has the reverse effect. Where land is at all valuable $A$. quadrimaculatus swamps and ponds are drained.

In the river valleys of New Mexico, Califormia and Oregon, A. maculipennis is the malaria vector of importance. This mosquito breeds in waters which are constantly refreshed, such as overflows, seepage areas, continuous-flow-rice-irrigation and stream pools below the river level. It, therefore, is apparent that much of our malaria is man-made although such factors as climate, topography, and movements of population play an important part in its distribution and spread.

\section{Economic Resources}

Cities and large towns are normally prosperous and they have been quick to take advantage of modern conveniences, such as water and sewerage systems, improved streets and drainage. Sereens are almost universally used as protection from insects. The utilization of these conveniences has resulted in the elimination of anophelines 
and the consequent restriction of malaria to rural districts.

The rural areas adjacent to the flood plains of rivers are, on the contrary, essentially agricultural and tenant farming is often practiced. Modern improvements are not available, screens are not normally used, housing is poor, wages are low, and the tenant farmers live largely from hand to mouth. In the southeastern states where anophelines are abundant, malaria still remains a serious economic burden to this class.

As serious as may appear to be the economic plight of sections of the United States where malaria is seriously prevalent, it is my belief that they could show a profit by controlling malaria, even if they chose the most expensive method. Fisher (1920) has investigated the question of how much a community can afford to pay for malaria control. Working from the angle of the probable return on the investment, he deduces a formula to indicate what return might be expected from an investment in control measures.

Anopheles control measures are sometimes classed as permanent or temporary; mechanical or chemical; natural or artificial; naturalistic, etc. In this paper they will be considered under the general items of Water Level Fluctuation; Clearing; Drainage ; Filling ; Use of Minnows ; Larvicides; Screening; Naturalistic, and Miscellaneous.

\section{Water Level Fluctuation}

The shallow vegetation-covered areas of impounded waters present ideal conditions for the propagation of $A$. quadrimaculatus because, under such conditions, an abundant food supply exists and protection is afforded from natural enemies. Water level fluctuation should be used as a mosquito control method wherever the level can be suitably regulated for it has been demonstrated that without its aid other methods are unable to suppress breeding.

Fundamentally a suitable fluctuation schedule commences with a high water level at the beginning of the anopheles breeding season, followed by a rapid lowering of the level a foot more or less each 10 days until the end of the breeding season. Such a schedule strands floatage and assures a reasonably vegetation-free edge. By thus creating clean banks and open water conditions, anopheline production is minimized. This ideal schedule cannot always be put into effect.

Water level fluctuation is rendered vastly more effective as a mosquito control measure by a preliminary clearing of vegetation and floatable material in the proposed basin prior to impounding, and, indeed, most states in the malarious belt require such clearing before a permit to impound will be granted. After impoundage, it often becomes necessary to employ secondary measures of control, such as larvicide application, on parts of the shore line not adequately controlled by fluctuation alone.

Anopheles production may also be curtailed in smaller ponds and lakes by a downward fluctuation of water level during the mosquito breeding season with or without vegetation clearing, although provision for fluctuation was not originally made. Natural fluctuation caused by drying or evaporation during the anopheles producing season is often responsible for a great reduction in anopheles propagation in many smaller water deposits.

\section{Clearing}

In the larval form, mosquitoes find protection from natural enemies under dense aquatic vegetation and among floatage. Both favor anopheles production by slowing down the current, creating still, rippleless water, affording direct larval protection, providing food, and hindering the proper distribution of larvicides. Low vegetation having horizontal leaflets, as certain grasses and weeds, are ideal for larvae protection as contrasted with the upright, tall, straight emergent types, as rushes and bushes.

Clearing, or the removal of this protective vegetation and floatage, is an important part of any program directed against 
anopheles larvae. It is practiced on impounded waters by pre-impoundage clearing of the basin, water level fluctuation, direct removal and poisoning. In smaller lakes mechanical removal is often advantageous. Vegetation growth (other than grass) along drainage ditches is often productive of conditions favoring anopheles breeding and it should be systematically removed and the channel regraded, often as frequently as twice during the anopheles producing season.

\section{Drainage}

Anopheles control drainage has during the past 25 years been highly developed in its special field and today it remains of prime importance as an anopheles control measure.

The purpose of anti-malarial drainage is to remove the proven breeding places of malaria vectors and is therefore directed at residual water rather than general runoff. Consequently the ditches are usually small or medium sized and should be cut to an even, regular grade. Although open earth ditches are not durable, they have a wide field of usefulness where swamps, sheet water, or other large areas are to be eliminated (particularly in rural areas). Under such conditions after the first rush of water is over the flow is intermittent.

Probably the greatest recent advance in anti-anopheles drainage in the United States has been the development of durable ditch linings and stabilized banks. While the first cost of a lined ditch is of course greater, the total cost over a period of years is usually much less than that of earth ditches. A durable invert of concrete, brick and mortar, or stone and mortar, is installed in the ditch bottom and the sides stabilized by sodding. This practically permanent type of construction is being used wherever the density of population can meet the first cost. It is particularly applicable to drainage systems in urban and semi-rural areas. In the rural areas of at least one state it has been found economically feasible to construct sod lined ditches and to install durable linings of concrete only at such places in. the ditch as may be subject to scour. This construction adds greatly to the life of a ditch at a minimum of extra cost.

The development of durable linings with stabilized banks has necessitated some changes in the established practices which are applied to earth ditches, such as permitting smaller cross sections, necessitating flatter bank slope, and providing lined inlets. Many miles of ditches with durable linings have been installed in malarious sections and their use is rapidly increasing as more economical methods of installation are being perfected.

Tile and similar underground drains constructed with poles, large rocks, or even old tin cans, are an important method for the elimination of anopheles producing areas. They are of use in intercepting seepage outcrops, drying marshes, or other wet areas, or lowering the ground water level. They are relatively simple and inexpensive to construct; they keep the water under ground; they operate satisfactorily over long periods, and of course do not disfigure the surface or take up valuable space. Their proper installation requires considerable engineering skill and a study of the underground flow of water. Randle (1940) has described the construction of this type of anopheles control drainage as developed in Mississippi.

In a flat country with pervious soil a line of open joint tile laid directly beneath the ditch bottom has often been found very effective in drying ditches having almost no fall which would normally produce anopheles. When a tile line is utilized in this manner, weeds and grass in the ditch are not cut and no maintenance work on the ditch itself is needed.

Under some conditions, particularly in limestone districts, vertical drainage may be utilized to remove surface water and isolated ponds. The method depends on locating a limestone fissure to which the water can be led, and consequently is not always successful. 


\section{Finling}

Going hand in hand with drainage as an anopheles control method is that of filling, thereby eliminating once for all some breeding areas. It has the advantage of not disturbing the ground water table. This is a method always to be considered but ofttimes overlooked.

\section{Use of Minnows}

In the early days of mosquito control it was noted that top minnows (Gambusia affinis or holbrooki), abundant in rivers, streams and ponds, consumed large numbers of mosquito larvae. Stocking of impounded lakes, ponds, and almost all types of water deposits with these fish was heralded as an effective naturalistic method available for the elimination of mosquito larvae. To be effective against larvae the water must be sufficiently free of vegetation to permit the fish considerable freedom of movement, because mosquito larvae are admirably adapted for camouflage against twigs, plant stems, etc. When water has been rendered clear and unobstructed by removal of vegetation and floatage so that minnows can be effective, this very condition in itself has minimized or prevented mosquito breeding.

Minnows, therefore, should not be relied upon as a prime measure against anopheline production in this region. Rather their use constitutes an auxiliary or secondary method which is of some value. Minnows when propagated in ponds, lakes and large reservoirs located at a distance from habitations often reduce breeding of mosquitoes in these deposits sufficiently to minimize their importance as producing areas.

\section{LARVICIDES}

The value of oil (kerosene) against mosquito larvae was demonstrated by $\mathrm{L}$. $\mathrm{O}$. Howard in 1892. This was probably the first use of a larvicide against mosquitoes and the application of various larvicides to the surface of water still remains one of the most effective methods of controlling malaria.
In order to insure economy and effectiveness larvicides should be applied under careful entomological supervision. Their application should be limited to places where actual breeding is known to occur. A check for the presence of adult anopheles often gives valuable clues to hidden breeding places which can usually be controlled with larvicides, pending the application of more permanent methods.

\section{Screening}

The screening of rural houses, while not a measure directed against the production of anopheles, is a measure directed at the control of the adult insects, in so far as their association with man is concerned. Its field of usefulness is in highly endemic rural areas where conditions are such that other methods of malaria control are not practicable. The deplorable condition of the majority of rural farm houses makes it necessary to mosquito-proof the walls, roof and floors, in addition to providing screens if anophelines are to be kept out. As difficult as an effective screening program may at first sight appear to be, it has been found economically feasible in many rural areas. The biting period of anopheline mosquitoes commences at twilight, hence the protection offered by a screened house is doubtless greater than that of bed nets not ordinarily utilized until some time after dark.

Around some impounded water projects where mosquito control failed, it was necessary to screen and mosquito-proof the houses within one mile of the shore line. Following the Mississippi River flood in 1927 much rural screening was done in the adjacent territory and statistics are available tending to demonstrate its anti-malarial value. However, the data are not conclusive and it must be remembered the responsibility for maintenance rests upon the occupant. The Tennessee Valley Authority has found the screening and mosquito-proofing of rural houses in one special area an effective malarial control measure where the prevention of anopheline breeding was impossible. 


\section{Naturalistic Methods Other Than Top Minnows}

These methods consist of so altering the environmental or the chemical or physical characteristics of breeding areas that they are no longer attractive to anophelines. Methods which possibly may be effective against the Nearctic fauna may be represented by the following : increasing salinity of the water; polluting the water by sewage, laundry or other wastes; filling by deposition of silt; introducing predacious insects; shading, or other method of controlling food supply, but their possibilities with our fauna have not been explored.

Under certain conditions the above methods might be utilized to change the mosquito fauna, but the possible substitution of very bothersome noxious species for Anopheles larvae should be considered. These methods can now be made effective only under certain conditions, but when factors limiting Anopheles production are more thoroughly understood, naturalistic methods of control may become of decided importance. Certainly the possibilities of naturalistic control possess potentialities which merit study.

\section{Miscellaneous}

While the foregoing methods are in general use for anopheline control, certain other methods or combination of methods may be useful under special circumstances.

Instead of draining a swamp or seepage area, it is sometimes desirable to deliberately increase the retention of water as a mosquito control measure, in order to facilitate the application of Anopheles control measures at the prepared edges.

The value of shading by tall bushes or trees as an anopheline control measure has been the subject of much discussion. Shading is a recognized measure for the control of some Anopheles of the old world and has been suggested for the control of $A$. quadrimaculatus. Long standing densely shaded swamps, never lumbered, do not support A. quadrimaculatus. Reshading of the lumbered swamps with quick growing low bushes has not been effective. Other types of shade should be tried.

A. maculipennis, the malaria vector of the west and southwest, is often produced in seepage water resulting from poorly applied or excessive irrigation water. It is much more practical to correct the conditions either at the source or by drainage, than to render them inoperative by creating shade. Where large seepage areas from irrigation cannot be prevented, the production of A. maculipennis can be controlled by the liberal use of larvicides aided possibly by minnows.

Nearctic anopheline fauna are attracted to animals as well as to man, and this fact has led to the trial of animal barriers (stabling of animals) between known anopheles producing areas and a population it was desired to protect. This might be termed a biological method of control and there is evidence to show that under ideal conditions some protection is afforded. However, opportunities for using this method effectively are so few in the area of the Nearctic fauna that it is at present of little practical importance.

An effective method of preventing the spread of malaria by controlling adult anophelines consists in. systematically killing them in the home each morning. Spraying with pyrethrum extract serves a similar purpose, and has been used in connection with the control of Anopheles gambiae in Brazil. These methods are designed to prevent the flight of infected Anopheles and were used very successfully by J. A. Le Prince (1926) at Panama when other means of malaria control (particularly screening) were not available. Its chief use would naturally be in temporary labor or military camps in malarious areas where anopheles were abundant and other means of malaria prevention impracticable. Although the method has apparently not been tried in North America, there are circumstances when it would seem to offer possibilities.

In discussing the applicability of the various measures of mosquito control for 
malaria reduction, naturally only the basic principles have been mentioned. It should be borne in mind that every Anopheles control problem is a new one and in each case a study of all factors affecting production should be made. Analysis of the data will indicate to a competent malariologist which measures are most appropriate. Usually a combination of methods is required.

Occasionally it develops that malaria control by anopheline reduction is not practicable. Rural areas are often in this class. It is regrettable that efficient, practical, cheap malaria control has not yet been developed for such use; for here the need is greatest.

In connection with Anopheles control activities it must be borne in mind that complete elimination of the vectors is unnecessary, for below a certain minimum density malaria will disappear of its own accord. The minimum number of vectors necessary to perpetuate malaria depends on many factors and no formula is available from which this number may be determined. Suffice it to say that in the case of A. quadrimaculatus, reduction to the point where only occasional adults are found in the suitable diurnal shelters, is effective.

\section{Desirable Future Studies Affecting ANOPHELES CONTROL}

Basic measures for the control of Anopheles production were developed by Howard and by Ross and were first applied on a large scale during the construction days of the Panama Canal. Most of the ensuing study has resulted mainly in a refinement of measures there used. The lining of ditches, while new in the United States, was done at Panama more than 20 years ago and by the British in the Federated Malay States over 30 years ago.

Dust larvicides and the pyrethrum larvicides represent recent developments, but do not open any new or basic approach to the problem. Likewise, screening recently developed to a high degree for rural communities in the United States, was recognized as a valuable malaria control method in the construction days of the Panama Canal.

The gross biology of anophelines is known but the minimal factors necessary for rapid production require detailed study. It is probable that a careful study of such factors as chemical content of the water, plankton, daily and seasonal changes in $\mathrm{pH}$ of the water, and associated phenomena either singly or collectively, may offer an answer to the question, Why do Anopheles breed in some waters while others, apparently similar, never produce them?

Further investigation may disclose the operation of some factors during larval development profoundly affecting the capacity of the adult insect to develop or transmit plasmodias.

It is also desirable that there be studies directed at the possible control of anophelines without seriously interfering with other forms of water utilizing life, upsetting as little as possible the delicate biological balance set up by nature in all water deposits. 


\title{
THE ADAPTABILITY OF CONTROL MEASURES TO THE MALARIA VECTORS OF THE CARIB- BEAN REGION
}

\author{
By HENRY W. KUMM \\ INTERNATIONAL HEALTH DIVISION, ROCKEFELLER FOUNDATION, BALTIMORE, MD.
}

Mosquito control in the Caribbean region was initiated in the early years of this century by General Gorgas in Cuba and Panama. At first, similar work in neighboring countries yielded disappointing results, so that for some time it was concluded that only localities having financial resources comparable to those of the Panama Canal region could hope for suceess. In this paper I will show that while adequate control of malaria has been maintained by the Health Department of the Canal Zone, other comntries and organizations in the Caribbean region are now making signifieant progress.

At the present time there is no known means of eradicating malaria from large rural areas of the American tropies. It is possible, however, to control malaria in circumseribed zones which are of sufficient importance to justify the necessary expense.

Most authorities agree that at the present state of our knowledge permanent measures are the most economical. The use of oil or Paris green to destroy anopheline larvae is at best only temporary. These measures undoubtedly have their place and in certain circumstances are the only ones available, but whenever possible measures should be taken to "build out" malaria. Such a program has its admitted limitations, but it is better than nothing.

At least 8 countries in the Caribbean region have malaria control programs and some of the larger commercial enterprises, such as the United Fruit Company and the Tropical Oil Company of Colombia, are obtaining highly significant results under their own control programs. In 1906 over 80 per cent, and in 1938 only 1 per cent, of the employees of the Canal Zone contracted malaria. Yet in the last 24 years the morbidity rate for the Zone has never fallen below the 1 per cent which seems to be the irreducible minimum.

The work of many investigators has shown that $A$. albimanus is the prineipal vector in 17 out of the 20 countries of the Caribbean region but much remains to be done before our knowledge will be complete. An accurate recognition of the principal malarial vectors in each country is important, because control measures directed against one species may be useless against another. A. albimanus, for instance, breeds in ground pools in the sun, but $A$. darling $i$ prefers the shady margins of slowly running streams. In Cuba, breeding of $A$. albimanus has been discouraged by the planting of shade trees, an excellent deterrent, but one that would not be effeetive against $A$. darlingi.

Dr. Gabaldon reports that in Venezuela A. albimanus is the principal vector, especially along the sea coast and around Lake Valencia. However, he has initiated eontrol measures against $A$. darling $i$ at three places, Cabudare, Acarigua and Maturin in the provinees of Lara, Portuguesa and Monagas. He feels that it is too early, as yet, to evaluate the effectiveness of these projects. In the island of Trinidad, de Verteuil and Spence (1937) attempted to control malaria transmitted by $A$. tarsimaculatus breeding in salt water.

A malaria control program has been in operation in the Panama Canal Zone for the past 35 years and the number of employees has at times exceeded 50,000. The malaria rates are given in Table I for persons living in the Panama Canal Zone and for the tropical employees of the United 
TABLE I

Malarial Rates of Employees of the Panama Canal, the United Fruit CoMpany and the Tropical OIL CoMpany of COLOMBIA

\begin{tabular}{|c|c|c|c|c|c|c|}
\hline \multirow[b]{2}{*}{ Years } & \multicolumn{2}{|c|}{ Panama } & \multicolumn{2}{|c|}{$\begin{array}{c}\text { United Fruit } \\
\text { Company }\end{array}$} & \multicolumn{2}{|c|}{$\begin{array}{c}\text { Tropieal Oil } \\
\text { Company }\end{array}$} \\
\hline & 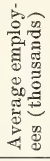 & 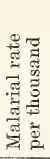 & 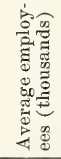 & 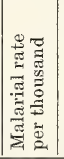 & 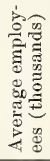 & 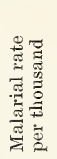 \\
\hline 1906 & 26.5 & 821 & $\ldots \ldots \ldots$ & $\ldots$ & $\ldots . .$. & $\ldots . .$. \\
\hline 1907 & 39.2 , & 424 & & & $\ldots . .$. & $\ldots . .$. \\
\hline $1908-9$ & 45.5 & 248 & . & 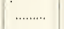 & $\ldots . .$. & $\ldots . .$. \\
\hline $1910-12$ & 50.2 & 160 & & $\ldots \ldots \ldots . .$. & $\ldots . .$. & ....... \\
\hline $1913-15$ & 45.3 & 70 & 30.7 & 208 & ..... & $\ldots$. \\
\hline $1916-18$ & 30.4 & 16 & 30.4 & 120 & $\ldots . .$. & ...... \\
\hline $1919-21$ & 19.8 & 22 & 50.1 & 153 & $\ldots . .$. & $\ldots . .$. \\
\hline $1922-24$ & 11.0 & 17 & 56.8 & 160 & 3.0 & 500 \\
\hline $1925-27$ & 12.8 & 17 & 54.1 & 163 & 3.3 & 623 \\
\hline $1928-30$ & 15.3 & 20 & 62.9 & 71 & 4.6 & 312 \\
\hline $1931-33$ & 13.2 & 20 & 51.5 & 58 & 2.4 & 38 \\
\hline $1934-36$ & 12.4 & 14 & 59.2 & 73 & 3.1 & 59 \\
\hline $1937-39$ & 14.5 & 12 & 62.5 & 82 & 3.9 & 43 \\
\hline
\end{tabular}

Fruit Company and of the Tropical Oil Company. The reduction in incidence is most evident among those living in the Canal Zone, but it is also apparent, after 1927, in the personnel of the United Fruit Company and after 1930 among those of the Tropical Oil Company.

In a recent letter Dr. D. P. Curry, who has for many years directed the mosquito control work in the Panama Canal Zone, says, "I would stress that, in spite of all our sanitation, we still insist on screened living quarters and screened offices for those working at night. Even temporary buildings of construction camps are screened throughout. This includes rooms for sleeping, recreation, dining, bath, toilet and any other space. We require all contractors to do the same. More and more confirmed is our belief here in the extremely long range of flight of $A$. albimanus from vast, far away, uncontrolled, and economically uncontrollable areas."

Dr. Curry wrote last October that the 1940 malaria morbidity rate to that date was 18.3 based on an average of $24,028 \mathrm{em}$ - ployees. New construction was being pushed both night and day on a 24 hour basis, so that there was more exposure at night outside screened quarters than in normal times.

The rate for employees of the United Fruit Company in 1937 was 55, but rose to 95 in 1938-39 because of new banana developments on the Pacific Coast of the Republics of Guatemala and Costa Rica.

During the construction days of the Panama Canal, anopheline breeding was largely controlled by the extensive use of larvicides, but with the advent of more stable conditions permanent drainage was introduced. The precast sectional inverts, which were first developed by Curry for paving drainage ditches in the Canal Zone (Panama Canal Health Department 1933) are now being used in many of the neighboring countries.

Simmons et al. (1939) gave an excellent description of the methods in use for the control of malaria in the Canal Zone. They discussed cement pipe for subsoil drainage and the types of molds used in Panama to make inverts, tiles and wall slabs. Several photographs are presented in this paper to illustrate these phases of the work.

At the present time the Health Department of the Panama Canal is making 150 three-foot inverts every other day, or over a mile of inverts per month. Even at that rate, Curry writes that he can hardly meet the demand for them by the Health Depart-

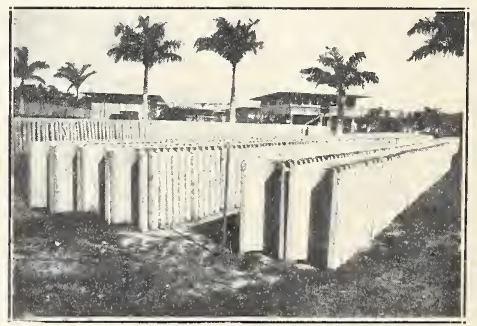

FIG. 1. Inverts for lining drainage ditehes, produced by the Health Department of the Panama Canal. 


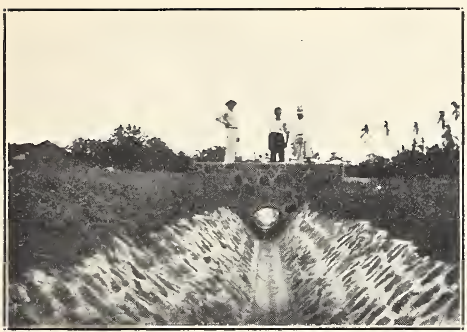

Fig. 2. A culvert over a large drainage channel at La Chorrera, Republie of Panama. This diteh 'was paved with inverts and the sides faced with stones set in cement.

ment, the Army, the Navy, engineers, and contractors. In the Canal Zone inverts cost about 45 cents apiece, including the expense of plant amortization and supervision.

The medical department of the United Fruit Company lays considerable emphasis on repeated applications of larvicides to anopheline breeding places. Screened houses are widely used and concrete gutters and drains, always in evidence on the Fruit Company property, do much toward reducing mosquito breeding.

An intensive antimosquito campaign started in the larger camps of the Tropical Oil Company of Colombia, in 1926, reached its maximum in 1927 and 1928, and since that time the mosquito control program has

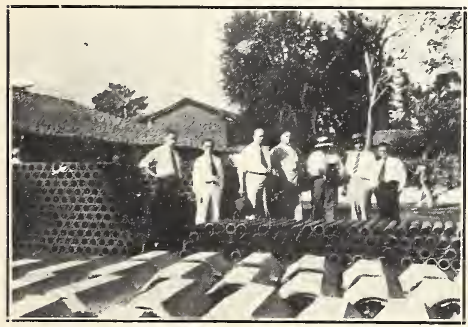

FrG. 3. Conerete inverts and pipe made in the "Plantel" at San Miguel, Republic of El Salvador. The pipe was used for subsoil drainage.

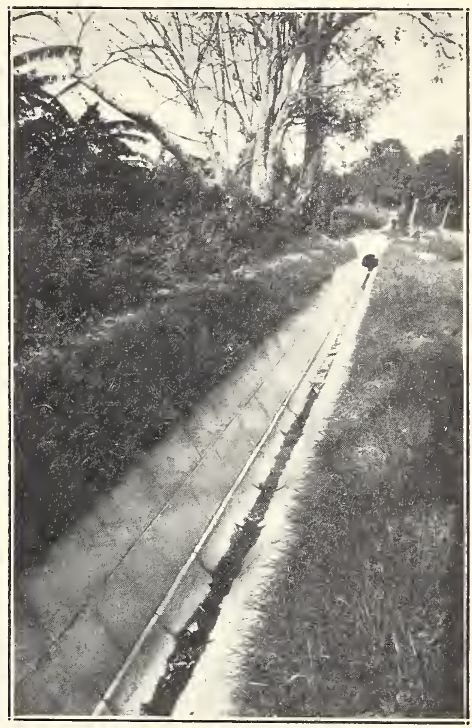

FIG. 4. A ditch lined with inverts, side slabs and grass at Liberia, Costa Rica.

been actively maintained. The work consisted primarily of drainage, oiling, and spraying with Paris green where drainage was not practical.

In the Republic of Nicaragua a coopera-

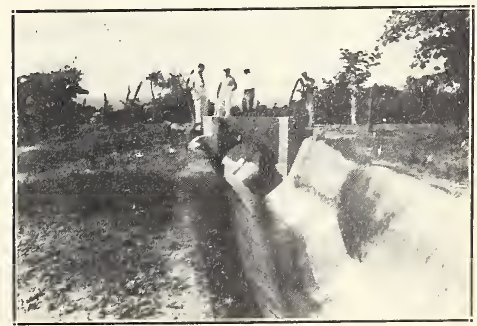

FIg. 5. This ditch at Santiago, in the Republic of Cuba, is lined with thirds of conerete pipe 36 inches in diameter instead of inverts. 
TABLE II

Expenditures for MaLARIA Control

\begin{tabular}{|c|c|c|c|c|c|}
\hline Year & Costa Rica & Cuba* & El Salvador $\dagger$ & Panama $\ddagger$ & Puerto Rico \\
\hline 1935 & $\ldots \ldots \ldots \ldots \ldots$ & $\ldots$ & $\$ 2,711$ & $\$ 23,000$ & $\$ 52,987$ \\
\hline 1936 & ......................... & $\$ 34,056$ & 1,527 & 23,000 & 53,883 \\
\hline 1937 & & 30,097 & 692 & 110,510 & 56,177 \\
\hline 1938 & $\$ 7,071$ & 22,115 & 390 & 99,480 & 100,528 \\
\hline 1939 & 13,592 & 22,490 & 1,000 & 74,840 & 93,887 \\
\hline
\end{tabular}

* From data provided by Dr. H. P. Carr.

† From data provided by Dr. V. A. Sutter.

† From data provided by Dr. Osear Vargas.

\& From data provided by Dr. Garrido Morales and Engineer L. D. Palacios.

tive malaria control demonstration by drainage was earried on from 1921 to 1925 in the adjoining towns of La Puebla and Rivas. The blood parasite index was reduced in 3 years from 64 per cent to less than 1 per cent, but when supervision of the drainage channels was relaxed, the index rose again. Ditches were not at first paved with inverts or side slabs and rapidly became choked with Pará grass. The per capita cost of the entire project was about $\$ 1.25$, though the annual cost of maintenance was very much less.

In the island of Puerto Rico, the town of Salinas was studied for 8 years from 1928 to 1935 . Earle (1937) reported that it was necessary to bring all breeding areas under control before the mosquito density was definitely reduced. The malaria prevalence was not affected until this mosquito density had been brought down and main-

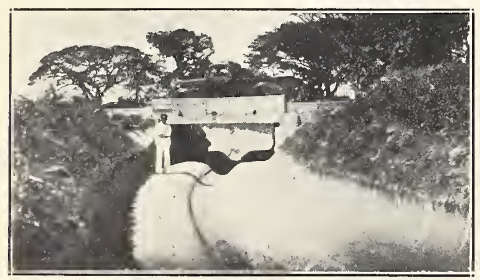

FIG:-6. Maracay, Venezuela. This large collecting ditch was lined with three rows of third rounds of pipe 36 inches in diameter, two rows of sixth rounds of pipe 36 inches in diameter, two rows of flat slabs 16 inches by 24 inches. tained at an extremely low figure. Extensive seepages in cane fields near Salinas were drained by subsoil tiling. Large breeding areas in pools in the bed of the Lapa river were not eliminated until a concrete pipe had been laid well beneath the surface of the river bed itself. During the four years from 1935 to 1938 inclusive, 3.9 per cent of the total expenditures of the Health Department of Puerto Rico were for malaria control. During this same period about 3.5 miles of open ditches, lined with precast concrete inverts, were constructed and almost 5 miles of subsoil pipe were laid. In addition, about 300 acres of swamp land were filled and a number of drainage pumps were installed.

Drainage projects for malaria control were drawn up for 5 localities in the Republic of Costa Rica, Central America. Three of these have been eompleted and in 2 others the work is still in progress.

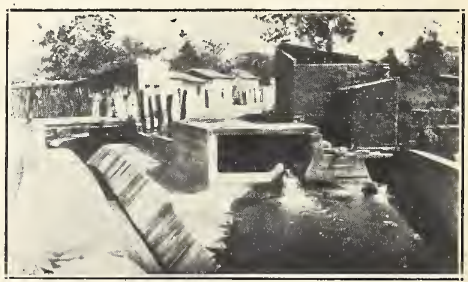

FIg. 7. The junction of two side drains with a main drain in Maracay in the Republic of Venezuela is shown in this eut. A central channel has been eut in the main drain to increase the velocity of the residual flow. 


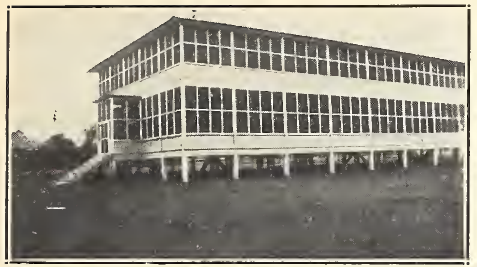

Fig. 8. Compania Agricola de Ulua, La Lima, Republic of Honduras. A well-screened house built by the United Fruit Company for bachelor quarters.

Liberia, the capital of the province of Guanacaste, is one of the latter. At Liberia the spleen index among school children has been reduced to one-fifth of its initial figure and the blood parasite index to about onefifteenth.

The exact amount spent for malaria control is not always accurately recorded, but some data on the expenditures made for this purpose during the past 5 years by the governments of Panama, Puerto Rico, El Sal-

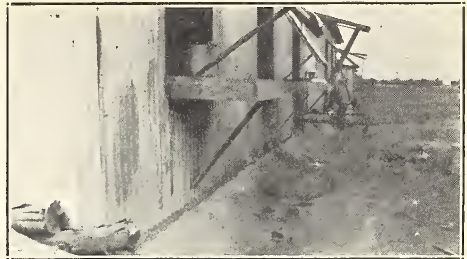

Fıg. 9. Compania Agricola de Ulua, Zapote Camp, Republic of Honduras. Conerete gutters for slops at the rear of the kitchens of the laborers' houses. Waste water from pumps goes into these same gutters.

vador, Costa Rica, and Cuba are presented in Table II.

From 1935 to 1939 the average annual expenditure for malaria control in the Republic of Panama has been about 5 per cent of its budget. Some drainage work has been done in at least 18 localities and in several of these towns the necessary ditches have been practically completed. Dr. Craw-

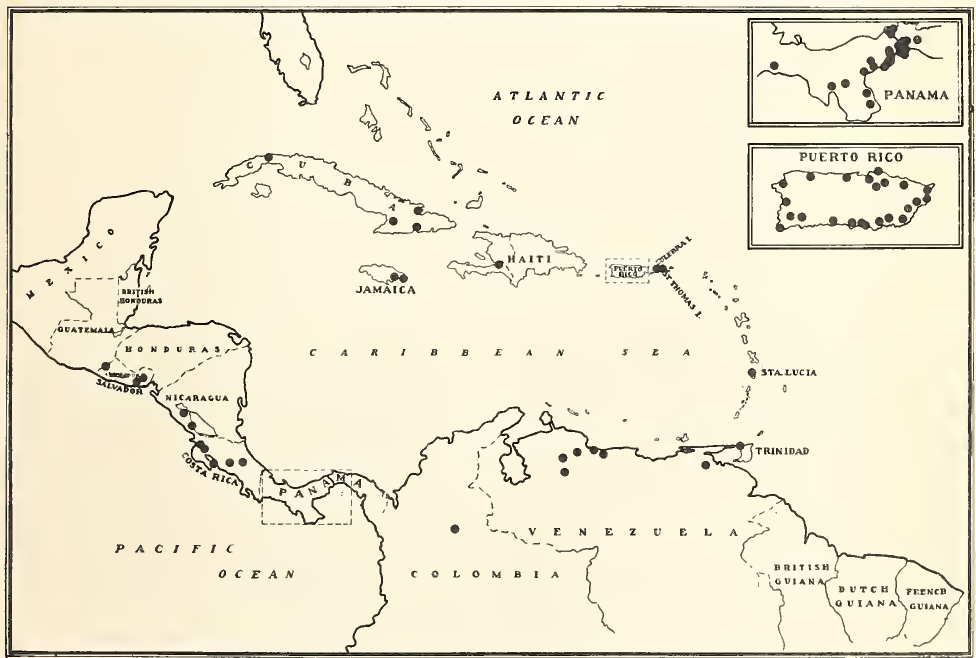

FIG. 10. Localities in the Caribbean area where drainage work for the control of malaria has been done in the past or is still in progress. 
ford reported that parasite and spleen. indices were definitely lowered in the towns of Antón, La Chorrera, Santiago, Chitré and especially in Soná.

The Republic of El Salvador has undertaken work of this type also, and during the four years from 1935 to 1938 inclusive, about 1 per cent of the total budget of the Health Department was spent for antimalaria drainage. This problem was attacked in three localities and more than 4000 inverts and 25,000 lengths of concrete pipe for subsoil drainage were manufactured.

Until May 1940 the Marianao area near Havana was the only control zone under the direction of the Malaria Commission of Cuba. At that time similar work was begun in Bayamo and Santiago in the Province of Oriente. The number of linear meters of ditches constructed each year in Marianao has varied from 1000 to 7000 , with an average annual figure of 5100. Dr Carr says that while larvicides were employed as temporary expedients, reliance was placed on permanent measures such as drainage and filling.

Gabaldon (1938, 1939a, b) mentions 6 places in Venezuela where projects for ma- laria control by drainage are either well advanced or planned for the immediate future. Up to the end of last year each of the drainage programs in the cities of Maracay and Puerto Cabello had cost more than $\$ 100,000$. The per capita cost of malaria control in Maracay up to the end of 1939 amounted to $\$ 3.36$, and in Puerto Cabello, to $\$ 5.13$. Dr. Gabaldon says that in Puerto Cabello the only malaria vector is $A$. albimanus. The average annual number of deaths from malaria in this eity in the interval 1926-36 was 66.5; for the interval 1937-39 after the beginning of drainage for malaria control the average was 15 .

\section{Summary}

1. Malaria ean be controlled in the Caribbean region, but not eradicated, in circumscribed areas by measures directed against the anopheline vectors.

2 . Wherever possible drainage is recommended, though there are places where screening and the temporary use of larvicides are necessary in addition.

3. Methods found successful in the Panama Canal Zone have proved effective in neighboring countries. 


\title{
THE ANTI-MALARIA PROGRAM IN NORTH AMERICA
}

\author{
By LOUIS L. WILLIAMS, Jr. \\ UNITED STATES PUBLIC HEALTH SERVICE, WASHINGTON, D. C.
}

Malaria is the disease of greatest economic importance in the southern portion of the United States and in the lowlands and river valleys of Mexico, Central America, and the West Indies. The temperate climate of the northern United States with its short transmission season, makes this our only republic in which the natural processes of settlement could, and did, automatically remove malaria from a large portion of the richest section of the country. Drainage caused the major part of this recession; residual malaria left the area because of the screening of homes (Maps 1 and 2).

\section{MÅLARIOUS AREA OF THE UNITED STATES}

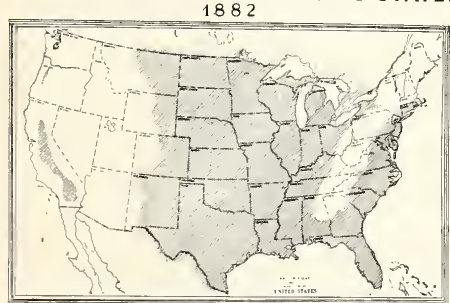

MAP 1. Probable endemic area.

1 IALARIOUS AREA OF THE UNITED STATES

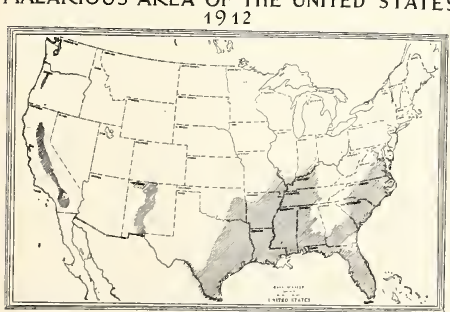

MaP 2. Endemic area.
Although the growth of malaria control in the southern states increased rapidly between its inception in 1912 and the present time, the malarious territory did not decrease commensurately. The infected territory contracted, expanded and contracted, each time defining a smaller area until 1932, when it reached what is probably its minimal endemic section. Since then, although less in intensity and more scattered in its endemic area, it is still found in the same portions of the same states as in 1912 (Maps 1912, 1932, and $1934-35)$.

In tropical America today, with its long

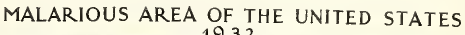
1932

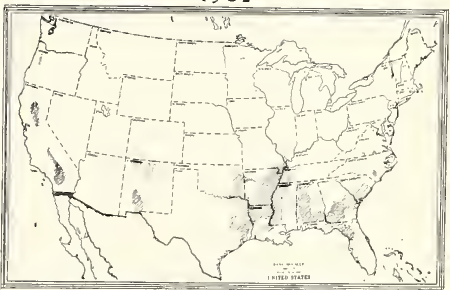

MAP 3. Endemic area.

MALARIOUS AREA OF THE UNITED STAIES $1934 \cdot 5$

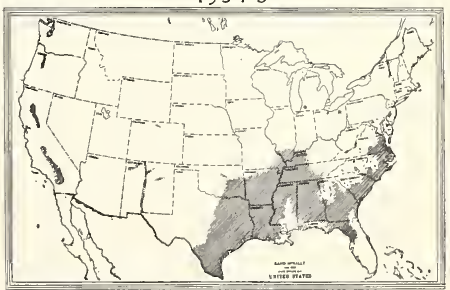

MAP 4. Endemic area. 
transmission season, malaria covers the approximate area it covered a century ago. There is no record of its natural recession from infected territory. On the contrary, the extension of industry, with its very low wage-scale has produced in some places a population of high density, inadequately fed and housed and poorly protected against disease. A'griculture in the tropies, in developing irrigation, has added greatly to the malarial burden, for control has been confined to the larger cities and to a few of the industrial settlements and to a very few of the larger agricultural projects.

From Mexico to Panama and in the islands the intensity of malaria varies with the altitudes, with the vector species and with the density of the population. On the coastal plains it tends to be of moderate endemic intensity with frequent epidemies, though in portions of Mexico and British Honduras high endemic rates have been found. In the highlands, it is moderately endemic with scattered epidemics up to elevations of five thousand feet and in the very high mountain valleys it tends to be a low grade endemic with severe epidemics during the occasional very hot summers. It is hyperendemic in many of the interior valleys at low elevations, particularly in southern Mexico. In the United States, it is of moderate endemic intensity in the sixteen southeastern states, with brief periods of high endemicity accompanied by scattered epidemies at intervals of approximately seven years, as shown in Chart I. This chart is a schematic representation of the estimated number of cases each year from

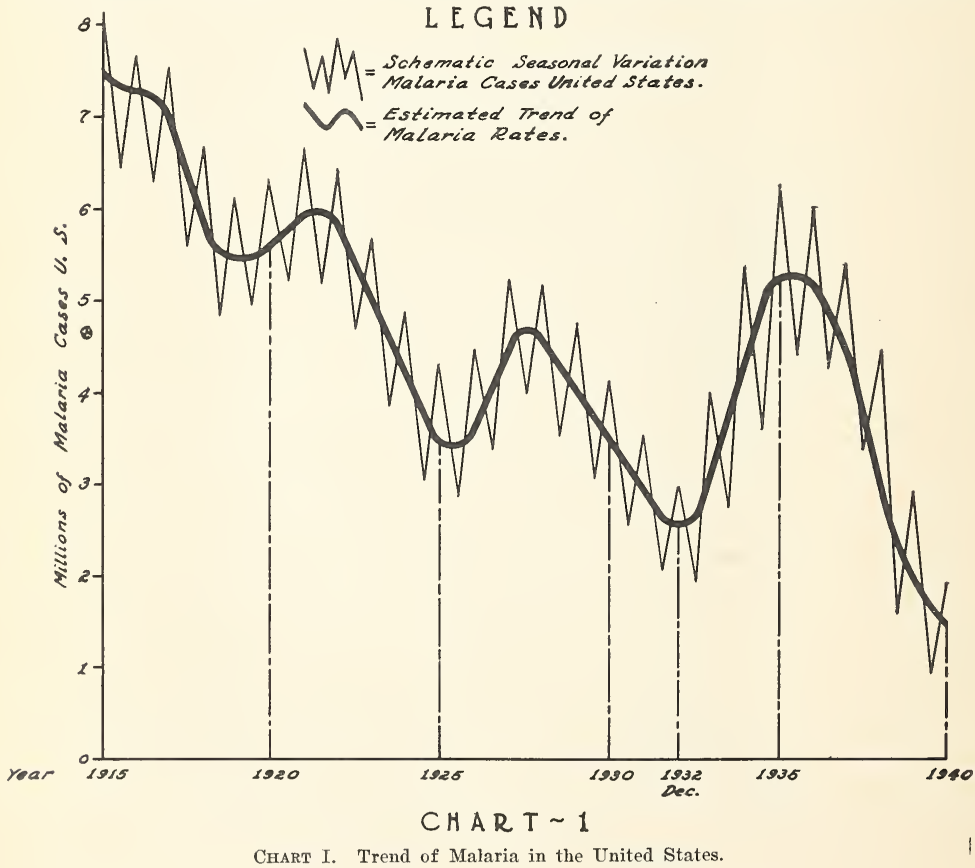


1914 through 1940 . The heavy line illustrates the cyclic waves of malaria. As the rate declines the area of infection shrinks. During the sudden subsequent rise there is an increase in severity of the disease, the appearance of epidemics, and a re-invasion of the territory which may have been free of infection for a number of years. The distinct decline to its low points in 1932 and 1940 is the result of drainage, larvicide application, screening, better housing, and control of impounded waters. During each half decade of declining rates it is probable that mass immunity falls, thus leaving a population ready for subsequent peaks. The steady decline between 1914 and 1932 probably allowed this immunity to reach its lowest ebb and may be the reason for the prompt rise during the summers of 1934 and 1935. The intensity of malaria has steadily fallen since the peak of 1935 and is still falling. The next peak is due during the summer of 1941 or 1942.

Apparently the cycle in our tropies is of shorter duration. It is approximately two years in the Chagres River Valley of Panama (Clark et al. 1940). When the cycle is worked out in all our other republies, their health administrations could be prepared for epidemic peaks and could attack them as the British have done in the Punjab, though the British predict their epidemics not on periodic occurrence, but from the previous flood season (Gill 1938b). Foci of infection should be located and as many as possible eliminated through anopheline control during the periods of falling rates; treatment facilities should be provided for epidemic control, when the cycle is due for a peak.

In our tropical countries morbidity and mortality records of malaria are incomplete and inaccurate, so an estimate of the number of infections is impossible. In the United States mortality records are of some value. In a few states morbidity records are accurate enough to indicate relative intensities. From the deaths and known cases in the United States, and from an index of 130,000 school children (Williams 1935) it is estimated that the number of infections annually varies from one to six million; the average is believed to be four million cases (Williams 1938).

Although it has been frequently stated that the infection is widespread, it is not possible to calculate the cost of malaria in Central America or the West Indies. Such estimates of cost have been made for the United States; first, in 1917 the annual loss in southern industry and agriculture was considered by H. R. Carter (1919) to be over $\$ 100,000,000$ annually and 20 years later (1937) the yearly cost of sickness was estimated by Williams (1938) to be about $\$ 51,000,000$ and the economic loss at $\$ 500$,000,000 . Bearing in mind the high cost of malaria, the expenditure by governments of millions for control seems reasonable.

Nevertheless active suppressive measures did not commence in the United States until 1912 and did not become a recognized part of State health work until about the time of the first World War. Its growth was slow as the cost of drainage seemed high to the people living in infected territory. The extended use of oil and screening and the introduction of Paris green reduced the cost so that some control measures at last became possible for nearly all people.

The program for control in the United States received a great stimulation during the time of the first World War in connection with extra-cantonment sanitation. During this emergency period the Government financed some rural work for camp protection which was so expensive that the localities, unaided, would not have attempted it. It drew attention to the necessity for expanding the research program to find cheaper methods. In addition to stimulating research, the War work brought about the development of some form of malarial control units in each of the southern states and gave a great impetus to the formation of county health units through which control could be further extended. More recently the Relief Administration, during the depression, created malaria control projects to give employment to hundreds of thousands of men. Under this 
program a daily average of 211,000 men for 6.5 years have worked on anti-malaria drainage in an average of 250 counties. They have dug 33,655 miles of ditch, removing 544,414 acres of anopheline breeding surface in the sixteen southeastern states.

Today our defense program is creating many new camps in areas where malaria control is necessary. Two large projects to protect 4 camps and their maneuver area (costing nearly $\$ 1,000,000$ ) have already been started. Several others of similar scope will be necessary as most of the larger training camps are located in the malarious southern states. Smaller projects will have to be undertaken at many flying fields. As the range of the soldier, on pass, has increased from the 5 miles of the World War to 50 miles, malaria control will have to be affected in much greater areas.

In Mexico, Central America, and the West Indies there has been no such stimulation either for research or for extension of control measures. Nevertheless in cooperation with the Rockefeller Foundation and through fruit and oil companies, farreaching malaria control projects have been completed and others are under way in 8 of the Caribbean countries of North America.

The United States Public Health Service has worked out a Plan for State-Wide Malaria Control (Williams 1937b) which has four objectives: elimination of the major foci of infection in each county, prevention of man-made malaria, maintenance of existing and new control projects, and an educational campaign.

The state boards of health press on continuously toward these four objectives through a malaria control and investigational unit within their own organization. This unit, in most of the southern states, is composed of a medical malariologist for epidemiological work, a sanitary engineer to plan for drainage, larvicides, and screening, an entomologist to make anopheline surveys and one or more technicians to examine thick blood films. These last are attached to the state laboratory.
The chief of the malarial unit chooses those counties considered most malarious for the first work. As early as possible in the autumn, thick film blood smears are taken from the 6 to 12-year age group of all school children in the rural areas and small villages. The slides go to the state laboratory for examination. The epidemiologist spots all positives by homes, thus revealing the foci of heaviest infection. The entomologist makes a complete anopheline survey of each focus. Based on this information, the sanitary engineer draws up a detailed plan of attack with an accurate estimate of cost. The chief of the unit determines whether the attack is to be by drainage, larvicide application, screening, or through treatment. This survey and plan are then laid before the authorities, to secure financing and operation of the control project.

To prevent man-made malaria, the malarial unit of each state makes contact with the builders of dams and insists on malaria control wherever waters are to be impounded. It cooperates with the highway department and the railroads traversing the state, to secure elimination of existing borrow pits and prevention of the formation of new ones. It shows the companies engaged in lumbering the swamps, the malaria hazard they are creating and induces them to construct an outlet channel to all swamps where lumbering operations are in progress.

As we have found that earth ditches are too expensive to maintain, units now urge that permanent lining be an integral part of anti-malaria drainage. We have learned from the work in Panama, Salvador, Nicaragua, and from our own field experiments, that concrete inverts and bank sodding make a more permanent ditch requiring a minimum of maintenance. Ditch lining is now included in most of the anti-malaria drainage projects in the United States and of the other republies.

The educational activities of the malarial unit include revision of the State Board of Health educational bulletins to keep them up to date, distribution of these bulletins to 
schools and universities through boards of education, and frequent lectures throughout the malarious portions of the state. The malarial unit also teachers the district and county health organizations the best methods of malaria control.

In all of these state activities the U. S. Public Health Service has cooperated and has even secured inter-departmental cooperation in federal activities. It has arranged with the Division of Soil Erosion Control and with the Rehabilitation Administration to supply all of their field workers with necessary instructions whereby malaria control may be secured at soil erosion control dams and recreational ponds. The Service protects our seaports from introduction, by airplane, of new anopheline species by requiring that all planes from other countries accomplish disinsectization at the last port of call before entry. Through the Tennessee Valley Authority the government is developing one entire drainage basin with malaria control built into the original plans.

In Mexico the state governments are becoming stronger and, responding to efforts of the central administration, are building up their own control work, but as yet have no fully organized units. Cuba and Puerto Rico, in cooperation with the Rockefeller Foundation, have a well-rounded plan similar to that of the United States. During the few years that Haiti was under the administration of the United States Navy, study and control of anophelines commenced at Port au Prince and other towns.

In the smaller republies and islands the whole program of control is under the central governments. Research and control units have been established in cooperation with the Rockefeller Foundation, but, as yet, are understaffed. They have made notable studies of their anopheline fauna. They attempt to control epidemic death rates by treatment and they practice anopheline control in some population centers where malaria is especially severe. No rural work is done except by the oil and fruit companies, for as yet no republic-wide or island-wide plan has been adopted. The
U. S. Public Health Service plan, which has become established in our southern states, may be adapted easily to any other republic. Our state boards of health, which have grown phenomenally in the last 23 years through the establishment of hundreds of county health departments and the strengthening of the special divisions of the central administrations, have demonstrated the efficacy of, mobile well-trained malaria units.

Every republic of North America is engaged in some research on malaria. Since Brazil is now suffering from an invasion of $A$. gambiae, we should amplify our study of airplane disinsectization, for North America can well do without this highly androphylic species. We need ironclad legislation for control of new and established impoundments in the eastern half of the continent and for better planning and supervision of irrigation projects in the western and tropical part.

In the field of chemotherapy, we need to develop a true causal prophylactic so that people may visit or even live in an area where anopheline control is, as yet, impossible. We need to discover a drug which not only cures the clinical attack but sterilizes the patient and so prevents chronic malaria and suppresses gametocyte production, thus reducing the sporozoite rate in the mosquito. For this purpose our researches should open up the almost unknown field of the exo-erythrocytic phase of the plasmodium in the human host.

For testing such chemicals, sporozoite malaria is necessary. In experimental work there is a great paucity of sporozoite-induced malaria. Blood-inoculation malaria is the favorite as it is easy to maintain and tends to produce a standardized type of infection, whereas sporozoite-induced malaria, both in man and birds is variable in intensity and uncertain of duration. The individualistic character of sporozoite infection should not be an irritation but a very desirable characteristic, as this malaria most nearly simulates natural infection. The average laboratory worker finds it more difficult to use and the results less 
clean-cut and so tends to discard it. However, sporozoite malaria should be used in all final tests, whether or not blood-inoculation malaria also is used.

In the field of immunology we are working to develop a serum and a vaccine.

In the field of bionomies and ecology new studies are underway seeking to solve the numerous problems of the botanical, hydrological, and physiological interdependencies which determine mosquito production. The solution of these problems may lead to the development of new larvicides, cheaper methods of distributing them and control of waters with the least disturbance. Study of the adult mosquito as to its feeding, flight, oviposition habits, and, most particularly as to its micro-habitat may lead to the discovery of other and simpler methods of preventing malaria transmission. Through these investigations in the bionomics and ecology of anophelines we may well achieve the ideal of the malariologist, biological control. 


\section{REFERENCES CITED ${ }^{1}$}

Abercrombie, T. F. 1940. State of Georgia Department of Public Health. Annual Report for the Year 1939. Atlanta.

Abrami, P. and Senevet, G. 1919. Pathogénie de l'accés Palustre. La Crise Hemoclasique Initiale. Bull. Soc. Med. Hôp, Paris, 43: 530, 537.

ACton, H. W. and Chopra, R. N. 1927. The Action of Quinine on the Malarial Parasites. Trans. 7th Congress F. E. A. T. M. (Calcutta), 2: 894. Cited from Boyd and Dunn 1939.

Aguilar, S. G. 1931. Claves Para Identificar Mosquitoes en El Salvador. Tesis de Doctorado en Medicina. San Salvador, El Salvador.

Aitken, T. H. G. 1939. The Anopheles maculipennis Complex of Western America. Pan-Pacif. Ent., 15: 192.

1941. Studies on the Anopheline Complex in Western America. In Press.

Alabama State Board of Health. See Regulations of Alabama State Board of Health.

AlleN, G. P. F. 1926. Some Post-Mortem Observations on Twenty-six Cases of Subtertian Malaria. Trans. Roy. Soc. trop. Med. Hyg., 20: 119.

ANayan, S. A. 1929. Sur la Biologie d'Anopheles pseudopictus Grassi en 1'oneze d'Etchmiadsine en Armenie et sur la Lutte Conduite Contre les Mostiques Hibernants. Russ. J. trop. Med., $7: 425$.

ANDREWS, J. M. 1938. Geographical distribution by Counties of Reported Malaria Deaths in Georgia (1928-37). Georgia Mal. Bull., 1: 26.

Anon. 1832-33. Medical Topographical Survey of the State. Trans. Med. Soc. St. N. Y., 1: 41 . App.

Anon. 1861. St. Louis med. surg. J., 19: 491.

Anon. 1940. Malaria and Drainage Ditches Combined Effectively. Amer. City, 62: 74.

ANTIC, D. 1925. Immunität der Malariker Gegen Superinfektion. Zbl. Bakt., Orig., 94: 134.

ANTUNeS, P. C. A. 1937. Informe Sobre Una Investigacion Entomologica Realizada en Colombia. Rev. Fac. Med., 6: 3 .

ARĨGAO, H. DE B. 1930. Evolution of the Gametes of Plasmodium falciparum (Preliminary Note). Mem. Inst. Osw. Cruz., 24: 41.

Ascногғ, L. 1924. Das Reticulo-endotheliale System. Ergeb. inn. Med. Kinderheilk., 26: 1.

AYroza, Galvão, A. L. 1938. Sobre a infeç̧ão experimental do Anopheles strodei pelo Plasmodium vivax. Rev. Biol. Hyg., S. Paulo, 9: 133. -1940. Contribuicão ao Conhecimento dos Anofelinos do Grupo Nyssorhynchus de São Paulo e Regiões Vizinhas. Arq. de Zool. do Estado de S. Paulo (Tomo 24 de Rev. Mus. paul.) 1: 399 .

1 Abbreviations of names of periodicals are those used in "A World List of Scientific Periodicals," second edition, 1934.
Atroza Galvão, A. L. and Barretto, M. P. 1938. Contribuicão ao Conhecimento dos Primeiros Estadios dos Anofelinos de São Paulo. Rev. Biol. Hyg., S. Paulo, 9: 110.

AzIz, M. 1939. The Water-Paris Green Mixture in Anopheline Control of the Tillaria Area. Cyprus. Med. Dept. Papers No. V. Printing Office Gov't. of Cyprus, Nicosia.

Balfour, M. C. 1928. Studies on the Bionomics of North American Anophelines. Winter Activities of Anophelines in Coastal North Carolina (36 N. Lat.). Amer. J. Hyg., 8: 68.

1936. Some Features of Malaria in Greece and Experience with its Control. Riv. Malariol., 15: 114.

Bang, F. G., Quinby, G. E. and Simpson, T. W. 1940. Anopheles walkeri (Theobald): A Wildeaught Specimen Harboring Malaria Plasmodia. Publ. Hith. Rep., Wash., 55: 119.

Barber, M. A. 1925. Some Further Experiences with Paris Green as a Larvicide. Compte-rendu du Premier Congrès International du Paludisme, Rome, Oct. 1925. p. 153. Publié par le Sécretaire général du Congrès, 1926.

1927. The Food of Anopheline Larvae -Food Organisms in Pure Culture. Publ. Hlth. Rep., Wash., 42 : 1494.

- 1936a. A Survey of Malaria in Cyprus. Amer. J. trop. Med., 16: 431.

1936b. Degeneration of Sporozoites of the Malaria Parasite in Anopheline Mosquitoes in Nature and in Its Relation to the Transmission of Malaria. Amer. J. Hyg., 24: 45.

- 1939. Further Observations (1938) on the Anopheles of New Mexico. Amer. J. trop. Med., 19: 345 .

. 1940. The Present Status of Anopheles gambiae in Brazil. Amer. J. trop. Med., 20: 249.

BARbEr, M. A. and Forbrich, L. R. 1933. Malaria in the Irrigated Regions of New Mexico. Publ. Hlth. Rep., Wash., 48: 610.

BARBER, M. A. and HAYNE, T. B. 1921. Arsenic as a Larvicide for Anopheline Larvae. Publ. Hlth. Rep., Wash., 36: 3027.

- 1924. Some Observations on the Dispersal of Adult Anopheles. Publ. Hlth. Rep., Wash., 39: 195.

BArber, M. A. and Komp, W. H. W. 1927. Some Observations in the Limon and Panama Divisions of the United Fruit Company, with Special Reference to Certain Measures for the Control of Malaria. 16th Ann. Rep. United Fruit Co. Med. Dept., p. 54.

- 1929a. Season and Regional Ineidence of Types of Malaria Parasites. Publ. Hlth. Rep., Wash., 44: 2048.

1929b. Method of Preparing and Examining Thick Blood Films for the Diagnosis of Malaria. Publ. Hlth. Rep., Wash., 44: 2330. 
1929c Breeding Places of Anopheles in the Yazoo-Mississippi Delta. Publ. Hlth. Rep., Wash., $44: 2457$.

BARBER, M. A., KOMP, W. H. W. and HAYNE, T. B. 1924. Some Observations on the Winter Activities of Anopheles in Southern United States. Publ. Hlth. Rep., Wash., 39: 231.

- 1927. The Susceptibility to Malarial Parasites and the Relation to the Transmission of Malaria of the Species of Anopheles Common in the Southern United States. Publ. Hlth. Rep., Wash., 42: 2487.

Barber, M. A., Komp, W. H. W. and King, C. H. 1929. Malaria and the Malaria Danger in Certain Irrigated Regions of the Southwestern United States. Publ. Hlth. Rep., Wash., 44: 1300.

BARBER, M. A., KoMP, W. H. W. and NeWMAN, B. M. 1929a. Observations and Experiments in the Panama Division of the United Fruit Company, with Special Reference to Certain Measures for the Control of Malaria. 18th Ann. Rep. United Fruit Co. Med. Dept., p. 34.

- 1929b. Effect of Small Doses of Plasmochin on Viability of Gametocytes of Malaria as Measured by Mosquito Infection Experiments. Publ. Hith. Rep., Wash., 44 : 1409.

Barber, M. A. and Olinger, M. T. 1931. Studies on Malaria in Southern Nigeria. Ann. trop. Med. Parasit., 25: 461.

BARBER, M. A. and Rice, J. B. 1935. The Malaria Infection Rate in Nature and in the Laboratory of Certain Species of Anopheles of East Macedonia. Ann. trop. Med. Parasit., 29: 329.

Barber, M. A., Rice, J. B. and Mandekos, A. 1936. A Dustless Method of Diluting and Spreading Paris Green in Malaria Control. Amer. J. Hyg., 24 : 41.

BARKER, L. F. 1895. A Study of Some Fatal Cases of Malaria. Johns Hopk. Hosp. Rep., 5: 219.

BARnes, M. E. 1925. The Toxic Effect of Oil Films upon Mosquito Larvae with Particular Reference to Pine Oil Film. Amer. J. Hyg., 5: 315.

BarratT, J. O. W. and Yorke, W. 1909. An Investigation into the Mechanism of Production of Blackwater. Ann. trop. Med. Parasit., 3: 1.

—. 1 1914. The Production of General Symptoms in Hemoglobinuria. Brit. med. J., 1: 235.

BArRETo, J. DE B. 1929. Do Espurgo por Nebulisacāo de Insecticidas na Lueta Contra a Malaria. Sci. Med., 7 : 527.

Barretto, M. P. 1938. Observações Sobre a Ecologia do $A$. darlingi Root 1926 var. paulistensis Galvão, Lane and Correia 1937. Rev. Biol. Hyg., S. Paulo, 9: 116.

Barrowman, B. 1936. Notes on a Demonstration of Malaria Control. Malay. med. J., 11: 2 .

Bass, C. C. 1912. Successful Cultivation of Malarial Plasmodia. J. Amer. med. Ass., 59: 936.

\footnotetext{
1915. A Method for Coneentrating
}

Malaria Plasmodia for Diagnostic and Other Purposes. Amer. J. trop. Dis., 3: 298.

1919. Studies in Malaria Control. VIII. Some Observations Indicating that Effective Immunity Against Malaria Parasite Infection Does Not Occur. Sth. Med. J., 12: 465.

- 1920. Studies on Malaria Control. XI: Control of Malaria by Quinine Sterilization of the Human Host. Sth. Med. J., 13: 250.

Bass, C. C. and JoHns, F. M. 1912. The Cultivation of Malarial Plasmodia (Plasmodium vivax and Plasmodium falciparum) in vitro. J. prev. Med., Baltimore, 16 : 567.

Bastedo, W. A. 1937. Materia Medica, Pharmacology and Therapeutics. Ed. IV., p. 483 . W. B. Saunders Co., Philadelphia.

Bates, J. P. 1913. A Review of a Clinical Study of Malaria Fever in Panama. J. trop. Med. (Hyg.), 14: 145.

Bates, M. 1940. The Nomenclature and Taxonomic Status of the Mosquitoes of the Anopheles maculipennis Complex. Ann. ent. Soc. Amer., $33: 343$.

BaTH, C. H. 1931. The Practical and Research Value of Mosquito Traps. Amer. J. trop. Med., 11: 147 .

Beach, Ted de Vinne. 1936. Evidence of Binary Fission of Ring Forms in Plasmodium vivax Grassi and Feletti. Amer. J. trop. Med., 16: 147.

Beattie, Mary V. F. 1932. The Physico-Chemical Factors of Water in Relation to Mosquito Breeding in Trindad. Bull. ent. Res., 23: 477.

BeaUperthay, L. D. 1854. Transmission of Yellow Fever and Other Diseases by Mosquitoes. Gaz. oficial de Cumaná, año 4, no. 57, May 23. Reproduced in R. Boyce. 1909. Mosquito or Man, p. 98. J. Murray, London.

BEck, M. 1911. Diagnosen der Schlafkrankheit. Arb. GesundhAmt., Berl., 31: 59.

Beeson, P. B. and Hoagland, C. L. 1940. The Use of Calcium Chloride in the Treatment of Chills. N. Y. St. J. Med., 40: 803.

Bella my, R. E. 1939. An Anopheline from Inland Georgia Resembling the Brackish-Water Race of Anopheles crucians. Supplement to Symposium on Malaria, Nat. Mal. Comm., 1938. (mimeographed) Tallahassee, Fla. (Also in $J$. Parasit., 25: 186.)

Bellamy, R. E. and Andrews, J. 1938. On the Occurrence of Anopheles walkeri Theobald in Georgia. Sth. Med. J., 31: 797.

Bennaroch, E. I. 1928. Thesis Univ. Cent. Venezuela. Summ. Trop. Dis. Bull., 26: 366. 1931. Studies on Malaria in Venezuela. Amer. J. Hyg., 14: 690 .

Berkeley, W. N. 1901. Some Further Work on the Mosquito Malaria Theory, with Special Reference to Conditions Around New York. Med. Rec. N. Y., $59: 124$.

Beyer, G. E. 1923. A New Species of Anopheles in Louisiana. Anopheles atropos, D. and $\mathrm{K}$. Amer. J. trop. Med., 3: 351. 
Beyer, G. E., Pothier, O. L., Couret, M. and LEMANN, I. I. 1902a. Experimental Investigations with Malaria in Connection with the Mosquitoes in New Orleans. New Orleans Med. Surg. J., 54: 419.

1902b. Bionomies, Experimental Investigations with Bacillus Sanarelli and Experimental Investigations with Malaria in Connection with the Mosquitoes of New Orleans. New Orleans Med. Surg. J., 54: 463.

BIGGaM, A. G. 1929. Malignant Malaria Associated with the Administration of Heroin Intravenously. Trans. Roy. Soc. trop. Med. Hyg., 23: 147.

Bishopp, F. C., Cory, E. N. and Stone, A. 1933. Preliminary Results of a Mosquito Survey in the Chesapeake Bay Section. Proc. ent. soc. Wash., $35: 1$.

Black, J. B. 1940. The Accidental Transmission of Malaria Through Intravenous Injections of Neoarsphenamine. Amer. J. Hyg., 31: 37.

BLACKLOCK, D. B. 1939. Notes on Siphon Action, with Special Reference to Antimosquito Work. Ann. trop. Med. Parasit., 33: 141.

BLACKLOCK, D. B. and GoRDON, R. M. 1925. Malaria Parasites in the Placental Blood. Ann. trop. Med. Parasit., 19: 37.

Blacklock, B. and Macdonald, G. 1928. The Mechanism of Blackwater Fever and Certain Allied Conditions. Brit. med. J., 2: 145.

Blanton, W. B. 1931. Medicine in Virginia in the Eighteenth Century. W. Byrd Press, Richmond.

Blaze, J. R. and Simeons, A. T. W. 1935. Preliminary Observations on New Soluble Atebrin Compounds. Indian med. Gaz., 70: 185.

BLooM, W. 1938a. Lymphocytes and Monocytes: Theories of Hematopoiesis. In H. Downey. Handbook of Hematology, vol. 1, p. 373. P. B. Hoeber, New York.

- 1938b. Fibroblasts and Macrophages. In H. Downey. Handbook of Hematology, vol. 2, p. 1336 . P. B. Hoeber, New York.

Booker, C. G. 1935. Annual Report of the South African Railways and Harbours Health Organization 1934-35. Rep. Dept. Publ. Hlth. South Africa, 1934-35.

- 1936. Annual Report of the South African Railways and Harbours Health Organization 1935-36. Rep. Dept. Publ. Hith. South Africa, 1935-36.

BoRChaRDT, W. 1930. Utber die chemo-therapeutische Wirkung von Chinin bzw. Plasmochin in vitro auf. Proteosoma praecox (Vogel Malaria). Arch. Schiffs. TropenHyg., 34: 360.

Botsford, R. C. and TuRner, N. 1933. Mosquito Control in Connecticut, 1932. Bull. Conn. agric. Exp. Sta., 349: 439.

BoyD, G. H. 1929. Experimental Modification of the Reproductive Activity of Plasmodium cathe. merium. J. Exp. Zool., 54: 111.

Boyd, G. H. and Allen, C. H. 1934. Adult Size in Relation to Reproduction of the Avian Malaria
Parasite, Plasmodium cathemerium. Amer. J. Hyg., 20: 73.

Boyd, G. H. and DunN, M. 1939. Effects of Quinine and Plasmochin Administration upon Parasite Reproduction and Destruction in Avian Malaria. Amer. J. Hyg., C, 30: 1.

BoyD, M. F. 1926. Studies of the Epidemiology of Malaria in the Coastal Lowlands of Brazil. Amer. J. Hyg., Monograph Ser., 5: 102.

- 1927. Studies on the Bionomies of North American Anophelines. I. The Number of Annual Broods of A. quadrimaculatus. Amer. J. Hyg., $7: 264$.

1929a. Malaria Survey of the Island of Jamaica, B. W. I. Amer. J. trop. Med., 9: 309 .

- 1929b. Studies on the Bionomies of North American Anophelines: Physical and Chemical Factors in their Relation to the Distribution of Larvae in Northeastern North Carolina. Amer. J. Hyg., $9: 346$.

1930a. Introduction to Malariology. Harvard Univ. Press, Cambridge.

- $1930 \mathrm{~b}$. Introduction to Malariology. Pp. 34-48, 147-174. Harvard Univ. Press, Cambridge.

1930c. Introduction to Malariology. P. 438. Harvard Univ. Press, Cambridge.

1930d. Studies on the Bionomics of North American Anophelines. VI. Some Observations on Imagines. Amer. J. Hyg., 12: 449.

- 1930e. The Cage Rearing of Anopheles quadrimaculatus. Amer. J. trop. Med., 10: 165. -1932. A Note on the Preparation of Anopheline Dissections for Examination. Amer. J. Hyg., 16: 836.

- 1934. Observations on Naturally Induced Malaria. Sth. Med. J., 27: 155.

-1935a. The Comparative Morphology of the Sporozoites of the Human Species of Plasmodium, Marchiafava and Celli, 1885. $J$. Parasit., 21: 255.

1935b. On the Schizogonous Cycle of Plasmodium vivax Grassi and Feletti. Amer. J. trop. Med., 15 (6): 605.

1937. On the Susceptibility of Anopheles quadrimaculatus to Plasmodium vivax after Prolonged Insectary Cultivation. Amer. $J$. trop. Med., 19: 593 .

- 1938. The Threshold of Parasite Density in Relation to Clinical Activity in Primary Infections with $P$. vivax. Amer. J. trop. Med., 18: 497.

1939. Malaria: Retrospect and Prospect. Amer. J. trop. Med., 19: 1 .

- 1940a. On Strains or Races of the Malaria Parasites. Amer. J. trop. Med., 20: 69. 1940b. Observations on Naturally and Artificially Induced Quartan Malaria. Amer. $J$. trop. Med., 20: 749 .

1940c. On the Correlation Between the Incidence of Stomach and Gland Infection in 
Anopheles quadrimaculatus Infected with Plasmodium vivax. Amer. J. trop. Med., 20: 129.

- 1940d. Some Characteristies of Artificially Induced Malaria. Amer. J. trop. Med., 20: 269.

- 1940e. The Influence of Sporozoite Dosage in Vivax Malaria. Amer. J. trop. Med., $20: 279$.

ㄴ. 1940f. Personal communication.

-1940g. Further Observations on the Comparative Suseeptibility of Nearetic and Neotropical Anophelines to Coindigenous Strains of Plasmodium falciparum. Amer. J. trop. Med., 20: 423 .

BoYD, M. F. and ARIs, F. W. 1929. A Malaria Survey of the Island of Jamaica, B. W. I. Amer. J. trop. Med., 9: 309 .

Boyd, M. F., CARR, H. P. and Rozeboom, L. E. 1938. On the Comparative Susceptibility of Certain Species of Nearetic and Neotropical Anophelines to Certain Strains of $P$. vivax and $P$. falciparum from the same Regions. Amer. J. trop. Med., 18: 157.

Boyd, M. F. and Earle, W. C. 1939. On the Susceptibility of a Neotropical Anopheles pseudopunctipennis Theobald, 1901, to Nearetic and Neotropical Strains of Plasmodium falciparum. Amer. J. trop. Med., 19: 405.

Boyd, M. F. and Foote, Helen. 1928. Studies on the Bionomics of American Anophelines: The Alimentation of Anopheline Larvae and Its Relation to Their Distribution in Nature. J. Prev. Med., 2: 219.

BoxD, M. F. and JobBins, D. M. 1940. Further Observations on the Comparative Susceptibility of Nearctic and Neotropical Anophelines to $\mathrm{Co}$ indigenous Strains of Plasmodium falciparum. Amer. J. trop. Med., 20: 423.

Boyd, M. F. and KrTCHeN, S. F. 1936a. The Comparative Susceptibility of Anopheles quadrimaculatus Say and Anopheles punctipennis Say, to Plasmodium vivax, Grassi and Feletti, and Plasmodium falciparum, Welch. Amer. J. trop. Med., 16: 67 .

-1936b. Is the Acquired Homologous Immunity to $P$. vivax Equally Effective Against Sporozoites and Trophozoites 9 Amer. J. trop. Med., 16: 317.

-1936c. On the Efficiency of the Homologous Properties of Acquired Immunity to $P$. vivax. Amer. J. trop. Med., 16: 447.

1937a. Observations on Induced Faleiparum Malaria. Amer. J. trop. Med., 17: 213.

1937b. A Further Note on the Infectiousness of Anopheline Mosquitoes Infected with P. vivax and P. falciparum. Amer. J.trop. Med., $17: 245$.

- 1937e. A Consideration of the Duration of the Intrinsic Incubation Period in Vivax Malaria in Relation to Certain Factors Affecting the Parasites. Amer. J. trop. Med., 17: 437.

- $1937 \mathrm{~d}$. Recurring Clinical Activity in
Infections with the MeCoy strain of Plasmodium vivax. Amer. J. trop. Med., 17: 833.

- 1937e. The Duration of the Intrinsic Incubation Period in Falciparum Malaria in Relation to Certain Factors Affecting the Parasites. Amer. J. trop. Med., 17: 845 .

—. 1937f. Simultaneous Inoculation with Plasmodium vivax and Plasmodium falciparum. Amer. J. trop. Med., 17: 855.

- 1938a. Vernal Vivax Activity in Persons Simultaneously Inoculated with $P$. vivax and P. falciparum. Amer. J. trop. Med., 18: 505.

1938b. Demonstrable Maturity of Gametocytes as a Factor in the Infection of Anopheles with Plasmodium vivax and Plasmodium falciparum. Amer. J. trop. Med., 18: 515.

1939. The Demonstration of Sporozoites in Human Tissues. Amer. J. trop. Med., 19: 27.

Boyd, M. F., KITchen, S. F. and KuPPER, W. H. 1937. The Employment of Multiply Infected A. quadrimaculatus to Effect Inoculation with Plasmodium vivax and $P$. falciparum. Amer. $J$. trop. Med., 17: 849.

Boyd, M. F., Kitchen, S. F. and MUENCH, H. 1936. Seasonal Variations in the Characteristics of Vivax Malaria. Amer. J. trop. Med., 16: 589.

Boyd, M. F., Kitchen, S. F. and Mulrennnan, J. A. 1936. On the Relative Susceptibility of the Inland and Coastal Varieties of $A$. crucians, Wied to $P$. falciparum, Welch. Amer. J. trop. Med., 16: 159.

Boyd, M. F. and MaTrhews, C. B. 1939. An Observation on the Incubation Period of $P$. falciparum. Amer. J. trop. Med., 19: 69.

Boyd, M. F. and Ponton, G. 1933. The Recent Distribution of Malaria in the Southeastern United States. Amer. J. trop. Med., 13: 143.

Boyd, M. F. and Proske, H. O. 1941. Observations on the Blood Proteins During Malaria Infections. Amer. J. trop. Med., 21: 245.

Boyd, M. F. and Stratman-Thomas, W. K. 1933a. A Controlled Technique for the Employment of Naturally Induced Malaria in the Therapy of Paresis. Amer. J. Hyg., 17 : 37.

- 1933b. A Note on the Transmission of Quartan Malaria by Anopheles quadrimaculatus. Amer. J. trop. Med., 13: 265.

-1933c. Studies on Benign Tertian Malaria. 1. On the Occurrence of Acquired Tolerance to Plasmodium vivax. Amer. J. Hyg., 17: 55.

laria. 1933d. Studies on Benign Tertian MaInoculation with $P$. vivax. Amer. J. Hyg., 18: 485.

- 1934a. On the Duration of Infectiousness in Anophelines Harboring Plasmodium vivax. Amer. J. Hyg., 19 : 539.

- 1934b. The Comparative Susceptibility of A. quadrimaculatus, Say, and A. crucians, 
Wied (inland variety) to the Parasites of Human Malaria. Amer. J. Hyg., 20: 247.

- 1934c. Studies on Benign Tertian Malaria. 7. Some Observations on Inoculation and Onset. Amer. J. Hyg., 20: 488.

- 1936. The Transmission of Quartan Malaria Through Two Consecutive HumanAnopheline Passages. Amer. J. trop. Med., 16: 63.

Boyd, M. F.. Stratman-Thomas, W. K. and Kitchen, S. F. 1935. On the Relative Susceptibility of Anopheles quadrimaculatus to Plasmodium vivax and Plasmodium falciparum. Amer. J. trop. Med.. 15: 485.

1936a. On Acquired Immunity to Plasmodium falciparum. Amer. J. trop. Med., 16: 139.

1936b. On the Duration of Infectiousness in Anophelines Harboring Plasmodium falciparum. Amer. J. trop. Med., 16: 157.

- 1936c. On the Duration of Acquired Homologous Immunity to Plasmodium vivax. Amer. J. trop. Med., 16: 311.

Bradley, G. H. 1926. Observations on the Emergence of Anopheles Mosquitoes. Amer. J. trop. Med., 6: 283.

- 1932. Some Factors Associated with the Breeding of Anopheles Mosquitoes. J. agric. Res., 44: 381.

-1936. On the Occurrence of Anopheles walkeri Theobald in Florida (Diptera, Culicidae). Sth. Med. J., $29: 857$.

Bradlex, G. H. and McNeel, T. E. 1935. Mosquito Collections in Florida with the New Jersey Light Trap. J. econ. Ent., 28: 780 .

Branch, GEORge C. 1940. Therapeutic Quartan Malaria in the Treatment of Neurosyphilis among Negroes. Amer. J. Psychiat., 96: 967.

Breind, V. and Jirovec, O. 1932. Einige Bemerkungen ueber die Nuklealreaktion bei Lavernia malariae und Proteosoma praecox. Zbl. Bakt., Orig., 126: 184.

Brown, H. 1940. The Problem of Malaria Mortality. Amer. J. publ. Hlth., 30: 1199.

Brown, J. Y. 1934. Safe Mosquito Nets for Use in Nigeria. W. Afr. med. J., 7: 147.

Brown, W. H. 1911a. Malarial Pigment (SoCalled Melanin): Its Nature and Mode of Production. J. exp. Med., 13: 290.

. 1911b. The Relation of Hematin to Pathological Pigment Formation. J. exp. Med., 14: 612 .

1912. Malarial Pigment (Hematin) as a Factor in the Production of the Maiarial Paroxysm. J. exp. Med., 15: 579.

- 1913a. The Renal Complications of Hematin Intoxication and Their Relation to Malaria. Arch. intern. Med., 12: 315.

- 1913b. Malaria Pigment (Hematin) as an Active Factor in the Production of the Blood Picture of Malaria. J. exp. Med., 18: 96.

Brown, W. H. and LoevenharT, A. S. 1913. The
Effect of Hematin on the Circulation and Respiration. J. exp. Med., 18: 107.

Bruetsch, W. L. 1927. Ein Beitrag zur Wirkungsweise der Impfmalaria auf den Histopathologisehen Prozess bei Progressiver Paralyse. Z. ges. Neurol. Psychiat., 110: 713.

- 1932a. The Histopathology of Therapeutie (Tertian) Malaria. Amer. J. Psychiat., 12: 19.

1932b. Activation of the Mesenchyme with Therapeutic Mälaria. J. nerv. ment. Dis., 78: 209.

Bull, C. G. and Reynolds, B. D. 1924. Preferential Feeding Experiments with Anopheline Mosquitoes II. Amer. J. Hyg., 4: 109.

Bull, C. G. and Root, F. M. 1923. Preferential Feeding Experiments with Anopheline Mosquitoes I. Amer. J. Hyg., 3: 514.

BUNKer, H. A., JR. 1926. Serial Hourly Leueoeyte Counts in Tertian Malaria. Amer. J. med. Sci., 172: 681.

Bunker, W. O. and HirschFelder, A. D. 1925. Mosquito Repellents. Amer. J. trop. Med., 5: 359.

Bustamente, M. E. 1939. Epidemiologia del Paludismo en el sur Del Valle de Mexico, Zona de Xochimilco. Gaz. Med. Mexico, 49: 1.

Buts, D. C. A. 1937. Malaria in Camden County, N. J. Report of a Recent Outbreak. Amer. J. trop. Med., 17: 279.

California State Department of Health. 1940. The Present Status of Malaria in California. Wkly. Bull. Calif. Bd. Hlth., 18: 197.

Cannon, P. R., Sullivan, F. L. and Neckerman, E. F. 1932. Conditions Influencing the Disappearance of Living Bacteria from the Blood Stream. J. exp. Med., 55: 121.

Cannon, P. R. and Taltaferro, W. H. 1931. Acquired Immunity in Avian Malaria. III. Cellular Reactions in Infection and Superinfection. J. prev. Med., $5: 37$.

Cardamatis, J. P. 1919. Le Paludisme en Macédoine. Malariologia, 12: 65.

Carley, P. S. 1931. Results of the Dissection of 1017 Wild-caught Anopheles in Jamaica. Amer. J. trop. Med., 11: 293.

CARnahaN, C. T. 1939. A Two Year Record of Adult Mosquito Trapping in Dade County, Florida. Publ. Hlth. Rep., Wash., 54: 608.

Carpenter, S. J. 1939. The Mosquitoes of Arkansas. Ark. State Bd. Hlth., Little Rock, Ark.

CARR, H. P. 1938. A. albimanus Breeding in Relation to Degree of Shade in Breeding Places. Sth. Med. J., 31: 803.

-1940. Informe de los Trabajos Cooperativos llevados a cabo en Cuba durante 1939 por la Secretaria de Sanidad y Beneficencia con la Fundacion Rockefeller. Bol. Secret. Sanid., Habana, 43: 1 .

Carr, H. P., Mrlendez, J. F. and Ros, A. 1940. Malaria Reconnaissance of the Province of Oriente in Cuba. Amer. J. trop. Med., 20:81. 
CARTer, H. R. 1914. Impounded Waters. Some General Considerations of Its Effect on the Prevalence of Malaria. Publ. Hlth. Rep., Wash., 29: 3458 .

1919. The Malaria Problem in the South. Publ. Hlth. Rep., Wash., 34: 1929.

1924. Proc. Inter. Conf. Health Problems Trop. Amer. P. 155. United Fruit Co., Boston.

Carter, H. R., LePrince, J. A. and Griffitts, T. H. D. 1916. Impounded Waters. Surveys in Alabama and South Carolina During 1915 to Determine Its Effect on Prevalence of Malaria. Publ. Hlth. Bull., Wash., 79: 34.

CAsinI, G. 1939. La fase Apigmentata di Evoluzione dei Plasmodidi nella Malaria Cronica. Riv. Malariol., 18: 73 .

Castellani, A. and Chalders, A. J. 1919. Manual of Trop. Med. 3rd Ed. William Wood and Co., New York.

Celli, A. 1900. Malaria. (Translated by Eyre.) Longman's, New York.

Celli, A. and Sanfelice, F. 1891. Sui Parassiti Del Globulo Rosso Nell'uomo et Negli Animali. Ann. Ist. Igiene Sper. Univ. Roma, 1 (N.S.) 3363. Ueber die Parasiten des Rothen Blutkorpercheres im Menschen und in Thieren. Fortsch. Med., 9: 581-586.

Chagas. 1904. In M. F. Boyd, 1926.

Chapdan, R. N. 1925. Animal Ecology with Especial Reference to Insects. McGraw-Hill Book Co., New York.

Chapman, E. A. 1940. World Trade Notes on Chemicals and Allied Producis. Dept. Commerce Weekly Bulletin, May 25.

Chessa, F. 1938. La Funzionalita Surrenale nella Malaria. Riv. Malariol., 17 (Sez. 1): 438.

ChoprA, B. L. 1938. Anti-malarial Measures in the Railway Area at Delhi. Indian med. Gaz., $73: 150$.

Chopra, R. N. and Das Gupta, B. M. 1938. Note on Therapeutic Efficiency of Soluseptasine in Sinian Malaria. (P. knowlesi). Indian med. Gaz., 73 : 395.

- 1939. M. and B. 693 . (2 sulfanilylamino pyridine) in Ape Malaria. Indian med. Gaz., 74: 201.

Chopra, R. N., Das Gupta, B. M., Sen, B. and HAYTER, R. T. M. 1939. Prontosil in Indian Strains of Malaria. Indian med. Gaz., 74: 321.

Chopra, R. N., Hayter, R. T. M. and Sen, B. 1939. M. and B. 693 in Indian Strains of Malaria. Indian med. Gaz., 74: 658.

Chopra, R. N., MukherJee, S. N. and Sen, B. 1935. Studies on the Protein Fractions of Blood Sera. III. Malarial Sera During and After the Rigor Stage. Indian J. med. Res., 22: 571.

Chopra, R. N., Roy, A. C. and Das Gupta, B. M. 1934. On the Concentration of Quinine in Blood After Intravenous and Intramuseular Injections. Indian med. Gaz., 69: 561.

Chorine, V. 1938. La Reaction de Henry n'est due qu'a des Modifications Quantitatives dans le Serum. Bull. Soc. Path. exot., 31: 838.

Chorine, V. and Gillier, R. 1934. Le Méchanisme de la Reaction de Henry. Ann. Inst. Pasteur, 52: 193.

Christophers, R. 1924. The Mechanism of Immunity Against Malaria in Communities Living Under Hyperendemic Conditions. Indian $J$. med. Res., 12: 273.

- 1933. Family Culicidae Tribe Anophelini. Fauna of British Inđ̌ia, Diptera IV. Taylor and Francis, London.

Christophers, R. and Fulton, J. D. 1939. Experiments with Isolated Malaria Parasites (Plasmodium knowlesi) Free from Red Cells. Ann. trop. Med. Parasit., 33 (2): 161.

Christophers, R. and Missrroli, A. 1933. Report on Housing and Malaria. Quart. Bull. Hlth. Org. L. o. N., 2. Ex. 6: 357 .

Christophers, R. and Sinton, J. A. 1938. The Correct Name of the Malignant Tertian Malaria Parasite. Brit. med. J., 2: 1130.

Christophers, R., Sinton, J. A. and Covell, G. 1936. How to do a Malaria Survey. Hlth. Bull. No. 14, Caleutta.

Ciuca, M., Ballif, L. and Chelarescu, M. 1928. Etudes sur l'immunité dans le Paludisme. Arch. roum. path. exp. Microbiol., 1: 577.

- 1933. Contribution à l'étude de l'infection Paludisme Intentionellement Provoqué par Inoculation de Sang Virulent de Tierce Benign, de Fièvre Quarte et de Laverania. Bull. Soc. Path. exot., 26: 300 .

- 1934. Immunity in Malaria. Trans. Roy. Soc. trop. Med. Hyg., $27: 619$.

Guca,M., Ballif, L., Chelarescu, M., Isanos, M. and Gluaser, L. 1937a. Contributions à 1'étude de la Tierce Maligne Expérimentale. Pouvoir Infectant du sang au cours de l'incubation. Riv. Malariol., 16: 85 .

- 1937b. On Drug Prophylaxis in Therapeutic Malaria. Trans. Roy. Soc. trop. Med. Hyg., 31: 241.

Ciuca, M., Thomescu, P. and Badenski, G. 1937. Contribution à l'étude de la Virulence du $P$. knowlesi chez l'homme. Caractères de la Maladie et Biologie du Parasite. Arch. roum. path. exp. Microbiol., 10: 5 .

Clark, B. B., Cominole, B. and Martin, S. J. 1939. Effect of Atebrin on Liver and Kidney Function. J. Pharmacol., 65: 166.

Clark, H. C. 1916. The Diagnostic Value of the Placental Blood Film in Aestivo-Autumnal Malaria. J. exp. Med., 22: 427.

- 1926. Anopheles Mosquitoes in our Tropical Divisions. 15th Ann. Rep. United Fruit Co. Med. Dept., p. 45.

- 1928. Spleen and Parasite Rates as Measures of Malaria in the Caribbean Area. Amer. J. trop. Med., 8: 423.

-1932. Sth. Med. J., 25: 528.

-1937. The First Twelve Months of 
Infancy as a Test for the Community Incidence of Initial Attacks of Malaria. Sth. Med. J., 30: 848.

Clatk, H. C. and Komp, W. H. W. 1932. A Second Year's Observation on Malaria in Some Unsanitated Chagres River Villages with Special Reference to the Use of Quinine and Plasmochin. Gorgas Memorial Lab., Panama.

ClaAk, H. C., Komp, W. H. W. and Jobbins, D. M. 1940. A Ninth Year's Observations on Malaria in Panama, with Reference to the Occurrence of an Epidemic Following Continued Treatment with Atabrine and Plasmochin. Amer. J. trop. Med., 20 : 47.

Clatke, J. Lyall. 1936. Progress of the Mosquito Control Campaign in the Desplaines Valley Area. Proc. N. J. Mosq. Ext. Ass., 23: 98.

Coatney, G. R. and Young, M. D. 1939. The Effect of Colchicine on Bird Malaria. $J$. Parasit., 25: 446.

Coggeshald, L. T. 1926. Relationship of Plankton to Anopheline Larvae. Amer. J. Hyg., 6: 556.

- 1930. Report of a Malarial Survey and Control Method on Lake Murray, Columbia, B. C. Sth. Med. J., 23: 442.

1938a. The Quantitative Relationship Between Immune Serum and Infective Dose of Parasites as Demonstrated by the Protection Test in Monkey Malaria. J. exp. Med., 68: 29.

1938b. The Cure of Plasmodium knowlesi Malaria in Rhesus Monkeys with Sulfanilamide, and Their Susceptibility to Reinfection. Amer. J. trop. Med., 18: 715 .

1938c. Prophylactic and Therapeutic Effect of Sulfanilamide Compounds in Experimental Malaria. Proc. Soc. exp. Biol., 38: 768. 1940a. The Selective Action of Sulfanilamide on the Parasites of Experimental Malaria in Monkeys in vivo and in vitre. J. exp. Med., 71: 13.

1940b. The Occurrence of Malarial Antibodies in Human Serum Following Induced Infeetion with Plasmodium knowlesi. J. exp. Med., 72 : 21.

Cocesshall, L. T. and Eaton, M. D. 1938a. The Complement Fixation Reaction in Malaria. $J$. exp. Med., 67: 871 .

- 1938b. The Quantitative Relationship Between Immune Serum and Infeetive Dose of Parasites as Demonstrated by the Protection Test in Monkey Malaria. J. exp. Med., 68: 29.

Coggeshall, L. T. and KumM, H. W. 1937. Demonstration of Passive Immunity in Experimental Monkey Malaria. J. exp. Med., 66: 177.

- 1938. Effect of Repeated Superinfection upon the Potency of Immune Serum of Monkeys Harboring Chronic Infections of Plasmodium knowlesi. J. exp. Med., 68: 17.

Coggeghall, L. T. and Mater. 1941. Determination of the Activity of Various Drugs Against the Malaria Parasite. J. Infect. Dis. In press.
Coнnнeiм, J. 1867. Utber Entzundung und Eiterung. Virchow's Archiv., 40: 1.

Comes, S. 1922. Appunti di Biologia Malarica Mode di Nutrizione e Genesis del Pigmento nel Parassita Malarico (Nota terza). Arch. Protist., 45: 401-409. 1922.

Concannon, C. C. 1940. Personal communication. Conway, E. A. 1939. Reaction of Lymphatic Tissue of Rabbits to Repeated Injections of Bacterium Monocytogenes. J. infect. Dis., 64: 217.

Coogle, C. P. 1925. Mosquito Repellants and Mosquitocides. Publ. Hlth. Bull., Wash., 156: 136.

1928. The Sereening and MosquitoProofing of Tenant Houses. Mississippi State Board Health Bull. 2.

CooK, S. S. 1930. Malaria Control in Haiti. Sth. Med. J., 23 : 454.

Correa, R. R. 1938. O Anopheles (N) strodei Root, 1926 como Provavel Vector de Malaria. Rev. Biol. Hyg., S. Paulo, 9: 104.

CoRT, E. C. 1929. Epidemiology of Blackwater Fever in Siam. Amer. J. trop. Med., 9: 105.

Coulston, F. and Manwell, R. D. 1941. SingleParasite Infections and Exoerythrocytic Schizogony in Plasmodium circumflexum. Amer. $J$. Hyg. (in press).

Counchman, W. T. and Аввотт, A. C. 1885 . A Contribution to the Pathology of Malarial Fever. Am. J. med. Sci., 89: 416.

Covell, G. 1927. A Critical Review of the Data Recorded Regarding the Transmission of Malaria by the Different Species of Anopheles; with Notes on Distribution, Habits, Breeding Places. Indian med. res. Mem, No. 7. Thacker, Spink and Co., Caleutta.

-1931a. Malaria Control by AntiMosquito Measures. Thacker, Spink and Co., Calcutta.

. 1931b. The Present State of Our Knowledge Regarding the Transmission of $\mathrm{Ma}$ laria by the Different Species of Anopheline Mosquitoes. Rec. Malar. Surv. India, 2: 1.

Covell, G., Muluigan, H. W. and AFrid, M. K. 1938. An Attempt to Control Malaria by the Destruction of Adult Mosquitoes with Insecticidal Sprays. J. Malar. Inst. India, 1: 105.

Craig, C. F. 1900. Report of the Surgeon General U. S. Army. Gov't. Print. Off., Wash. 1909. The Malarial Fevers. William Wood and Co., New York.

- 1926. A Manual of Parasitic Protozoa of Man. Pp. 569. J. B. Lippineott and Co., Philadelphia.

. 1928. Malaria. Arch. Path., 6: 645.

1933. The Nomenclature of Plasmodium ovale Stephens, 1922. Amer. J. trop. Med., 13: 539.

Univ. Press, London and New York.

Oxford 1939. The Modern Treatment of Malarial Infections. Tex. St. J. Med., 35: 213. 
Craig, C. F, and Faust, E. C. 1940. Clinical Parasitology. Pp. 772. Lea and Febiger, Philadelphia.

Cropper, J. 1908. Phenomenal Abundance of Parasites in a Fatal Case of Pernicious Malaria. Lancet, 2: 16.

Cumming, F. 1810. Sketches of a Tour to the Western Country, Through the States of Ohio and Kentucky, a Voyage Down the Ohio and Mississippi Rivers and a Trip Through the Mississippi Territory, and a Part of West Florida. Reprinted in Thwaites, R. G. 1940. Early Western Travels, 1748-1846. Vol. IV. A. H. Clark Co., Cleveland.

Curry, D. P. 1925. Some Observations on Mosquito Control in the Canal Zone, with Especial Reference to the Genus Anopheles. Aimer. $J$. trop. Med., 5: 1.

- 1928. A New Anopheline Mosquito, Anopheles (Chagasia) bathanus Dyar, Discovered in the Canal Zone. Amer. J. trop. Med., 8: 243. - 1931a. Anopheles (Anopheles) neomaculipalpus, a New Species of the Arribalzagia Group of Anopheles from Panama. Amer. $J$. Hyg., 13: 643.

1931b. The Anopheline Mosquitoes of the Isthmus. Rep. Hlth. Dept. Panama Canal.

- 1932. Some Observations on the Nyssorhynchus Group of the Anopheles (Culicidae) of Panama. Amer. J. Hyg., 15: 566.

- 1934. Breeding of Anopheline Mosquitoes Among Aquatic Vegetation of Gatun Lake, Accompanied by Periodic Long Flights of A. albimanus Wied. Sth. Med. J., 27: 644 .

DAMPF, A. 1935. The Occurrence of Anopheles maculipennis in Mexico. Science, 82: 171.

1936. El Anopheles occidentalis Dyar and Knab (A. maculipennis Auct.) en la Mesa Central de Mexico. Rev. mex. Biolv 18: 91. (Quoted in Rev. appl. Ent. B, 26: 140.)

DANIELL, W. C. 1826. Observations upon the Autumnal Fevers of Savannah. Collins and Hannay, New York.

DANIELS, C. W. 1901. Notes on Blackwater Fever in Central Africa. Rep. Malar. Comm. roy. Soc., 5th Ser., p. 44.

Danzel, L. 1929. A propos de l'hélianthe un grand solẹil plante industrielle médicinale. Rev. Méd. Hyg. trop., 21: 158.

Darline, S. T. 1910. Studies in Relation to Malaria. Rep. Isthmian Canal Commission, Government Printing Office, Washington.

- 1925. Discussion on the Relative Importance in Transmitting Malaria of Anopheles quadrimaculatus, punctipennis and crucians, etc. Sth. Med. J., 18: 452 .

DAUER, C. C. and FAUST, E. C. 1936. Malaria Mortality in the Southern United States for 1934, with Supplementary Data for Previous Years. Sth. Med. J., 29: 757 .
- 1937. Malaria Mortality in the United States, with Special Reference to the Southeastern States. Sth. Med. J., 30: 939.

DaveY, T. H. and Gordon, R. M. 1933. The Estimation of the Density of Infective Anophelines as a Method of Calculating the Relative Risk of Inoculation with Malaria from Different Species or in Different Localities. Ann. trop. Med. Parasit., 27: 27.

- 1938. The Size of the Aperture Necessary in Screencloth Intended for the Protection of Dwellings in West Africa. Ann. trop. Med. Parasit., 32: 413.

Davis, N. C. 1926. A Field Study of Mountain Malaria in Brazil. Amer. J. Hyg., 6: 119.

- 1927. Anopheles pseudopunctipennis as a Malaria Transmitter in Northern Argentine Republic. Amer. J. trop. Med., 7: 167.

-1931. A Note on the Malaria-Carrying Anophelines in Belem, Para, and in Natal, Rio Grande do Norte, Brazil. Riv. Malariol., 10: 43.

Davis, N. C. and KuMM, H. W. 1932. Further Incrimination of Anopheles darlingi Root as a Transmitter of Malaria. Amer. J. trop. Med., 12: 93.

Davis, N. C. and Shannon, R. C. 1928. The Blood Feeding Habits of Anopheles pseudopunctipennis in Northern Argentina. Amer. J. trop. Med., 8: 443 .

Dawson, W. T. 1930. Cinchona Alkaloids and Malaria. Int. Clin. (40th Ser.) 2: 121.

1932. Antimalaria Value of Cinchona Alkaloids Other than Quinine. Sth. Med. J., 25: 529.

Dawson, W. T., Gingrich, W. and Hollar, E. D. 1935. Intravenous Toxicity of Atabrine. Amer. J. trop. Med., 15: 515.

Dawson, W. T., SANDERS, J. P. and TomLinson, L. M. 1933. Differentiation of Optically Isomeric and Related Cinchona Alkaloids by Quinine-sensitive Subjects. J. Immunol., 24: 173.

DeAderick, W. H. 1914. Blackwater Fever. An Analysis of Thirty-Four Cases. N. Y. med. J., $100: 873$.

DeBezerra, A. 1936. Habits dos Anophelineos do Brazil. Folba Med., 17: 125.

DE BUCK, A. 1935. Infection Experiments with Quartan Malaria. Ann. trop. Med. Parasit., 29: 171.

- 1936. Some Results of Six Years Mosquito Infection Work. Amer. J. Hyg., 24: 1.

DE BurCA, B. 1939. Note on Anti-malaria Measures in Quetta Cantonment during 1938. J. Malar. Inst. India, 2: 121.

Deeks, W. E. and JaMes, W. M. 1911. A Report of Hemoglobinuric Fever in the Canal Zone, a Study of Its Etiology and Treatment. Isthmian Canal Commission Press, Mount Hope, C. Z.

Delangen, C. D. and Btork, C. J. 1935. A Com- 
parative Clinical and Experimental Study of the Action of Quinine, Plasmochin and Atebrin. Mededeel. Dienst. Volksgezondh. Ned.-Ind., 24: 27.

DELEON, A. D. 1937. Treatment of Malaria with Sulfonamide Compounds. Publ. Hlth. Rep., Wash., 52: 1460 .

DE LEON, R. 1933. Los Anopeles de la Cuidad de Guatemala. Bol. Sanit. Guatemala, 4: 778 .

1936a. El Paludismo de Altura en Guatemala. Bol. San. Guat. Año 7, 44: 67. 1936b. Greve Estudio de la Fauna Anofelica de Guatemala. Bol. San. Guat. Año $7,44: 77$.

Guatemala. Bol. San. Guat. Año 9, 46: 411.

DE SAnctis Monaldi, T. 1935. Ricerche sulla Malaria Sperimentale da Inoculazione di Sporozoiti. Riv. Malariol., 14, Sez. 1: 344.

De Saussure, H. W. 1860. Quinine as a Prophylactic of Intermittent and Remittent Fevers. Charleston Med. J. Rev., 15: 433.

DE Verteuil, E. 1925. -According to Covell, 1927.

1933. Malaria Survey of 1932, Trinidad and Tobago. Council Paper No. 32 . Govt. Printing Office, Port-of-Spain, Trinidad.

DE VERTEUIL, E. and SPEnce, T. 1937. Malaria in Trinidad. Low Tide Level Culvert System in Coastal Drainage. Trans. Roy. Soc. trop. Med. Hyg., 30 : 449.

Dock, G. 1894. Pernicious Malarial Fever. Amer. J. Med. Sci., 107: 379.

- 1931. The Medicinal Use of Cinchona. Proc. of the Celebration of the Three Hundredth Anniversary of the First Recognized Use of Cinchona, p. 149. Botanical Garden, St. Louis, Mo.

Doty, Dr. 1912. As quoted in Howard, Dyar and Knab, "The Mosquitoes of North and Central America and the West Indies," Vol. 1, p. 444. Carnegie Institution, Washington.

Dudgeon, L. S. and Clarke, C. 1917. A Contribution to the Microscopical Histology of Malaria. Lancet, 2: 153.

DUNN, L. H. 1934. Entomological Investigations in the Chiriqui Region of Panama. Psyche, 16: 166.

DyaR, H. G. 1922. The Mosquitoes of the United States. Proc. U. S. natl. Mus., 62: 1.

- 1928. The Mosquitoes of the Americas. Carnegie Inst. Wash. Publ., No. 387.

EARLE, W. C. 1925. The Relation of Irrigation on Cane Fields to the Malaria Problem. Agri. Notes, Puerto Rico Agri. Exp. Sta., Mayaguez, 21: 1-3.

- 1930a. Malaria in Porto Rico. Amer. J. trop. Med., 10: 207.

1930b. Malaria in Porto Rico in Its Relation to the Cultivation of Sugar Cane. Sth. Med. J., 23: 499.

- 1932. Some Observations of Anti-mosquito Screening and Screening Materials. Puerto Rico J. Publ. Hlth., 8: 227.
1934. Summary of Malaria Activities in Grenada, B. W. I., 1929-1932 inclusive. Rep. Med. Sanit. Dep. Grenada 1932, p. 44.

1936a. Anopheles grabhamii (Theobald), a Possible Vector of Malaria. Bol. Asoc. med. P. Rico, 28: 228.

- 1936b. The Relative Importance of Anopheles tarsimaculatus, Anopheles argyritarsis, and Anopheles pseudopunctipennis as Vectors of Malaria in the Windward Group of the West Indies. Amer. J. trop. Med., 16: 459.

- 1937. The Relation between Breeding Area, Anopheles albimanus Density, and Malaria in Salinas. Puerto Rico J. Publ. Hlth., 30: 946. . 1939. The Epidemiology of Malaria with Special Reference to Puerto Rico. Puerto Rico J. Publ. Hlth., 15: 3 .

EARLE, W. C. and How ARD, H. H. 1936. The Determination of Anopheles Mosquito Prevalence. Bol. Asoc. med. P. Rico, 28: 233.

EARLE, W. C. and Perez, Manuel. 1932. Enumeration of Parasites in the Blood of Malarial Patients. J. Lab. clin. Med., 17: 1124.

EATON, M. D. 1938. The Agglutination of Plasmodium knowlesi by Immune Serum. J. exp. Med., 67: 857.

Eaton, M. D. and Coggeshali, L. T. 1939. Complement Fixation in Human Malaria with an Antigen Prepared from the Monkey Parasite Plasmodium knowlesi. J. exp. Med., 69: 379.

Eaton, Paul. 1934. Susceptibility of Red Cells to Malaria. A Preliminary Note. Amer. $J$. trop. Med., 14: 431.

Edwards, F. W. 1930. Mosquito Notes. IX. Bull. ent. Res., 21: 287.

. 1932. Diptera. Fam. Culicidae. In Wyssman, P., Genera Insect., Fasc. 194. DesmetVertenuiel, Bruxelles.

EDWARDS, F. W. and Box, H. E. 1940. Notes on Mosquitoes. Ann. Mag. nat. Hist., 5: 314.

EJercito, A. 1935. Biological Control of Anopheline Vectors of Malaria in the Philippines: Preliminary Report. J. P. I. med. Ass., 15: 177.

- 1938. Report of Inspection in Malaria Control Unit No. 3 with Headquarters at Callag, Dalig-Ziffu Region, Isabela. Bull. Bur. Hlth. Philippines, 18: 249.

Emin, A. 1914. Une Varieté Nouvelle de Parasite de Laveran. Bull. Soc. Path. exot., $7: 385$.

Fabry, A., Guillerm, J. and Ragiot, C. 1937. Syndrome Lipoidique et Parapaludisme. Bull. Soc. Path. exot., 30: 90.

FAGET, G. H. 1933. Malarial Fever in Narcotic Addicts: Its Possible Transmission by the Hypodermic Syringe. Publ. Hlth. Rep., Wash., 48: 1031.

Fatrley, N. H. 1938. Methaemalbumin (Pseudomethaemoglobin). Nature, 142: 1156.

. 1939. Methaemalbumin in Man (PseudoMethaemoglobin). Proc. Roy. Soc. Med., 32: 1278.

FaIrLey, N. H. and Bromfield, R. J. 1933. Laboratory Studies in Malaria and Blackwater 
Fever. Part I. Malaria. Trans. Roy. Soc. trop. Med. Hyg., 27: 289.

- 1934. Laboratory Studies in Malaria and Blackwater Fever. Part III. A New Blood Pigment in Blackwater Fever and Other Biochemical Observations. Trans. Roy. Soc. trop. Med. Hyg., 28: 307.

- 1937. Pseudomethaemoglobin in Blackwater Fever and Its Clinical Significance. Trans. Roy. Soc. trop. Med. Hyg., 31: 139.

FARINAUd, E. and RAgiot, C. 1939. Récherches sur l'emploi des Dérives de la Sulfamide dans le Traitement du Paludisme. Pr. Méd., 47: 385.

FAUST, E. C. 1932. The 1930 Status of Malaria in the Southern United States as Determined by Mortality Data. Sth. Med. J., 25: 544.

- 1933. Malaria Mortality in the Southern United States. Sth. Med. J., 26: 454; 27 : $457 ; 29: 757 ; 33: 897$.

1939a. What the Life Insurance Companies Think of Malaria. Sth. Med. J., 32: 689. - 1939b. Malaria Mortality in the United States for the Year 1937. Amer. J. trop. Med., 19: 447 .

1940. Malaria Mortality in the Southern United States for the Year 1938. Sth. Med. J., $33: 897$.

Faust, E. C. and Dibols, C. G. 1935. Malaria Mortality in the Southern United States for the Year 1933. Sth. Med. J., 28: 757.

Felett, R. and Grassi, B. 1890. Sui Parassiti Della Malaria. Arch. ital. Biol., 13: 287.

Fenner, E. D. 1850. Editorial Comment. Sth. Med. Rep., 2: 349 .

Field, J. C. and LeFleming, H. 1939. Morphology of Malarial Parasites in Thick Blood Films. Trans. Roy. Soc. trop. Med., 32: 467 and 33: 507.

Fischer, O. and RheindorF, G. 1928. Zur Frage der Plasmochin-nebenwirkung. Arch. Schiffs. TropenHyg., 32: 594.

Fischl, V. and Schlossberger, H. 1932. Handbuch der Chemotherapie, p. 153. Fischers, Leipzig.

Fischl, V. and Singer, E. 1934. Gewinnung und Verholten Arzneifester Recurrensspirochäten. $Z$. Hyg. Infekt., 116: 138.

Fisher, L. M. 1920. What Can A Community Afford to Pay to Rid Itself of Malaria. Publ. Hlth. Rep., Wash., 35: 1269.

Fletcher, W. 1928. Notes on the Treatment of Malaria with the Alkaloids of Cinchona. John Bale Sons and Danielson, Ltd., London. Cited from Dawson, 1932.

Fonde, G. H. and Fonde, E. C. 1939. Chronic Malaria. Arch. intern. Med., 64: 1156.

Fox, H. and Kond, A. 1936. Researches on Blackwater Fever in Greece. IV. Experimental Investigations into the Existence of Hemolytic Strains of Malaria and/or other Specific Parasites in Blackwater Fever. Ann. trop. Med. Parasit., 30: 423.

-1937. Blackwater Fever in Macedonia. Trans. Roy. Soc. trop. Med. Hyg., 31: 123.
1938. Spectrographic Analysis of Pig. ments in Serum and Urine of Blackwater Fever. Trans. Roy. Soc. trop. Med. Hyg., 32: 49.

François-Julien, G. 1930. De la Persistence du Paludisme à la Guadeloupe. Les Causes-La Prophylaxie. Thèse Fac. Méd. Paris (Rev. Appl. ent. B. 19: 58).

Freeborn, S. B. 1923. The Range Overlapping of Anopheles maculipennis Meig. and Anopheles quadrimaculatus Say. Bull. Brooklyn ent. Soc., 18: 157.

1926. The Mosquitoes of California. Univ. Cal. Publ. Ent., 3: 333.

- 1932. The Seasonal Life History of Anopheles maculipennis with Reference to Humidity Requirements and "Hibernation." Amer. $J$. Hyg., 16: 215.

Freeborn, S. B. and Atstat, R. F. 1918. The Effect of Petroleum Oils on Mosquito Larvae. J. econ. Ent., 11: 299.

Frohne, W. C. 1939. Anopheline Breeding: Suggested Classification of Ponds Based on Characteristic Desmids. Publ. Hlth. Rep., Wash., 54: 1363.

Fuller, A. T. 1937. Is p-aminobenzenesulphonamide Active Agent in Prontosil Therapy? Lancet, 1: 194.

Fullerton, H. R. and Bishop, E. L. 1933. Improved Rural Housing as a Factor in Malaria Control. Sth. Med.J., 26: 465.

Futton, J. D. 1939. Experiments on the Utilization of Sugars by Malarial Parasites (Plasmodium knowlesi). Ann. trop. Med. Parasit., 33: 217.

Fulton, J. D. and Christophers, R. 1938. The Inhibitive Effect of Drugs upon Oxygen Uptake by Trypanosomes (Trypanosoma rhodesiense) and Malaria Parasites (Plasmodium knowlesi). Ann. trop. Med. Parasit., 32: 77.

Gabaldon, A. 1938. Primer Informe anual (1937) de la Division de Malariologia. Pub. Div. Mal., No. 1. Prim. Inf. An. (1937) Div. Mal., Caracas, Venezuela.

- 1939a. Segundo Informe anual (1938) de la Division de Malariologia. Pub. Div. Mal., No. 4. Segundo Inf. An. (1938) Div. Mal., Caracas, Venezuela.

- 1939b. Generalidades Sobre la Malaria y Organización de la Lucha Antimalarica en Venezuela. Bol. Minist. Sanidad Asist. Soc., 4: 69. Caracas, Venezuela.

Gabaldon, A., Cova-Garcia, P. and Arevalo, A. 1940. Observaciones Sobre Numero de Huevos, Salinidad de Criaderos y Tiempo de Desarrallo de Tres Especies de la Sub-serie oswaldoi. Publ. Div. Mal., 5: 25.

Gabaldon, A., Cova-Garcia, P. and Lopez, J. A. 1940. Anopheles (Nyssorhynchus) rangeli, una Nueva Especie de la Sub-serie oswaldoi (Diptera, Culicidae) de Amplia Distribucion en Venezuela. Publ. Div. Mal., 5: 9.

Gabaldon, A., Herrera, J., Perez-Vivas, M. A. and RAUSSEO, J. A. 1940. Chagasia bathanus 
Dyar, 1928: su Hallazgo en Venezuela, y Nota Sobre Variaciones Morfologicas de Las Pupas. Publ. Div. Mal., 5: 57.

Galvão, A. L. and Barretto, M. P. 1938. Observacoes Sobre o Anopheles albitarsis Arribalzaga, 1878 e A. triannulatus Neiva and Pinto, 1922, de São Paulo. Rev. Biol. Hyg., S. Paulo, 9: 144.

Galvão, A. L. and LANE, J. 1937a. Notas Sobre os "Nyssorhynchus", de S. Paulo. IV. Sobre a Infeccão Experimental do Anopheles albitarsis e A. strodei de Cidade de S. Paulo Pelo Plasmodium vivax. Folia clin. biol., S. Paulo, 3: 65.

- 1937b. Nota Sobre os Nyssorhynchus de S. Paulo. VII. Estudo Sobre as Variedades Deste Grupo com a Descricao de Anopheles (Nyssorhynchus) albitarsis Arribalzaga, 1878 var. limae n. var. Ann. Fac. Med. S. Paulo, 13: 211.

GarnhaM, P. C. C. 1935 . Hyperendemic Malaria in a Native Reserve of Kenya and the Influence upon Its Course of Atebrine and Plasmoquine. Trans. Roy. Soc. trop. Med. Hyg., 29: 167.

- 1938. The Placenta in Malaria with Special Reference to Reticulo-endothelial Immunity. Trans. Roy. Soc. trop. Med. Hyg., 32: 13.

GarRISH, Doctor. 1879. Discussion, St. L. Med. Surg. J., 37: 112.

Gaskell, J. F. and Millar, W. L. 1919-20. Studies on Malignant Malaria in Macedonia. Quart. J. Med., 13: 381.

GAteR, B. A. R. 1933. Notes on Malayan Mosquitoes. II. Seasonal Distribution. Malay. med. J., $8: 42$.

Geiger, J. C. and Purdy, W. C. 1920. The Malaria Problem of the Rice Field of the United States. Sth. Med.J., 13: 577.

Geiger, J. C., Purdx, W. C. and TARbett, R. E. 1919. Effective Malaria Control in a Ricefield District, with Observations on Experimental Mosquito Flights. J. Amer. Med. Ass., 72: 844.

Ghosh, B. N. and NAtH, M. C. 1934. The Chemical Composition of Malaria Pigment (Haemozoin). Rec. Malar. Surv. India, 4: 321.

GHosh, B. N. and Sinton, J. A. 1934. Studies of Malarial Pigment (Haemozoin). Part II. The Reactions of Haemozoin to Tests for Iron. Rec. Malar. Surv. India, $4: 43$.

- 1935. Quantitative Changes in the Proteins of the Blood Sera of Monkeys Infected with Malarial Plasmodia. Rec. Malar. Surv. India, 5: 173.

Giaquinto, Mira M. 1931. Una Nueva Especie de Anopheles en Guatemala: Anopheles hectoris Mihi. Biol. Direc. Salub. Guatemala, 1: 606.

- 1936. La Malaria en Guatemala. Estudios Epidemiologico y Desarrollo de la Campana Antipaulidica. Riv. Malariol. (Rev. appl. Ent., B. 49 : 292.)

Gibbons, E. G. 1933. Morphological Study of Malaria Pigment in Oocysts of Naturally Infected Anopheles. J. Parasit., 25: 428.

GibsoN, A. 1935. Mosquito Suppression Work in
Canada in 1934. Proc. N. J. Mosq. Ext. Ass., 22: 77.

GiguroLI, G. 1929. Malarial Nephritis, Etiological, Epidemiological and Clinical Studies on Bright's Disease in British Guiana, 1922-29; British Guiana Rep. of the Sur. Gen. for 1929. Appen$\operatorname{dix} 2$.

1932. Clinical Notes, Autopsy and His topathological Findings from Five Fatal Cases of Quartan Malarial Nephritis from British Guiana. Trans. Roy. Soc. trop. Med. Hyg., 26: 177.

1938a. Malaria on the Coast-land of British Guiana. Health Conditions in the British Guiana Sugar Estates, Nov. 21, App. I and II, pp. 1-26.

1938b. Malaria in British Guiana, Pts. I, II. Agric. J. Brit. Guiana, 9: 75, 135.

- 1938c. Malaria in British Guiana. Pt. II. Agric. J. Brit. Guiana, 9: 137.

1940. Malaria in British Guiana. Report Dir. Med. Surv. (1938) Append. II: 118. Georgetown, Demerara, British Guiana.

Gist, C. A. 1914. Epidemic or Fulminant Malaria Together with a Preliminary Study of the Part Played by Immunity in Malaria. Indian J. med. Res., 2: 268.

- 1933. Immunity in Malaria. Trans. Roy. Soc. trop. Med. Hyg., 27: 281.

- 1938a. The Seasonal Periodicity of Malaria. J. and A. Churchill, Ltd., London. 1938b. Prediction of Epidemies. J. and $\mathrm{A}$. Churchill, Ltd., London.

GINGRICH, W. 1934. The Effect of an Increased Burden of Phagocytosis upon Natural and Acquired Immunity to Bird Malaria. J. Parasit., $20: 332$.

- 1941. The Role of Phagocytosis in Natural and Aequired Immunity in Avian Malaria. J. Infect. Dis., 68: 37 .

GinsBURG, J. M. 1927. The Effect of Various Chemicals on the Spreading and-Penetration of Oils in Different Mosquito Breeding Places. Proc. N. J. Mosq. Ext. Ass., 14: 52.

1928. Studies of Mosquito Oils and Dust Larvicides. Proc. N. J. Mosq. Ext. Ass., 15: 53 .

1929. Relation Between Toxicity of Oil and Its Penetration into Respiratory Siphon of Mosquito Larvae. Proc. N. J. Mosq. Ext. Ass., 16: 50 .

1930. Studies of Pyrethrum as a Mosquito Larvicide. Proc. N. J. Mosq. Ext. Ass., $17: 57$.

1934. Mosquito Larvicides. Proc. N.J. Mosq. Ext. Ass., 21: 121.

1935. Larvicides and a Method for Temporary Protection from Adult Mosquitoes in Limited Areas. Proc. N.J. Mosq. Ext. Ass., 22: 147.

1936. Protection of Outdoor Gatherings from the Mosquito Pest. Proc. N. J. Mosq. Ext. Ass., 23: 166. 
- 1937a. Studies in Reducing Volume of Oil Necessary to Kill Mosquito Larvae. J. econ. Ent., 30: 328.

- 1937b. Principles Underlying the Proteetion of Outdoor Meetings from the Mosquito Pest and Methods of Applieation. Proc. N.J. Mosq. Ext. Ass., 24: 5 .

- 1939. New Jersey Mosquito Larvicide. N. J. Agri. Exp. Sta. Cir. 382 .

Giovannola, A. 1935. Plasmodium immaculatum (Grassi and Feletti 1892) Sehaudinn 1902. The Correct Name for the Parasite Causing Malignant Tertian Malaria. Proc. Hel. Soc. Wash., 2: 90.

- 1938. Ceppi Tropicali e Ceppi Europei di Plasmodium immaculatum. Acta. Con. Tertii Trop. Mal. Morb., 2: 567.

GIRNDT, O. 1929. Ưber den Angriffespunkt von Antipyreticis: Chinin and Chininähnlichen Stoffen. Arch. exp. Path. Pharmak., 140: 91.

Godor, A. and Pinto, C. 1923. Estudos Sobre Malaria. Brazil-med., 37: 29.

GoLDIE, H. 1930. Notes on the Association of Malaria with Nephritis. Trans. Roy. Soc. trop. Med. Hyg., 23: 503.

GoLGI, C. 1886a. Sull 'infezione Malarica. Arch. Sci. méd., 10: 109.

- 1886b. Sulla Infezione Malarica. Opera Omnia, III: 989 . U. Hoepli, Milano.

-1886e. Aneora sulla Infezione Malariea. Gas. degli Osp. Clin., $7: 419$.

-. 1888. Il Fagocitismo nell' Infezione Malarica. Rif. Med., 4: Reprint p. 17.

Gordon, R. M. and LuMsDen, W. H. R. 1939. A Study of the Behavior of the Mouth-Parts of Mosquitoes When Taking up Blood from Living Tissue; Together with Some Observations on the Ingestion of Mierofilariae. Ann. trop. Med. Parasit., 33: 259.

Gore, R. N. 1937a. A Modified Village Mosquito Trap. Indian med. Gaz., 72: 674 .

1937b. An Improved Feather Duster Mosquito Trap. Rec. Malar. Surv. India, 7: 209.

Gorgas, W. C. 1910. In Ross, R., Prevention of Malaria, p. 346 . E. P. Dutton and Co., New York.

- 1915. Sanitation in Panama. D. Appleton and Co., New York and London.

GranETT, P. 1940. Studies of Mosquito Repellents II. Relative Performance of Certain Chemicals and Commercially Available Mixtures as Mosquito Repellents. J. econ. Ent., 33: 566.

GrAssI, B. 1891. Weiteres zur Malariafrage. II. Verschiedene Untersuchungen. Zbl. Bakt. Orig., $10: 517$.

10: 1901. Die Malaria. Studien eines Zoologen. Jena.

Grassi, B., Bignami, A. and Bastianeli, G. 1898. Coltivazione delle Semilune Malariche Dell'uomo nell' Anopheles claviger Fabr. (Sinonimo $A$. maculipennis Meig.). Atti. R. C. Accad. Lincei Sed Solenni, 295, 5 ser. v. 7, sem. 2, pp. 313-314. -1899. Ciclo Evolutivo delle Semilune nell' "Anopheles claviger" e Altri Studi sulla
Malaria Dell' ottobre 1898 al Maggio 1899. Ann. d'Ig. sper. (Roma), new ser. Vol. 9, pp. 258-271. 1899. Also in Atti Soc. Studi Malar., 1: 14-27.

Grassi, B. and Feletti, R. 1890a. Parasites Malariques chez les Oiseaux. Arch. Ital. Biol., $13: 297$.

1890b. Sunti Delle Memorie Ancora in Parassiti Malarici Degli Ucelli. Bol. Accad. gioenia, Mar. 24, 13: 3-6 and Apr. 27, 14: 2-7. 1892. Contribuzione allo Studio dei Parassiti Malariei. Atti. Accad. gioenia, Series 4, 5: 1-81.

Grassi, B. and Sella, M. 1920. Seconda Relazione della Lotta Antimalarica a Fiumicino. Ann. Igiene, 30 suppl.

Green, H. W. 1921 and 1922. Preliminary Report on Anopheles Mosquito Reduction in Aguirre, Puerto Rico. Rockefeller Foundation unpublished reports.

- 1922. Second Preliminary Report on Malaria Control in Aguirre, Puerto Rico. Rock. Found. (Quoted in Earle and Howard, 1936.)

- 1924. Effect of Oil Upon Anopheles Mosquito Larvae. Amer. J. Hyg., 4: 12. published data.

Green, R. 1931. Notes on the Use of Thick Blood Films in the Diagnosis of Malaria. Bull. Inst. Med. Res. Fed. Malay States, No. 1.

Grieg, E. D. W., van Rooyen, C. E. and Hendry, E. B. 1934. Observations on the Melano-Preeipitation Serological Reaction in Malaria. Trans. Roy. Soc. trop. Med. Hyg., 28: 175.

GriffitTs, T. H. D. 1925. Method of Applying Oil Under Pressure as a Larvicide. Publ. Hlth. Bull., Wash., 156: 15.

1926. In Aquafax, 2: 10.

-1933. Air Traffic in Relation to Public Health. Amer. J. trop. Med., 13: 283.

1927. Moist Sand Method of Applying Paris Green for Destruction of Subsurface-Feeding Mosquito Larvae. Publ. Hlth. Rep., Wash., 42: 2701.

Gutzevich, A. V. and Podolyan, V. YA. 1935. Pyrethrum Smoke Candles as a Means of Controlling Mosquitoes and Sandflies. Rev. Microbiol., $14: 87$.

HACKETT, L. W. 1925. The Importance and Uses of Paris Green (Copper Aceio Arsenite) as an "Anophele"' Larvicide. Compte-rendu du Premier Congrès International du Paludisme, Rome, Oct. 1925, Publié par le Sècretaire Général du Congrès, 1926. p. 158.

- 1937. Malaria in Europe. Oxford Univ. Press, London.

HACKETT, L. W. and BATES, M. 1938. The Laboratory for Mosquito Research in Albania. Acta. Conv. Tertii Mal. Morbis. Pars II, 113.

HACKETT, L. W., RUssell, P. F., ScharfF, J. W. and Senion-White, R. 1938. The Present Use of Naturalistic Measures in the Control of Malaria. Bull. Hlth. Org. L. o. N., 7: 1016. 
HANAFIN, J. B. 1928. Further Results in Mosquito-Proofing Barracks. J. R. Army med. Cps., 51: 127.

Hanson, H., Boyd, M. F. and Griffitts, T. H. D. 1935. Some Factors in the Epidemiology of Malaria. Amer. J. publ. Hlth., 25: 156.

HARDENBURG, W. E. 1922. Mosquito Eradication. MeGraw Hill Book Co., New York.

HaRgreaves, H. 1936. Annual Report of the Government Entomologist for 1935. Rep. Med. Uganda 1935, p. 69.

HaRkins, W. D. and Davies, E. C. H. 1917. The Orientation of Molecules in the Surface of Liquids. J. Amer. chem. Soc., 39: 541.

Hasell, P. G. and Weathersbee, A. A. 1938. A Preliminary Report on the Manufacture and Use of Small Bore Butt Joint Tile for Dewatering Drainage Ditches. (Unpublished report.)

HATCHER, R. A. and GoLD, H. 1927. IV. Studies on Quinine. J. Pharmacol., 30: 347.

Hatcher, R. A. and WeIss, S. 1926. Studies on Quinine. J. Pharmacol., 29: 279.

Headlee, T. J. 1932. The Development of Mechanical Equipment for Sampling the Mosquito Fauna and Some Results of its Use. Proc. N.J. Mosq. Ext. Ass., 19: 106.

Неснт, G. 1933. Pharmakologisches über Atebrin. Arch. Exp. Path. Pharmak., 170: 328.

-. 1936. Die Verteilung des Atebrins im Organismus. Arch. Exp. Path. Pharmak., 183: 87.

HEGNER, R. W. 1938. Relative Frequency of RingStage Plasmodia in Reticulocytes and Mature Erythrocytes in Man and Monkey. Amer. J. Hyg., 27: 690.

Hegner, R. W. and Eskridge, L. 1938. Passive Immunity in Avian Malaria. Amer. J. Hyg., 28: 367.

HegNeR, R. W., SHAw, E. H., JR. and MANWELL, R. D. 1928. Methods and Results of Experiments on the Effects of Drugs on Bird Malaria. Amer. J. Hyg., 8: 564.

HeHIR, P. 1927. Malaria in India. Oxford Univ. Press, London.

HELPER, M. 1934. An Epidemic of EstivoAutumnal and Quartan Malaria Among Drug Addicts in New York City Transmitted by the Use of Contaminated Syringes. Publ. Hlth. Rep., Wash., 49: 421.

Henry, A. F. X. 1927. Contribution à l'étude Sérologique de l'infection Palustre. Rapport presenté au 5le Congrès de l'association français pour l'advancement des Sciences. Gaz. hebd. Sci. méd., 48: 311.

-1934. Seroflokkulation bei Malaria. Arch. Schiffs. TropenHyg., 38: 93.

HERMS, W. B. 1919. Occurrence of Malaria and Anopheline Mosquitoes in Northern California. Publ. Hith. Rep., Wash., 34: 1579.

- 1929. Anopheline Mosquito Investigations in California. 4th Int. Congress of Ent. Trans., 2: 708 .
Macmillan Co., New York.

Herms, W. B. and Freeborn, S. B. 1920. The Egg Laying Habits of Californian Anophelines. J. Parasit., 7: 69.

Herms, W. B. and Frost, F. M. 1932. A Comparative Study of the Eggs of Californian Anophelines. J. Parasit., 18: 240.

HeRMS, W. B. and GRAY, H. F. 1940. Mosquito Control. Practical Methods for Abatement of Disease Vectors and Pests. Commonwealth Fund, New York.

Herms, W. B. and Hewite, R. 1940. Bird Malaria. Amer. J. Hyg. Mon. Ser. No. 15. Johns Hopkins Press, Baltimore.

HewitT, R. 1940. Bird Malaria. Amer. J. Hyg., Mon. Ser. 15. Johns Hopkins Press, Baltimore. Hildebrand, S. F. 1921. Top Minnows in Relation to Malaria Control, with Notes on Their Habits and Distribution. Publ. Hlth. Bull., Wash., 114: 1.

Hill, R. A. and Goodwin, M. H., JR. 1937. "Prontosil" in Treatment of Malaria; Report of 100 Cases. Sth. Med.J., 30: 1170.

HrLL, R. B. 1934. Feeding Habits of Some Venezuelan Anopheles. Amer. J. trop. Med., 14: 425. 1937. The Malaria Problem in Portugal. Sth. Med. J., 30: 953.

HimmelsbacH, C. K. 1933. Malaria in Narcotic Addicts at United States Penitentiary Annex, Fort Leavenworth, Kansas. Publ. Hlth. Rep., Wash., 48: 1465 .

Hinman, E. H. 1932. The Role of Solutes and Colloids in the Nutrition of Anopheline Larvae. Amer. J. trop. Med., 12: 263.

- 1934a. Predators of the Culicidae (Mosquitoes) I. The Predators of Larvae and Pupae, Exclusive of Fish. J. trop. Med. (Hyg.), 37: 129.

- 1934b. Predators of the Culicidae (Mosquitoes) I. The Predators of Larvae and Pupae, Exclusive of Fish. J. trop. Med. (Hyg.), 37: 129. II. Predators of Adult Mosquitoes, $37: 145$.

1934c. Preliminary Observations on the "Hibernation" "of Anopheles quadrimaculatus in Southern Louisiana. Sth. Med. J., 27: 461.

- 1938. Biological Effects of Fluctuation of Water Level on Anopheline Breeding. Amer. J. trop. Med., 18: 483.

Hinman, E. H. and Hurlbut, H. S. 1940. A Study of Winter Activities and Hibernation of Anopheles quadrimaculatus in the Tennessee Valley. Amer. J. trop. Med., 20: 431.

. 1941. A Collection of Anopheline Mosquitoes from Southern Ontario. Canad. Ent. (In press.)

HIRsch, August. 1883. Handbook of Geographical and Historical Pathology. Translation by Charles Creighton, Vol. I, p. 197. New Sydenham Society, London. 
HIRShbERG, L. K. 1904. An Anopheles Mosquito Which Does Not Transmit Malaria. Johns Hopk. Hosp. Bull., 15: 53.

HODGKIN, E. P. 1938. Naturalistic Methods of Malaria Control. J. Malaya Br. Brit. med. Ass., 2: 24.

Hofrman, W. A. 1926. Resting Position of Haitian Anopheles. Amer. J. trop. Med., 6: 377. 1930. From San Juan to Aruba. Puerto Rico J. Publ. Hlth., 5: 357.

-1940. Personal Communications.

Hofrmann, C. C. 1928. Contribucion al Conocimiento del Paludismo y de sus Transmisores en el Estado de Sinaloa. Bol. Dept. Sal. Pub., 1: 31.

1929a. Los Mosquitos Transmisores Sal. Pub., 2: 11.

- 1929b. La Distribucion Geografica de los Mosquitos Anopheles en el Estado de Veracruz. Dept. Sal. Publ., Mexico, 76 pp.

-1932. On Anopheles pseudopunctipen$n i s$ and its Relation to Malaria in Mexico. Sth. Med. J., 25: 523.

-1934. Contribucion al Conocimiento del Paludismo en la Peninsula de Yucatan. Bol. Inst. Hig. Mex., 2: 57.

-1935. La Formacion de Razas en los Anopheles Mexicanos. I. A. maculipennis y $A$. quadrimaculatus y una Raza Nueva del maculipennis. Ann. Inst. Biol. Univ. Mex., 6: 3.

1936. Algunas Palabras Acerca de las

Razas Americanas del Anopheles maculipennis Meigen. Rev. Paras. Clin. Lab., 2: 403.

- 1937. Contribucion al Conocimiento de los Mosquitos del Valle del Mezquital. Ann. Inst. Biol. Mex., 8: 207.

- 1938a. La Formacion de Razas en los Anopheles Mexicanos. II. Anopheles albimanus y sus Variedades en la Republica Mexicana. Ann. Inst. Biol. Mex., $9: 167$.

-1938b. Los Criaderos Invernales de Anopheles pseudopunctipennis en el Estado de Oaxaca. Ann. Inst. Btol. Mex., 9: 181.

-1938c. Nota Acerca de los Criaderos Invernales de Anopheles albimanus Wied. on los Pantanos de Veracruz. Ann. Inst. Biol. Mex., 9: 193.

Hormann, C. C. and Samano, B. A. 1938. Los Criaderos Invernales de Anopheles pseudopunctipennis en el Estado de Oaxaca. Amer. Inst. Biol. Mex., 9: 181.

Hogstad, A. 1931. The Three Hundredth Anniversary of the First Recognized Use of Cinchona. Mo. Botanical Garden Bull., 18 No. 9, 141.

Hoops, G. L. 1934. The History of Malaria. Malay. Med. J., 9: 123.

Hopkins, G. H. E. 1940. Afforestation as a Method of Drying up Swamps. E. Afr. med. J., $17: 189$.

HowARD, L. O. 1892. An Experiment Against Mosquitoes. Insect Life, 5: 12.

- 1900. Notes on the Mosquitoes of the
United States. U. S. Dept. of Agri., Div. Ento., Bull. 25, N. S.

1901. Mosquitoes. MeClure, Phillips \& Co., New York.

. 1916. Mosquitoes and Bats. Proc. N. J. Mosq. Ext. Ass., 3: 69.

Howard, L. O., DyaR, H. G. and KNAB, F. 19121917a. The Mosquitoes of North and Central America and the West Indies. Systematic Description. Part II. 4: 525. Carnegie Institution, Wash.

1912b. The Mosquitoes of North and Central America and the West Indies. Vol. 1: p. 444. Carnegie Institution, Wash.

p. 1917b. The Mosquitoes of North and Central America and the West Indies. Vol. 4. Carnegie Institution, Wash.

How ARD, R. S., JR. and ANDREWS, J. 1940. Studies on the Concentration and Distribution of Paris Green-Lime Mixtures Applied as Anopheles Larvicides. In press.

HoYt, R. N. 1935. Malaria Epidemic in Aurora, Ohio. Publ. Hlth. Rep., Wash., 50: 895.

Hoyt, R. N. and Worden, R. E. 1935. A Report of an Outbreak of Malaria in Aurora, Ohio. Publ. Hlth. Rep., Wash., 50: 895.

HUFr, C. G. 1927. Studies on the Infectivity of Plasmodia of Birds for Mosquitoes, with Special Reference to the Problem of Immunity in the Mosquito. Amer. J. Hyg., 7: 706.

- 1929. The Effects of Selection upon Susceptibility to Bird Malaria in Culex pipiens Linn. Ann. trop. Med. Parasit., 23: 427.

-1930. Individual Immunity and Sus. ceptibility of Culex pipiens to Various Species of Bird Malaria as Studied by Means of Double Infectious Feedings. Amer. J. Hyg., 12: 424.

- 1931. The Inheritance of Natural Immunity to Plasmodium cathemerium in Two Species of Culex. J. prev. Med., 5 : 249.

- 1934. Comparative Studies on Susceptible and Insusceptible Culex pipiens in Relation to Infections with Plasmodium cathemerium and P. relictum. Amer. J. Hyg., 19: 123.

1940. Quantitative Studies on Size, Variability and Growth Rates of Oöcysts of Different Strains of Avian Malaria. Amer. J. Hyg., 32C: 71.

1941. Comparative Importance of Various Factors Upon the Regulation of Size of Avian Malarial Oöcysts in Mosquitoes. Amer. $J$. Hyg. In press.

Hulse, F. E. 1922. Sketch Mapping for Public Health Problems. Amer. J. publ. Hlth., 12: 1034.

Humboldt, A. voN. 1852. Personal Narrative of Travels to the Equinoctial Regions of America During the Years 1799-1804. Trans. by Thomasina Ross. Vol. 2; p. 312. Bell and Daldy, London.

HuRlBUt, H. S. 1938a. A Study of the Larval Chaetotaxy of Anopheles walkeri Theobald. Amer. J. Hyg., 28: 149. 
tering of the Eggs of Anopheles walkeri Theobald, with a Description of the Eggs. J. Parasit., 24: 521.

Hutron, E. L. and Shute, P. G. 1939. The Risk of Transmitting Malaria by Blood Transfusion. J. trop. Med. (Hyg.), 42: 309.

HrLkema, B. 1920. The Development of the Parasites of Quartan Malaria in the Myzomia Ludlowi and Their Transmission on Man. Meded. burgerl. geneesk. ienst. Ned.-Ind., 6: 50.

IrEDELL, A. W. 1933. An Account of MosquitoProofing Carried Out by the Royal Air Force in India. J. R. Army med. Cps., 60: 33.

IRELAND, M. W. 1925. Medical and Casualty Statistics. Medical Department of the U. S. Army in the World War. 15, Pt. 2.

Iranic, M. 1935. Ueber die zwei Allerfrühesten Kernteilungsstadien des Tertianaparasiten (Plasmodium vivax Grassi et Feletti) und deren Bedetung. Zbl. Bakt., Orig., 133: 274.

- 1937. Neue Beitrage zur Kenntnis der Promitotischen Kernteilung beim Tertianaparasiten (Plasmodium vivax Grassi et Feletti). Z Zbl. Bakt., Orig., 138: 254.

IYENGAR, M. O. T. 1932. Experimental Infection of Anopheline Mosquitoes. Indian J. med. Res., $20: 841$.

JACKson, R. 1791. A Treatise on the Fevers of Jamaica, with Some abservations on the Intermitting Fevers of America. Printed for J. Murray, London.

JACOBSthal, E. 1936. Die Infektion der Reticulocytem durch Malaria Plasmodien. Klin. Wschr., 15: 942 .

JAMES, J. F. 1935. Fumigation and Trapping of Mosquitoes. J.R. Army med. Cps., 65: 267.

JAMES, S. P. 1922. Malaria: Pathology. In The Practice of Medicine in the Tropies, vol. II. Byam and Archibald, London.

- 1926. Report on the First Laboratory Work in Malaria in England. L. o. N. Health Org. Mal. Comm., Geneva.

- 1929. The Disappearance of Malaria from England. Proc. Roy. Soc. Med., 23: 1.

- 1931. Some General Results of a Study of Induced Malaria in England. Trans. Roy. Soc. trop. Med. Hyg., 24: 477.

- 1933. Antimalarial Chemotherapeutic Tests at Devon Mental Hospital. Trans. Roy. Soc. trop. Med. Hyg., 27: 289.

- 1937. The Treatment of Malaria. Bull. Hlth. Org. L. o. N., 6: 899.

- 1939. The Incidence of Exo-erythrocytic Schizogony in $\boldsymbol{P}$. gallinaceum in Relation to the Mode of Infection. Trans. Boy. Soc. trop. Med. Hyg., 32: 743 .

JAMES, S. P. and Christophers, R. 1922. Malaria. Byam and Archibald's The Practice of Medicine in the Tropies. Henry Frowde and Hodder and Stoughton, London.

JAMES, S. P. and CrucA, M. 1938. Species and
Races of Human Malaria Parasites and a Note on Immunity. Acta con. Tertii Trop. Mal. Morb., 2: 269.

JAMES, S. P., NICOL, W. D. and ShUTE, P. G. 1927. Note on a New Procedure for Malaria Research. Trans. Roy. Soc. trop. Med. Hyg., 21: 233.

- 1932. A Study of Induced Malignant Tertian Malaria. Proc. Roy. Soc. Med., 25: 1153.

- 1933. Plasmodium ovale Stephens 1922. Parasit., 25: 87.

- 1936. Clinical and Parasitological Observations on induced Malaria. Proc. Roy. Soc. Med., 29: 27.

JAMES, S. P. and ShuTE, P. G. 1926. Report on the First Results of Laboratory Work on Malaria in England. Publ. Hlth. Org. L. o. N., 3: 57.

JAMES, W. M. 1910. Quartan Malaria and Its Parasite. Proc. Canal Zone med. Ass., 3: 1.

J ANscó, N. 1904. Zur Frage der Infektion des Anopheles claviger mit Malariaparasiten bei niederer Temperatur. Zbl. Bakt., Orig., 36: 624.

JeFFeries, L. D. 1936. Concrete Silo Staves Used to Pave Peoria Streambed. Engng. News Rec., Oct. 1,1936, p. 470.

JERACE, F. 1934. Osservazioni sui Rapporti tia Intensita dell Infezione, Durata deI Periodo di Incubazione, tipo Febbrile e Decorso Clinico della Malaria Umana Indotta con Anofeli o con Sangue. Riv. Malariol., 13: 694.

-1939. Raro reperto Ematico e Parassitario Nella Malaria Umana. (A Rare Blood and Parasite Finding in Human Malaria). Riv. Malariol., 18: 153.

Jirovec, O. and Cerny, W. 1932. Die Ergebnisse der Nuklealreaction bei Vogelmalaria. $\mathrm{Zbl}$. Bakt., Orig., 126: 181.

Jobrins, D. M. 1940. Ann. Rep. Gorgas Mem. Lab., 1939: 5. U. S. Gov. Print. Off., Washington.

Јонмs, F. M. 1931. Influence of Dextrose and of Low Temperatures on Preservation, Transportation and Viability of Malaria Parasites. Proc. Soc. exp. Biol., 28: 743.

Johnson, H. A. 1926. Occurrence of Anopheles vestitipennis in Puerto Rico. Amer. J. trop. Med., 6: 157.

- 1936. Notes on the Occurrence of $A$. walkeri. Sth. Med. J., $29: 856$.

1937. Attractivity of Light for Anopheles Mosquitoes. J. Tenn. Acad. Sci., 12: 104.

JoNEs, W. H. S. 1909. Malaria and Greek History. University Press, Manchester.

- 1923. Hippocrates, with an English Translation. V. 1, p. 181. Wm. Heinemann, London.

JoRDAN, J. 1937. Mosquito Larvicidal Measures. Chin. med. J., 51: 927.

JUAN, G. and ULLUA, A. DE. 1806. A Voyage to South America; Describing at Large the Spanish Cities, Towns, Provinces, ete., on that Extensive Continent. Trans. by John Adams, 4th ed. V. 2, Longman and Co., London. 
JUNIOR, P. and BRANDÃo, P. P. 1937. Insufficiencia Suprarenal no Impaludismo. Commentarios Acerca de 10 Casos Clinicos. Brasil-méd., 51: 1047. Abstr. Trop. Dis. Bull., 1938, 35: 657.

KALM, PETER. 1770. Travels in North America. The English Version of 1770 Revised from the Original Swedish by Adolph B. Benson. 1937. Wilson-Erickson, New York.

KAUDERS, O. 1927. Immunitätsstudien bei Impfmalaria. Zbl. Bakt., Orig., 104: 158.

KEDZIE, R. C. 1874. The Influence of Drainage on Public Health in Michigan. Trans. Amer. med. Ass., 25: 401.

Kehar, N. D. 1936. Some Physico-Chemical Factors and Their Relationship to Protein Fractions, Blood Cells and Parasite Counts, in the Blood Sera of Monkeys Infected with Plasmodium knowlesi. Rec. Malar. Surv. India, 6: 499.

- 1937. Cholesterin and Lecithin in Malaria. Rec. Malar. Surv. India, 7: 117.

KEHAR, N. D. and HARBHAGWAN, S. A. S. 1937. Sedimentation Rate of Erythrocytes in Human and Monkey Malaria. Rec. Malar. Surv. India, 7: 131 .

KHelevin, N. V. 1938. Sur les Lieux d'Hibernation des Anophèles dans la Nature Libre dans les Conditions de la Région d'Ivanovo. Med. Parasit., 7: 144.

KibBex, C. H. 1923. Malaria Control as Approached by an Alabama Industrial Corporation. Trans, 4th Ann. Conf. Malaria Field Workers. Publ. Hlth. Bull., Wash., 137: 25.

- 1925. Larvicides. Trans, 5th Conf. Malaria Field Workers. Publ. Hlth. Bull., Wash., 156: 141.

Kiker, C. C. and Breedlove, H. E. 1941. Mosquito-Proofing for Malaria Control from the Standpoint of Construction and Maintenance Cost. Amer. J. Hyg., (in press).

Kiker, C. C., FAIrer, C. D. and Flanaky, P. N. 1938. Further Observations on Airplane Dusting for Anopheles Larvae Control. Sth. Med. J., 31: 808 .

Kiker, C. C. and Stromeuist, W. G. 1939. Importance of Reservoir Preparation in Mosquito Control of Impounded Water. Sth. Med. J., 32 : Suppl. July issue, 60-71.

KIкUTH, W. 1932. Zur Weiterentwicklung Synthetisch Dargestellter Malariamittel; über die Chemotherapeutische Wirkung des Atebrin. Dtsch. med. Wschr., 58: 530.

KIKUTH, W. and GIovannolA, A. 1933. Zur Frage der Medikamentösen Malaria Prophylaxe auf Grund von Experimentellen Untersuchungen aus des Vogel Malaria Riv. Malariol., 12: 657.

KING, A. F. A. 1883. Inseets and Diseases-Mosquitoes and Malaria. Pop. Sci. Mon., 23: 644.

KING, W. V. 1916a. Anopheles punctipennis a Host of Tertian Malaria. Amer. J. trop. Dis., $3: 426$.

- 1916b. Experiments on the Development of Malaria Parasites in Three American Species of Anopheles. J. exp. Med., 23: 703.
- 1917. The Effect of Cold on the Malaria Parasites in the Mosquito Host. J. exp. Med., 25: 495.

-1921. Natural Malaria Infection in Anopheles Mosquitoes. Amer. J. trop. Med., 1: 39.

-1924. Anopheline Infection under Natural Conditions. Sth. Med. J., 17: 596.

1929. Additional Notes on the Infection of Anopheles with Malaria Parasites. Amer. J. Hyg., 10: 565 .

-1937. On the Distribution of Anopheles albimanus and its Occurrence in the United States. Sth. Med.J., 30: 943. (Reprint, p. 25.) - 1939. The Varieties of Anopheles crucians Wied. Amer. J. trop. Med., 19: 461.

KING, W. V. and Bradlex, G. H. 1926. Airplane Dusting in the Control of Malaria Mosquitoes. U. S. Dept. of Agrie., Dept. Circular 367, pp. $1-16$.

King, W. V., Bradley, G. H. and McNeel, T. E. 1937. Experiments in Florida in Repelling Mosquitoes by Outdoor Spraying. Proc. N.J. Mosq. ext. Ass., 24: 163.

- 1939. The Mosquitoes of the Southeastern States. U. S. Dept. Agric., Misc. Pub. No. 336, pp. 1-91.

King, W. V. and BuLL, C. G. 1923. The Blood Feeding Habits of Malaria-Carrying Mosquitoes. Amer. J. Hyg., 3: 497.

KInG, W. V. and McNeet, T. E. 1938. Experiments with Paris Green and Calcium Arsenite as Larvicides for Culicine Mosquitoes. $J$. econ. Ent., 31: 85 .

KingsburY, A. N. 1927. Some Investigations of Malarial Fevers. IV. The Complement Fixation Reaction. Trans. Roy. Soc. trop. Med. Hyg., 20: 359 .

-1934. Psychoses in Cases of Malaria Following Exhibition of Atebrin. Lancet, 2: 979.

Kirschbaum, W. 1923. Methoden und Kautelen einer Malaria-Blut-Konservierung und Versendung usw. Klin. Wschr., 2: 1404.

KITCHeN, S. F. 1938. The Infection of Reticulocytes by Plasmodium vivax. Amer. J. trop. Med., 18: 347 .

1939a. The Infection of Mature and Immature Erythrocytes by $P$. falciparum and $P$. malariae. Amer. J. trop. Med., 19: 47.

-1939b. The Differential Infection of Mature and Immature Erythrocytes by the Plasmodia of Human Malaria. Sth. Med. J., 32 : 679 .

- 1940. Personal communication.

Kitchen, S. F. and Bradley, G. H. 1936a. Anopheles walkeri Theobald as a Vector of Plasmodium falciparum Welch. Amer. J. trop. Med., 16: 579 .

- 1936b. Anopheles walkeri Theobald as a Vector of Plasmodium vivax Grassi and Feletti. Amer. J. Hyg., 17 : 515.

KrTchen, S. F., WebB, E. L. and KupPER, W. H. 
1939. The Influence of Malarial Infections on the Wassermann and Kahn Reactions. J. Amer. med. Ass., 112: 1443.

KLIGLER, I. J. 1930. Immunity to an Infection with Malaria Following Treatment. Trans. Roy. Soc. trop. Med. Hyg., 24: 331.

Kligler, I. J. and MER, G. 1933. The Development of Immunity Against Malaria in Children under 10 years of Age. Trans. Roy. Soc. trop. Med. Hyg., 27 : 269.

-1937. Studies on the Effect of Various Factors on the Infection Rate of Anopheles elutus with Different Species of Plasmodium. Ann. trop. Med. Parasit., 31: 71.

KNowles, R. and BAsU, B. C. 1935. Nuclear Division in Malarial Sporozoites. India $J$. med. Res., 22: 443.

Knowles, R. and DAS GuPTA, B. M. 1930. Studies in Untreated Malaria. 1. A Case of Experimentally Induced Quartan Malaria. In. dian med. Gaz., 65: 301.

KNowles, R. and SENIOR-WHite, R. 1927. Malaria: Its Investigation and Control with Special Reference to Indian Conditions. Thacker, Spink and Co., Calcutta.

KомP, W. H. W. 1926. Observations on Anopheles walkeri and Anopheles atropos. Insec. Inscit. Menst., 14: 168.

1929a. Observations on Malaria in the Panama Division, 1930. 18th Ann. Rep. United Fruit Co., Med. Dept., p. 65.

- 1929b. Observations on Malaria in the Chiriqui Land Company Division, 1930. 18th Ann. Rep. United Fruit Co. Med. Dept., p. 70. -1933. Additional Notes on the Preparation and Examination of Thick Blood Films for Malaria Diagnosis. Publ. Hlth. Rep., Wash., 48: 875 .

1936a. Anopheles (Anopheles) chiriquiensis, a New Species of Anopheles from Panama (Diptera, Culicidae). Proc. ent. Soc., Wash., 38: 156.

- 1936b. Anopheles (Anopheles) anomalophyllus, A New Species of Anopheles from Panama and Costa Rica (Dipt., Culicidea). Proc. ent. Soc., Wash., 38: 160.

- 1937a. The Species of the Subgenus Kerteszia of Anopheles. Ann. ent. Soc. Amer. $30: 492$.

—_. 1937b. Sth. Med. J., 30: 946. 1940a. The Oceurrence of Anopheles darlingi Root in British Honduras and Guatemala. Science, 91: 522.

- 1940b. Personal Communications.

Kомp, W. H. W. and ClaRK, H. C. 1936. A Fifth Year's Observation on Malaria in Panama with Reference to the Failure of Atebrin to Control and Epidemic. Amer. J. trop. Med., 16: 109.

Kоте, Chin. 1937. Pharmacology of Atebrin. Formosa, J. Amer. Med. Ass., 36: 138.

Kremer, B. I. and KuvichinsKII, B. S. 1937. Anabadust-nouvelle Méthode pour la Lutte
Contre les Moustiques Adultes. Med. Parasit., 6: 550 .

KRISHNAN, K. V. 1933. Observations on the Action of Quinine in Malaria. Indian J. med. Res., 21: 331 .

- 1939. Annual Report of the All-India Institute of Hygiene and Public Health. Calcutta.

KrishnaN, K. V., GHosh, B. M. and Bose, P. N. 1936. Cholesterol Changes in Monkey Malaria and Haemoglobinuria. Rec. Malar. Surv. India, $6: 1$.

KRITSchewski, I. L. and DemidowA, L. W. 1934. Ueber eine noch unbekannte Funktion des Retikuloendothelial Systems XXII. Ueber die Bedeutung des Retikuloendothelial Systems in der Therapie der Malaria. Z. ImmunForsch., 84: 14.

KRUSE, W. 1892. Der Gegenwartige Stand Unserer Kenntnisse von den Parasitaren Protozoen. Hyg. Rdsch., 2: 357-380 and 453-500.

KUMM, H. W. 1929a. Studies in the Dispersion of Anopheles Mosquitoes. Amer. J. trop. Med., $9: 67$.

1929b. The Geographical Distribution of the Malaria Carrying Mosquitoes. Amer. $J$. Hyg. Mon. Ser., No. 10: 1-178.

1932. Observations on two Malaria Vectors and Distribution Records of Other Anopheles in the States of Bahia and Sergipe, Brazil. Amer. J. trop. Med. Protoz., 26: 1.

- 1940a. The Eggs of Some Costa Rican Anophelines. (In press.)

E. Rozeboom.

1940c. Personal communication to Watson and Hewitt.

S. Simmons.

KUMM, H. W. and RUIz, H. 1928. See Covell, 1927.

-1939a. Informe anual del reconocimiento del paludismo y de mosquitos en la Republica de Costa Riea. Mem. Sec. Salub. Pub. Proteccion Social (1938), pp. 103-133.

1939b. A Malaria Survey of the Republic of Costa Rica. Amer. J. trop. Med., 19: 425.

KUMм, H. W., Kомp, W. H. W. and RUi, H. 1940. The Mosquitoes of Costa Rica. Amer. J. trop. Med., 20: 385 .

LABBE, A. 1894. Récherches Zoologiques et Biologiques sur les Parasites Endoglobulaires du Sang des Vertebrates. Arch. Zool. exp., 2: 55.

Lancisi. 1717. From Ross R., 1910. Prevention of Malaria.

LANGMUIR, I. 1917. The Constitution and Fundamental Properties of Solids and Liquids. $J$. Amer. chem. Soc., $39: 1848$.

Larde Arthes, C. R. 1921. Anofeles de El Salvador y Profilaxia Paludica. Tesis, Doct. Med. Univ. Salvador.

LAVERAN, A. 1881. Nature Parasitaire des Acci- 
dents de l'impaludisme; Description d'un Noveau Parasite Trouvé dans le Sang des Malades Atteintes de Fievre Palustre, 104 pp. Baillière et Cie, Paris.

- 1889. Des Hematozoaires du Paludisme. Arch. Med. exp., 1: 798.

- 1901. Essai de Classification des Hematozoaires Endoglobulaires ou Haemocytozoa. C. R. Soc. Biol., 53: 798.

1908. Paludisme et Trypanosomiase. Nouveau Traité de Médecine et de Therapeutique. J. B. Baillière et fils, Paris.

League of Nations. 1937. The Treatment of Malaria. Study of Synthetic Drugs, as Compared with Quinine, in the Therapeutics and Prophylaxis of Malaria. Fourth General Report of the Malaria Commission. Bull. Hlth. Org., L. o. N., 6: 897 .

Legwen, W. A. and Kirby, A. J. 1939. The Practical Application of $\mathrm{pH}$ on Larvicidal Operations. Supplement to Symposium on Malaria, pp. 72-91, mimeographed. Tallahassee, Fla.

LeHeUX, J. W. and deLind van WrNdgaARden, C. 1928. Utber Plasmochin. Arch. exp. Path. TropenHyg., 128: 105.

- 1929. Uber die Pharmakologisches Wirkung des Plasmochins. Arch. exp. Path. TropenHyg., 144: 341.

LeISHMAN, W. 1912. The Etiology of Blackwater Fever. Trans. Roy. Soc. trop. Med. Hyg., 6: 1.

LePrince, J. A. 1926. Destroying Engorged Mosquitos as a Malaria Control Measure. Publ. Hlth. Rep., Wash., 41: 1220.

LePrince, J. A. and Grifritss, T. H. D. 1917. Flight of Mosquitos. Publ. Hlth. Rep., Wash., 32: 656

LePrince, J. A. and Johnson, H. A. 1929. Development of a Power Dusting Device for Applying Paris Green as an Anopheline Larvicide. Publ. Hlth. Rep., Wash., 44: 1001.

LePrince, J. A. and Orenstein, A. J. 1916. Mosquito Control in Panama. G. P. Putnam's Sons, New York and London.

LePrince, J. A., Orenstein, A. J. and Howard, L. O. 1916. Mosquito Control in Panama. G. P. Putnam's Sons, New York.

Lewkowicz, X. 1897. Ueber den Entwickelungsgang und die Einteilung der Malariaparasiten. Zbl. Bakt., Orig., 21: 130.

LischetTI, A. B. 1927. The Poisoning of Adult Anophelines. Soc. Argentina Patol Regional Norte, 1926.

LORANDO, N. and SOTERIADES, D. 1936. Immunity in Malaria. Therapeutic Results Obtained from Subcutaneous Injection of Immunized Blood. J. trop. Med., 39: 197.

- 1937. Treatment of Malaria with Immune Serum. Trans. Roy. Soc. trop. Med. Hyg., 31: 227.

LoURIE, E. M. 1934. Studies on Chemotherapy in Bird Malaria. II. Observations Bearing on the Mode of Action of Quinine. Ann. trop. Med. Parasit., 28: 255.
Ludlow, C. S. 1907. Mosquito Notes, No. 5. Canad. Ent., 39: 267.

LÜнE, M. 1900. Ergebnisse der Neuren Sporozoenforschung. G. Fischer, Jena.

MacCaLluM, W. G. 1897. On the Flagellated Form of the Malarial Parasite. Lancet, 2: 1240.

McCrachen, I. 1904. Anopheles in California with a Description of a New Species. Ent. News, 15: 9 .

MacCreary, D. 1939. Comparative Results Obtained by the Use of Several Mosquito Traps in a Limited Area. J. econ. Ent., 32: 216.

MacCreary, D. and Stearns, L. A. 1937. Mosquito Migration Across Delaware Bay. Proc. $N$. J. Mosq. ext. Ass., 24: 188.

MACCULlOCH, J. 1829. Malaria: An Essay on the Production and Propagation of this Poison, and on the Nature and Localities of the Places by Which It is Produced. Thomas Kite, Philadelphia.

MCDonald, G. 1939. A design of Flushing Siphon for Control of Anopheline Breeding. J. Malar. Inst. India, 2: 63.

MCFiE, J. W. 1917. Limitation of Kerosene as a Larvicide with Some Observations on the Cutaneous Respiration of Mosquito Larvae. Bull. ent. Res., 8: 277.

McGown, Thompson. 1849. A Practical Treatise on Most Common Diseases of the South. Grigg, Elliot and Co., Philadelphia.

MacNay, C. G. 1939. Studies on Repellents for Biting Flies. Canad. Ent., 71: 38.

Madras Cinchona Commission 1867 and 1868. Cited from Dawson, 1930.

Magoon, E. H. 1935. A Portable Stable Trap for Capturing Mosquitos. Bull. ent. Res., 26: 363.

Magoon, E. H. and Molloy, D. M. 1936. Construcción de Secciones de Concreto Para la Pavimentación de Fosos o Zanjas de Desaqüe. Bol. Ofic. sanit. Pan-amer., 15: 944.

MaLaret, P. S. 1929. The Control of Malaria in the Preston Division. 18th Ann. Rep. United Fruit Co. Med. Dept., 83.

Manalane, C. 1931. Malaria Transmission in the Philippines. II. Philipp. J. Sci., 45: 367.

MANIFOLD, J. A. 1939. Comments on R. Christophers" "Malaria in War." Trans. Roy Soc. trop. Med. Hyg., 33: 272.

MANNABERG, J. 1905. Malaria. Nothangel's Practice. English Trans. W. B. Saunders, Philadelphia.

Mansell, R. A. 1930. Note on Experiments Toward the Production of a Cheap Insecticide for Anti-mosquito Work. J. R. Army med. Cps., $54: 123$.

Manson-BaHR, P. H. 1931. Manson's Tropical Diseases, 9th Ed. William Wood and Co., New York.

MaNWell, R. D. 1934. Quinine and Plasmochin Therapy in Infections with Plasmodium circumflexum. Amer. J. trop. Med., 12: 123.

Manwell, R. D. and Goldstein, F. 1940. Pag- 
sive Immunity in Avian Malaria. J. exp. Med., $71: 409$.

MaRchand, F. 1924. Die örtlichen Reaktiven Vorgänge (Lehre von der Entzündung). In L. Krehl and F. Marchand. Hand. allgem. Path., 4: 78.

Marchiafada, E. 1931. Pernicious Malaria. Amer. J. Hyg., 13: 1.

Marchiafava, E. and Bignami, A. 1894. Malaria. New Sydenham Society, London.

1901. Malaria, Twentieth Century Practice of Medicine. William Wood and Co., New York.

Marchiafava, E. and Celli, A. 1885. Weitere untersuchungen über die Malariainfection. Fortschr. Med., 3: 787.

- 1887. Sulla Infezione Malarica. Atti R. Accad. Med. Roma, 13: 1 .

Marchoux, E. and Chorine, V. 1931. Fecondation des Gametes d'Hemoproteus paddae. Conditions Nécessaires et Suffisantes pour qu'elle se Produise. C. R. Soc. Biol., 106: 530 .

Markham, Clements R. 1880. Peruvian Bark. J. Murray, London.

Marotta, G. and Sandicchi, G. 1939. Contributo all'infezione Sperimentale di Anofili con Plasmodium malariae e Inoculazione della Malattia All'uomo. Riv. Malariol., 18: 89.

Martin, S. J., Cominole, B. and Clark, B. B. 1939. Chronic Oral Administration of Atabrin. J. Pharmacol., 65: 156.

Martini, E. 1933. The Hypopygia of Certain Anophelines (Diptera: Culicidae). Proc. ent. Soc. Wash., 35: 61.

- 1935. Los Mosquitos de Mexico. Bol. Tec. Dept. Salub. Publ. Mexico, 1: 65.

Martirano, F. 1902. La Malaria nel Mezzogiorno d'Italia. Atti Soc. Studi Malaria Roma, $3: 475$.

Marzinowskr, E. J. 1916. De differentes espëces du parasite de la Malaria. Ann. Inst. Pasteur, $30: 243$.

Matheson, R. 1929. A Handbook of the Mosquitoes of North America. C. C. Thomas, Springfield, Ill.

- 1930. The Utilization of Aquatic Plants as Aids in Mosquito Control. Amer. Nat., 64: 56.

- 1932. Medical Entomology. C. C. Thomas, Springfield, Ill.

Matheson, R., Boyd, M. F. and StratmanTномаs, W. 1933. Anopheles walkeri Theobald, as Vector of Plasmodium vivax Grassi and Feletti. Amer. J. Hyg., 17: 515.

Matheson, R. and HURLbUt, H. S. 1937. Notes on Anopheles walkeri Theobald. Amer. J. trop. Med., 17: 237.

Maxcr, K. F. 1923. The Distribution of Malaria in the United States as Indicated by Mortality Reports. Publ. Hlth. Rep., Wash., 38: 1125.

- 1928. Limitations to the Use of Quinine Intravenously in the Treatment of Malaria. J. Amer. med. Ass., 91: 1372.
Maximow, A. 1927a. Bindegewebe und Blutbildende Gewebe. In W. v. Möllendorff. Handb. mikr. Anat. Menschen, 2: 232.

- 1927b. Morphology of the Mesenchymal Reactions. Arch. Path. Lab. Med., 4: 557.

MAYNE,1 B. 1919. Infeetivity of Anopheles crucians in Nature. Publ. Hlth. Rep., Wash., 34: 1355.

- 1922. How Long Does a Mosquito Retain Malaria Parasites? Publ. Hlth. Rep., Wash., 37: 1059.

- 1926a. Notes on the Influence of Temperature and Humidity on Oviposition and Early Life of Anopheles. Publ. Hlth. Rep., Wash., 41: 986.

- 1926b. Report of a Survey to Determine the Malaria Prevalence in the Okefenokee Swamp. Publ. Hlth. Rep., Wash., 41: 1652.

1930a. Tests on the Effects of Coumarin on the Life of the Mosquito and the Malaria Parasite. Indian J. med. Res., 17: 963. - 1930b. A Study of the Influence of Relative Humidity on the Life and Infectibility of the Mosquito. Indian J. med. Res., 17: 1119. - 1932. Note on Experimental Infection of Anopheles punctipennis with Quartan Malaria. Publ. Hlth. Rep., Wash., 47: 1771.

Mayne, B. and Griffitts, T. H. D. 1931. Anopheles atropos D. and K.- a New Potential Carrier of Malaria Organisms. Publ. Hlth. Rep., Wash., 46: 3107.

MaYne, B. and YounG, M. D. 1938. Antagonism between Species of Malaria Parasites in Induced Mixed Infections. Publ. Hlth. Rep., Wash., 53: 1289.

Melener, H. E. 1937. The Problem of Malaria Mortality in the United States. Amer. J. trop. Med., 17: 15 .

Melenet, H. E., Bishop, E. L. and Roberts, F. L. 1929. Observations on the Malaria Problem of West Tennessee. Sth. Med. J., 22: 382.

Melenet, H. W. and Crabtree, J. A. 1934. Results from Screening Rural Homes in Lake County, Tennessee. Sth. Med. J., 27:552.

MER, G. 1933. Observations on the Development of Plasmodium malariae Lav. in Anopheles elutus Edw. Experimental Transmission of Quartan Malaria to Man from the Mosquito. Ann. trop. Med. Parasit., 27: 483.

MERMoD, C. and Dock, W. 1935. Fragility and Maturation of Reticulocytes. Arch. Intern. Med., 55: 52.

MerritT, J. King. 1861. Quinine as a Prophylactic in Malarious Regions. Am. med. Times, $3: 305$.

Messini, M. 1928. Die Wirkung des Plasmochin auf Hemoglobinemia in vivo. Bull. Soc. ital. Biol. sper., 3 : 1123.

Metchnikofr, E. 1887. A Contribution to the Study of Malaria. Russk. Meditsina, 12: 207.

- 1892. Leçons sur la Pathologie Comparée de I'Inflammation. G. Masson, Paris.

1 Before 1919, Mitzmain. 
Metz, C. W. 1918. Anopheles crucians, Habits of Larvae and Adults. Publ. Hlth. Rep., Wash., 33: 2156.

- 1919a. Observations on the Food of Anopheles Larvae. Publ. Hlth. Rep., Wash., 34; 1783 .

- 1919b. Some Aspects of Malaria Control Through Eradication. Publ. Hlth. Rep., Wash., 34: 167.

- 1919c. Anopheles crucians Wied as an Agent in Malaria Transmission. Publ. Hlth. Rep., Wash., 34: 1375.

Michelson, L. and Wilcox, A. 1940. A Rapid Thick Film Blood Stain. Publ. Hlth. Rep., Wash., 55: 1221.

Mretzch, F. and Mauss, H. 1933. Atebrin, ein Neues Heilmittel gegen Malaria. Klin. Wschr., 12: 1276 .

Middleton, W. S. 1928. The Yellow Fever Epidemic of 1793 in Philadelphia. Ann. med. Hist., $10: 434$.

Milam, D. F. and Coggeshall, L. T. 1938. Duration of $P$. knowlesi Infections in Man. Amer. J. trop. Med., 18: 331.

MudM, D. F. and KuscH, E. 1938. Observations on P. knowlesi Malaria in General Paresis. Sth. Med. J., 31: 947.

Mink, O. J. 1933. Mosquito Control in Haiti. U. S. Nav. Med. Bull., 31: 323.

MirA, M. G. 1936. La Malaria en Guatemala. Estudios Epidemiologicos y Desarrolo de la Compana Antipaludica. Edizioni " Riv. di Malariol.", Stamperia Moderna S. A., Rome.

Missiroli, A. 1917. Nuove Richerche Sulla Volatilizzatione Fermentiva Dell'arsenico. Boll. $R$. Acad. Med. Roma, 43: Fase. V. (Quoted from Hackett 1925).

- 1932. Tipi Epidemici delle Febbri Malariche. Riv. Malariol., 11: 1.

- 1934. Sullo Svilluppo dei Parassiti Malarici, Nota 2 2a. Riv. Malariol., 13: 539.

1939. Sullo Svilluppo dei Parassiti Malarici, Nota 5. Riv. Parassit., 3: 339.

MrtzMaIn, ${ }^{2}$ M. B. 1916a. Anopheles punctipennis Say-Its Relation to the Transmission of Malaria. Publ. Hlth. Rep., Wash., 31: 301.

- 1916b. Anopheles crucians-Their Infectibility with the Parasite of Tertian Malaria. Publ. Hith. Rep., Wash., 31: 764.

- 1916c. Tertian Malarial Fever-Transmission Experiments with Anopheles punctipen. nis. Publ. Hlth. Rep., Wash., 31: 1172.

- 1916d. Anopheles Infectivity Experiments. Publ. Hlth. Rep., Wash., 31: 2325.

-1916e. Is Mosquito of Man the Winter Carrier of Malaria Organisms P Pub. Hlth. Bull., Wash., 84: 1.

-1917a. Anopheline Mosquitoes, Their Distribution and Infectivity Under Natural Conditions. Publ. Hlth. Rep., Wash., 32: 536.

- 1917b. The Malaria Parasite in the

2 After 1918, Mayne.
Mosquito. The Effects of Low Temperature and Other Factors on its Development. Publ. Hlth. Rep., Wash., 32: 1400.

- 1919. Hibernation. Trans. First Ann. Conf. of Sanitary Engineers and Other Officers of the Public Health Service Directing Antimalaria Campaign. Publ. Hlth. Bull., Wash., 104: 96.

Molloy, D. M. 1932. Sth. Med. J., 25: 528.

Montestruc, E. 1936. Le Paludismo à la Martinique. Bull. Soc. Path. exot., 29: 193.

Moon, V. H. 1939. The Occurrence and Clinical Significance of Hemoconcentration. Ann. intern. Med., 13: 451.

Mosna, E. 1938. Contributo allo Studio dell' Immunita nell' Infezione Malarica. Riv. Parassit., $2: 327$.

Most, H. 1940a. Malignant Malaria Among Drug Addicts. Epidemiological, Clinical and Laboratory Studies. Trans. Roy. Soc. trop. Med. Hyg., 34: 139.

- 1940b. Falciparum Malaria in Drug Addicts. Amer. J. trop. Med., 20: 551.

MоттA, G. 1937. Il Comportamento dell' elettrocardiogramma nel Coniglio in Seguito ad Iniezioni Intramuscolari di Atabrin. Riv. Malariol., 16: 1 .

MulHern, T. D. 1940. Summary of Mosquito Control Accomplishments in N. J. in 1939. Proc. N. J. Mosq. ext. Ass., 27: 53.

Mulligan, H. W. 1929. Studies of the Reticuloendothelial System with Special Reference to Malaria. Part II. The Large Mononuclear Cells in the Peripheral Blood in Malaria. Indian J. med. Res., 16: 1107.

MUlligan, H. W. and MaJid, A. 1932. Some Experiments to Determine the Efficacy of Wire Gauze Screens of Different Apertures in Excluding Anophele Mosquitoes in India. Reo. Malar. Surv. India, 3: 163.

- 1936. Some Notes on the Care, Transportation, and Use of Gambusia affinis under Indian Conditions. Rec. Malar. Surv. India, 6: 537.

Mulligan, H. W. and Russell, P. F. 1940. Ag. glutination of Sporozoites of $P$. gallinaceum. J. Malar. Inst. India, 3: 199.

MURRAY, D. R. P. 1939. Problems Concerning the Efficieney of Oils as Mosquito Larvicides. II. Spreading Power of Oils and Methods of Increasing It. Bull. ent. Res., 30: 173.

NABoKov, V. A. 1929. Sur la Desinsectisation \&े Froid par l'anhydride Sulfuleux. Russ. J. trop. Med., $7: 349$.

Nabokov, V. A. and TiburskayA, N. A. 1936. On the Use of Pyrethrum in Insect Control. Med. Parasit., 4: 486.

NATALI, C. 1934. Histologische Untersuchungen über Nebennierenveränderungen bei Einem Fall von Malaria Tropica und Vergleichende Untersuchungen an Nebennieren Experimentell Infizierter Affen (Plasmodium knowlesi). Arch. Schiffs. Tropen Hyg., 38: 243. 
Nerva, A. and Pinto, C. 1922. Consideracães Sobre o Genero "Cellia" Theobald, com a Descripção de Una Nova Especie. Brazil-méd, 2: 355

NELSON, E. E. 1927a. Studies on Quinine and Quinidine. II. Their Action upon the Blood Vessels. Arch. int. Pharmacodyn., 33: 186.

-1927b. Studies on Quinine and Quinidine. III. The Antagonism of Quinine and Quinidine for the Circulatory Action of Epinephrine. Arch. int. Pharmacodyn., 33: 198.

Nicolaew, B. P. and YakoWleWA, W. W. 1929. Le sort des Formes Sexuées du Plasmodium vivax dans le Cavité Abdominale des Culex, Theobaldia, et Aëdes. Russian J. trop. Med., 7: 581 .

Nicholas, W. A. 1939. A Four-Day Automatic Sluice. J. Malaya Br. Brit. med. Ass., 3: 44.

Nicholls, L. 1912. Some Observations on the Bionomics and Breeding Places of Anopheles in St. Lucia, British West Indies. Bull. ent. Res., 3: 251.

NiJkamp, J. A. and Swellengrebel, N. H. 1934. Experiments on the Destruction of Anopheles. with Preparations of Pyrethrum. Med. Tijdschr. Geneesk., 78: 2327.

Nocht, B. and MAYER, M. 1937. Malaria. John Bale, London.

NoтT, I. C. 1848. Yellow Fever Contrasted with Bilious Fever, etc. New Orleans Med. surg. J., 4: 563 .

Osler, WILliam. 1923. The Evolution of Modern Medicine, p. 223. Yale Univ. Press, New Haven.

OverbeeK, J. G. and Stoker, W. J. 1938. Malaria in the Netherlands Indies and Its Control. J. Malaya Br. Brit. med. Ass., 1: 281.

OWEN, W. B. 1937. The Mosquitoes of Minnesota, with Special Reference to Their Biologies. Minn. Agr. Expt. Sta. Tech. Bull., 126: 1.

Paissead, G. and Lemaire, H. 1916. De l'insuffisance Surrénale dans le Paludisme. Pr. Méd., 24: 545 .

Pampana, E. J. and Fletcher, W. 1934. The Therapeutic Efficacy of Totaquina in Human Malaria. Quart. Bull. Hlth. Org. L. o. N., 3 : 325.

Panama Canal Health Department. 1933. Precast Concrete Sectional Inverts for Paving Drainage Ditches.

Parrot, L., Catanei, A. and Ambialet, R. 1937. Comparative Experiments in Mass Prophylaxis of Malaria by Means of Quinine and of Synthetic Drugs. Bull. Hlth. Org. L. o. N., 6: 683.

PatTy, R. L. 1936. The Relationship of Colloids in Soils to its Favorable Use in Pisé or Rammed Earth Walls. South Dakota Agri. Exper. Sta. Bull. 298.

Patty, R. L. and Minimum, L. W. 1933. Rammed Earth Walls for Buildings. South Dakota AgriExper. Sta. Bull. 27.

PAWAN, J. L. 1931. Feulgen Nucleal Reaction and the Malarial Nucleus. Ann. trop. Med. Parasit., 25: 185.

Paz Soldan, C. E. 1938. Las Tercianas del
Conde de Cinchon. Ediciones de La Reforma Medica, Lima.

Peckolt, T. 1899a. Heil und Nutzpflanzen Brasiliens. Ber. Deutsch. Pharm. Ges., 9: 226.

- 1899b. Heil und Nutzpflanzen Brasiliens. Ber. Deutsch. Pharm. Ges., 9 : 328.

Pereira Barretro, M. 1938. Observacōes Sobre a Ecologia do A. darlingi Root (1926) Var. paulistensis Galvão, Lane and Correia, 1937. Rev. Biol. Hyg., S. Paulo, 9: 116.

Perez, M. 1930. An Anopheline Survey of the State of Mississippi. Amer. J. Hyg., 11: 696.

Perrine, H. 1826. Fever Treated with Large Doses of Sulphate of Quinine in Adams County, near Natchez, Miss. Philad. J. med. phys. Sci., 13 (n.s., IV): 36.

Petrocchi, J. 1925. Descripcion de un Nuevo Anopheles. Rev. Inst, bact. Dept. Nac. Hig. Argent., 4: 69.

PEWNy, W. 1918. Prazipitinversuche bei Malaria. Wien. klin. Wschr., 31: 205.

Prvovarov, V. M. and Guterman, E. M. 1937. L'emploi de l'anabadust dans la Lutte Contre les Moustiques Hibernants. Med. Parasit., 6: 424.

PleHN, A. 1926. Immunisierung gegen Malaria. Arch. Schiffs. TropenHyg., 30: 18.

Pöch, R. 1903. Ueber das Verhalten der Weissen Blutkörperchen bei Malaria. Z. Hyg., Infektkr., 42: 563.

Polayes, S. H. and Derby, I. M. 1934. Blood Groups and Therapeutic Malaria. J. Amer. Med. Ass., 102: 1126.

Proske, H. O. and Watson, R. B. 1939. The Protein Tyrosin Reaction: A Biochemical Diagnostic Test for Malaria. Publ. Hlth. Rep., Wash., 54: 158 .

PURI, I. M. 1931. Larvae of Anopheline Mosquitoes, with Full Description of Those of the Indian Species. Indian Med. Res. Memoirs No. 21.

RADOSAVlJEvić, A. and Ristić, L. 1926. Ergebnisse der Senkungsreaktion und der Bluteiweiszbestimmung bei Malaria. Z. ges. exp. Med., 51: 48.

Rarraele, G. 1937a. Sullo Sviluppo Iniziale dei Parassiti Malarici Nell'ospite Vertebrato. Riv. Malariol., 16: 185.

- 1937b. Ricerche sul ciclo di Evoluzione Iniziale dei Parassiti Malarici Umani. Riv. Malariol., Sez. I: 413.

1939. Sulla Struttura dei Gameti Maschili dei Plasmodidi. Riv. Malariol. (Sez. I:) $18: 141$.

1940. Ulteriori ricerche sulla fase monogonica primaria dei plasmodidi nell'uomo e negli uccelli. Riv. Malariol., 19: 193.

RAFFAELE, G. and LEGA, G. 1937. Osservazioni su di un ceppo Etiopico di Plasmodium falciparum. Riv. Malariol., 16: 3 .

RAM, R. 1935. Anti-malarial Engineering. Control of Anopheline Larvae in Running Water by Means of Mechanical Devices for Flushing and Flooding. Indian Engr., 98: 122. 
RAMSAY, G. C. and CARPENTER, J. A. 1932. An Investigation on Petroleum Oils for Malaria Control Purposes. Rec. Malar. Surv. India, 3: 203.

RAMSAY, G. C. and MACDONALD, G. 1936. The Species Control of Anophelines in India. Indian med. Gaz., 71: 699 .

RANDLE, T. A. 1940. A New Development in Underground Drainage for Mosquito Control. Amer. J. publ. Hlth., 30: 736.

RAO, B. A. and SweET, W. C. 1937. Paris Green and Paddy. Rec. Malar. Surv. India, 7: 185.

RATCLIFFE, H. L. 1927. The Relation of Plas. modium praecox and Plasmodium vivax to the Red Cells of Their Respective Hosts as Determined by Sections of Blood Cells. Amer. J. trop. Med., 7 : 383.

- 1928. The Relation of Plasmodium faloiparum to the Human Red Blood Cell as Determined by Sections. Amer. J. trop. Med., 8: 559 .

RAYNAL, J. 1932. Note sur le Paludisme d'altitude. Marseille méd., 69: 245.

ReDMoNd, W. B. 1939. The Cross-Immune Relationship of Various Strains of Plasmodium cathe. merium and $P$. relictum. J. infect. Dis., 64: 273.

REED, A. C. 1940. The Treatment of Malaria. $J$. Amer. med. Ass., 115: 602.

Regulations Governing the Impounding of Waters. 1927. Alabama State Board of Health, Montgomery, Ala.

Report of Works Progress Administration Official Project 466-24-241, Lewes, Del., Mosquito Control Commission, Delaware, 1939.

Rice, J. L. 1936. Mosquito Control in New York City. Proc. N. J. Mosq. ext. Ass., 23: 174.

RICE, J. B. and BARBER, M. A. 1935. Malaria Studies in Greece. J. Lab. clin. Med., 20: 876.

ROBERTS, J. I. 1940. A New Malaria Parasite of Man in East Africa. E. Afr. Med. J., 17: 212.

Robertson, G. E. and Moore, W. 1923. Blackwater Fever. U. S. Nav. Med. Bull., 19: 413.

RoBson, G. C. and RICHARDs, O. W. 1936. The Variations of Animals in Nature. Longmans Green and Co., New York.

Rockefeller Foundation, International Health Board. 1924. The Use of Fish for Mosquito Control. Internat. Health Bd. of the Rockefeller Foundation, New York.

Ror, M. A., Linlie, R. D. and Wricox, A. 1940. American Azures in the Preparation of Satisfactory Giemsa Stains for Mrlaria Parasites. Publ. Hlth. Rep., Wash., 55: 1272.

Roz, M. A., Wrlcox, A. and LILliE, R. D. 1941. Eosinates of the Azures and Methylene Blue in the Preparation of a Satisfactory Giemsa Stain from Dyes of American Manufacture. (In press).

RoEEL, W, 1926. Die Wirkung des Plasmochins auf die Vogel Malaria. Arch. Schiffs. TropenHyg., 30: 311. (Suppl., p. 11.)

Roor, F. M. 1922. Notes on Mosquitoes and Other Blood-Sucking Flies from Porto Rico. Amer. $J$. Hyg., 2: 394. toes, Their Classification and Their Relation to the Transmission of Malaria. Proc. Inter. Conf. Health Problems Trop. Amer., United Fruit Co., Boston.

- 1924b. American Anopheline Mosquitoes, Their Classification and Their Relation to the Transmission of Malaria. Proc. Inter. Conf. Health Problems Trop. Amer., p. 149, United Fruit Co., Boston.

-1924c. Notes on Blood-sucking Arthropods Collected at.Tela, Honduras, and Port Limon, Costa Rica, during the Summer of 1924. 13th Ann. Rept. United Fruit Co., Med. Dept., p. 207, Boston.

1924d. Notes on the Mosquitoes of Lee County, Georgia. Amer. J. Hyg., 4 : 449.

- 1926. Studies on Brazilian Mosquitoes. I. The Anophelines of the Nyssorhynchus Group. Amer. J. Hyg., 6: 684.

- 1927. Note on the Mosquito Fauna of the Republic of Haiti. Amer. J. Hyg., 7: 463.

RooT, F. M. and ANDREWS, J. 1938. Malaria and Anopheline Survey of Grenada, B. W. I. Amer. J. Hyg., $27: 549$.

Ross, G. A. P. 1936. Report of the Pan-African Health Conference Held at Johannesburg, November 20-30, 1935. Quart. Bull. Hlth. Org. L. o. N., 5: 114 .

- 1938. La Destruction Automatique des Moustiques dans les Aéronefs et le Vecteur de ls Fièvre Jaune dans les Traversées Aériennes en Afrique. Bull. off. int. Hyg. Publ., 30: 2002.

Ross, G. 1903. The Improved Method of Microseopical Diagnosis of Intermittent Fever. Lancet, 164: 86 .

Ross, G. R. 1932. Researches on Blackwater Fever in Southern Rhodesia. London School of Hyg. and Trop. Med., Mem. 6. London.

Ross, R. 1897. On Some Peculiar Pigmented Cells Found in Two Mosquitoes Fed on Malarial Blood. Brit. med. J., 2: 1786.

- 1898. Pigmented Cells in Mosquitoes. Brit. med. J., 1: 550 .

- 1910. The Prevention of Malaria. E P. Dutton and Co., New York.

RoubaUd, E. 1920. Emploi du Trioxyméthylène en Poudre Pour la Destruction des Larves d'Anophèles. C. R. Aciad. Sci. Paris, 170: 1521; 171 : 51.

Row, R. 1931. Precipitin Reaction in Malarial Sera. Trans. Roy. Soc. trop. Med. Hyg., 24: 623.

RozeBoom, L. E. 1935. Infection of Anopheles bachmanni, Petrocchi, with Plasmodium vivax, Grassi and Feletti, and Observations on the Bionomics of the Species. Amer. J. trop. Med., 15: 521.

- 1936. The Life Cycle of Laboratory. bred Anopheles albimanus Wiedemann. Ann. ent. Soc. Amer., 29: 480.

-1937a. On Anopheles albitarsis Lynch Arribalzaga in Panama. Sth. Med. J., 30: 950. - 1937b. The Egg of Anopheles pseudopunctipennis in Panama. J. Parasit., 23: 538. 
1938a. The Role of Some Common Anopheline Mosquitoes of Panama in the Transmission of Malaria. Amer. J. trop. Med., 18: 289. . 1938b. The Eggs of the Nyssorhynchus Group of Anopheles (Culieidae) in Panama. Amer. J. Hyg., 27 : 95.

RozeBoom, L. E. and Gabaldon, A. 1941. A Summary of the "Tarsimaculatus" Complex of Anopheles (Diptera: Culicidae). Amer. J.Hyg., (In press).

Rubitschung, O. 1925. Die Wiederholung des Kurventyps der Leukozyten für akute Infektionen bei jedem Anfall von Malaria Tertiana. Arch. Schiffs. TropenHyg., 29: 217.

RUDOLFs, WILlem. 1923. Observations on the Relations Between Atmospheric Conditions and the Behavior of Mosquitoes. N. J. Agri. Expt. Sta. Bull., 388.

- 1926. Investigations of Mosquito Problems Carried on at the N. J. Agricultural Experiment Station During the Past Year. Proc. N.J. Mosq. ext. Ass., 13: 33.

-1930. Effeets of Chemicals upon the Behaviour of Mosquitoes. N. J. Agric. Exp. Sta. Bull., 496.

RUGE, H. 1935. Leberfunction bei Frischer Malaria. Arch. Schiffs. TropenHyg., 39: 14.

1939. Leberfunetion bei Friseher Malaria Nebst Einigen Klinisehen Bemerkungen. Z. klin. Med., 136: 311.

Russell, P. F. 1932. The Control of Anopheles minimus Mosquito Larvae in the Philippines by Stranding and Flushing. Philipp. J. Sci., 47: 439.

- 1934. Zooprophylaxis Failure, an Experiment in the Philippines. Rev. Malariol., 13: 610.

1935. The Spleen in Malaria Surveys. Amer. J. trop. Med., 15: 11.

Amer. 1936. A Survey of Malaria in Cyprus. Amer. J. trop. Med., 16: 431.

- 1938. Malaria Due to Defective and Untidy Irrigation-a Preliminary Discussion. J. Malar. Inst. India, 1: 339.

RUssell, P. F. and JACOB, V. P. 1939a. Some Experiments in the Naturalistic Control of Anopheles Breeding in Casuarina-pits. J. Malar. Inst. India, $2: 293$.

- 1939b. Some Experiments in the Use of Fish to Control Anopheles Breeding in Casuarina-pits. J. Malar. Inst. India, 2: 273.

Russell, P. F. and KNIPE, F. W. 1939. Malaria Control by Spray Killing Adult Mosquitoes. $J$. Malar. Inst. India, 2: 229.

Russer., P. F. and Moran, B. N. 1939. Experimental Infections in Anopheles stephensi (type) from Contrasting Larva Environments. Amer. J. Hyg., C, 30: 73 .

- 1940. Further Observations on Ex. perimental Malaria Infections in A. stephensi from Contrasting Larva Environments. Amer. J. Hyg., C, 31: 19.

- 1941. Further Observations on Experi- mental Malaria Infections in A. stephensi from Contrasting Sea-water and Tap-water Larva Environments. Amer. J.trop. Med. (In press.)

Russell, P. F. and Nono, A. M. 1934. A Mosquito Net for Use in the Philippine Islands. Experimental Studies and Canvass of Materials. Philipp. J. Sci., 53: 107.

Russell, P. F. and Santiago, D. 1934. An Earthlined Trap for Anopheline Mosquitoes. Proc. ent. Soc., Wash., 36: 1 .

Russo, G. 1927. Informe de Entomologia Agricola Dominicana. Inf. Estae. Nae, agron. Col. Agric. Moca, 2: 3 (Rev. appl. Ent. B., 20: 118.)

ST. Jонn, J. H. 1928. The Gametocytes of Tertian Malaria and Their Early Appearance in Malaria Transmitted by Anopheles punctipennis. Amer. J. trop. Med., 8: 305.

Salisbury, E. I. and Corrigan, J. A. 1927. Control of Malaria in Costa Riea. 18th Ann. Rept. United Fruit Co. Med. Dept., p. 88.

SAMBON, L. W. 1902. Remarks Coneerning the Nomenelature, Etiology, and Prophylaxis of the Intermittent Fevers. Brit. med. J., 2: 964.

SAPEIKA, N. 1934. The Action of Atebrin on the Uterus. Quart. J. Pharm., 7: 41.

ScharFF, J. W. 1935. Antimalaria Drainage from the Point of View of the Health Officer. Malay. med.J., 10 : 135.

Schaudinn, F. 1902. Studien über Krankheitserregende Protozoen. II. Plasmodium vivax Grassi u. Feletti der Erreger des Tertian Fiebers beim Mensehen. Arb. Gesundhamt, 19: 169.

Schuling, V. 1924. Malaria. In C. Mense. Handb. der Tropenkrankheiten, 1: 565-583 and 670-685. 3rd ed. J. A. Barth, Leipzig.

Schiluina, V., Jossmann, Horfmann, K., RUbitSCHUNG, O. and VAN DER SPEK. 1924. Biologisch-klinisehe Blutstudien über Allgemeine Infektionsfragen an der Impfmalaria der Paralytiker, besonders über ihre Unspezifische Wirksamkeit. Z. klin. Med., 100: 742 .

Scноо, H. J. M. 1903. La Malaria in Olanda nel 1902. Atti Soc. Studi Malaria Roma, 4: 85.

SCHÜ PFNER, W. A. P. 1938. Two Subjects Relating to the Epidemiology of Malaria. J. Malar. Inst. India, 1: 3 .

SchÜ FFNER, W. A. P., KorTEWEG, P. C. and SWELIJENGREBEL, N. H. 1929. Experimental Malaria with Protracted Incubation. Proc. Kon. Akad. Wetensch. Amsterdam, 32: 903.

SchÜFfNer, W. A. P. and Swellengrebel, N. H. 1938. Report for 1936 and 1937 of the Malaria Commission of the Health Couneil of Holland. Versl. Meded. Volksgezondh., July 1938.

Schulemann, W. 1940. Zur Pathologie der Malaria. Deutsche med. Wschr., 66: 404.

SchulemanN, W., SchozNHOEFER, F. and WINGLER, A. 1927. Plasmochin. Abh. Auslandsk., 26: 507.

BchulemanN, W. and SpIes, K. 1940. Zu Ursprung und Entwicklund der pigmentfreien formen der Malaria-Parasiten. Dtsch. med. Wschr., 15: 404 . 
Seagar, E. A. 1928. Malaria in Barbados. Trop. Agriculture, Trinidad, 5: 48. (Rev. appl. Ent. B., 16: 119).

Senevet, G. 1936. Les Moustiques de la Martinique. Arch. Inst. Pasteur Algér, 14: 123.

- 1938. Les Moustiques de la Guadeloupe. Arch. Inst. Pasteur Algér, 16: 176.

Senior-White, R. 1928. Physical Factors in Mosquito Ecology. Indian J. med. Res., 16: 11.

Sergent, Ed., Sergent, Et. and Catanei, A. 1929. Une Question de Nomenclature le nom de Plasmodium praecox Doit-il Désigner un Parasite du Paludisme Humain ou un Parasite du Paludisme Aviare 9 Arch. Inst. Pasteur Algér, 7: 223.

Sergent, Ed., Sergent, Et., Parrot, L. and Catanei, A. 1939. Nomenclature of the Malaria Parasites. Brit. Med. J., 1: 747 and Arch. Inst. Pasteur Algér, 17 : 242.

Sexparth, C. 1926. Die Malaria. In F. Henke and O. Lubarsch. Handbuch der speziellen pathologisehen Anatomie und Histologie, p. 178.

ShaFFER, G. O. 1911. How Contact Insecticides Kill. Mich. Agri. Exp. Sta. Tech. Bull. 11.

Shannon, R. C. 1930 . Observations on Anopheles pseuciopunctipennis in Peru. Amer. J. Hyg., 12 : 422.

-1933. Anophelines of the Amazon Valley. Proc. ent. Soc. Wash., 35: 117.

-1939. Methods for Collecting and Feeding Mosquitoes in Jungle Yellow Fever Studies. Amer. J. trop. Med., 19: 131.

Shannon, R. C. and Davis, N. C. 1930. Observations on the Anophelini (Culicidae) of Bahai, Brazil. Ann. ent. Soc. Amer., 23: 467.

Shannon, R. C., Davis, N. C. and deL Ponte, E. 1927. La Distribucion del Anopheles pseudopunctipennis y su Relacion con el Paludismo, en la Argentina. Rev. Inst. Bact., 4: 679.

SHAW, E. H. 1928. The Absorption of Chemical Compounds by Red Blood Corpuseles and Its Therapeutic Significance in the Treatment of Bird Malaria. Amer. J. Hyg., 8: 583.

ShaW, E. B. 1932. St. Croix's Rainiest Year Causes an Epidemic of Malaria. Science, 76: 566.

ShIELdS, S. E. 1938. Tennessee Valley Mosquito Collections. J. econ. Ent., 31: 426.

ShortT, H. E. and Menon, K. P. 1940. Experimental Production of Monkey and Avian Malaria by an Unusual Route in Infection. J. Malar. Inst. India, $3: 195$.

Shute, P. G. 1937. A Technique for the Inoculation of Known Numbers of Sporozoites as an Aid to Malarial Research. Ann. trop. Med. Parasit., 31 : 85 .

Simmons, J. S. 1936a. Anopheles (Anopheles) punctimacula Naturally Infected with Malaria plasmodia. Amer. J. trop. Med., 16: 105.

1936b. Anopheles (Anopheles) punctimacula (D. and K.) Experimentally Infected with Malaria plasmodia. Science, 83: 268.

- 1936c. The Infection of Anopheles (Anopheles) punctimacula with Malaria. Rev. Parasit., Clin. Lab., Habana, 2: 455. -1936d. Anopheles (Anopheles) neomaculipalpus Curry Experimentally Infected with Malaria plasmodia. Science, 84: 202.

- 1937. Observations on the Importance of Anopheles punctimacula as a Malaria Vector in Panama, and a Report of Experimental Infection in $A$. neomaculipalpus, $A$. apicimacula and A. eiseni. Amer. J. trop. Med., 17: 191.

. 1939a. Malaria in Panama. Johns Hopkins Press, Baltimore.

- 1939b. Observations on the Vectors of Malaria in Panama with Experimental Studies of A. punctimacula and Other Recently Incriminated Anophelines. Thesis Harvard School of Public Health.

Simmons, J. S., Callender, G. R., CURRY, D. P., Schwartz, S. C. and RANDALL, R. 1939. Malaria in Panama. Amer. J. Hyg., Mon. Ser. 13, $326 \mathrm{pp}$.

SineH, J, and SINeH, H. 1940. Agglutination Reactions with Plasmodium knowlesi. J. Malar. Inst. India, 3 : 53.

SintoN, J. A. 1930. A Suggested Standard Treatment of Malaria Based on the Results of the Controlled Investigations of Over 3,700 Cases. Indian med. Gaz., 65: 603.

- 1938. The Effect of Treatment upon the Development and Degree of Immunity Acquired in Malarial Infections. Acta Conv. Tertii Mal. Morbis, pars II, 312.

- 1939a. Malignant Tertian Parasite: Correction. Brit. med. J., 1: 146.

- 1939b. Studies of Infections with $P$. ovale. III. Resistance to the Inoculation of Sporozoites as Compared with Trophozoites. Trans. Roy. Soc. trop. Med. Hyg., 33: 305.

1940a. Studies of Infections with $P$. ovale. V. The Effects of Multiple Inoculations upon the Degree and Nature of the Immunity Developed. Trans. Roy. Soc. trop. Med. Hyg., 33 : 585 .

- 1940b. Studies of Infections with $P$. ovale. IV. The Efficacy and Nature of the Immunity Acquired as a Result of Infections Induced by Sporozoite Inoculations as Compared with Those by Trophozoite Injections. Trans. Roy. Soc. trop. Med. Hyg., 33: 439.

Sinton, J. A. and BANERJEA, A. C. 1925. Notes on the Thick Film Method of Examination of Malarial Parasites. Indian J. med. Res., 12: 537. Sinton, J. A. and Bird, W. 1929. Studies in Malaria with Special Reference to Treatment, Part XI. The Cinchona Alkaloids in the Treatment of Benign Tertian Malaria. Indian J. med. Res., 16: 725

Sinton, J. A. and Ghosh, B. N. 1934a. Studies of Malarial Pigment (Haemozoin). Part I. Investigation of the Action of Solvents on Haemozoin and the Spectroseopical Appearances Observed in the Solutions. Rec. Malar. Surv. India, 4: 15.

- 1934b. Studies of Malarial Pigment (Haemozoin). Part III. Further Researches into the Action of Solvents and the Results of 
Observations on the Action of Oxidising and Reducing Agents, on Optical Properties, and on Crystallization. Rec. Malar. Surv. India, 4: 205.

Sinton, J. A., HarbHagwan and Singh, J. 1931. The Numerical Prevalence of Parasites in Relation to Fever in Chronic Benign Tertian Malaria. Indian J. med. Res., 18: 871.

Sinton, J. A. and Hughes, T. A. 1924. The Functional Capacity of the Liver in Malaria, with Special Reference to the Levulose Tolerance Test. Indian J. med. Res., 12: 409.

Sinton, J. A., Hutton, E. L. and Shute, P. G. 1939a. Some Successful Trials of Proseptasine as a True Causal Prophylactic Against Infection with Plasmodium falciparum. Ann. trop. Med. Parasit., 33: 37.

1939b. Studies of Infeetions with $P$. ovale. II. Acquired Resistance to ovale Infections. Trans. Roy. Soc. trop. Med. Hyg., 33: 47.

Sinton, J. A. and KeHAR, N. D. 1931. Changes in the Amount of Blood Sugar in Malaria. Rec. Malar. Surv. India, 2: 287.

Sinton, J. A., ORR, W. B. F. and AHMAD, B. 1928. Some Physico-chemical Changes in the Blood, Produced by the Malarial Paroxysm. Indian $J$. med. Res., $16: 341$.

Sinton, J. A. and WATS, R. C. 1935. The Efficacy of Various Insecticidal Sprays in the Destruetion of Adult Mosquitoes. Rec. Malar. Surv. India, 5: 275 .

SMALT, F. H. 1937. The Periodic Draining of Rice Fields to Control Malaria. Meded. Dienst. Volksgegondh. Med.-Ind., 26: 285.

Shillie, W. G. 1927. Studies of an Epidemic of Malaria at the Gantt Impounded Area, Covington County, Alabama. Amer. J. Hyg., 7: 40.

Smith, J. M. 1860. Report on the Medical Topography and Epidemics of the State of New York. Trans. Amer. med. Ass., 13: 188.

SмiтH, L. 1932. One Year's Experience with Pyrethrum Larvicide. Proc. N. J. Mosq. Ext. Ass., 19: 128.

Sмith, T. and KILBORNE, F. L. 1893. Investigations into the Nature, Causation, and Prevention of Southern Cattle Fever. U. S. Dept. Agri. Bur. Animal Ind. Rep. for the Years 1891 and 1892, p. 177.

Somogri, J. C. 1939. Agglutination of Plasmodium knowlesi by Serum of Chronically Infected Monkeys. Riv. Parassit., 3: 157.

Soteriades, D. 1917. Essais de Sérothérapie dans la Malaria. Grèce Méd., 19: 27.

Stage, H. H. and GJullin, C. M. 1935. Anophelines and Malaria in the Pacific Northwest. Northw. Sci., 9: 7 .

Stadber, L. A. 1939. Factors Influencing the Asexual Periodicity of Avian Malarias. $J$. Parasit., 25: 95.

Stearns, L. A., MacCreary, D. and NeWhouse, N. P. 1933. The Problem of Mosquito Control in Delaware. Bull. Del. Agric. Exp. Sta., 181.

Stephens, J. W. W. 1913. Studies in Blackwater Fever. Ann. trop. Med. Parasit., 7: 479.
1914. A New Malaria Parasite of Man. Proc. Roy. Soc., B., 87: 375 and Ann. trop. Med. Parasit., 8: 119.

1921. Malaria in a Venezuelan Oil

Field. Ann. trop. Med. Parasit., 15: 435.

-1922. A New Malaria Parasite of Man. Ann. trop. Med. Parasit., 16: 383.

- 1937. Blackwater Fever. University Press of Liverpool. Hodder and Stoughton, London.

Stephens, J. W. W. and Christophers, R. 1904. The Practical Study of Malaria and Other Blood Parasites. Univ. of Liverpool Press, Liverpool. - 1908. The Practical Study of Malaria and Other Blood Parasites. III Ed. Univ. of Liverpool Press, Liverpool.

Stephens, J. W. W. and Stotт, W. 1915. Studies in Blackwater Fever. III. The Relationship of Quinine to Blackwater Fever. Ann. trop. Med. Parasit., 9: 201.

STERNBERG, G. M. 1884. Malaria and Malarial Diseases. William Wood and Co., New York. 1886. The Malarial "Germ" of Laveran. Med. Rec., 29: 489

Stitt, E. R., Clough, P. W. and Clough, N. C. 1938. Practical Bacteriology, Hematology and Animal Parasitology. P. Blakiston's Son \& Co., Philadelphia.

StoRm, C. J. 1935. Anwendung von Adrenalin auf Intravenous Injection Atebrin im Affen. Klin. Wschr., 1: 756 .

1935. On the Use of Adrenalin in Intravenous Injection of Atebrin. Acta brev. Neerl. Physiol., 5: 60.

Stratman-Thomas, W. K. 1931. On the Supposed Antagonism Between Alfalfa and Malaria. Amer. J. Hyg., 14: 394.

- 1935. Studies on Benign Tertian Malaria. 8. Observations on Splenomegaly. Amer. J. Hyg., 21: 361.

- 1940. The Influence of Temperature on Plasmodium vivax. Amer. J. trop. Méd., 20: 703 . Stratman-Thomas, W. K. and BaKer, F. C. 1936. Anopheles barberi Coquillet, as a Vector of Plasmodium vivax Grassi and Feletti. Amer. J. Hyg., 24: 182.

Stratman-Thomas, W. K. and Dulaner, A. D. 1940. Complement Fixation in Human Malaria. II. Diagnostic Application. J. Immunol., 36: 257.

Sullivan, F. L., NeckermanN, E. F. and Cannon, P. R. 1934. The Localization and Fate of Bacteria in the Tissues. J. Immunol., 26: 49.

Sutter, V. A. 1939. Primer Informe de los Tra. bajos del Quinto Departamento (Malariologia). Bol. sanit. Salvador, 8: 225.

SWeET, W. C. 1938. Irrigation and Malaria. Proc. Nat. Inst. Sci. India, 4: 185.

Sweet, W. C. and RAo, B. A. 1934. Notes on Malaria in Mysore State. Part V. The Control of Anopheline Breeding in Bangalore City and Its Cost in Mysore State. Rec. Malar. Surv. India, 4: 95 . 
SWEETMaN, H. L. 1936. The Biological Control of Insects. Comstock Pub. Co., Inc., Ithaca, N. $Y$.

SWELlengrebel, N. H. 1933. Report on a Small Experimental Epidemic of Benign Tertian Malaria Started in September, 1931, and Followed up till January, 1933. Proc. Kon. Akad. Wetensch. Amsterdam, 36: 234.

- 1934. Seven Years Experience in Work Against Anopheles at Medemblik. Ned. Tijdschr. Geneesk., 78: 345.

- 1940. The Efficient Parasite. Proc. Third Internat. Congr. Microbiol, p. 119. Waverly Press, Baltimore.

SWellengrebel, N. H. and DE BUCK, A. 1938. Malaria in the Netherlands. Scheltema and Holkema, Ltd., Amsterdam.

SWELlengrebel, N. H., DE BUCK, A. and KraAN, H. 1937. Further Investigations on "Healthy", Human Carriers of $P$. vivax in North-Holland. Proc. Kon. Alcad. Wetensch. Amsterdam 40:368.

SYMEs, C. B. 1930. Anophelines in Kenya. Kenya E. Afr. med. J., $7: 2$.

-1937. Les Insects dans les Aéronefs. Bull. Office Int. Hyg. Publ., 29: 1150.

TAKATsUKI, A. 1917. An Essential Property of Petroleum for Mosquito Control. Kyato Igaku, Vassi Kioto, 14, No. 7. Also in Rev. appl. Ent. B, 6: 135 .

TALIAFERRo, L. G. 1928. Return to Normal of the Asexual Cycle in Bird Malaria After Retardation by Low Temperatures in vitro. J. prev. Med., 11: 525 .

TALIAFERRO, L. G. and TALIAFERRO, W. H. 1934. Alteration in the Time of Sporulation of Plasmodium brasilianum in Monkeys by Reversal of Light and Dark. Amer. J. Hyg., 20: 50.

TALIAPERRO, W. H. 1929. The Immunology of Parasitic Infections. The Century Co., New York.

- 1934. Some Cellular Bases for Immune Reactions in Parasitic Infections. J. Parasit., $20: 149$.

- 1941. The Immunology of the Parasitic Protozoa. In G. N. Calkins. Protozoa and Biological Research, Ch. 18. Columbia Univ. Press, New York.

Taltatrerro, W. H. and Cannon, P. R. 1936. The Cellular Reactions During Primary Infections and Superinfections of Plasmodium brasilianum in Panamanian Monkeys. J. Infect. Dis., 59 : 72.

TALIAFERRO, W. H. and Kü̈vER, C. 1940. The Hematology of Malaria (Plasmodium brasilianum) in Panamanian Monkeys. I. Numerical Changes in Leucocytes. II. Morphology of Leucocytes and Origin of Monocytes and Macrophages. J. Infect. Dis., 67: 121, 162.

Taliaflerio, W. H. and Mulligan, H. W. 1937. The Histopathology of Malaria with Special Reference to the Function and Origin of the Macrophages in Defence. Indian Med. Res. Mem., 29: 1-138.

TAliafirro, W. H. and TALtaFerRo, L. G. 1928. 2nd Report. A Precipitin Test in Malaria. $J$. prev. Med., 2: 147.

1940. Active and Passive Immunity in Chickens Against Plasmodium lophurae. J. Infect. Dis., 66: 153.

TERry, R. J. 1931. Dr. John Sappington, Pioneer in the Use of Quinine in the Mississippi Valley, p. 165. Proc. of the Celebration of the Three Hundr. Anniv. First Recognized Use of Cinchona. Botanical Garden, St. Louis, Mo.

THAYER, W. S. 1897. Lectures on the Malarial Fevers. D. Appleton Co., New York.

1900. On Recent Advances in Our Knowledge Concerning the Etiology of Malarial Fever. Philad. med. J., 5: 1046.

THAYER, W. S. and Hewetson, J. 1895. The Malarial Fevers of Baltimore. Johns Hopk. Hosp. Rep., 5: Nos. 1-5, pp. 1-218.

Theobald, F. V. and GrabHam, M. 1905. The Mosquitoes or Culicidae of Jamaica. Inst. Jamaica, Kingston.

Thibault, J. K., JR. 1918. Vegetable Powder as a Larvicide in the Fight Against Mosquitoes. A Preliminary Note. J. Amer. med. Ass., 70: 1215.

Thomson, D. 1914. The Origin and Development of Gametes (Crescents) in Malignant Tertian Malaria: Some Observations on Exflagellation, etc. Ann. trop. Med. Parasit., 8: 85.

Thomson, J. G. 1924. Researches on Blackwater Fever in Southern Rhoderia During the Years 1922 and 1923. Proc. Roy. Soc. Med., 17: 47.

- 1932. Some Observations on the Nuclear Structure of the Malignant Tertian Parasite (Plasmodium falciparum). J. trop. Med. Hyg., 35: 1.

1933. Immunity in Malaria. Trans. Roy. Soc. trop. Med. Hyg., 26: 483.

- 1934. Malaria in Nyasaland. Proc. Roy. Soc. Med., 28: 391.

Thomson, J. G. and Robertson, A. 1929. Protozoology. William Wood and Company, New York.

_. 1935. The Strueture and Development of Plasmodium falciparum Gametocytes in the Internal Organs and Peripheral Circulation. Trans. Roy. Soc. trop. Med. Hyg., $29: 31$.

Thomson, J. G. and Thomson, D. 1913. The Growth and Sporulation of the Benign and Malignant Tertian Malarial Parasites in the Culture Tube and in the Human Host. Proc. Roy Soc., B., $87: 77$.

THoMPsoN, J. D. and Woodcock, H. M. 1922. The Parasites of Malaria. In Byam and Archibald, The Practice of Medicine in the Tropies, Vol. II. Oxford Med. Publ., London.

Thompson, R. C. M. 1940. Studies of the Behavior of Anopheles minimus. J. Malar. Inst. India, 3 : 265.

Thornton, E. N. 1934. Malaria. Rep. Dept. Pub. Health South Africa, 1933-34, p. 45. _. 1936. Malaria. Rep. Dept. Pub. Health South Africa, 1935-36, p. 31. 
Toumanoff, C. and Hu, S. M. K. 1935. Sur le Comportment Trophique d'Anopheles hyrcanus var. sinensis dans la Region de Shanghai. Bull. Soc. Path. exot., 28: 832.

Tournier. 1937. Les Moustiques à la Guyane. Ann. Med. Pharm., 35: 227.

TownsEnd, C. H. T. 1934. Mosquitoes of the Rio Tapajos. Rev. Ent., S. Paulo, 4: 486.

TRÉfouËL, J., TrÊFoü̈L, MME. J., NITT, F. and Bovkt, D. 1935. Activité du p-aminophenylsulfamide sur les Infections Streptococeiques Expérimentales de la Souris et du Lapin. C. R. Soc. Biol., 120: 756 .

Treillard, M. 1934. Destruction Sainsonnière Domestique des Anophèles Adultes (H. minima) Pour la Prophylaxie Antipaludique en Indochine Méridionale. Bull. Soc. Path. exot., 27 : 937.

TropP, C. and WEISs, W. 1933. Untersuchungen über die Ausseheidung von Atebrin dureh Harn und Fazes. Arch. exp. Path., 170: 339.

TskimanaURI, G. 1931. Zur Pharmakologie des Plasmochins. Arch. Schiffs. TropenHyg., 35: 89.

Tulloch, G. S. 1937. The Brackish Water Mosquitoes of Puerto Rico. J. Agric. Univ. Puerto Rico, 21: 581.

Twinn, C. R. 1931. Notes on the Biology of Mosquitoes of Eastern Canada. Proc. N. J. Mosq. Ext. Ass., 10.

UNGo-MUGDAN, A. 1938. La reazione nucleare di Feulgen negli stadi exoeritrociti del $P$. gallinaceum, Brumpt (1935). Riv. Parassit., 2: 323.

Urbino, C. M. 1936. The Density of Anopheles minimus var. flavirostris Ludl. by Trapping with Human Baits and Its Relation with the Factors that Influenced its Variations. Bull. Bur. Hlth. Philipp., 16: 471.

1938. Malaria Control and Agricultural Settlements. Mon. Bull. Bur. Hlth. Philipp. I., $18: 301$.

VAN DER WIELEN, Y. 1937. Prontosil in Quartan Malaria. Ned. Tijdschr. Geneesk., 81: 2905.

VAN DINE, D. L. 1922. Impounding Water in a Bayou to Control Breeding of Malaria Mosquitoes. U. S. Dept. Agri. Bull., 1098: 22.

VANnote, R. L. 1931. Practical Application of Pyrethrum Mosquito Larvicide. Proc. $N$. J. Mosq. Ext. Ass., 18: 111.

VARGAS, L. 1938. Observaciones Sobre la Preferencia Alimenticia Sanguinea de la Pseudopunctipennis en Temisco, Morelos. Ann. Inst. Biol. Mex., 9: 201.

- 1939a. Anofelismo sin Malaria en Mexico. Med. Rev. Mex., 19: 334.

- 1939b. Datos Acerca del A. pseudopuncti pennis y de un Anopheles Nueva de California. Med. Rev. Mex., 19: 356. (Quoted in Rev. Appl. Ent., B., 28: 46.)

- 1940a. Clave para Identificar las Larvas de Anopheles Mexicanos. Ciencia, 1: 66.

-1940b. Nuevas Observaciones Sobre Anopheles Mexicanos. Ciencia, 1: 256.

- 1940e. Personal communication.

VERTEUIL, E. DE. 1933. Malaria Survey for 1932.
Pap. Legis. Coun. Trin. Tab. No. 32 of 1933 ; Rev. appl. Ent. B. 22 : 29.

Vertedil, E. DE. and Spence, T. 1937. Malaria in Trinidad, Low Tide Level Culvert System in Coastal Drainage. Trans. Roy. Soc. trop. Med. Hyg., $30: 449$.

Von EzDoRf, R. H. 1914. Prevention of Malaria. Publ. Hlth. Rep., Wash., 29: 503.

- 1916. Demonstrations of Malaria Control. Publ. Hlth. Rep., Wash., 31: 614.

WAFER, LIONEL. 1699. A New Voyage and Deseription of the "Isthmus of America." Ed. G. P. Winship, 1903. Burrows Bros. Company, Cleveland.

WALDERT, ALBERT. 1901. Cultivation of the Estivo-Autumnal Malarial Parasite in the Mosquito, Anopheles quadrimaculatus. J. Amer. med. Ass., 36: 559.

W ARREN, A. J. and CogGeshall, L. T. 1937. Infectivity of Blood and Organs in Canaries after Inoculation with Sporozoites. Amer. J. Hyg., 26: 1.

WASHBURN, B. E. 1933. An Epidemic of Malaria at Falmouth, Jamaica, B. W. I. Amer. J. Hyg., $17: 656$.

Watson, M. 1903. A Note on the Parasites of a Case of Malignant Malaria with Discussion on the Development of the Crescent. J. trop. Med. Hyg., 6: 221.

. 1915. Rural Sanitation in the Tropies. John Murray, London.

WATson, R. B. and Bishop, E. L. 1940. The Control of Anopheles quadrimaculatus in the Tennessee Valley. Proc. N. J. Mosq. Ext. Ass., 27: 145.

Watson, R. B., Kiker, C. C. and JoHnson, H. A. 1938. The Role of Airplane Dusting in the Control of Anopheles Breeding Associated with Impounded Waters. Publ. Hlth. Rep., Wash., 53: 251.

Watson, R. B. and Maher, Helen C. 1940. An Evaluation of Mosquito-Proofing for Malaria Control Based on One Year's Observations. Amer. J. Hyg. (in press).

Watson, R. B. and Rice, Margaret. 1941. Further Observations on Mosquito-Proofing for Malaria Control, submitted for publication. Amer. J. Hyg.

WATSON, R. B. and SpaIN, E. L., JR. 1937. Studies on Malaria in the Tennessee Valley. The Influence of Physiography on the Occurrence of Breeding Places of Anopheles quadrimaculatus in Northern Alabama. Amer. J. trop. Med., 17 : 289.

WebBER, H. J. 1920. Malaria-mosquito Survey. Ann. Rep. 1919-1920 Univ. California Agric. Expt. Sta., pp. 60-61.

WeIsS, S. and HATCHER, R. A. 1927. Studies on Quinine. J. Pharmacol., 30: 327.

WeLCH, E. V. 1939. Insects Found in Aireraft at Miami, Florida, in 1938. Publ. Hlth. Rep., Wash., 54: 561 .

WeLCH, W. H. 1897. Malaria: Definition, Syno- 
nyms, History, and Parasitology. Loomis and Thompson, Syst. Practice Med., V. I, pp. 17-76. Lea Bros. and Co., New York.

WeLls, C. W. 1930. The Identification of the Anopheline Mosquitoes of Porto Rico. Amer. J. trop. Med., 10 : 243.

WENYoN, C. M. 1921. The Incidence and Aetiology of Malaria in Macedonia. J. Roy. Army med. Cps., $37: 81$.

1926a. Protozoology. Two vols. William Wood and Co., New York.

1926b. Protozoology, vol. 2, p. 924. William Wood and Co., New York.

—_. 1941. Trop. Dis. Bull., 38: 30.

WhIPPLE, G. H. 1927. The Pathology of Blackwater Fever. Amer. J. trop. Med., $7: 1$.

WhITMORE, E. R., RoBERTS, C. M. and JANTZEN, W. 1929. The Action of Plasmoquin in Rendering Subtertian Gametocytes Non-infectious for Mosquitoes. 18th A'nn. Rept. United Fruit Co. Med. Dept., p. 37.

WILLE, J. 1933. Estudio Entomologico de la Epidemia del Paludismo en los Valles de la Convencion y Lares. (Dept. del Cuzco) Bol. Direc. Agric. Ganad., Peru, 3: 303.

Wrulams, C. L. 1940. Disinsectization of Aircraft. Publ. Hlth. Rep., Wash., 55: 1005.

Wruliams, C. L. and Dreessen, W. C. 1935. A Non-inflammable Pyrethrum Spray for Use in Airplanes. Publ. Hlth. Rep., Wash., 50: 1401.

WILLIAMs-Ehuis-Clovar. 1920. Cottage Building in Cob, Pisé, Chalk and Clay. Chas. Scribners \& Son, New York.

Williams, L. L., JR. 1935. Civil Works Administration Emergency Relief Administration Malaria Control Program in the South. Amer. J. Publ. Hlth., 25: 11.

- 1937a. Mosquitoes and Malaria. $J$. econ. Entomol., 30 : 20.

-1937b. A Plan for State-Wide Control of Malaria. Publ. Hlth. Seri. Document, B-2534, Feb., 1937.

- 1938. Economic Importance of Malaria Control. Proc. N. J. Mosq. Ext. Ass., 25: 148.

1. 1940. Personal Communication.

WILliams, L. L., JR. and CooK, S. S. 1927. Paris Green Applied by Airplane in the Control of Anopheles Production. Publ. Hlth. Rep., Wash., 42: 459 .

- 1928. Airplanes and Paris Green in Control of Anopheles Production. Sth. Med. J., 21: 754 .

Wiuliamson, K. B. 1935. The Control of Rural Malaria by Natural Methods. League of $\mathrm{Na}$ tions, Eastern Bureau, Singapore, pp. 1-26. Also in Malay. Agri-Hort. Ass. Mag., 1933-34, 3 : 145; 3: $201 ; 4: 224 ; 4: 281$. Also 1936 League of Nations Eastern Bureau, Singapore, pp. 1-89.

Wriliamson, K. B. and ScharFF, J. W. 1936. Anti-larval Sluicing. Malaya med. J., 11: 123.
Wilson, D. B. 1936. Rural Hyper-Endemic Malaria in Tanganyika Territory. Trans. Roy. Soc. trop. Med. Hyg., 29: 583.

- 1939. Implications of Malarial Endemicity in East Africa. Trans. Roy. Soc. trop. Med. Hyg., 32 : 435.

Wilson, D. B. and Wirson, M. E. 1937. The Manifestations and Measurement of Immunity to Malaria in Different Races. Trans. Roy. Soc. trop. Med. Hyg., 30: 431.

Woldert, A. 1901. Cultivation of the EstivoAutumnal Parasite in the Mosquito Anopheles quadrimaculatus. J. Amer. med. Ass., 36: 559.

Wolfson, F. 1940. Successful Cultivation of Avian Plasmodia in Duck Embryos. Amer. $J$. Hyg., 32: 60.

Works Progress Administration, see under " Report of.'"

YoRKe, W. and MACFIE, J. W. S. 1924a. Observations on Malaria Made During Treatment of General Paralysis. Trans. Roy. Soc. trop. Med. Hyg., 18: 13.

1924b. Certain Observations on Malaria during Treatment of General Paralysis. Lancet, 1: 1017 (May 17).

YoRke, W., MURGATROYD, F. and OWEN, D. U. 1930. Observations on Five Cases of Blackwater Fever. Trans. Roy. Soc. trop. Med. Hyg., 23: 335.

YoRKE, W. and NAUSs, R. W. 1911. The Mechanism of the Production of the Suppression in Blackwater Fever. Ann. trop. Med. Parasit., 5: 287.

Young, M. D. 1938. A Rapid Method of Drying Thick Blood Films. Publ. Hlth. Rep., Wash., 53 : 1256.

Young, M. D. and Coatney, G. R. 1940. Personal communication.

Youna, M. D., Coatney, G. R. and StubBs, T. H. 1940. Studies on Induced Quartan Malaria in Negro Paretics. II. The Effect of Modifying the External Conditions. Amer. J. Hyg., C, 32: 63.

Young, M. D., Stubbs, T. H. and Coatnet, G. R. 1940. Studies on Induced Quartan Malaria in Negro Paretics. I. Periodic Phenomena of the Asexual Cycle. Amer. J. Hyg., C, 31: 51.

ZIEGLER, E. 1891. Utber die Beteiligung der Seukocyten an der Geweosneubildung. Verhand. Intern. Med. Cong. Berlin, 2: 1.

ZIEMANN, H. 1915. Uber eingenartige Malariaparasitenformen. Zbl. Bakt. Orig., 76: 384.

1924. Malaria und Schwarzwasserfieber. Handbuch der tropen-krankheiten, V. III, P. 592. J. A. Barth, Leipzig.

- 1938. Gibt es Verschiedene Rassen, Varietaten oder Species bei Plasmodium falciparum. Acta Con. Tertii Trop. Mal. Morb., 2: 515.

ZWEMER, R. L., Sims, E. A. H. and Coggeshal, L. T. 1940. The Plasma Potassium Level During Malaria Infection in Monkeys and Man. Amer. J. trop. Med., 20: 687. 
. 



\section{.}





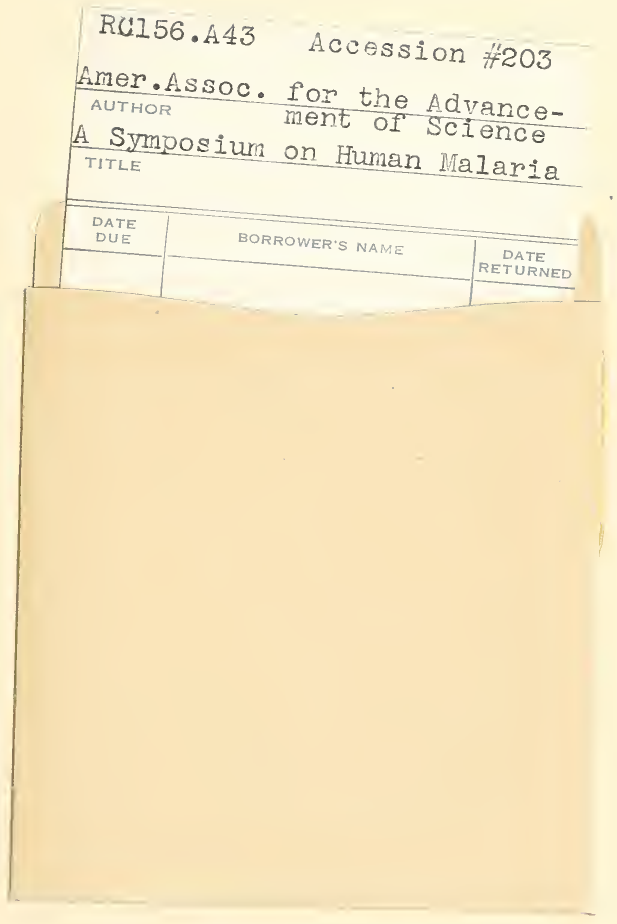


